NASA Technical Memorandum 105210

\title{
Experimental Investigation of Turbulent Flow Through a Circular-to-Rectangular Transition Duct
}

David O. Davis

Lewis Research Center

Cleveland, Ohio

$=\overline{(N A S A-T A-105210) \text { EXPERTMENTAL INVESTIOATION N91-31100 }}$ JF TURGULENT FLOW THROUGH A

CIRCULAR-TO-RECTANUULAR TRANSITIUN DUCT

Ph.n. rhesis - Washington Univ. (HASA) Unclas 217

CSCL OIA G3/O2 0040346

September 1991 


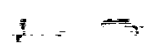




\title{
EXPERIMENTAL INVESTIGATION OF TURBULENT FLOW THROUGH A
}

\section{CIRCULAR-TO-RECTANGULAR TRANSITION DUCT}

\author{
David O. Davis \\ National Aeronautics and Space Administration \\ Lewis Research Center \\ Cleveland, Ohio 44135
}

\begin{abstract}
Steady, incompressible, turbulent, swirl-free flow through a circular-torectangular transition duct has been studied experimentally. The transition duct has an inlet diameter of $20.43 \mathrm{~cm}$, a length-to-diameter ratio of 1.5, and an exit plane aspect ratio of three. The cross-sectional area remains the same at the exit as at the inlet, but varies through the transition section to a maximum value approximately $15 \%$ above the inlet value. The cross-sectional geometry everywhere along the duct is defined by the equation of a superellipse. Mean and turbulence data were accumulated utilizing pressure and hot-wire instrumentation at five stations along the test section. Data are presented for operating bulk Reynolds numbers of 88,000 and 390,000 . Measured quantities include total and static pressure, the three components of the mean velocity vector and the six components of the Reynolds stress tensor. The results show that the curvature of the transition duct walls induces a relatively strong pressure-driven crossflow that produce a contra-rotating vortex pair along the diverging side-walls of the duct. The vortex pair significantly distorts both the mean flow and turbulence field. Local equilibrium conditions at the duct exit, if they exist at all, are confined to a very small region near the wall indicating that care must be taken when using wall functions to predict the flowfield. Analysis of the Reynolds stress tensor at the exit plane shows that streamline curvature and lateral divergence effects distort the non-dimensional turbulence structure parameters.

In addition to the transition duct measurements, a hot-wire technique which relies on the sequential use of single rotatable normal and slant-wire probes has been proposed. The technique is applicable for measurement of the total mean velocity vector and the complete Reynolds stress tensor when the primary flow is arbitrarily skewed relative to a plane which lies normal to the probe axis of rotation. Measurement of the mean flow has been verified in fully-developed pipe flow under simulated pitch angles up to $\pm 20^{\circ}$. Measurements of the Reynolds stress tensor has been verified for the unskewed condition. Under skewed conditions, systematic deviations of the stress tensor were observed which are attributed to the geometry of the slant-wire probe. A new slant-wire probe is proposed which is designed to reduce, if not eliminate, the observed deficiencies.
\end{abstract}





\section{TABLE OF CONTENTS}

Page

LIST OF FIGURES $\ldots \ldots \ldots \ldots \ldots \ldots \ldots \ldots \ldots \ldots \ldots \ldots \ldots \ldots \ldots \ldots, \mathbf{v}$

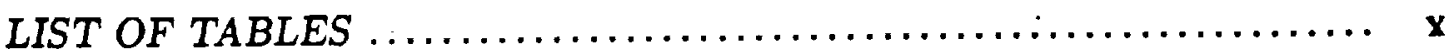

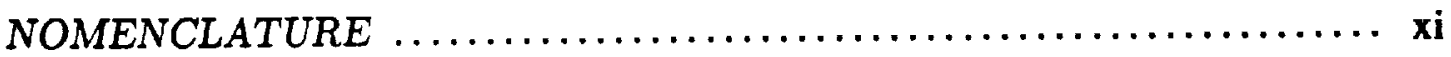

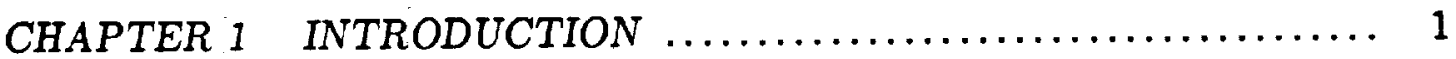

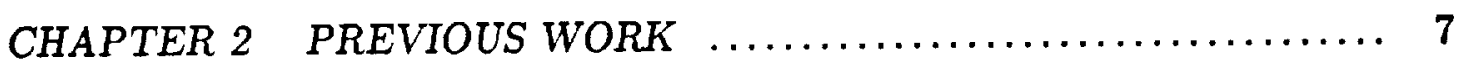

CHAPTER 3 EXPERIMENTAL PROGRAM .................. 15

3.1 Introduction $\ldots \ldots \ldots \ldots \ldots \ldots \ldots \ldots \ldots \ldots \ldots \ldots \ldots \ldots \ldots \ldots \ldots, 15$

$3.2 \quad$ Flow Facility $\ldots \ldots \ldots \ldots \ldots \ldots \ldots \ldots \ldots \ldots \ldots \ldots \ldots, 15$

3.3 Test Section $\ldots \ldots \ldots \ldots \ldots \ldots \ldots \ldots \ldots \ldots \ldots \ldots \ldots \ldots \ldots \ldots \ldots \ldots, 16$

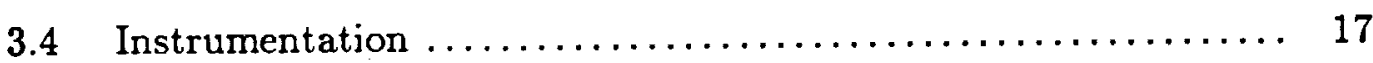

3.5 Data Reduction ................................ 18

CHAPTER 4 INLET CONDITIONS (Developing Pipe Flow) ........ 31

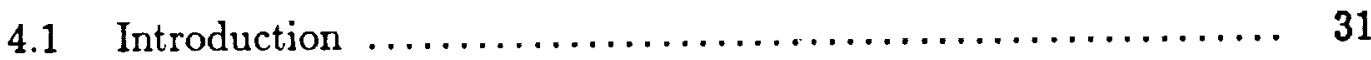

4.2 Preliminary Results $\ldots \ldots \ldots \ldots \ldots \ldots \ldots \ldots \ldots \ldots \ldots \ldots, \quad 32$

4.3 Range of Operating Conditions ...................... 32

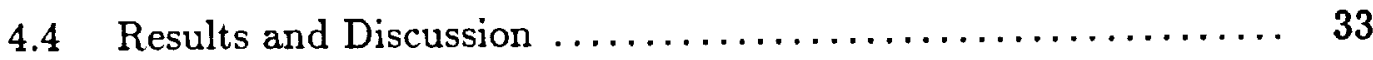

CHAPTER $5 \quad$ RESULTS AND DISCUSSION .................. 47

5.1 Introduction $\ldots \ldots \ldots \ldots \ldots \ldots \ldots \ldots \ldots \ldots \ldots \ldots \ldots \ldots \ldots \ldots \ldots \ldots \ldots, 47$

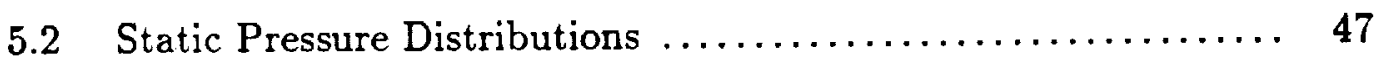

5.3 Effect of Varying Reynolds Number $\ldots \ldots \ldots \ldots \ldots \ldots \ldots \ldots, 48$

5.4 Mean Flow Results ................................ 49

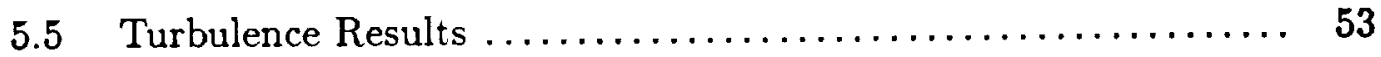

5.6 Turbulence Modelling Considerations $\ldots \ldots \ldots \ldots \ldots \ldots \ldots \ldots, 59$

5.7 Wall Function Beharior $\ldots \ldots \ldots \ldots \ldots \ldots \ldots \ldots \ldots \ldots \ldots, 63$

CHAPTER 6 CONCLUSIONS AND RECOMMENDATIONS ...... 125

BAEE í INTENTIONALLY BLANK iii 
REFERENCES

APPENDIX A Transition Duct Geometry ..................... 135

APPENDIX B Hot-Wire Data Acquisition System ................ 139

APPENDIX $C \quad$ Hot-Wire Techniques $\ldots \ldots \ldots \ldots \ldots \ldots \ldots \ldots \ldots \ldots, 145$

APPENDIX D Supplementary on Mean Flow Equation Development . 171

APPENDIX E Supplementary on Turbulence Equation Development . 179

APPENDIX $F \quad$ Verification of the Hot-Wire Technique $\ldots \ldots \ldots \ldots \ldots, 187$

APPENDIX $G \quad$ Uncertainty Estimates ........................ 209 


\section{LIST OF FIGURES}

Page

1.1 Schematic of typical propulsion system $\ldots \ldots \ldots \ldots \ldots \ldots \ldots \ldots \ldots$

1.2 Circular-to-rectangular transition duct $\ldots \ldots \ldots \ldots \ldots \ldots \ldots \ldots \ldots \ldots$

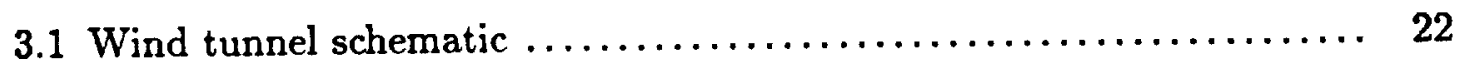

3.2 Transition duct test section schematic $\ldots \ldots \ldots \ldots \ldots \ldots \ldots \ldots \ldots \ldots \ldots \ldots \ldots \ldots$

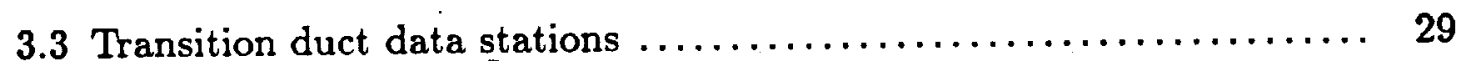

4.1 Tripped and non-tripped boundary layer profiles at

Station $1, R e_{b}=234,000$ and $403,000 \ldots \ldots \ldots \ldots \ldots \ldots \ldots \ldots \ldots \ldots \ldots \ldots \ldots$

4.2 Friction velocity vs. centerline Reynolds number at Station $1 \ldots \ldots . .39$

4.3 Tripped boundary layer profiles at Station 1 ,

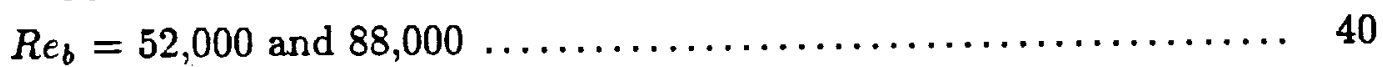

4.4 Boundary layer profiles at Station $1, R e_{b}=88,000 \ldots \ldots \ldots \ldots \ldots \ldots \quad 41$

4.5 Boundary layer profiles at Station $1, R e_{b}=390,000 \ldots \ldots \ldots \ldots \ldots .42$

4.6 Reynolds stress distributions at Station $1 \ldots \ldots \ldots \ldots \ldots \ldots \ldots \ldots \ldots$

4.7 Turbulence kinetic energy distributions at Station $1 \ldots \ldots \ldots \ldots \ldots .44$

4.8 Production of turbulence kinetic energy

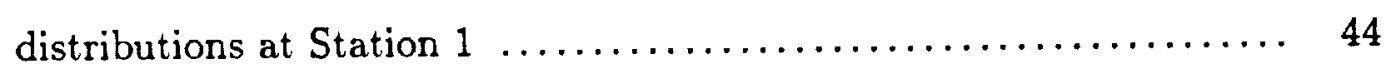

4.9 Shear stress correlation $\left(R_{u v}=\overline{u v} / u^{\prime} v^{\prime}\right)$ at Station $1 \ldots \ldots \ldots \ldots \ldots \quad 45$

4.10 Shear-energy ratio parameter $(a 1=\overline{u v} / 2 k)$ at Station $1 \ldots \ldots \ldots \ldots$

5.1 Peripheral wall pressure coefficient distributions $\ldots \ldots \ldots \ldots \ldots \ldots .66$

5.2 Total pressure contours at Station $5 \ldots \ldots \ldots \ldots \ldots \ldots \ldots \ldots \ldots \ldots$

5.3 Axial mean velocity contours at Station $5 \ldots \ldots \ldots \ldots \ldots \ldots \ldots$

5.4 Transverse mean velocity vectors at Station $5 \ldots \ldots \ldots \ldots \ldots \ldots$ 
5.5 Total pressure contours $\ldots \ldots \ldots \ldots \ldots \ldots \ldots \ldots \ldots \ldots \ldots \ldots \ldots \ldots \ldots \ldots \ldots \ldots \ldots, \quad 70$

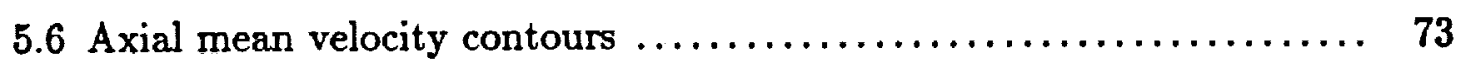

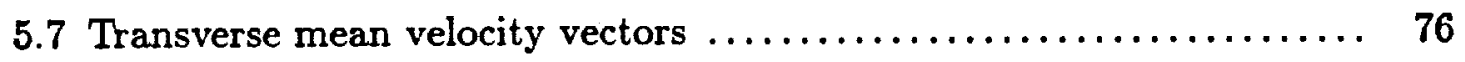

5.8 Static pressure contours at Station $5 \ldots \ldots \ldots \ldots \ldots \ldots \ldots \ldots \ldots . \ldots \ldots$

5.9 Axial distribution of strength of divergence parameter $\ldots \ldots \ldots \ldots \ldots, 80$

5.10 Surface oil flow visualization by Reichert $[4] \ldots \ldots \ldots \ldots \ldots \ldots \ldots, 80$

5.11 Total pressure profiles along the semi-major and semi-minor axes $\ldots \ldots \ldots \ldots \ldots \ldots \ldots \ldots \ldots \ldots \ldots \ldots, 81$

5.12 Axial mean velocity profiles along the semi-major and semi-minor axes $\ldots \ldots \ldots \ldots \ldots \ldots \ldots \ldots \ldots \ldots \ldots \ldots \ldots, 82$

5.13 Axial mean vorticity contours $\ldots \ldots \ldots \ldots \ldots \ldots \ldots \ldots \ldots \ldots \ldots . \ldots \ldots$

5.14 Axial turbulence intensity contours $\ldots \ldots \ldots \ldots \ldots \ldots \ldots \ldots \ldots \ldots$

$5.15 \overline{u^{2}} / U_{b}^{2} \times 10^{4}$ Reynolds stress contours $\ldots \ldots \ldots \ldots \ldots \ldots \ldots \ldots \ldots . . \ldots 7$

$5.16 \overline{v^{2}} / U_{b}^{2} \times 10^{4}$ Reynolds stress contours $\ldots \ldots \ldots \ldots \ldots \ldots \ldots \ldots \ldots .88$

$5.17 \overline{w^{2}} / U_{b}^{2} \times 10^{4}$ Reynolds stress contours $\ldots \ldots \ldots \ldots \ldots \ldots \ldots \ldots \ldots . . \ldots 9$

$5.18 \overline{u v} / U_{b}^{2} \times 10^{4}$ Reynolds stress contours $\ldots \ldots \ldots \ldots \ldots \ldots \ldots \ldots \ldots \ldots .90$

$5.19 \overline{u w} / U_{b}^{2} \times 10^{4}$ Reynolds stress contours $\ldots \ldots \ldots \ldots \ldots \ldots \ldots \ldots \ldots . . \ldots 1$

$5.20 \overline{v w} / U_{b}^{2} \times 10^{4}$ Reynolds stress contours $\ldots \ldots \ldots \ldots \ldots \ldots \ldots \ldots \ldots . . \ldots 2$

5.21 Axial and transverse normal stress anisotropy $\left(\overline{u^{2}}-\overline{v^{2}}\right) \ldots \ldots \ldots \ldots 93$

5.22 Axial and transverse normal stress anisotropy $\left(\overline{u^{2}}-\overline{w^{2}}\right) \ldots \ldots \ldots . .64$

5.23 Transverse normal stress anisotropy $\left(\overline{v^{2}}-\overline{w^{2}}\right) \ldots \ldots \ldots \ldots \ldots \ldots . \ldots 5$

5.24 Turbulence kinetic energy contours $\ldots \ldots \ldots \ldots \ldots \ldots \ldots \ldots \ldots \ldots, 96$

5.25 Production of turbulence kinetic energy contours $\ldots \ldots \ldots \ldots \ldots \ldots .97$

5.26 Reynolds normal stress profiles at Station $1 \ldots \ldots \ldots \ldots \ldots \ldots \ldots . \ldots 8$ 
5.27 Normal stress profiles along semi-major axis

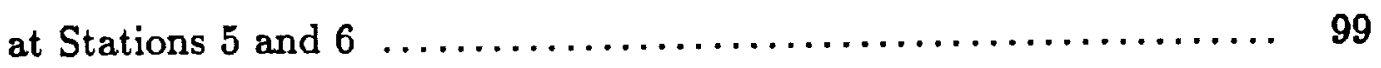

5.28 Shear stress and kinetic energy profiles along semi-major axis $\ldots \ldots .100$

5.29 Normal stress profiles along semi-minor axis

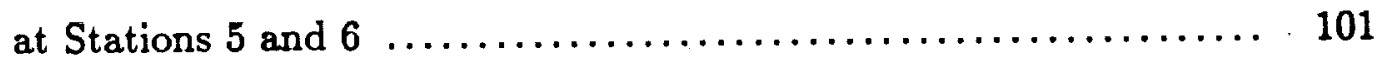

5.30 Shear stress and kinetic energy profiles along

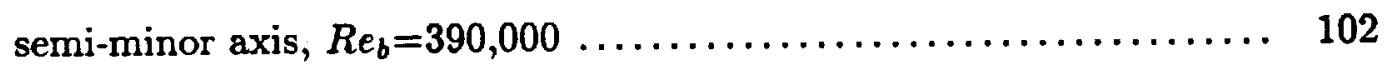

5.31 Shear stress and kinetic energy profiles along

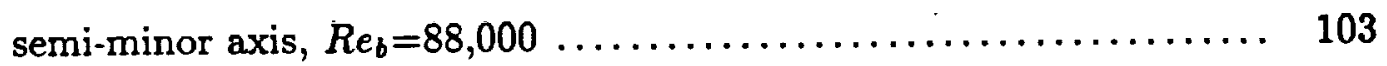

5.32 Primary rates-of-strain at Station $5 \ldots \ldots \ldots \ldots \ldots \ldots \ldots \ldots \ldots \ldots . \ldots \ldots$

5.33 Component eddy viscosity distributions at Station $5 \ldots \ldots \ldots \ldots .105$

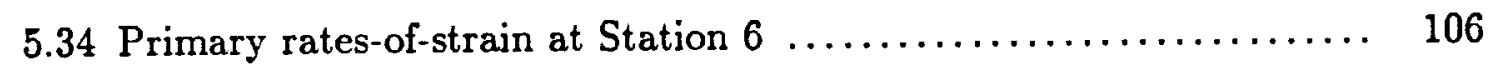

5.35 Component eddy viscosity distributions at Station $6 \ldots \ldots \ldots \ldots, 107$

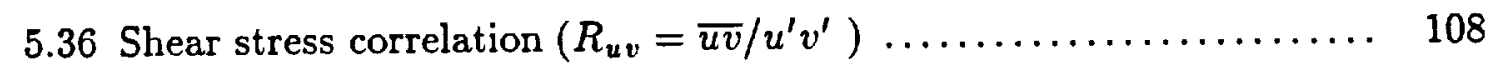

5.37 Shear stress correlation $\left(R_{u w}=\overline{u w} / u^{\prime} w^{\prime}\right) \ldots \ldots \ldots \ldots \ldots \ldots \ldots \ldots . \ldots \ldots$

5.38 Shear stress correlation $\left(R_{v w}=\overline{v w} / v^{\prime} w^{\prime}\right) \ldots \ldots \ldots \ldots \ldots \ldots \ldots \ldots$

5.39 Shear-energy structure parameter $\left(a 1_{y}=\sqrt{\overline{u v}^{2}+\overline{v w}^{2}} / 2 k\right) \ldots \ldots \ldots 111$

5.40 Shear-energy structure parameter $\left(a 1_{z}=\sqrt{{\overline{u w^{2}}}^{2}+\overline{v w}^{2}} / 2 k\right) \ldots \ldots \ldots 112$

5.41 Physical realizability limits of turbulence invariant map ......... 113

5.42 Anisotropic stress tensor invariants at Station $1 \ldots \ldots \ldots \ldots \ldots \ldots . \ldots \ldots$

5.43 Anisotropic stress tensor invariants at Station $5 \ldots \ldots \ldots \ldots \ldots \ldots .115$

5.44 Anisotropic stress tensor invariants at Station $6 \ldots \ldots \ldots \ldots \ldots \ldots, 116$

5.45 Reference coordinates for wall function behavior $\ldots \ldots \ldots \ldots \ldots \ldots, 117$

5.46 Peripheral skin friction coefficient distributions $\ldots \ldots \ldots \ldots \ldots \ldots \ldots, 117$

$5.47 \mathrm{Law}$-of-the-wall velocity profiles $\ldots \ldots \ldots \ldots \ldots \ldots \ldots \ldots \ldots \ldots \ldots \ldots \ldots \ldots \ldots \ldots$ 
$5.48 \sqrt{\left(\overline{u v}^{2}+\overline{u w}^{2}\right)} / U_{\tau}^{2}$ distributions parallel to semi-minor axis $\ldots \ldots \ldots \quad 119$

$5.49 k / U_{\tau}^{2}$ distributions parallel to semi-minor axis $\ldots \ldots \ldots \ldots \ldots \ldots \ldots .120$

$5.50 P y_{w} / U_{\tau}^{3}$ distributions parallel to semi-minor axis $\ldots \ldots \ldots \ldots \ldots \ldots \ldots 121$

$5.51 \sqrt{\left(\overline{u v}^{2}+\overline{u w}^{2}\right)} / U_{\tau}^{2}$ distributions along semi-major axis $\ldots \ldots \ldots \ldots \ldots \quad 122$

$5.52 k / U_{\tau}^{2}$ distributions along semi-major axis $\ldots \ldots \ldots \ldots \ldots \ldots \ldots \ldots \ldots, 122$

$5.53 P_{y_{w}} / U_{\tau}^{3}$ distributions along semi-major axis $\ldots \ldots \ldots \ldots \ldots \ldots \ldots \ldots$

B.1 Block schematic of hot-wire data acquistion system .......... 144

C.1 Hot-wire probe configurations for Method $A \ldots \ldots \ldots \ldots \ldots \ldots \ldots$

C.2 Hot-wire positions for mean flow determination $\ldots \ldots \ldots \ldots \ldots \ldots \ldots$

C.3 Reference coordinates for Reynolds stress determination ........ 166

C.4 Behavior of Reynolds stress coefficients, normal-wire, Method A ... 167

C.5 Behavior of Reynolds stress coefficients, slant-wire, Method A . . .. 168

C.6 Behavior of Reynolds stress coefficients, normal-wire, Method B .... 169

C.7 Behavior of Reynolds stress coefficients, slant-wire, Method B ..... 170

D.1 Effective cooling velocity for mean flow calculations $\ldots \ldots \ldots \ldots \ldots .176$

D.2 Tangential cooling coefficient behavior $\ldots \ldots \ldots \ldots \ldots \ldots \ldots \ldots \ldots$

E.1 Effective cooling velocity for turbulence calculations $\ldots \ldots \ldots \ldots \ldots \ldots$

F.1 Schematic of Method A hot-wire verification setup ........... 194

F.2 Hot-wire orientation for Method A .................... 194

F.3 Typical probe construction for Method A ................. 195

F.4 Detail of probe prong geometry for Method A ............. 195

F.5 Offset prong slant-wire calibration $\ldots \ldots \ldots \ldots \ldots \ldots \ldots \ldots \ldots \ldots \ldots$

F.6 Conjectured probe prong influence for offset prong slant-wire . . . . 197

F.7 Fully-developed mean flow for unskewed conditions . . . . . . . . 198 
F.8 Fully-developed pipe flow Reynolds stress profiles for unskewed conditions $\ldots \ldots \ldots \ldots \ldots \ldots \ldots \ldots \ldots \ldots \ldots \ldots \ldots \ldots \ldots$

F.9 Fully-developed mean flow for skewed conditions ............ 201

F.10 Transformed Reynolds stress profiles $\ldots \ldots \ldots \ldots \ldots \ldots \ldots \ldots \ldots, 203$

F.11 Proposed offset prong slant-wire probe $\ldots \ldots \ldots \ldots \ldots \ldots \ldots \ldots \ldots, 208$

F.12 Interference region for proposed slant-wire probe $\ldots \ldots \ldots \ldots \ldots \ldots 208$ 


\section{LIST OF TABLES}

Page

4.1 Boundary layer parameters at Station $1 \ldots \ldots \ldots \ldots \ldots \ldots \ldots \ldots, 34$

5.1 Velocity deficit in the vicinity of the vortex cores $\ldots \ldots \ldots \ldots \ldots \ldots .54$

5.2 Normalized boundary layer thickness $(\delta / R)$ at Stations 1,5 and $6 \quad \ldots \quad 57$

5.3 Normalized friction velocity $\left(U_{\tau} / U_{b} \times 100\right)$ at mid-planes, $R e_{b}=390,000 \quad 58$

A.1 Transition duct polynomial coefficients $\ldots \ldots \ldots \ldots \ldots \ldots \ldots \ldots \ldots, 136$

A.2 Transition duct geometry variables $\ldots \ldots \ldots \ldots \ldots \ldots \ldots \ldots \ldots, 137$

B.1 DMA Interface Cable pin connections $\ldots \ldots \ldots \ldots \ldots \ldots \ldots \ldots, 143$ 


\section{NOMENCLATURE}

\section{ENGLISH SYMBOLS}

\begin{tabular}{|c|c|}
\hline $\boldsymbol{a}$ & $=$ semi-major axis of superellipse \\
\hline$a 1$ & $=$ shear-energy ratio parameter $(\overline{u v} / 2 k)$ \\
\hline$A_{c s}$ & $=$ transition duct cross-sectional area \\
\hline$A R$ & $=$ transition duct aspect ratio \\
\hline$b$ & $=$ semi-minor axis of superellipse \\
\hline$B$ & $=$ slope of calibration characteristic \\
\hline$C$ & $=$ law-of-the-wall constant (5.0) \\
\hline$C_{f}$ & $=$ skin friction coefficient $\left(\tau_{w} /\left(\frac{1}{2} \rho U_{b}^{2}\right)\right)$ \\
\hline$C_{p}$ & $=$ pressure coefficient $\left(\left(P_{w}-P_{i}\right) / \frac{1}{2} \rho U_{b}^{2}\right)$ \\
\hline$C_{\mu}$ & $=$ empirical constant $(0.09)$ \\
\hline$d$ & $=$ Preston tube outside diameter \\
\hline$D$ & $=$ transition duct inlet diameter \\
\hline$e$ & $=$ fluctuating bridge voltage component \\
\hline$E$ & $=$ instantaneous bridge voltage \\
\hline $\bar{E}_{0}$ & $=$ bridge voltage intercept at zero velocity \\
\hline$h$ & $=$ dynamic pressure head $\left(P_{t}-P\right)$ \\
\hline$h_{B N}$ & $=$ binormal cooling coefficient \\
\hline$h_{N}$ & $=$ normal cooling coefficient \\
\hline$H_{12}$ & $=$ first boundary layer shape factor $\left(\delta_{1} / \delta_{2}\right)$ \\
\hline$H_{32}$ & $=$ second boundary layer shape factor $\left(\delta_{3} / \delta_{2}\right)$ \\
\hline$k$ & $=$ kinetic energy of turbulence \\
\hline$k$ & $=$ tangential cooling coefficient \\
\hline$k_{r}$ & $=$ reference tangential cooling coefficient \\
\hline$l_{p}$ & $=$ Prandtl's length scale \\
\hline$L$ & $=$ distance from begining to end of transition \\
\hline$m$ & $=$ generalized cooling law exponent \\
\hline$n$ & $=$ non-linearized cooling law exponent $(0.45)$ \\
\hline$P$ & $=$ static pressure \\
\hline$P$ & $=$ production of turbulence kinetic energy \\
\hline
\end{tabular}




\begin{tabular}{|c|c|}
\hline$P_{t}$ & $=$ total pressure \\
\hline$Q_{\text {ref }}$ & $=$ reference dynamic pressure $\left(\frac{1}{2} \rho U_{b}^{2}\right)$ \\
\hline$r, \theta, z$ & $=$ cylindrical (pipe) coordinates \\
\hline$R$ & $=$ inlet pipe radius \\
\hline$R_{\mathrm{c}}$ & $=$ radius of curvature \\
\hline$R_{u v}$ & $=$ shear stress correlation $\left(\overline{u v} / u^{\prime} v^{\prime}\right)$ \\
\hline$R_{u w}$ & $=$ shear stress correlation $\left(\overline{u w} / u^{\prime} w^{\prime}\right)$ \\
\hline$R_{v w}$ & $=$ shear stress correlation $\left(\overline{v w} / v^{\prime} w^{\prime}\right)$ \\
\hline$R e$ & $=$ Reynolds number $(U D / \nu)$ \\
\hline$s$ & $=$ peripheral curvilinear coordinate (Fig. 3.2 ) \\
\hline$T$ & $=$ temperature \\
\hline$u^{\prime}, v^{\prime}, w^{\prime}$ & $=\mathrm{rms}$ Reynolds normal stress components \\
\hline$\overline{u^{2}}, \overline{v^{2}}, \overline{w^{2}}$ & $=$ Reynolds normal stress components \\
\hline$\overline{u v}, \overline{u w}, \overline{v w}$ & $=$ Reynolds shear stress components \\
\hline$U, V, W$ & $=$ mean velocity components in the $x, y, z$-directions \\
\hline$U_{e}$ & $=$ effective cooling velocity \\
\hline$U_{r}$ & $=$ resultant yaw velocity vector in the $x-z$ plane \\
\hline$U_{\tau}$ & $=$ friction velocity $\left(\sqrt{\tau_{w} / \rho}\right)$ \\
\hline$V_{r}$ & $=$ resultant transverse velocity vector in the $y-z$ plane \\
\hline$x, y, z$ & $=$ primary cartesian coordinate system \\
\hline$x_{p}$ & $=$ streamwise coordinate measured from pipe inlet \\
\hline$x^{\prime}, y, z^{\prime}$ & $=$ secondary cartesian coordinate system (Method $A)$ \\
\hline$x, y^{\prime}, z^{\prime}$ & $=$ secondary cartesian coordinate system $($ Method $B)$ \\
\hline$x_{w}, y_{w}, z_{w}$ & $=$ local wall coordinate system ( $y_{w}$ normal to duct surface) \\
\hline \multicolumn{2}{|c|}{ GREEK SYMBOLS } \\
\hline$\alpha$ & $=$ angle between probe axis and wire normal \\
\hline$\beta$ & $=$ flow angle $\left(\beta=\tan ^{-1}(V / U)\right)$ \\
\hline$\gamma$ & $=$ flow angle $\left(\gamma=\tan ^{-1}(W / U)\right)$ \\
\hline$\Gamma$ & $=$ Gamma function \\
\hline$\delta$ & $=$ boundary layer thickness \\
\hline$\delta_{1}$ & $=$ axisymmetric displacement thickness \\
\hline
\end{tabular}




\begin{tabular}{|c|c|}
\hline$\delta_{2}$ & $=$ axisymmetric momentum thickness \\
\hline$\delta_{3}$ & $=$ axisymmetric energy thickness \\
\hline$\Delta p$ & $=$ Preston tube pressure head \\
\hline $\boldsymbol{\epsilon}$ & $=$ dissipation rate of turbulence kinetic energy \\
\hline$\zeta$ & $=$ pitch flow angle $\left(\zeta=\tan ^{-1}\left(V / U_{r}\right)\right)$ \\
\hline$\eta$ & $=$ exponent of superellipse equation \\
\hline$\theta$ & $=$ wire rotation angle (relative to secondary coordinates) \\
\hline$\kappa$ & $=$ Von Karman's constant $(0.41)$ \\
\hline$\lambda$ & $=$ wire rotation angle (relative to primary coordinates) \\
\hline$\mu$ & $=$ molecular viscosity \\
\hline$\mu_{t}$ & $=$ turbulent viscosity \\
\hline$\nu$ & $=$ kinematic viscosity $(\mu / \rho)$ \\
\hline$\rho$ & $=$ density \\
\hline$\tau_{\boldsymbol{w}}$ & $=$ wall shear stress \\
\hline$\phi$ & $=$ total mean flow skewness angle \\
\hline$\psi$ & $=$ wire orientation angle \\
\hline$\Omega_{x}$ & $=$ mean streamwise vorticity $(\partial W / \partial y-\partial V / \partial z)$ \\
\hline \multicolumn{2}{|c|}{ SUBSCRIPTS } \\
\hline$a m b$ & $=$ ambient condition \\
\hline$b$ & $=$ bulk condition \\
\hline cl & $=$ centerline condition \\
\hline$F D$ & $=$ fully-developed condition \\
\hline$i$ & $=$ inlet condition \\
\hline$L$ & $=$ linearized quantity \\
\hline$N L$ & $=$ non-linearized quantity \\
\hline$B N$ & $=$ binormal effective cooling velocity component \\
\hline$N$ & $=$ normal effective cooling velocity component \\
\hline$P$ & $=$ parallel (tangential) effective cooling velocity component \\
\hline$n$ & $=$ normal-wire \\
\hline$s$ & $=$ slant-wire \\
\hline$w$ & $=$ wall condition \\
\hline
\end{tabular}


SPECIAL NOTATIONS

$\overline{(~)} \quad=$ time-averaged quantity 


\section{CHAPTER 1}

\section{INTRODUCTION}

The term "transition duct" refers to a class of internal flow configurations in which the cross-sectional shape changes in the streamwise direction. Examples include ducts which change cross section from square-to-round, round-to-square, square-to-rectangular, etc. Transition ducts are commonly used in ventilation systems, in aircraft propulsion systems, as wind tunnel components and as diffusers in hydro-electric turbines. High-performance military aircraft often utilize transition ducts as components in engine inlet and exhaust systems. A schematic of a typical propulsion system is shown in Fig. 1.1. The flow through these ducts is three-dimensional and viscous effects are not negligible. Transverse pressure gradients induced by streamline curvature, coupled with viscous effects, can induce streamwise vortices and regions of local flow separation which adversely affect overall performance. The propulsion system designer must be able to predict the flow through a given configuration accurately in order to avoid undesirable flow characteristics.

One particular transition duct that is currently of interest is the circularto-rectangular (CR) transition duct. Nonaxisymmetric engine exhaust nozzles, usually of rectangular cross section, are being developed in order to improve the performance of military aircraft. An example is the Two-Dimensional/Convergent-Divergent $(2-D / C-D)$ multi-function engine exhaust nozzle. This nozzle, first proposed by Pratt and Whitney Aircraft Group, provides conventional jet area variations as well as thrust vectoring and reversing capabilities [1]. The three modes of operation of the 2-D/C-D nozzle are illustrated in Fig. 1.1. To connect a $2-D / C-D$ nozzle to the engine, a circular-to-rectangular transition section is needed. A typical transition duct for this purpose is shown in Fig. 1.2. The ideal transition duct should provide a uniform, subsonic flow over as short a distance as possible, without incurring unacceptable pressure losses.

Another application of the CR transition duct is as a component in hydroelectric turbines. Hydraulic draft tubes, which are $\mathrm{CR}$ transition ducts with a $90^{\circ}$ bend, are used to diffuse the water exiting the vertical turbine runner at hydro-electric power plants. The inlet flow to the draft tubes typically contains a significant degree of swirl. Pressure recovery in the draft tube is the primary parameter of interest for this application.

Developments in computational fluid mechanics have made it possible to calculate the three-dimensional turbulent flows generated in these transition ducts. A duct which changes from a circular to a rectangular configuration, while maintaining a nearly constant cross-sectional area, offers a particularly stringent test 
for code validation purposes because one set of opposite walls converges while the other set diverges at a given streamwise location. This behavior leads to regions of convex and concave streamline curvature in the flow which tend to respectively suppress and amplify turbulence intensities. In addition, transverse pressure gradients of opposite sign are generated by the curvature which induce secondary flow that can lead to streamwise vortex formation. If swirl is imparted to the flow, then additional helical streamline curvature will be present which can have a significant effect on the local turbulence structure.

An ongoing program at NASA-Lewis Research Center is the sponsorship of benchmark quality experimental studies for the purpose of validating threedimensional viscous flow solvers. A variety of inlet and exhaust flow components, including the circular-to-rectangular transition duct, are being investigated. The Computational Fluid Dynamics Branch at NASA-Lewis has designated a series of circular-to-rectangular transition ducts of specific geometry to be of interest. These ducts cover a wide range of aspect ratios $(A R)$, length ratios $(L / D)$ and cross-sectional area variations. To facilitate mesh generation for numerical computations, the cross sections of all these ducts are defined by superellipses (see Appendix A). A recent study by Patrick and McCormick [2,3], which was under sponsorship by NASA-Lewis, has provided a limited experimental database for two of the these ducts, the $A R 310(A R=3, L / D=1.5)$ and $A R 630$ $(A R=6, L / D=3.0)$ transition ducts. The particular duct configuration chosen for the present study has an aspect ratio of three and a length-to-diameter ratio of 1.5. An isometric view of the duct is shown in Fig. 1.2. At any given streamwise location, the cross-sectional configuration is defined by the equation of a superellipse. The local cross-sectional area ratio increases from unity at the inlet to a maximum of 1.15 at the midpoint before decreasing back to unity at the duct exit. This distribution is intended to model the area variation of a duct constructed of flat surfaces and conical sections more typical of the manufactured product. The exit plane cross-sectional shape does not have sharp corners but a variable radius fillet as seen in Fig. 1.2. A flow visualization study by Reichert et al. [4] shows that the flow remains wholly attached within this configuration for subsonic flow in the presence of the adverse pressure gradient induced by the $15 \%$ area expansion. The complete geometric description of this duct is given in Appendix A. 

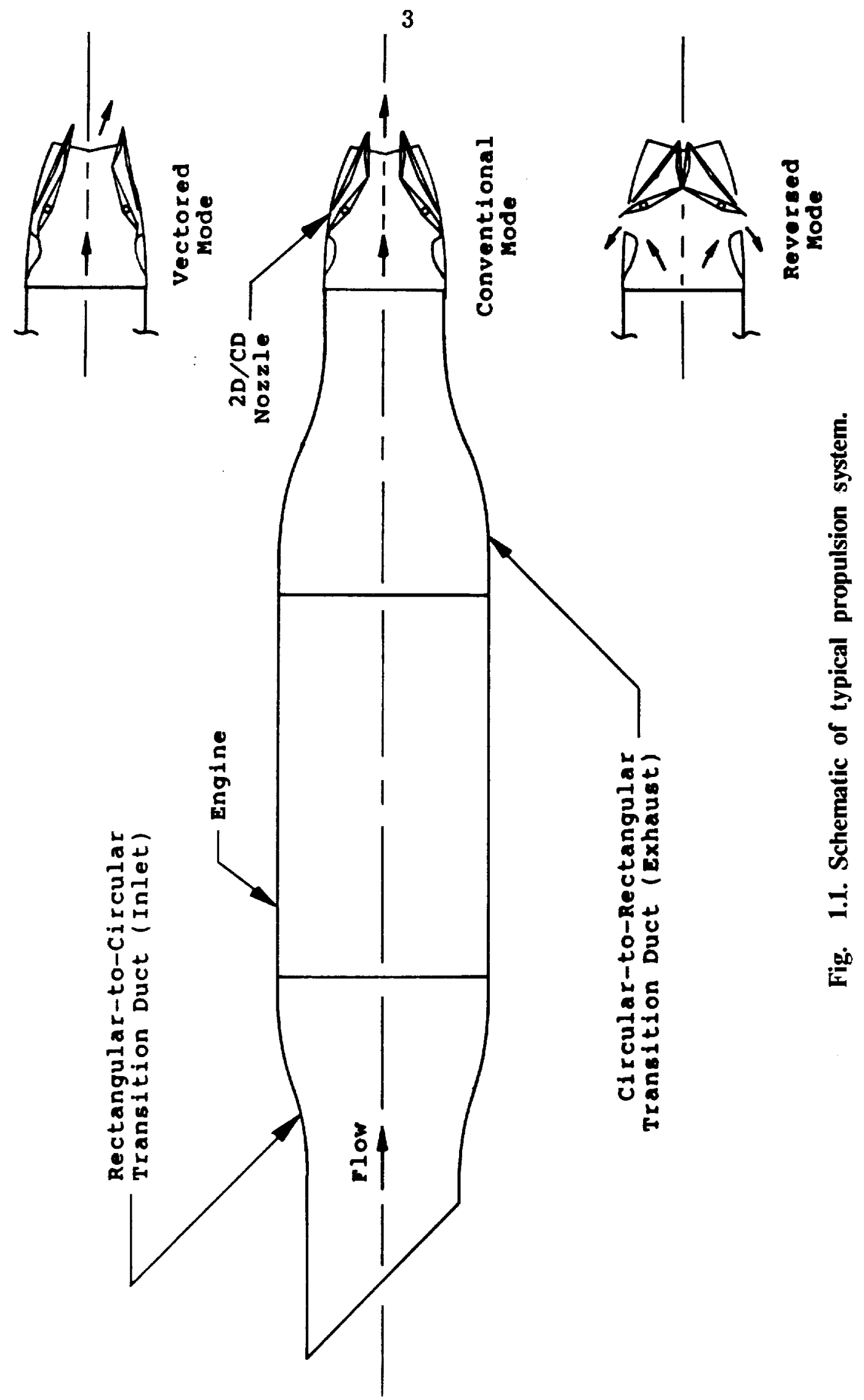

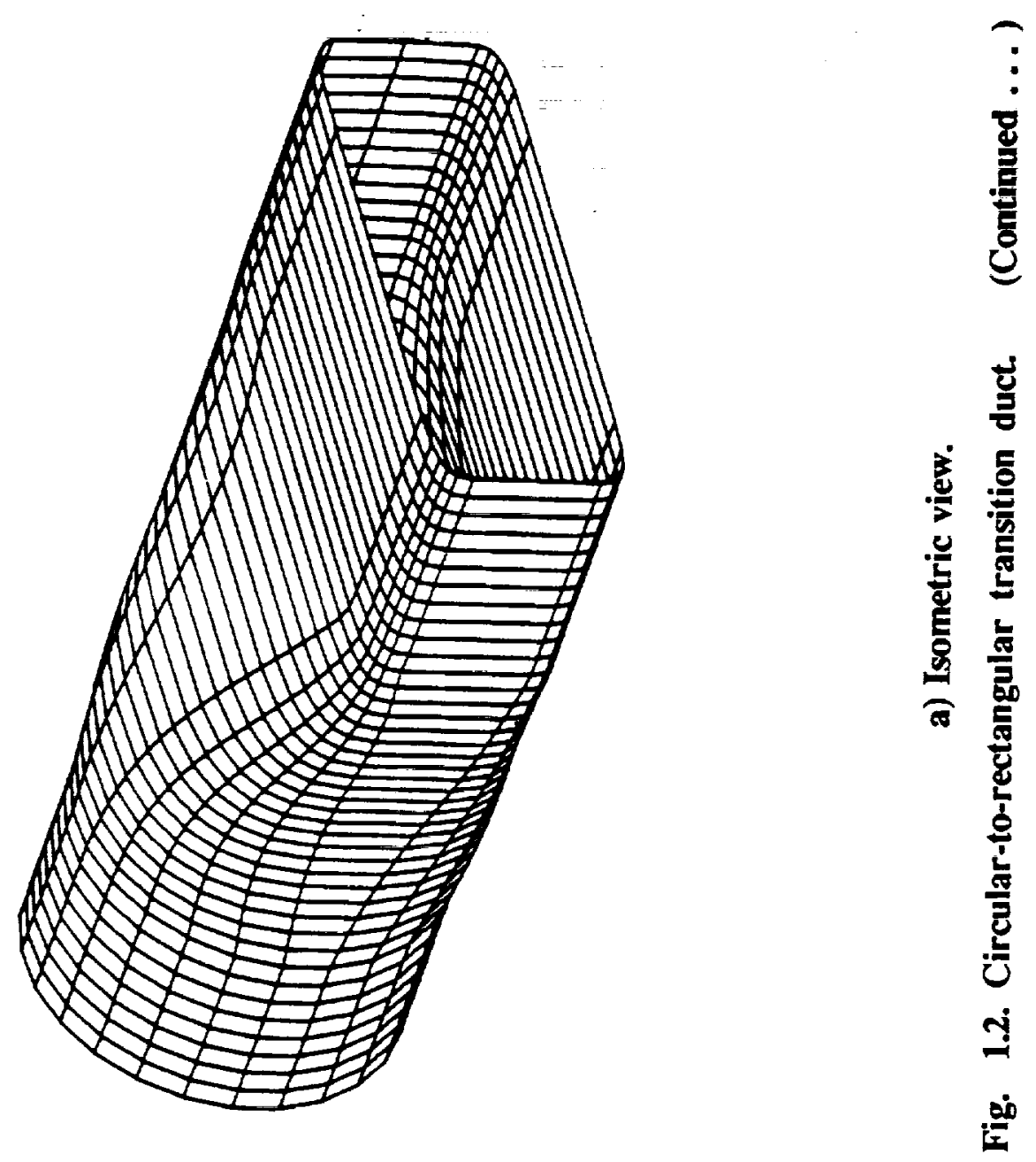

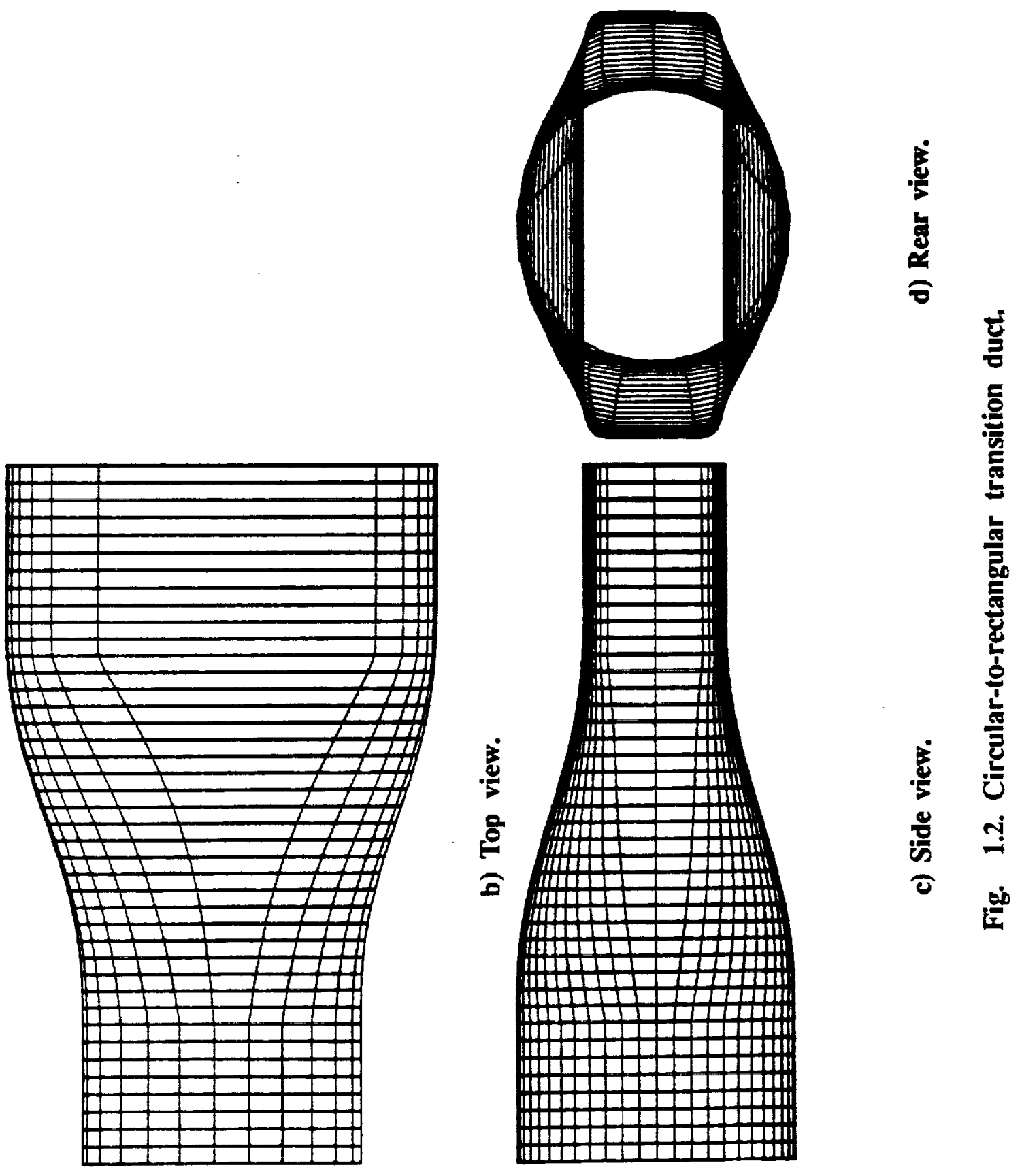


\section{CHAPTER 2}

\section{PREVIOUS WORK}

Turbulent flow through a circular-to-rectangular transition duct is characterized by streamline curvature and streamwise vorticity embedded in the boundary layer. In addition, the boundary layer is subjected to both lateral convergence and divergence. This chapter will begin by reviewing previous transition duct studies, followed by a discussion of the effect that streamline curvature, embedded vorticity and lateral divergence have on turbulent boundary layer flow.

\subsection{Flow Through Transition Ducts}

The earliest study on transition duct flow was an experimental investigation done by Mayer [5] in 1939. In his study, he investigated flow through two rectangular-to-circular (and vice versa) transition ducts of constant crosssectional area. The ducts had transition lengths of 0.69 and 2.76 hydraulic diameters. The data included streamwise static pressure distributions, total pressure contours and the three-dimensional velocity field. In a similar study, Taylor et al. [6] investigated turbulent flow through a square-to-round transition duct which had a $21.5 \%$ reduction in cross-sectional area over the length of the transition. This decrease in area was the result of the hydraulic diameter $(40 \mathrm{~mm})$ being held constant along the duct. The transition occurred over two hydraulic diameters. LDV techniques were used to measure streamwise and transverse velocity components along the duct for an operating Reynolds number of 35,350 . The results of these studies have shown that the length of the transition section is influential on flow development and that pressure-driven crossflows $(\approx 10 \%$ of maximum streamwise velocity), can lead to significant distortion of the primary flow.

During the early development stages of the 2-D/C-D nozzle, Pratt and Whitney Aircraft developed a design procedure for circular-to-rectangular transition ducts intended to minimize both pressure losses and axial length. This procedure is governed by the following criteria [1]:

1) Constant cross-sectional area

2) Corner radius decreasing linearly with length

3) Straight sidewalls

4) Sidewall divergence angle limit $=45$ degrees

In a recent combined experimental and numerical study by Burley et al. $[7,8]$, the original PWA guidelines were examined to determine if expanded design criteria could be established so that shorter transitions are possible, thereby reducing exhaust system weight. Five different circular-to-rectangular transition 
duct configurations were investigated to explore the effects of duct length, wall shape and cross-sectional area distribution on performance. All of the ducts were defined by super-elliptic cross sections (see Appendix A). The transition ducts were installed in a transonic wind tunnel with a high aspect ratio, nonaxisymmetric nozzle and the overall internal performance was measured. In addition, one duct was tested with swirl vanes installed. Discharge coefficient and thrust ratio versus nozzle pressure ratio were used as performance criteria. The results of their investigation show that for length ratios less than or equal 0.75 , large regions of separated flow are present. However, because the flow reattached before the entrance to the nozzle, only a small decrease in performance was observed. They also found that swirling the flow had a positive effect on performance for low nozzle pressure ratios, but that performance was decreased when the nozzle was near a choked condition. Finally, these researchers reported that decreasing the cross-sectional area along the duct reduced flow separation and provided a modest increase in performance.

Patrick and McCormick [2,3] were the first to make turbulence measurements within a circular-to-rectangular transition duct. LDV and total pressure measurements were made at the inlet and outlet stations of two different ducts at an operating Reynolds number of 420,000 . The first duct, designated the AR310, had an aspect ratio of three, a length-to-diameter ratio of one, and constant cross-sectional area through the duct. The second duct, designated the AR630, had an aspect ratio of six and a length-to-diameter ratio of three. The local cross-sectional area ratio increased from unity at the inlet to a maximum of 1.10 at the midpoint before decreasing back to unity at the duct exit. In order to facilitate grid generation for numerical comparisons, the cross-sectional shape everywhere along the ducts was prescribed by the equation of a superellipse. Measured quantities included all three mean velocity components and the three normal Reynolds stress components at the inlet and outlet planes. The results for the AR310 duct showed that the axial mean flow did not develop uniformly but had a convex profile along the major axis at the duct exit plane. Outward transverse velocities, nominally parallel to the major axis, were observed that peaked at about $10 \%$ of the bulk velocity. No streamwise vorticity was observed except deep in the corner region, but the measurement grid was too coarse to discern discrete vortical motion. The AR630 duct behaved quite differently. Here the flow developed much more uniformly, and a pair of discrete vortices along the duct sidewalls, centered about the duct semi-major axis, were observed. The origin of these vortices is in the first half of the transition where the wall curvature creates a pressure gradient which causes a crossflow from the upper and lower walls to the sidewalls. The crossflow meets at the duct centerline and turns inward along it. In the second half of the duct, where the curvature changes sign, the pressure gradient is reversed, counteracting the secondary motion. If a vortical pattern was established in the first half of the AR310 duct, then the reversed 
pressure gradient was effective in stopping it.

Miau et al. [9] experimentally investigated three CR ducts with length-todiameter ratios of $1.08,0.92$ and 0.54 , under low subsonic flow conditions. The aspect ratio was equal to two and the cross-sectional area was constant for all three ducts. Mean flow and turbulence data were taken at the inlet and exit planes. Secondary flow patterns indicative of streamwise vortex formation were observed at the exit plane of the ducts. From these results, all the terms in the axial mean vorticity equation were computed. Their analysis showed that the generation of streamwise vorticity is due primarily to transverse pressure gradients induced by geometrical deformation.

With the exception of the performance data reported by Burley et al. $[7,8]$, none of the above studies considered the case where a swirl velocity component is imparted to the inlet flow. The addition of swirl may have several benefits. First, swirl will impart a radial velocity component to the flow, thus improving it's ability to follow steeply sloped sidewalls in the transition duct. Secondly, Schwartz [10] has observed that noise associated with axisymmetric jet exhaust can be reduced by swirling the flow. Finally, in an axisymmetric jet, the rate of decay of the axial velocity component can be substantially increased (reduced thermal plume) by swirling the flow, with minimal loss of thrust [11]. Der et al. [12] performed a water tunnel flow visualization study of swirling flow through a CR duct. Later, Chu et al. $[13,14]$ analyzed these data and found that swirling the flow dramatically reduced the thermal plume. Recently, Reichert et al. [4], using a duct identical to the one in the present study, compared the mean flow field for the cases of swirling and non-swirling inlet flow at an operating Mach number of 0.35 and a Reynolds number of $1.5 \times 10^{6}$.

Related studies have been undertaken which include the effects of a turbine centerbody and axial centerline curvature. Sobota and Marble [15] performed a detailed experimental and numerical investigation of a CR transition duct with a large centerbody and various degrees of inlet swirl. This study provided insight into vorticity generation mechanisms and the ability to tailor vorticity distributions. CR transition ducts with a $90^{\circ}$ bend in the transition section (hydraulic turbine draft tubes) have recently been analyzed with and without swirl by $\mathrm{Vu}$ and Shyy [16]. The flow through the draft tube was predicted using a finitevolume approximation to the full Navier-Stokes equations in conjunction with a $k-\epsilon$ turbulence model and the results were compared to wind and water tunnel experimental data. In general, pressure recovery, as well as the three-dimensional velocity field, agreed well between experiment and numerical predictions.

The review of the literature has revealed that there is only a small experimental database for flow through a circular-to-rectangular transition duct that is of sufficient detail to be useful for CFD code calibration/validation purposes. At the present, only Miau's data set include measurements of the complete Reynolds 
stress tensor in a CR transition duct. The present study is intended to help fill this void by providing complete mean flow and Reynolds stress measurements at the inlet and outlet stations, supplemented by mean flow data at intermediate stations, for a duct with an aspect ratio larger than that considered by Miau. On the basis of previous work in this area, it was anticipated that skew induced secondary flow would have a dominating influence on the primary flow and on the local turbulence structure.

\subsection{Streamline Curvature Effects}

A great deal of work has been published on the effects of streamline curvature on turbulent boundary layer development. The bulk of the studies have been for the quasi-two-dimensional case with the curvature induced by a constant radius bend in a square or rectangular wind tunnel. Although the transition duct flow is considerably more complex, it is useful to examine previous related results in order to gain some insight into the mechanisms operating within the transition duct.

In 1973, Bradshaw [17] published a comprehensive review of the effects of streamline curvature on turbulent flow. His work was motivated by what he referred to as "the surprisingly large effect exerted on shear-flow turbulence by curvature of the streamlines in the plane of the mean shear". Flows with streamline curvature are characterized by the presence of extra rates of strain, that is, rates additional to the simple shear $\partial U / \partial y$. When the equations of motion are written in semi-curvilinear coordinates (e.g., the $s, n$ system of reference [17]), extra explicit terms appear which account for the presence of curvature. Experimental measurements have shown, however, that the effects of extra rates of strain are an order of magnitude larger than would appear when calculation methods for simple shear flows are extended to curved flows. Bradshaw explains this discrepancy by concluding that streamline curvature directly causes large changes in the higher-order parameters of the turbulence structure.

Convex and concave curvature are often referred to as stabilizing and destabilizing curvature, respectively. Laminar flow over a destabilizing (concave) surface is subject to centrifugal instability which is characterized by the presence of streamwise vortices within the boundary layer; the so-called Taylor-Görtler vortices. For turbulent flows over a concave surface, the presence of vortices analogous to the laminar Taylor-Görtler type have been observed experimentally by So and Mellor [18,19], Meroney and Bradshaw [20] and Hoffmann et al. [21], as well as others; and numerically, by direct simulation of the Navier-Stokes equations, by Moser and Moin [22]. In addition, the stabilizing and destabilizing effect on turbulence acts, respectively, to attenuate and amplify the turbulence intensities. 
In regions sufficiently close to curved walls, mean velocity profiles have been observed to follow the flat plate law-of-the-wall for both convex and concave curvature [18,19,23]. Hoffman and Bradshaw [24] have suggested that the lawof-the-wall applies when $y / R_{c}$ is small. This is important in that it allows for the use of law-of-the-wall based wall functions in numerical computations. The agreement with law-of-the-wall behavior is apparently where the similarity between convex and concave curved flows end. In contrast to flat plate flows, turbulent flow over a convex surface is characterized by slower boundary layer growth, lower wall shear stress, reduced turbulence intensities and reduced heat transfer rates. Conversely, turbulent flow over a concave surface is characterized by increased boundary layer growth, higher wall shear stress, increased turbulence intensities and increased heat transfer rates, as well as the aforementioned streamwise vortices. The most significant difference in the turbulence statistics appears in the Reynolds shear stress. Measurements by So and Mellor [18], Gillis and Johnston [25] and Smits et al. [26] show a sharp decrease in the turbulent shear stress to near-zero levels in the outer region of the boundary layer when a flat plate boundary layer is suddenly subjected to a strong $\left(\delta / R_{c} \approx 0.10\right)$ convex curvature. For the concave case, the turbulent shear stress dramatically increased to a near two-fold level as compared to flat plate results. Hunt and Joubert [27] found similar behavior in flows subjected to mild streamline curvature $\left(\delta / R_{c} \approx 0.01\right)$, but to a lesser degree. The two flow cases also respond differently when they are subjected to a flat plate recovery region. Whereas the Reynolds stresses recovering from convex curvature do so in a monotonic fashion, the stresses in concave flows drop well below their entry region values before recovering [26].

The dramatic differences between the convex and concave curvature cases have hindered development of adequate turbulence models because the mechanisms that produce them are not well understood. Indeed, Muck et al. [28] have concluded that, although governed by the same dimensional analysis, there is no other useful connection between the two cases. From this conclusion, they imply that allowances for the effect of streamline curvature in calculation methods for turbulent flows should be formulated separately for the stabilizing and destabilizing cases.

The above discussion serves to illustrate factors which may add to the complexity of transition duct flow. The degree to which streamline curvature affects transition duct flow depends on the thickness of the incoming boundary layer. For the present study, a boundary layer thickness of $\delta / R \approx 0.25$ is anticipated. This corresponds to a maximum curvature parameter of $\delta / R_{c} \approx 0.085$ for both convex and concave walls. On the basis of previous experimental results, it was expected that streamline curvature would influence the development of the flow in the transition duct. 


\subsection{Embedded Streamwise Vortices}

The transition duct studies reported in Refs. 2,3 and 9 have shown that streamwise vorticity is generated within CR transition ducts. The results for the AR630 duct (Refs. 2 and 3) show that a discrete vortex pair (common flow away from the surface) develops in the duct sidewall boundary layer. Streamline vortices embedded in a boundary layer are as often beneficial as they are detrimental. On aircraft flight surfaces, streamwise vortices are purposefully generated to promote mixing between the freestream and the boundary layer in order to forestall flow separation. In combustor applications, streamwise vortices are used to enhance mixing between fuel and oxidant. In turbomachinery, however, streamwise vortices generated by blade-hub junctures may sweep away the protective film cooling on adjacent blades causing damaging hot spots. In transition duct applications, streamwise vortices result in undesirable pressure losses, although they may inhibit flow separation.

Streamwise vortices in boundary layers can be generated by transverse pressure gradients or by gradients of the Reynolds stresses. Pressure-gradient induced streamwise vorticity occurs whenever a shear layer (laminar or turbulent) with spanwise vorticity is deflected laterally by transverse pressure forces. If the deflection occurs over a short spanwise distance, then a discrete vortex is formed. Vortices generated in this manner occur in strut-endwall (junction) configurations and in flow through curved ducts. Reynolds stress induced vorticity occurs in turbulent flow through non-circular ducts, even when the ducts are straight, i.e., uncurved in the streamwise direction. Because both types of vorticity generation occur in many practical engineering flows, a large body of literature exits on the subject. Some of the more comprehensive experimental studies on the effect that embedded streamwise vortices have on the mean flow and turbulence structure include those due to Shabaka et al. [29], Mehta and Bradshaw [30] and Pauley and Eaton $[31,32]$. These researchers studied the effects of single and paired vortices in an otherwise two-dimensional boundary layer flow. Mean flow and turbulence (one-point double and triple correlations) were measured. The vortices in all these studies were generated by half-delta wings, although the placement of the generators in the wind tunnels differed. Whereas Pauley and Eaton placed the generators on the floor of the 2-D channel, Shabaka and Mehta placed the generators upstream of the contraction in the settling chamber. By placing the generators in the plenum, the velocity deficit in the wake of the delta wing is reduced to a small percentage of the freestream velocity as the flow accelerates through the contraction. Pauley and Eaton obtained data in measurement planes 97 and $188 \mathrm{~cm}$ downstream from the generators. Shabaka and Mehta present results in measurement planes between 60 and $255 \mathrm{~cm}$ downstream. The results of these investigations showed that thickening of the boundary layer occurred in upwash regions and thinning occurred in downwash regions. Paired vortices with the common flow away from the surface were attracted and moved 
away from the surface. In contrast, vortex pairs with the common flow towards the surface moved away from each other and stayed in close proximity to the wall. In the vicinity of the vortex core(s), a concentrated maxima of turbulence intensity occurs, but no large-scale unsteadiness in the flow was detected. When compared to the surrounding 2-D boundary layer, large changes in the dimensionless turbulence structure parameters were observed and eddy viscosities were reported to be very ill-behaved in the vortex region. These observations led the researchers to conclude that full Reynolds stress transport modelling would be required for prediction purposes.

Liandrat et al. [33] used the data of Refs. 29 and 30 for comparison with numerical simulations based on mixing length and $k-\epsilon$ turbulence models. In addition, calculations based on two forms of the Reynolds stress transport equations were performed. The results of this investigation showed simple turbulence models provide good estimations of overall mean flow properties for the case of a single embedded vortex. For the case of paired vortices with common flow away from the surface, the mean flow results were found to be largely unsatisfactory. For both cases, details of the predicted turbulence structure required Reynolds stress transport models. However, even these higher-order models did not give adequate predictions of the transverse normal stresses and the secondary shear stresses that control the diffusion of streamwise vorticity. In a review of turbulent secondary flows, Bradshaw [34] concludes that the primary inadequacy in the Reynolds stress transport models is in the modelling of the pressure-strain term.

As a final note, Patrick and McCormick [2,3] made mention of the similarity between the generation of the vortex pair in the AR630 CR transition duct and in a circular pipe with an S-shaped bend. Experimental mean flow results and a discussion of vorticity generation for the latter case is presented by Bansod and Bradshaw [35]. Limited turbulence measurements in a circular S-shaped duct with embedded vortices are presented by Taylor et al. [36].

\subsection{Laterally Diverging Boundary Layer}

Along the converging walls of a CR transition duct, the boundary layer is subjected to lateral divergence. Conversely, along the diverging walls, the boundary layer converges laterally. Boundary layer divergence is another example of a shear flow with extra strain rates $(\partial V / \partial y, \partial W / \partial z)$. As in the case of streamline curvature, the effect that lateral divergence has on the turbulence structure of a boundary layer is much larger than, and sometimes opposes, what is predicted by explicit terms that appear in the Reynolds stress transport equations. The strength of divergence in a boundary layer is typically characterized by the rateof-strain parameter $(\partial W / \partial z) /(\partial U / \partial y)$. Smits et al. [37] reviewed the effect that

lateral divergence and convergence have on boundary layer flow. In addition, 
they obtained mean flow and turbulence measurements in a diverging boundary layer that develops on a cylinder-flare where $((\partial W / \partial z) /(\partial U / \partial y) \approx 0.1)$ midway through the layer. In the transition region between the cylinder and flare, the flow was subjected to relatively strong concave longitudinal curvature. Although they found that the effects of divergence and curvature in the transition region could not be quantitatively separated, they argue that the memory of the curvature is short-lived and that the downstream flowfield is primarily a result of divergence effects alone. They note that boundary layers subjected to lateral divergence and convergence tend to, respectively, thin and thicken. Turbulence kinetic energy is amplified for the diverging case and attenuated for the converging case. The shear stress in a diverging boundary layer is elevated and a peak occurs which moves outward from the surface as the flow develops. Pauley and Eaton $[31,32]$ obtained mean flow and turbulence measurements in the diverging boundary layer that develops between an embedded vortex pair with the common flow towards the surface. Although the strength of divergence was fairly weak $\left((\partial W / \partial z) /(\partial U / \partial y) \approx 10^{-3}\right)$, the flow is unique, inasmuch as complicating factors such as streamwise pressure gradients and curvature are absent. Unlike the results of Smits (and others), these researchers found no significant difference in either the mean flow or the Reynolds stresses when compared to their counterparts in the two-dimensional boundary layer outside the vortex pair.

\subsection{Concluding Remarks}

Turbulent flow through a circular-to-rectangular transition duct represents a practical engineering flow of interest where multiple complicating effects are present. In particular, the transition duct geometry imposes extra rates-of-strain on an initially two-dimensional boundary layer possessing only the simple strain $\partial U / \partial y$. Although Smits et al. [37] have shown that individual effects cannot be quantitatively separated due to non-linear interactions, qualitative assessment of the flowfield should certainly be possible. By studying this flow two goals are hoped to be achieved. First, a sufficiently detailed mean flow and turbulence data set will be provided that may be used for direct comparison with numerically generated results. Secondly, the results will be analyzed in a way that will aid predictors and modellers in determining the level of sophistication required in their computational efforts. 


\section{CHAPTER 3}

\section{EXPERIMENTAL PROGRAM}

\subsection{Introduction}

The present study is primarily of an experimental nature. The goal of the study is to provide a comprehensive set of mean and turbulence measurements at inlet, intermediate and outlet stations of a CR transition duct. These data are intended for use in CFD code calibration/validation and turbulence model development. The wind tunnel flow facility, test section instrumentation and some data reduction methods are described in this chapter.

\subsection{Flow Facility}

The Square Duct Flow Facility in the Heat Power Laboratory in the Mechanical Engineering Building has been modified to a configuration appropriate for the present study. The inlet to the Square Duct Flow Facility has been replaced by the new ductwork illustrated in Fig. 3.1. Atmospheric air enters the wind tunnel through a 2.8:1 elliptic bellmouth contraction and then passes through a settling chamber which consists of an Alfco combination filter/honeycomb flow straightener (Model 36"0 CYL,9FG-B), two fine $(20 \times 20)$ mesh screens, and a $20: 1$ concentric contraction. This inlet section was designed to provide a uniform, low turbulence level, axisymmetric exit flow using design criteria developed by Morel [38]. Prior to construction, the flow through the 20:1 contraction was computed using NASA-Lewis' VISTA program [39], which is an axisymmetric subsonic Navier-Stokes flow solver. The inlet boundary layer thickness was varied from $\delta_{i} / R_{s}=0.001$ to 0.05 , where $R_{s}$ is the settling chamber radius, and the inlet velocity was varied from $U_{i}=0.762$ to $1.524 \mathrm{~m} / \mathrm{s}$, which corresponds to velocities at the exit plane from $U_{e}=15.2$ to $30.5 \mathrm{~m} / \mathrm{s}$. For all cases, the results showed that the inviscid core velocity at the exit plane of the contraction was uniform to within $0.1 \%$ of the centerline value.

From the 20:1 contraction, the flow enters a $20.42 \mathrm{~cm}$ diameter pipe of variable length. Pipe sections are made in lengths of $L / D=3$ and can be added or removed so as to vary the boundary layer thickness $(\delta)$ at the inlet to the transition duct. To promote transition to turbulent flow, a $2.54 \mathrm{~cm}$ wide strip of \#36 sandpaper was placed at the beginning of the first pipe. At the end of the pipe section is a probe access ring which has provisions for making detailed measurements of the test section inlet flow. From the probe access ring the flow enters the test section which consists of the transition duct and a removable transition duct extension. The rectangular duct downstream of the test section supports a probe traversing mechanism. Finally, a diffuser section (2 degrees divergence) provides the link to the existing $0.254 \times 0.254$ meter square duct. 
Air is drawn through the facility by means of a two-speed centrifugal fan located at the exit of the Square Duct Flow Facility. The fan discharges the air back into the laboratory through a set of remotely actuated shutters which provide a means for varying the mass flow rate through the wind tunnel. Details of the remaining Square Duct Flow Facility are described by Eppich [40].

The primary materials used to build the flow facility are as follows. The two inlet contractions are constructed of polyester resin fiberglass with embedded aluminum mounting flanges. The settling chamber, which houses the filter/honeycomb and screens, is constructed of wood and Formica. The 0.203 meter diameter inlet pipes, the probe access ring, the probe access duct and the diffuser are all fabricated of aluminum. The transition duct and the transition duct extension are constructed of epoxy resin fiberglass in halves which part in the $x-y$ plane. The patterns for molding the duct halves were provided by the NASA-Lewis Research Center.

\subsection{Test Section}

A side view of the test section is shown in Fig. 3.2.a. Cross-sectional views at each of the six data stations indicated in Fig. 3.2.a are shown in Figs. 3.2.b through 3.2.f. The origin of the coordinate system shown in Fig. 3.2.a. was chosen so as to be in agreement with NASA-Lewis' definition of the transition duct. Station 1 is located one inlet duct diameter upstream from where the beginning of transition occurs (Station 2). Although the cross section at Station 2 is still circular, it was anticipated that some distortion of the flow field will occur due to the influence of the changing downstream geometry. At Stations 3 and 4 the geometry is changing rapidly and relatively large transverse velocities were expected. Station 5 is located at the end of transition and data Station 6 is located two diameters downstream from the end of transition. An isometric view of the six data station cross sections is shown in Fig. 3.3.

The probe traversing mechanism located just downstream of the test section in Fig. 3.2.a was used for acquiring data at Stations 5 and 6. This mechanism holds the probe axis parallel to the duct centerline and has provisions for rotating the probe about it's longitudinal axis by means of a spring-loaded (anti-backlash) bevel gear arrangement. Dial indicators were used to position the probes in each direction to within an estimated accuracy of $\pm 0.025 \mathrm{~mm}$, and the probes were rotated to fixed angular positions to within an estimated accuracy of \pm 0.5 degree. The vertical and horizontal traversing capabilities are such that the probe can be positioned anywhere within the cross section at Stations 5 and 6 . However, due to the convergence of the upper and lower walls of the transition duct, there is an increasingly larger area upstream from Station 5 which cannot be accessed. Therefore, at Stations 1 through 4 probes were inserted through holes in the duct wall which are normal to the duct axial centerline. These access holes are illustrated in Figs. 3.2.b through 3.2.e. 
For this study, two identical transition ducts were constructed. The first of these was used when taking data at Stations 1,5 and 6. This duct has only static pressure taps along the periphery of the lower half of the duct at Station 5 (see Fig. 3.2.f). The second duct has probe access holes along the periphery of the upper half of the duct and static pressure taps along the periphery of the lower half of the duct at Stations 2, 3 and 4 (see Figs. 3.2.c through 3.2.e). Two ducts were constructed to insure that there would be no influence on the flow field at Stations 5 and 6 from probe access holes at upstream stations.

\subsection{Instrumentation}

The test section is instrumented to facilitate measurement of both mean and fluctuating quantities. Mean quantities were measured by means of static and total pressure instrumentation and hot-wire anemometry. All fluctuating quantities were measured using hot-wire anemometry. Local skin friction was measured with Preston tubes, which are simply circular Pitot tubes resting on the duct wall.

A variety of pressure probes were used to measure the various mean quantities of interest. Total pressure contours were measured with two types of probes. At Stations 1,2,5 and 6, where streamlines in the cross section are everywhere nominally parallel to the axial centerline of the duct, circular Pitot tubes having an outside tip diameter of $0.635 \mathrm{~mm}$ were used. At intermediate data Stations 3 and 4 , where the streamlines were skewed by as much as 20 degrees relative to the probe centerline, a United Sensor Model KAC12 Kiel probe was used. According to Chue [41], Kiel probes are able to measure total pressure accurately for skew angles as high as $40^{\circ}$. Boundary layer profiles were measured by means of flattened Pitot tubes having outer dimensions of $0.812 \times 0.406 \mathrm{~mm}$ at the tip. Static pressure distributions on the duct axial centerline were measured by means of a static pressure probe which was traversed along the centerline. Transverse flow angles were measured by means of a two-tube Conrad probe and a normal hot-wire. All pressure data were measured with a 10 torr Barocel electronic manometer, Model 571D-10T-1C2-V1, coupled with a Datametrics digital display unit, Model 1174.

Hot-wire probes consisted of single, rotatable normal and slant-wires. The sensing element of the hot-wire probes is $0.00381 \mathrm{~mm}(4 \mu)$ diameter platinum coated tungsten wire. The ends of the wire are copper plated to facilitate soldering and to define the sensing element length, which typically has a length-todiameter ratio near 300 . The prongs of the probes are similar to configurations recommended by Comte- Bellot et al. [42], for minimizing aerodynamic disturbances. The fluctuating turbulence signal was processed by means of a TSI Intelligent Flow Analyzer (IFA) system, which consists of an IFA 100 constant temperature anemometer and an IFA 200 analog-to-digital (A/D) converter. The 
digitized signal is recorded on a VAX Workstation, via a DRQ3B DMA card, where the various turbulence correlations are computed. Appendix B contains a description of the setup and operation of this equipment.

The probe access ring (see Fig. 3.2.a) was used to measure the flow conditions at the transition duct inlet (Station 1). The ring contains four probe access holes, four static pressure taps and four Preston tubes equally spaced around the periphery of the ring as shown in Fig. 3.2.b. To ensure that Preston tube data were taken within the law-of-the-wall region of the boundary layer, each Preston tube was of a different diameter; $2.769,3.962,5.537$ and $6.350 \mathrm{~mm}$. The tubes were semi-permanently installed and were removed before data were taken at the downstream stations.

\subsection{Data Reduction}

This section contains the data reduction methods for the pressure probes, hot-wire probes and equations for computing boundary layer parameters.

\subsubsection{Pressure Probe Data Reduction}

\subsubsection{Mean Velocity}

The total mean velocity along a flow streamline can be deduced from a Pitot probe aligned with the streamline or, if alignment is not practical, from measurements with a Kiel probe. The total velocity is related to the probe pressure by Bernoulli's relation and the ideal gas law, namely:

$$
U_{0}=\left(2 h R_{a i r} T_{a m b} / P_{a m b}\right)^{1 / 2}
$$

where:

$$
\begin{array}{ll}
U_{0} & =\text { total velocity }(\mathrm{m} / \mathrm{s}) \\
h & =\text { measured pressure head }\left(P_{t}-P\right) \quad(\mathrm{mmHg}) \\
R_{a i r} & =\text { gas constant for air }\left(287.0 \mathrm{~J} /\left(\mathrm{kg} \cdot{ }^{\circ} \mathrm{K}\right)\right) \\
T_{a m b} & =\text { ambient temperature }\left({ }^{\circ} \mathrm{K}\right) \\
P_{a m b} & =\text { ambient static pressure }(\mathrm{mmHg})
\end{array}
$$

The Cartesian velocity components are related to the total velocity vector by:

$$
\begin{aligned}
& U=U_{0} \cos \beta \cos \gamma \\
& V=U_{0} \sin \beta \cos \gamma \\
& W=U_{0} \cos \beta \sin \gamma
\end{aligned}
$$

where $\beta$ and $\gamma$ are the flow angles in the $x-y$ and $x-z$ planes, respectively. These flow angles can be measured directly by performing a differential pressure nulling 
technique with a two-tube Conrad probe, first in the $x-y$ plane and then in the $x-z$ plane.

\subsubsection{Skin Friction}

Head and Vasanta Ram's [43] tabulated presentation of Patel's [44] Preston tube calibration was used to deduce local skin friction values. Their table presents the calibration in the functional form:

$$
\frac{\Delta p}{\tau_{w}}=f\left(\frac{\Delta p d^{2}}{\rho \nu^{2}}\right)
$$

where $\Delta p$ is the difference between the Preston tube and local wall static pressure and $d$ is the Preston tube outside diameter. Head and Vasanta Ram estimate that, even without interpolation, their tables should give values that are accurate to within \pm 1 per cent. For the present study, a linear interpolation was used. This method of evaluating shear stress presumes that the two-dimensional form of the law-of-the-wall is valid and that streamwise pressure gradients are small. It was anticipated that this method would be applicable to data taken at Stations 1,5 and 6 , inasmuch as there is no longitudinal wall curvature or cross-sectional area change at these locations.

\subsubsection{Hot-Wire Probe Data Reduction}

For reasons given in section 3.3, the positioning of the hot-wire probe is dependent upon the particular station being investigated. At Stations 1 through 4 , the probe body centerline was positioned in a direction normal to the axial centerline of the duct, while at Stations 5 and 6 the probe was positioned parallel to the axial direction. To simplify the following discussion, the former positioning method shall hereafter be designated $M$ ethod $A$, while the latter will be referred to as Method $B$

For the hot-wire measurements, single-wire techniques will be employed rather than the more complicated two or three-wire methods. This approach eliminates some of the difficulties associated with multi-wire techniques, such as extensive calibration requirements, possible wire interference, multi-wire drift and poor spatial resolution. Single-wire techniques, however, limit turbulence measurements to second-order correlations (Reynolds stresses).

For Method $B$, a hot-wire technique developed by Al-Beirutty $[45,46]$ and Arterberry [47] was used to relate the three mean velocity components and six Reynolds stresses to the mean and mean-square anemometer output voltages. This technique uses a fixed normal-wire and a single, rotatable slant-wire and utilizes an empirical cooling velocity law. The method is applicable to flows of low-to-moderate turbulence intensity and zero-to-moderate flow skewness (up to 30 degrees total shewness in both pitch and yaw). Validation of the technique 
for low-intensity turbulent flows was accomplished by analyzing data obtained in fully-developed pipe flow under simulated skewed flow conditions [47]. For moderate-intensity turbulent flows, validation was accomplished by obtaining data under simulated skewed flow conditions in a free jet which issued from fully-developed pipe flow [45]. The working forms of the mean and turbulence response equations developed by Al-Beirutty are presented in Appendix C without derivation. For details of the development of these equations, the reader is referred to Refs. 45 and 46.

For Method $A$, appropriate hot-wire response equations are developed following a methodology similar to that used by Al-Beirutty. The working forms of the response equations will be presented in Appendix C, with the details of their derivation included in Appendices D and E. Also, following Al-Beirutty, the Method $A$ technique was partially validated in the present study by means of data obtained in fully-developed pipe flow under simulated skewed flow conditions. The verification procedure is described in Appendix F.

\subsubsection{Boundary Layer Parameters}

The following are definitions of some axisymmetric incompressible boundary layer parameters which are useful in characterizing the inlet flow condition:

Boundary Layer Thickness

$$
\delta=y @ \quad U / U_{c l}=0.995
$$

Displacement Thickness

$$
\delta_{1}=\int_{0}^{R}\left(1-\frac{U}{U_{c l}}\right)\left(\frac{r}{R}\right) d r
$$

Momentum Thickness

$$
\delta_{2}=\int_{0}^{R} \frac{U}{U_{c l}}\left(1-\frac{U}{U_{c l}}\right)\left(\frac{r}{R}\right) d r
$$

Energy Thickness

$$
\delta_{3}=\int_{0}^{R} \frac{U}{U_{c l}}\left(1-\frac{U^{2}}{U_{c l}^{2}}\right)\left(\frac{r}{R}\right) d r
$$

Blockage Factor

$$
B=\frac{2 \delta_{1}}{R}=1-\frac{U_{b}}{U_{c l}}
$$


First Shape Factor

$$
H_{12}=\frac{\delta_{1}}{\delta_{2}}
$$

Second Shape Factor

$$
H_{32}=\frac{\delta_{3}}{\delta_{2}}
$$

It should be pointed out here that equations (3.5), (3.6) and (3.7) are approximate, but that equation (3.8) is exact when the displacement thickness $\delta_{1}$ is calculated by equation (3.5). 


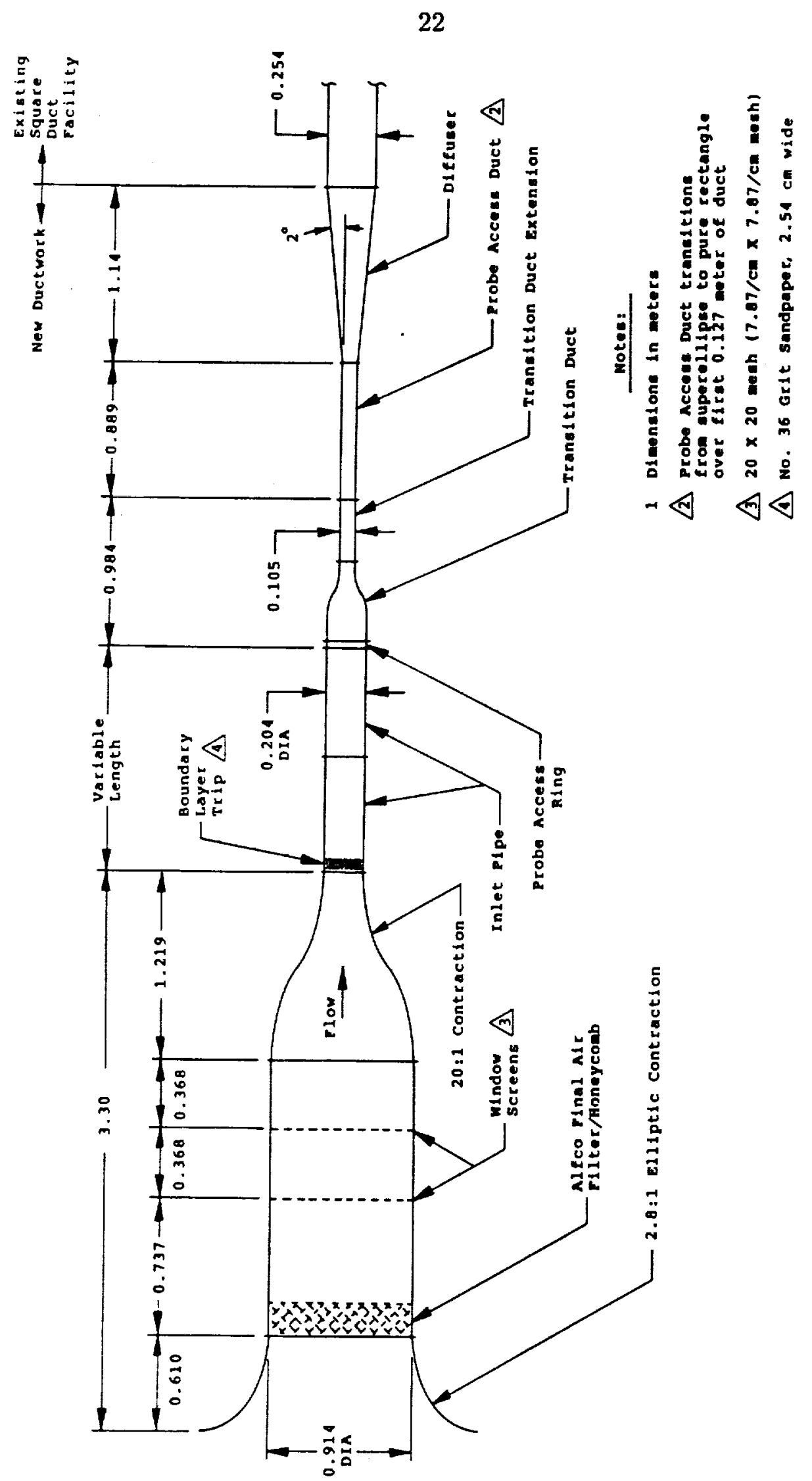

总 


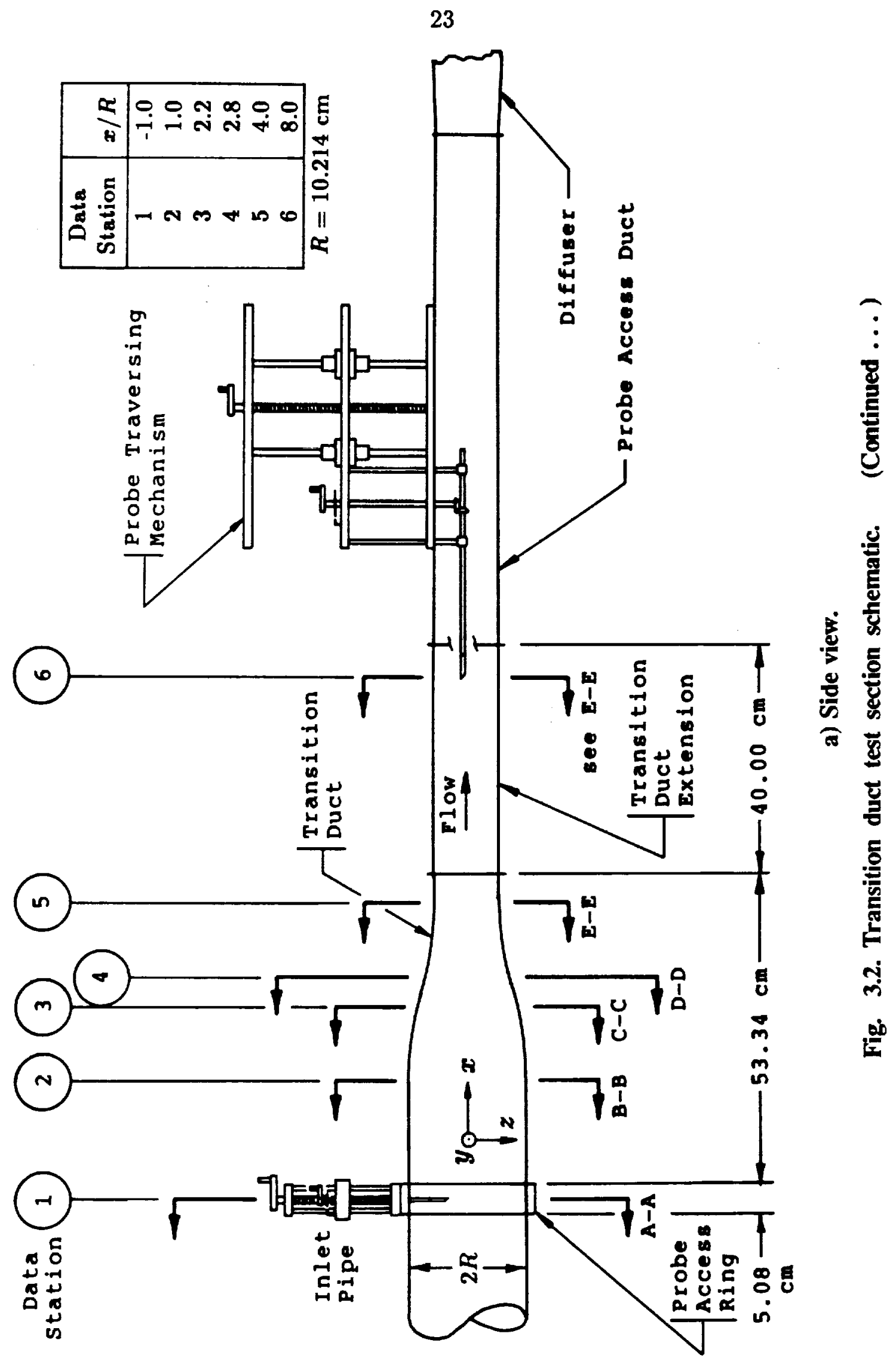



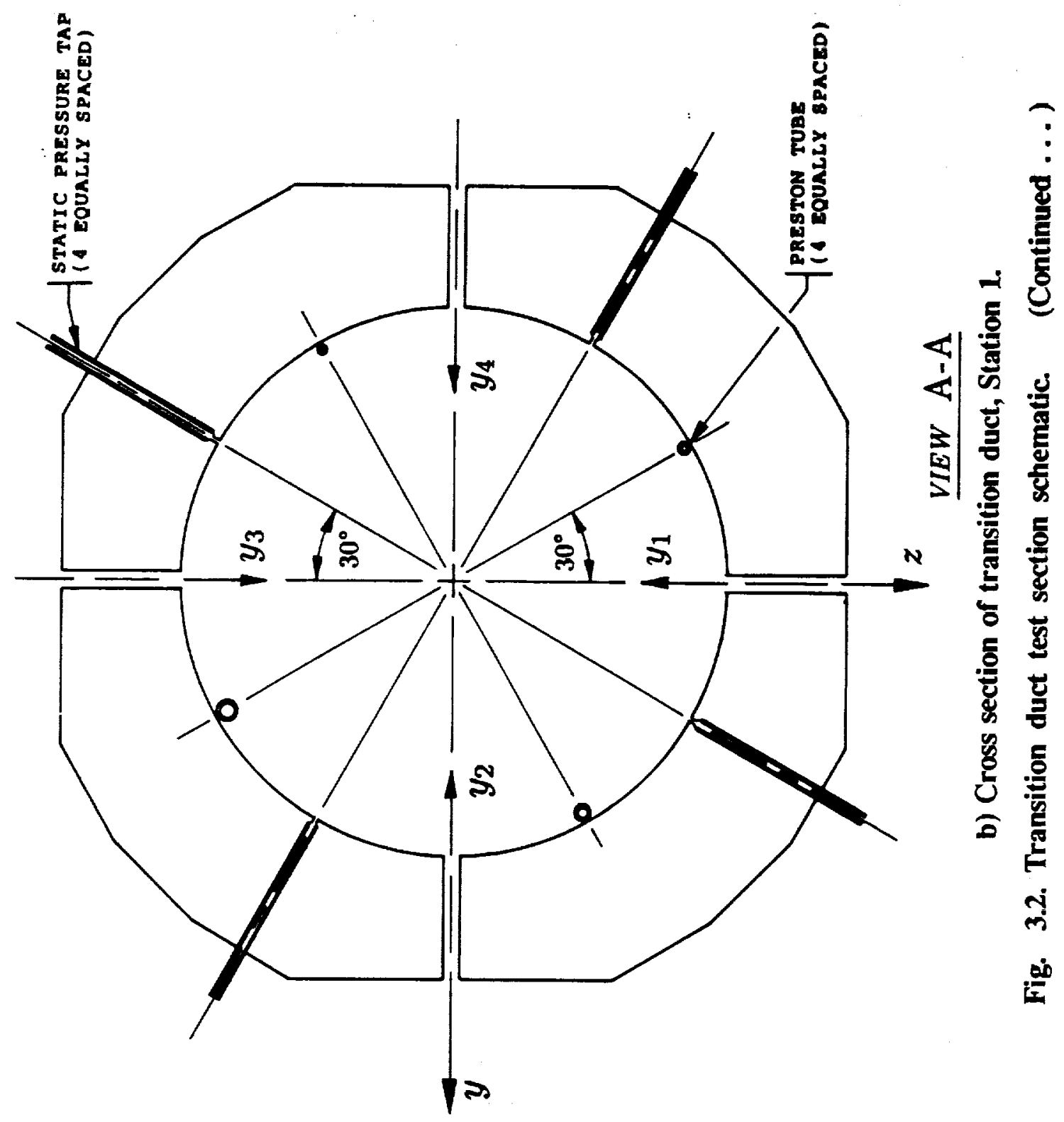


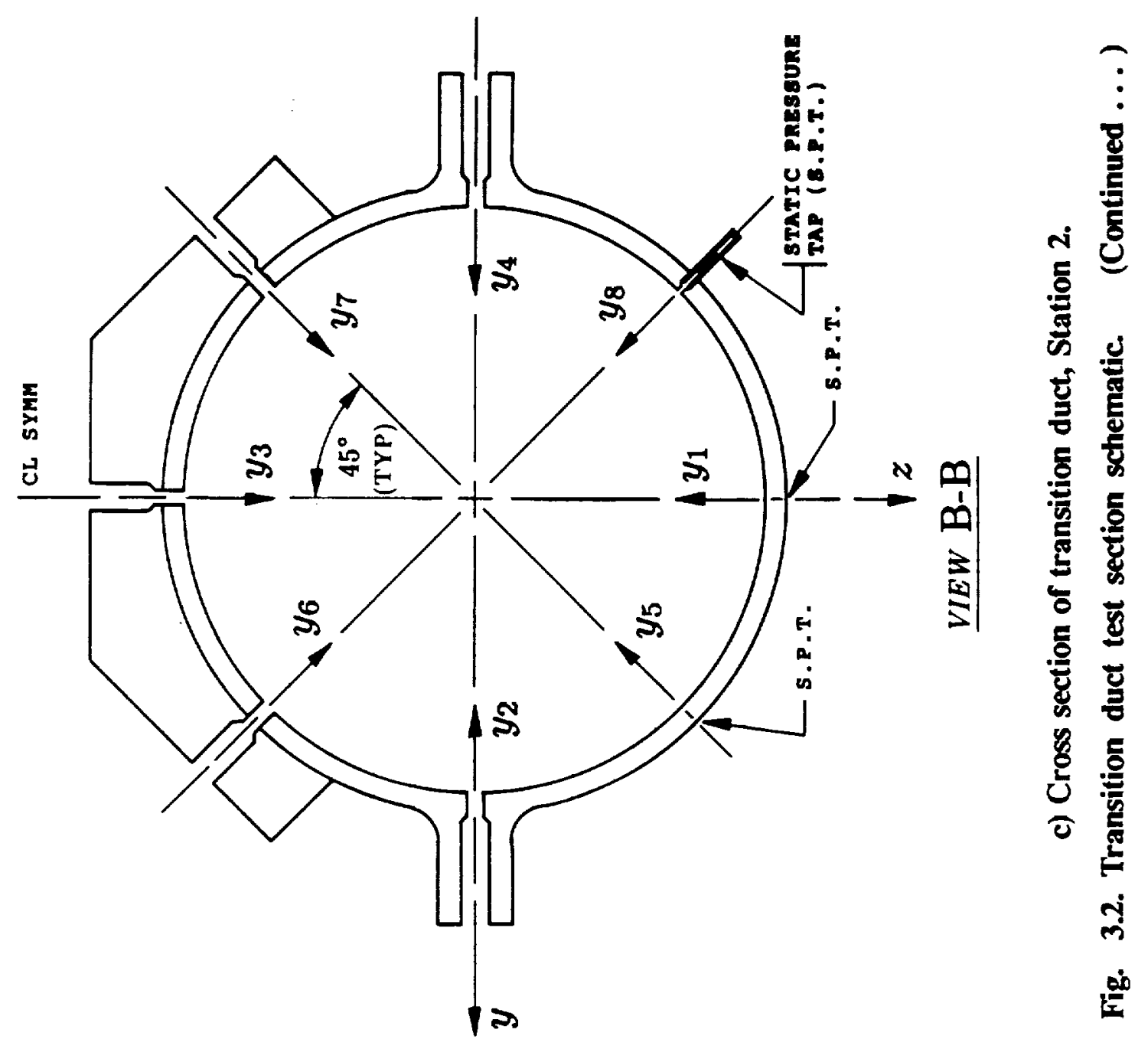



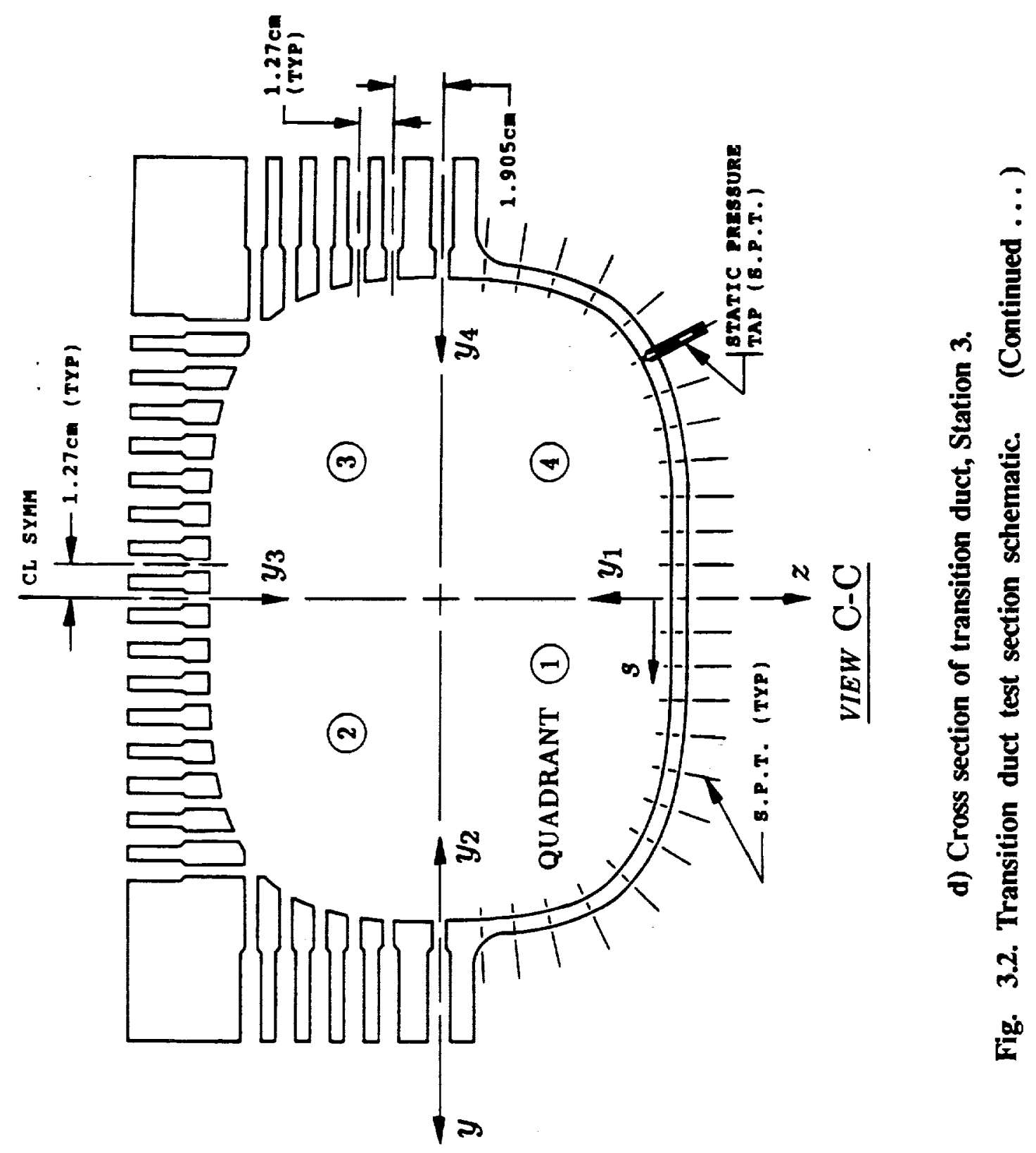

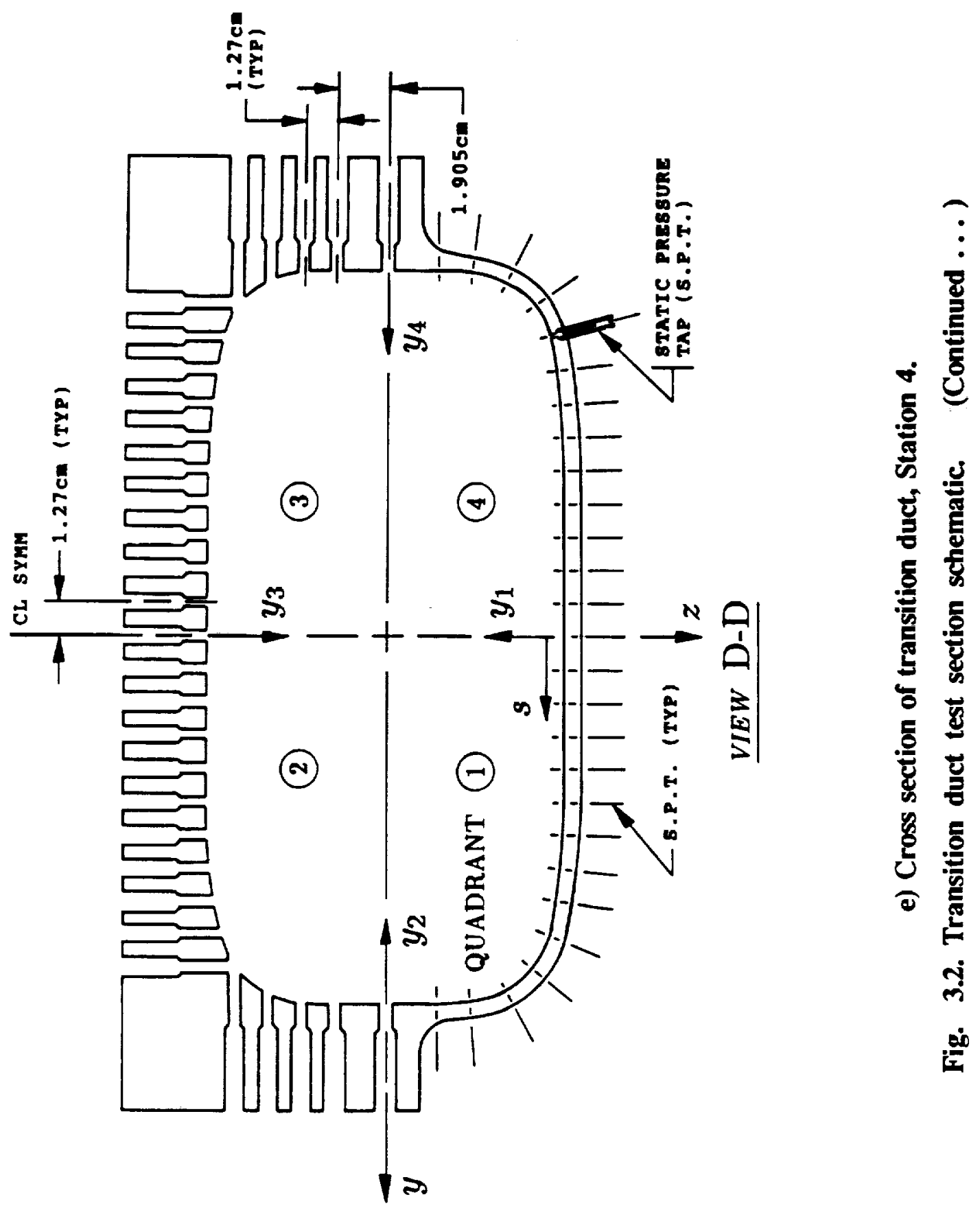


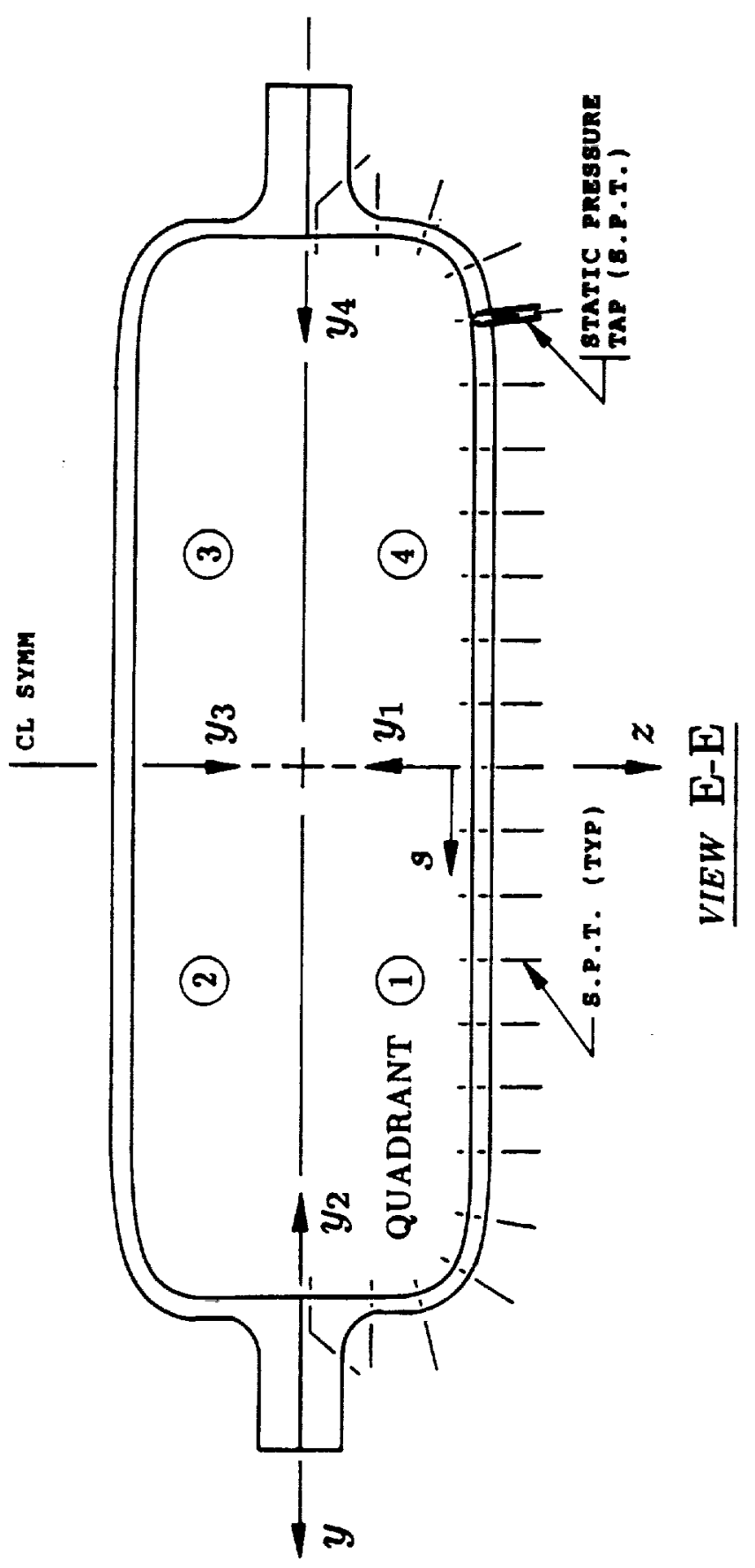

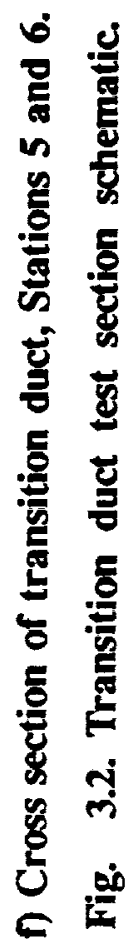




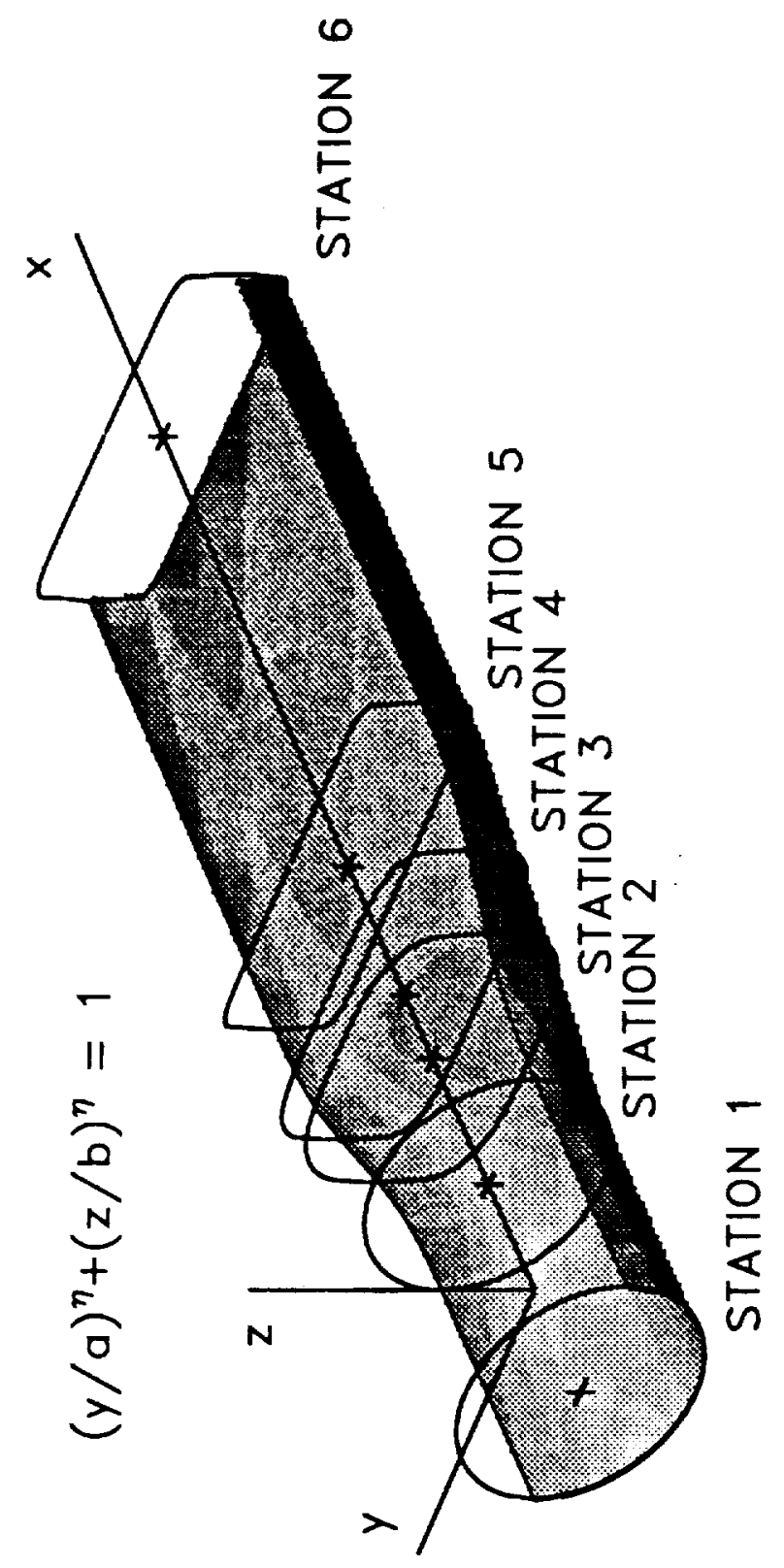




\section{CHAPTER 4}

\section{INLET CONDITIONS \\ (Developing Pipe Flow)}

\subsection{Introduction}

The flow condition at Station 1 corresponds to partially developed turbulent pipe flow. This seemingly simple flow case has been the subject of numerous experimental and theoretical studies. Probably the most outstanding feature of the work which has been done to date is the general lack of agreement among the results of the various experimental investigations. Klein [48], after reviewing more than a dozen turbulent developing pipe flow experiments, attributed the disparities to the extreme sensitivity of upstream flow conditions on flow development. Contraction ratio, boundary layer tripping devices and starting conditions (smooth contraction vs. annular bleed) all influence local flow development downstream of the pipe inlet. In addition, the use of a boundary layer trip makes specification of the virtual origin of the boundary layer difficult.

Of the experimental studies, those due to Barbin \& Jones [49], Richman \& Azad [50] and Reichert \& Azad [51] are the most complete. The facility used by Barbin \& Jones incorporated a 4:1 circular contraction with an annular bleed. A $2.54 \mathrm{~cm}$ wide strip of sand particles placed $5.1 \mathrm{~cm}$ downstream from the leading edge of the pipe served as a boundary layer trip. The coordinate origin was coincident with the leading edge of the pipe. Mean flow and turbulence data were accumulated over a development length of 40 diameters for a bulk Reynolds number of 388,000 . In contrast, the facility used by Richman \& Azad utilized a smooth 89:1 circular contraction connected directly to the pipe. The boundary layer was tripped with a $9 \mathrm{~cm}$ wide strip of \# 16 sandpaper located at the entrance to the pipe. The coordinate origin was chosen to coincide with the downstream edge of the boundary layer trip, although the authors estimate the virtual origin of the boundary layer to be $3 \mathrm{~cm}$ upstream of this location. Data for this study were collected over a development length of 70 diameters for bulk Reynolds numbers of 100,000, 200,000 and 300,000. Reichert \& Azad used the same facility as Richman \& Azad but report that a $5.1 \mathrm{~cm}$ wide strip of unspecified sandpaper was used as a trip. Data for this study were collected over a development length of 70 diameters for seven bulk Reynolds numbers between 112,000 and 306,000 .

The experimental data of Barbin \& Jones and Richman \& Azad were recently used by Martinuzzi \& Pollard $[52,53]$ for comparative purposes in a comprehensive evaluation of 11 turbulence models: 4 algebraic models, $2 \mathrm{k}-\epsilon$ models and 5 Reynolds stress models. All models were implemented in the same computer

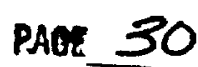


code using equivalent boundary conditions. Although each model had its own merits and drawbacks, the low Reynolds number form of the $k-\epsilon$ model overall performed best.

\subsection{Preliminary Results}

Preliminary measurements were made at Station 1. The purpose of these preliminary measurements was: 1) to establish the range of operating Reynolds number attainable and 2) to determine the lowest operating Reynolds number where fully turbulent flow exists at the first data station. The results of these measurements follow.

The wind tunnel was initially configured with three inlet pipes $(L / D=$ 9) and the boundary layer was allowed to develop naturally within the pipe, i.e. no boundary layer trip was present upstream. Pitot tube surveys of the bounidary layer at Station 1 were obtained for two arbitrary Reynolds numbers, $R e_{b}=234,000$ and 403,000 , the latter Reynolds number being very near the upper operating limit of the wind tunnel. Assuming constant static pressure across the data plane, mean velocity profiles were computed by equation (3.1). These results were plotted in law-of-the-wall coordinates using friction velocities deduced from measurements with the four different diameter Preston tubes shown in Fig. 3.2.b. Agreement with the theoretical law-of-the-wall profile was found to be poor, indicating that the boundary layer was not yet in a fully turbulent state. To promote transition to turbulence, a $2.54 \mathrm{~cm}$ wide strip of \# 36 grit sandpaper was applied around the periphery of the first inlet pipe, $1.27 \mathrm{~cm}$ downstream from the joint, as shown in Fig. 3.1. With the boundary layer trip in place the pitot tube surveys were repeated at nominally the same Reynolds numbers. A comparison of mean velocity profiles with and without the boundary layer trip at the two Reynolds numbers is shown in laboratory coordinates in Fig. 4.1.a. and in law-of-the-wall coordinates in Fig. 4.1.b. These results indicate that the sandpaper trip was effective in producing a fully-turbulent boundary layer at the inlet station.

\subsection{Range of Operating Conditions}

With the boundary layer trip installed, the range of operating Reynolds number was found to be $0<R e_{c l} \leq 479,000\left(0<R e_{b} \leq 442,000\right)$. The presence of the boundary layer trip, however, does not guarantee a turbulent boundary layer over the entire operating Reynolds number range. Indeed, the Preston tube calibration is valid only when law-of-the-wall behavior is present. Since the Preston tubes had to be removed before taking data at downstream stations, and the operating Reynolds numbers for data acquisition had as yet not been established, a correlation between friction velocity and centerline Reynolds number was obtained. This allows the inlet skin friction condition to be known for any operating Reynolds number, provided that the flow is fully turbulent. 
This correlation for the four Preston tubes is shown in Fig. 4.2. Agreement is generally good for the different diameter tubes, with the exception of results referred to the largest tube at centerline Reynolds numbers above 425,000 . The reason for this deviation is that the boundary layer thins as the Reynolds number is increased, allowing the largest tube to extend beyond the law-of-the-wall region where the Preston tube calibration is valid. Another small deviation, which was repeatable, occurs in the neighborhood of $R e_{c l}=220,000$. The cause for this is unknown, but may be associated with an unsteadiness in the wind tunnel at that operating condition. Also indicated in Fig. 4.2 is the approximate region where fully turbulent flow begins. The determination of this region will be discussed shortly. Using the points outside the transition region, an empirical correlation was determined that describes friction velocity behavior at Station 1:

$$
\frac{U_{\tau}}{U_{c l}}=0.255-0.0712 \log _{10}\left(R e_{c l}\right)+0.00577 \log _{10}^{2}\left(R e_{c l}\right)
$$

which is applicable in the range $97,000<R e_{c l}<460,000$.

A simple indication of fully turbulent flow is whether or not law- of-the-wall behavior is observed within the boundary layer. Fig. 4.1 shows that law-of-thewall behavior exists at $R e_{c l}=255,000\left(R e_{b}=234,000\right)$. To estimate the lower limit of fully turbulent flow, Pitot profiles were measured at two relatively low Reynolds numbers, $R e_{c l}=55,000$ and $97,000\left(R e_{b}=52,000\right.$ and 88,000). These results are plotted in laboratory coordinates in Fig. 4.3.a and in law-of-the-wall coordinates in Fig. 4.3.b. The mean profile at the lower Reynolds number is considerably thinner than the higher Reynolds number profile and appears to have a laminar-like shape. In addition, this profile clearly does not follow lawof-the-wall behavior. An examination of the boundary layer shape factor, $H_{12}$, indicates, however, that the flow is not fully laminar either. For the present profile a shape factor of $H_{12}=2.0$ was measured, whereas typical values for the fully laminar and fully turbulent profiles are 2.6 and 1.4, respectively [48]. Conversely, the higher Reynolds number profile corresponds to a thick boundary layer that agrees well with the law-of-the-wall and has a measured shape factor of 1.4 .

Based on the above results, it was decided that data for the turbulent flow case would be taken at bulk Reynolds numbers of 88,000 and 390,000 , for which the inlet flow at data Station 1 (refer to Fig. 3.2.a) should be fully turbulent. The upper value represents the maximum operating speed of the wind tunnel throttled back slightly to allow adjustment for variations in ambient conditions.

\subsection{Results and Discussion}

For the present study, data were collected at a development length $x_{p} / D$ $=9$, where $x_{p}=0$ corresponds to the pipe inlet. It is likely that the effective 
length is slightly larger, inasmuch as a boundary layer is already developing in the contraction and the sandpaper trip tends to thicken the boundary layer artificially. The transverse flow angle in the azimuthal direction was measured using a pressure-nulling technique with a two-tube Conrad probe. The probe was first nulled at the pipe centerline and then the flow angle along the $y_{1}$ traverse (see Fig. 3.2.b) was measured relative to the centerline null value. For both operating Reynolds numbers, the computed transverse flow velocity $\left(U_{\theta}\right)$ was found to be less than $0.25 \%$ of the local axial velocity component across the entire traverse.

Mean velocity profiles plotted in laboratory coordinates and in law-of-thewall coordinates along four equally-spaced radial traverses at Station 1 are shown for $R e_{b}=88,000$ and 390,000 in Figs. 4.4 and 4.5, respectively. The excellent peripheral symmetry of the flow and agreement with law-of-the-wall behavior are readily apparent. The non-dimensional Preston tube diameters $\left(d^{+}\right)$used to deduce skin friction are indicated on the law-of-the-wall plot. The largest tube for the higher Reynolds number case extends slightly into the wake region and, as a result, skin friction values deduced from this tube were slightly higher than those for the three smallest tubes. Friction velocities deduced from the three smallest tubes deviated by less than $0.3 \%$ from their mean value. Boundary layer thickness, integral parameters, skin friction and centerline turbulence intensity at Station 1 are summarized in Table 4.1. The integral parameters shown are averages of values computed from the four individual traverses.

Table 4.1. Flow condition at Station 1

\begin{tabular}{ccc}
\hline & $R e_{b}=88,000$ & $R e_{b}=390,000$ \\
\hline$\delta / R$ & 0.3141 & 0.2855 \\
$\delta_{1} / R$ & 0.0448 & 0.0383 \\
$\delta_{2} / R$ & 0.0312 & 0.0281 \\
$\delta_{3} / R$ & 0.0545 & 0.0497 \\
$B$ & 0.0896 & 0.0765 \\
$H_{12}$ & 1.438 & 1.364 \\
$H_{32}$ & 1.748 & 1.771 \\
$\tau_{w} / \tau_{w, F D}$ & 1.00 & 0.96 \\
$\left(u^{\prime} / U\right)_{c l}$ & 0.008 & 0.003 \\
$R=10.214 \mathrm{~cm}$ & &
\end{tabular}

As expected, the boundary layer is somewhat thicker for the lower Reynolds number case. The $H_{12}$ shape factors agree well with those reported by Klein for fully turbulent, developing pipe flow. The wall shear stress in Table 4.1. has been normalized by the fully-developed pipe flow shear stress $\tau_{w, F D}$ which was determined from a relation given by Eppich [40]. Data presented by Reichert \& Azad show that when a smooth contraction is used as the starting condition, 
the skin friction at the entrance to the pipe is below the fully-developed value and that the skin friction approaches the fully-developed value in an oscillatory manner. In contrast, Barbin \& Jones data show that when a sharp edge is used as a starting condition, the skin friction at the beginning of the pipe is above the fully-developed value but asymptotically reaches the fully-developed value within the first 15 diameters. The present results show that the wall shear stress at $x_{p} / D$ $=9$ is very near the fully-developed value. At the lower Reynolds number, the flow was much more susceptible to ambient disturbances and this is reflected in the larger data scatter and higher freestream turbulence levels.

Reynolds normal and shear stress distributions at Station 1 for $R e_{b}=88,000$ and 390,000 are shown in Fig. 4.6. Experimental results obtained by Barbin \& Jones and Richman \& Azad at a comparable location $\left(x_{p} / D=10\right)$ and operating Reynolds numbers are also shown for purposes of comparison. The turbulence levels shown in these plots near the pipe centerline should not be considered representative of the core flow turbulence intensity, inasmuch as the output from the probe used to measure the distributions exhibited a small sinusoidal trace on the oscilloscope when positioned in the core flow. This noise was attributed to vortex shedding from the probe prongs when the probe was positioned normal to the axial mean flow. The turbulence intensity values shown in Table 4.1. are correct and were measured independently using a forward-facing probe fixed at the duct centerline.

The Reynolds stress profiles in Fig. 4.6 are seen to be nearly Reynolds number independent over the range considered. Based on the turbulence results, it appears that the boundary layer in the present study is thinner than that of the other investigators. In the near-wall region, the normal stresses are seen to be significantly higher than those due to Barbin \& Jones. The shear stress in the near-wall region agrees well with the results of Richman \& Azad and with the fully-developed distribution.

The more rapid boundary layer growth associated with Richman and Azad's experiments can probably be attributed to the rougher and wider sandpaper tripping device used in their study, which extended over a streamwise width of 0.9 pipe diameters in comparison to the width used in the present study $(0.12$ pipe diameters). In Barbin \& Jones' experiments, a sand grain trip of the same relative width as that used in the present study was employed. By-pass bleed through an annular gap between the pipe and a 4:1 contraction ratio nozzle was used to promote spanwise flow uniformity across the pipe inlet. In reference to Fig. 1 of Ref. 49, the pipe in Barbin \& Jones' experiments extended upstream into the nozzle, so that the pipe inlet could have been in a region where the flow was still converging, and the flow may have separated at the wedge-shaped lip of the pipe. This event, if it occurred, could have promoted more rapid boundary layer growth in comparison to that observed in the present study. If local flow 
separation and reattachment did occur near the pipe inlet in Barbin \& Jones experiments, then one would also anticipate that flow in the near-entrance would not be in local equilibrium. This conjecture is supported to some extent by the behavior of the velocity profiles measured by Barbin \& Jones at $x_{p} / D=1.5,4.5$, 7.5 , all of which show significant departures from the law-of-the-wall across the width of the boundary layer (refer to Fig. 6 of Ref. 49).

The differences between corresponding distributions in Figs. 4.6.a and 4.6.b could be reconciled further if a code were available for predicting developing turbulent pipe flow with high accuracy starting from a low-turbulence level, uniform inlet flow condition. Unfortunately, predictions of a given variable in the entrance region based on various $k$ - $\epsilon$ type transport equation models show differences which are the same order of magnitude as those which exist among the various data sets for nominally the same operating conditions [52,53]. Further work will be required in order to: (1) provide comprehensive data which characterize disturbance-free, turbulent boundary layer growth in a circular pipe, and (2) develop a code which can predict this behavior, recognizing that the data may have to be corrected for virtual origin (streamwise displacement) effects. It should be noted here that the present data set at Station 1 appears to be relatively free of upstream disturbances, inasmuch as the core flow at this station is uniform and at a relatively low turbulence level (as indicated in Table 4.1) and velocity profiles measured along four radial traverses $90^{\circ}$ apart are symmetric and in excellent agreement with the law-of-the-wall (refer to Figs. 4.4 and 4.5).

The turbulence kinetic energy $k$ and its rate of production are shown for the two operating Reynolds numbers in Figs. 4.7 and 4.8, respectively. In Fig. 4.7 , the conventional wall function value based on $C_{\mu}=0.09$ is shown. The lower Reynolds number data agree well with the limiting value, but the higher Reynolds number data is slightly high. The higher kinetic energy in the near-wall region at the higher Reynolds number is supported by the kinetic energy production distributions shown in Fig. 4.8. Also indicated are results based on the data of Barbin and Jones, which lie considerably below the present results. The results shown in Figs. 4.7 and 4.8 will be used later as reference distributions to assess the distorting effect of the transition duct geometry on the turbulence field. Non-dimensional turbulence structure parameters, which will also be useful for latter comparisons, are the shear stress correlation $R_{u v}=\overline{u v} / u^{\prime} v^{\prime}$ and the shearenergy ratio parameter $a 1=\overline{u v} / 2 k$. These parameters are sometimes prescribed as constants in Reynolds-averaged turbulence models. Distributions measured at Station 1 are shown in Figs. 4.9 and 4.10, respectively. The shear stress correlation may be interpreted as the ability of the normal stresses to generate shear stress. In a two-dimensional boundary layer, this parameter is nearly constant within the layer with a typical value of about 0.45 [54]. The shear-energy ratio parameter has also been observed to be nearly constant in a two-dimensional boundary layer with a value of about 0.15 . With the exception of the scatter 
near the boundary layer edge (at $r / R \approx 0.72$ ) for the lower Reynolds number, the present results generally agree well with these values. 


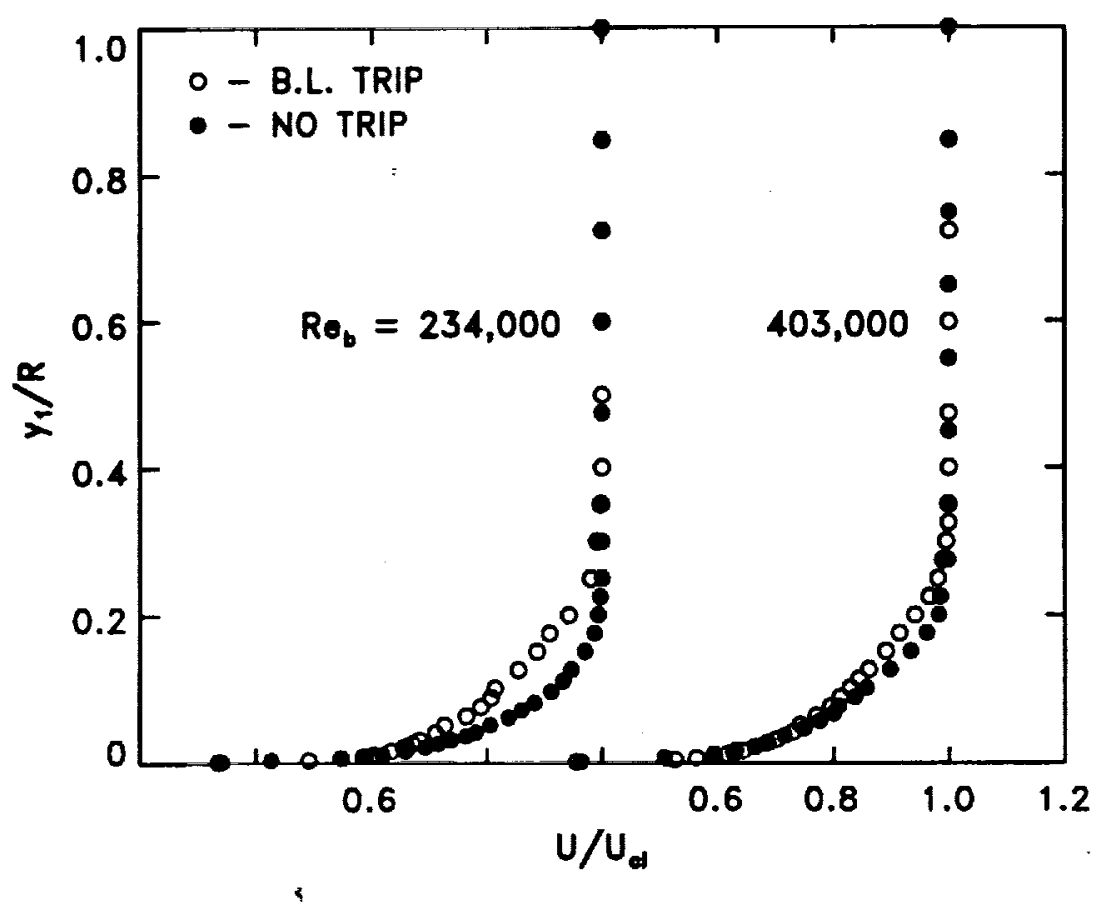

a) Mean velocity profiles.

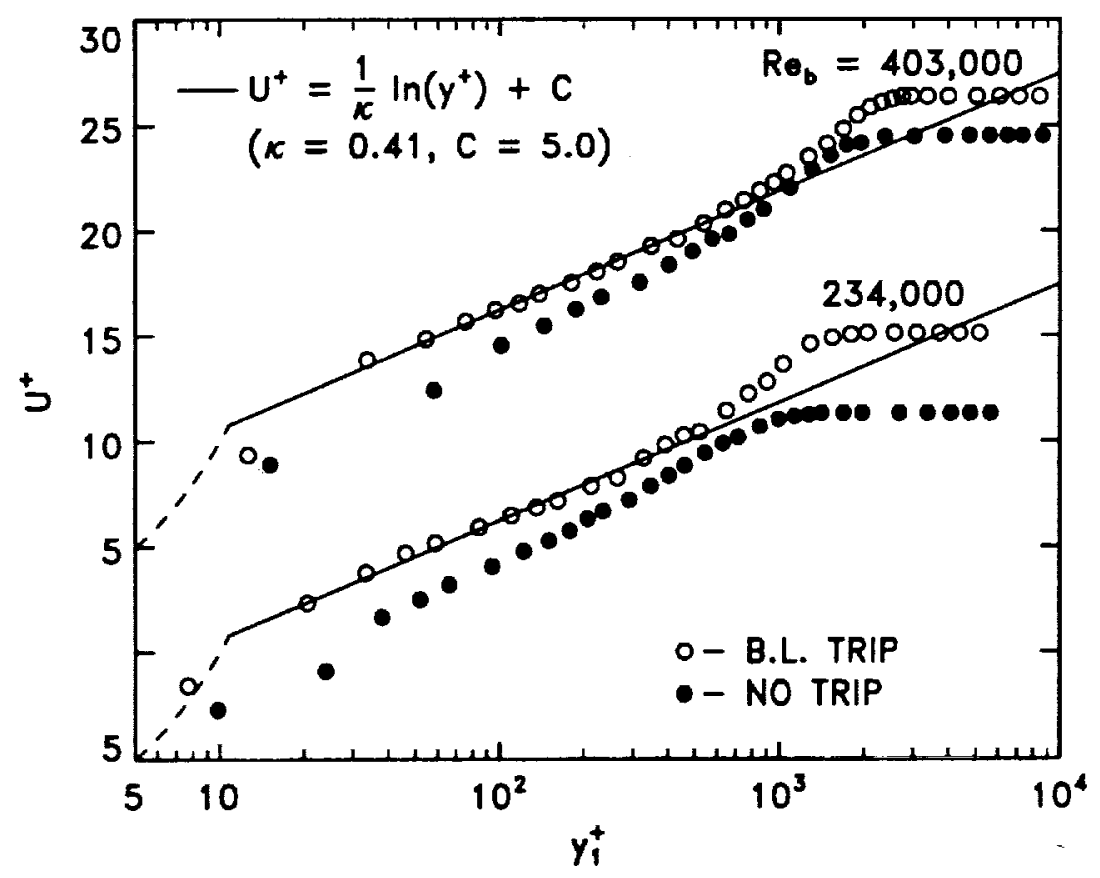

b) Law-of-the-wall profiles.

Fig. 4.1. Tripped and non-tripped boundary layer profiles at Station $1, \mathrm{Re}_{\mathrm{b}}=\mathbf{2 3 4 , 0 0 0}$ and 403,000 . 


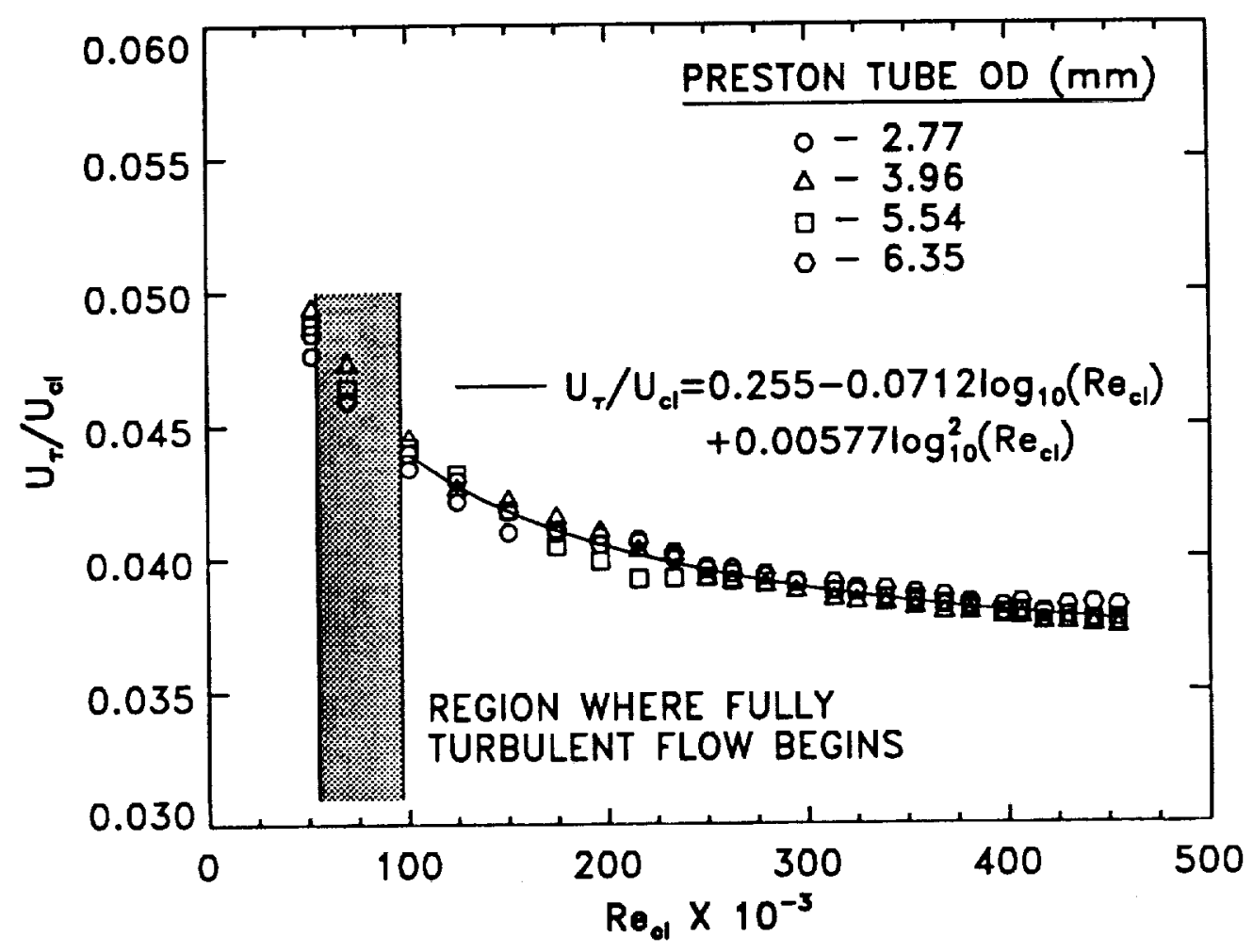

Fig. 4.2. Friction velocity vs. centerline Reynolds number at Station 1. 
40

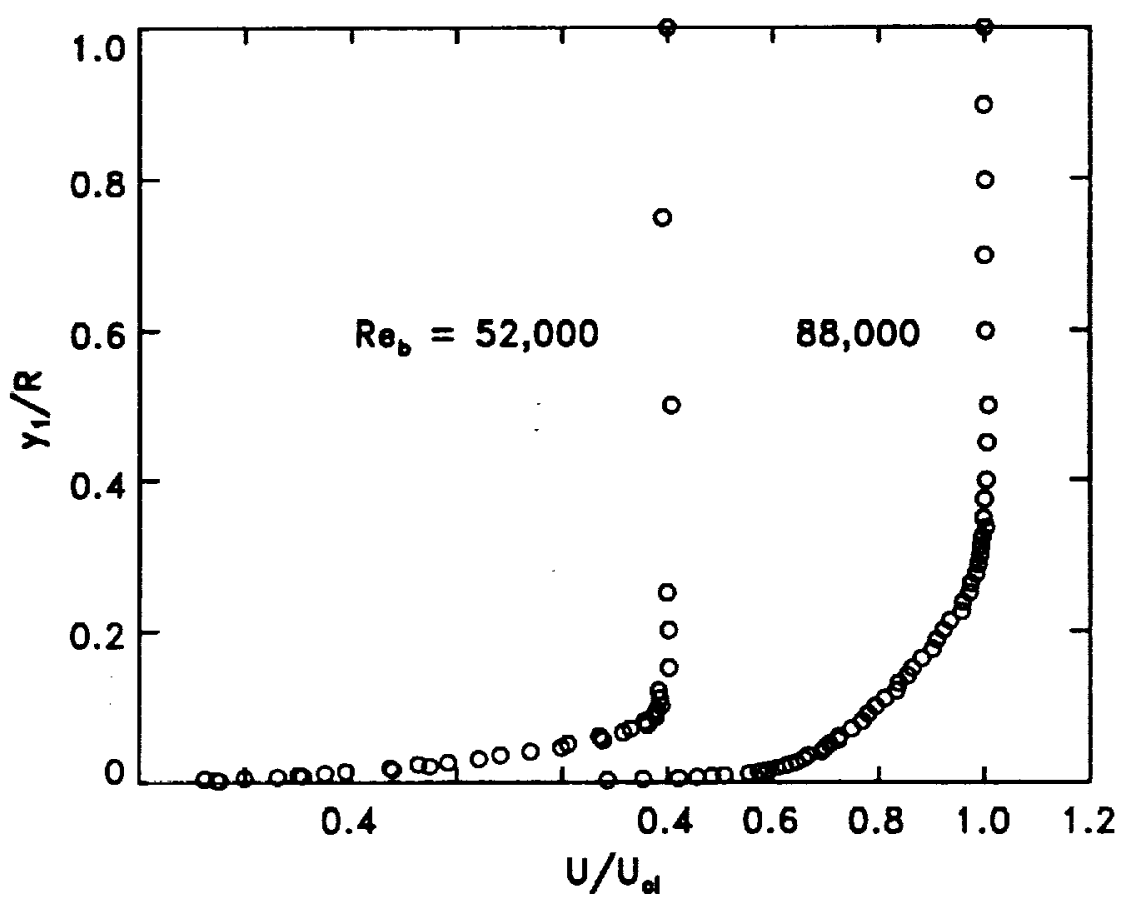

a) Mean velocity profiles.

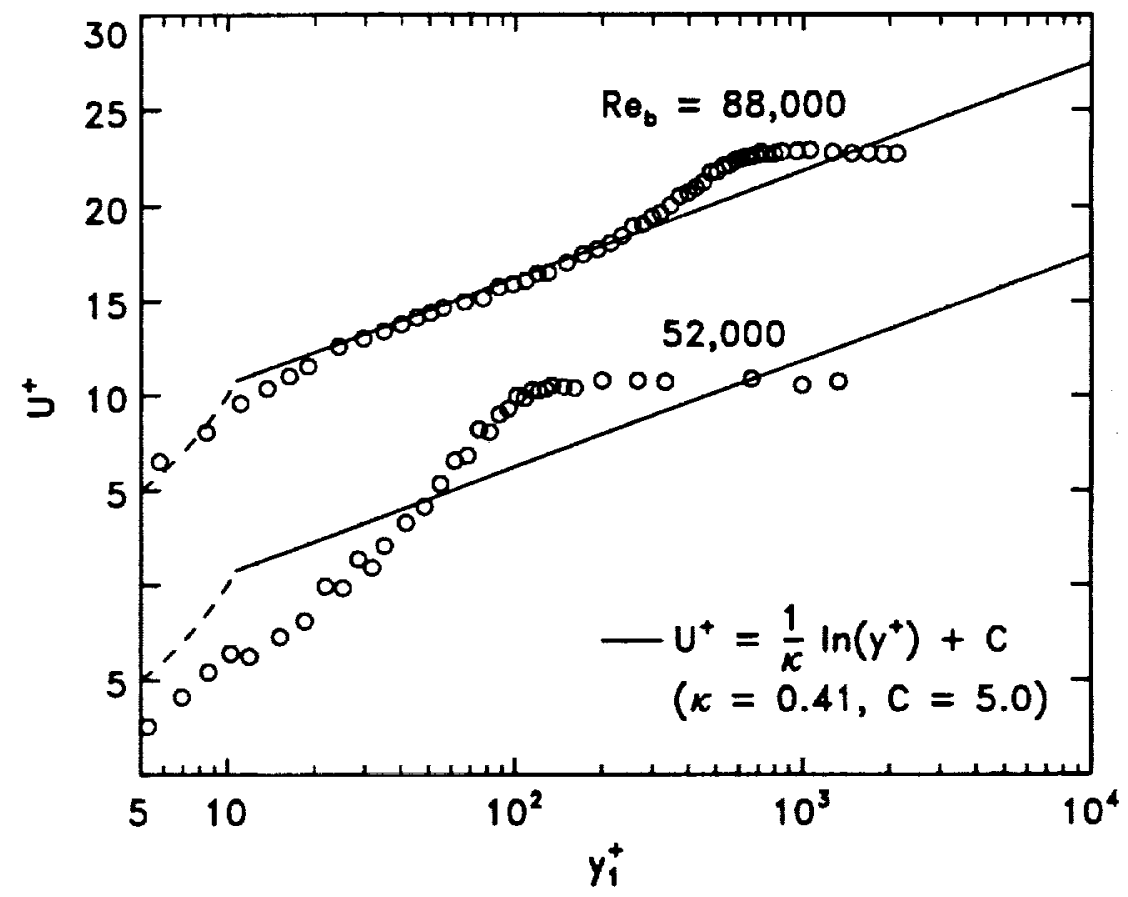

b) Law-of-the-wall profiles.

Fig. 4.3. Tripped boundary layer profiles at Station $1, \mathrm{Re}_{\mathrm{b}}=52,000$ and 88,000 . 


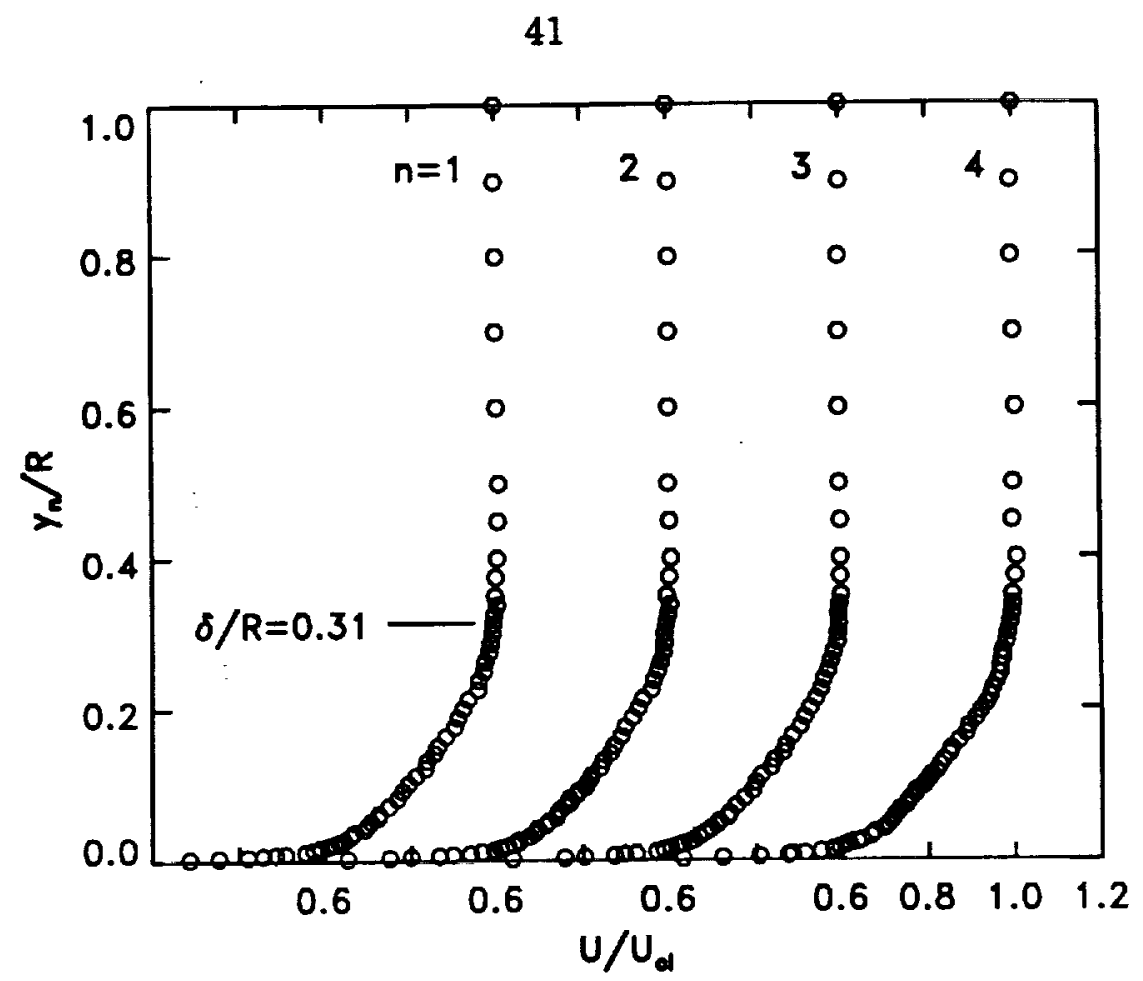

a) Mean velocity profiles.

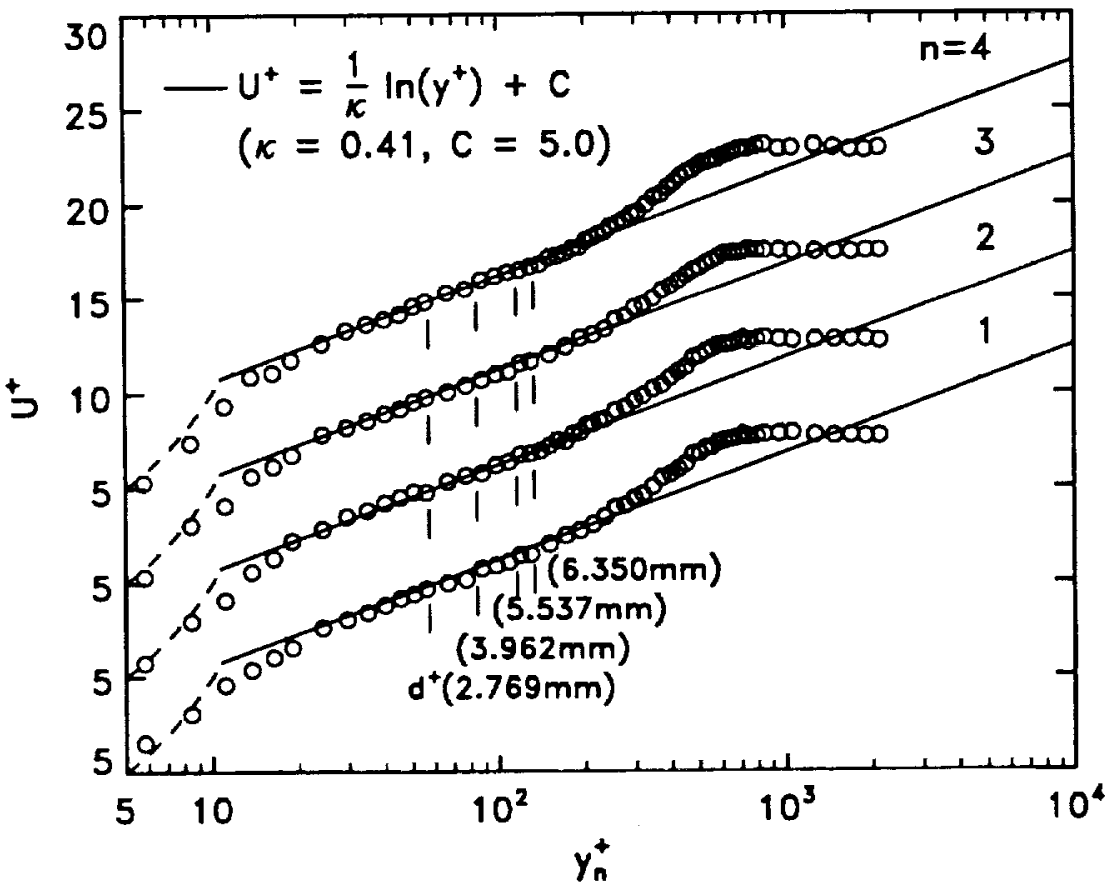

b) Law-of-the-wall velocity profiles.

Fig. 4.4. Boundary layer profiles at Station $1, R_{b}=88,000$. 


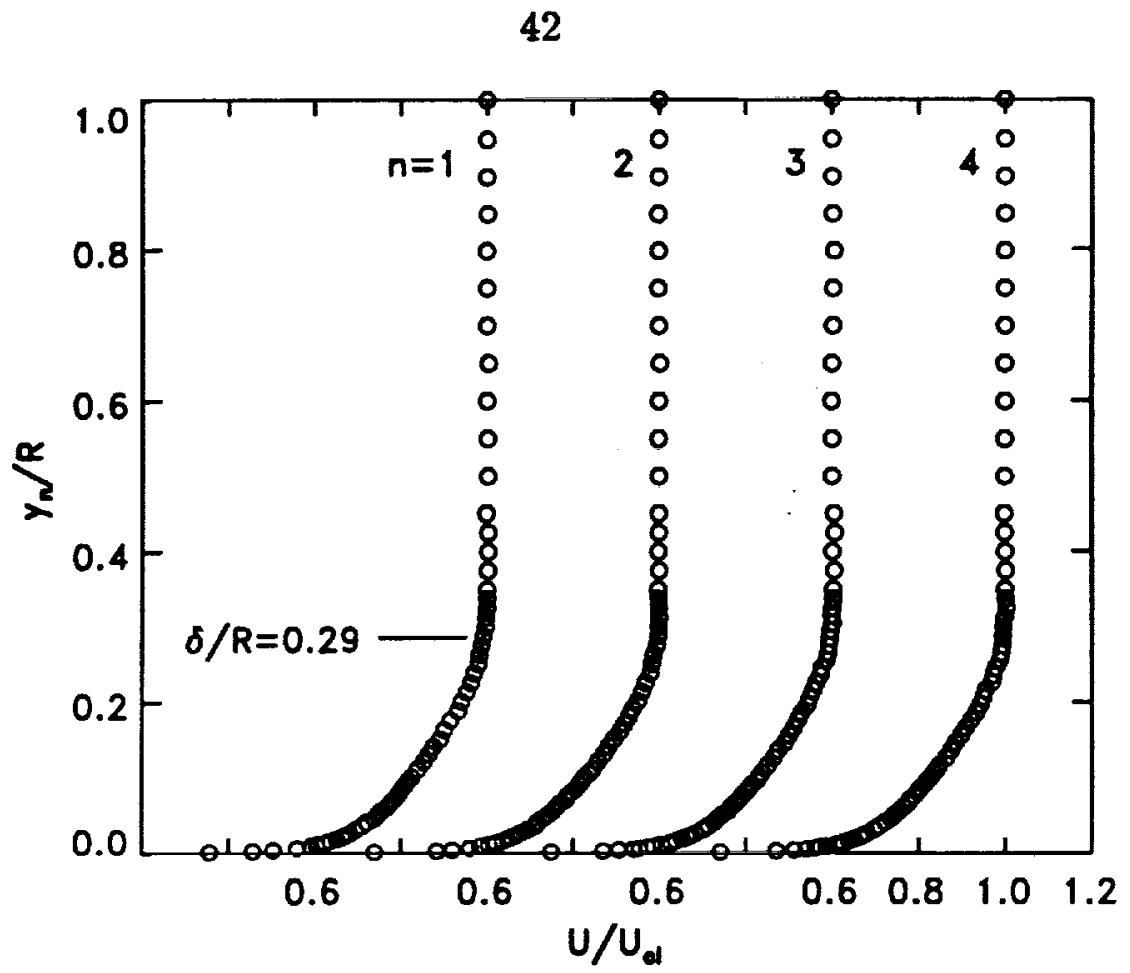

a) Mean velocity profiles.

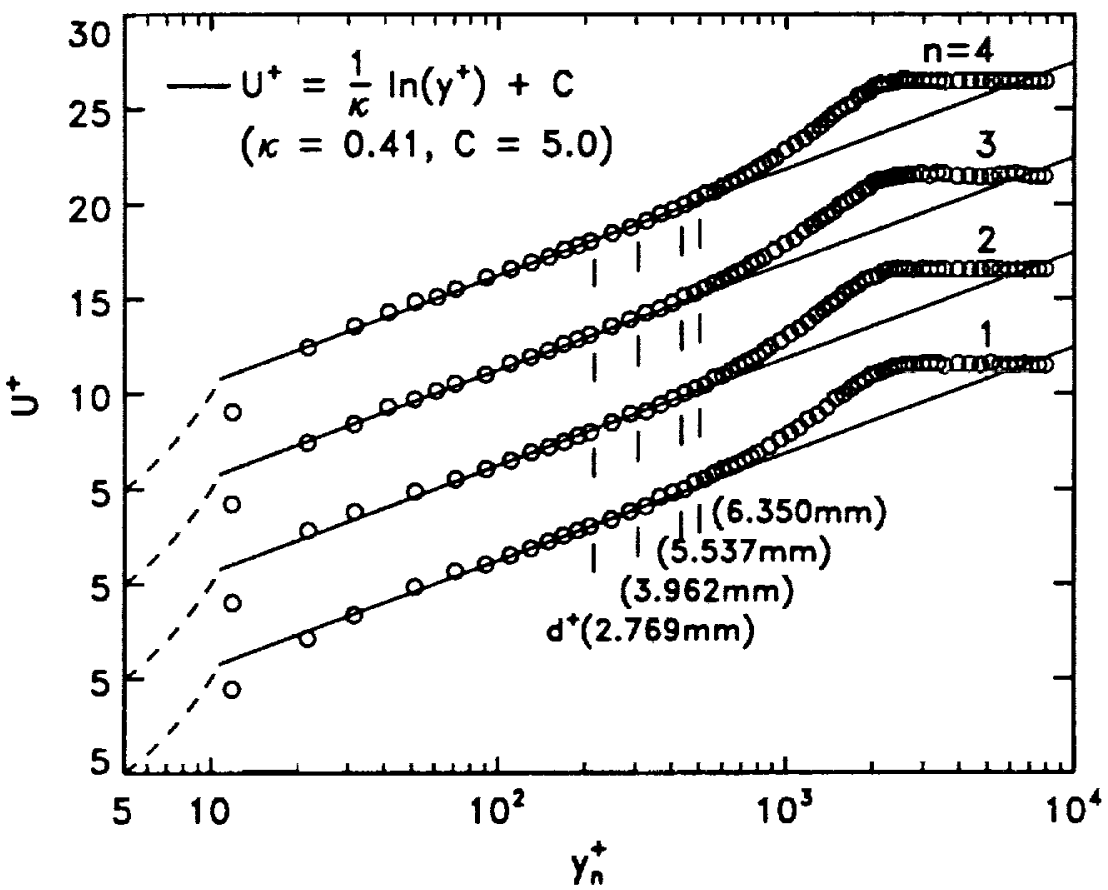

b) Law-of-the-wall velocity profiles.

Fig. 4.5. Boundary layer profiles at Station $1, R_{b}=390,000$. 


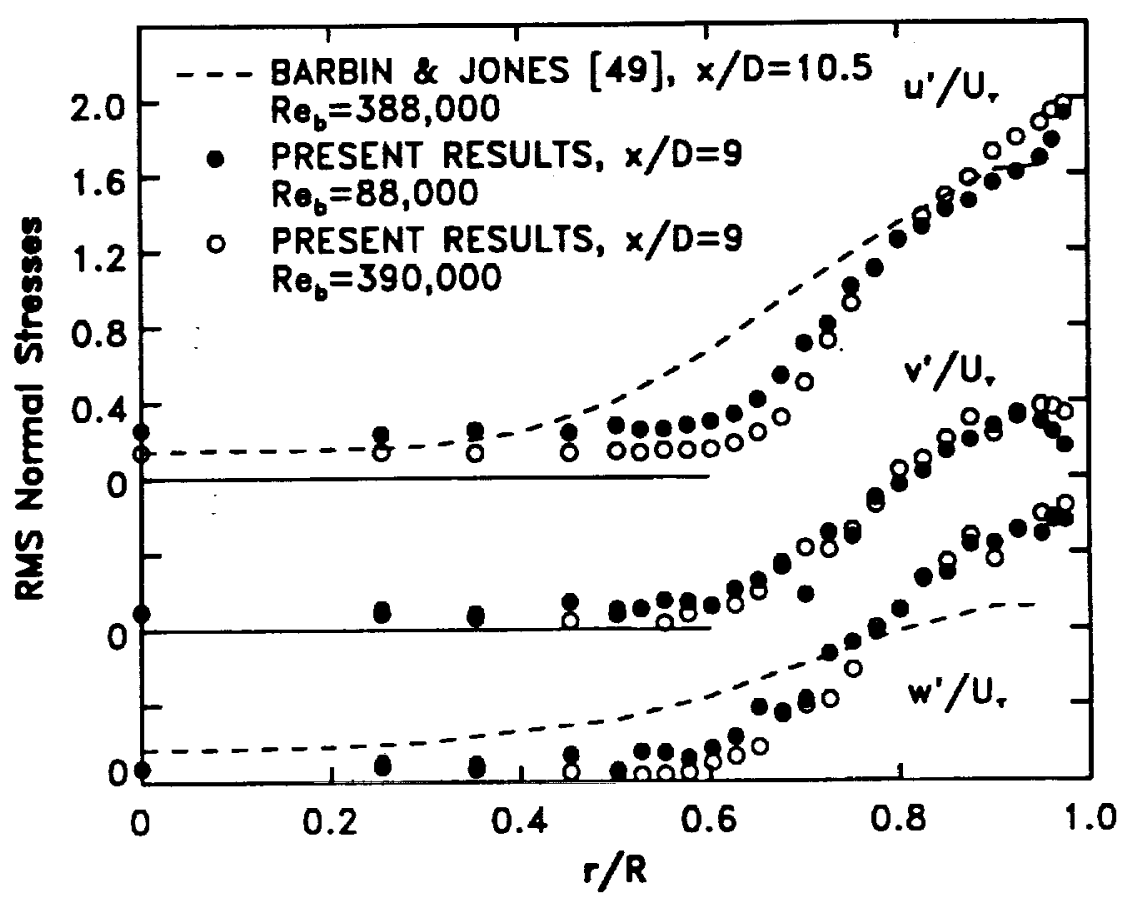

a) RMS normal stresses.

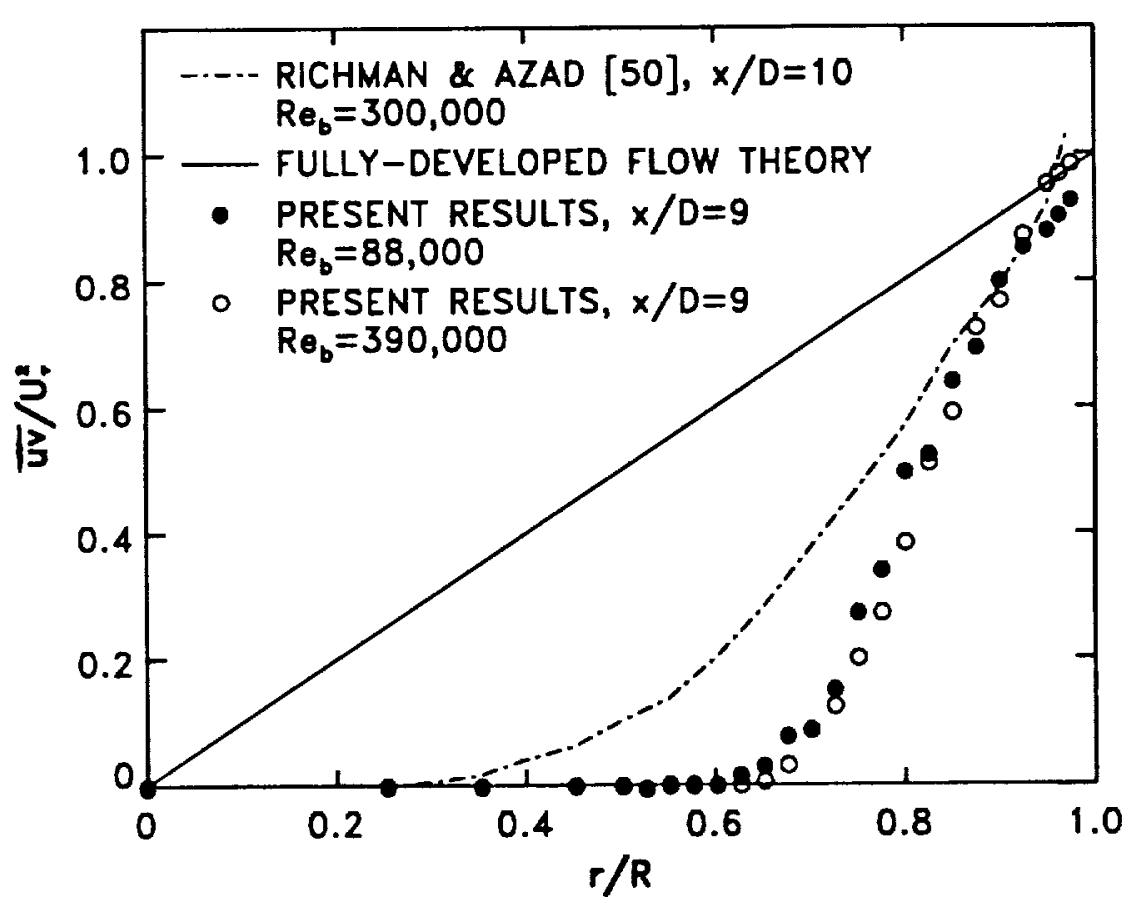

b) Shear stress.

Fig. 4.6. Reynolds stress distributions at Station 1. 
44

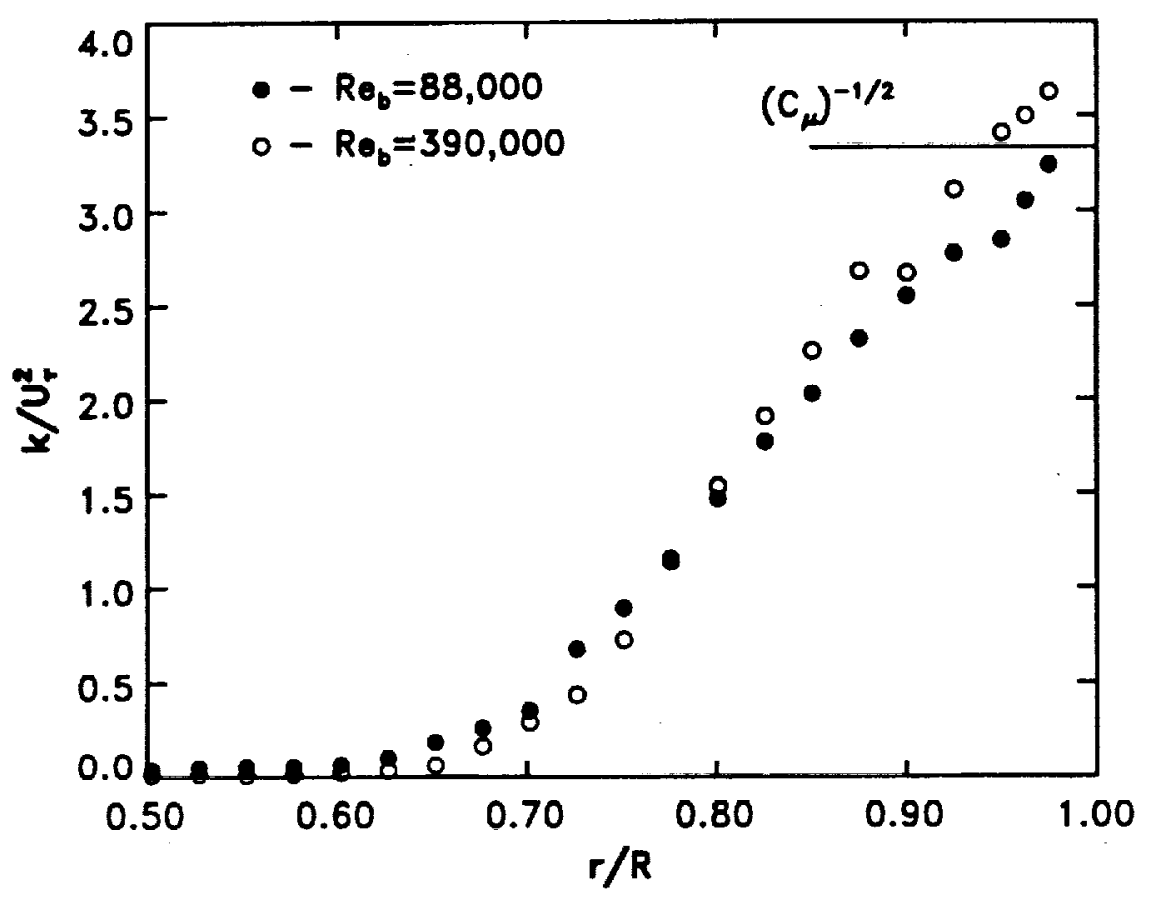

Fig. 4.7. Turbulent kinetic energy distributions at Station 1.

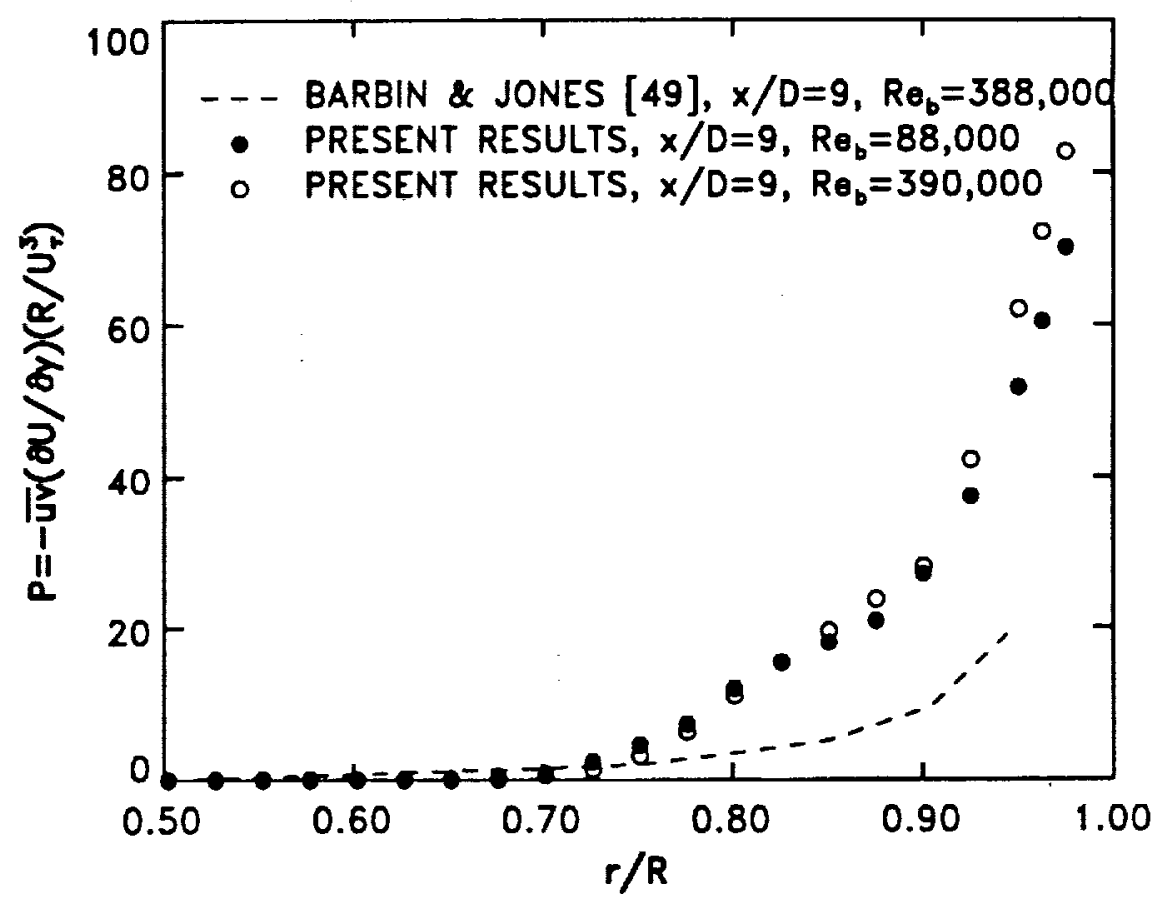

Fig. 4.8. Production of t.k.e. distributions at Station 1. 


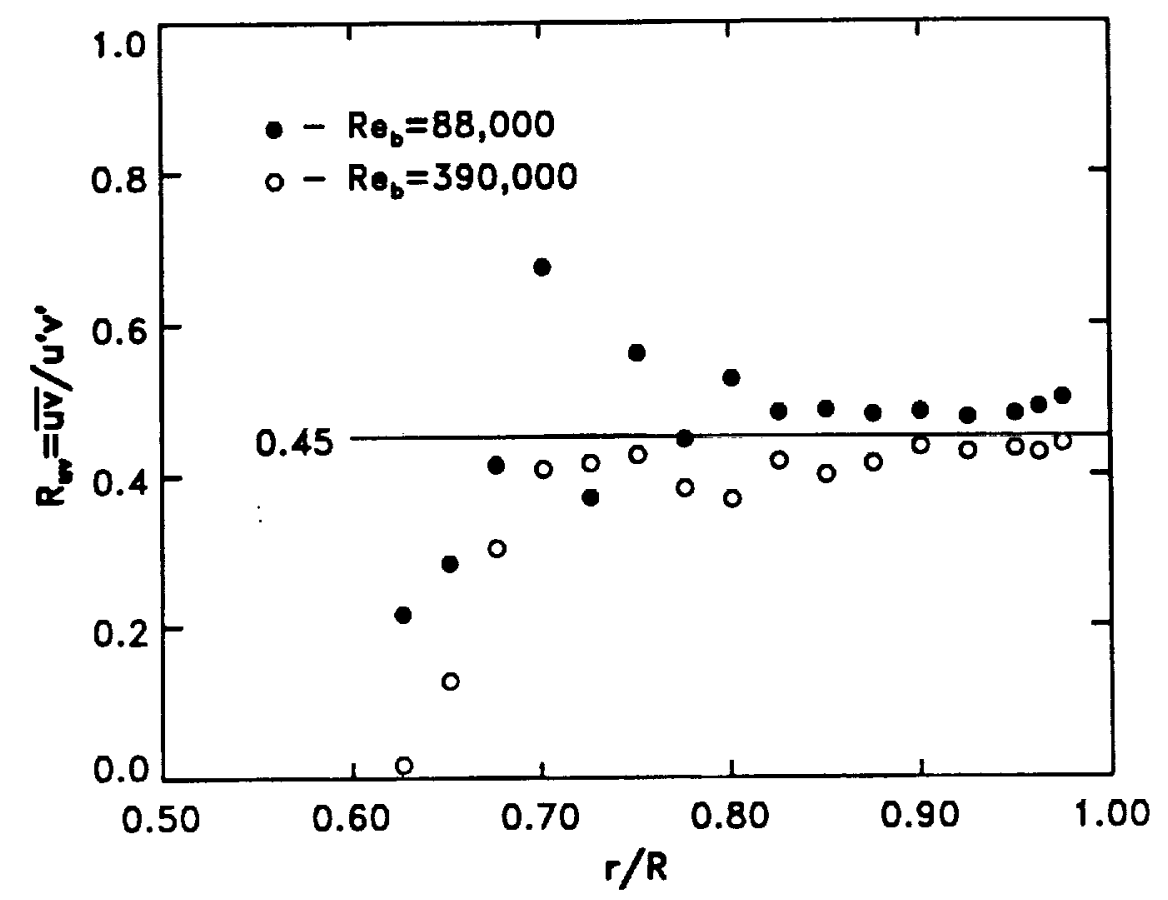

Fig. 4.9. Shear stress correlation distributions at Station 1.

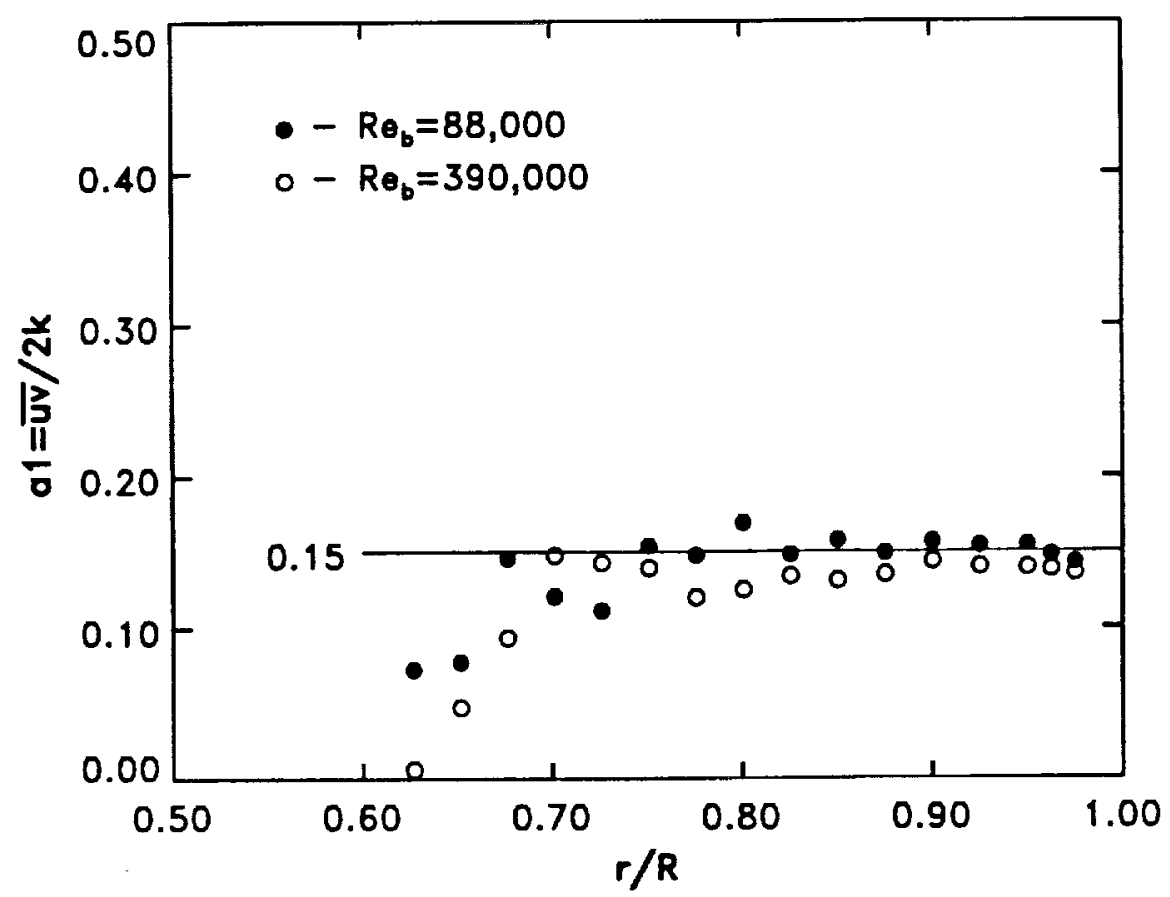

Fig. 4.10. Shear-energy ratio parameter distributions at Station 1. 


\section{CHAPTER 5}

\section{RESULTS AND DISCUSSION}

\subsection{Introduction}

The sequence in which data were collected in the transition duct is as follows. The first transition duct was installed and the peripheral wall static pressure distributions at each data station were obtained. Data were then accumulated at Station 5 to check flow symmetry and determine the extent of Reynolds number dependence of the flow. The transition duct extension was then installed and data were accumulated at Station 6 . Finally, the second transition duct was installed and measurements were made at the intermediate Stations 3 and 4 . No data were taken at Station 2. Total pressure and mean velocity were deduced from hot-wire measurements made at Stations $3,4,5$ and 6 . Turbulence quantities, however, were only measured at Stations 5 and 6 since the anticipated vortex structure was not well defined until Station 5.

At all stations, symmetry was assumed about the $x-z$ plane and, as such, data were accumulated only in Quadrants 1 and/or 2 shown in Figs. 3.2.d-3.2.f. At Stations 3 and 4, due to the arrangement of the probe access holes (see Figs. 3.2.d and 3.2.e), data were taken only in Quadrant 2. For presentation purposes, the data were imaged about the $y$-axis to show an entire duct half. All subsequent plots where data have been imaged are so indicated. The spacing between data points along individual traverses varied from $1.27 \mathrm{~cm}$ in the core region to 0.127 $\mathrm{cm}$ near the wall surface. At Stations 5 and 6 , rather than assuming symmetry about the midplane $z=0$, measurements were made in both Quadrants 1 and 2. Data were taken at selected points on a $63 \times 19$ rectangular grid $(0.254 \mathrm{~cm}$ spacing) in each quadrant. The spacing between data points varied from 2.54 $\mathrm{cm}$ in the core region to $0.254 \mathrm{~cm}$ in regions of large gradients. Approximately 400 data points were taken in the duct half. It should be emphasized here that all subsequent contour results for Stations 5 and 6 are based on data obtained in both quadrants, so that the level of symmetry about the $x-y$ plane of the duct is a direct indication of the quality of the flow and of the measurement techniques employed in this study. For plotting purposes, the measured results at all stations were interpolated onto an evenly spaced mesh by means of a monotonic derivative spline interpolant [55]. This method of interpolation performs no smoothing and guarantees no overshoot of the data.

\subsection{Static Pressure Distribution}

Wall static pressure distributions were measured along the periphery ( $s$ coordinate, refer to Figs. 3.2.d through 3.2.f) of the lower half of the duct at 
Stations $3,4,5$, and 6 . Peripheral wall static pressure coefficient distributions are shown for $R e_{b}=88,000$ and 390,000 in Fig. 5.1. The normalizing dimension $s_{r e f}$ is $1 / 4$ of the duct circumference at a given data station. Solid symbols represent static pressure measured along the duct centerline. The excellent spanwise regularity at all stations supports the assumption of symmetry about the $x-z$ plane. The primary difference between results referred to the two Reynolds numbers is that the net pressure coefficient drop along the duct is larger for the lower Reynolds number case and is the result of increased viscous losses associated with the more rapid boundary layer growth.

Local static pressure is a function of cross-sectional area, wall curvature and viscous forces. Station 3 is located at an axial position where streamwise diffusion of the flow is occurring. In reference to Fig. 5.1, this results in a net rise in static pressure above the inlet value $\left(C_{p}=0\right)$ which is reflected in the centerline pressure. Concave curvature along the upper wall $\left(s / s_{\text {ref }}=0\right)$ induces a positive pressure gradient $(\partial P / \partial r>0)$ which results in the observed pressure peak. Conversely, convex wall curvature along the sidewalls $\left(s / s_{\text {ref }}= \pm 1\right)$ induces a negative pressure gradient resulting in the pressure minima. A net decrease in static pressure occurs between Stations 3 and 4 which is the result of a slight decrease in cross-sectional area and viscous losses. Also, the radius of curvature of the walls changes sign between Stations 3 and 4 which causes maximum and minimum pressures to occur along the side and upper walls, respectively. At Station 5 the area has returned to the inlet value so that a net decrease in pressure relative to the inlet occurs which is due only to viscous effects. Although there is no curvature of the walls at this station, upstream curvature effects (the wall curvature peaks between Station 4 and 5) are still strongly present. At Station 6 , there is no area change or curvature effects, and the static pressure is nominally constant across the entire cross section.

\subsection{Effect of Varying Reynolds Number}

Results presented thus far have shown that, aside from a thicker boundary layer at the lower operating Reynolds number, no appreciable Reynolds number dependence exists over the limited range considered. In order to determine the influence of Reynolds number on downstream flow development, mean flow measurements and some limited turbulence data were accumulated at Station 5 for operating bulk Reynolds numbers of 88,000 and 390,000 . Total pressure contours, axial mean velocity contours and transverse velocity vectors for the two operating conditions are shown in Figs. 5.2, 5.3 and 5.4, respectively. These results are shown here only for comparison; the physical significance of the flowfield behavior will be discussed in the next section. In Fig. 5.4, the reference velocity vector represents the largest measured vector in the plane. The mean flowfields at the two operating conditions are observed to be very similar. One notable difference is that the vortex pair for the lower Reynolds number is more 
circular and centered further away from the wall. Overall, though, the differences observed were not significant enough to justify repeating all measurements for both operating conditions. It was decided, therefore, to restrict the bulk of the measurements at the remaining stations to one operating condition. Since, for most practical applications, the operating condition for a circular-to-rectangular transition duct would be closer to the higher operating condition, most of the remaining measurements are for the $R e_{b}=390,000$ case.

\subsection{Mean Flow Results}

\subsubsection{Mean flow contours}

Mean flow variables were measured in the transverse plane at Stations 3,4,5 and 6. Reference coordinates applicable to theses data stations are shown in Figs. 3.2.d through 3.2.f, respectively. Total pressure contours (Pitot probe) and axial velocity contours (hot-wire probes) at Stations $3,4,5$ and 6 are shown in Figs. 5.5 and 5.6, respectively. At Station 3, the total pressure and axial velocity contours generally follow the cross- sectional shape of the duct. At Station 4 , a distortion of the contours is seen to develop in the vicinity of the sidewall. The data at Stations 5 and 6 show that the distortion grows in the axial direction. The development of the distortion is qualitatively very similar to the results presented by Taylor et al. [39] for turbulent flow through a non-diffusing $\mathrm{S}$-shaped duct. Transverse velocity vectors measured by means of hot-wires are shown in Fig. 5.7. The magnitude of the reference velocity vector indicated in the plots represents the maximum vector magnitude observed at that station. The distortion of the primary flow is due to a secondary flow pattern which develops into a discrete vortex pair along the duct sidewalls. This secondary flow arises as a result of lateral skewing of the near-wall flow in the vicinity of the sidewall induced by transverse pressure gradients (refer to Fig. 5.1) associated with wall curvature. At Station 5 , the vortices are oblong in shape and their centers are positioned relatively near the duct sidewall. At Station 6 , the vortices have grown in lateral extent, are more circular and are centered further away from the sidewall. Between Stations 2 and 5, lateral divergence of the upper (lower) wall boundary layer and lateral convergence of the sidewall boundary layer causes thinning and thickening of the boundary layer, respectively. Between Stations 5 and 6 , the boundary layer everywhere thickens by natural growth and, in the vicinity of the sidewall, by lateral convergence.

The static pressure distribution at Station 5 was measured directly with a static pressure probe and calculated from the total pressure and velocity distributions:

$$
P=P_{t}-\frac{1}{2} P_{a m b} U_{0}^{2} /\left(R_{a i r} T_{a m b}\right)
$$

where the variables are defined as in equation (3.1). Measurement of the static pressure at Station 5 was possible since the total flow angle is less than $10^{\circ}$. 
The measured and calculated distributions are shown in Fig. 5.8. The measured static pressure in the near wall region agrees well with the wall tap measurements shown in Fig. 5.1.b. The calculated pressure field qualitatively agrees with the measurements, but doesn't show the saddle-shaped distributions as clearly.

\subsubsection{Boundary layer divergence}

The mean velocity distributions were used to estimate the strength of the divergence along the midplanes $y=0$ and $z=0$ (negative divergence is the same as convergence). On the midplane $y=0$ (upper wall), the divergence parameter is defined as $(\partial V / \partial y) /\left(\partial U / \partial y_{1}\right)$ evaluated midway through the boundary layer. On the midplane $z=0$ (side wall), the divergence parameter is defined as $(\partial W / \partial z) /\left(\partial U / \partial y_{2}\right)$ evaluated at the first data point from the wall $(\approx 0.5 \mathrm{~cm})$. An estimate of the axial distribution of these divergence parameters is shown in Fig. 5.9. Along the duct upper (lower) surface, the divergence is, for the most part, : confined to the actual transition section, peaking at around $20 \%$. To further illustrate the degree of divergence on the upper and lower surfaces of the duct, Fig. 5.10 reproduces surface oil flow results by Reichert et al. [4] obtained in an identical transition duct at an operating condition of $R e_{c l, i}=1.57 \times 10^{6}$. Along the duct sidewalls, the flow begins to converge in a manner similar to the divergence on the upper wall, but then a large jump is observed between $x / R=$ 2.8 and 4.0 (Stations 4 and 5). This jump is due to a reduction in the primary strain rate $\partial U / \partial y_{2}$ which is a direct result of the vortex pair. The results at $x / R=8.0$ shows that the boundary layer continues to converge well into the transition duct extension. Based on the strength of the divergence observed, it is anticipated that divergence effects will be reflected in the local turbulence structure.

\subsubsection{Mean flow profiles}

Total pressure profiles, at Stations $1,3,4,5$ and 6 , measured along the $y_{2}$ (sidewall) and $y_{3}$ (upper wall) traverses (see Fig. 3.2) are shown in Figs. 5.11.a and 5.11.b, respectively. Similarly, axial velocity profiles are shown in Figs. 5.12.a and 5.12.b. These results show that, between Stations 1 and 5, the boundary layer substantially thickens along the $y_{2}$ traverse and thins along the $y_{3}$ traverse. This is due to the secondary flow transferring boundary layer fluid along the duct periphery from the vicinity of the $y_{3}$ traverse to the vicinity of the $y_{2}$ traverse. Beyond Station 5, where the cross-sectional shape is constant, the boundary layer along both traverses thickens. Along the $y_{3}$ traverse, the thickening is due to natural boundary layer growth, but along the $y_{2}$ traverse, the thickening is due also to the common outward flow associated with the vortex pair.

At Stations 5 and 6 , the total pressure and velocity profiles along the $y_{2}$ traverse exhibit a double inflection behavior. The total pressure contours (Figs. 5.5.c and 5.5.d) and the axial velocity contours (Figs. 5.6.c and 5.6.d) indicate 
that along traverses adjacent and parallel to the $y_{2}$ traverse, a double peaking behavior is observed. This is a result of the vortex pair convecting a "ridge" of high momentum fluid from the potential core flow to the region along the duct sidewalls, re-energizing the boundary layer and very probably preventing flow separation. A break in the ridge occurs at the midplane ( $y_{2}$ traverse) due to a transfer of low momentum fluid from the boundary layer toward the centerline, creating a flat spot in the velocity field. This flat region is seen to be much larger at Station 6 (Fig. 5.6.d) than at Station 5 (Fig. 5.6.c).

\subsubsection{Streamwise vorticity}

The presence of the vortex pairs in the exit plane of the transition duct is undesirable, inasmuch as they cause significant regions of total pressure loss. Streamwise vorticity in non-circular ducts can be generated by two different mechanisms. The first is vorticity generation by the lateral deflection (by pressure gradients) of a shear layer with spanwise vorticity. This mechanism is often referred to as skew-induced streamwise vorticity. An example of this type is the horseshoe vortex generated by a blunt obstruction in a 2-D boundary layer. The second mechanism is streamwise vorticity created by the Reynolds stresses and is referred to as stress-induced vorticity. Streamwise vorticity generation by the Reynolds stresses occurs in turbulent flows through straight non-circular ducts. Generally, stress-induced vorticity is much weaker than skew-induced vorticity. These mechanisms are represented in the steady axial mean vorticity equation:

$$
\begin{array}{r}
U \frac{\partial \Omega_{x}}{\partial x}+V \frac{\partial \Omega_{x}}{\partial y}+W \frac{\partial \Omega_{x}}{\partial z}=\Omega_{x} \frac{\partial U}{\partial x}+\Omega_{y} \frac{\partial U}{\partial y}+\Omega_{z} \frac{\partial U}{\partial z} \\
+\frac{\partial}{\partial x}\left(\frac{\partial \overline{u v}}{\partial z}-\frac{\partial \overline{u w}}{\partial y}\right)+\left(\frac{\partial^{2}}{\partial y^{2}}-\frac{\partial^{2}}{\partial z^{2}}\right)(-\overline{v w})+\frac{\partial^{2}}{\partial y \partial z}\left(\overline{v^{2}}-\overline{w^{2}}\right)+\nu \nabla^{2} \Omega_{x}
\end{array}
$$

where,

$$
\Omega_{x}=\frac{\partial W}{\partial y}-\frac{\partial V}{\partial z}, \quad \Omega_{y}=\frac{\partial U}{\partial z}-\frac{\partial W}{\partial x}, \quad \Omega_{z}=\frac{\partial V}{\partial x}-\frac{\partial U}{\partial y}
$$

The LHS of equation (5.2) represents the increase in streamwise vorticity by convection. The first term on the RHS represents production of streamwise vorticity by vortex line stretching (streamwise acceleration causes amplification of vorticity). The second and third terms on the RHS represent the increase in vorticity due to lateral skewing (by transverse pressure gradients) of vorticity in the transverse directions. These are the terms associated with skew-induced vorticity. The fourth term represents the production of streamwise vorticity by the primary shear stresses and is often neglected since it contains a streamwise gradient. The fifth and sixth terms on the RHS represent the production of 
streamwise vorticity by inhomogeneity of the transverse normal stress anisotropy and by the secondary shear stress, respectively. These terms are responsible for stressed-induced vorticity. The last term on the RHS represents the diffusion of streamwise vorticity by viscous forces.

The significance of equation (5.2) can now be discussed relative to the present transition duct configuration. Since the sign of axial vorticity changes across planes of symmetry, to avoid confusion, the following discussion will be restricted to Quadrant 1 (refer to Fig. 3.2) of the transition duct. The axial vorticity component at Stations $3,4,5$ and 6 was calculated by interpolating the transverse velocity components onto a uniform grid $(0.508 \times 0.508 \mathrm{~cm})$ and then evaluating the derivatives by central difference approximations. Contours of axial vorticity at Stations 3,4,5 and 6 are shown in Fig. 5.13. Negative vorticity is represented by dashed contour lines. The filled circles in these plots mark the approximate location where the peak vorticity occurs. At Station 1, the vorticity field is comprised only of transverse vorticity components $\Omega_{y}$ and $\Omega_{z}$, the axial component $\Omega_{x}$ being zero. Beginning at Station 2, the wall curvature induces the transverse pressure gradients which were discussed in Section 5.3 and illustrated in Fig. 5.1. In the first half of transition, a global deceleration of the flow occurs due to the area expansion. Fig. 5.13.a (Station 3) shows that in Quadrant 1 the transverse pressure gradient creates primarily negative vorticity. The no-slip condition requires there to be a thin layer of positive vorticity in the very near-wall region which was not resolved in the present measurements. The generation of negative vorticity will occur in any straight $C R$ transition duct without swirl since the pressure gradient is primarily a function of wall curvature. In the second half of transition, the wall curvature changes sign which causes a reversal of the transverse pressure gradient (see Fig. 5.1). Also, the contracting area causes a global acceleration of the flow. It might be expected that the reversal of the pressure gradient would effectively cancel the vorticity generated in the first half of transition. This is not the case, however, as Figs. 5.13.b and 5.13.c (Stations 4 and 5), show that the negative vorticity migrates towards the midplane $z=0$ and intensifies. This strengthening of the vorticity is caused by streamwise acceleration (vortex stretching) and concave surface curvature along the duct sidewalls. Although the vortices are not generated by centrifugal instabilities, concave curvature will accentuate the vorticity in the same manner that Taylor-Görtler type vortices intensify. In addition, the reversed pressure gradient assists the inward (negative $y$ direction) flow between the developing vortex pair. In the transition duct extension (Fig. 5.13.d, Station 6), the vorticity is diffused by turbulent action. Between Stations 5 and 6 , the magnitude of the peak vorticity drops from $\Omega_{x} R / U_{b}=2.4$ to 0.09 .

Miau et al. [9] evaluated all the terms in equation (5.2) at the exit plane of two CR transition ducts of constant cross- sectional area. Both ducts had aspect ratios $(A R)$ of two, but the transition lengths differed: $L / D=0.54$ and 
$L / D=1.08$. The results of their analysis showed that the generation of axial vorticity at the exit plane was primarily skew-induced (terms 3 and 4), rather than turbulence induced (terms 5-7). A notable difference between Miau's results and the present study is that the axial vorticity in the corner region at the exit plane is of opposite sign. Whereas negative axial vorticity is observed in Quadrant 1 (upper quadrant, Fig. 5.13.c) of the present study, positive axial vorticity is observed in the equivalent quadrant of their ducts. Patrick and McCormick $[2,3]$ calculated the axial vorticity from measurements in the exit plane of two CR ducts. One duct had an aspect ratio of three and a length-to-diameter ratio of one, and the other duct had an aspect ratio of six and a length-to-diameter ratio of three. The results of this investigation showed small regions of positive vorticity for the $A R=3$ duct and large regions of negative vorticity for the $A R=6$ duct. A common feature between the ducts which exhibited positive vorticity is that the cross-sectional area through the duct was constant. In contrast, the ducts which exhibit negative vorticity in the exit plane had an area expansion followed by a contraction through the transition section. For all ducts, the inlet area equalled the exit area. Another distinguishing feature is the transition length was considerably shorter for the positive vorticity ducts than for the negative vorticity ducts: $L / D=0.54$ and 1.08 vs. 1.5 and 3.0 , respectively. These results suggest that the condition of the flowfield in the exit plane is very sensitive to the geometry of the transition. More specifically, the path that the vortex core takes through the duct will determine if the initial vorticity is amplified or attenuated.

\subsection{Turbulence Results}

The complete Reynolds stress tensor was measured at Stations 5 and 6. A distinguishing feature of the flowfield at these stations is the turbulence structure in the vicinity of the vortex pair. In Section 2.3, several detailed experimental investigations of embedded vortex flows were mentioned. In particular, the studies by Mehta and Bradshaw [30] and Pauley and Eaton [31,32] are relevant, inasmuch as they present detailed mean flow and turbulence results for embedded vortex pairs with the common flow away from the surface, as occurs in the transition duct. The present measurements afford the opportunity to make comparisons with these data sets to determine if modelling conclusions based on their results are applicable to the transition duct flow. For comparative purposes, the origin of the vortices in the transition duct is taken as the location where the geometry deformation begins (Station 2). Based on this, the development length for the vortices is approximately 30 and $70 \mathrm{~cm}$ at Stations 5 and 6 , respectively. The first measurement station reported by Pauley and Eaton was $97 \mathrm{~cm}$ downstream from the generators and Mehta and Bradshaw present most of their results at a development length of $135 \mathrm{~cm}$. In addition to development length, other differences between the studies should be noted. Whereas the other investigators studied vortices embedded in a two-dimensional boundary layer, the transition duct boundary layer in the region of the vortices is three-dimensional. Also, the 
velocity deficit in the vicinity of the vortex cores is much larger in the transition duct than in the other studies. Mehta and Bradshaw placed their generators in the settling chamber so that the velocity deficit was a small percentage of the potential core flow velocity by the time the vortices entered the test section. Pauley and Eaton, on the other hand, generated their vortices at the beginning of the test section, so that a significant deficit was initially present, but had diminished somewhat at the downstream data station where the turbulence measurements were made $(97 \mathrm{~cm})$. Velocity deficit in the vortex core region is summarized in Table 5.1.

Table 5.1. Velocity deficit in the vicinity of the vortex cores.

\begin{tabular}{lcc}
\hline & $x(\mathrm{~cm})$ & $U / U_{e}$ \\
\hline Present results & 30 & 0.40 \\
Present results & 70 & 0.50 \\
Pauley \& Eaton [31] & 66 & 0.50 \\
Pauley \& Eaton [31] & 97 & 0.75 \\
Pauley \& Eaton [31] & 142 & 0.90 \\
Mehta \& Bradshaw [30] & 60 & 0.95 \\
Mehta \& Bradshaw [30] & 90 & 0.95 \\
Mehta \& Bradshaw [30] & 135 & 0.95 \\
\hline
\end{tabular}

Based on these differences alone, it is expected that the present results will qualitatively be more similar to the results of Pauley and Eaton than to those of Mehta and Bradshaw.

\subsubsection{Turbulence contours}

Axial turbulence intensity $u^{\prime} / U$ contours measured at Stations 5 and 6 are shown in Fig. 5.14. Note that the turbulence intensity here is defined relative to the local axial velocity component and not the bulk velocity. Since the local intensity exceeds $10 \%$ in places, second-order response equations (see Appendix C) were used for reducing all of the hot-wire data. The filled circles in Fig. 5.14 and subsequent figures mark the location of the vortex cores (peak vorticity) as an aid to interpretation. In the vicinity of the sidewall, the vortex pair extrudes a tongue of moderate turbulence intensity; $\left(u^{\prime} / U\right)_{\max } \approx 14 \%$ and $11 \%$ at Stations 5 and 6 , respectively. The increase in boundary layer thickness and distortion by the vortex pair between Stations 5 and 6 is clearly evident. Along the midplane $z$ $=0$, the turbulence intensity exhibits a double peak behavior which is a result of the double inflection observed in the mean velocity profiles shown in Fig. 5.12.a.

Contours of the six Reynolds stress components at Stations 5 and 6 are shown in Figs. 5.15-5.20. Negative contour levels are represented by dashed 
lines. The level of symmetry for each stress component about the midplane $z / R=0$ is very good, even for the difficult-to-measure $\overline{v w}$ stress component shown in Fig. 5.20. As expected, the $\overline{u w}$ and $\overline{v w}$ stress components change sign between Quadrants 1 and 2. In general, Figs. 5.15-5.20 show that the Reynolds stress contours are more distorted at Station 6 than at Station 5, and that peak contour values are higher and displaced farther from the duct sidewall. The increase in peak magnitude of the stresses is in contrast to the decrease in peak axial turbulence intensity (Fig. 5.14) which resulted from an increase in the local axial velocity in the vicinity of the high turbulence levels.

At both Stations 5 and 6 , the peak magnitude of the transverse normal stress components $\frac{v^{2}}{v^{2}}$ and $\overline{w^{2}}$ are nearly equal and are roughly half the peak magnitude of the axial stress component $\overline{u^{2}}$. Qualitatively, the $\overline{u^{2}}$ stress component differs significantly between all the vortex studies. The results of Mehta and Bradshaw at $135 \mathrm{~cm}$ show that as the wall is approached along the plane of symmetry, the $\frac{u^{2}}{2}$ component monotonically increases. The results of Pauley and Eaton at 97 $\mathrm{cm}$ show a peak in the near-wall region, but also show an additional peak on the plane of symmetry near the outer edge of the vortices. They also show a pair of peaks in the vortex core region symmetrically located about the plane of symmetry. The present results show a large peak on the plane of symmetry at the edge of the vortex pair. These differences can be traced to the velocity deficit (or lack of) in the vortex core region. It is likely that the vortices in the Pauley and Eaton study exhibited similar behavior to the present results at a location closer to the generators since a large velocity deficit is present directly behind the generators. At Station 6, the present results show that the $\overline{w^{2}}$ stress component exhibits two peaks symmetrically located about the $y=0$ midplane which were not observed at Station 5. Pauley and Eaton's data at $97 \mathrm{~cm}$ suggest that all the normal stresses will eventually exhibit this double peak behavior.

Anisotropy between the axial stress component and the horizontal transverse component $\left(\overline{u^{2}}-\overline{v^{2}}\right)$ is shown in Fig. 5.21. At Station 5, the axial component is everywhere larger than the horizontal component. In the vicinity of the vortex core at Station 6, however, the transverse component exceeds the axial component by as much as $20 \%$. This is in contrast to a two-dimensional boundary layer where the axial component is greater than either transverse components. Pauley \& Eaton and Mehta \& Bradshaw both report similar behavior of the normal stresses in the vicinity of the vortex core. Anisotropy between the axial and vertical normal stress components $\left.\left(\overline{u^{2}}-\overline{w^{2}}\right)\right)$ is shown in Fig. 5.22. Near the vortex core at Station 5 , the stresses are observed to be nearly equal. In the vicinity of $y / R=1.4, \pm z / R=0.35$, the vertical stress exceeds the axial component. At Station 6 , the vertical stress is everywhere less than the axial component, but they are still nearly equal in the vortex core region. Anisotropy between the vertical and horizontal transverse normal stress components $\left(\overline{v^{2}}-\overline{w^{2}}\right)$ is important in the generation of streamwise vorticity in non-circular ducts (see Section 5.4.1). 
Contours of this quantity are plotted in Fig. 5.23. These plots show significant differences between Stations 5 and 6. It is well known that in two-dimensional boundary layers, $\overline{u_{1}^{2}}>\overline{u_{3}^{2}}>\overline{u_{2}^{2}}$, where $\overline{u_{1}^{2}}$ is the axial component, $\overline{u_{3}^{2}}$ is the transverse component tangential to the surface, and $\overline{u_{2}^{2}}$ is the component which acts normal to the surface (see, e.g., Fig. 18.5 of Ref. 54). For the transition duct flow, the above inequality is observed to hold true in the near-wall region at both Stations 5 and 6 . In the outer region of the upper and lower wall boundary layers at Station 5 , however, the component tangent to the surface exceeds the component which acts normal to the surface (negative anisotropy). In the region of the vortex pair at Station 5, a small pocket exists where the anisotropy is positive. By Station 6, the region of positive anisotropy has grown and intensified. The results at Station 6 are qualitatively very similar to the results reported by Pauley and Eaton for a vortex pair at a development length of $97 \mathrm{~cm}$ (see Fig. 4.39 of Ref. 31).

The primary shear stress $\overline{u v}$ shown in Fig. 5.18 is observed to be positive everywhere except for a small region in the vicinity of the vortex cores. These regions of negative stress are a result of the positive mean rate-of-strain $(\partial U / \partial y>$ 0 ) associated with the aforementioned velocity ridge (see Section 5.4.3). Along the midplane $z=0$ where the axial mean velocity profile flattens, the primary mean rate-of-strain in both the $y$ and $z$ directions is nearly zero and, as a result, stress levels in this region are depressed. In the $z$-direction, the primary shear $\overline{u w}$ shown in Fig. 5.19 also changes sign as the summit of the velocity ridge is crossed. The secondary shear stress $\overline{v w}$ is another quantity that plays an important role in the production of streamwise vorticity (see Section 5.4.1). In the vicinity of the vortex cores, this stress is of the same order of magnitude as the primary shear stresses.

The normal stress data were used to calculate the turbulence kinetic energy. These results are shown for Stations 5 and 6 in Fig. 5.24. The production of kinetic energy was also calculated. Neglecting streamwise derivatives, the production of kinetic energy is given by:

$$
P=-\overline{u v} \frac{\partial U}{\partial y}-\overline{u w} \frac{\partial U}{\partial z}
$$

Contours of this quantity are shown in Fig. 5.25. Generally, high levels of kinetic energy are associated with high production rates. A notable exception occurs on the duct upper and lower walls at Station 5. Here, the production in the near-wall region is significantly less than that observed at Station 6 , which seems disproportionate when compared to the relatively small difference in kinetic energy between these stations. Between Stations 5 and 6, the peak production is essentially the same, but the peak kinetic energy increases by approximately $35 \%$. 


\subsubsection{Turbulence profiles}

In this section, Reynolds stress profiles along the duct semi-major and semiminor axes are presented and compared with the initial distributions measured at Station 1. In the following profile plots, the designation of the Reynolds stress components are relative to wall coordinates and not the $x, y, z$ laboratory coordinates; that is, the $v$ fluctuating velocity component is always directed along the wall coordinate of interest, either $y_{2}$ or $y_{3}$ (see Fig. 3.2). All of the stresses are normalized by the bulk velocity and the wall coordinate is normalized by the local boundary layer thickness, as summarized in Table 5.2 .

Table 5.2. Normalized boundary layer thickness $(\delta / R)$ at Stations 1,5 and 6.

\begin{tabular}{ccccc}
\hline & \multicolumn{2}{c}{$R e_{b}=88,000$} & $R e_{b}=390,000$ \\
Station & $y_{2}$-axis & $y_{3}$-axis & $y_{2}$-axis & $y_{3}$-axis \\
\hline 1 & 0.31 & 0.31 & 0.29 & 0.29 \\
5 & - & 0.21 & 0.58 & 0.18 \\
6 & - & - & 0.65 & 0.25 \\
\hline
\end{tabular}

$R=10.214 \mathrm{~cm}$

Reference normal Reynolds stress distributions measured at Station 1 are shown in Fig. 5.26. Normal stress distributions measured at Stations 5 and 6 along the semi-major axis are shown in Fig. 5.27 and shear stress and kinetic energy profiles are shown in Fig. 5.28. It is readily apparent that the distributions along the semi-major axis deviate considerably from the initial profiles. The boundary layer on the duct sidewalls is subjected first to stabilizing convex curvature and stabilizing lateral convergence. In the second half of transition, the developing sidewall vortex pair creates stabilizing lateral convergence near the wall and destabilizing lateral divergence in the outer region of the boundary layer. In addition, the flow experiences destabilizing concave curvature. The common flow away from the wall creates a region of velocity deficit resulting in the double infection behavior of the mean velocity profiles shown in Fig. 5.12.a. At Station 5 , the axial normal and shear stress components show the largest deviation with strong attenuation in the near-wall region. Near the wall at Station 6, these stress components have increased to a certain extent, but are still below the initial levels. The transverse normal stress components in the near-wall region decrease between Stations 5 and 6 . In wall-bounded shear layers, the Reynolds stresses are known to scale with the local friction velocity. Preston tube measurements were made in the duct mid-planes at Stations 1,5 and 6 from which the local friction velocity was deduced. These results are summarized in Table 5.3. 
Table 5.9. Normalized friction velocity

$\left(U_{\tau} / U_{b} \times 100\right)$ at mid-planes, $R e_{b}=390,000$.

\begin{tabular}{ccc}
\hline Station & $y_{2}=0$ & $y_{3}=0$ \\
\hline 1 & 4.06 & 4.06 \\
5 & 2.70 & 4.89 \\
6 & 3.23 & 4.38 \\
\hline
\end{tabular}

The drop and subsequent rise in the axial Reynolds normal and shear stress components near the wall between Stations 1 and 6 correlates with the friction velocity behavior shown in Table 5.3. The transverse normal stresses appear to lag the development of the friction velocity, although it is presumed that all the stress components in the near-wall region will eventually increase as the vortex pair moves further away from the duct sidewalls. The decrease in the transverse normal stresses outweighs the increase in the axial normal stress so that a decrease in turbulence kinetic energy is observed between Stations 5 and 6. Several factors contribute to the attenuation of the turbulence in the nearwall region. First, the primary rate-of-strain $\left(\partial U / \partial y_{2}\right)$, and hence the wall shear stress, is reduced by the common outward flow of the vortex pair. Second, lateral convergence of the boundary layer acts to suppress turbulence. And finally, Fig. 5.12.a shows that between Stations 4 and 6, the near-wall flow is subjected to streamwise acceleration which suppresses turbulence generation. The high turbulence levels observed in the mid-region of the boundary layer are a result of high primary rates-of-strain associated with the velocity deficit.

The history of the flow along the $y=0$ mid-plane (semi-minor axis) is signifcantly different from the flow along the $z=0$ mid-plane (semi-major axis). In the first half of transition, the boundary layer is subjected to destabilizing concave curvature and destabilizing lateral divergence. In the second half of transition, the divergence decreases and the flow experiences stabilizing convex curvature. Normal stress distributions measured along the duct semi-minor axis at Stations 5 and 6 are shown in Fig. 5.29 and shear stress and kinetic energy profiles are shown in Fig. 5.30. Wall proximity measurements at Stations 5 and 6 were limited by the bevel gear arrangement necessary for probe rotation. This limitation was most serious along the upper and lower duct surfaces at Station 5 where the boundary layer is thinnest. Over the range of the boundary layer that was able to be measured, the net effect of the above flow conditioning is relatively small. The trends show an increase in the axial normal stress component and a decrease in the transverse normal stress components between Stations 1 and 6 . The combined effect on the turbulence kinetic energy is very small. At Station 5 , the shear stress value nearest the wall is observed to decrease, although it is recognized that one point is not statistically significant. To investigate this further, turbulence measurements along the $y_{3}$ axis were made at a bulk operat- 
ing Reynolds number of 88,000 where the boundary layer is thicker and a larger region could be resolved. Shear stress and kinetic energy profiles for this case are shown in Fig. 5.31. These results confirm both the unchanged kinetic energy levels and the decreasing behavior of the shear stress. The decrease in shear stress is in contrast to the increased wall shear stress measured by means of the Preston tubes. This indicates that either the shear stress recovers in the near-wall region or that the flow is not in local equilibrium near the wall. Without the benefit of turbulence measurements at the intermediate Stations 3 and 4 , it is difficult to speculate on the factors which lead to the flow condition at Station 5. Near-wall behavior will be examined more closely in Section 5.7.

\subsection{Turbulence Modelling Considerations}

The inviscid calculations by Burley et al. $[7,8]$ have shown that even for overall performance predictions, viscous effects cannot be neglected when calculating transition duct flows. For accurate predictions the effects of turbulence must also be included. The level of turbulence modelling required, of course, depends on the information desired. From a computational standpoint, the most difficult aspect of the present configuration is the flowfield in the neighborhood of the vortex pair. Since the initial generation of streamwise vorticity via pressure gradient effects is essentially an inviscid process, even simple models will predict the presence of the vortex pairs. Accurate prediction of the diffusion of vorticity, however, relies on accurate modelling of the Reynolds stress components. Modelling efforts for the present configuration can be divided into two groups. The first group is concerned primarily with overall performance parameters and prediction of flow separation. The computational work of Liandrat et al. [33] has shown that the primary features of the mean flowfield in the region of embedded vortices can be predicted reasonably well with simple mixing length models. The second group is concerned with the task of demonstrating the ability of particular turbulence models to predict detailed mean flow and Reynolds stress behavior. The present data set should be useful for this group.

The simplest turbulence model is the algebraic (zero-order) eddy viscosity model based on the concept of Boussinesq. Here, the Reynolds stresses are assumed to behave like the molecular viscosity stresses and the Reynolds stress tensor for incompressible flow is written as:

$$
\overline{u_{i} u_{j}}=-\nu_{t}\left(\frac{\partial U_{i}}{\partial x_{j}}+\frac{\partial U_{j}}{\partial x_{i}}\right)+\frac{2}{3} \delta_{i j} k
$$

where $\nu_{t}$ is the turbulent eddy viscosity, $\delta_{i j}$ is the Kronecker delta function and $k$ is the turbulence kinetic energy. Equation (5.4) is applicable to threedimensional flows where only the Reynolds shear stresses $\left(\overline{u_{i} u_{j}}, \quad i \neq j\right)$ are important, inasmuch as the Reynolds normal stresses predicted by this equation are not in agreement with even simple flows. For the present configuration, 
equation (5.4) may be adequate for prediction purposes through the end of the transition section, but will not be applicable if the flow is allowed to develop in the rectangular duct where the transverse Reynolds normal stresses are important in the generation and diffusion of corner-generated secondary flows.

Since many design-oriented flow solvers are implemented with algebraic eddy viscosity turbulence models, it is worthwhile to examine the behavior of the eddy viscosity for the present flow. For the primary shear stresses, if streamwise gradients are neglected, equation (5.4) reduces to:

$$
\begin{gathered}
\overline{u v}=-\nu_{t} \frac{\partial U}{\partial y} \\
\overline{u w}=-\nu_{t} \frac{\partial U}{\partial z}
\end{gathered}
$$

Rearranging these equations to solve for the eddy viscosity yields:

$$
\begin{aligned}
& \nu_{t, y}=-\overline{u v} /\left(\frac{\partial U}{\partial y}\right) \\
& \nu_{t, z}=-\overline{u w} /\left(\frac{\partial U}{\partial z}\right)
\end{aligned}
$$

where the $y$ and $z$ subscripts admit directional dependence (anisotropy) of the eddy viscosity. The behavior of the primary rates-of-strain (denominators in equations (5.7) and (5.8)) at Station 5 is shown in Fig. 5.32. Also indicated in these plots are horizontal traverses along which the component eddy viscosities were calculated. These results, plotted in terms of a viscosity ratio $\left(\nu_{t} / \nu\right)$, are shown for Station 5 in Fig. 5.33. Equivalent results for Station 6 are shown in Figs. 5.34 and 5.35. To avoid singular points where the strain-rates vanish (between solid and dashed lines in Figs. 5.32 and 5.34), the eddy viscosity is computed only where the denominator in equations (5.7) and (5.8) is greater than $5 \%$ of its maximum value in the cross plane. Although the eddy viscosity distributions shown in Figs. 5.33 and 5.35 show similar trends with respect to symmetry about the midplane $z=0$, the magnitude of the differences is enough so that the results should be considered only qualitative. In areas where the stress and strain in both transverse directions are nominally of the same magnitude, the eddy viscosity components are observed to be nearly equal. Most of the regions where large deviations occur can be traced to either a large difference in the component strain rates or to inadequate resolution of a large velocity gradient, e.g., in the vicinity of the velocity ridge near the vortex core. this flow. This is in contrast to the results of Mehta and Bradshaw, who reported that the $\nu_{t, y}$ component in their embedded vortices was so ill-behaved so as to preclude plotting. 
It was mentioned in Chapter 2 that boundary layer flows with extra rates-ofstrain often exhibit more spectacular behavior than what is predicted by explicit terms which account for the extra strain rates. It is argued that the reason for this is that the extra rates-of-strain cause large changes in higher-order terms which appear in the Reynolds stress transport equations. These higher-order terms are modelled in terms of the Reynolds stresses and require the specification of empirical constants. Dimensionless turbulence structure parameters such as the cross-correlation coefficient are related to constants in turbulence models. Often the constants are determined based on the results of simple flows such as two-dimensional boundary layers, resulting in poor performance when applied to more complex flowfields. Some of these dimensionless structure parameters were computed for the present flow. The shear stress correlations $R_{u v}=\overline{u v} / u^{\prime} v^{\prime}$, $R_{u w}=\overline{u w} / u^{\prime} w^{\prime}$ and $R_{v w}=\overline{v w} / v^{\prime} w^{\prime}$ evaluated at Stations 5 and 6 are shown in Figs. 5.36, 5.37 and 5.38, respectively. The primary shear stress correlations $R_{u v}$ and $R_{u w}$ may be compared with the two-dimensional value of 0.45 . Near the duct sidewalls and in the vicinity of the vortex cores, the $R_{u v}$ parameter is depressed due to strong lateral convergence of the boundary layer. At Station 5 , in the region where the peak turbulence intensities occur, a near constant value of approximately 0.55 is observed. By Station 6 , the value has decreased and is close to the two-dimensional value. Near the upper and lower walls, the $R_{u w}$ parameter generally is in agreement with the two-dimensional value. The secondary shear stress correlation $R_{v w}=\overline{v w} / v^{\prime} w^{\prime}$ at both stations shows values in the range of 0.25 for most of the flowfield, but this parameter is also depressed in the region of the vortex core. Another dimensionless parameter of interest is the shear-energy ratio parameter $a 1$. This parameter is defined as the ratio of the resultant shear stress in a plane normal to a wall surface to twice the turbulence kinetic energy. In a two-dimensional boundary layer, this parameter has also been observed to be constant with a value of 0.15 (see, e.g., Bradshaw et al. [56]). For the present flow, two shear-energy parameters were computed:

$$
\begin{aligned}
& a 1_{y}=\sqrt{\overline{u v}^{2}+\overline{v w}^{2}} / 2 k \\
& a 1_{z}=\sqrt{{\overline{u w^{2}}}^{2}+\overline{v w}^{2}} / 2 k
\end{aligned}
$$

which are applicable away from the corner region on the vertical and horizontal walls, respectively. The $a \mathrm{l}_{y}$ shear-ratio parameter at Stations 5 and 6 is shown in Fig. 5.39 and the $a 1_{z}$ parameter is shown in Fig. 5.40. In the regions where high turbulence levels are observed, the $a 1_{y}$ parameter is higher than the twodimensional value and, like the shear stress correlation, is well below the initial value in the region of the vortex pair. Along the horizontal walls, the $a 1_{z}$ parameter is elevated at Station 5, but returns to the initial value at Station 6 . These results show that the transition duct produces a distortion of the turbulence structure, more so at Station 5 than at Station 6 . The largest distortion occurs 
in the vicinity of the vortex core region where lateral convergence suppresses turbulence.

Further insight into the structure of the turbulence can be gained by examining the data in terms of the invariants of the anisotropic stress tensor. This analysis is based on the concept of physical realizability limits of turbulence which has been used extensively to study the return to isotropy of homogeneous turbulence (see, e.g., Lumley [57]). The anisotropic stress tensor, first proposed by Rotta [58], is defined as:

$$
b_{i j}=\frac{\overline{u_{i} u_{j}}-\frac{2}{3} k \delta_{i j}}{2 k}
$$

This tensor must satisfy the Cayley-Hamilton theorem:

$$
\lambda^{3}-I \lambda^{2}+I I \lambda-I I I=0
$$

where $I, I I$ and $I I I$ are the tensor invariants:

$$
\begin{gathered}
I=b_{i i} \\
I I=-\frac{1}{2} b_{i j} b_{j i} \\
I I I=\frac{1}{3} b_{i j} b_{j k} b_{k i}
\end{gathered}
$$

The first invariant, $I$, is identically zero by definition of the turbulence kinetic energy. Invariant $I I I$ defines the shape of the ellipsoid associated with the Reynolds stress tensor. A positive value of $I I I$ indicates that there is only one principle component that is large, and a negative value indicates that two principle components are large. Limits on the anisotropic stress tensor can be defined by applying the condition that the Reynolds stress in any direction must go to zero as the strain rate in that direction goes to infinity. For example, as $\partial U / \partial x$ goes to infinity, $\overline{u^{2}}$ goes to zero and $b_{11}$ goes to $-1 / 3$. The largest level of turbulence that can occur in any one direction is $2 k$, which occurs when the turbulence is one-dimensional. If all the turbulence is in the $x$ direction, then $\overline{u^{2}}=2 k$ and $b_{11}$ is equal to $2 / 3$. Following Lumley [57], the limits on allowable turbulence are recast in terms of the tensor invariants $I I$ and $I I I$. These limits are illustrated in Fig. 5.41. The shaded area on this plot represents the region within which all physically realizable turbulence must lie. The nature of the turbulence at the boundaries is also labeled. The Reynolds stress tensor at Stations 1,5 and 6 was recast in terms of the anisotropic stress tensor invariants. At Station 1, the turbulence is primarily contained in the axial component so it is expected that the III invariant will always be positive. The invariants at Station 1 are plotted in Fig. 5.42.a. Following Pauley and Eaton, the location of each data point on the 
invariant map relative to its position in the flowfield is represented by a vector which has its origin at $I I=I I I=0$ and the tip at the data point location on the invariant map. This vector is plotted with its origin coincident with the physical location in the flowfield in Fig. 5.42.b. As expected, all the points correspond to positive $I I I$ values. At the edge of the boundary layer the turbulence should approach an isotropic condition (zero vector length). The large vectors near the boundary layer edge indicate that the turbulence is approaching a more onedimensional nature which is not realistic. This should not be taken to seriously though, since the magnitude of the vector is not related to the magnitude of the turbulence. That is, experimental uncertainty in regions of low-turbulence levels can cause one component to be large relative to another resulting in an apparent high level of anisotropy. Invariant map plots of the Reynolds stress data at Stations 5 and 6 are shown in Figs. 5.43 and 5.44, respectively. All of the data are observed to lie within the limits of realizability. In the region of the vortex core, the turbulence is nearly isotropic. In regions where the primary strain rates are large, the turbulence structure is much like that of the initial two-dimensional boundary layer at Station 1 . Along the velocity ridge, where the primary strain rates are small, the $I I I$ invariant is negative. Between Stations 5 and 6 the data cluster more toward and spread further out along the axisymmetric contraction limit indicating that the axial stress component is becoming more dominant.

\subsection{Wall Function Behavior}

The use of wall functions for predicting the present flow was analyzed at Stations 5 and 6. Reference coordinates for this analysis are shown in Fig. 5.45. Since flow variables in the near-wall region scale with the local friction velocity, peripheral skin friction coefficient distributions were obtained at Stations 5 and 6. These distributions, which were deduced from Preston tube data measured along the periphery of the duct in Quadrant 1 (see Fig. 5.45), are shown in Fig. 5.46. The results based on different diameter Preston tubes at Station 5 show systematic variations with a change in diameter, particularly for $s / s_{\text {ref }}<0.6$, indicating that deviations from the law-of-the-wall exist at this station. Although only two Preston tubes were used at Station 6, the relatively good agreement suggests that the flow recovers to law-of-the-wall behavior downstream. Boundary layer profiles were measured along the $y_{n}$ traverses $(n=1,2,5,6,7,8)$ shown in Fig. 5.45 with both pitot and hot-wire probes. Results obtained at Stations 5 and 6, plotted in law-of-the-wall coordinates, are shown in Fig. 5.47. The friction velocities used for these plots are based on the average distribution lines shown in Fig. 5.46. The non-dimensional outside diameters of the individual Preston tubes are superimposed on these plots. At Station 5, deviations from the law-of-the-wall are apparent for $y^{+}$values greater than 80 . In the near-wall region, however, all profiles tend toward law-of-the-wall behavior within the interval $30 \leq y^{+} \leq 80$. At Station 6 , the law-of-the-wall is satisfied on all traverses for $y^{+}$values between 30 and 200 . This behavior implies that the law-of-the-wall 
may be used for prediction purposes, recognizing that the limiting $y^{+}$value on the first mesh line between the duct inlet (Station 1) and Station 5 may be less than the limiting value which applies at Station $5\left(y^{+} \cong 80\right)$.

Wall function behavior for the turbulence kinetic energy $(k)$ and the dissipation rate of turbulence kinetic energy $(\epsilon)$ was also analyzed at Stations 5 and 6. If only first-order effects are retained and terms containing secondary ratesof-strain are neglected in the reduced forms of the Reynolds stress transport equations, and the mean velocity profile along any normal to the duct surface is in accordance with the law-of-the-wall, then the following expressions (relative to the $x_{w}, y_{w}, z_{w}$ wall coordinate system) apply for turbulent flow through non-circular ducts in local equilibrium [59]:

$$
\begin{aligned}
\frac{k}{U_{\tau}^{2}} & =\frac{1}{\sqrt{C_{\mu}}} \frac{\sqrt{\left(\overline{u v}^{2}+\overline{u w}^{2}\right)}}{U_{\tau}^{2}} \\
\frac{\epsilon y_{w}}{U_{\tau}^{3}} & =\frac{1}{\kappa} \frac{\sqrt{\left(\overline{u v}^{2}+\overline{u w}^{2}\right)}}{U_{\tau}^{2}} \sqrt{F_{p}} \\
\frac{\sqrt{\left(\overline{u v}^{2}+\overline{u w}^{2}\right)}}{U_{\tau}^{2}} & =\frac{l_{p}^{2}}{\left(\kappa y_{w}\right)^{2}} F_{p}
\end{aligned}
$$

where

$$
F_{p}=1+\frac{\left(\partial U / \partial z_{w}\right)^{2}}{\left(\partial U / \partial y_{w}\right)^{2}}
$$

with $C_{\mu}=0.09$ and $\kappa=0.41$, and where $l_{p}$ is Prandtl's length scale. In the nearwall region along the transition duct horizontal and vertical walls at Stations 5 and 6, $\left(\partial U / \partial y_{w}\right) \gg\left(\partial U / \partial z_{w}\right)$, so that $F_{p} \cong 1.0$. Further, it is reasonable to assume that along these walls, but excluding the corner region, Prandtl's length scale can be specified as $l_{p}=\kappa y_{w}$. With these assumptions, the following alternate forms of equations (5.13)-(5.15) apply along the horizontal and vertical walls:

$$
\begin{aligned}
\frac{k}{U_{\tau}^{2}} & =\frac{1}{\sqrt{C_{\mu}}} \\
\frac{P y_{w}}{U_{\tau}^{3}} & =\frac{1}{\kappa} \\
\frac{\sqrt{\overline{u v}^{2}+{\overline{u w^{2}}}^{2}}}{U_{\tau}^{2}} & =1
\end{aligned}
$$

where $\epsilon$ has been replaced by $P$ (production) in accordance with the local equilibrium assumption. Distributions of $\sqrt{\overline{u v}}^{2}+{\overline{u w^{2}}}^{2} / U_{\tau}^{2}, \quad k / U_{\tau}^{2}$ and $P y_{w} / U_{\tau}^{3}$ along several traverses parallel to the $z$ axis at Stations 5 and 6 are shown in Figs. 5.48, 5.49 and 5.50 , respectively. Also indicated on these plots are the conventional 
wall function values. Although the results are restricted to $y^{+}$values greater than 400 because of limitations imposed by the probe rotation mechanism, some conclusions can still be drawn. For example, it can be seen that distributions measured along the vertical midplane traverse $y / R=0$ at Stations 5 and 6 generally tend toward the conventional limits. This same comment applies for all other distributions measured at Station 6. At Station 5, however, the trends are more difficult to discern, especially along the $y / R=1.37$ traverse, which lies within the sidewall boundary layer.

The behavior of $\sqrt{\overline{u v}^{2}+{\overline{u w^{2}}}^{2}} / U_{\tau}^{2}, \quad k / U_{\tau}^{2}$ and $P y / U_{\tau}^{3}$ along the semi-major traverse $y_{2}=0$ at Stations 5 and 6 is shown in Figs. 5.51, 5.52 and 5.53, respectively. The distributions measured at Station 6 approach the conventional limits. The deviation observed at Station 5 is probably attributable to the neglect of secondary rates-of-strain in the derivation of equations (5.13)-(5.15), inasmuch as Fig. 5.9 shows that secondary strain near the sidewall at Station 5 is a large percentage of the primary rate-of-strain, but that it decreases to a relatively small percentage by Station 6 . The first data point for Station 5 , however, is at $y_{2}^{+} \cong 300$ which is beyond the region where law-of-the-wall behavior is observed (refer to the $y_{2}^{+}$distribution of Fig. 5.47.a). In reference to predictions, if $y^{+}$ on the first mesh line is restricted to an interval between 30 and 50, and if secondary rates-of-strain are small within this interval, then wall function values on this mesh line may be close to the conventional limits at Station 5 and, indeed, at intermediate stations within the duct. Further measurements in the near-wall region will be required in order to verify this conjectured behavior. 
66

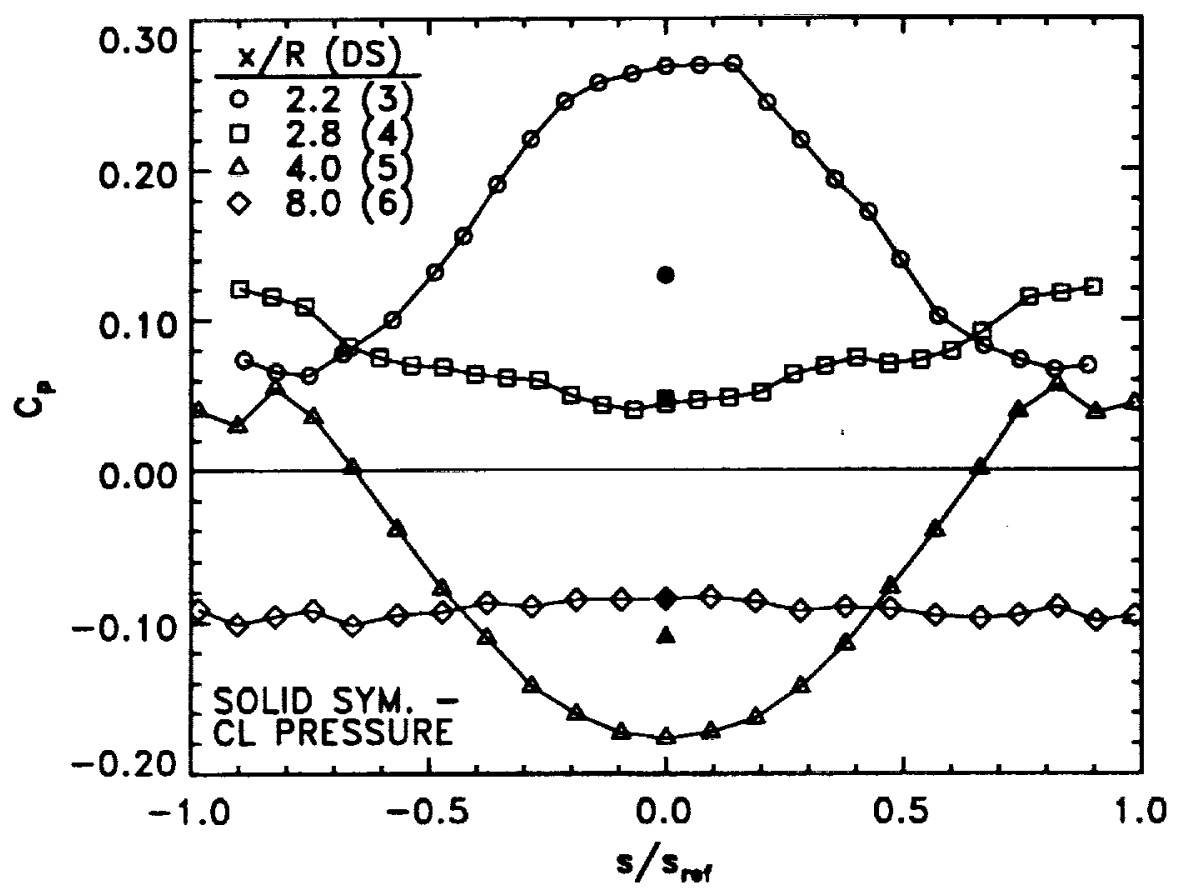

a) $R e_{b}=88,000$.

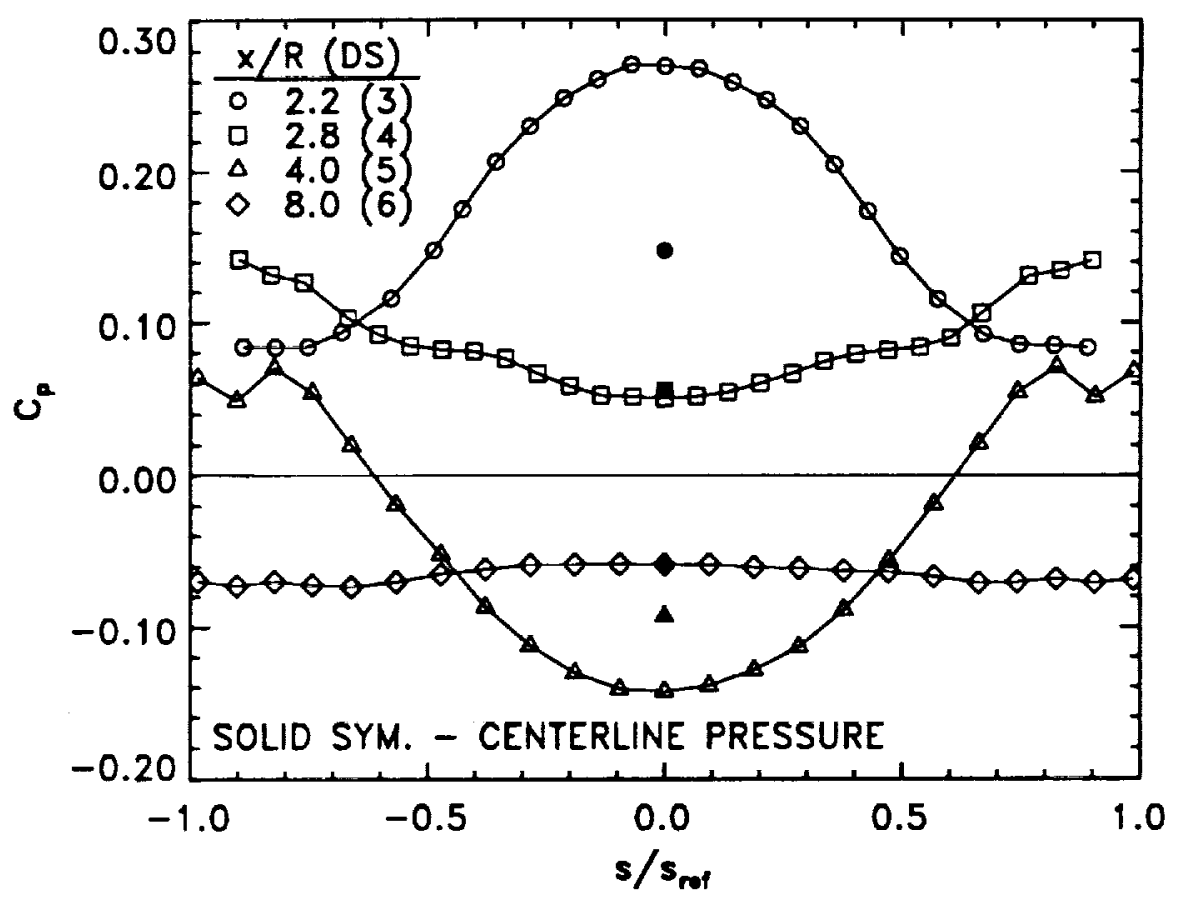

b) $\mathbf{R e}_{\mathrm{b}}=390,000$.

Fig. 5.1. Peripheral wall pressure coefficient distributions. 


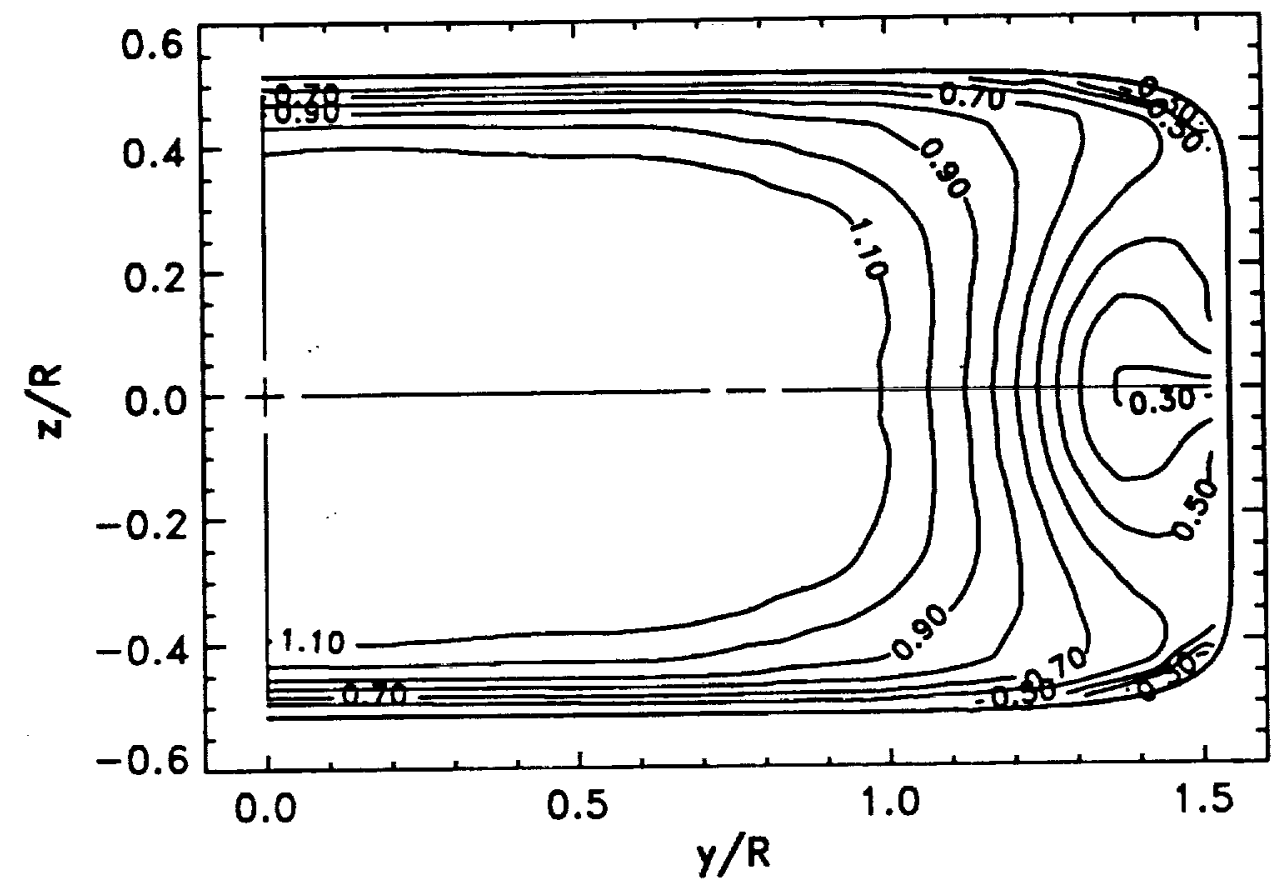

a) $R_{e_{b}}=88,000$.

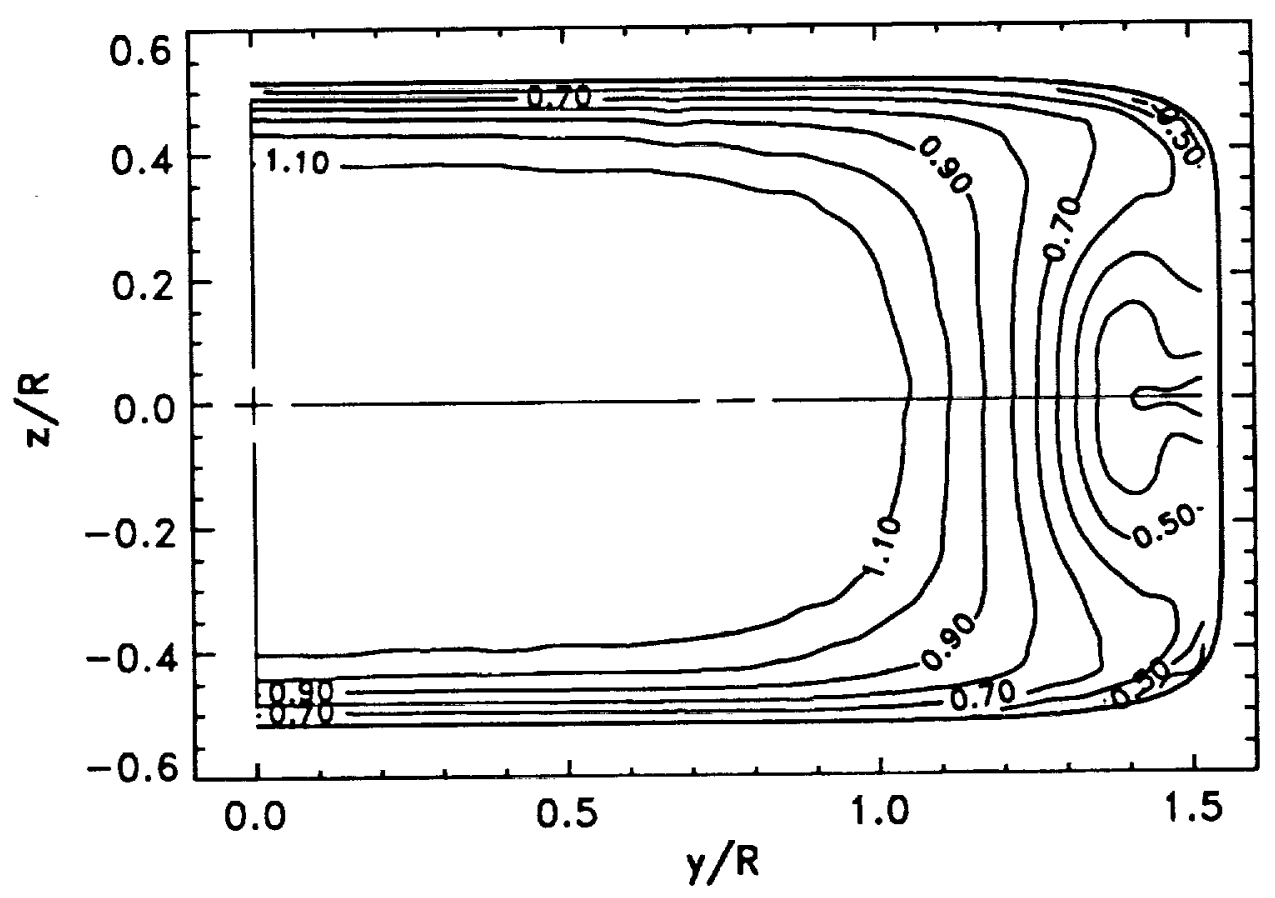

b) $\mathrm{Re}_{\mathrm{b}}=390,000$.

Fig. 5.2. Total pressure contours at Station $5\left(\left(P_{t}-P_{i}\right) / Q_{\text {ref }}\right)$. 


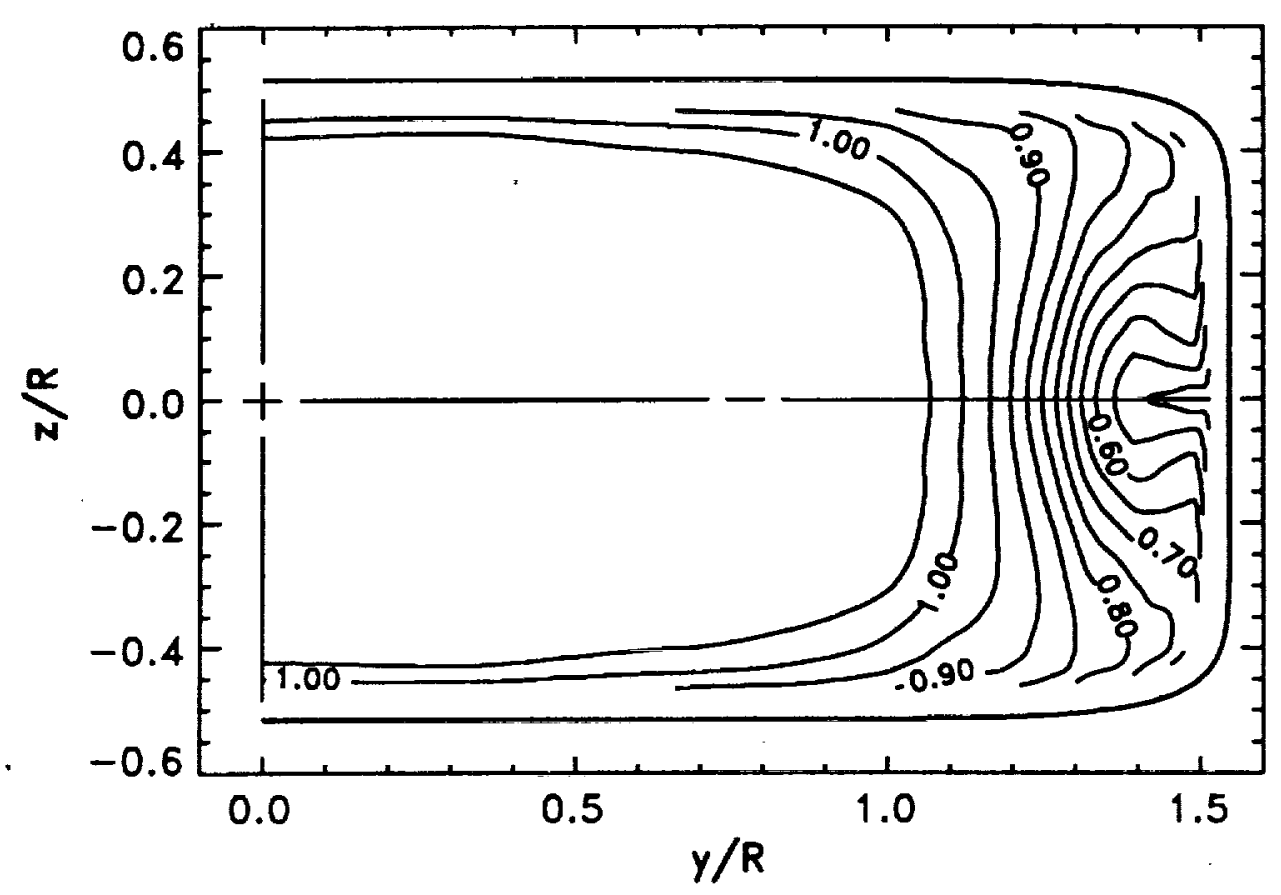

a) $\mathrm{Re}_{\mathrm{b}}=88,000$.

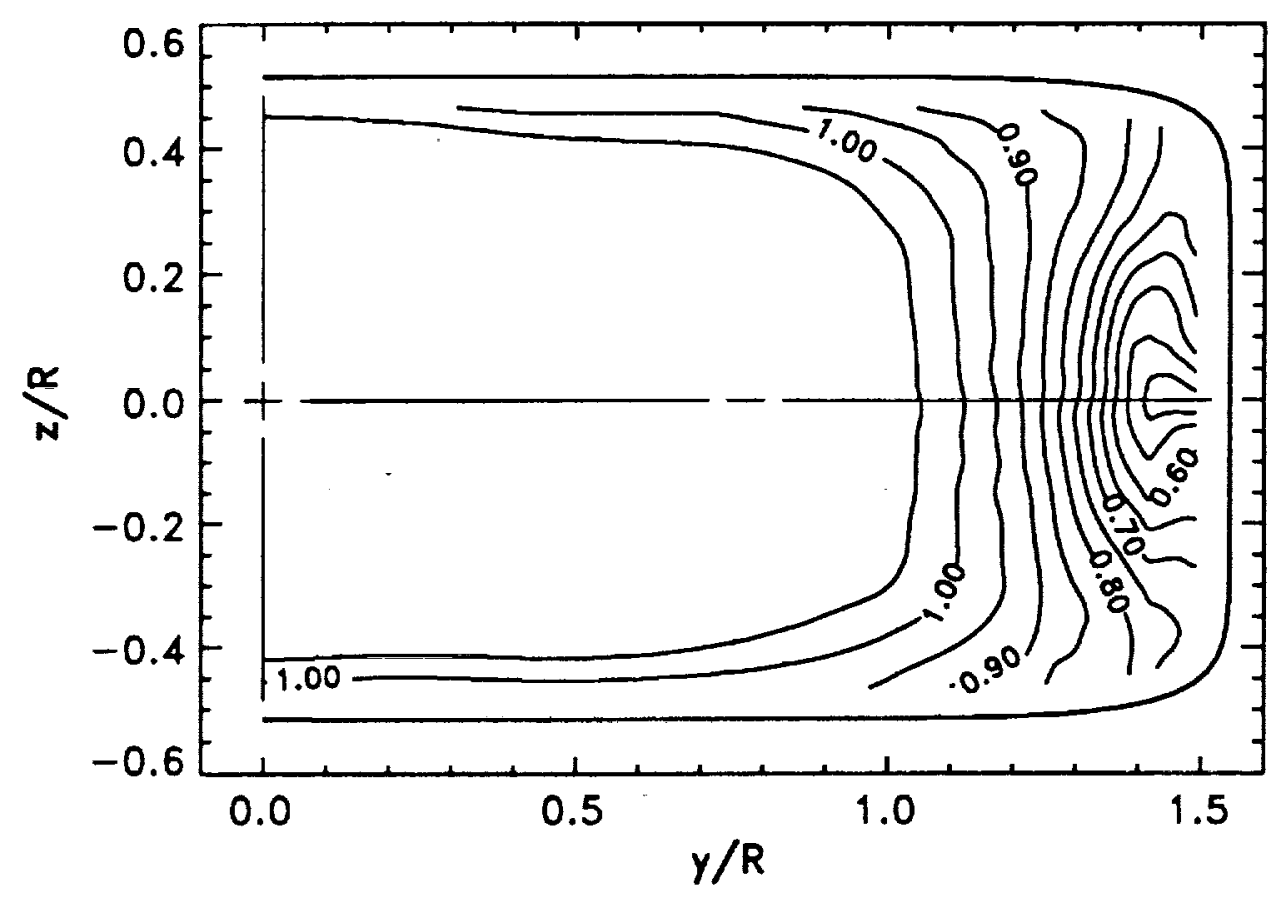

b) $\mathrm{Re}_{\mathrm{b}}=390,000$.

Fig. 5.3. Axial mean velocity contours at Station $5\left(U / U_{b}\right)$. 


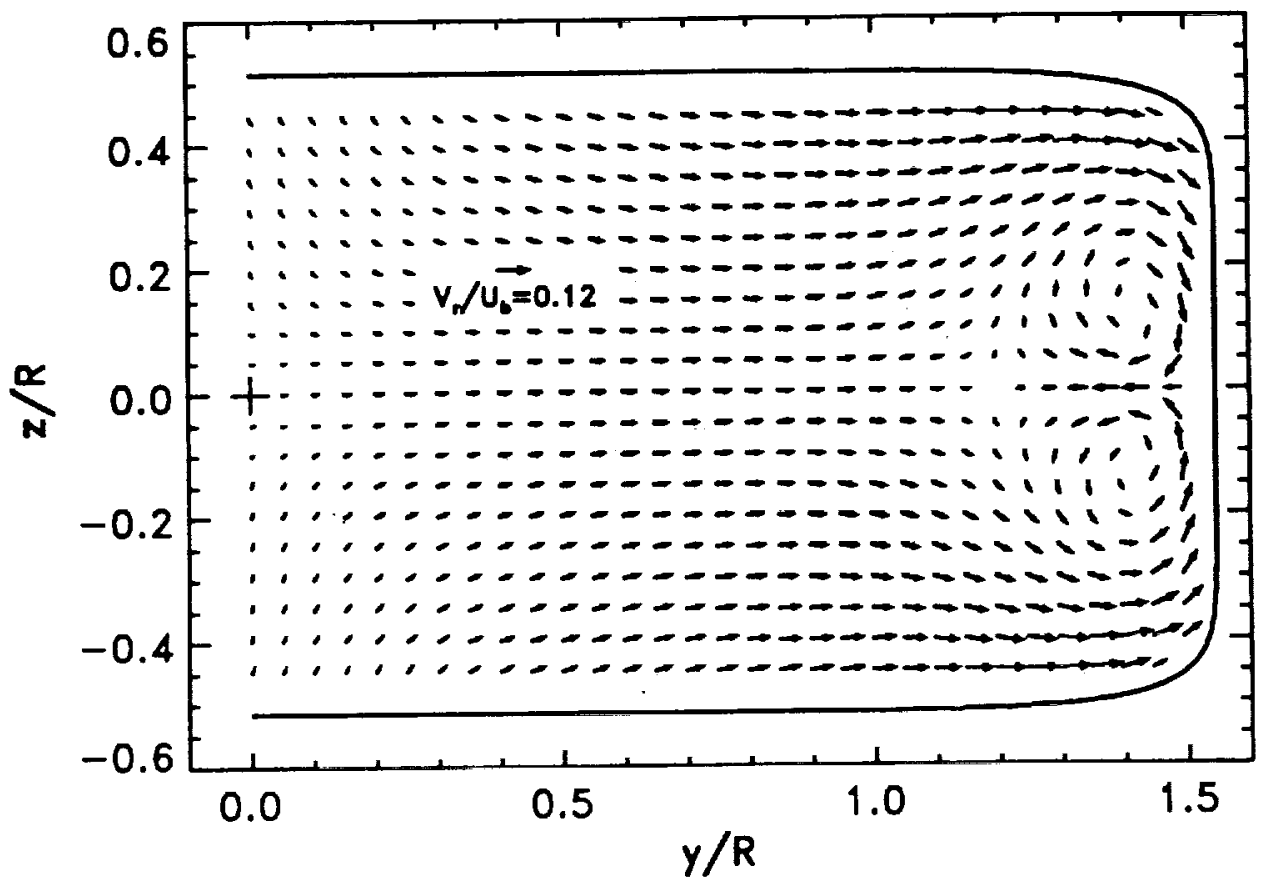

a) $R \mathbf{e}_{b}=88,000$.

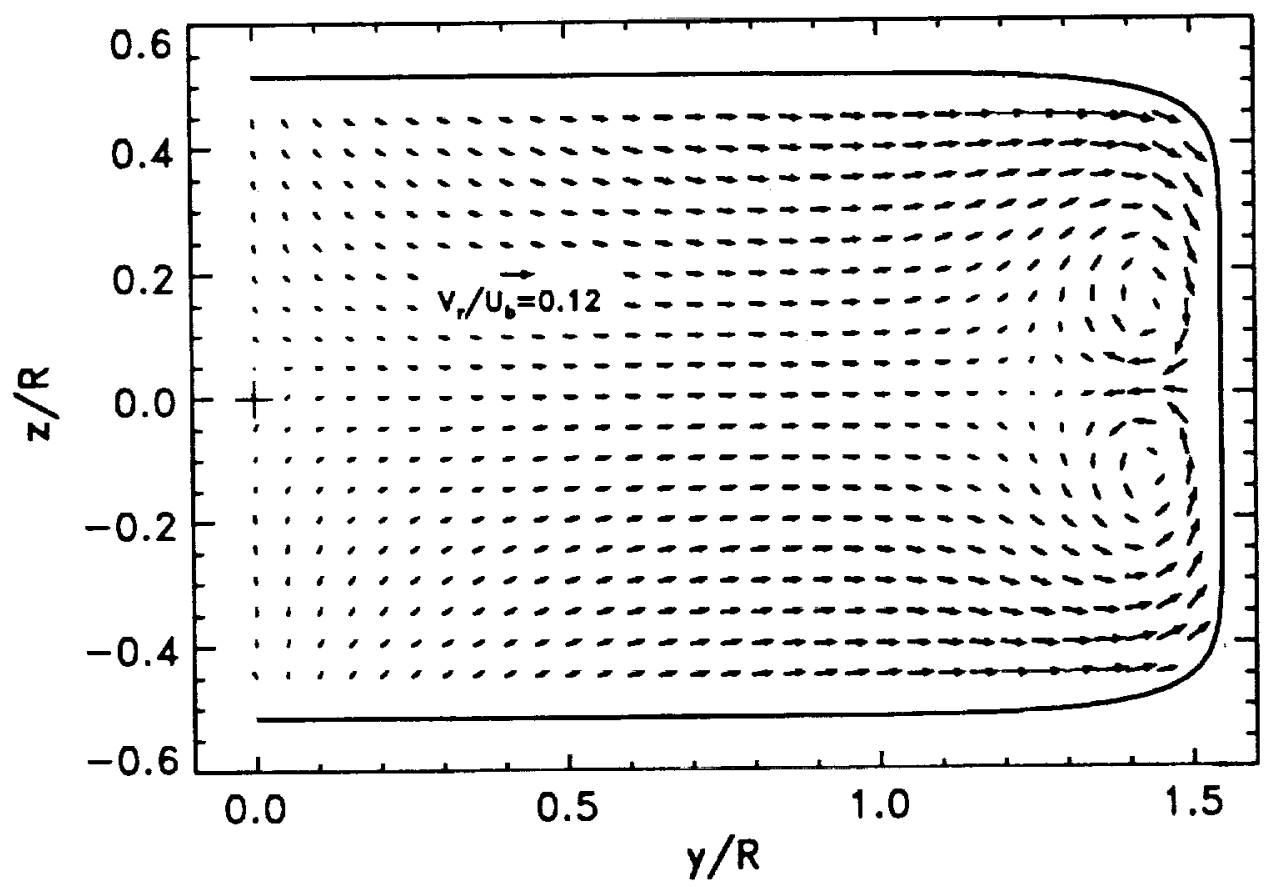

b) $\mathrm{Re}_{\mathrm{b}}=390,000$.

Fig. 5.4. Transverse mean velocity vectors at Station $5\left(V_{r} / U_{b}\right)$. 


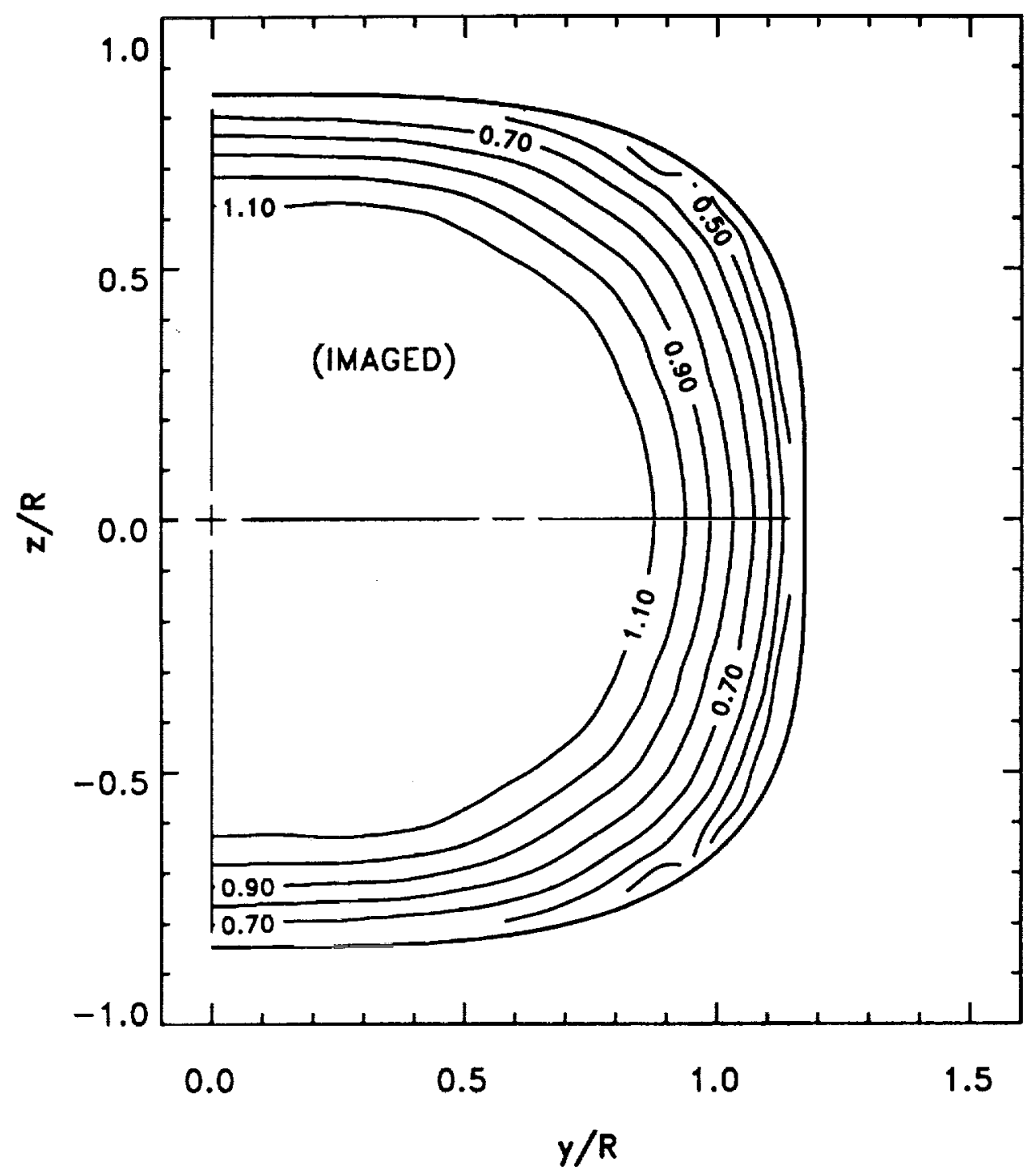

a) Station 3 .

Fig. 5.5. Total pressure contours $\left(\left(P_{t}-P_{i}\right) / Q_{\text {ref }}\right)$. (Continued ...) 


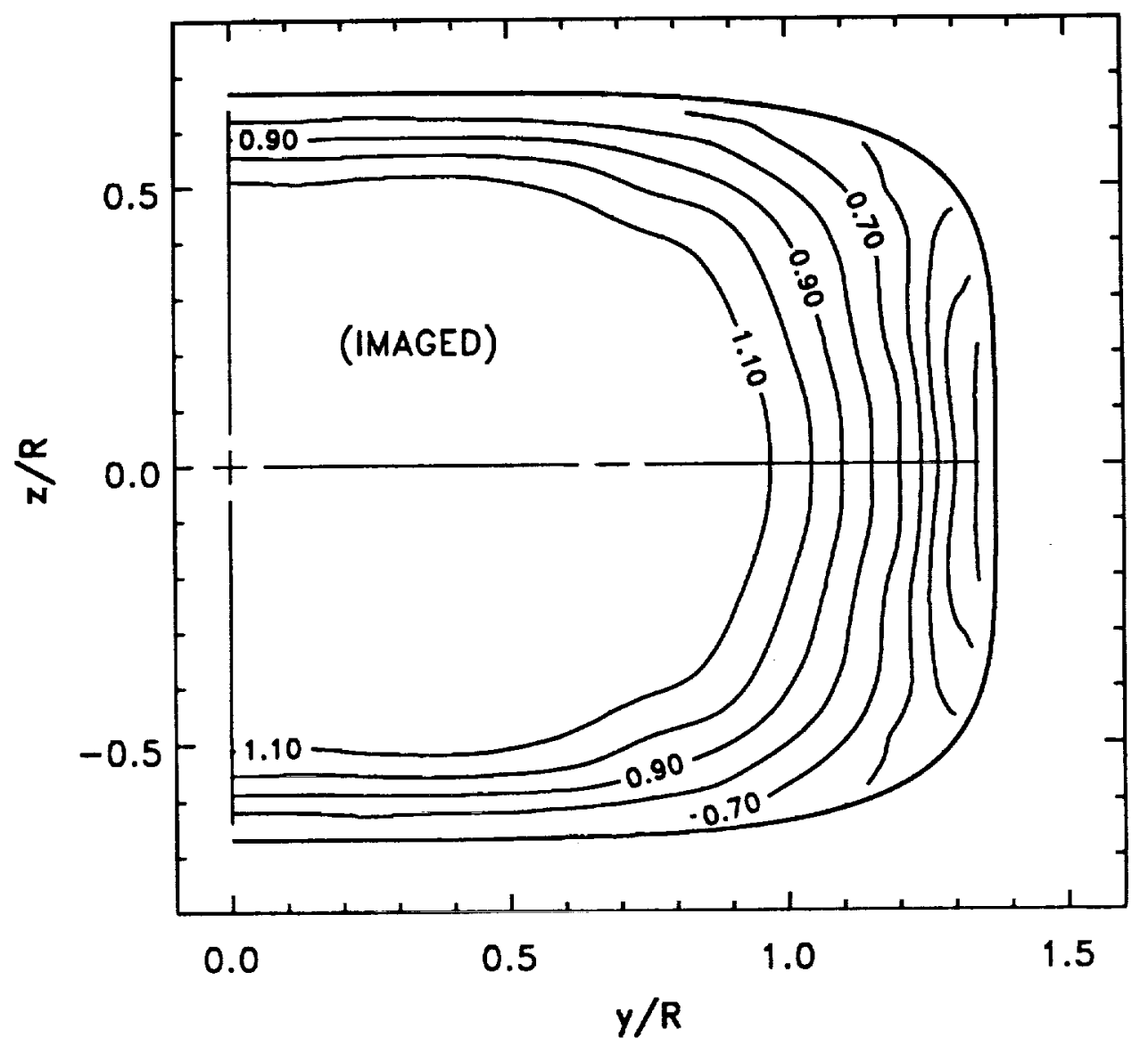

b) Station 4 .

Fig. 5.5. Total pressure contours $\left(\left(P_{t}-P_{i}\right) / Q_{\text {ref }}\right)$. (Continued ...) 


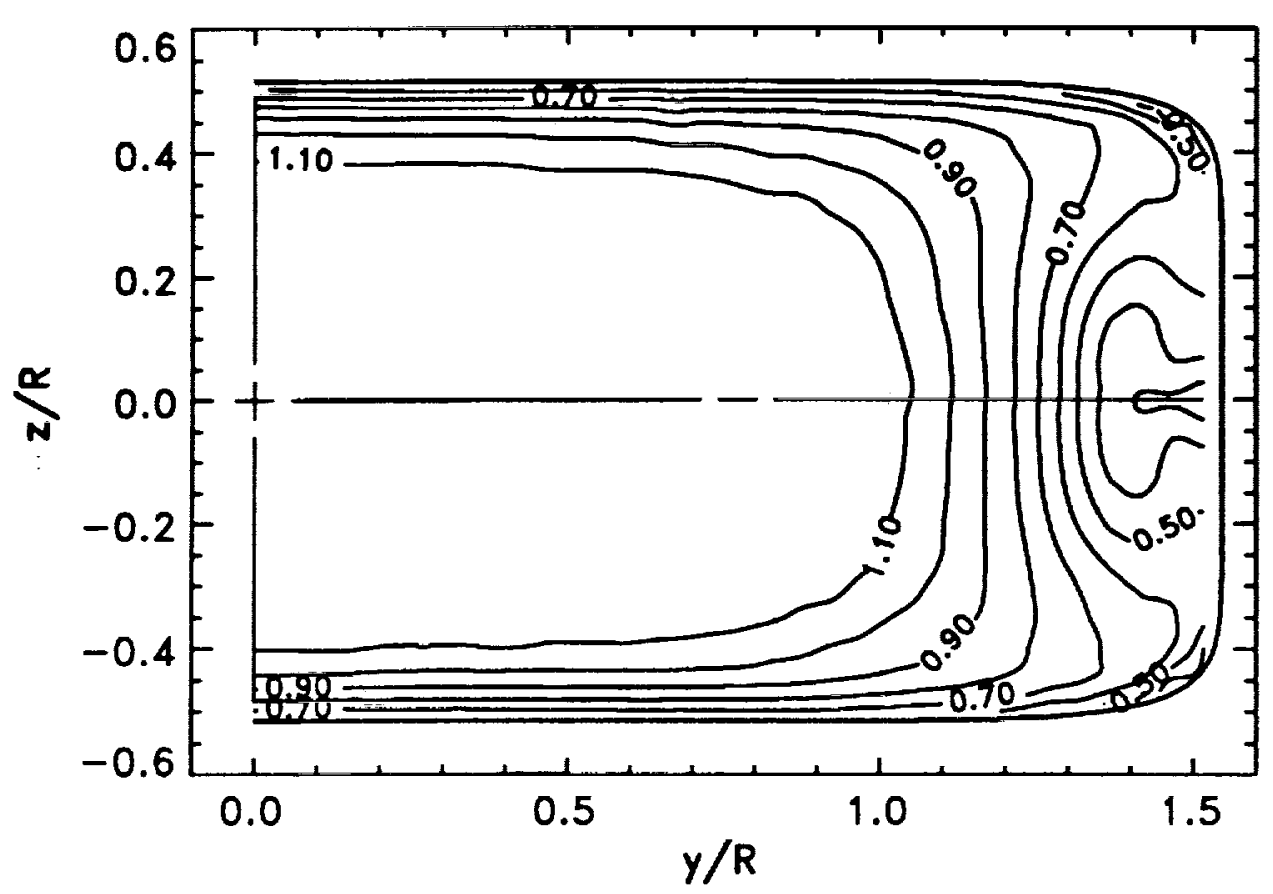

c) Station 5.

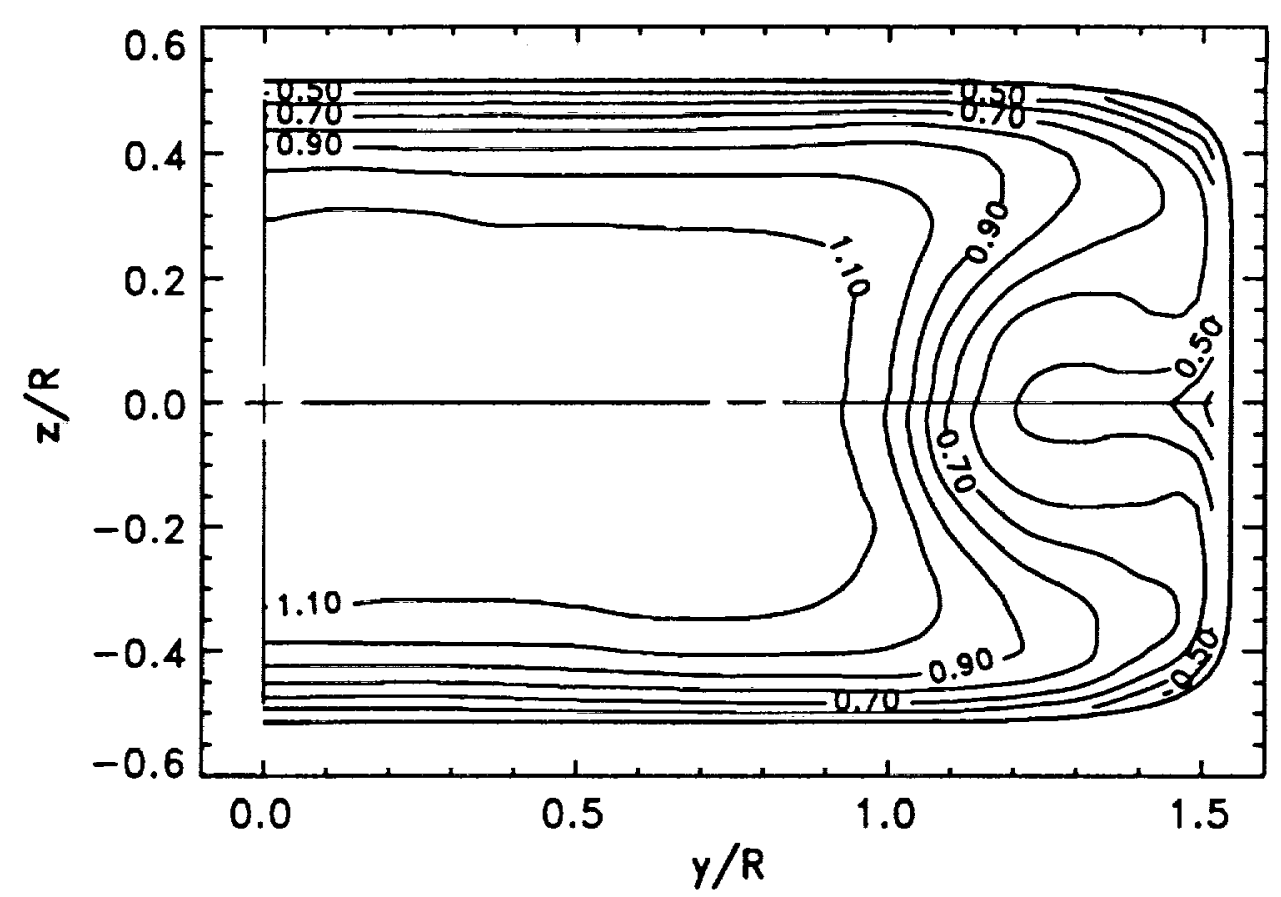

d) Station 6.

Fig. 5.5. Total pressure contours $\left(\left(P_{t}-P_{i}\right) / Q_{r e f}\right)$. 


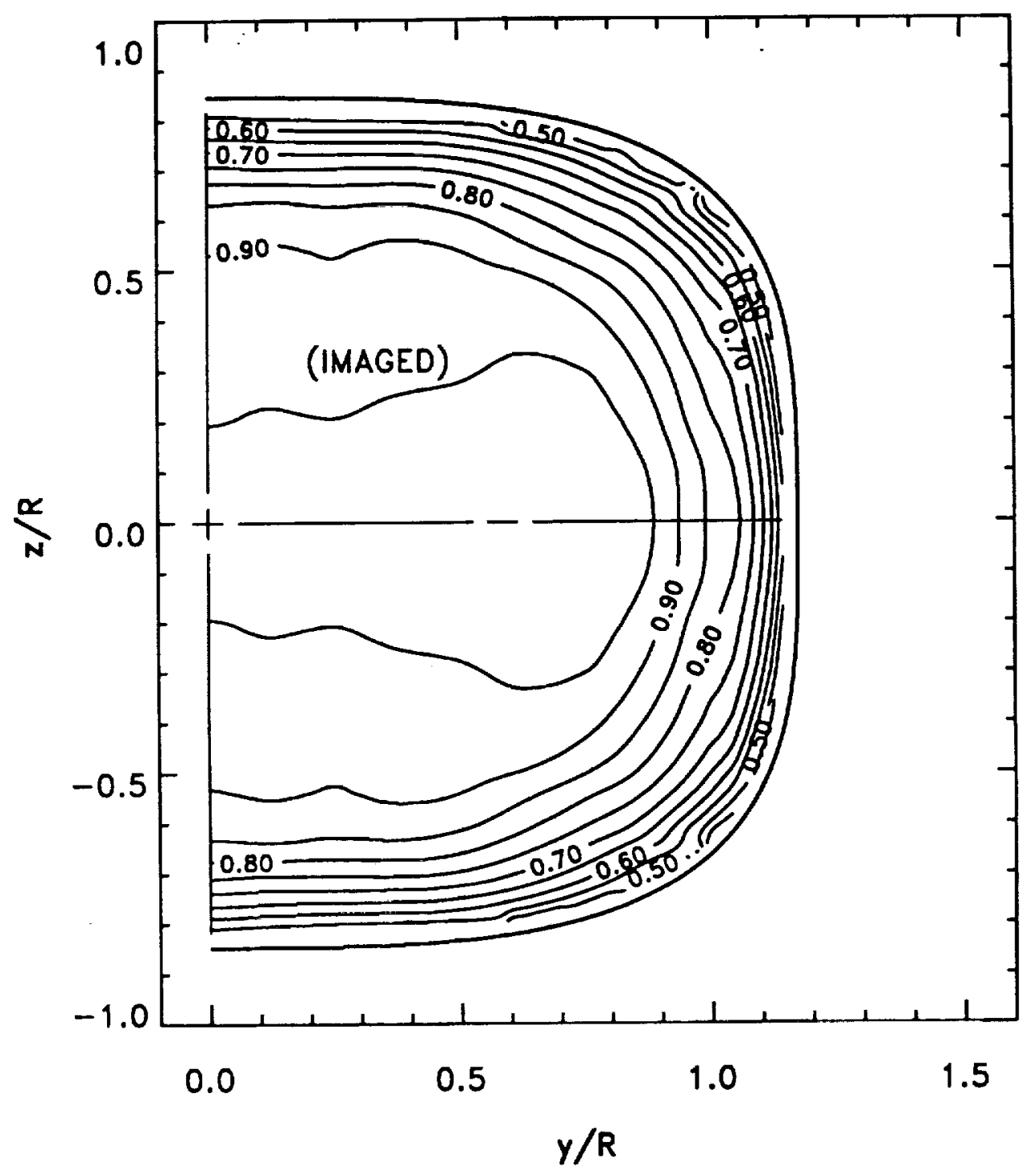

a) Station 3 .

Fig. 5.6. Axial mean velocity contours $\left(U / U_{b}\right)$. (Continued ...) 


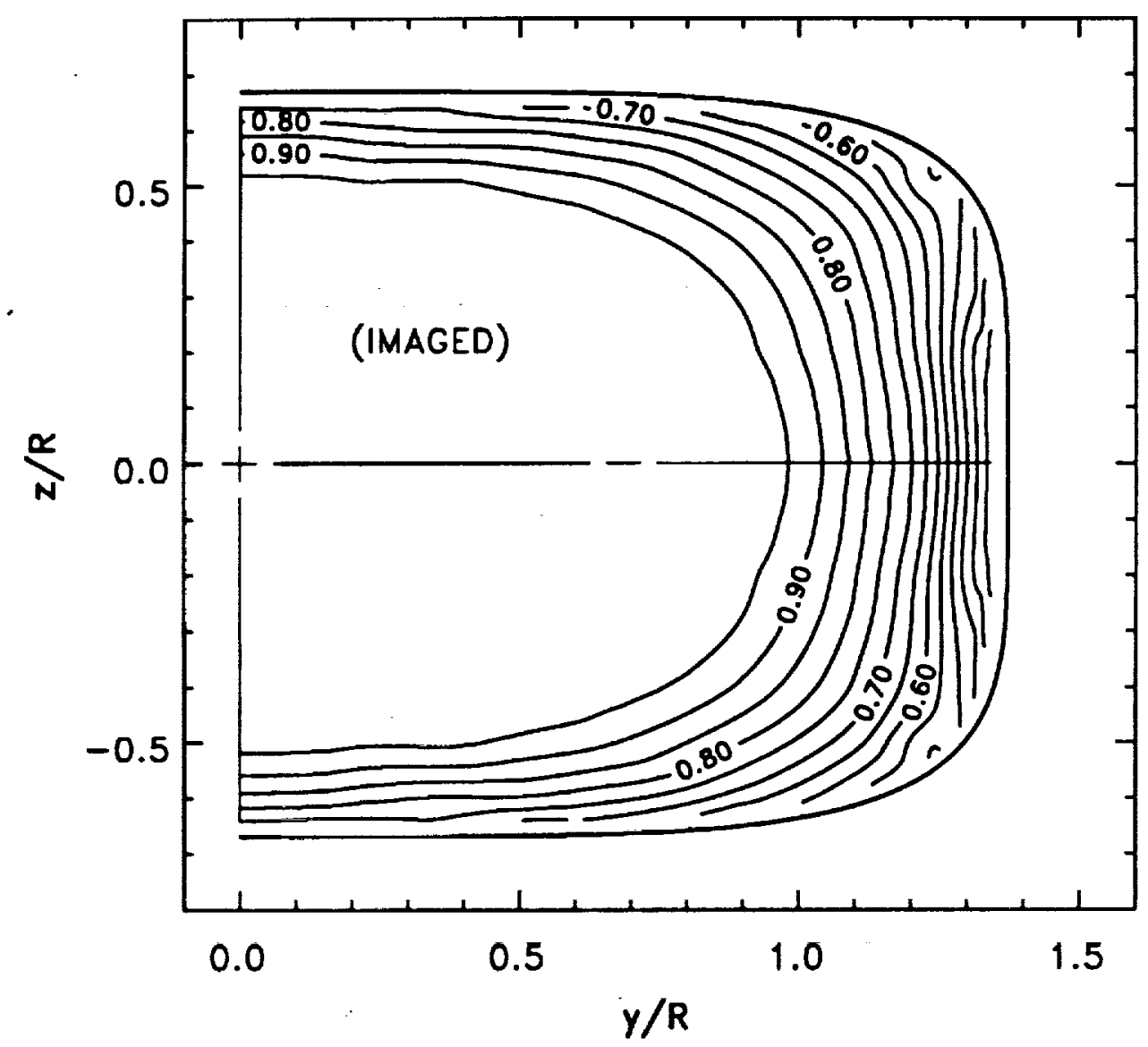

b) Station 4 .

Fig. 5.6. Axial mean velocity contours $\left(U / U_{b}\right)$. (Continued ...) 


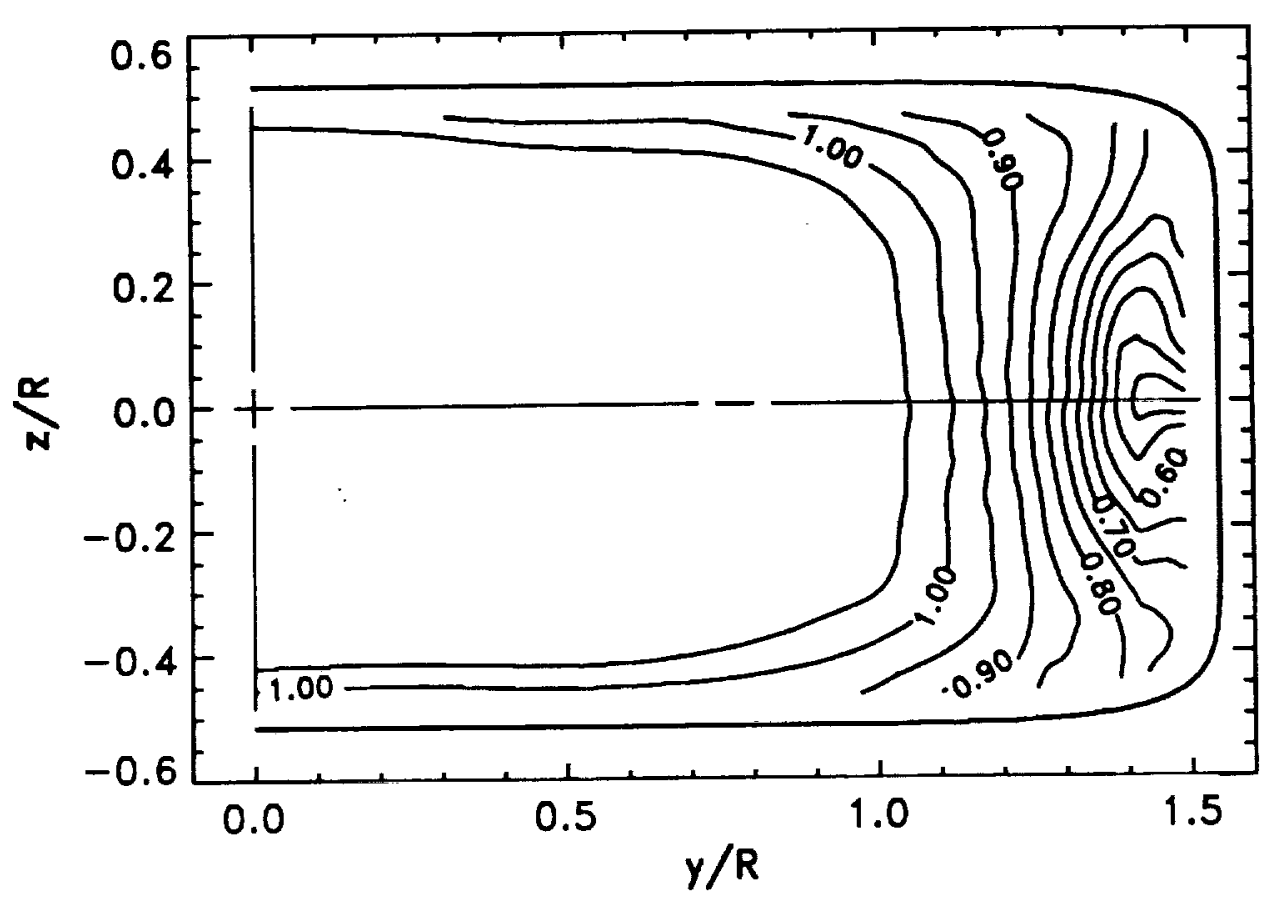

c) Station 5 .

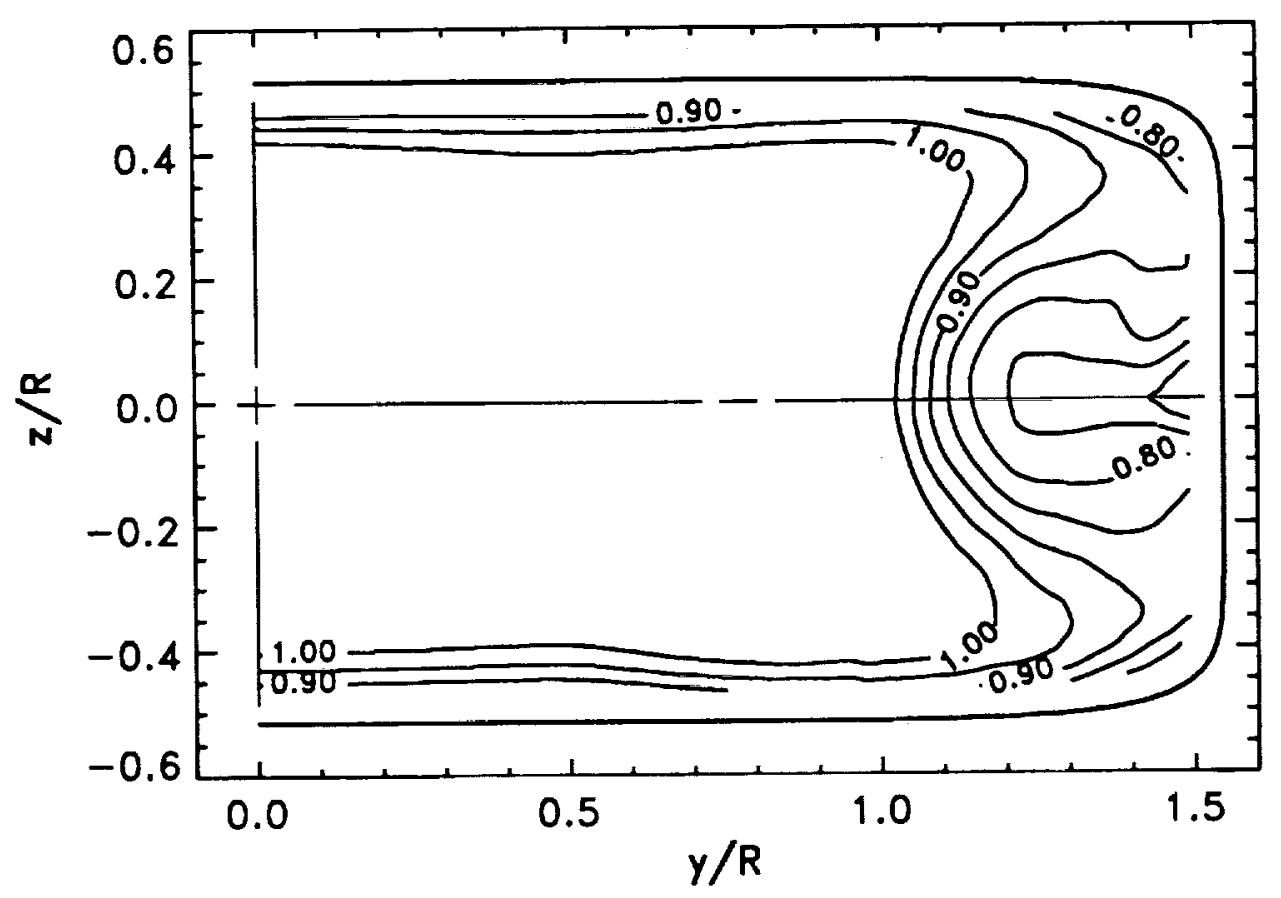

d) Station 6 .

Fig. 5.6. Axial mean velocity contours $\left(U / U_{b}\right)$. 


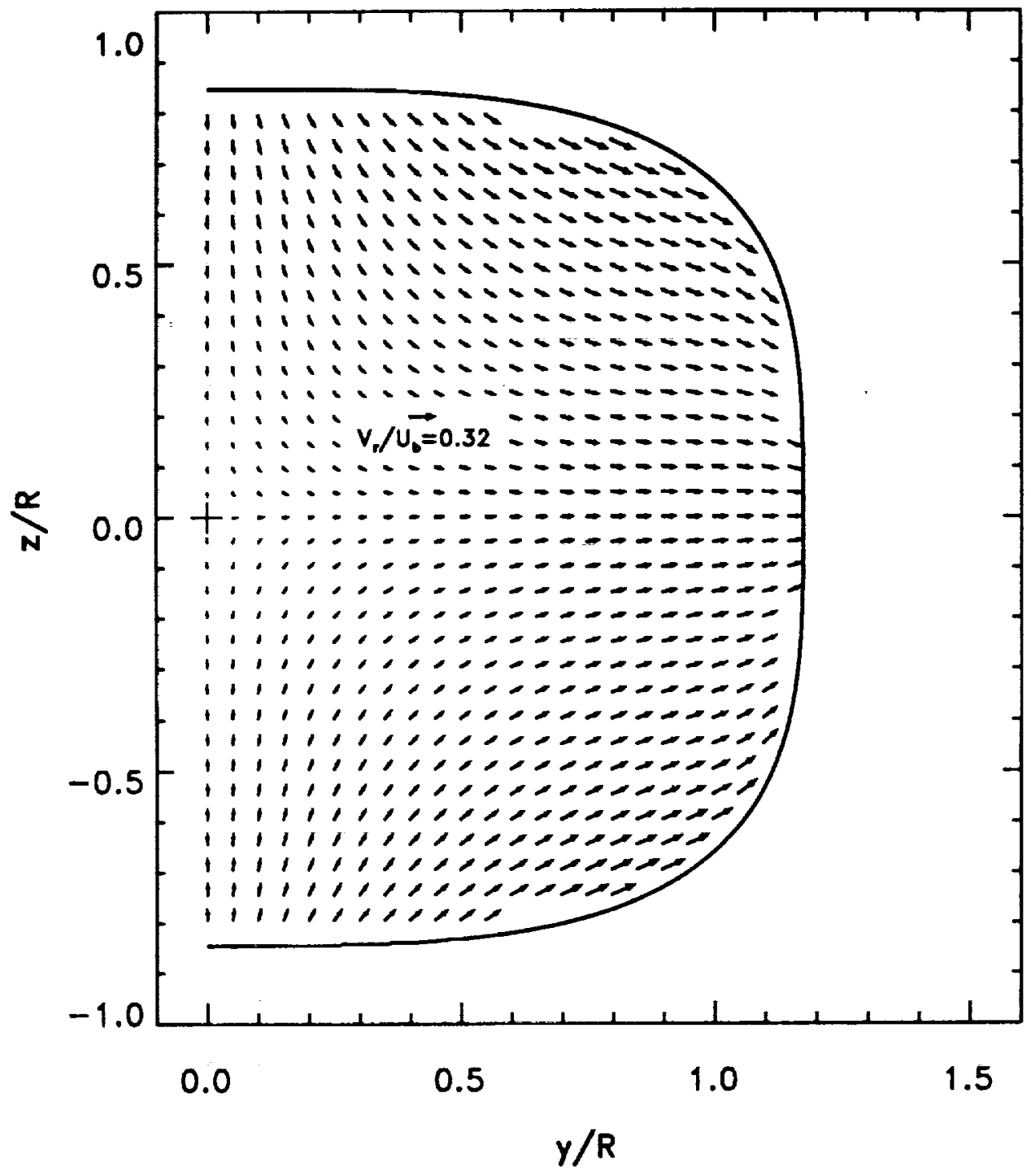

a) Station 3.

Fig. 5.7. Transverse mean velocity vectors $\left(V_{r} / U_{b}\right)$. (Continued ...) 


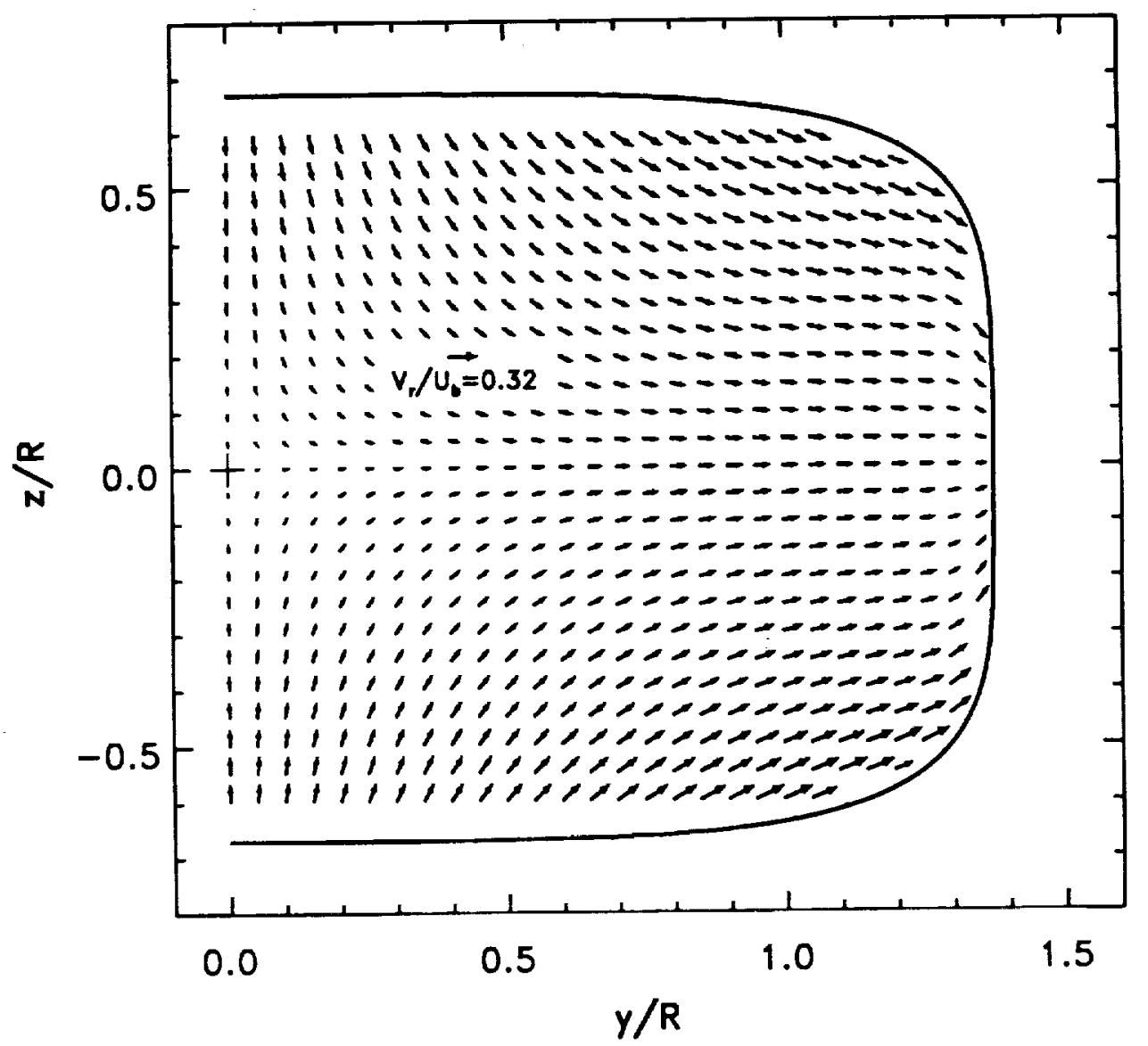

b) Station 4.

Fig. 5.7. Transverse mean velocity vectors $\left(V_{x} / U_{b}\right)$. (Continued ...) 


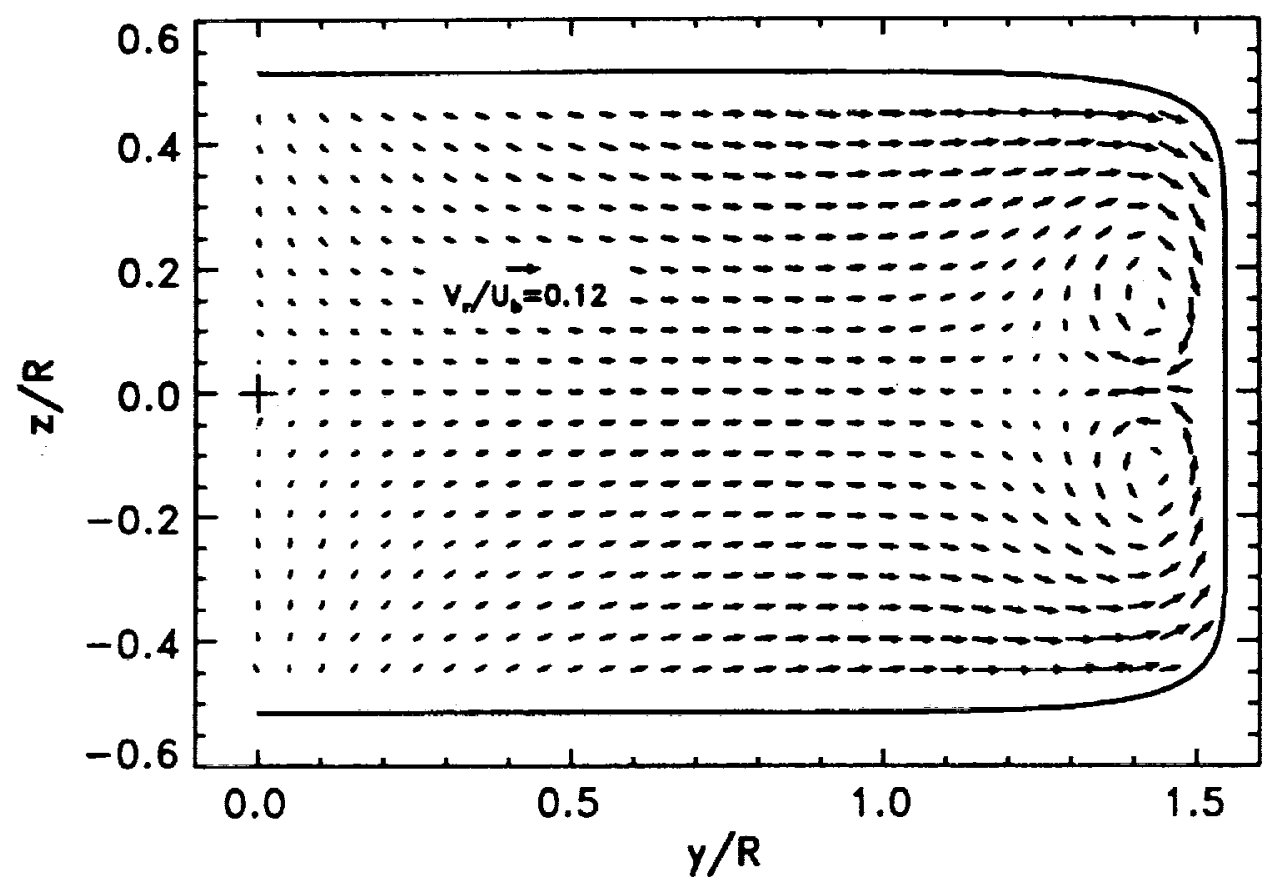

c) Station 5.

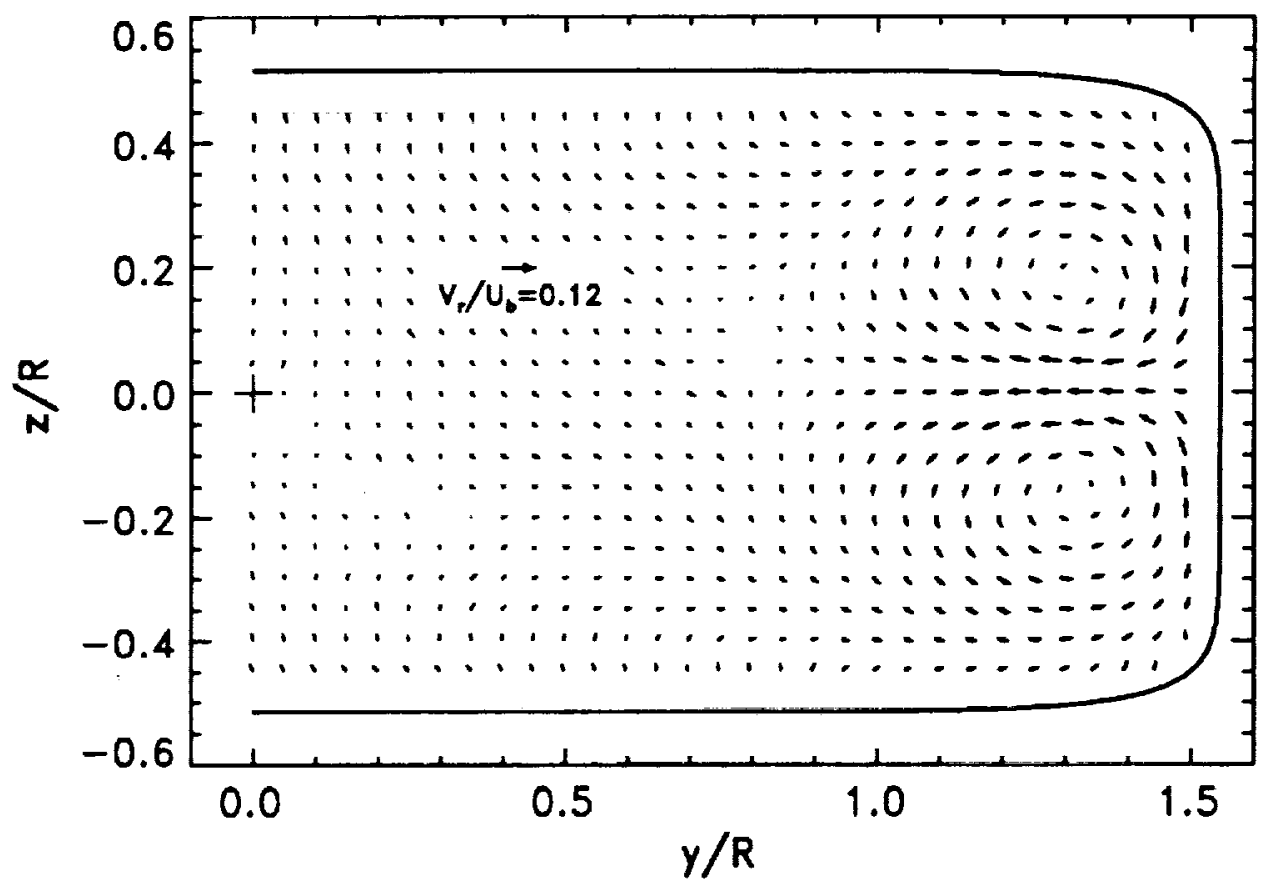

d) Station 6 .

Fig. 5.7. Transverse mean velocity vectors $\left(V_{r} / U_{b}\right)$. 


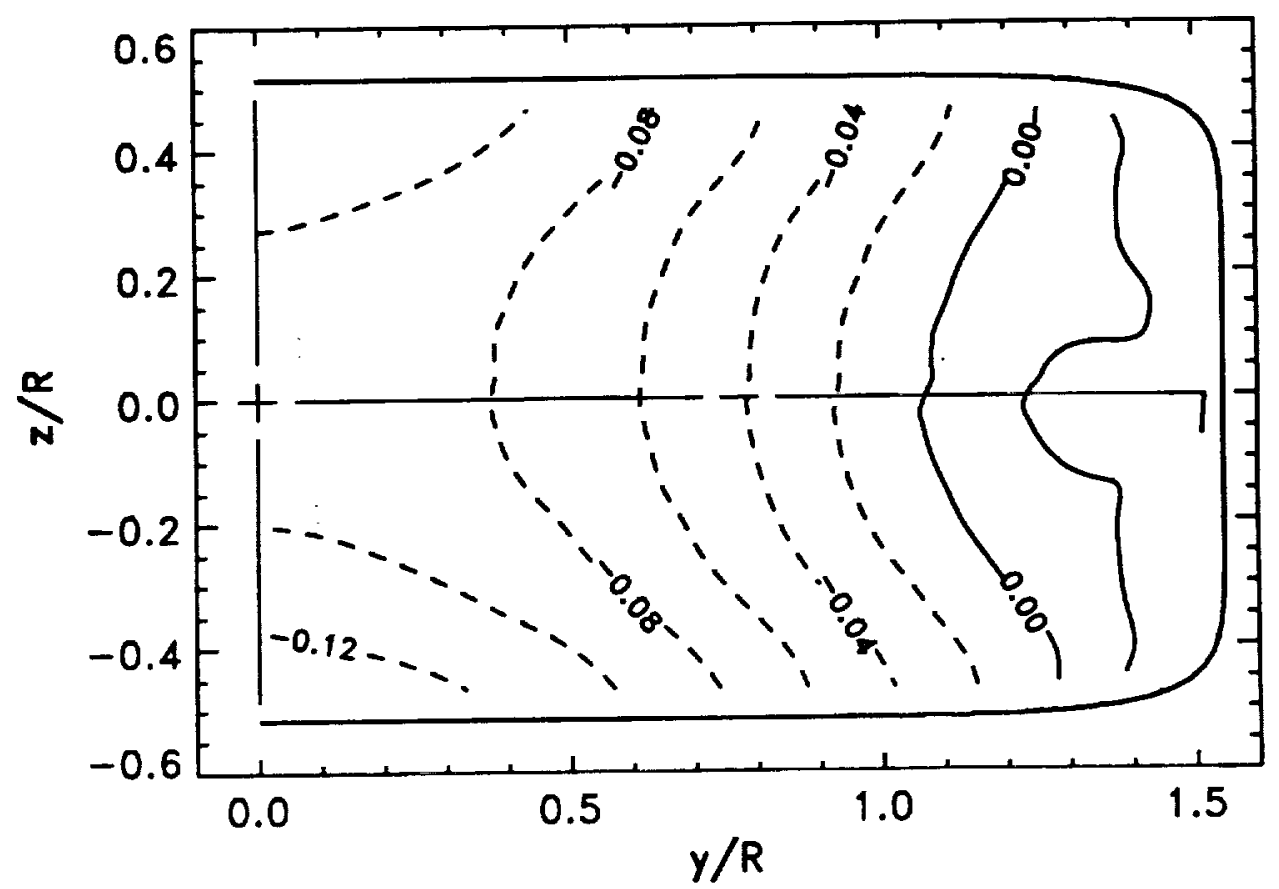

a) Measured.

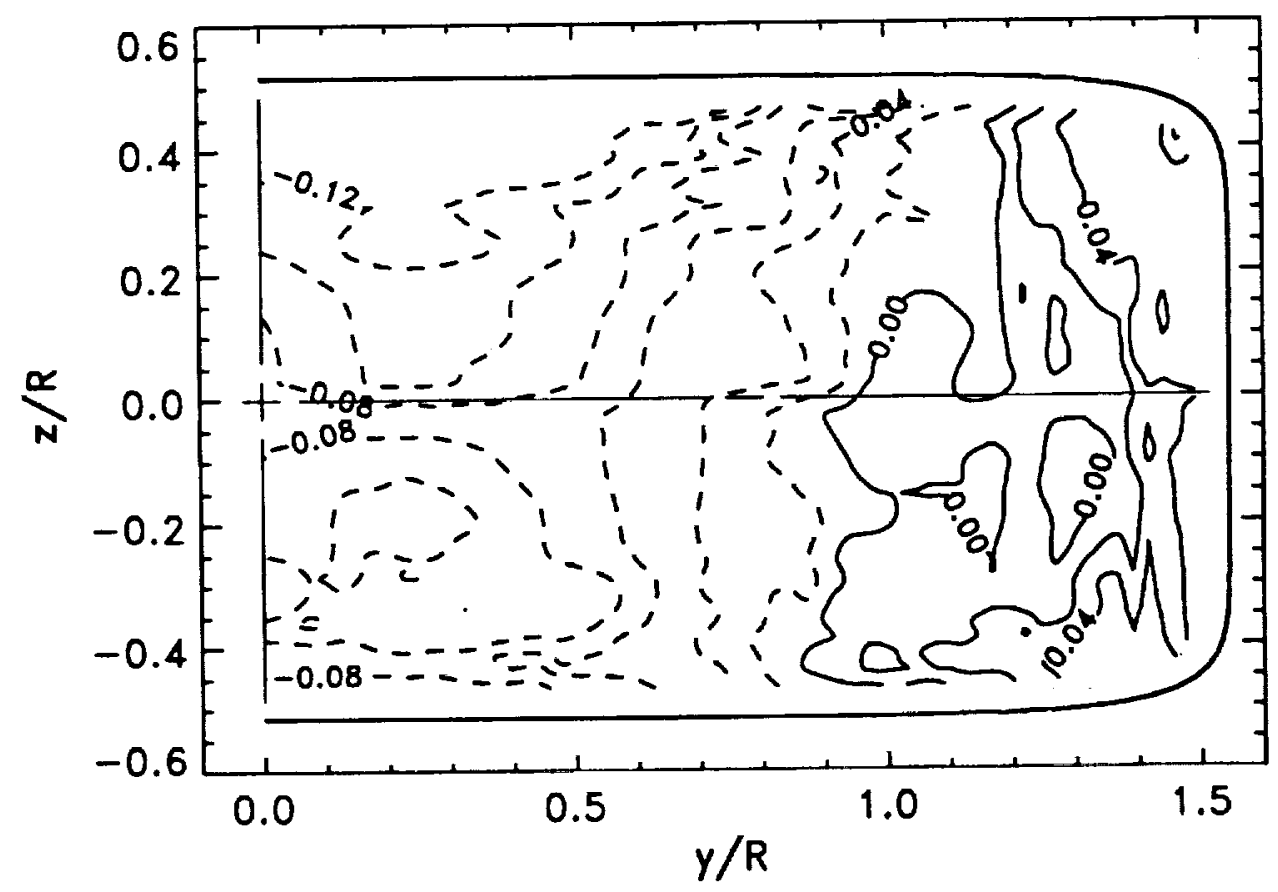

b) Calculated.

Fig. 5.8. Static pressure contours $\left(\left(P-P_{i}\right) / Q_{r e f}\right)$ at Station 5 . 
80

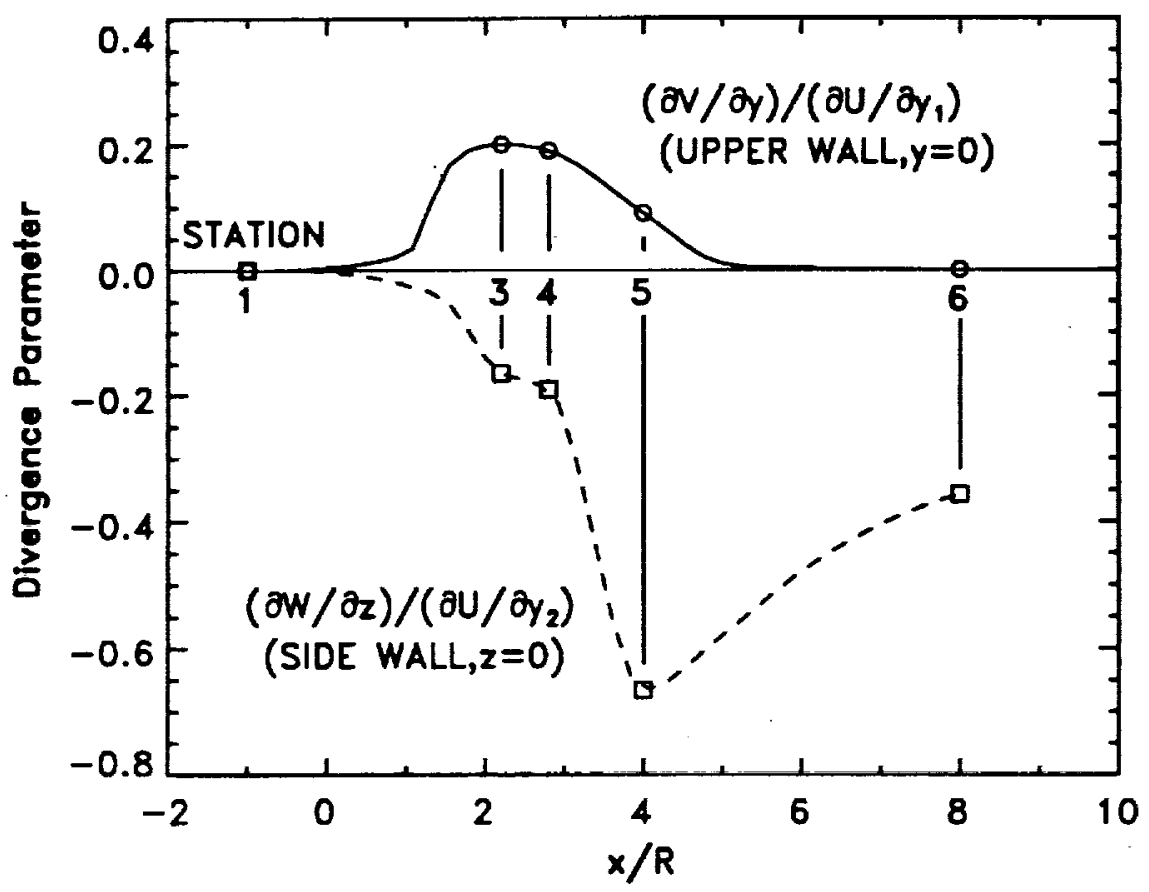

Fig. 5.9. Axial distribution of divergence rate-of-strain parameter along midplanes $y=0$ and $z=0$.

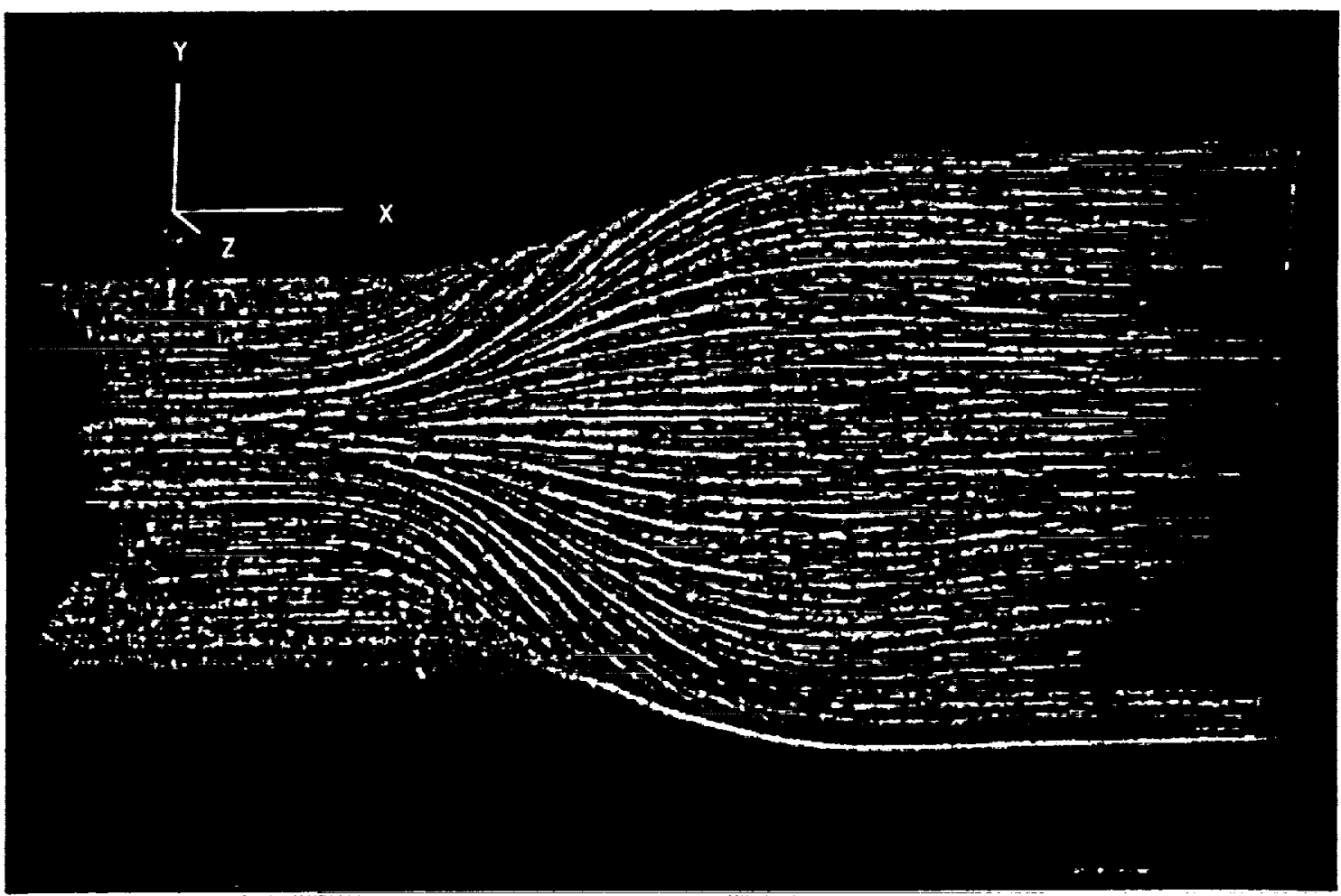

Fig. 5.10. Surface oil flow visualization by Reichert [4], $R e_{c l, i}=1.57 \times 10^{6}$. 
81

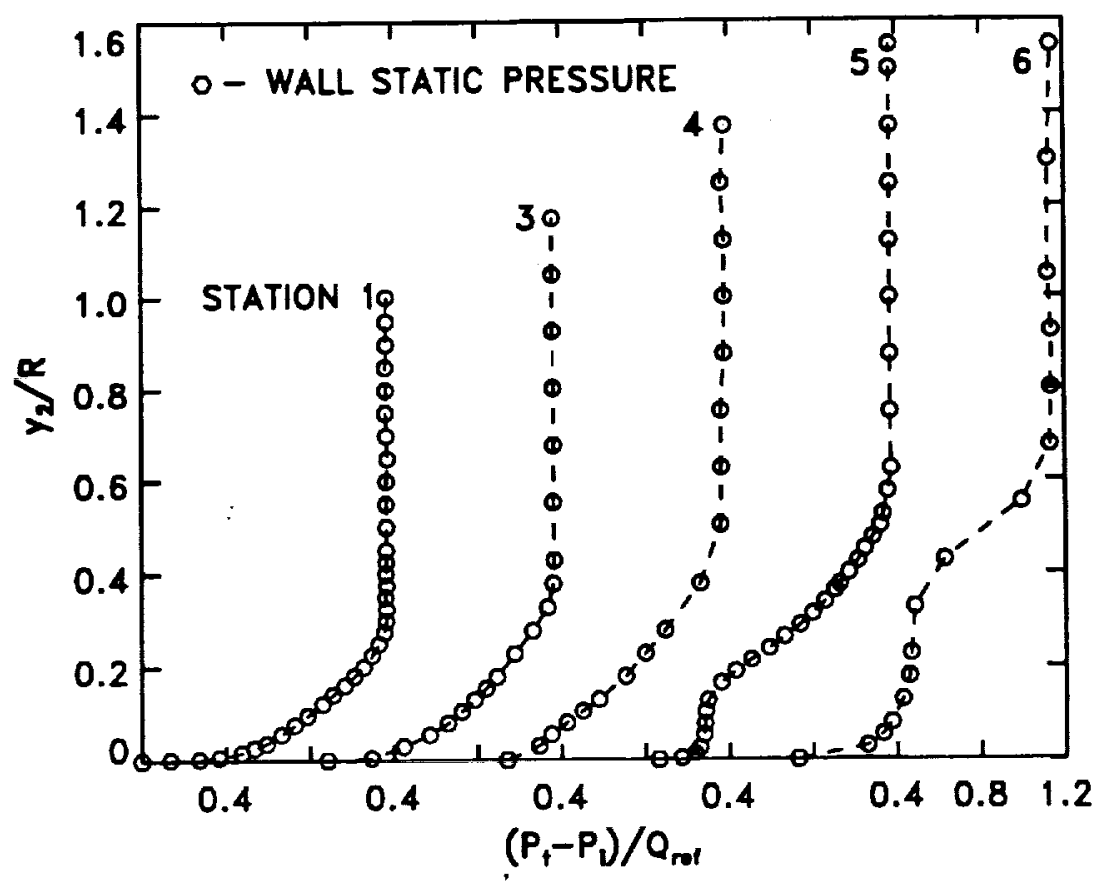

a) $y_{2}$ traverse (see Fig. 3.2).

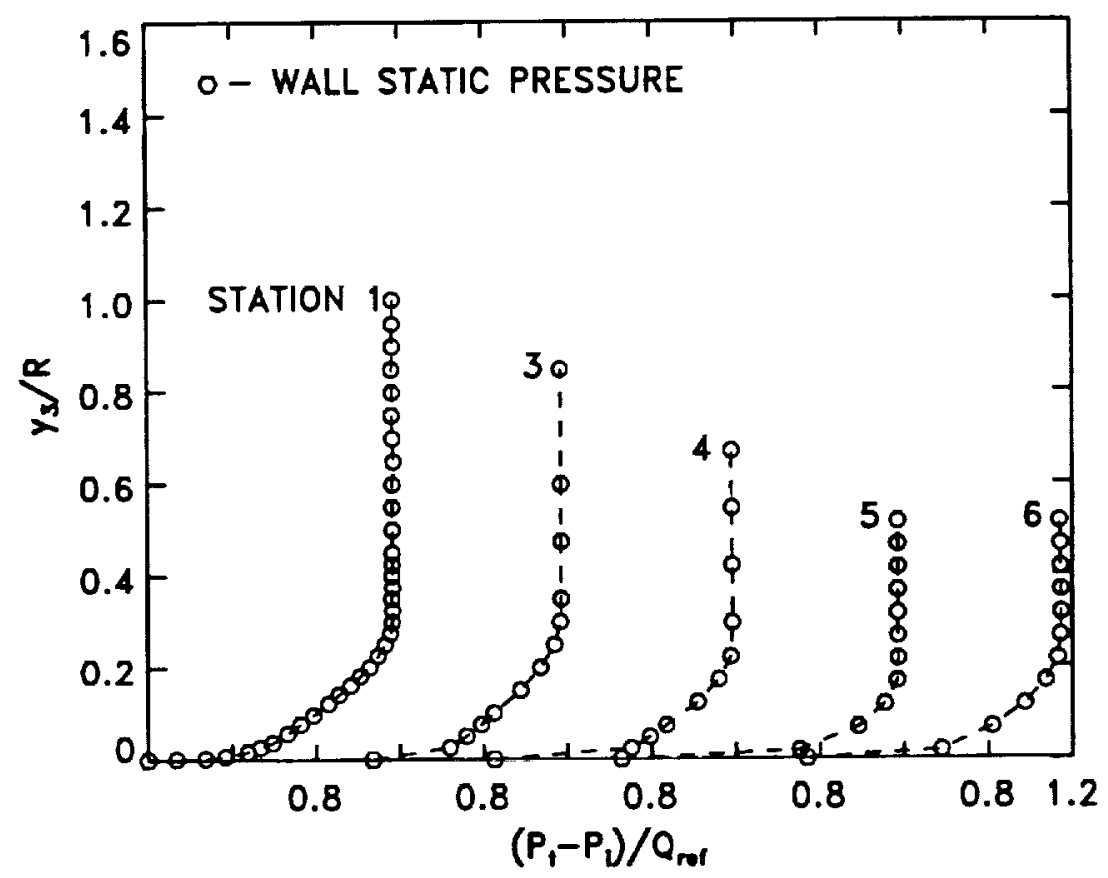

b) $y_{3}$ traverse (see Fig. 3.2).

Fig. 5.11. Total pressure profiles along the semi-major and semi-minor axes, $\mathrm{Re}_{b}=390,000$. 


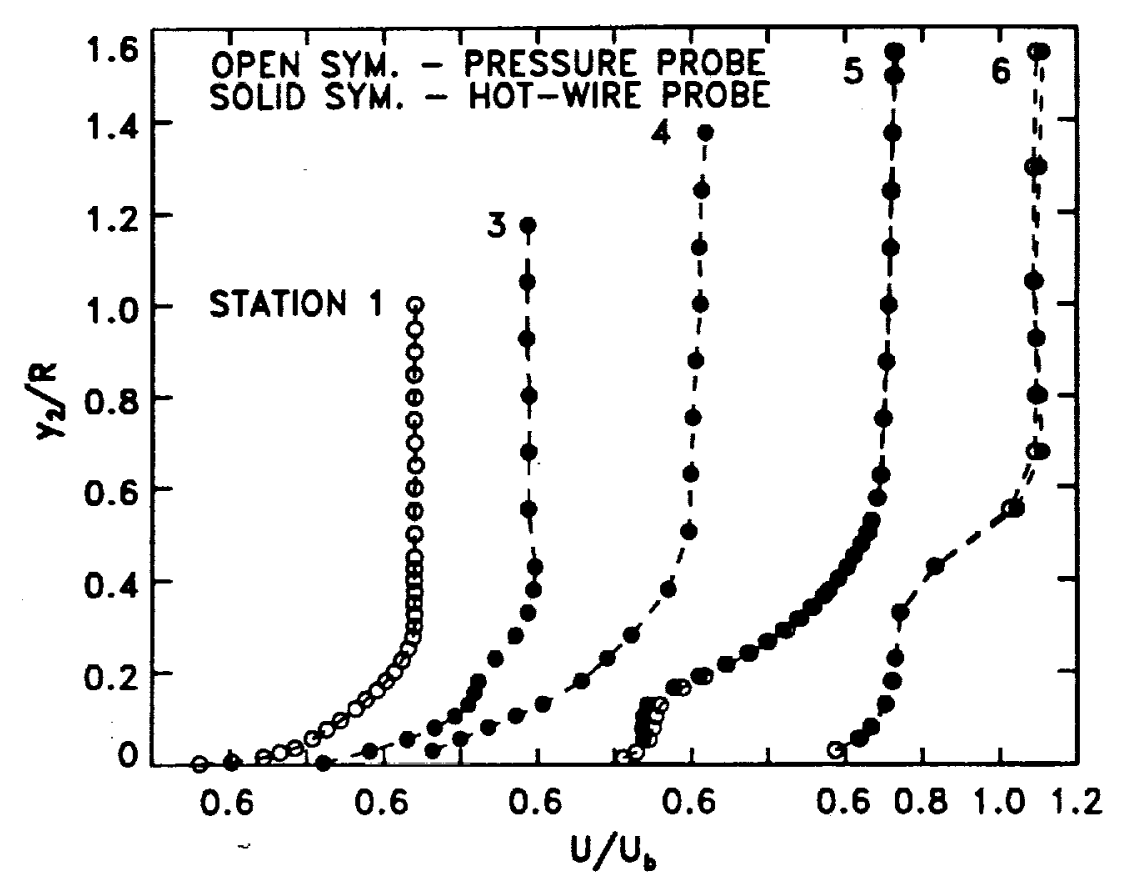

a) $y_{2}$ traverse (see Fig. 3.2).

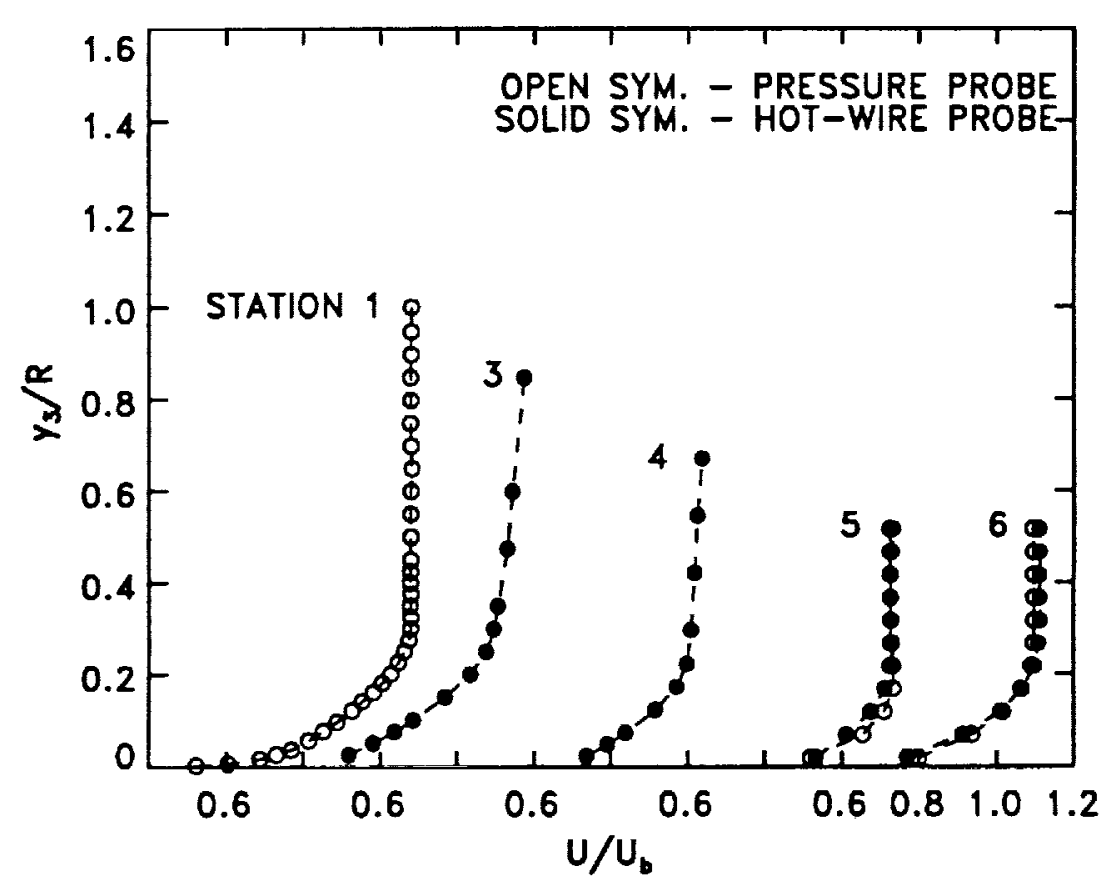

b) $y_{3}$ traverse (see Fig. 3.2).

Fig. 5.12. Axial velocity profiles along the semi-major and semi-minor axes, $\mathrm{Re}_{b}=390,000$. 


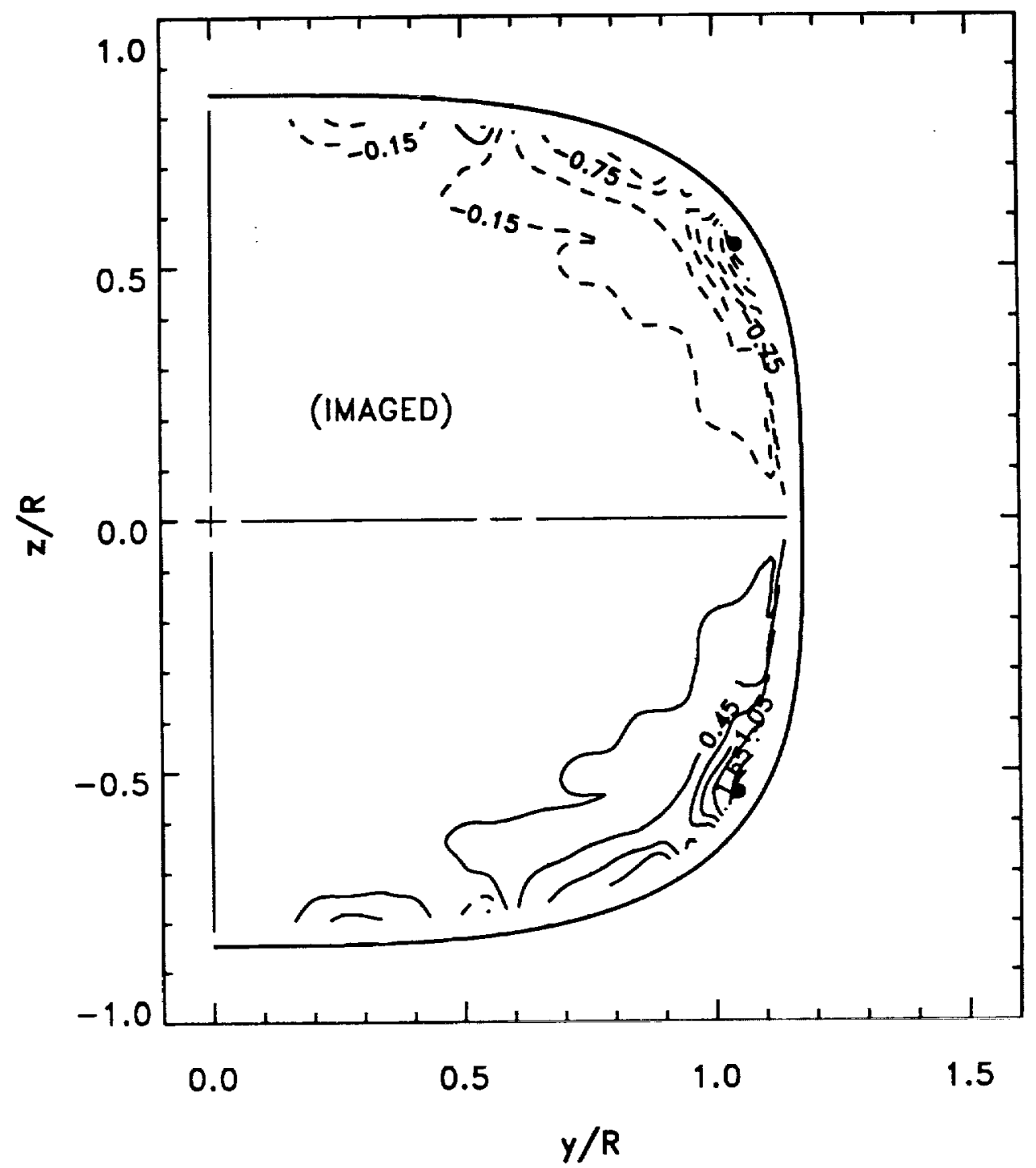

a) Station 3 .

Fig. 5.13. Axial mean vorticity contours $\left(\Omega_{x} R / U_{b}\right)$. (Continued ...) 


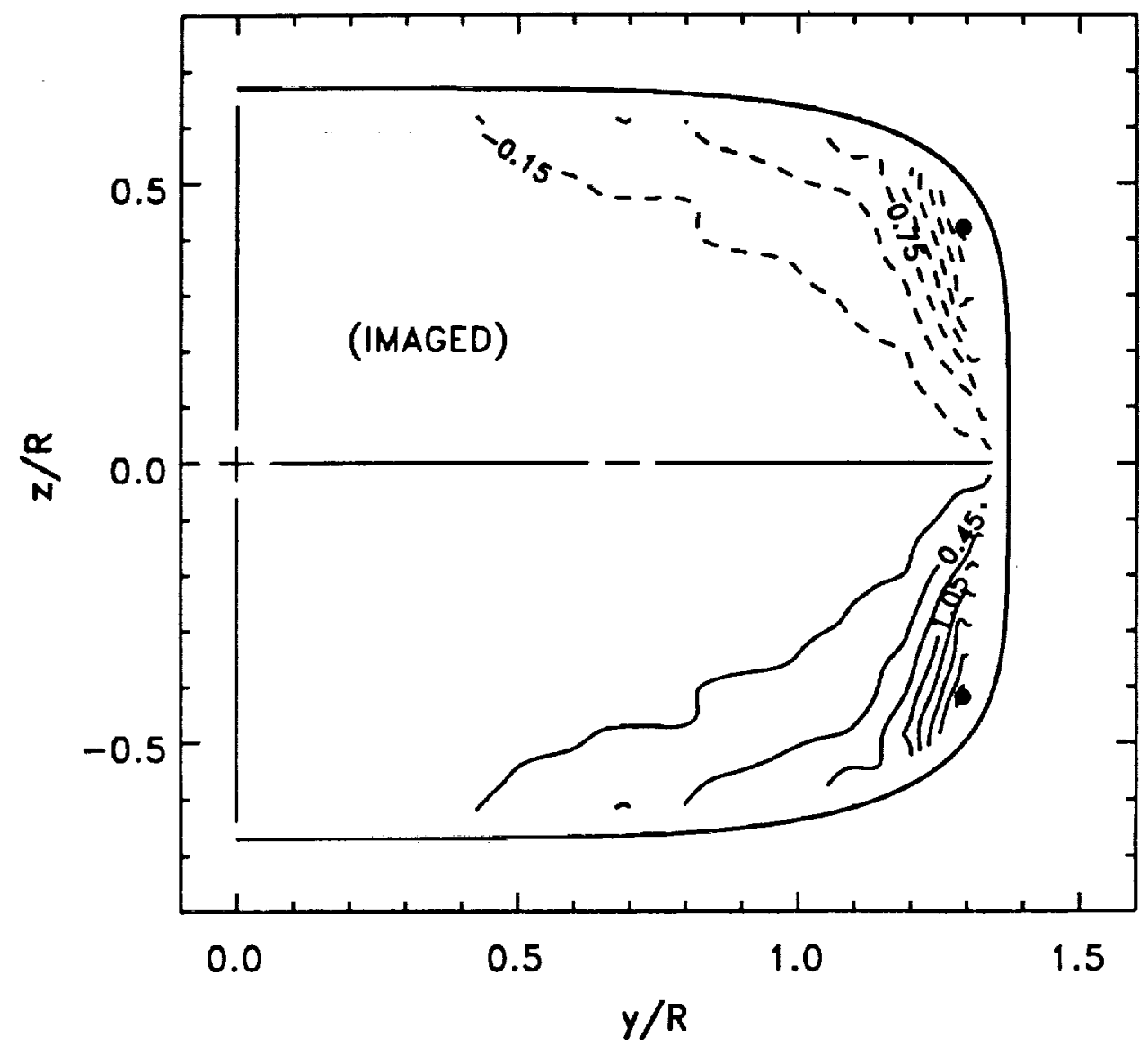

b) Station 4 .

Fig. 5.13. Axial mean vorticity contours $\left(\Omega_{x} R / U_{b}\right)$. (Continued ...) 


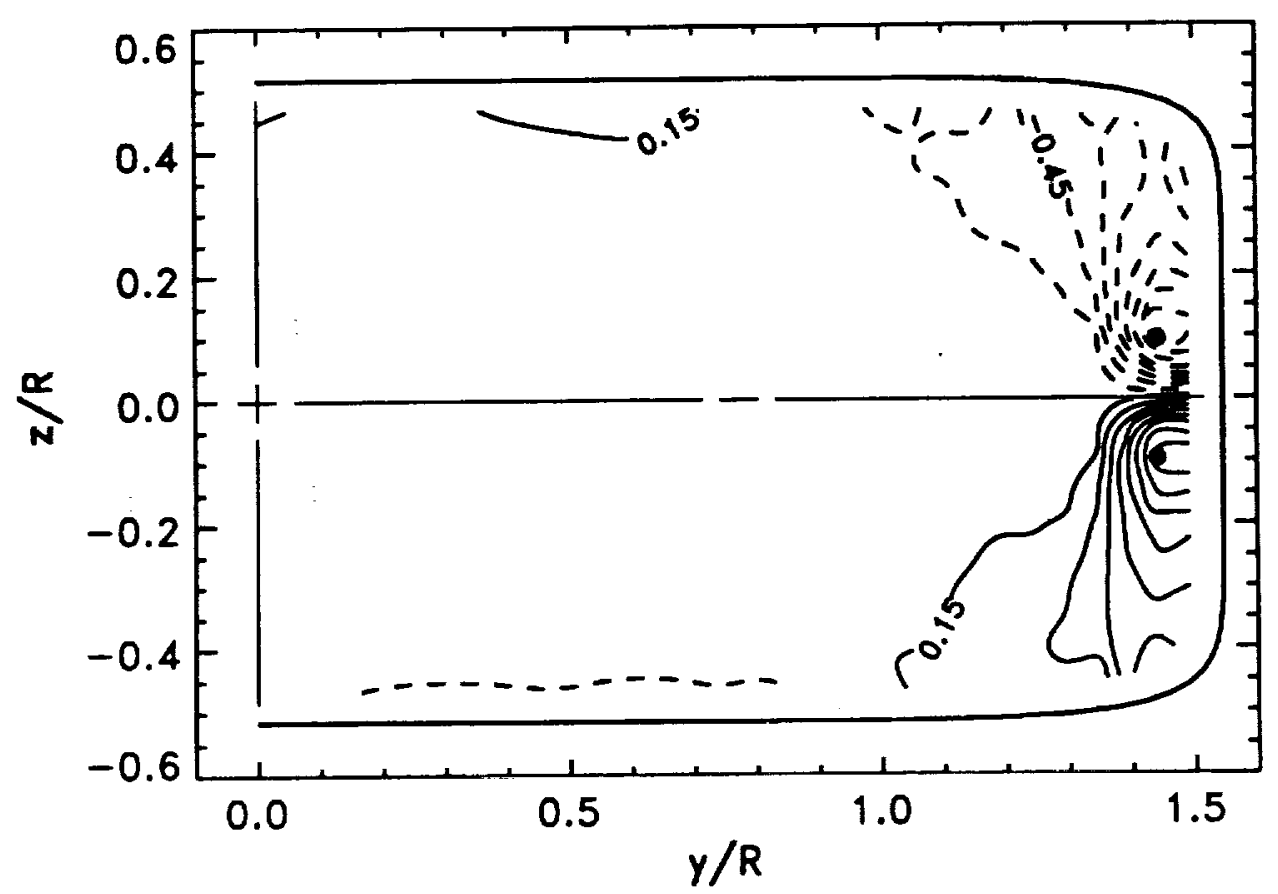

c) Station 5 .

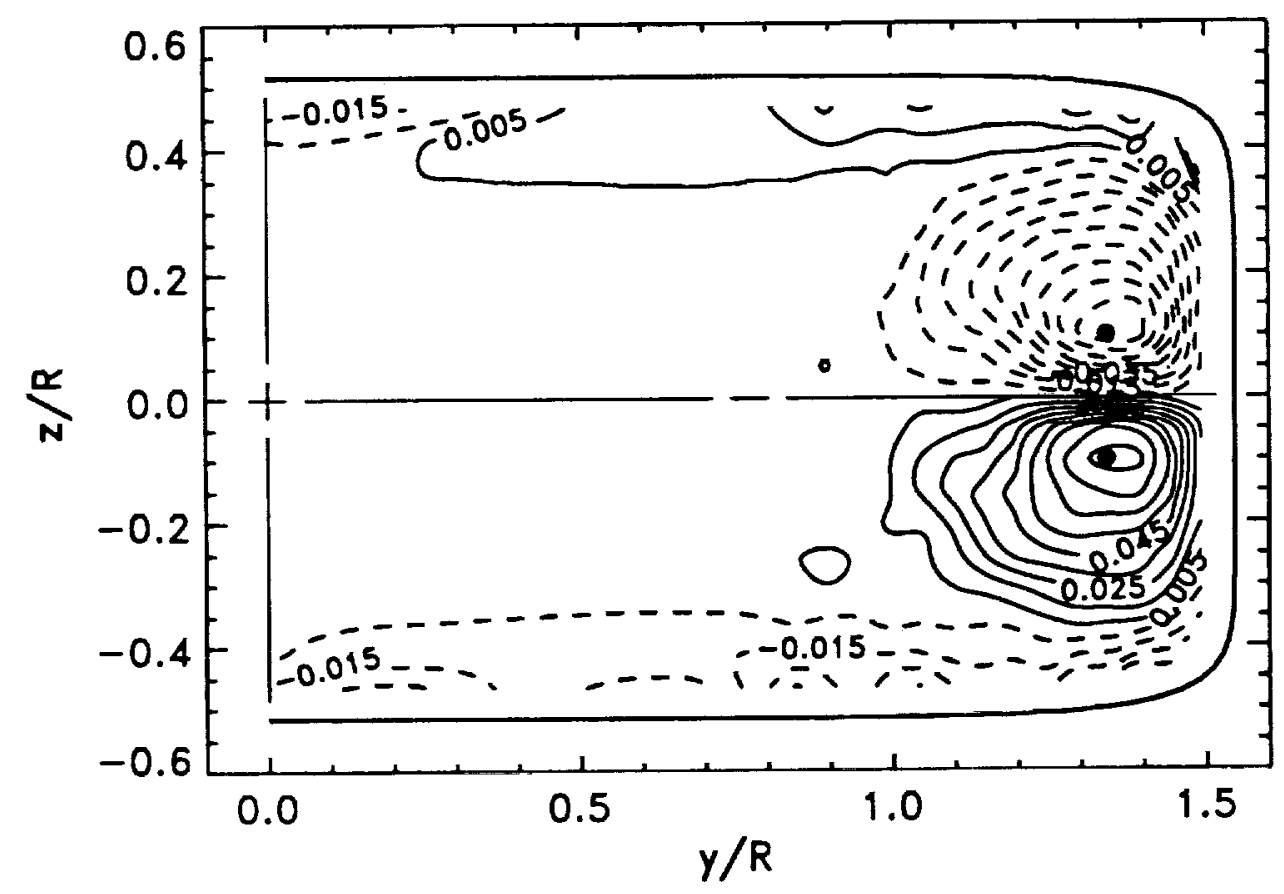

d) Station 6.

Fig. 5.13. Axial mean vorticity contours $\left(\Omega_{x} R / U_{b}\right)$. 


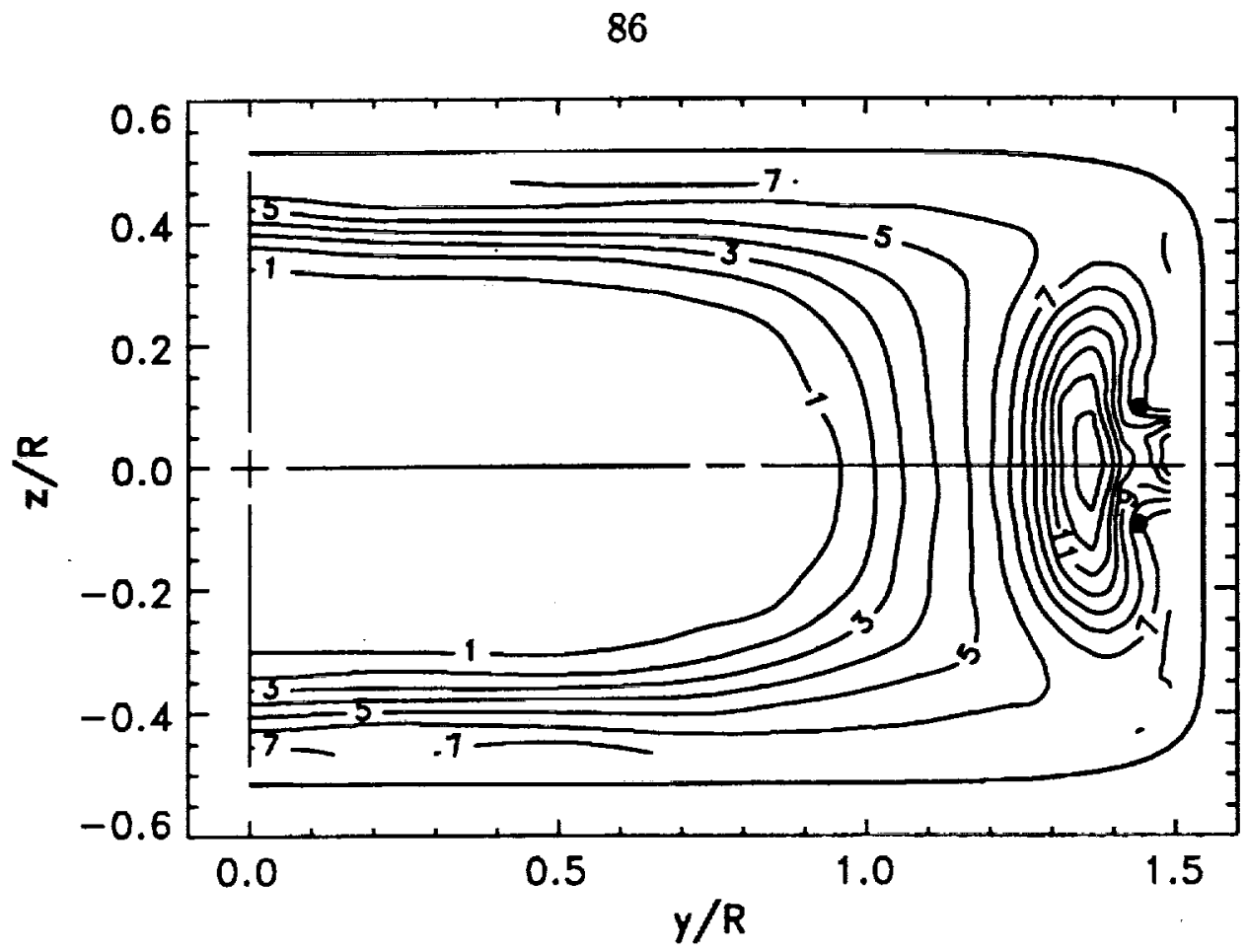

a) Station 5 .

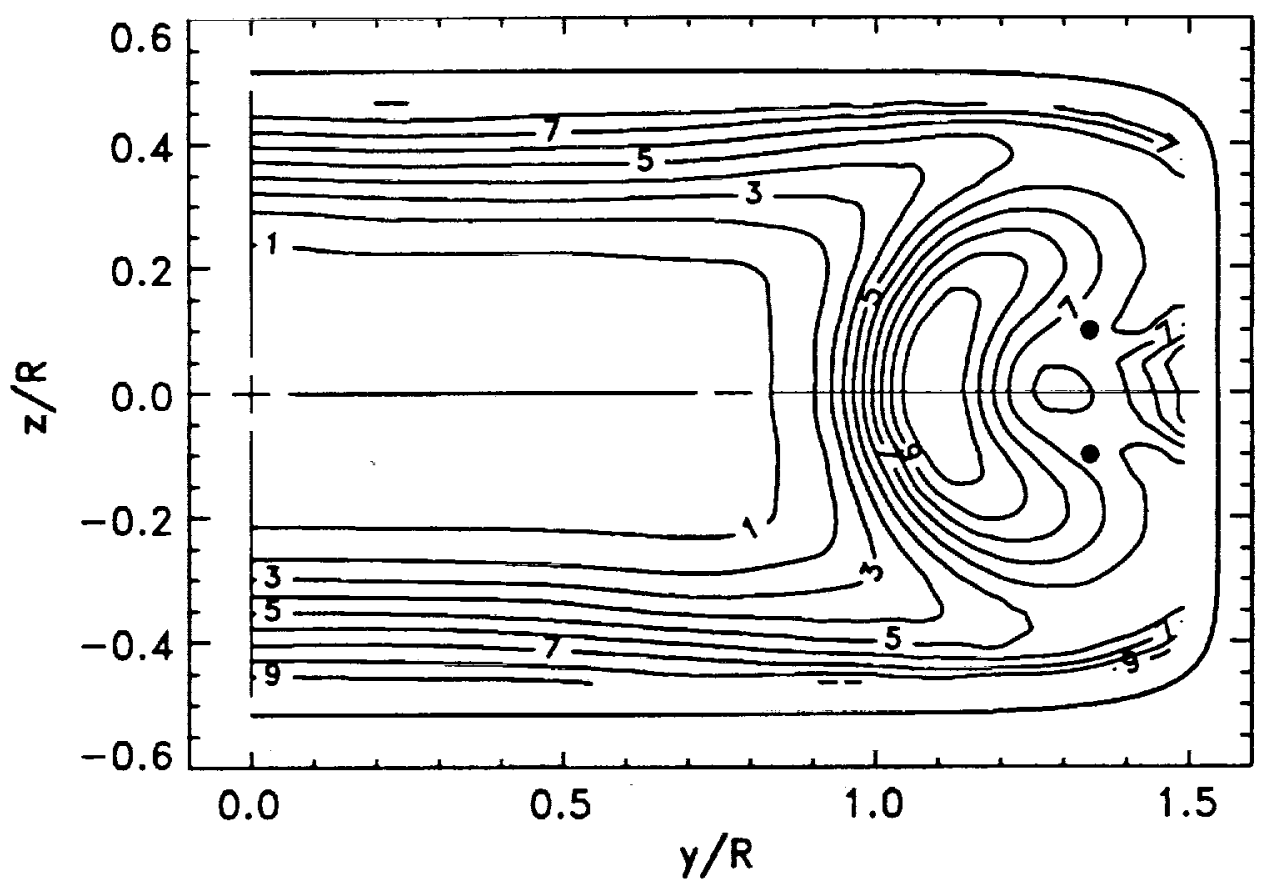

b) Station 6 .

Fig. 5.14. Axial turbulence intensity contours $\left(u^{\prime} / U \times 10^{2}\right)$. 


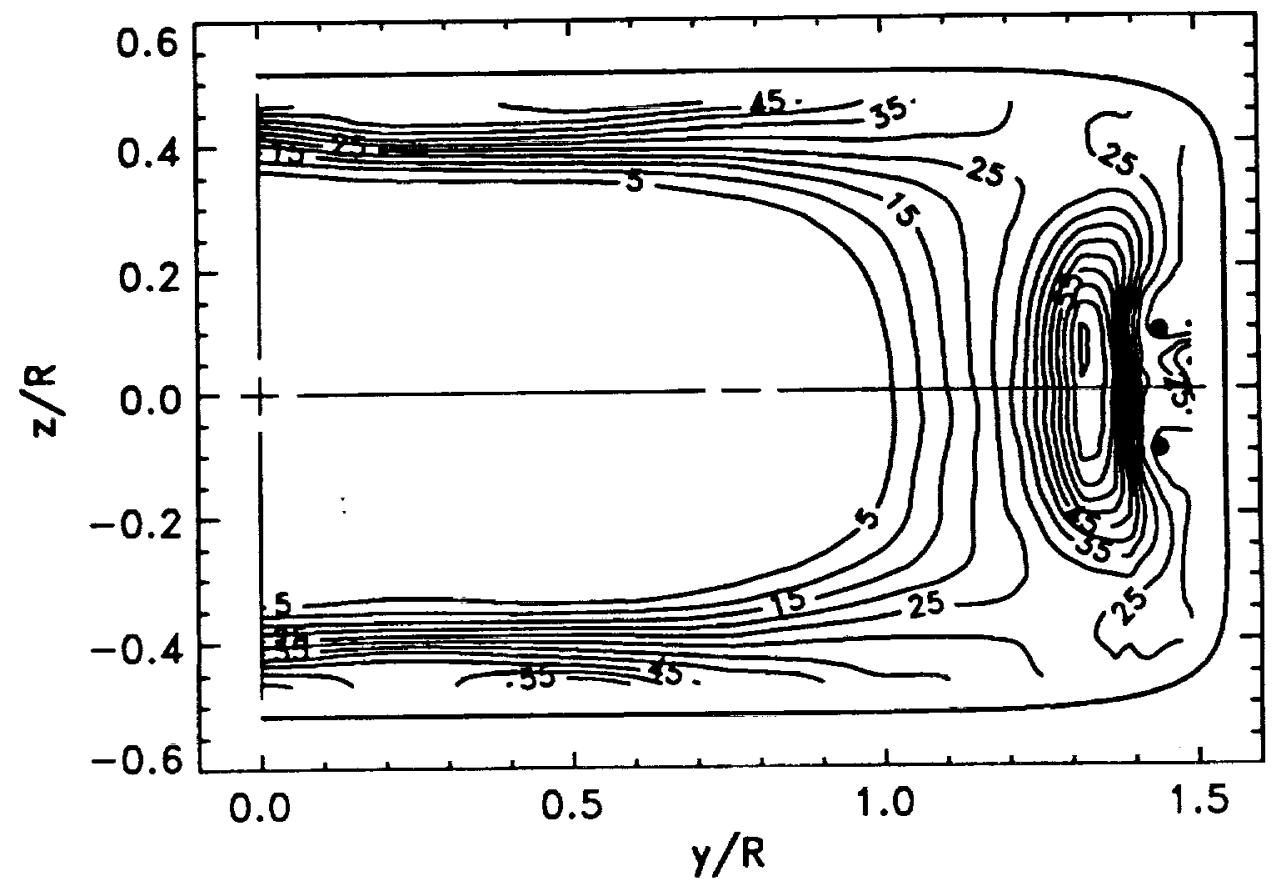

a) Station 5 .

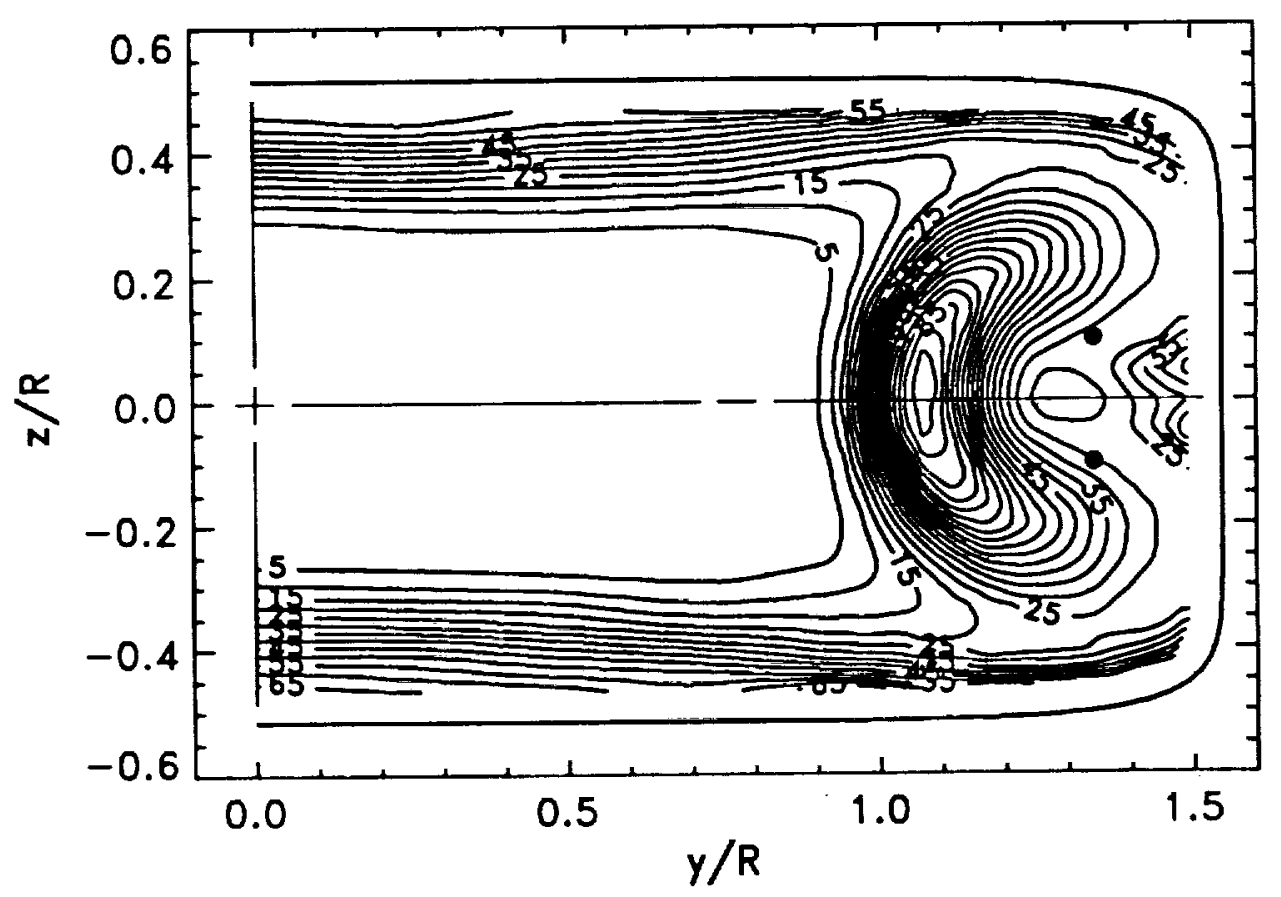

b) Station 6 .

Fig. 5.15. $\overline{u^{2}} / U_{b}^{2} \times 10^{4}$ Reynolds stress contours. 


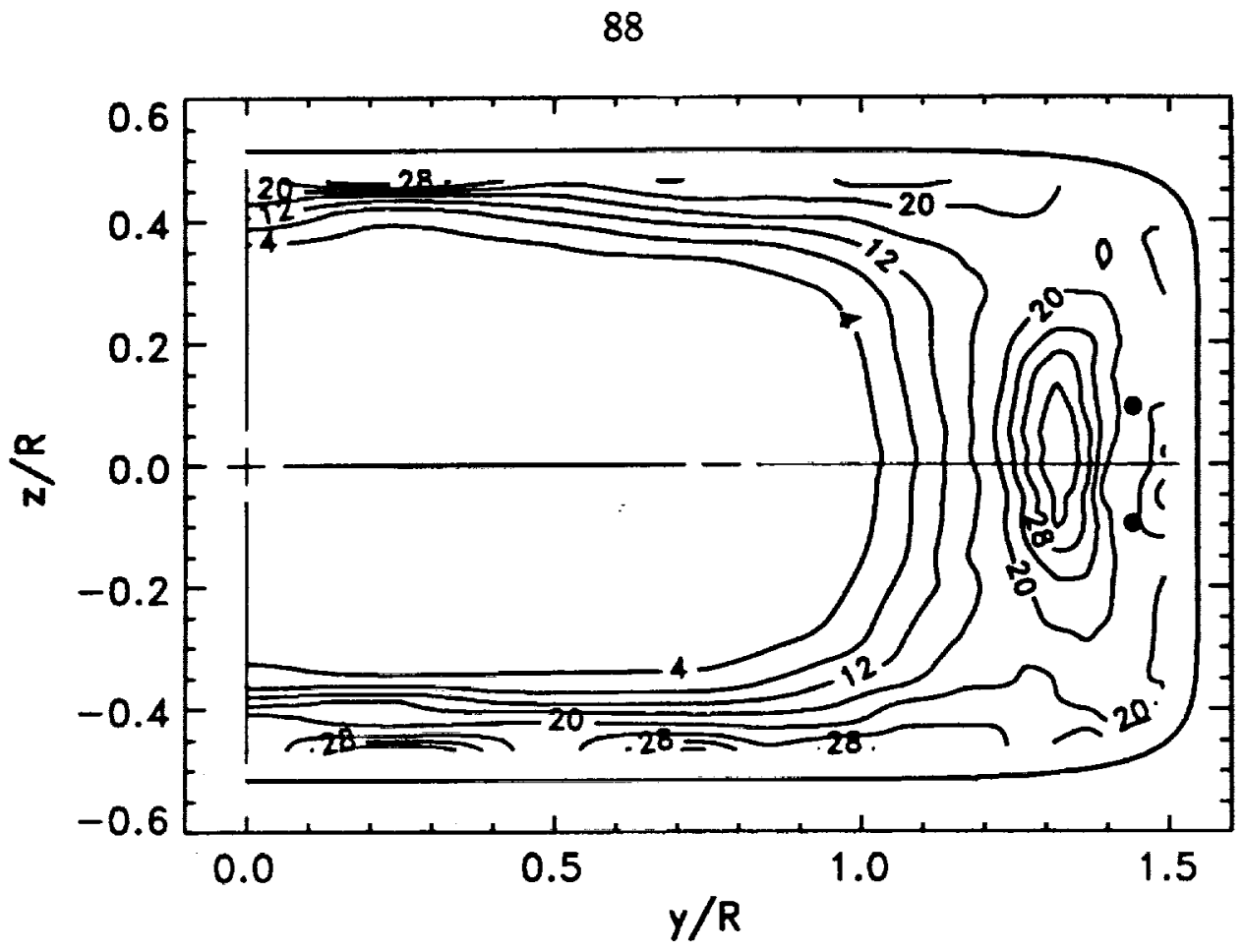

a) Station 5 .

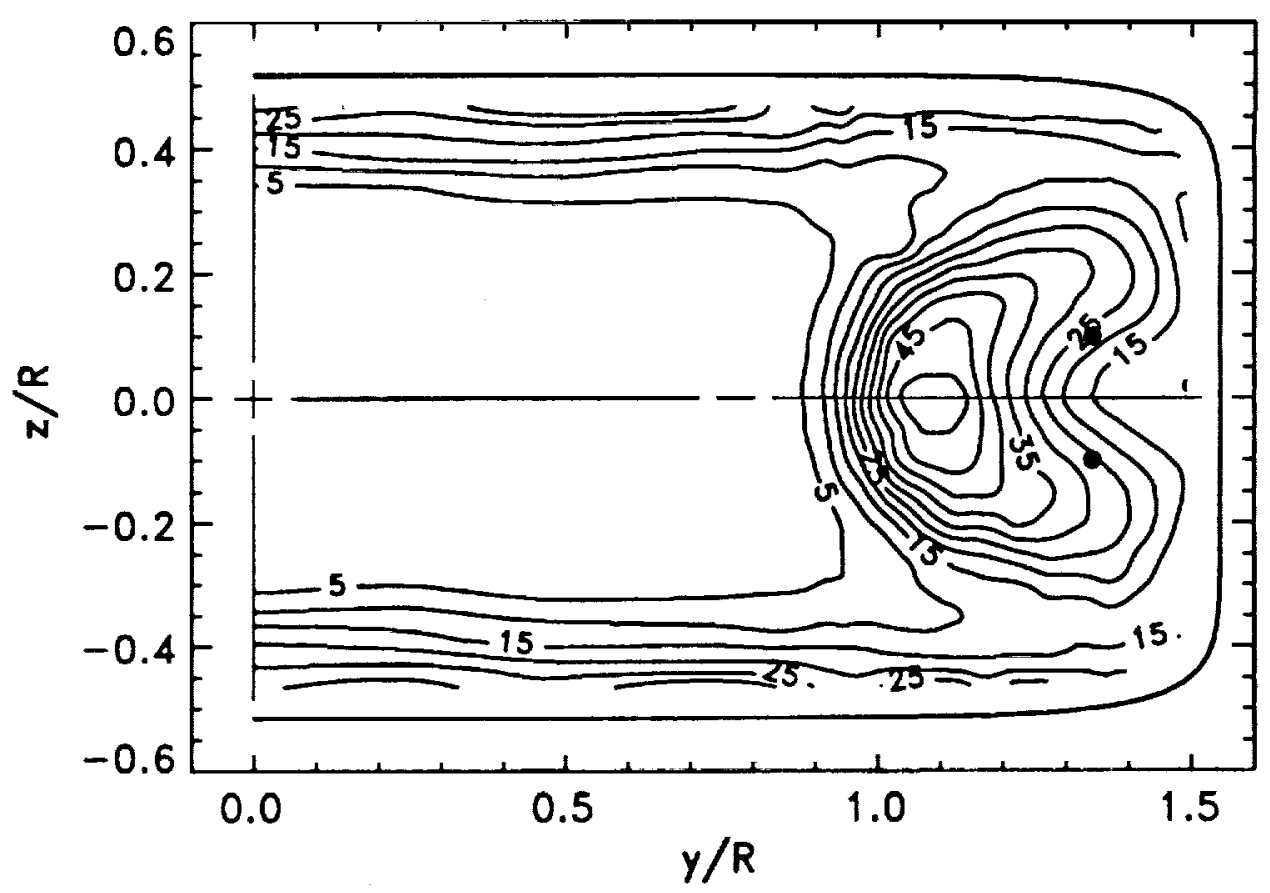

b) Station 6 .

Fig. 5.16. $\overline{v^{2}} / U_{b}^{2} \times 10^{4}$ Reynolds stress contours. 


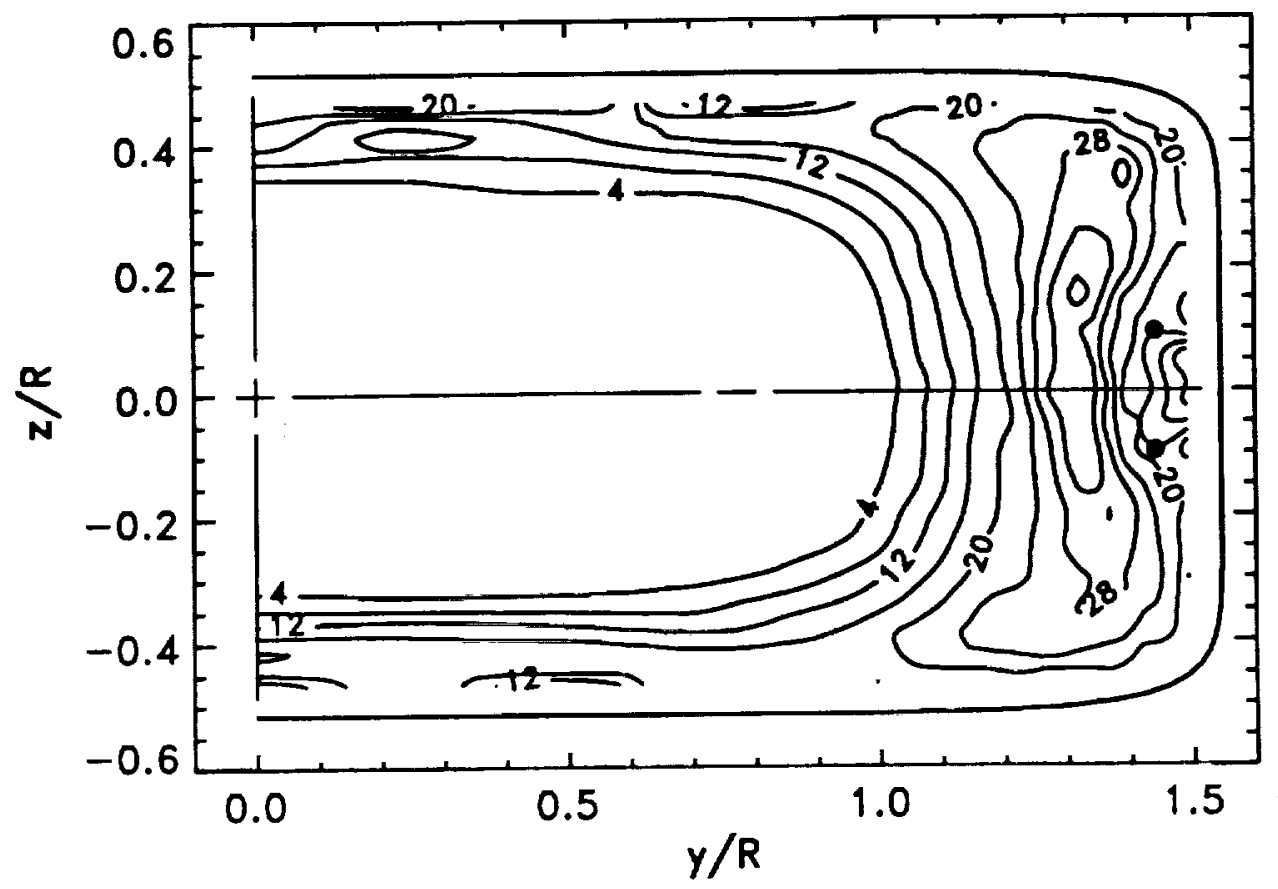

a) Station 5 .

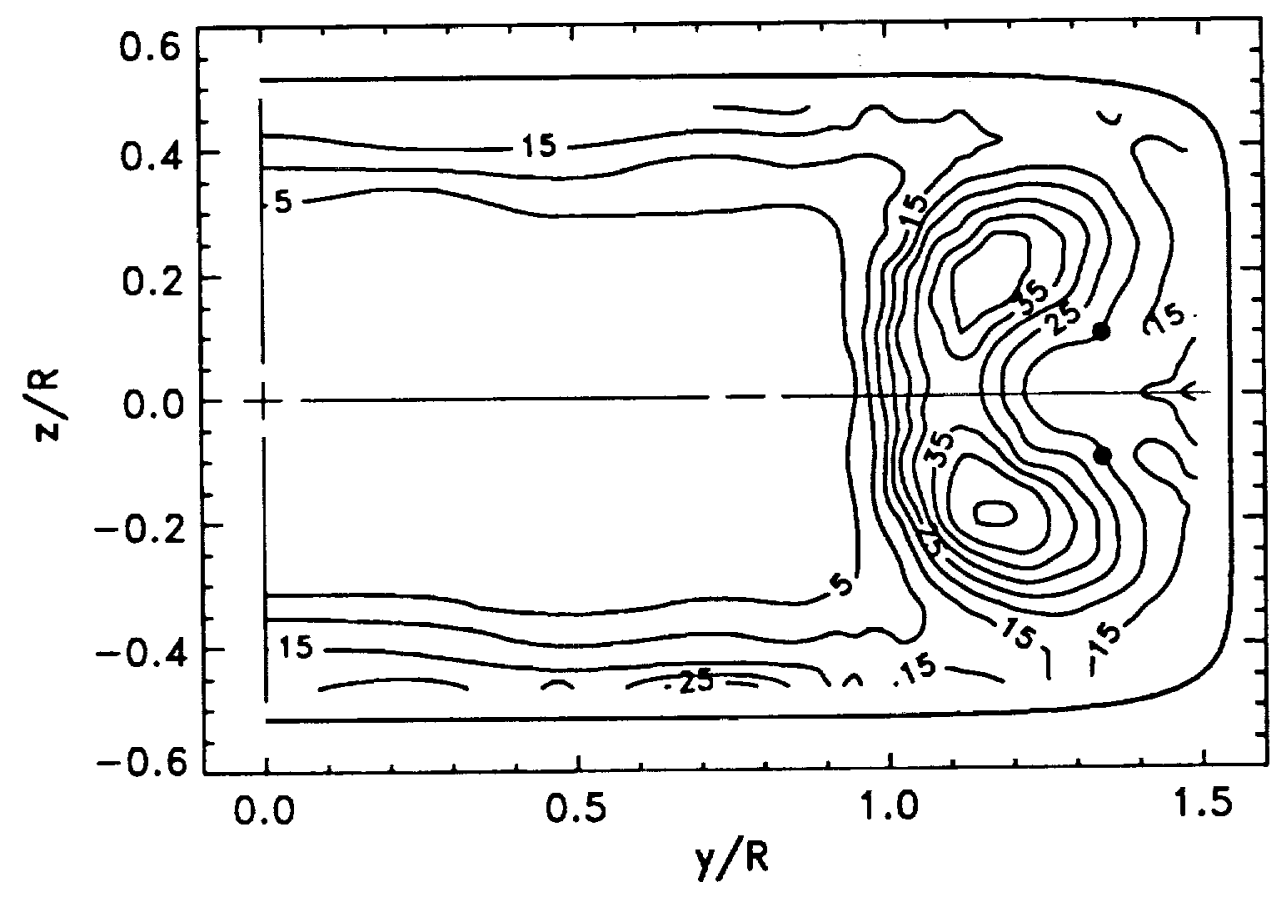

b) Station 6 .

Fig. 5.17. $\overline{\mathrm{w}^{2}} / \mathrm{U}_{\mathrm{b}}^{2} \times 10^{4}$ Reynolds stress contours. 
90

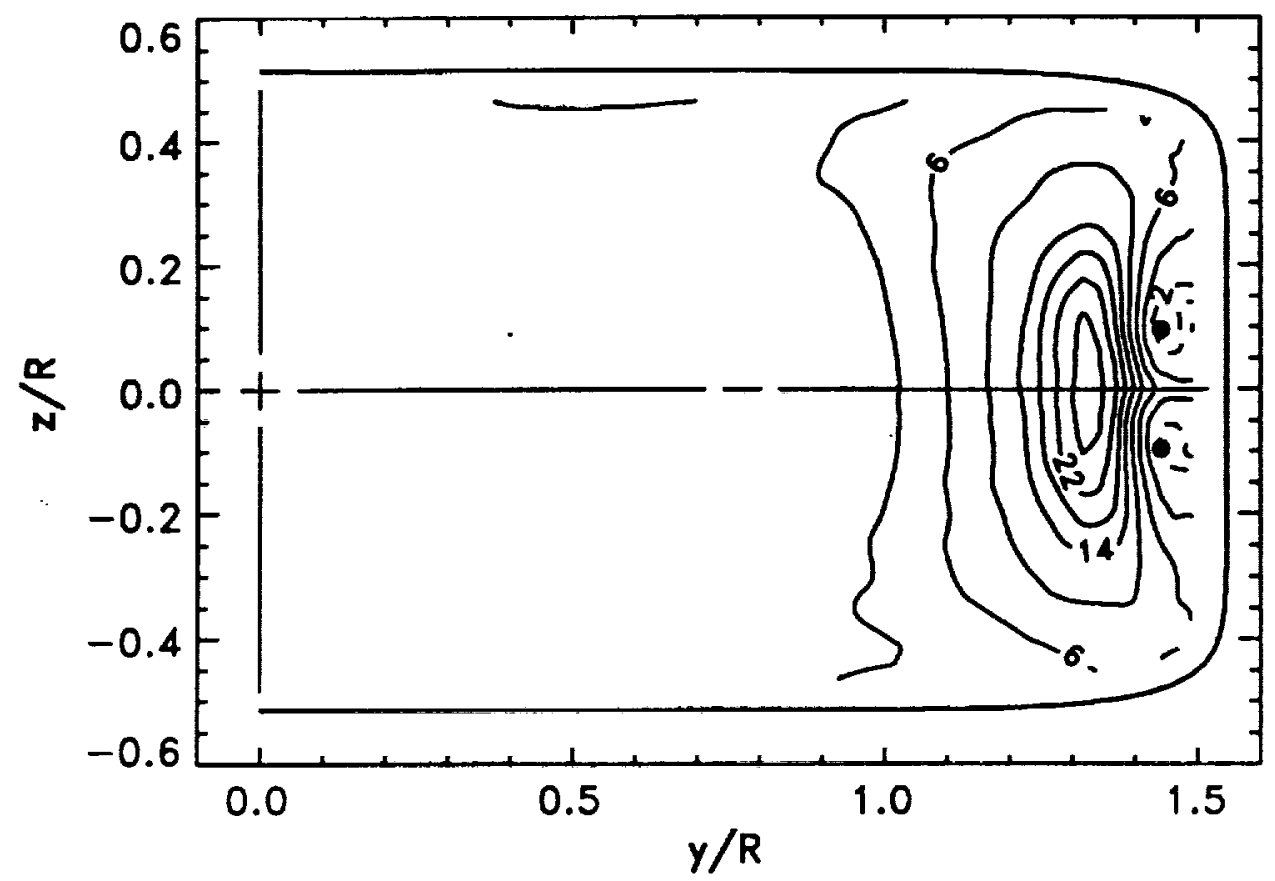

a) Station 5 .

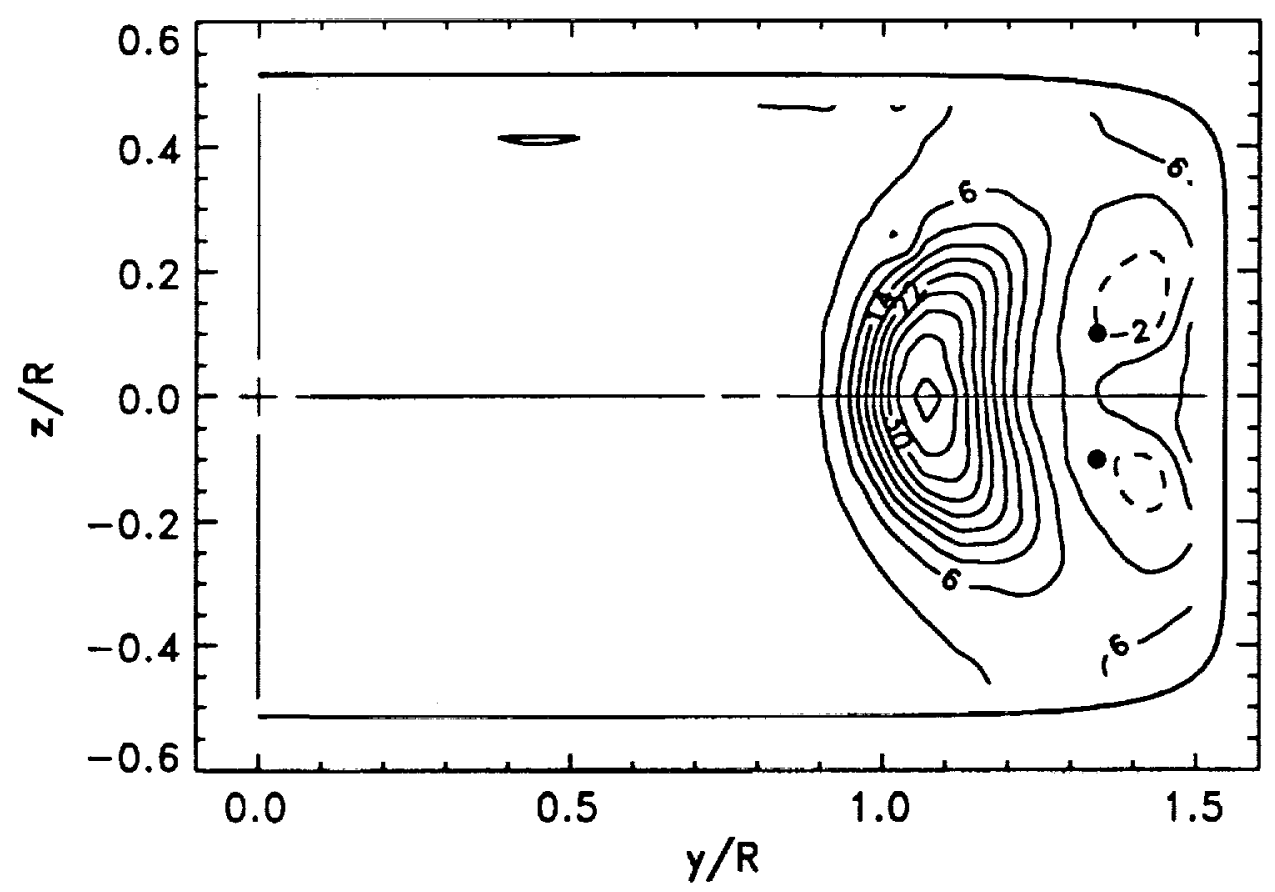

b) Station 6.

Fig. 5.18. $\overline{\mathrm{uv}} / \mathrm{U}_{\mathrm{b}}^{2} \times 10^{4}$ Reynolds stress contours. 


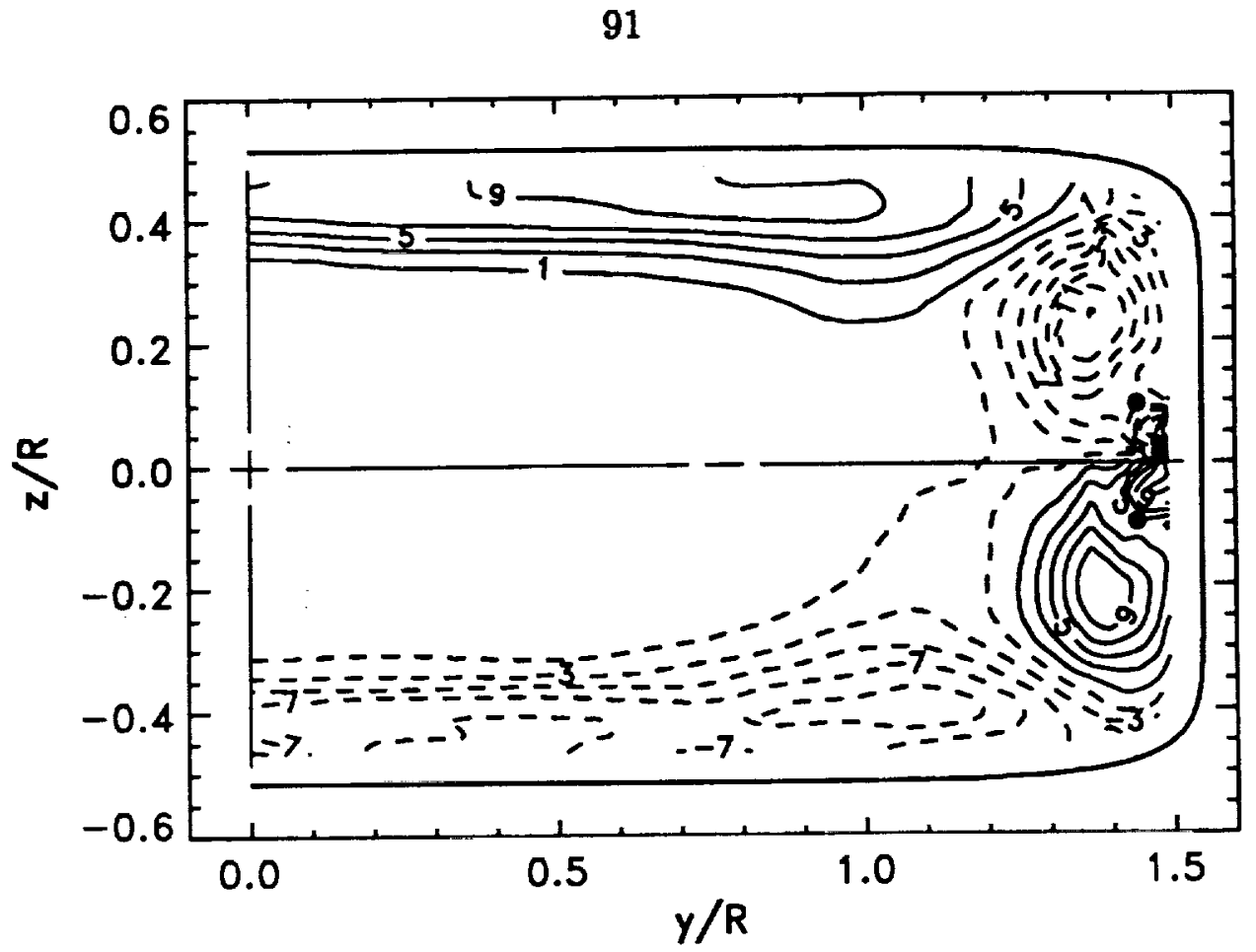

a) Station 5 .

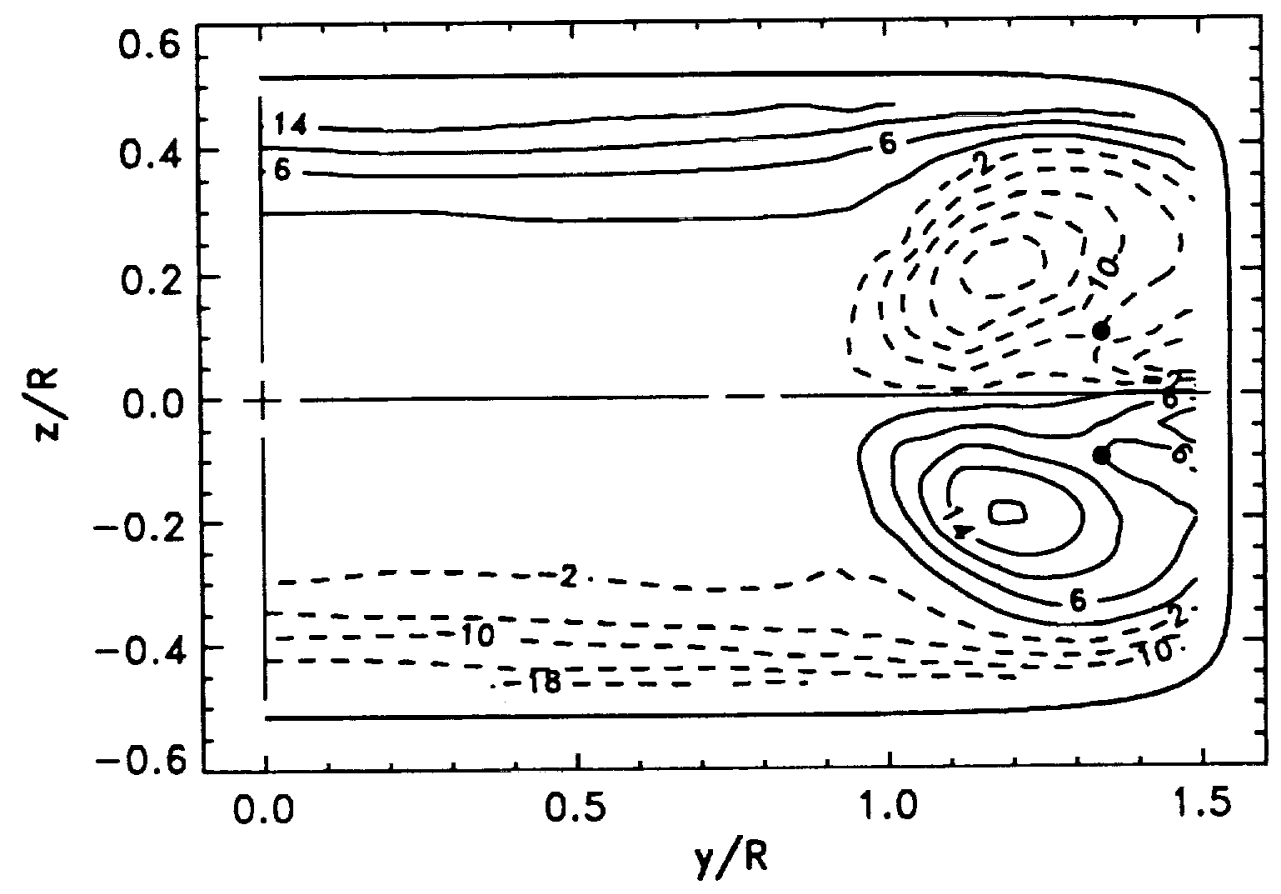

b) Station 6 .

Fig. 5.19. $\overline{\mathrm{uw}} / \mathrm{U}_{\mathrm{b}}^{2} \times 10^{4}$ Reynolds stress contours. 


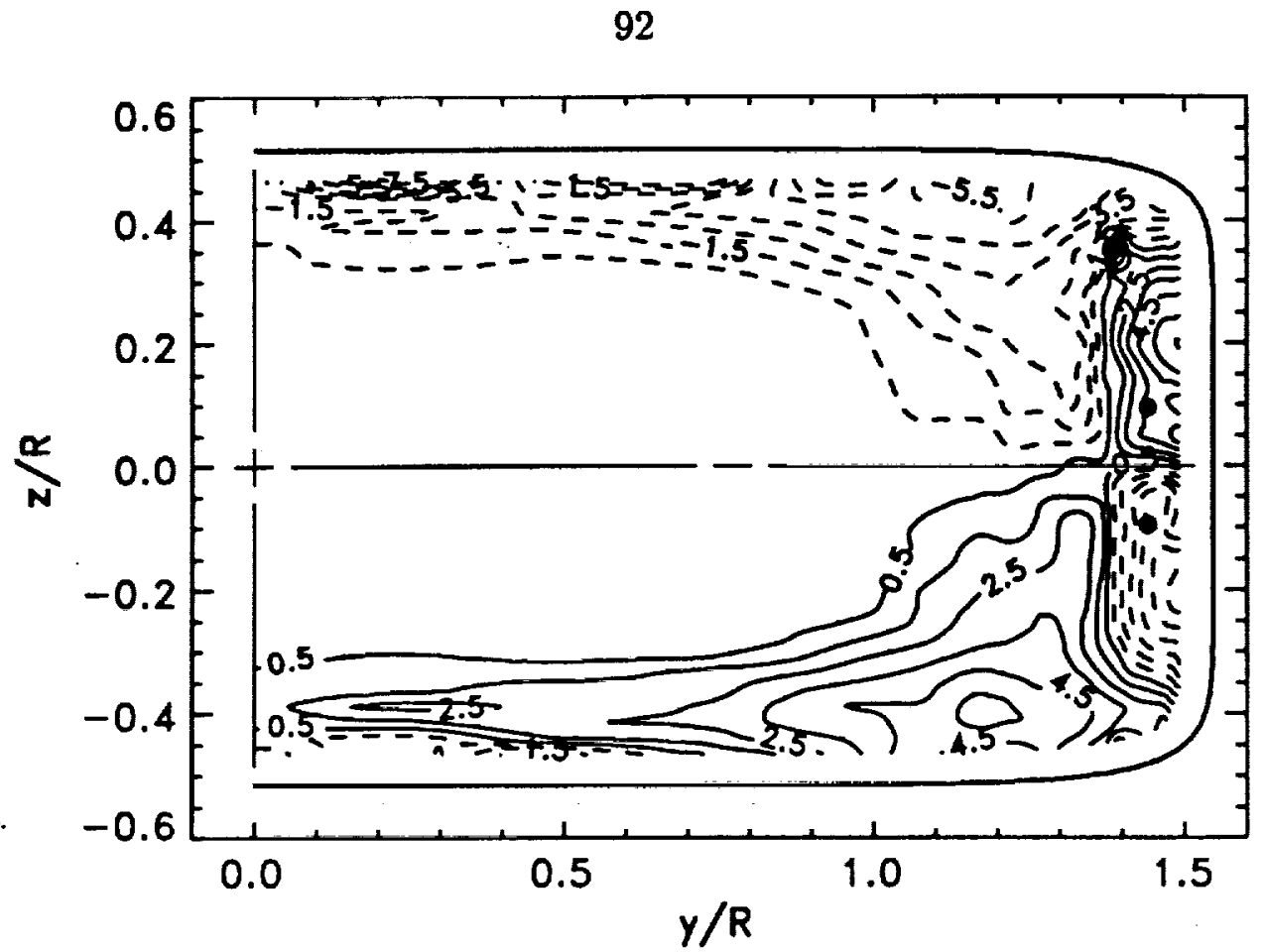

a) Station 5 .

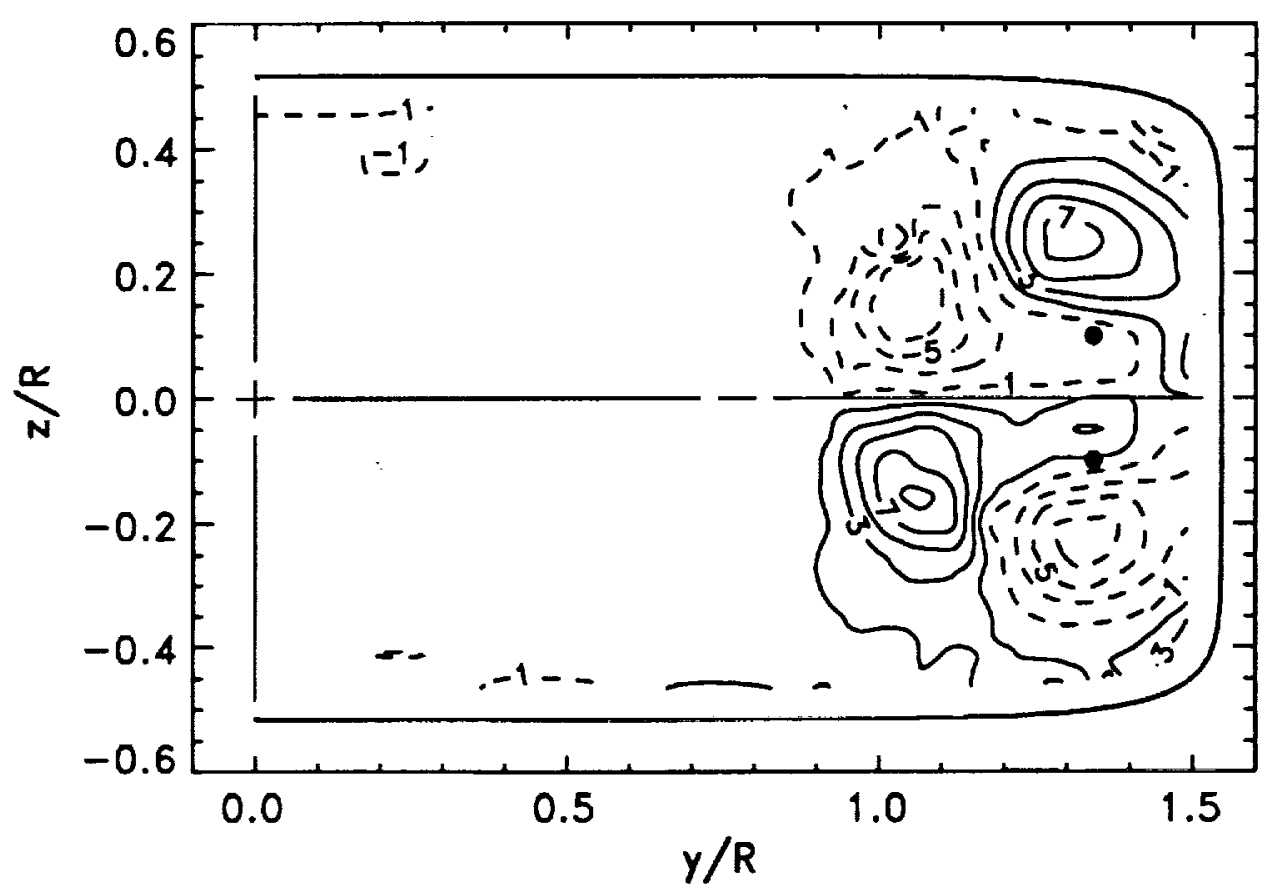

b) Station 6 .

Fig. 5.20. $\overline{\mathrm{vw}} / \mathrm{U}_{\mathrm{b}}^{2} \times 10^{4}$ Reynolds stress contours. 


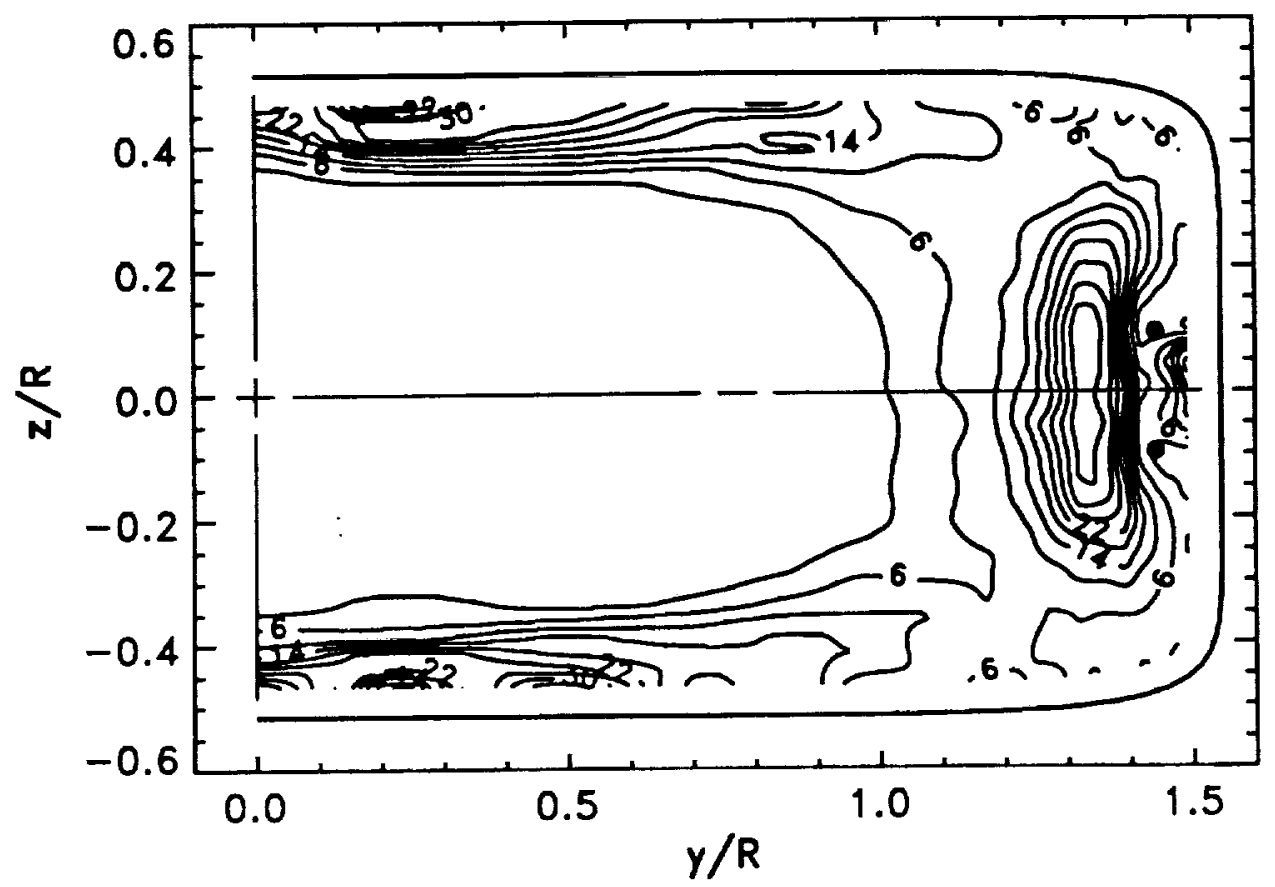

a) Station 5 .

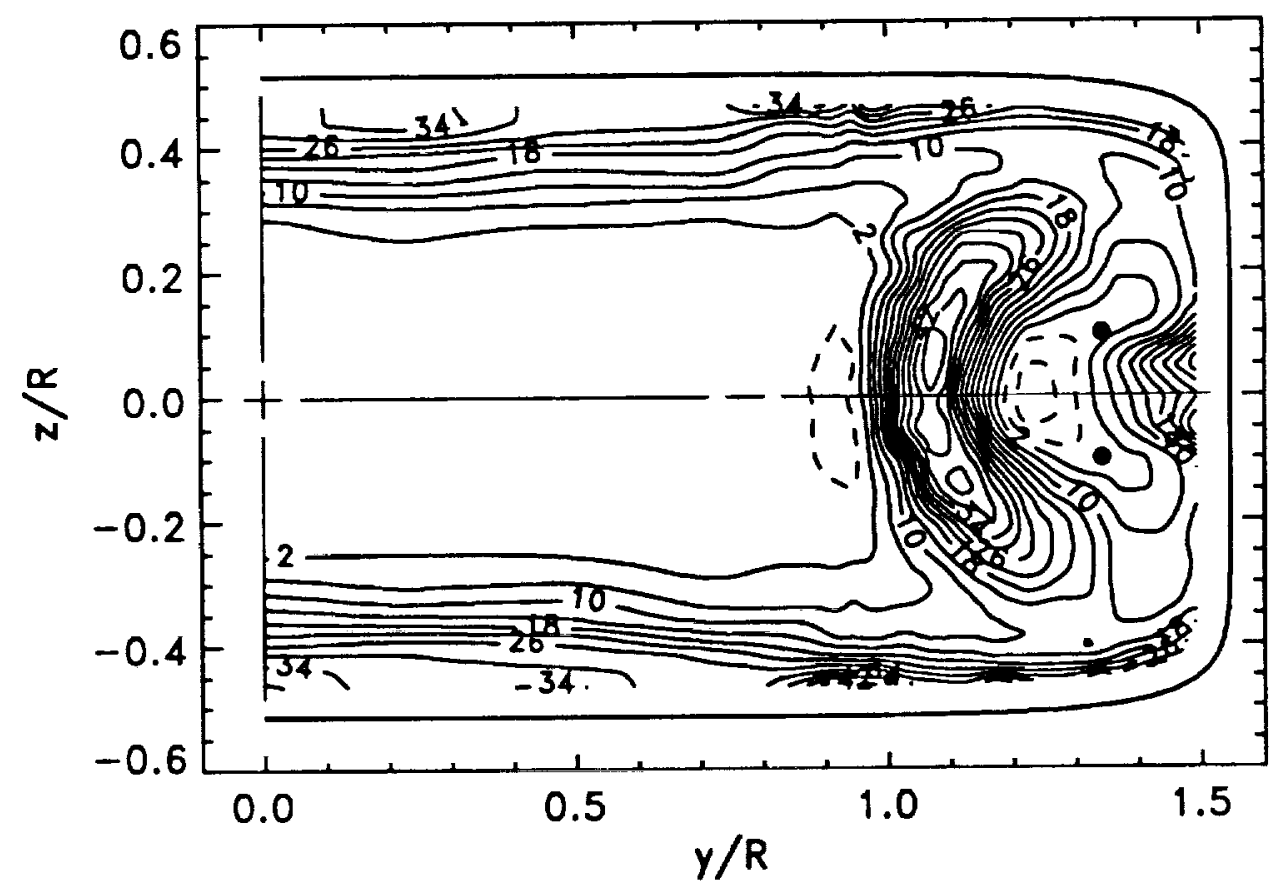

b) Station 6 .

Fig. 5.21. Axial and transverse normal stress anisotropy $\left(\left(\overline{u^{2}}-\overline{v^{2}}\right) / U_{b}^{2} \times 10^{4}\right)$. 


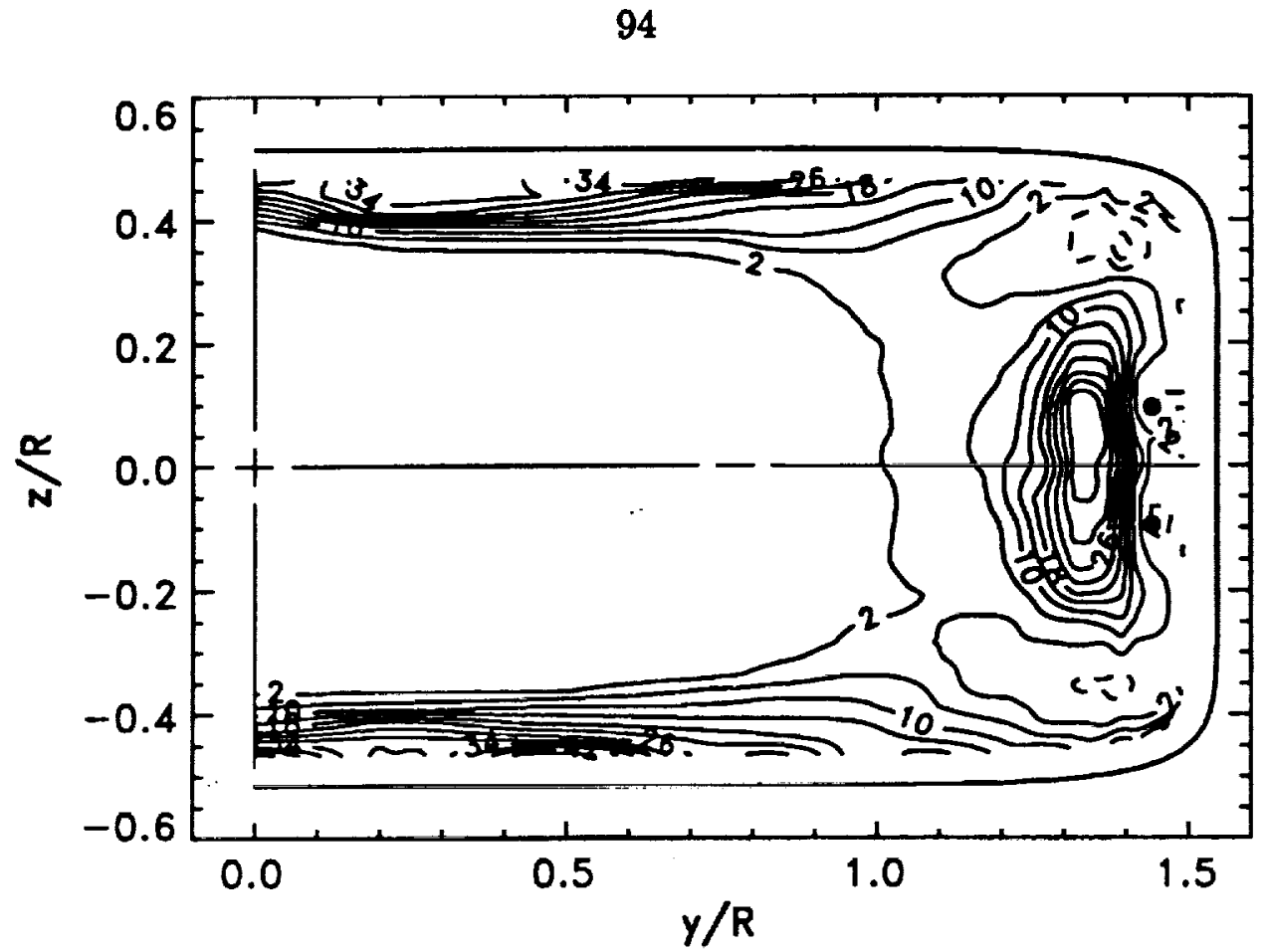

a) Station 5 .

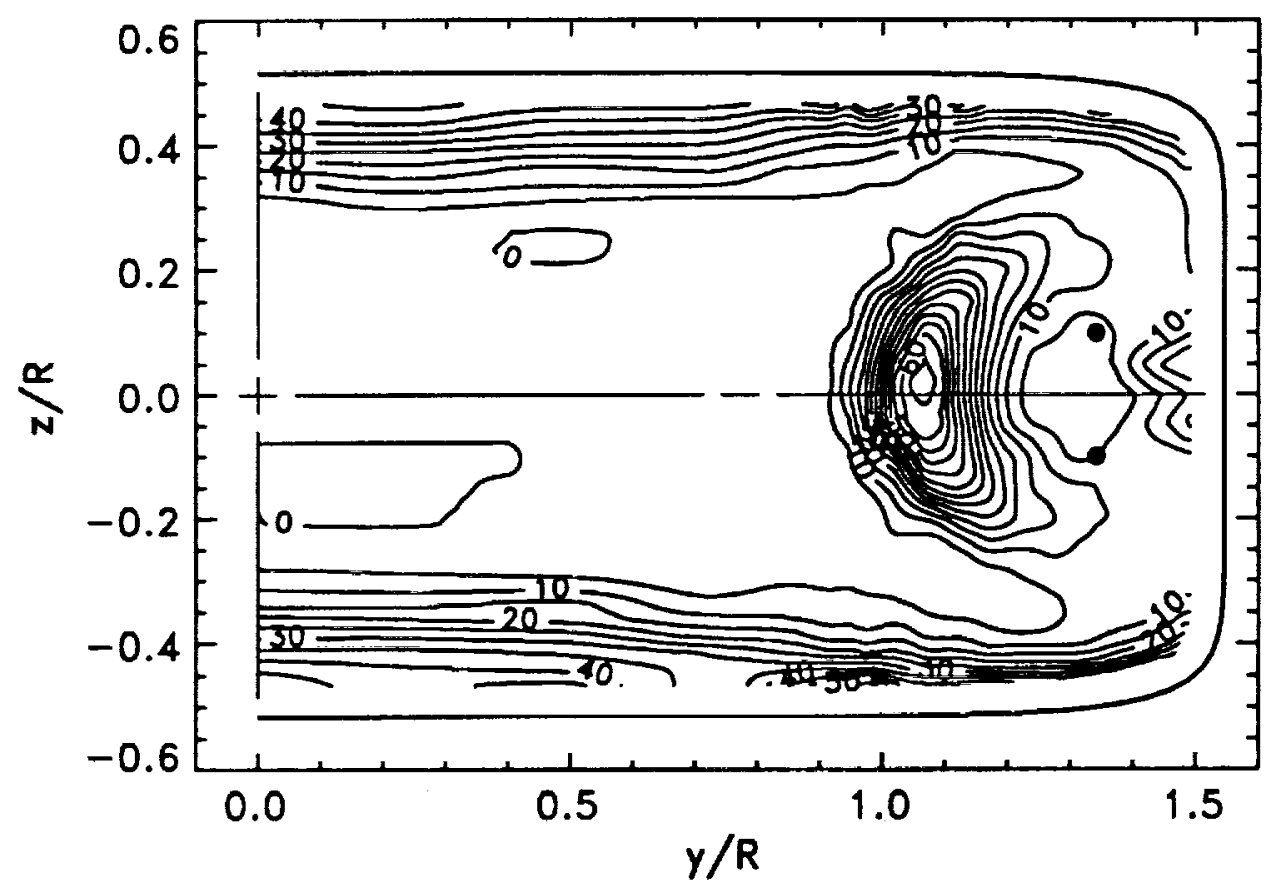

b) Station 6 .

Fig. 5.22. Axial and transverse normal stress anisotropy $\left(\left(\overline{u^{2}}-\overline{w^{2}}\right) / U_{b}^{2} \times 10^{4}\right)$. 


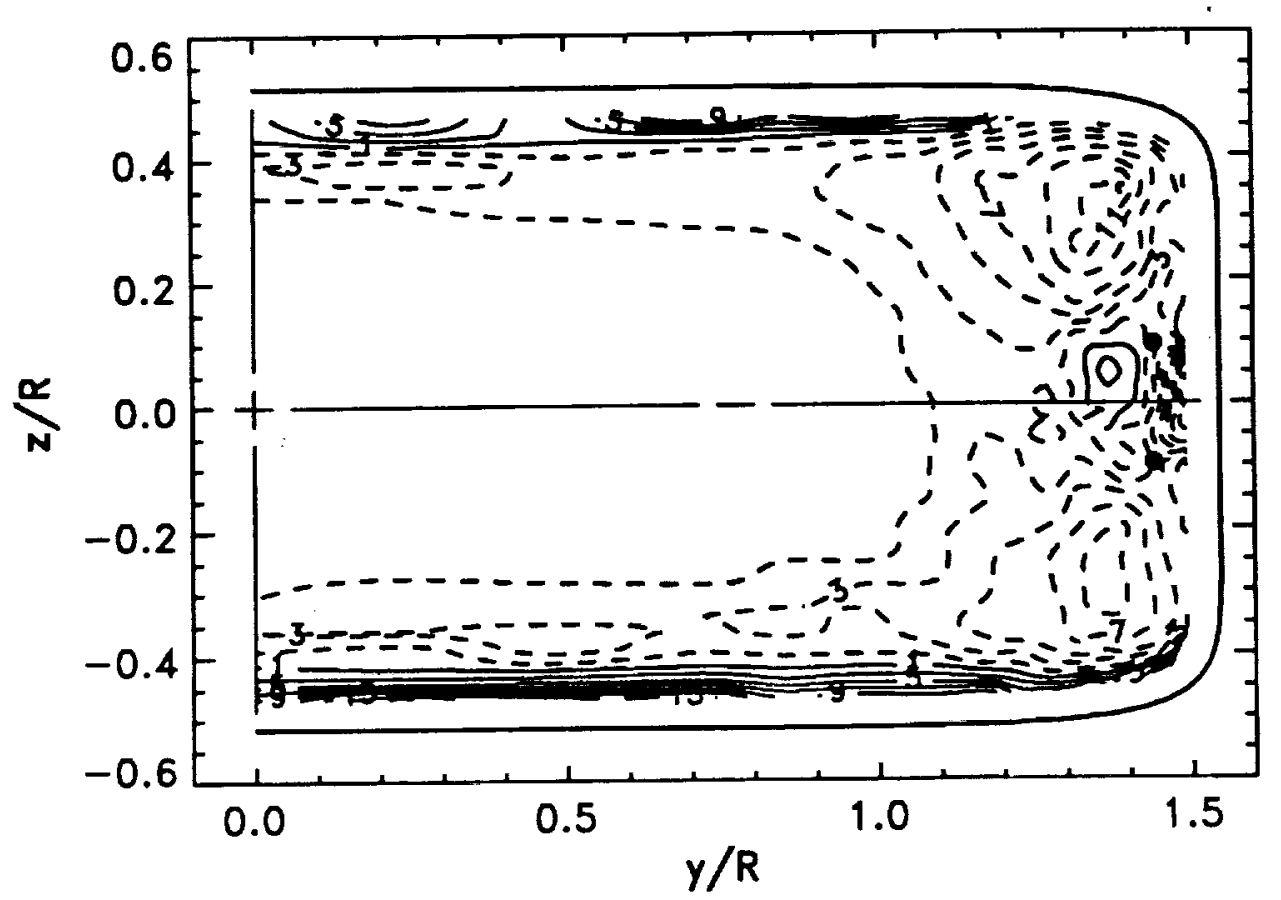

a) Station 5 .

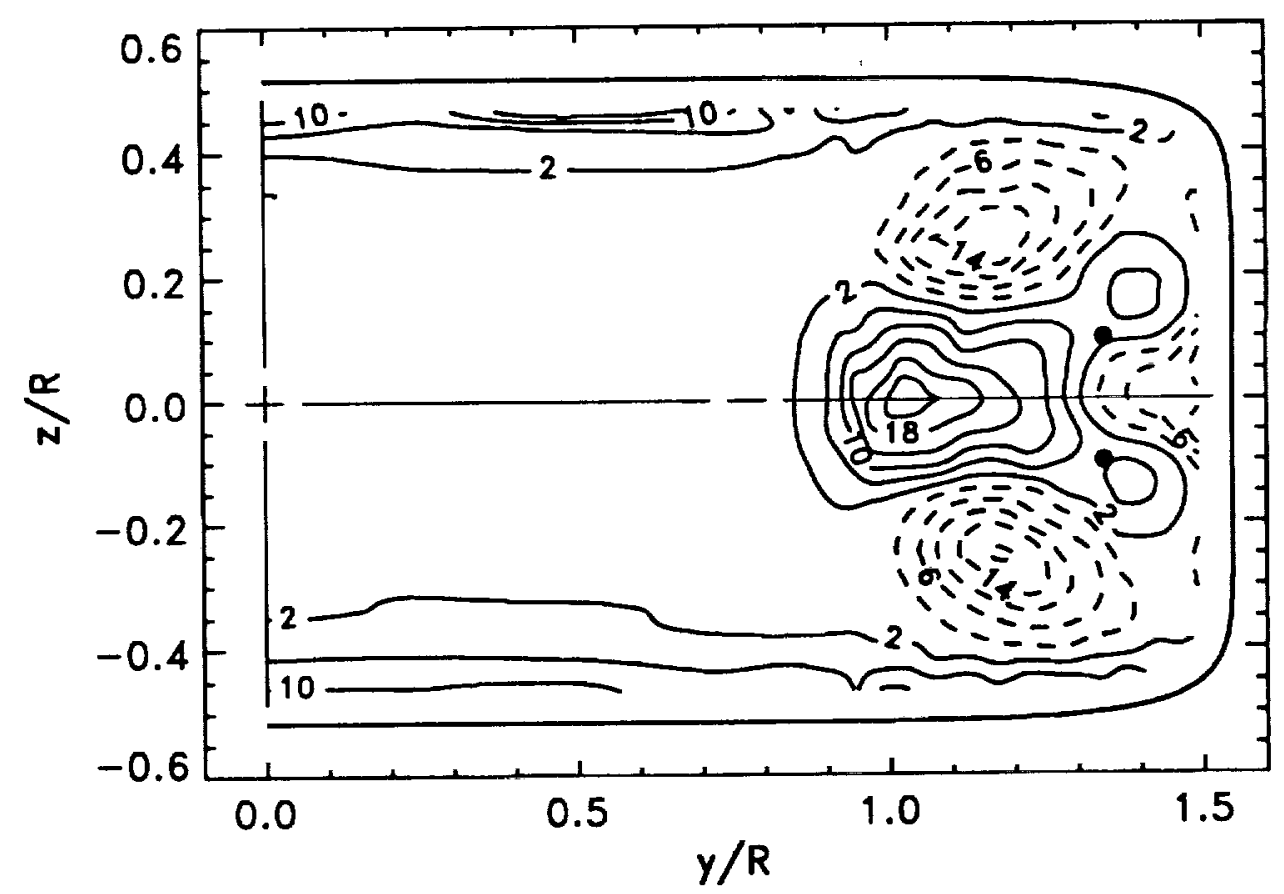

b) Station 6 .

Fig. 5.23. Transverse normal stress anisotropy $\left(\left(\overline{v^{2}}-\overline{w^{2}}\right) / U_{b}^{2} \times 10^{4}\right)$. 


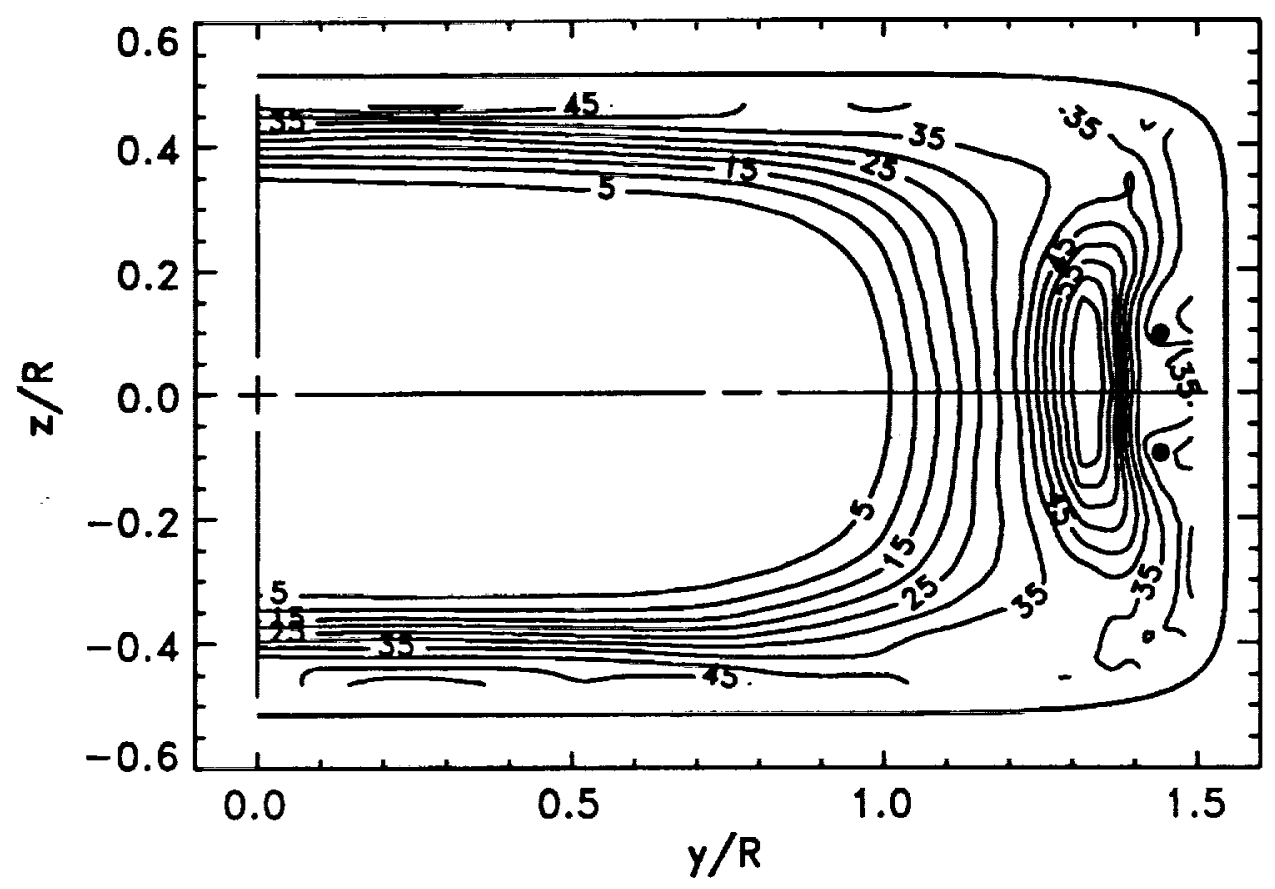

a) Station 5 .

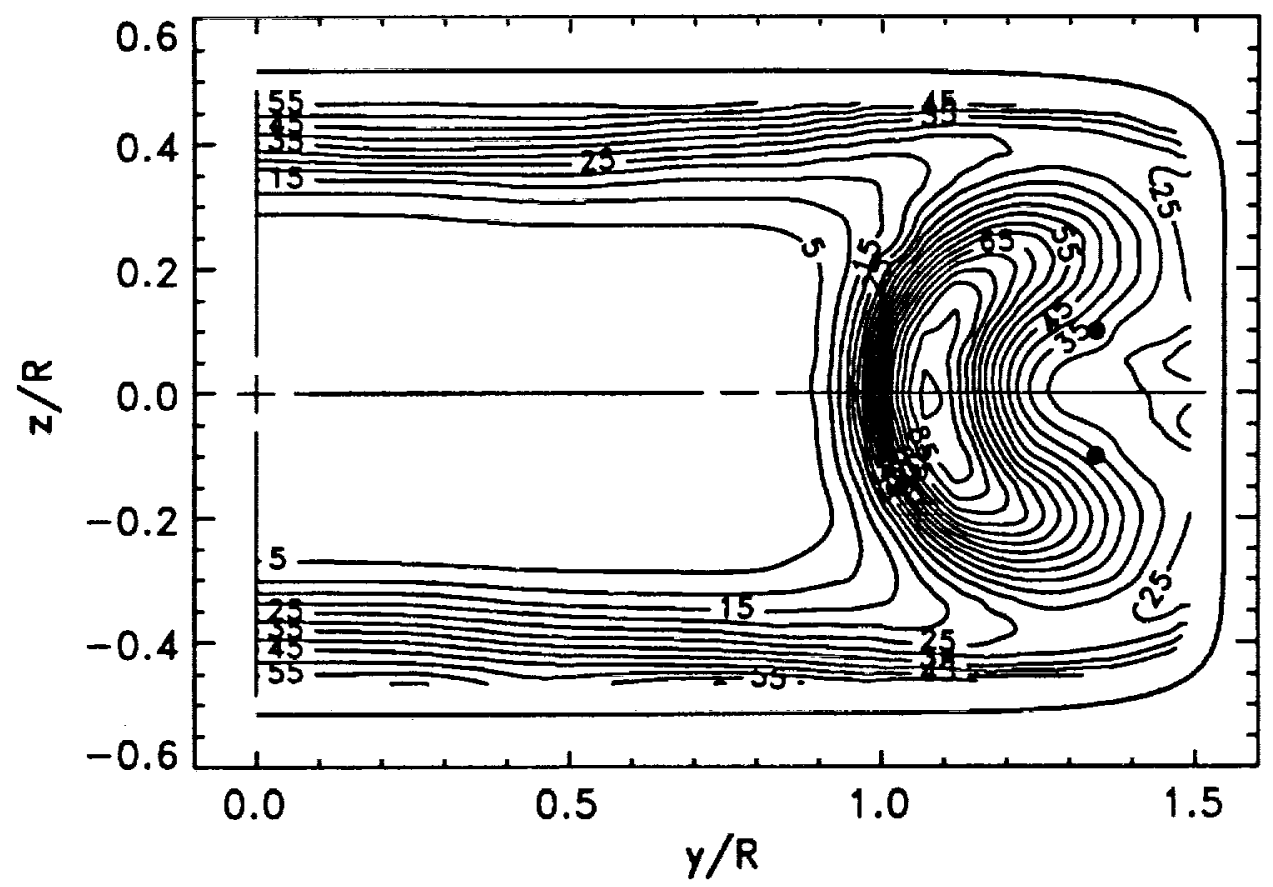

b) Station 6 .

Fig. 5.24. Turbulence kinetic energy $\left(k / U_{b}^{2} \times 10^{4}\right)$. 


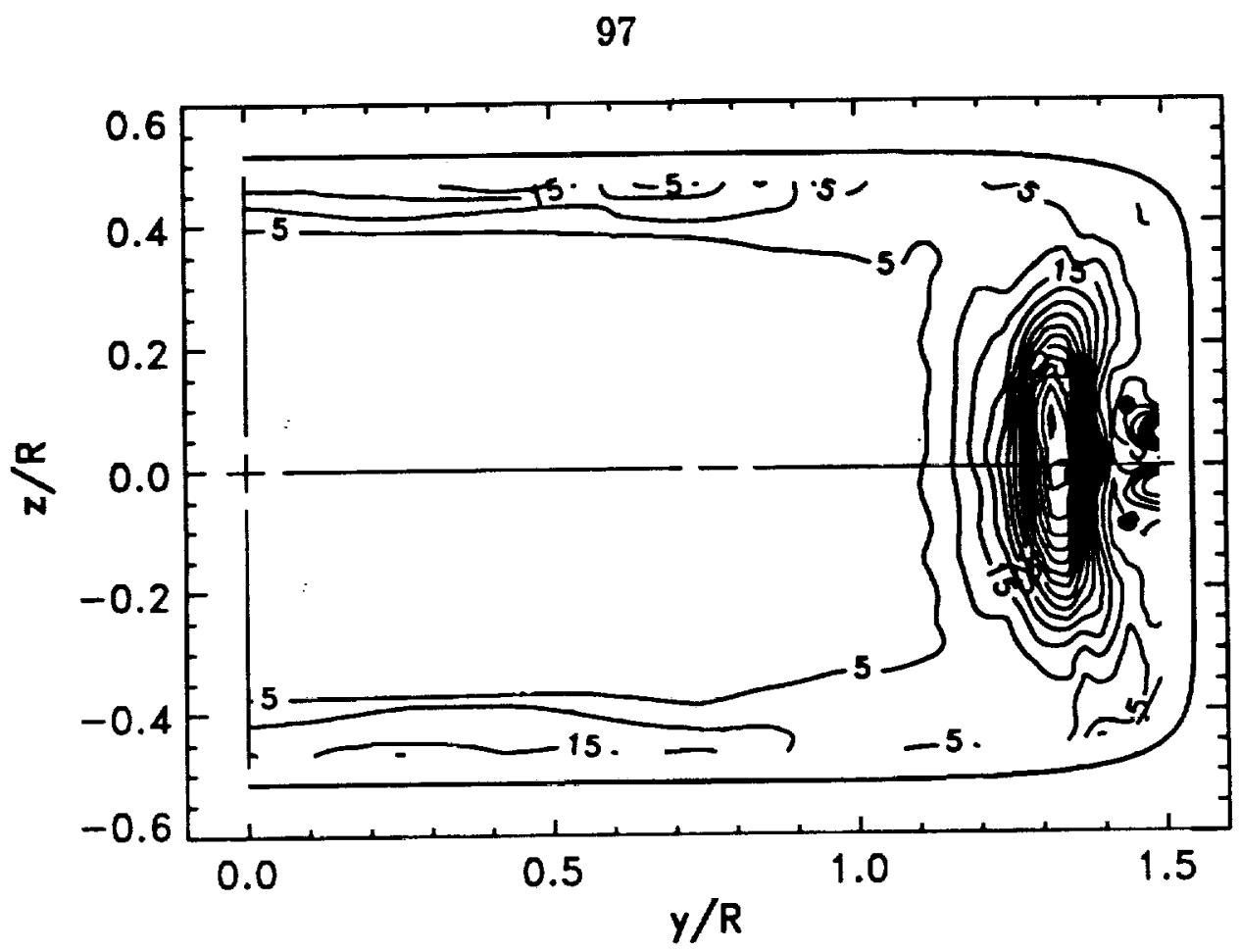

a) Station 5 .

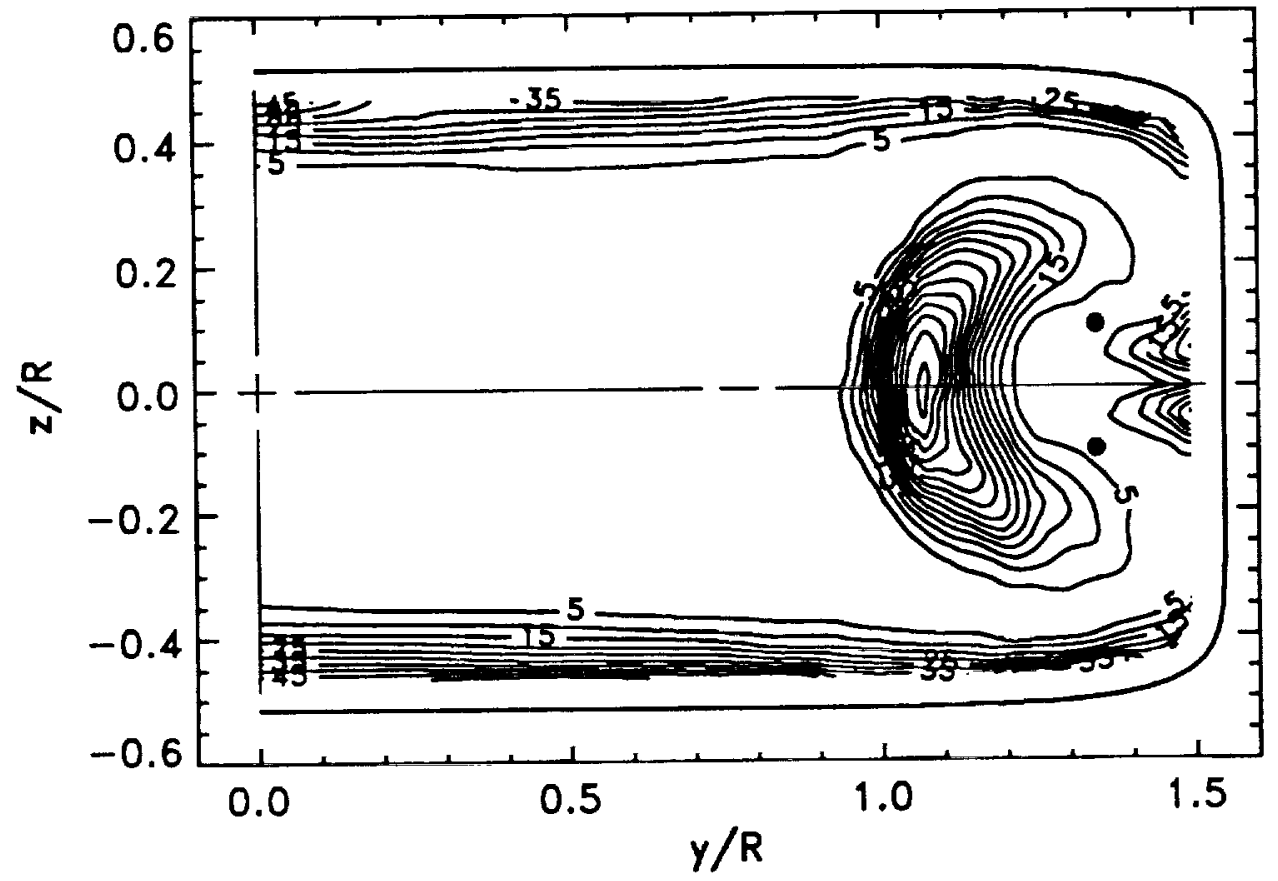

b) Station 6 .

Fig. 5.25. Production of t.k.e $\left(\left(-\overline{\mathbf{u v}} \frac{\partial U}{\partial y}-\overline{u w} \frac{\partial U}{\partial z}\right)\left(\frac{R}{U_{b}^{3}}\right) \times 10^{4}\right)$. 


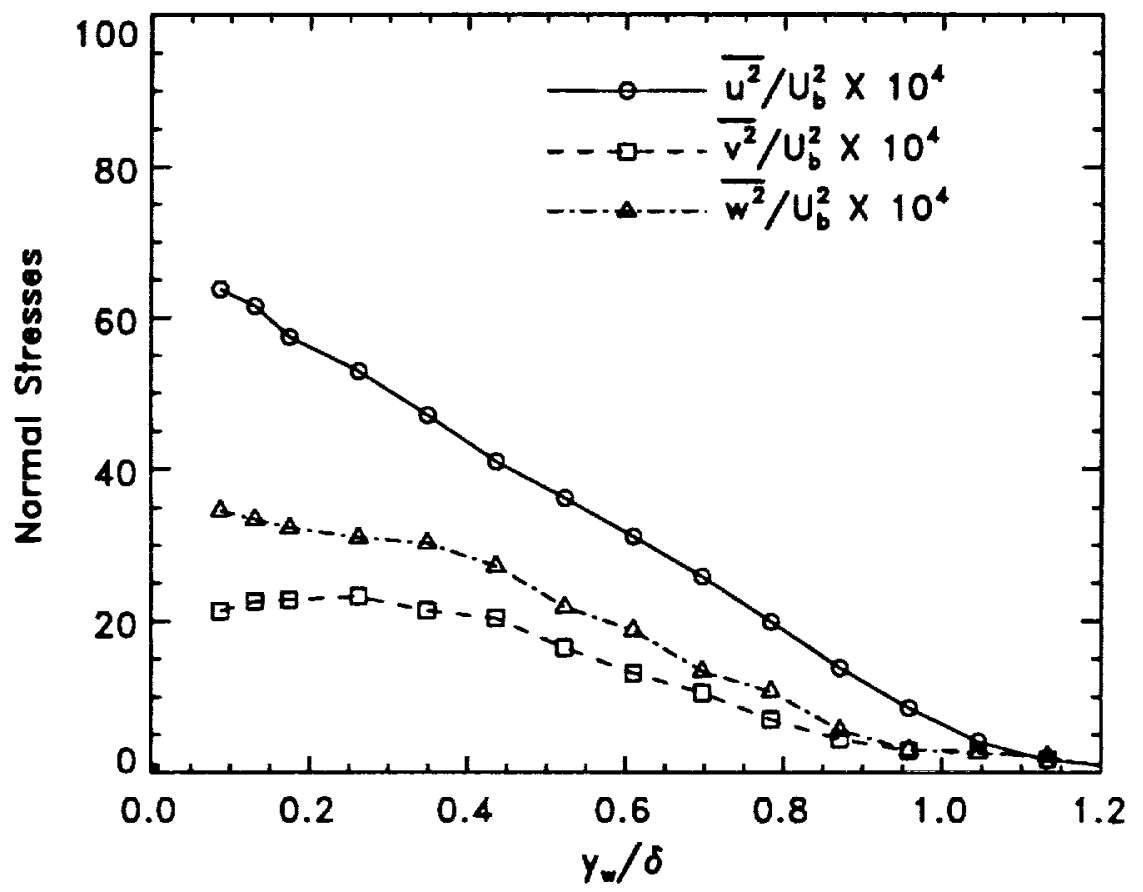

Fig. 5.26. Reynolds normal stress profiles at Station 1. 
99

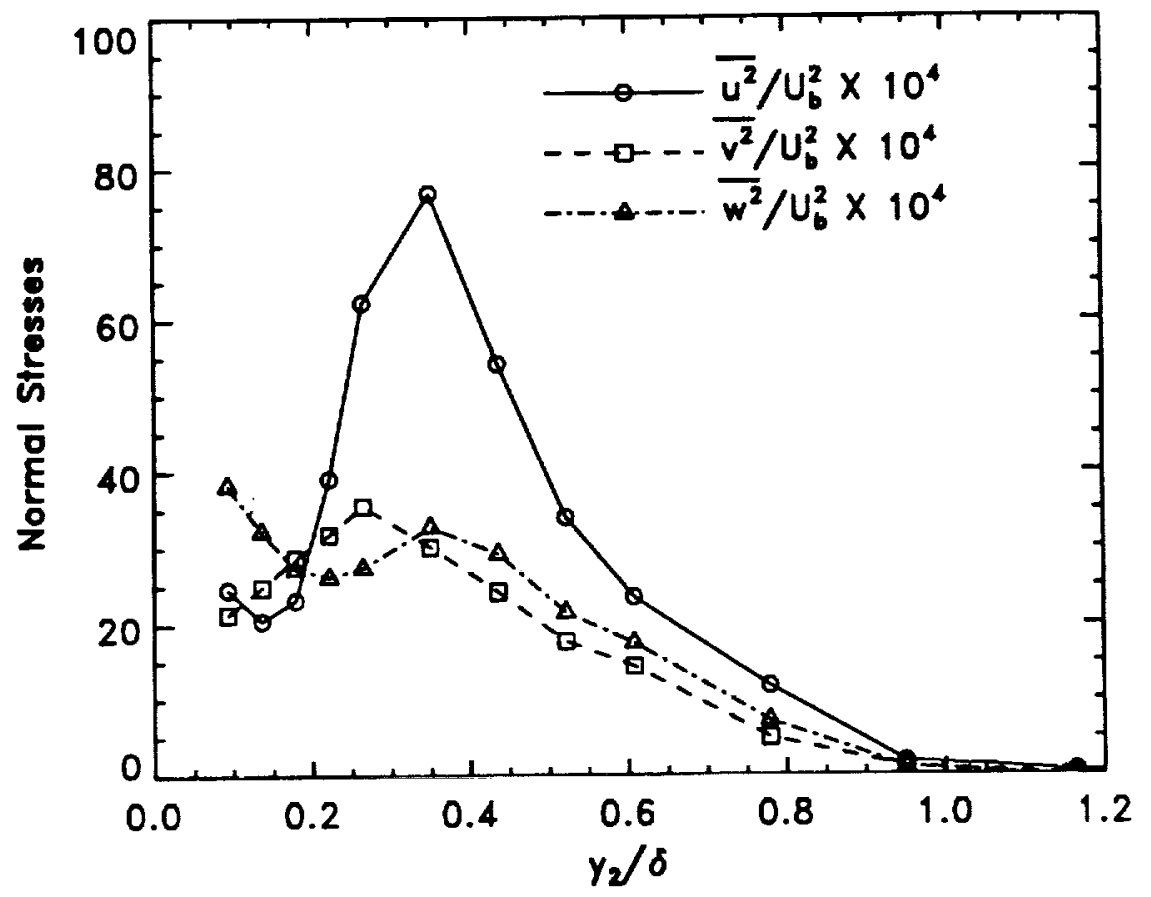

a) Station 5 .

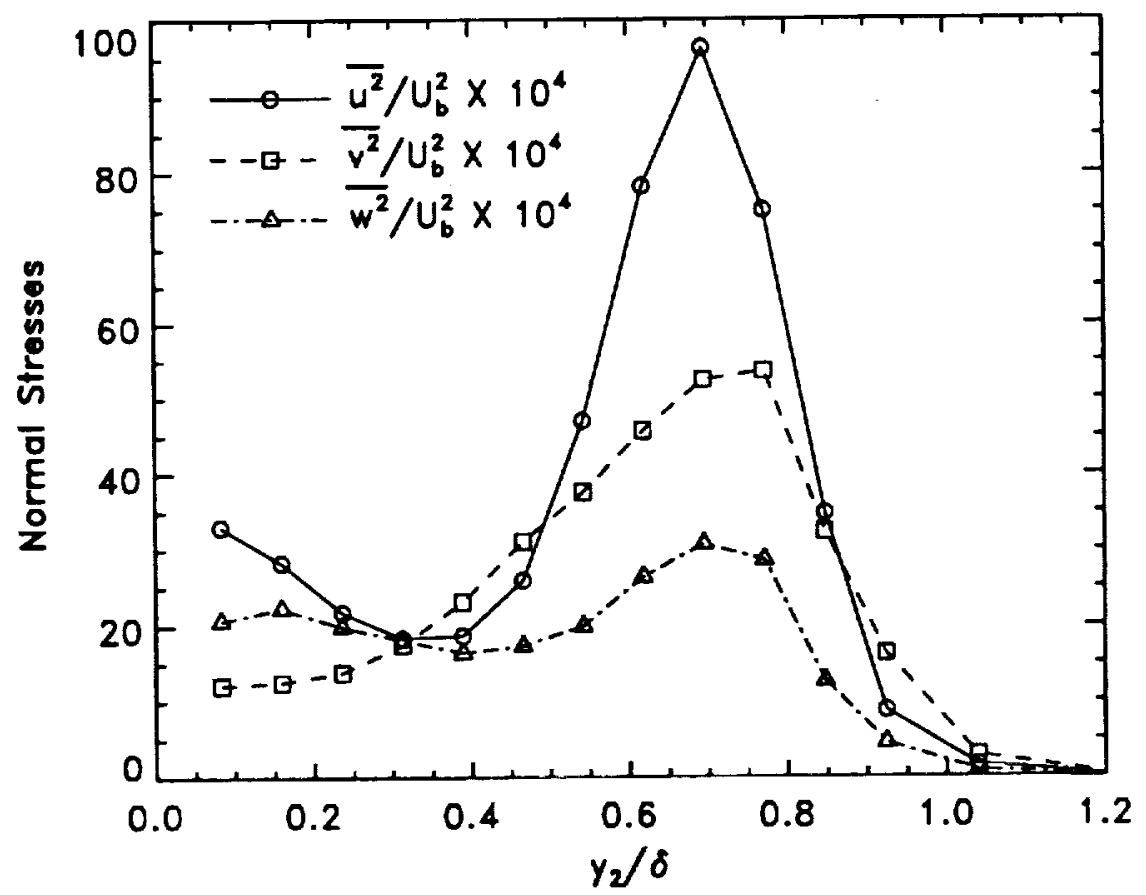

b) Station 6.

Fig. 5.27. Normal stress profiles along semi-major axis at Stations 5 and 6. 


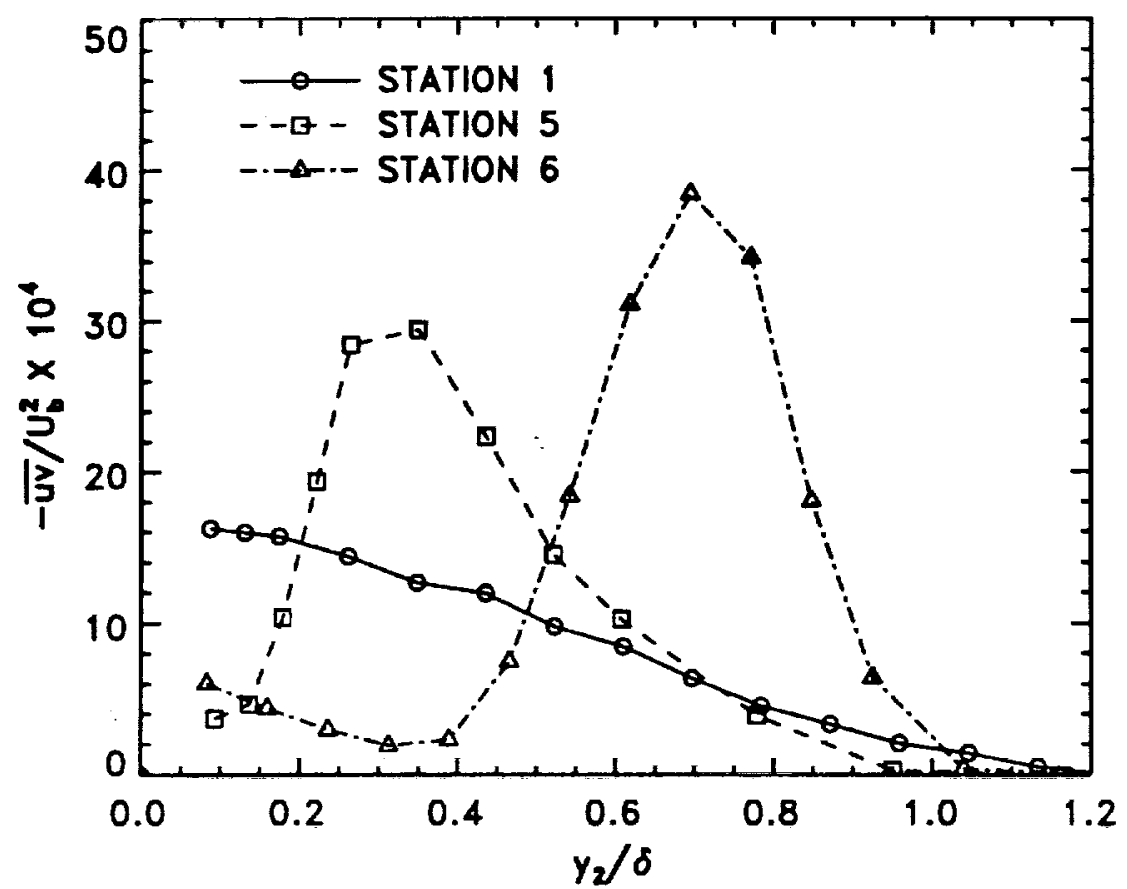

a) Shear stress.

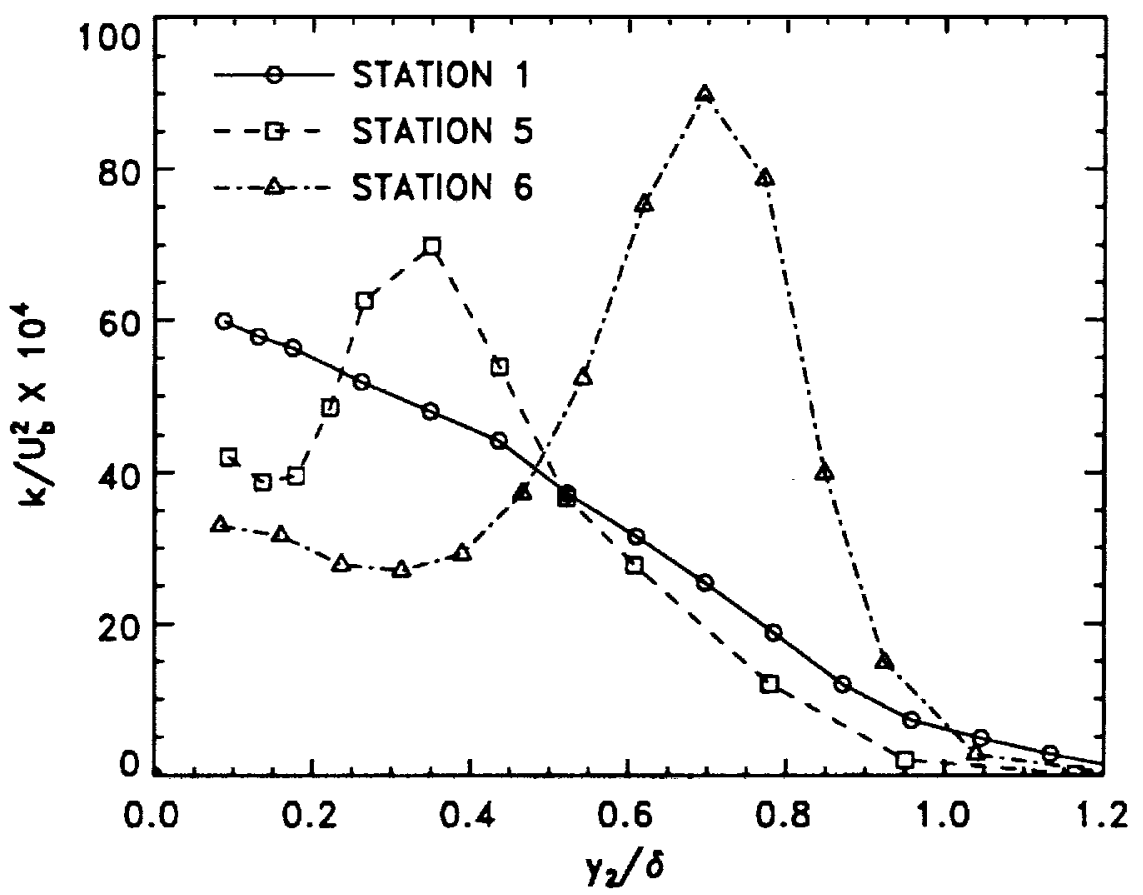

b) Turbulence kinetic enegy.

Fig. 5.28. Shear stress and kinetic energy profiles along semi-major axis. 


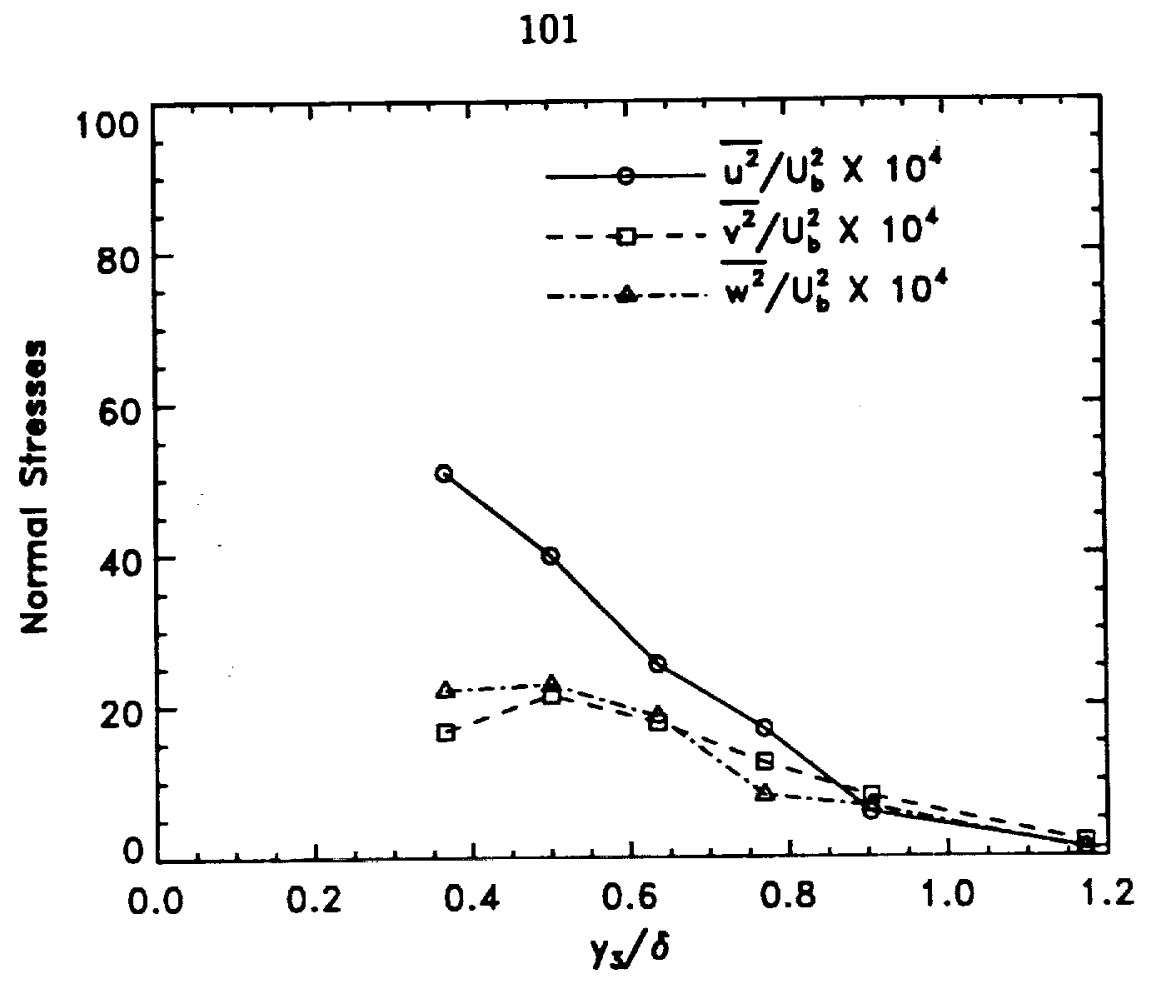

a) Station 5 .

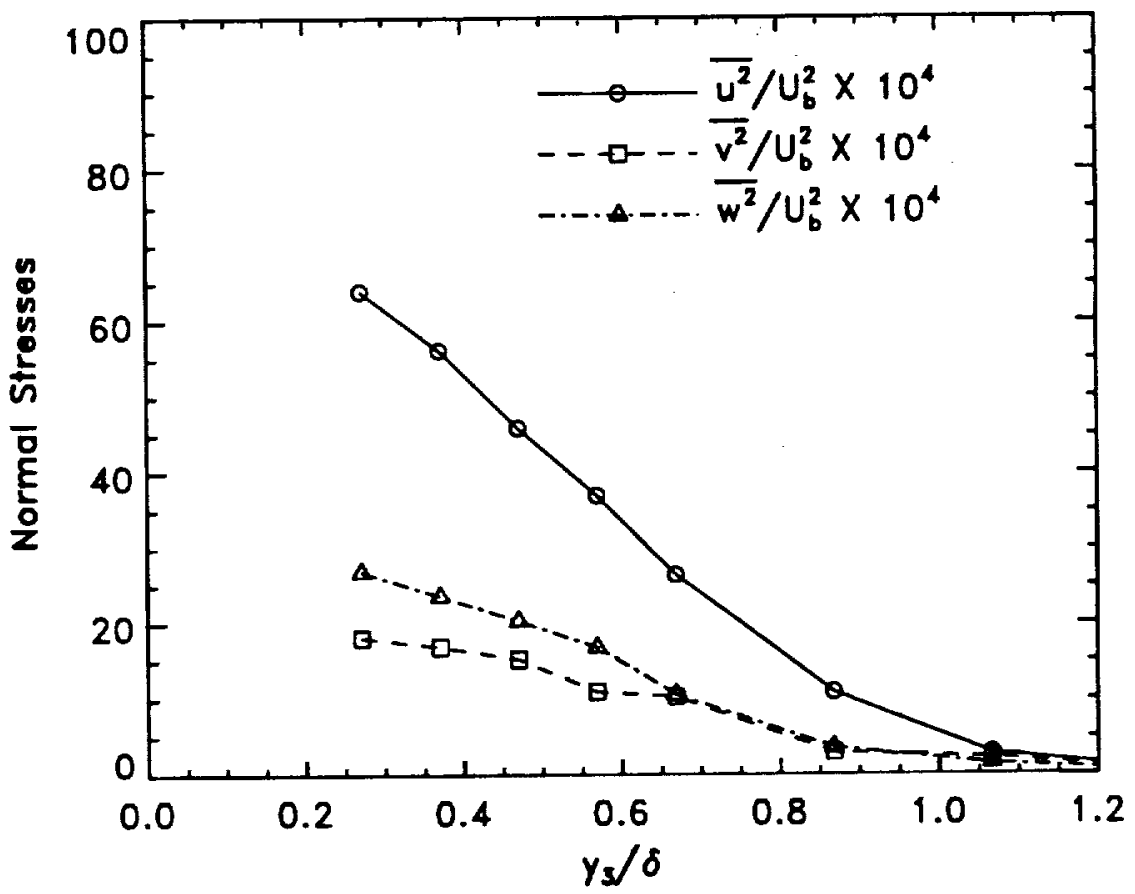

b) Station 6.

Fig. 5.29. Normal stress profiles along semi-minor axis at Stations 5 and 6 . 


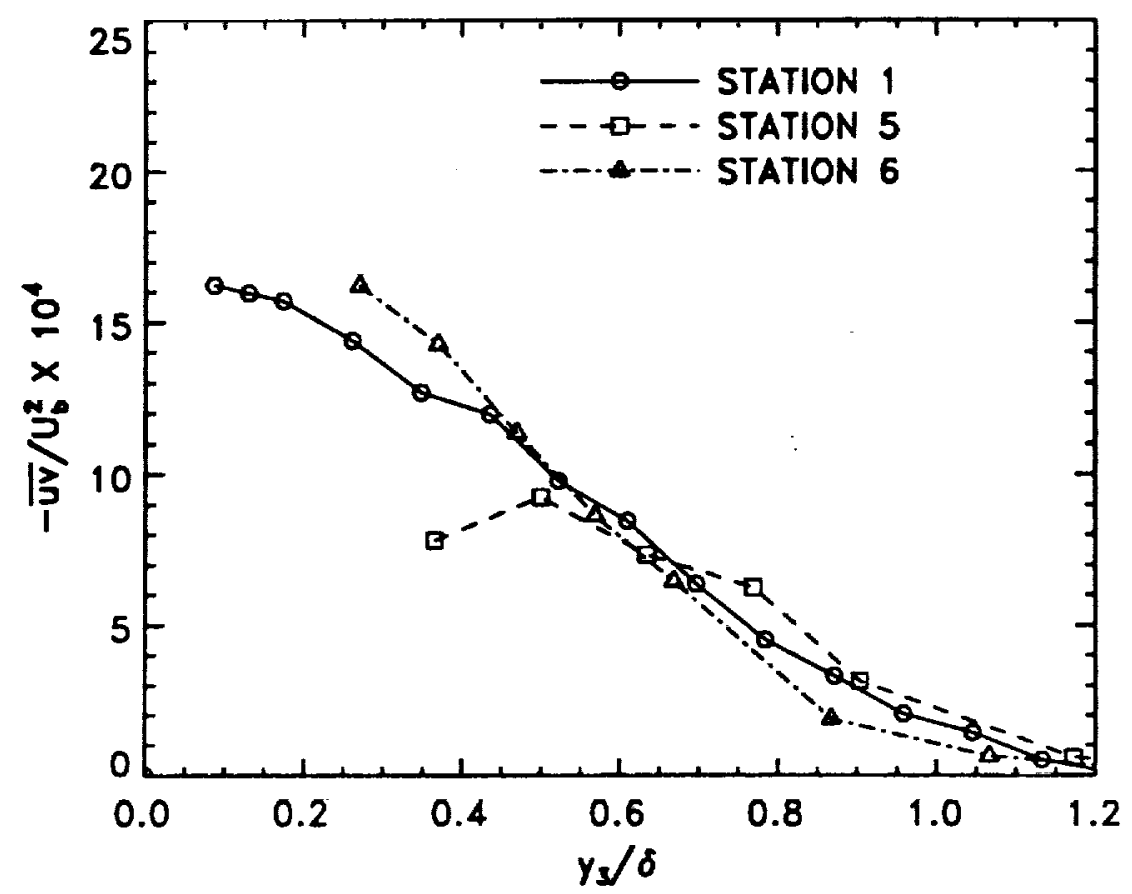

a) Shear stress.

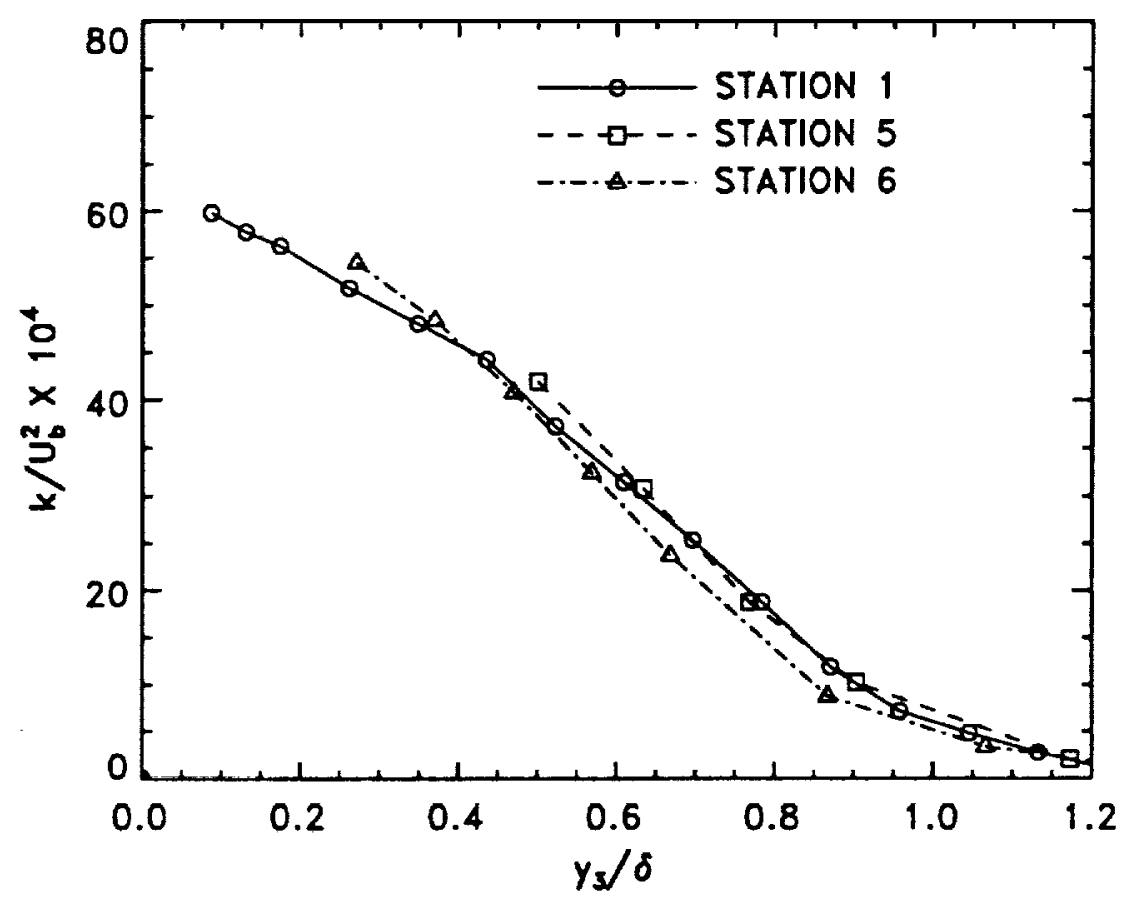

b) Turbulence kinetic enegy.

Fig. 5.30. Shear stress and t.k.e. profiles along semi-minor axis, $\mathbf{R e}_{b}=390,000$. 


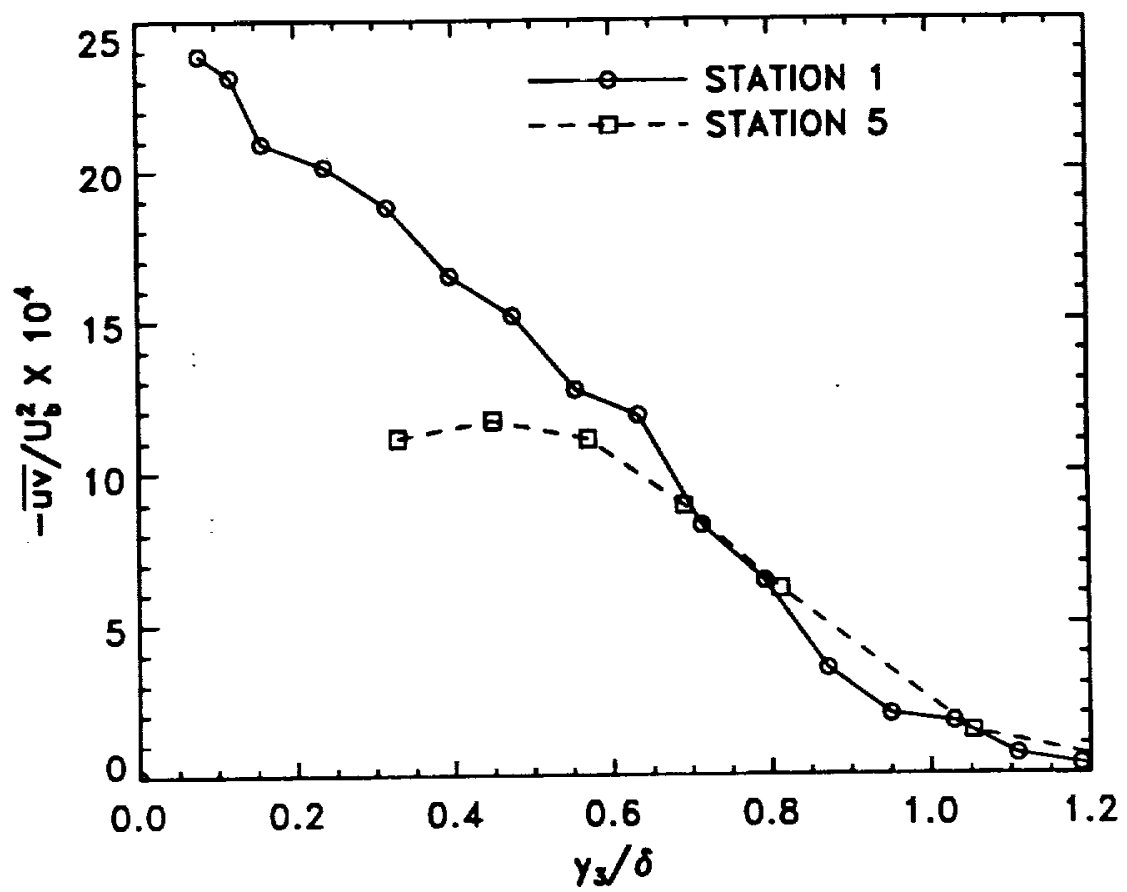

a) Shear stress.

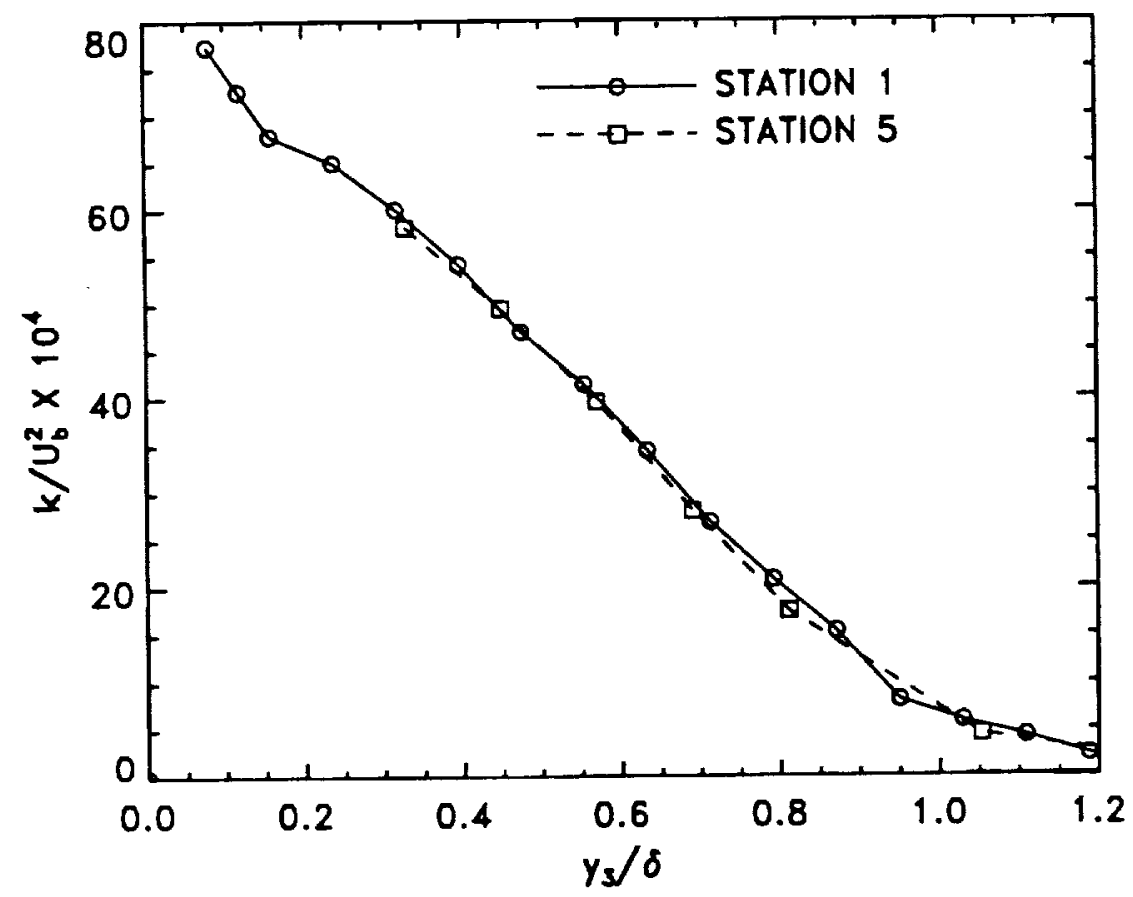

b) Turbulence kinetic enegy.

Fig. 5.31. Shear stress and t.k.e. profiles along semi-minor axis, $R_{b}=88,000$. 
104

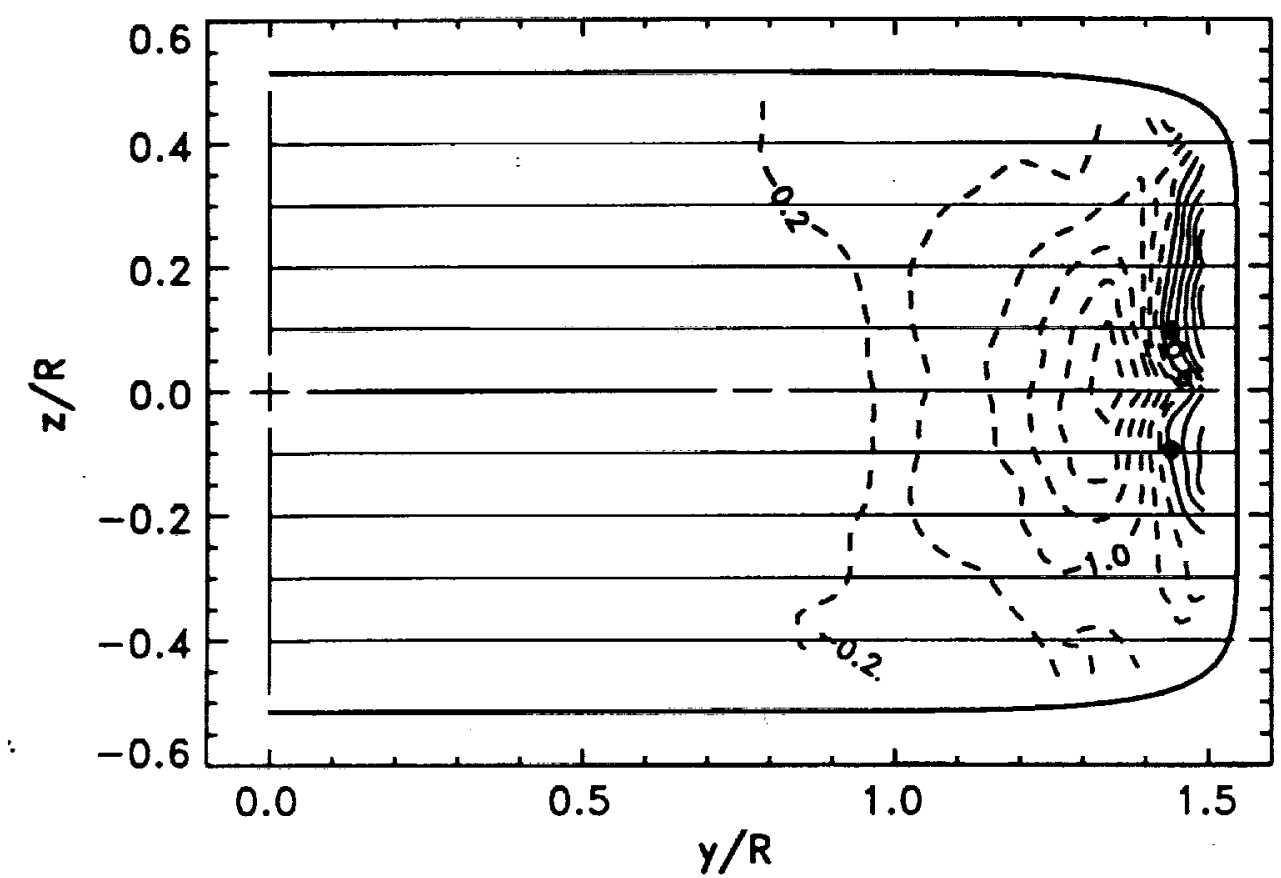

a) $(\partial \mathrm{U} / \partial \mathbf{y}) \mathbf{R} / \mathrm{U}_{\mathbf{b}}$.

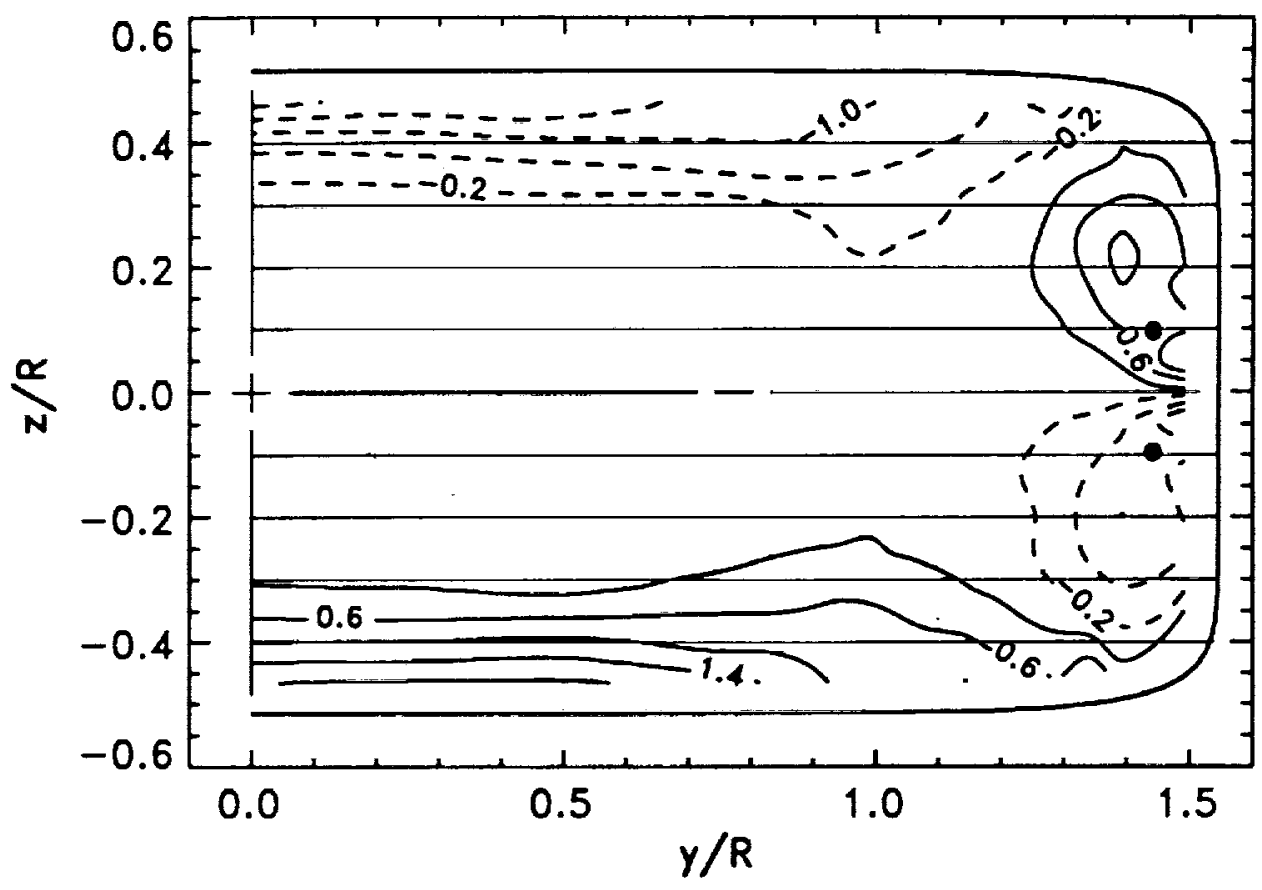

b) $(\partial \mathbf{U} / \partial \mathbf{z}) \mathbf{R} / \mathbf{U}_{\mathbf{b}}$.

Fig. 5.32. Primary rates-of-strain at Station 5. 


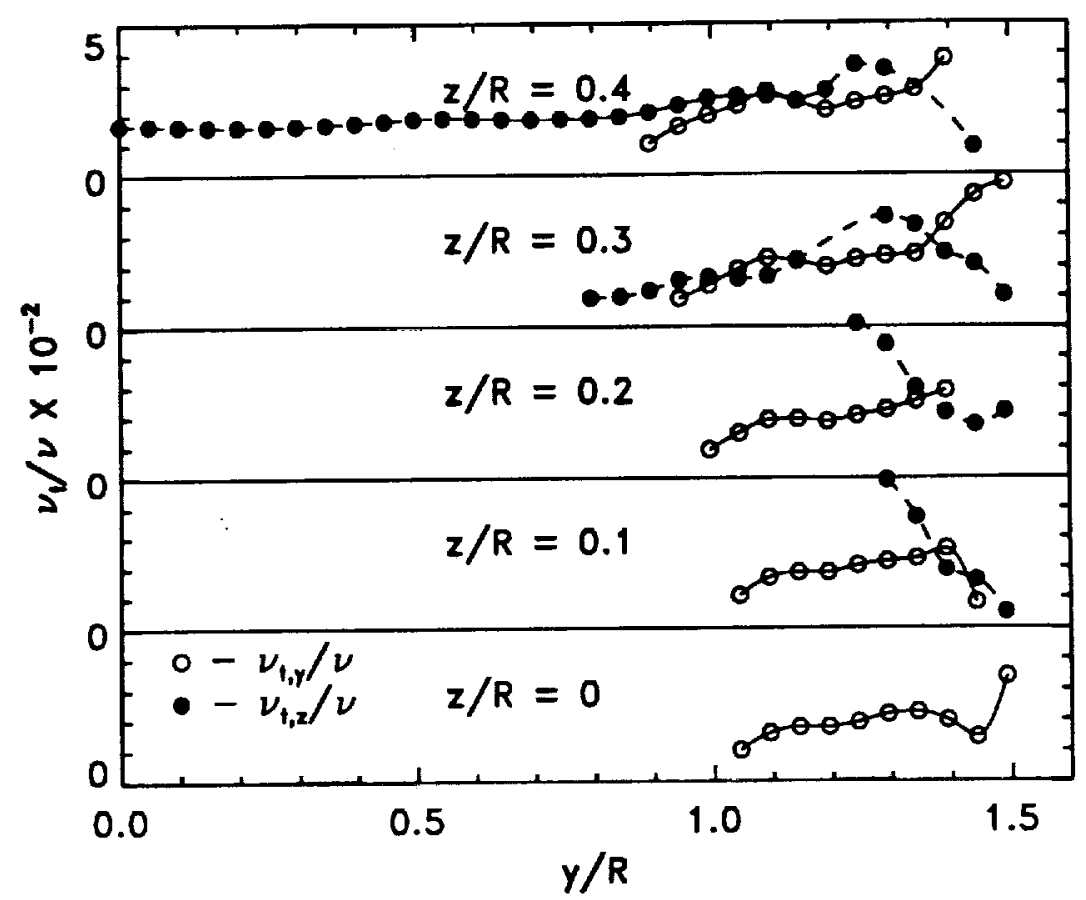

a) Quadrant 1.

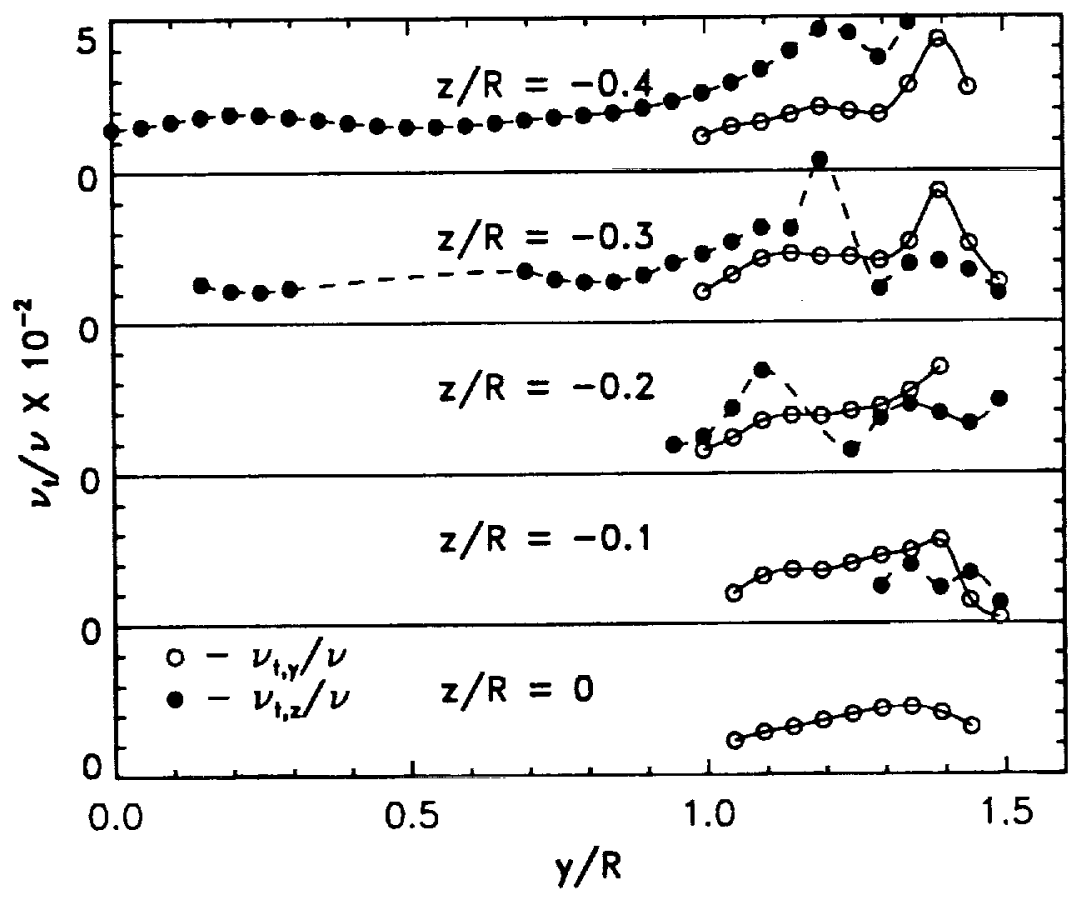

b) Quadrant 2.

Fig. 5.33. Component eddy viscosity distributions at Station 5 . 


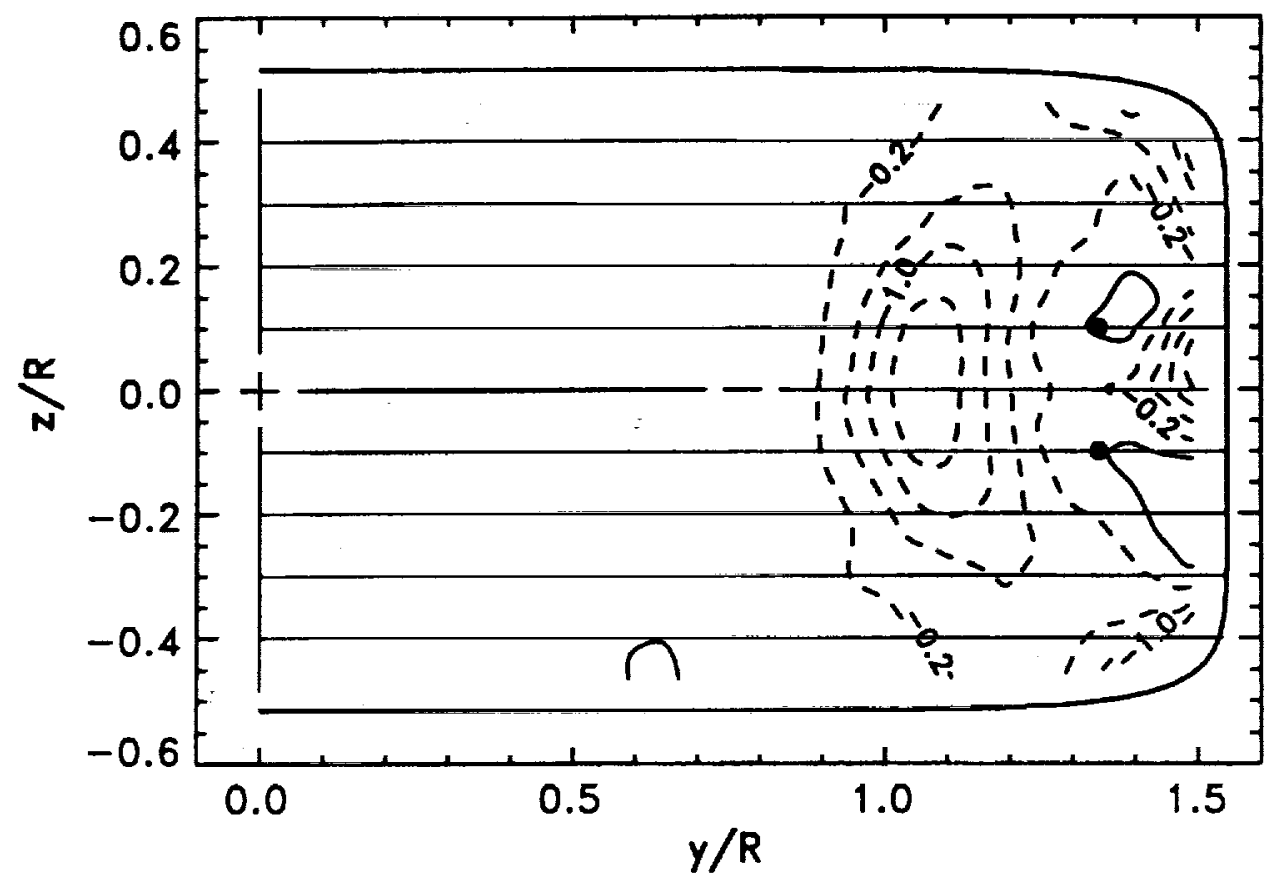

a) $(\partial \mathbf{U} / \partial \mathbf{y}) \mathbf{R} / \mathbf{U}_{\mathbf{b}}$.

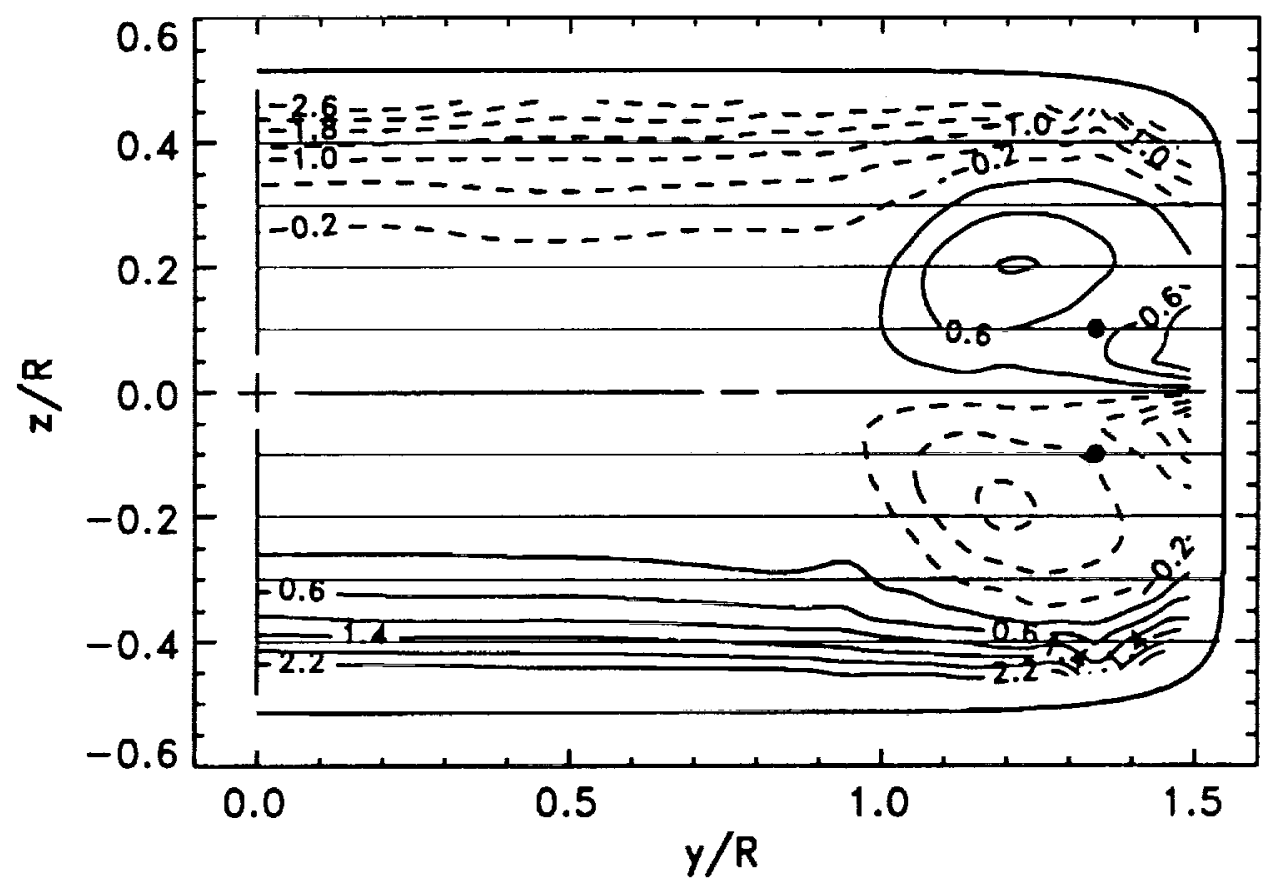

b) $(\partial \mathrm{U} / \partial \mathbf{z}) \mathbf{R} / \mathbf{U}_{\mathbf{b}}$.

Fig. 5.34. Primary rates-of-strain at Station 6. 


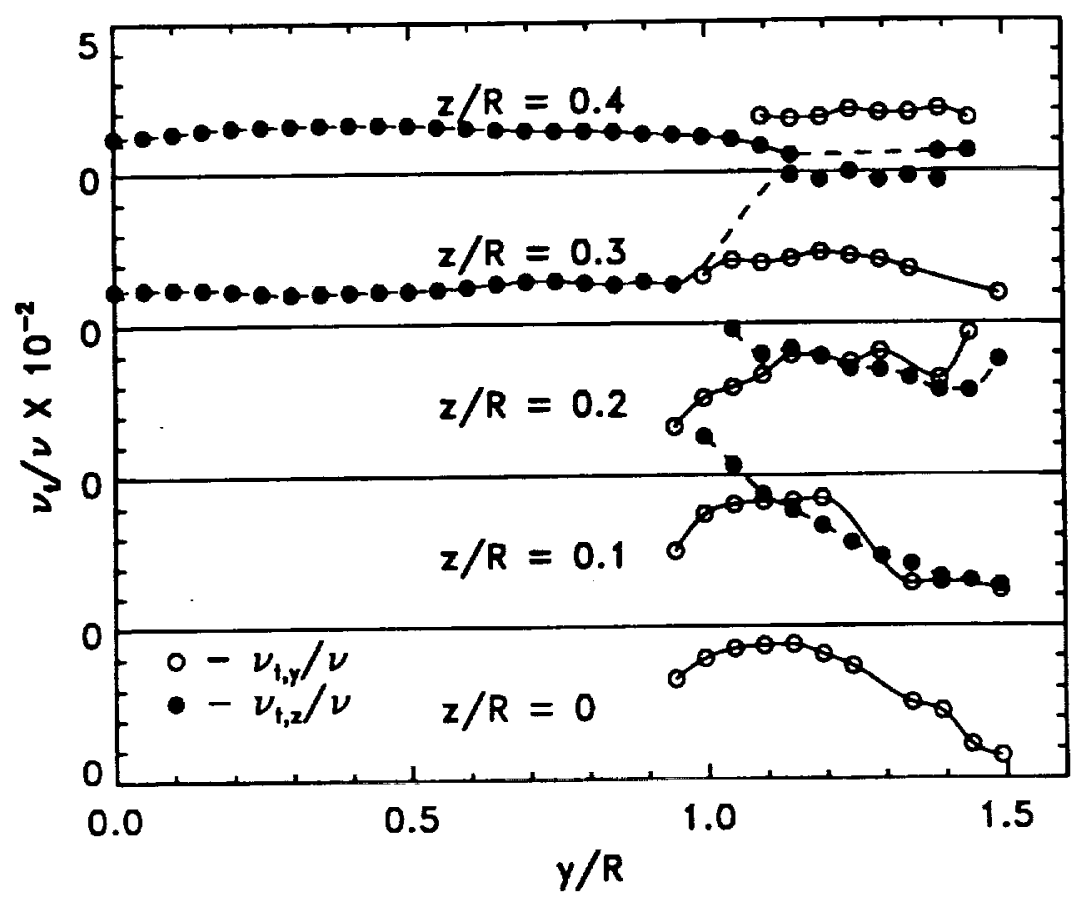

a) Quadrant 1.

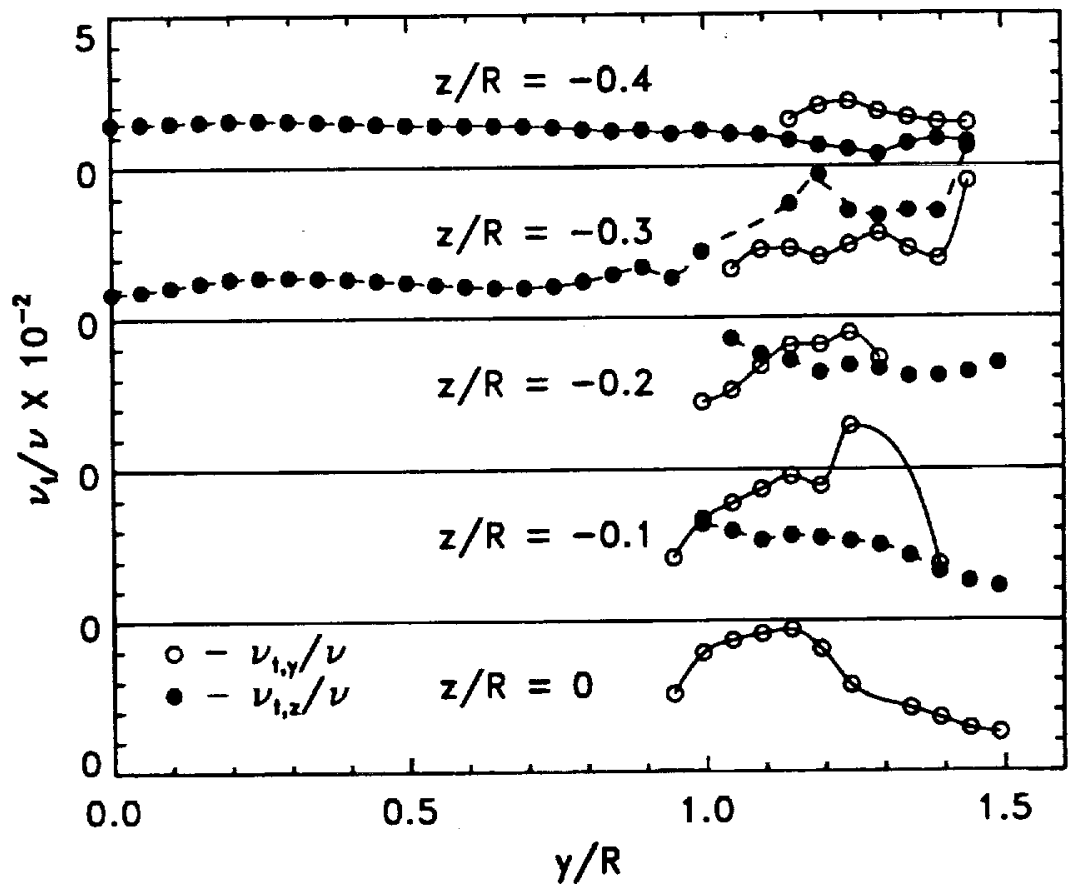

b) Quadrant 2.

Fig. 5.35. Component eddy viscosity distributions at Station 6 . 


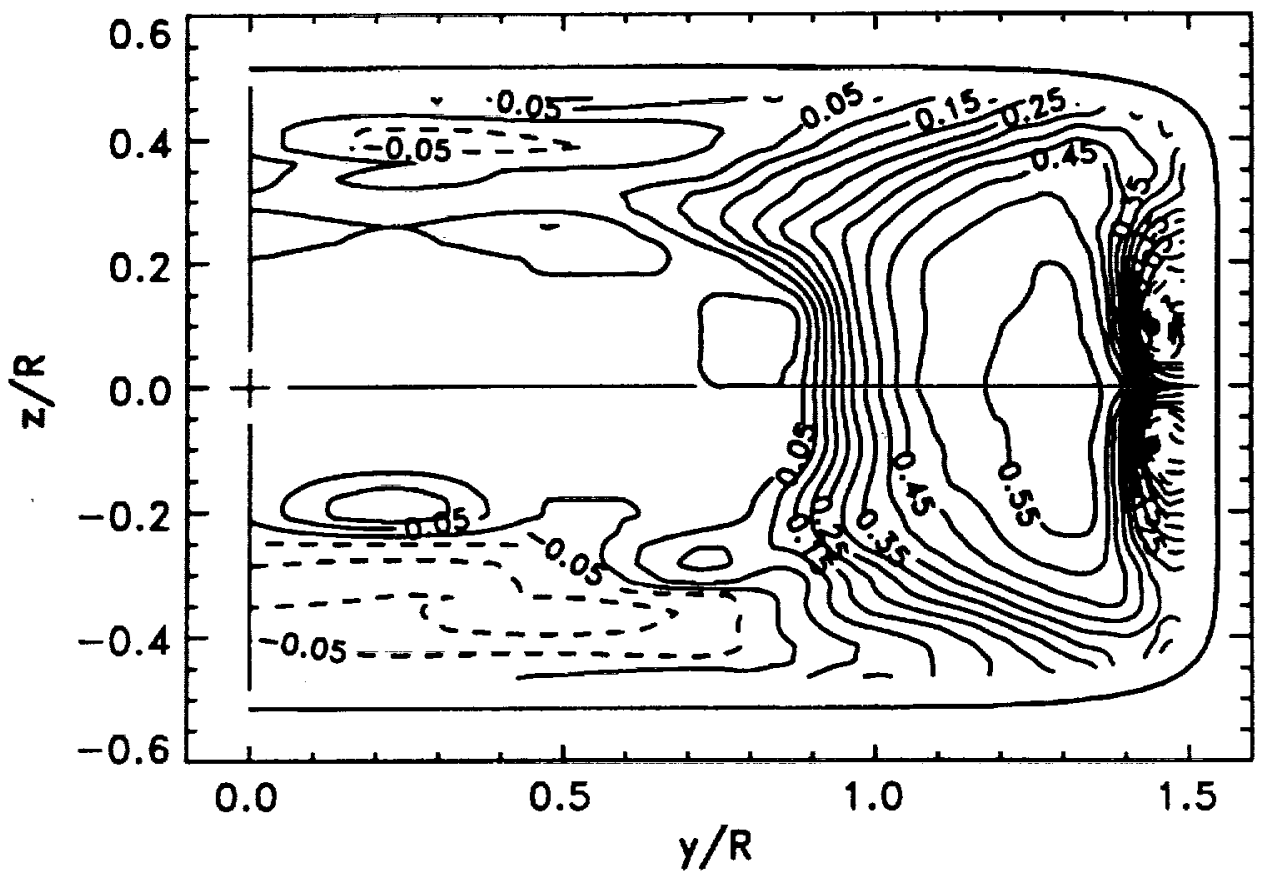

a) Station 5 .

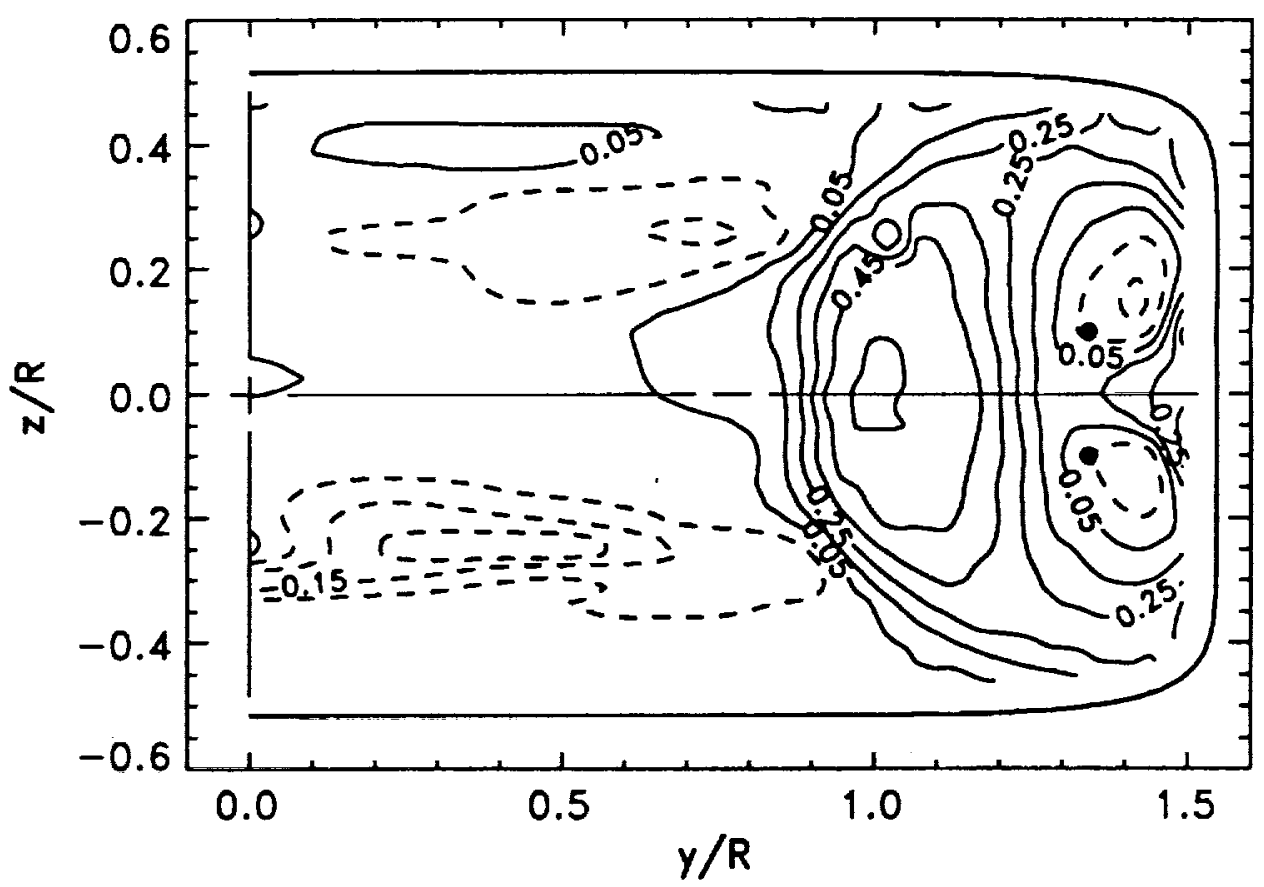

b) Station 6.

Fig. 5.36. Shear stress correlation $R_{u v}=\overline{u v} / u^{\prime} v^{\prime}$. 


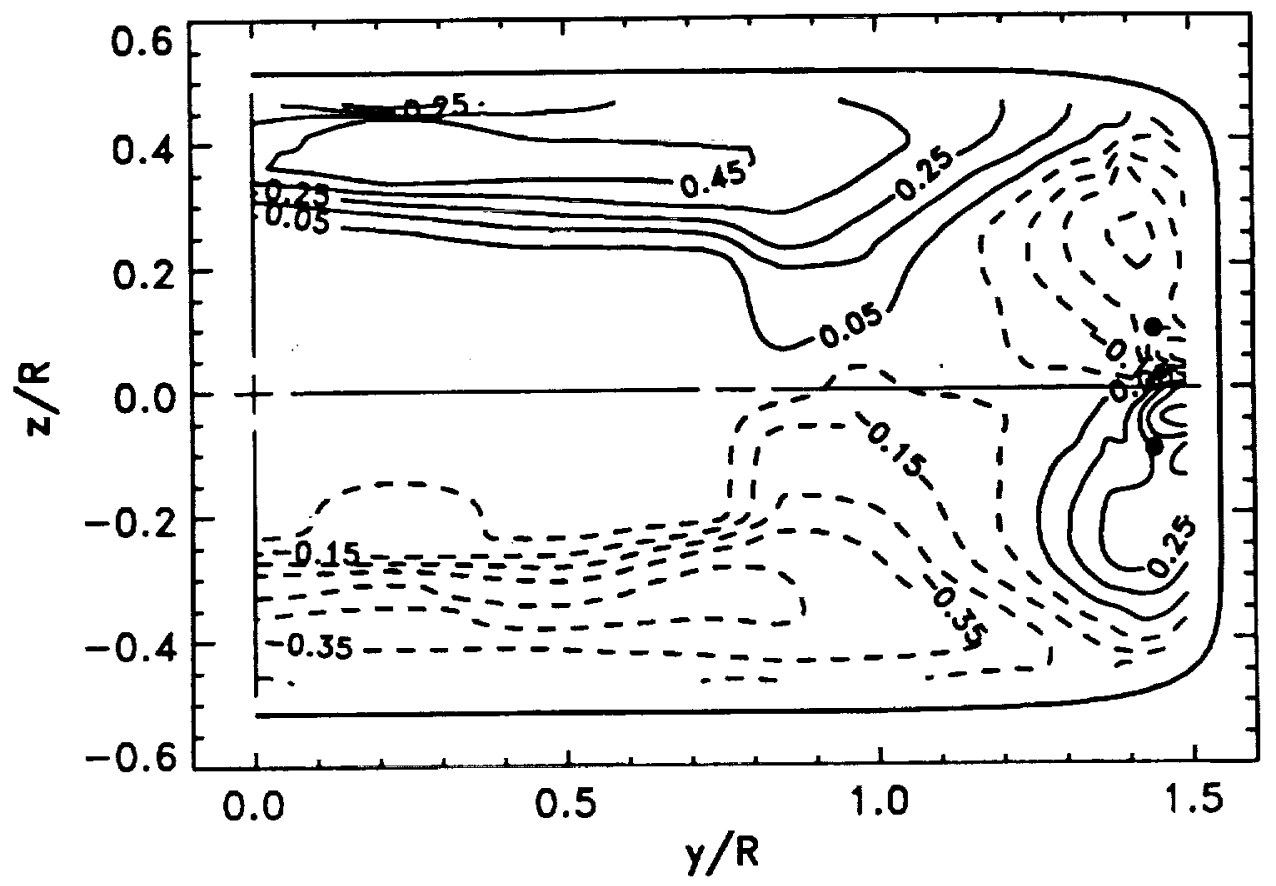

a) Station 5 .

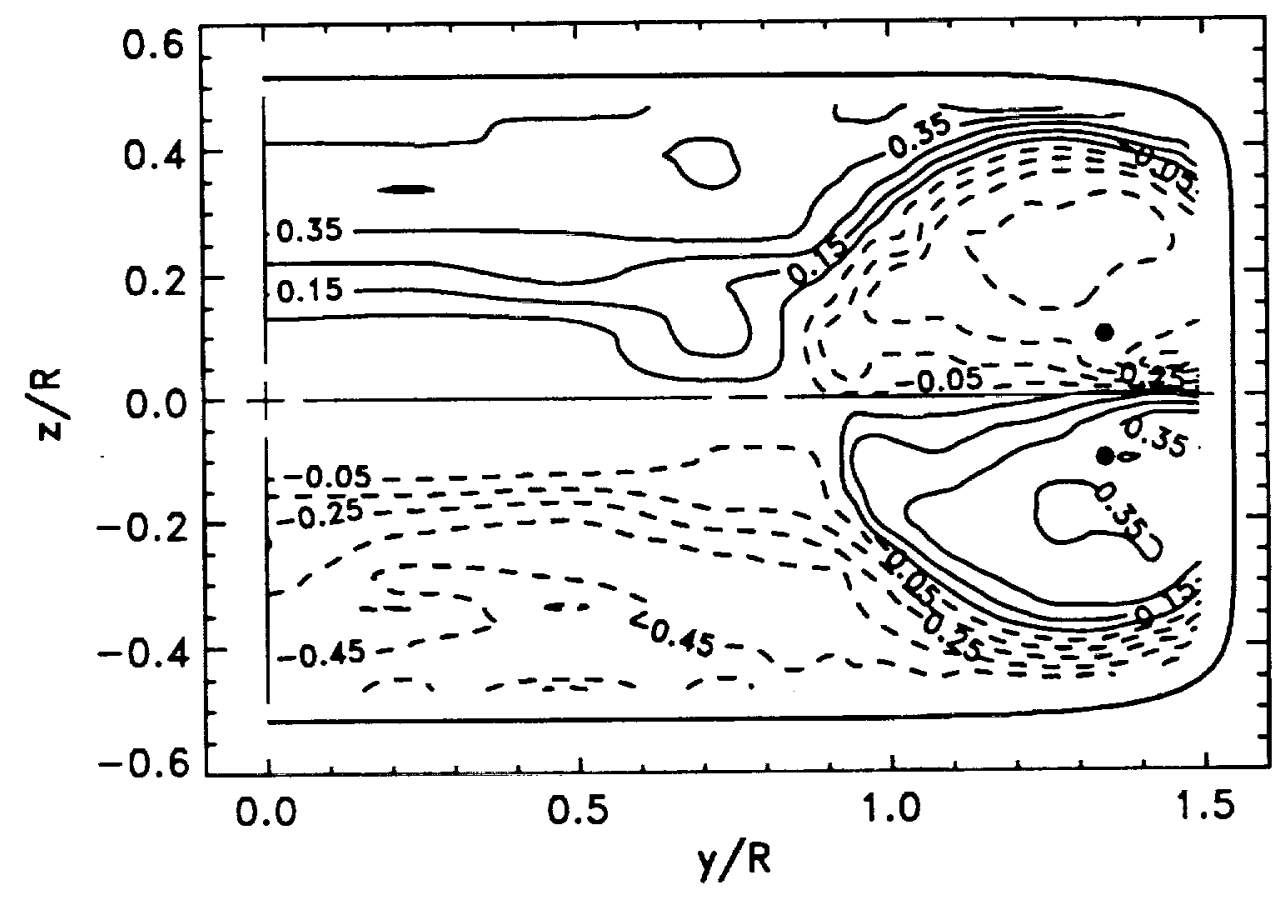

b) Station 6 .

Fig. 5.37. Shear stress correlation $R_{u w}=\overline{u w} / u^{\prime} w^{\prime}$. 


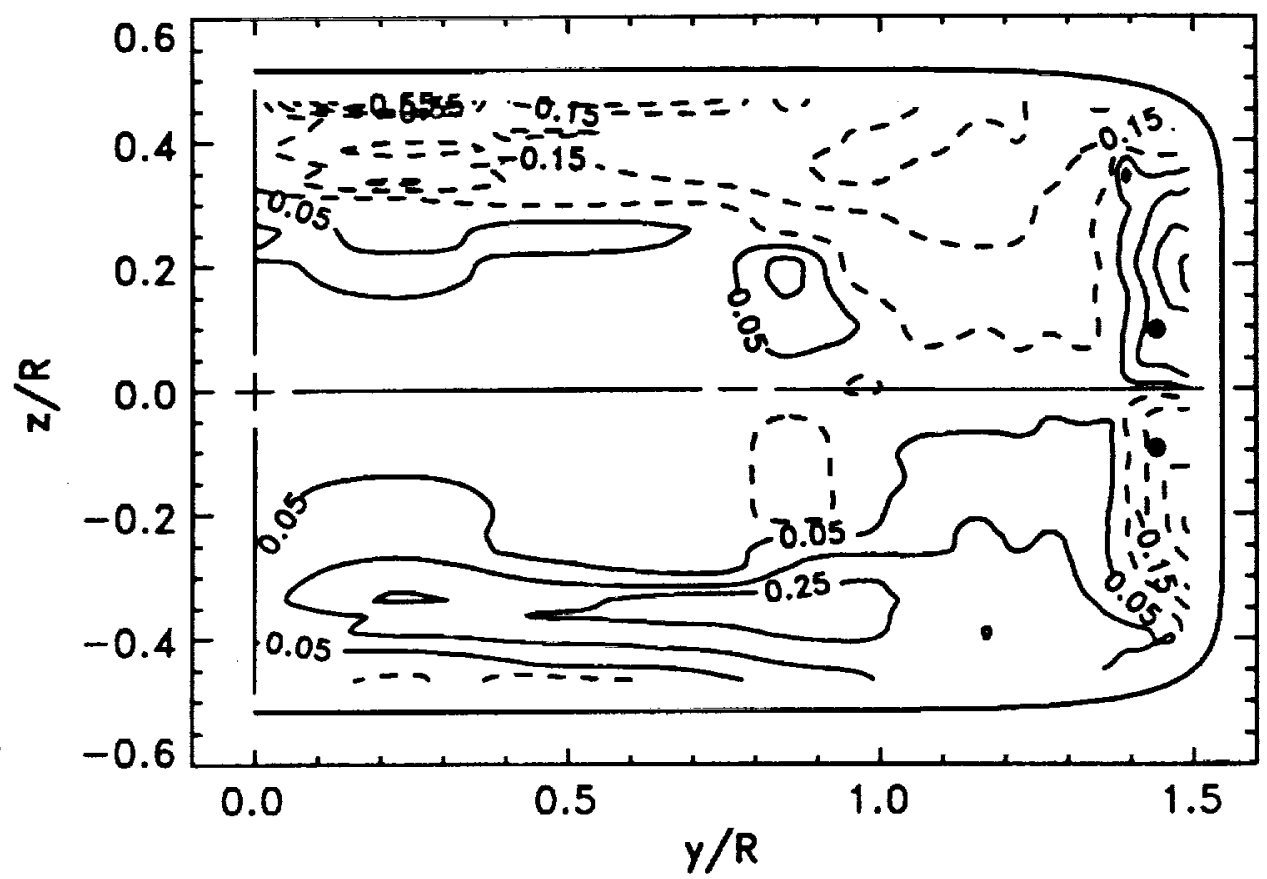

a) Station 5 .

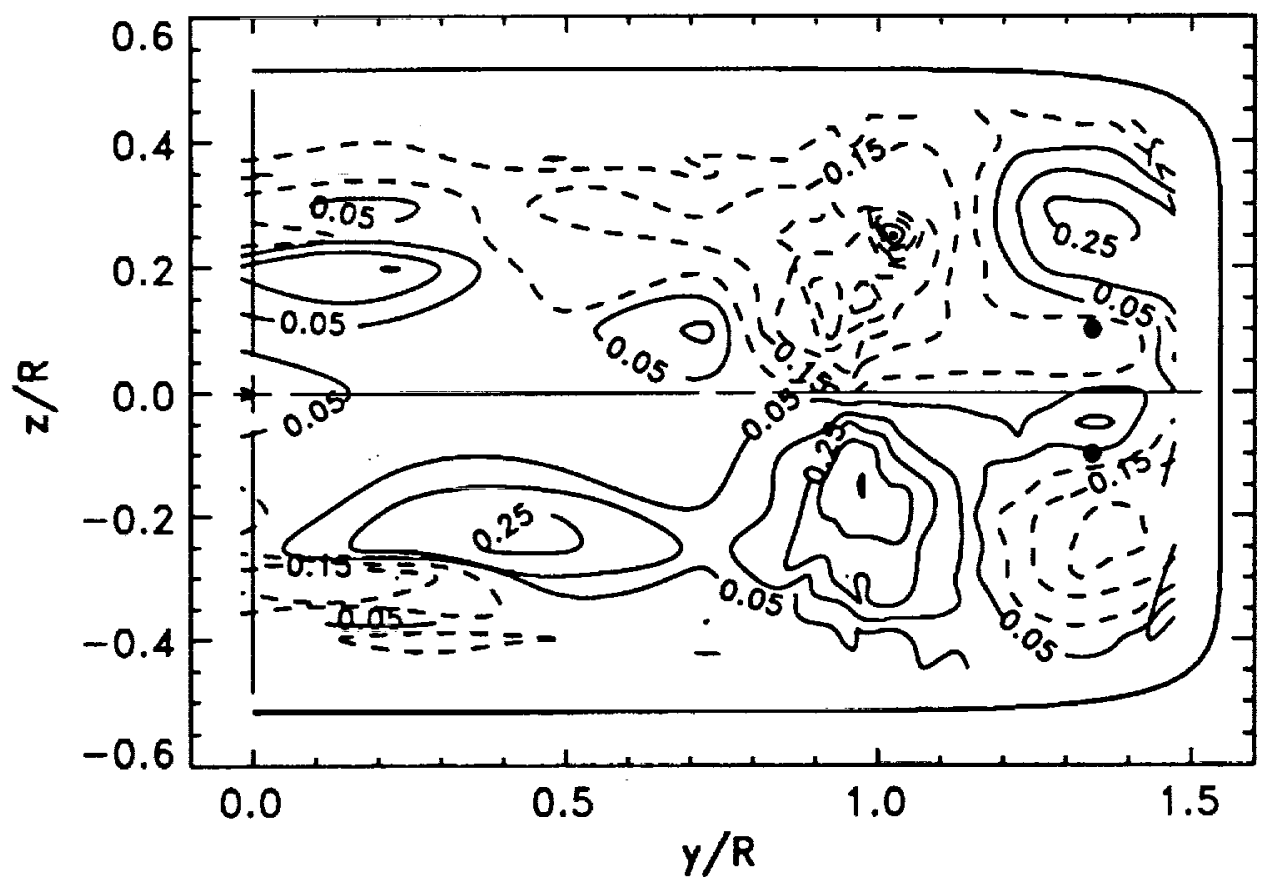

b) Station 6.

Fig. 5.38. Shear stress correlation $R_{v w}=\overline{v w} / v^{\prime} w^{\prime}$. 


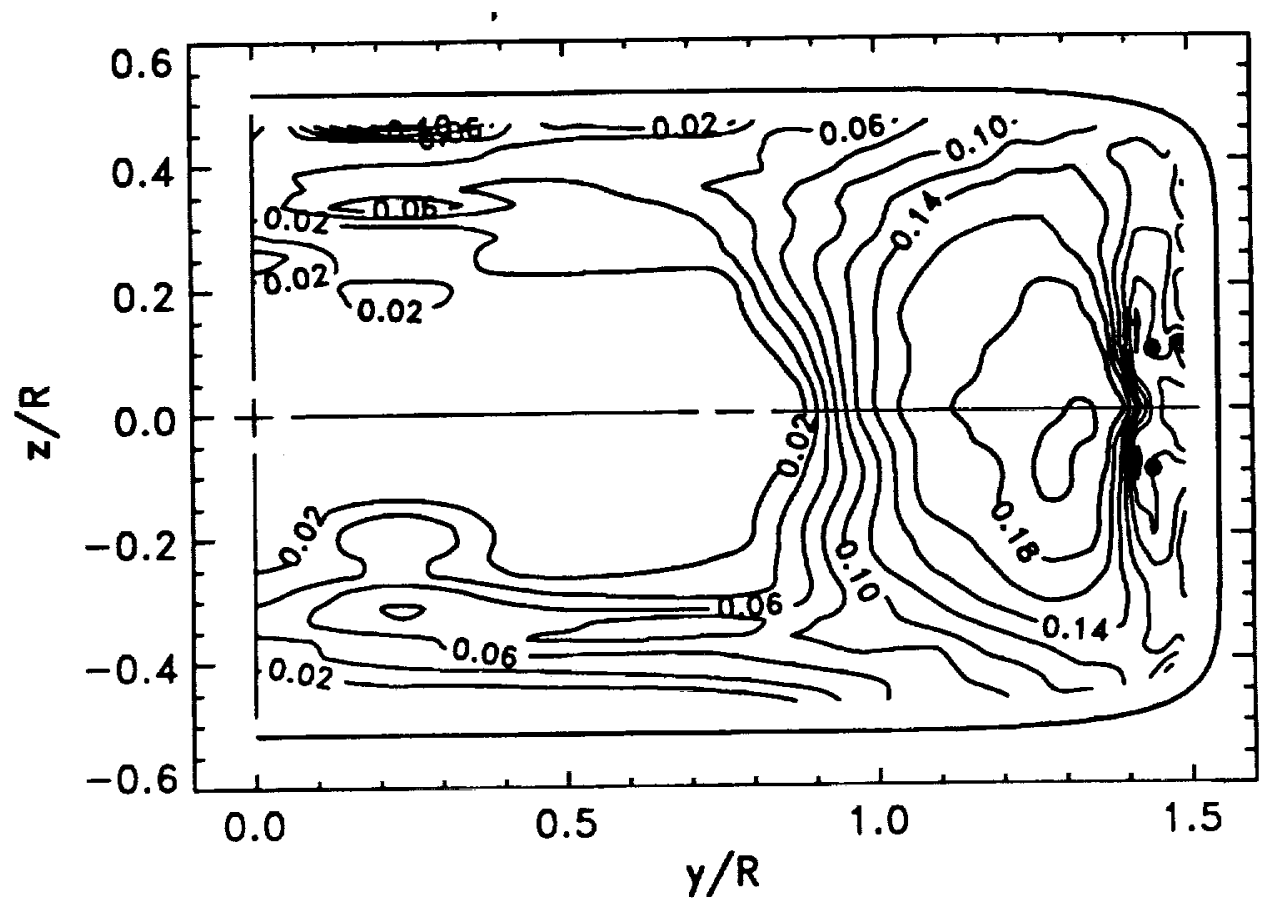

a) Station 5 .

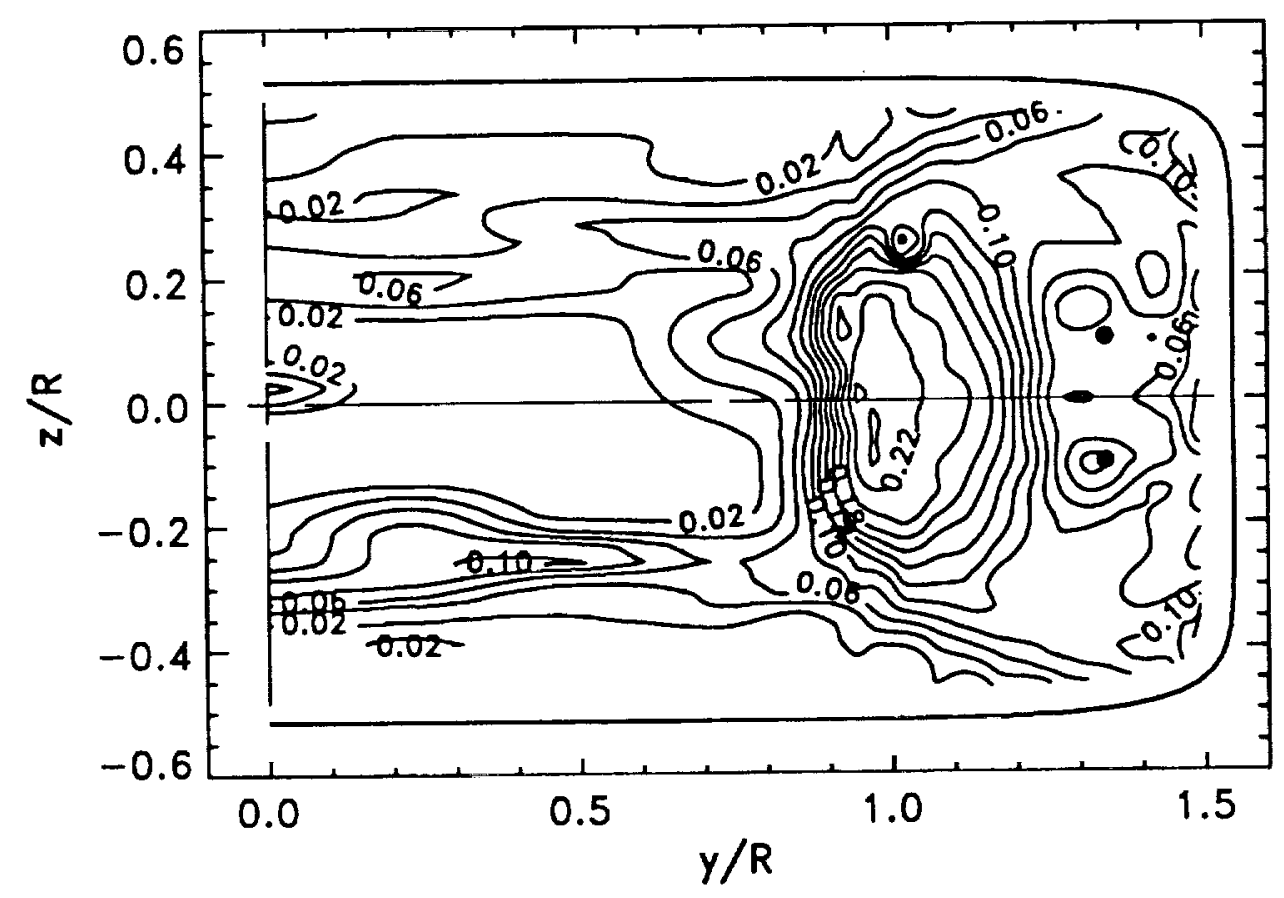

b) Station 6 .

Fig. 5.39. Shear-energy parameter $a 1_{y}=\sqrt{\overline{u v}^{2}+\overline{v w}^{2}} / 2 k$. 


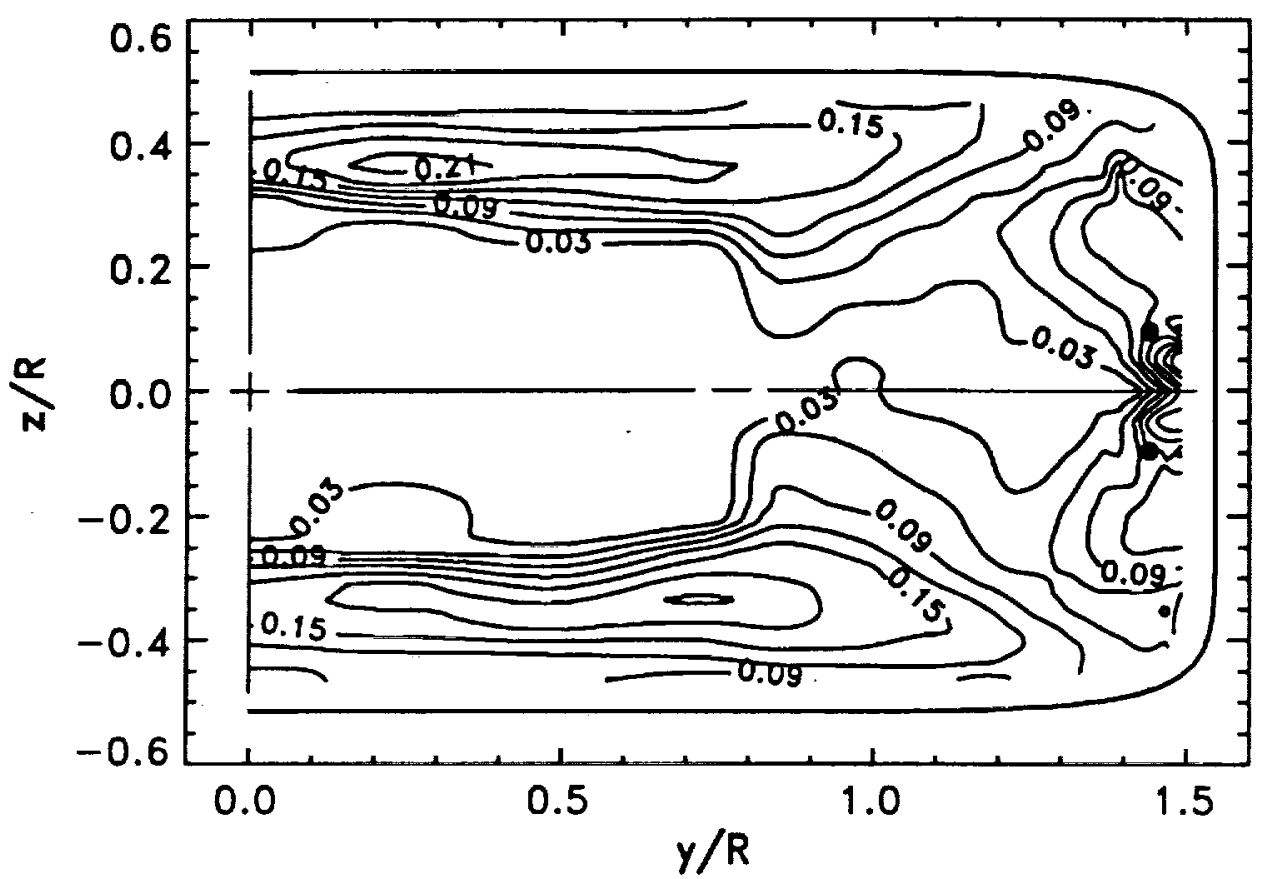

a) Station 5 .

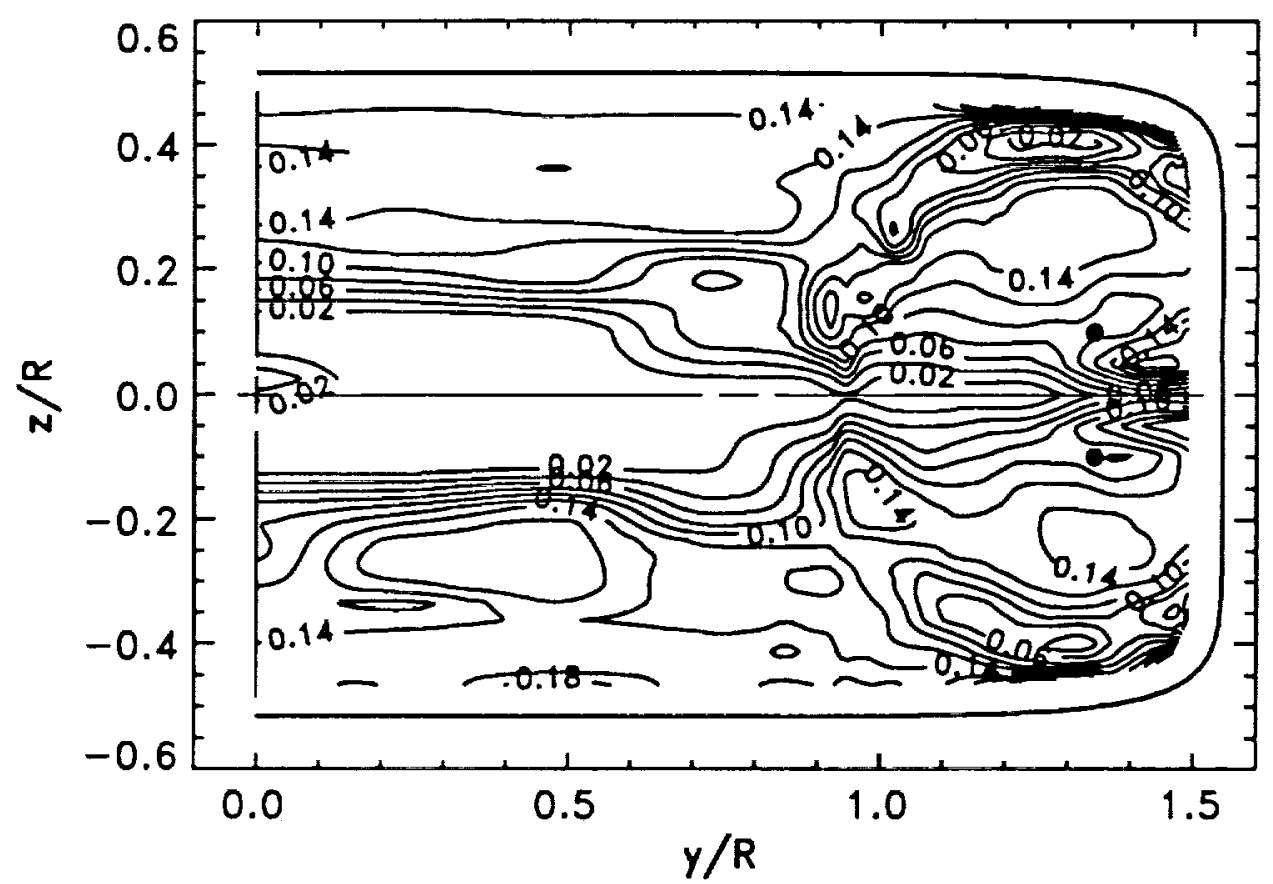

b) Station 6 .

Fig. 5.40. Shear-energy parameter $a 1_{z}=\sqrt{\overline{u w}^{2}+\overline{v w}^{2}} / 2 k$. 


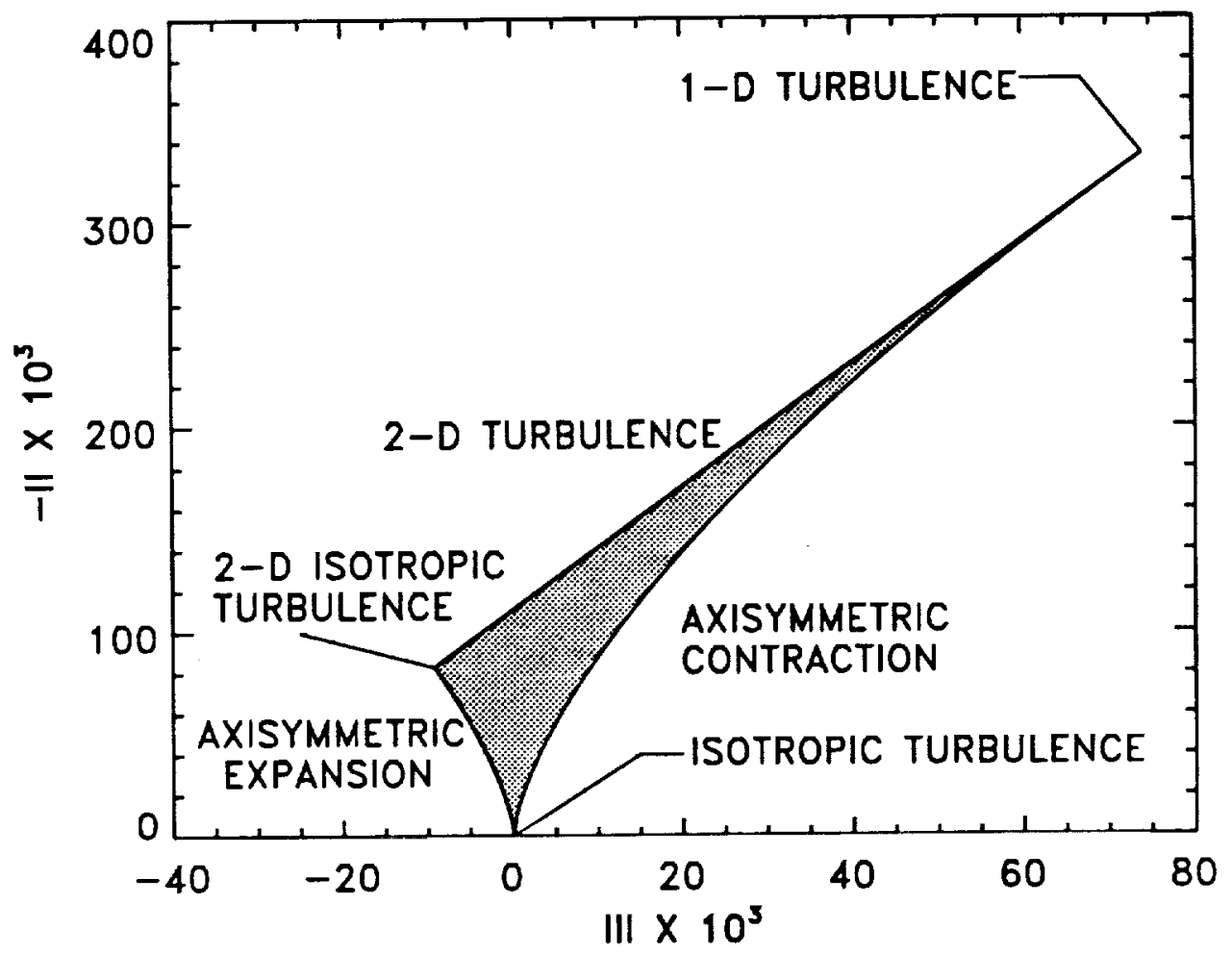

Fig. 5.41. Physical realizability limits of turbulence invariant map. 
114

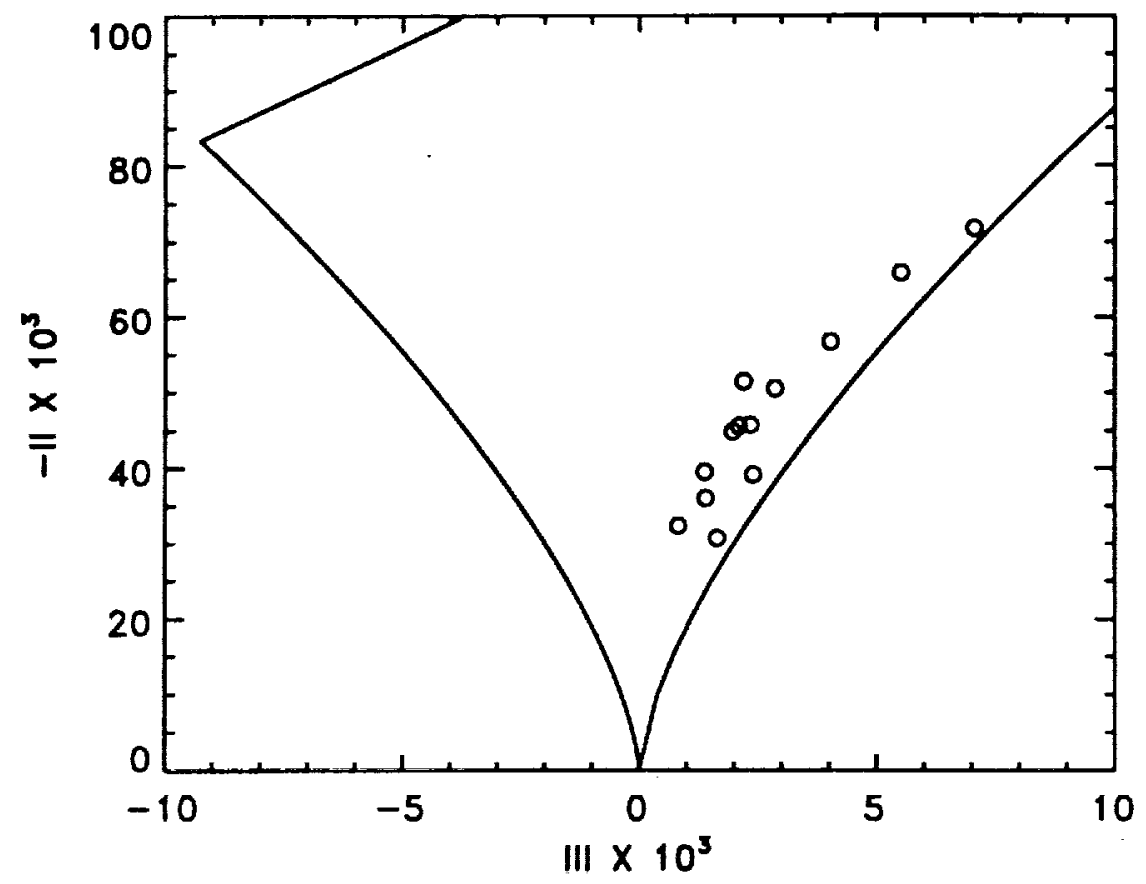

a) Invariant map.

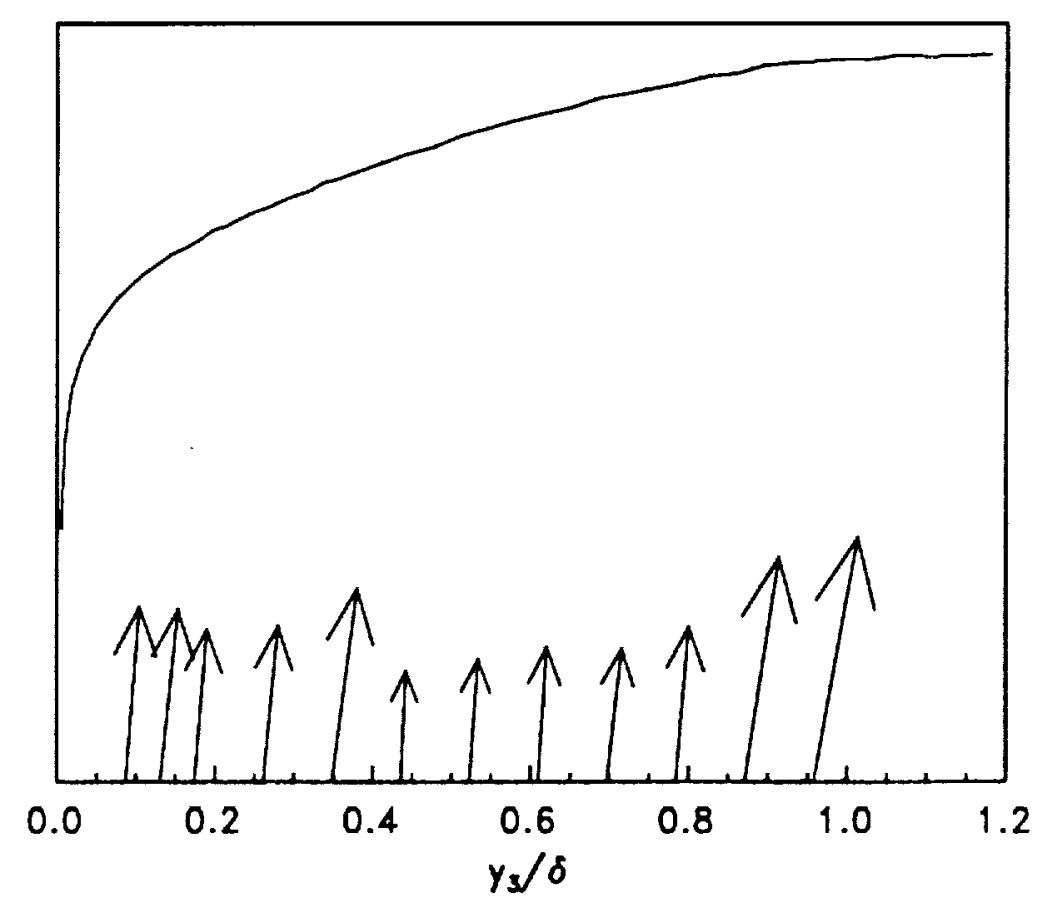

b) Location of data points on invariant map.

Fig. 5.42. Anisotropic stress tensor invariants at Station 1. 


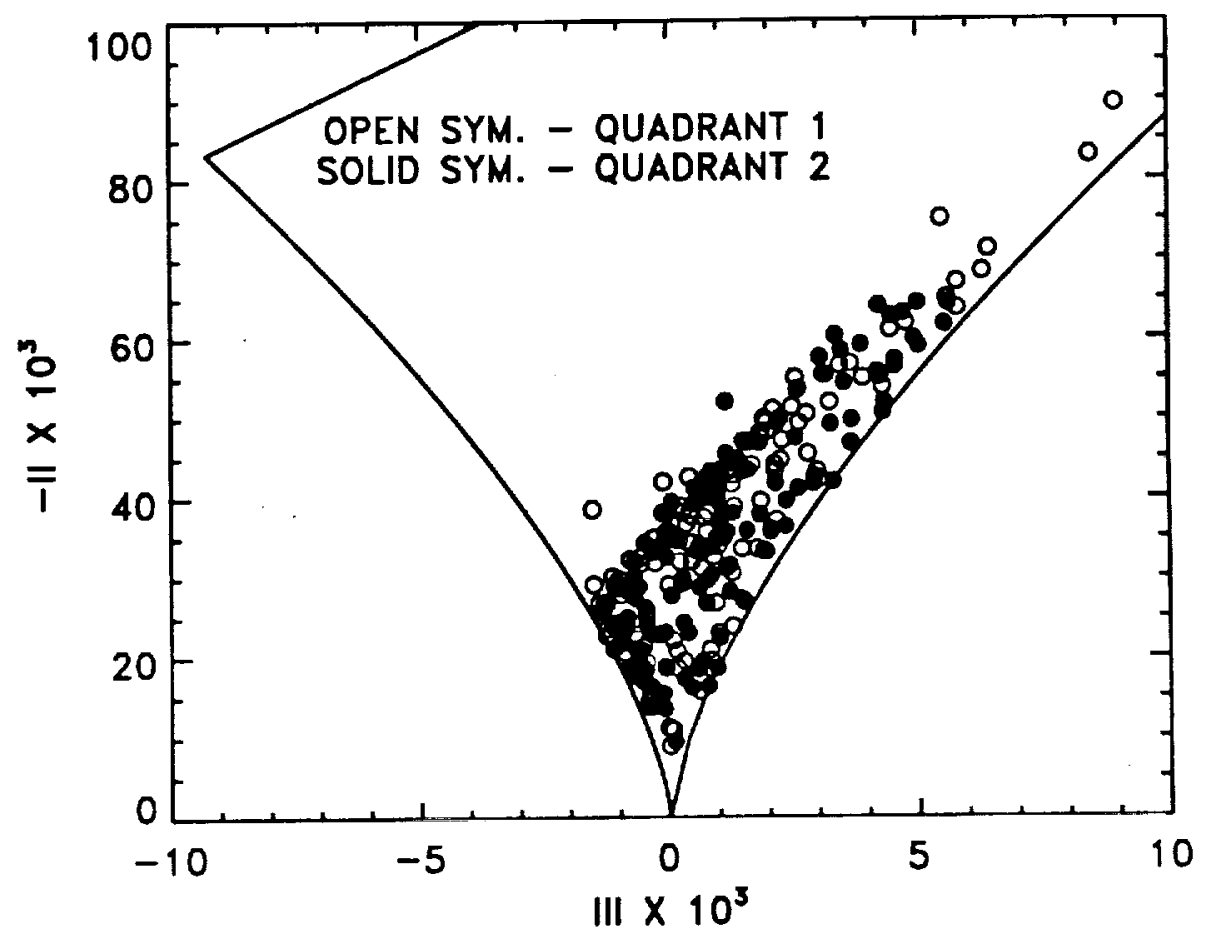

a) Invariant map.

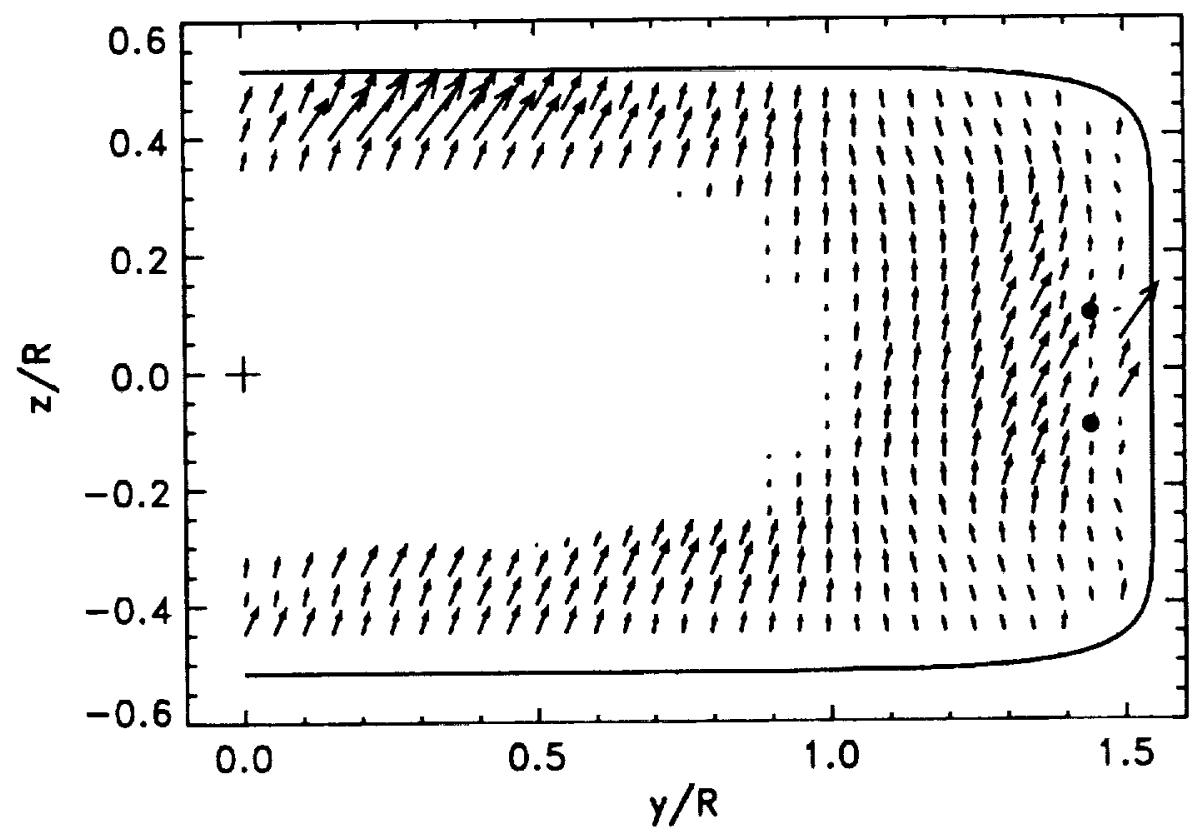

b) Location of data points on invariant map.

Fig. 5.43. Anisotropic stress tensor invariants at Station 5. 
116

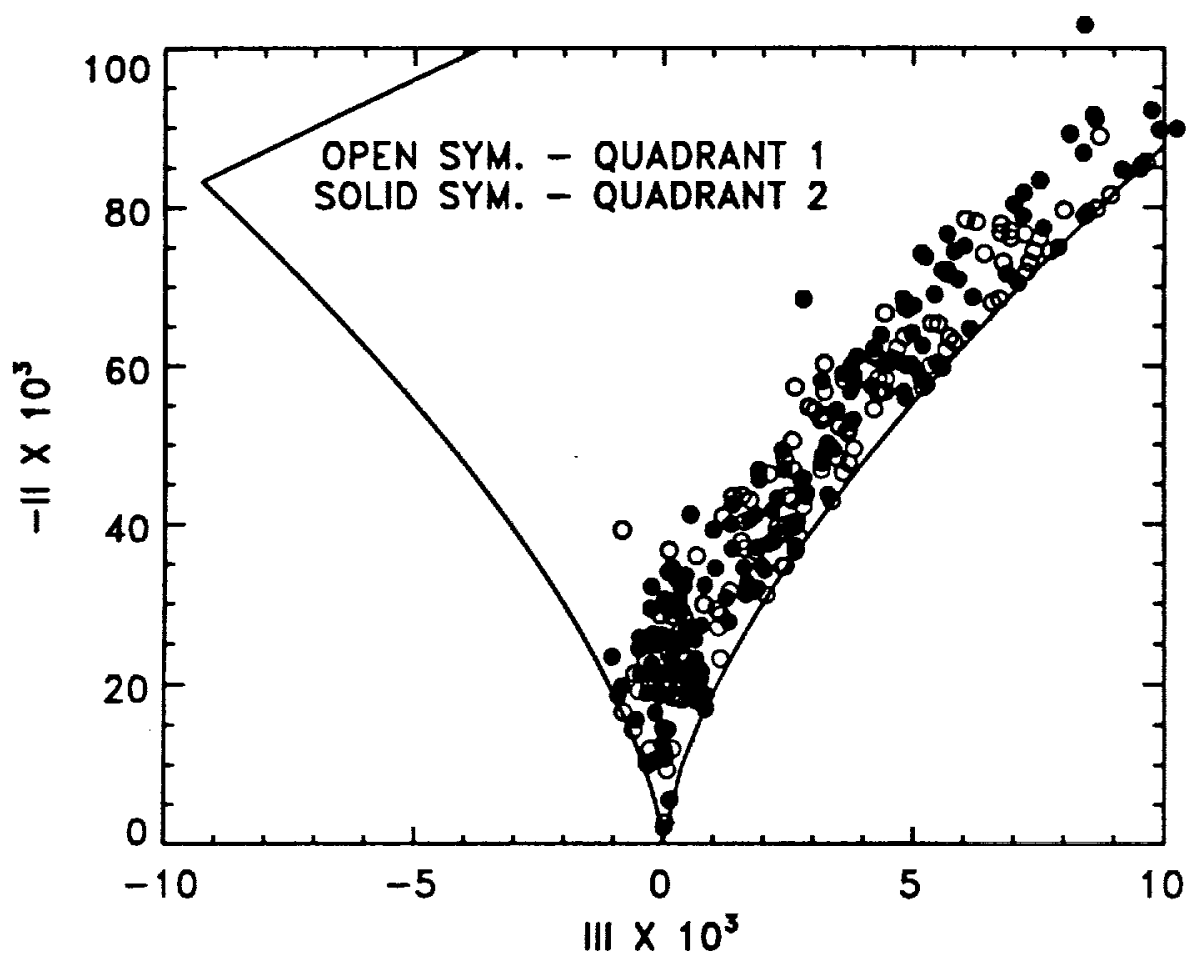

a) Invariant map.

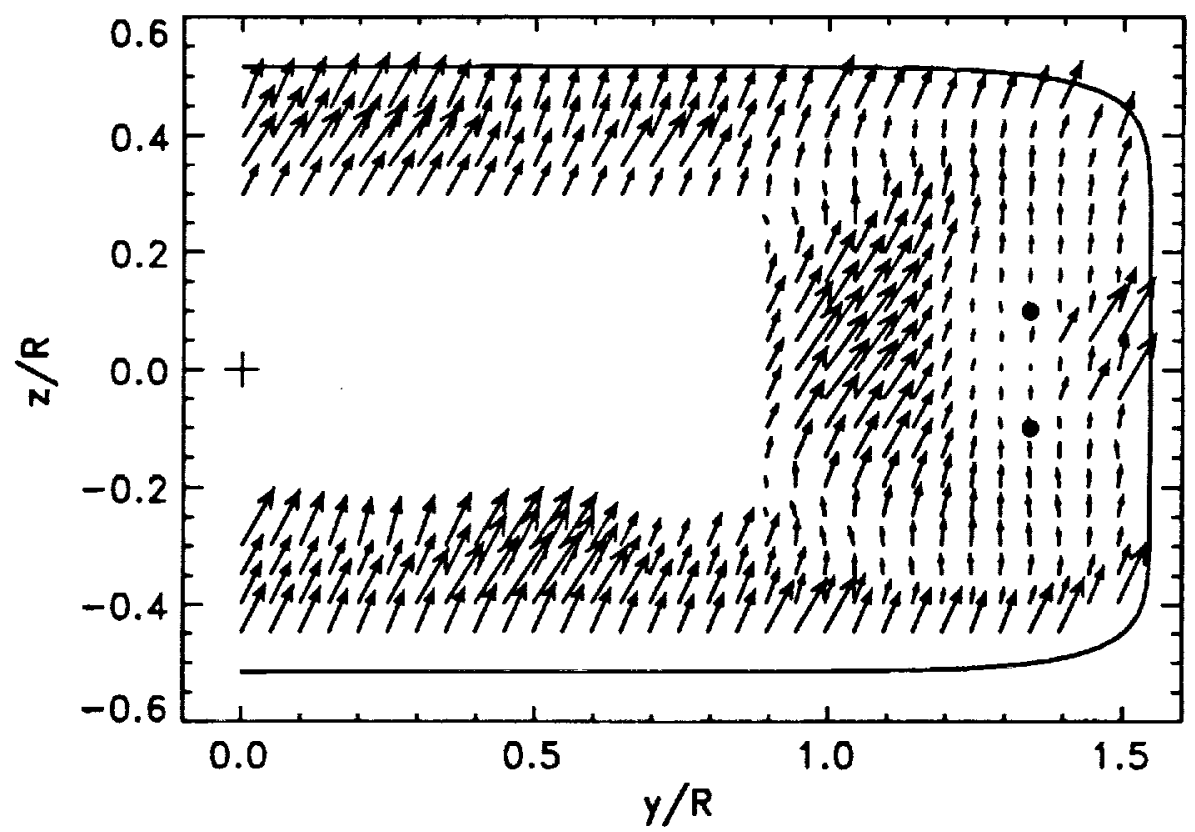

b) Location of data points on invariant map.

Fig. 5.44. Anisotropic stress tensor invariants at Station 6. 


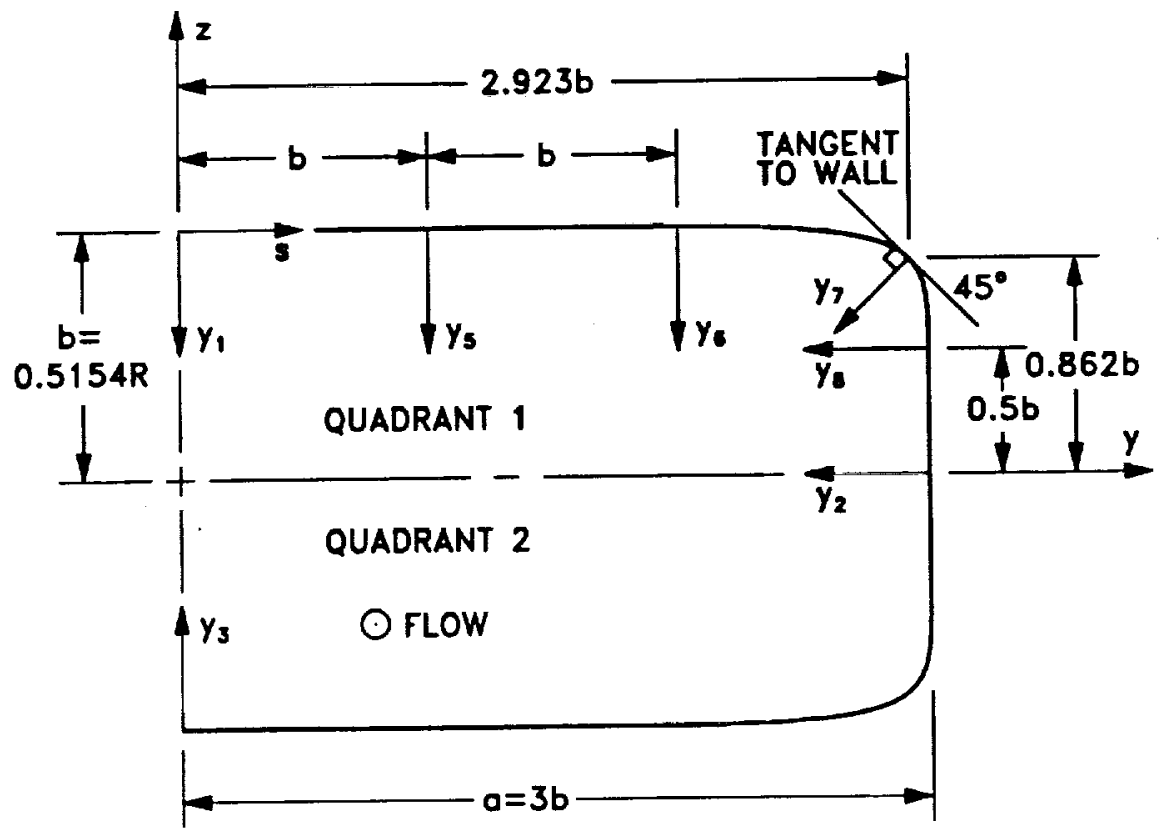

Fig. 5.45. Reference coordinates for wall function behavior.

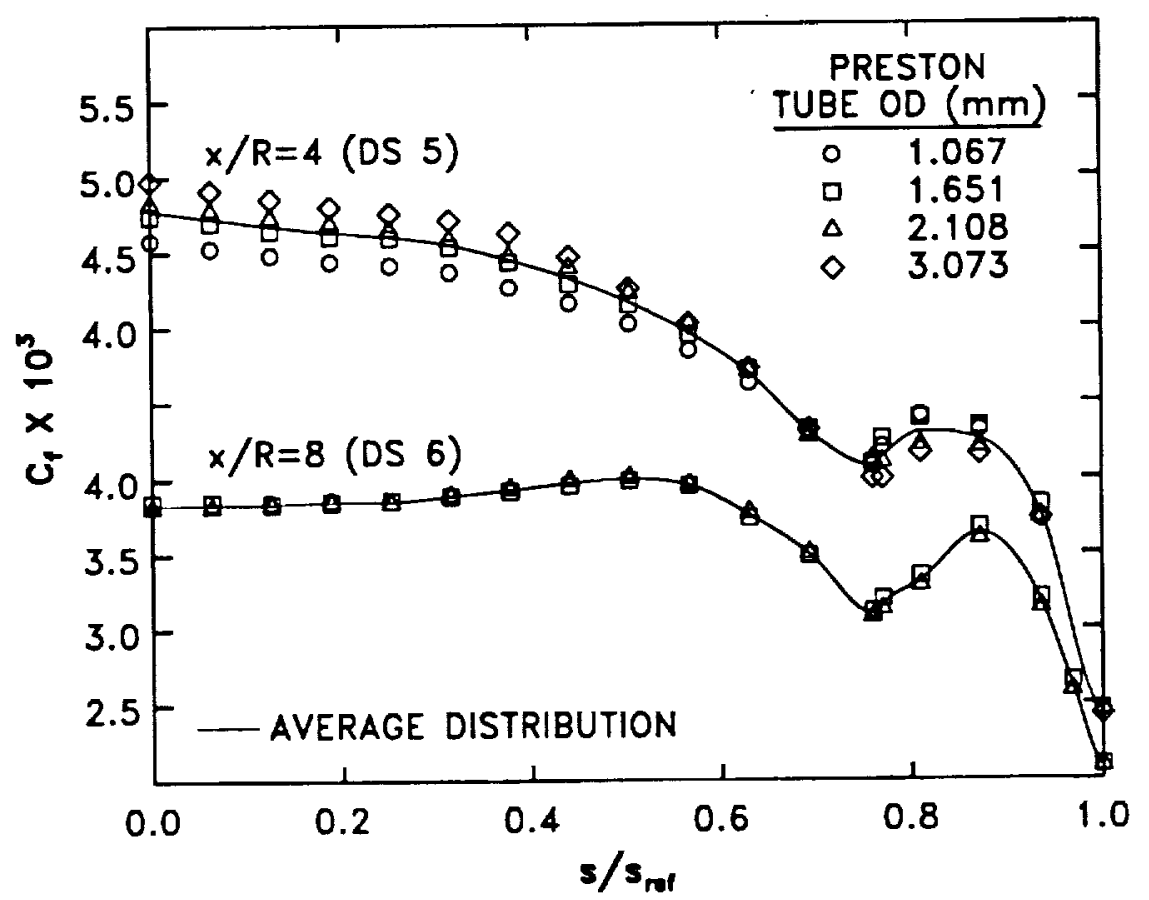

Fig. 5.46. Periperal skin friction coefficient distributions at Stations 5 and $6, \mathbf{R e}_{\mathrm{b}}=390,000$. 
118

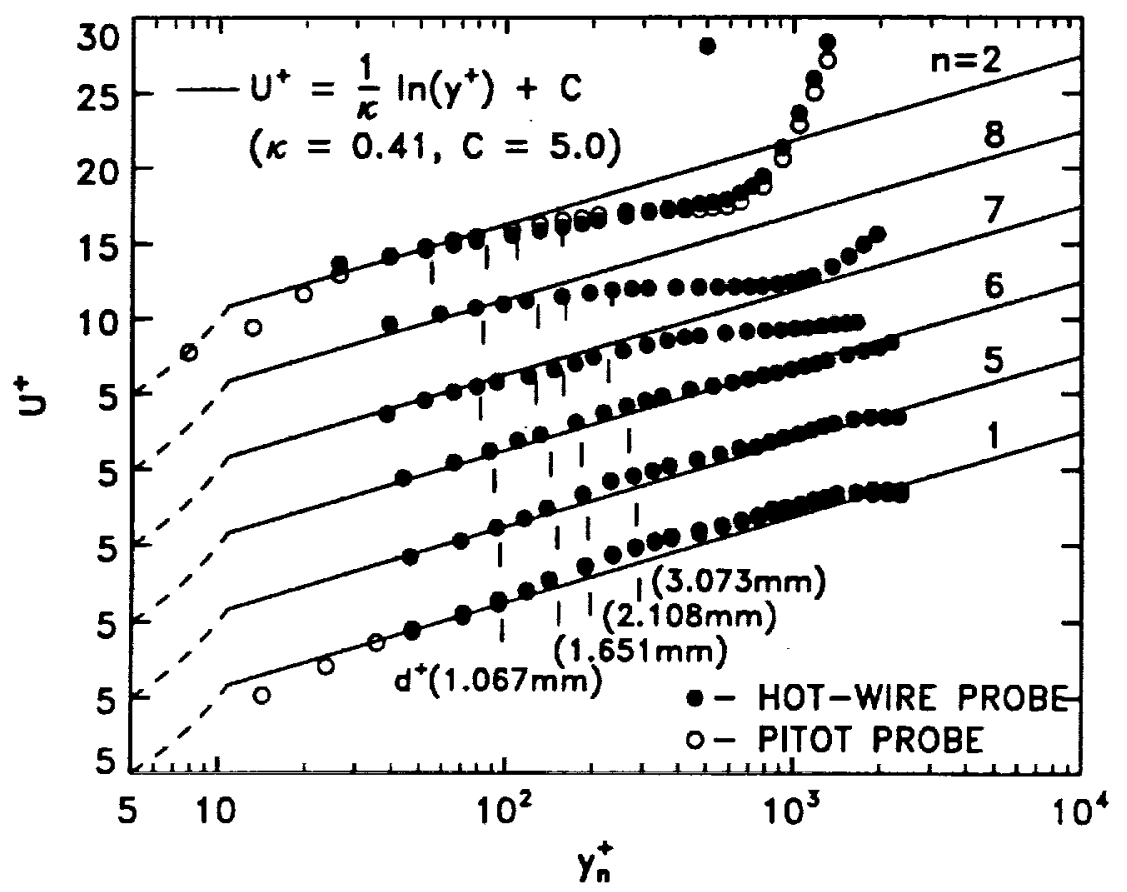

a) Station 5 .

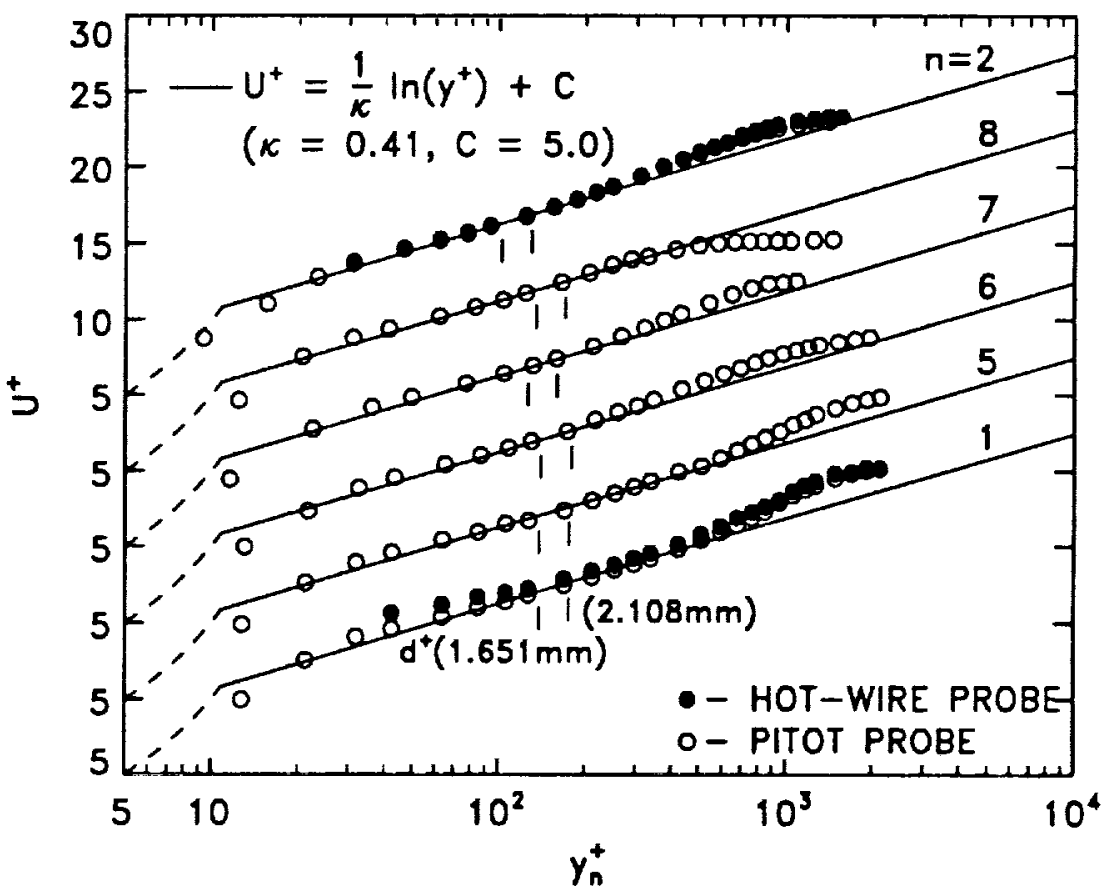

b) Station 6 .

Fig. 5.47. Law-of-the-wall velocity profiles at Stations 5 and $6, \mathbf{R e}_{b}=390,000$. 


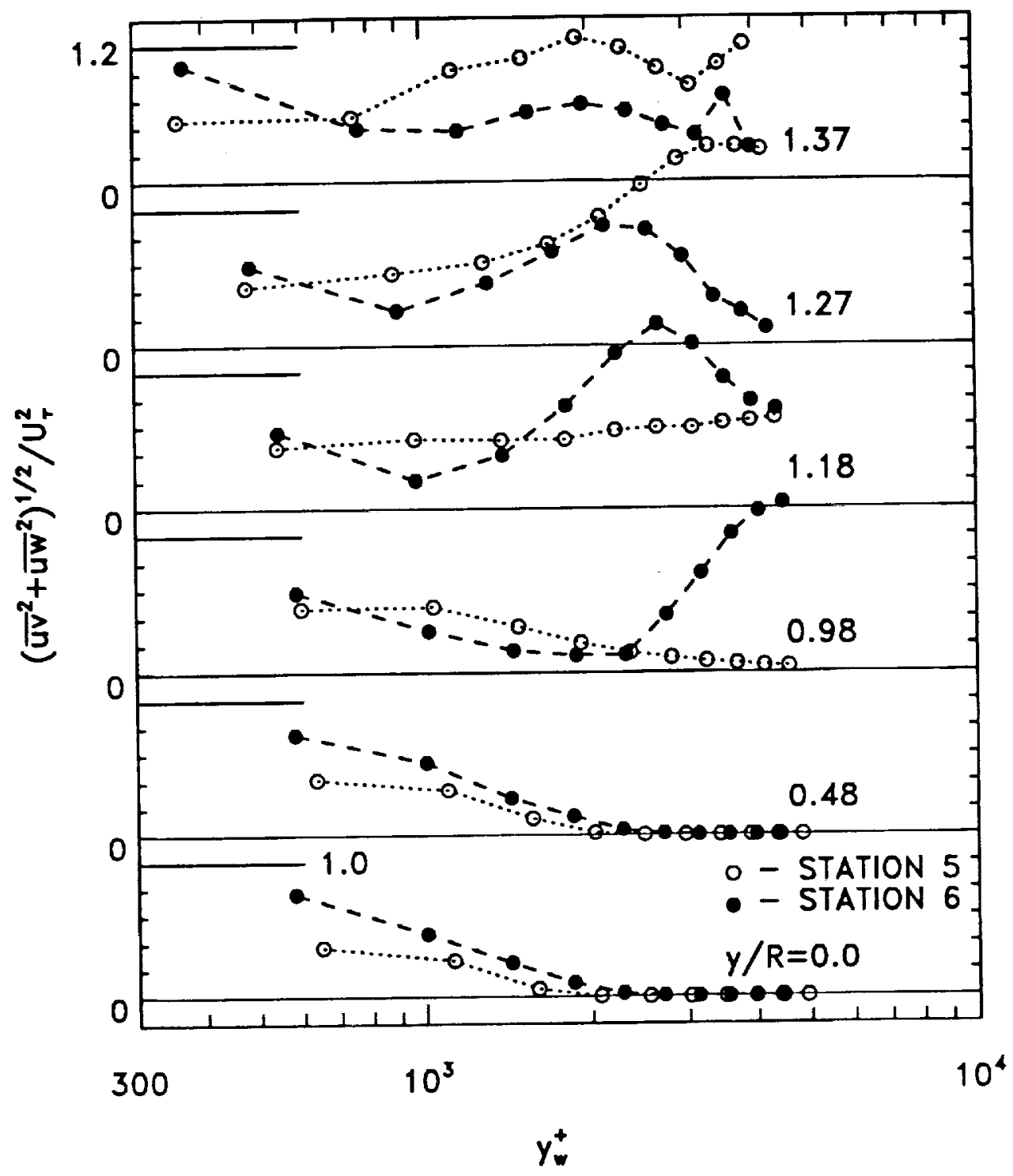

Fig. 5.48. $\left(\overline{\mathrm{uv}}^{2}+\overline{\mathrm{uw}}^{2}\right)^{1 / 2} / \mathrm{U}_{\tau}^{2}$ distributions parallel to semi-minor axis. 


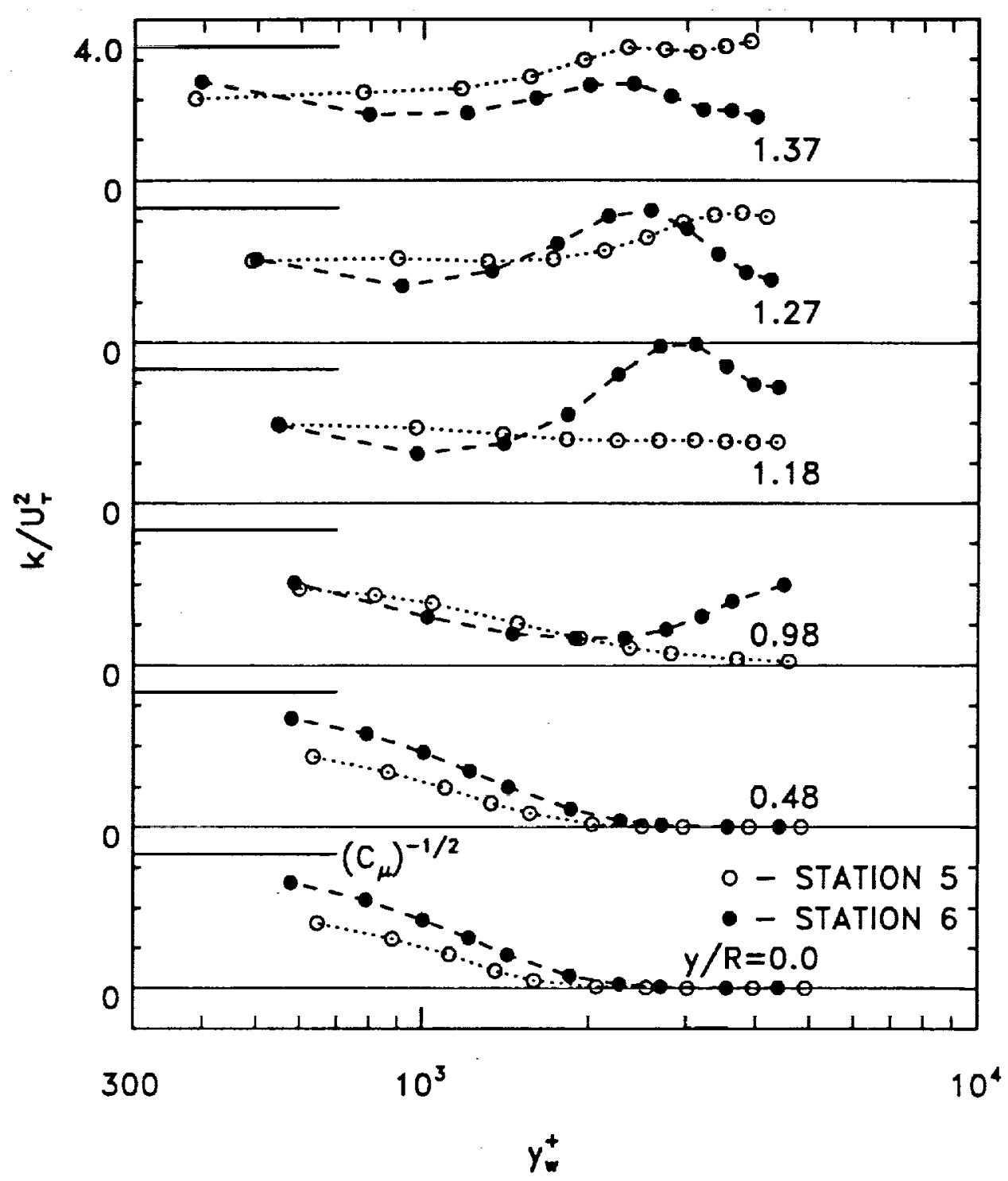

Fig. 5.49. $k / U_{\tau}^{2}$ distributions parallel to semi-minor axis. 


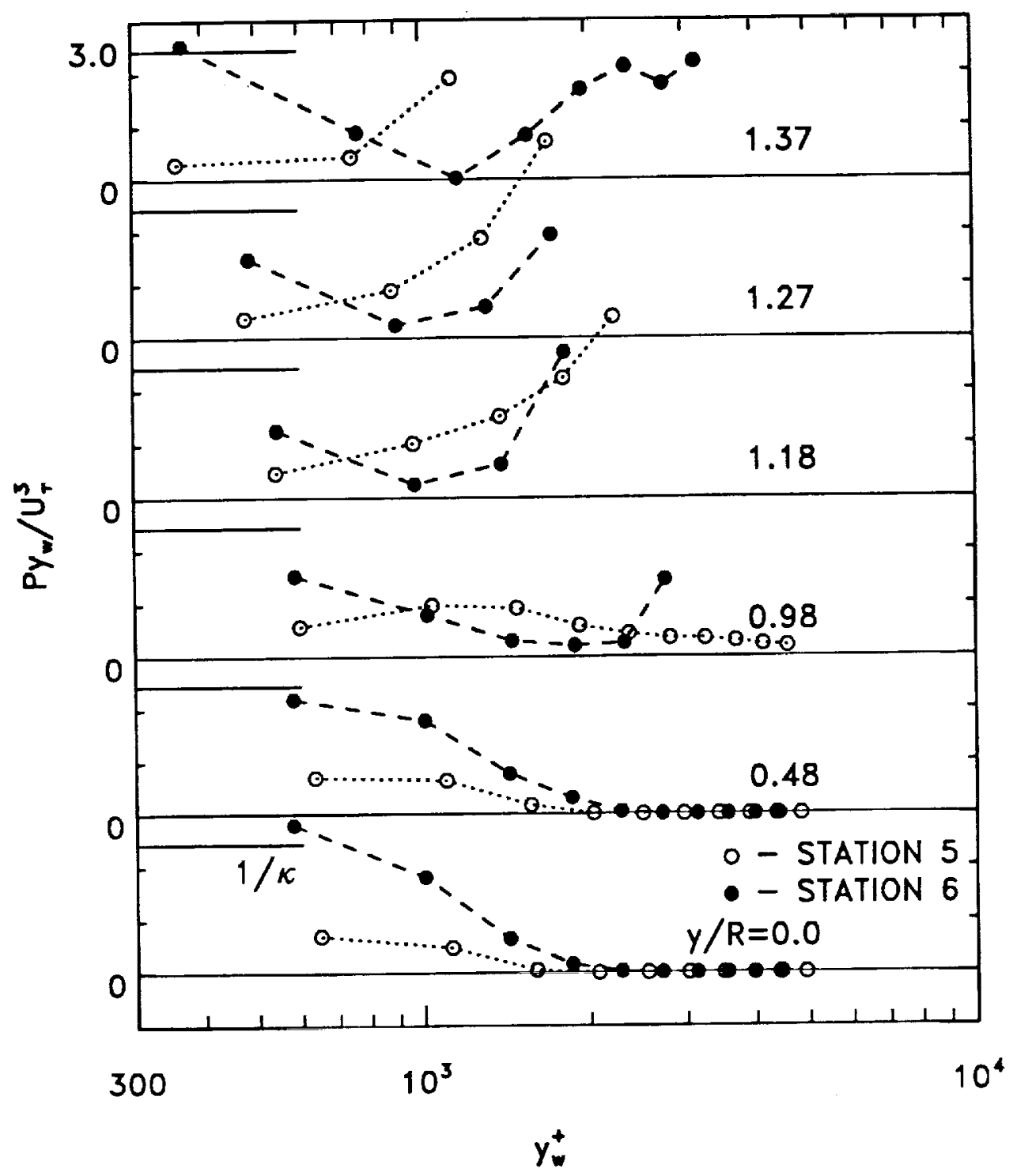

Fig. 5.50. $P y_{w} / U_{\tau}^{3}$ distributions parallel to semi-minor axis. 


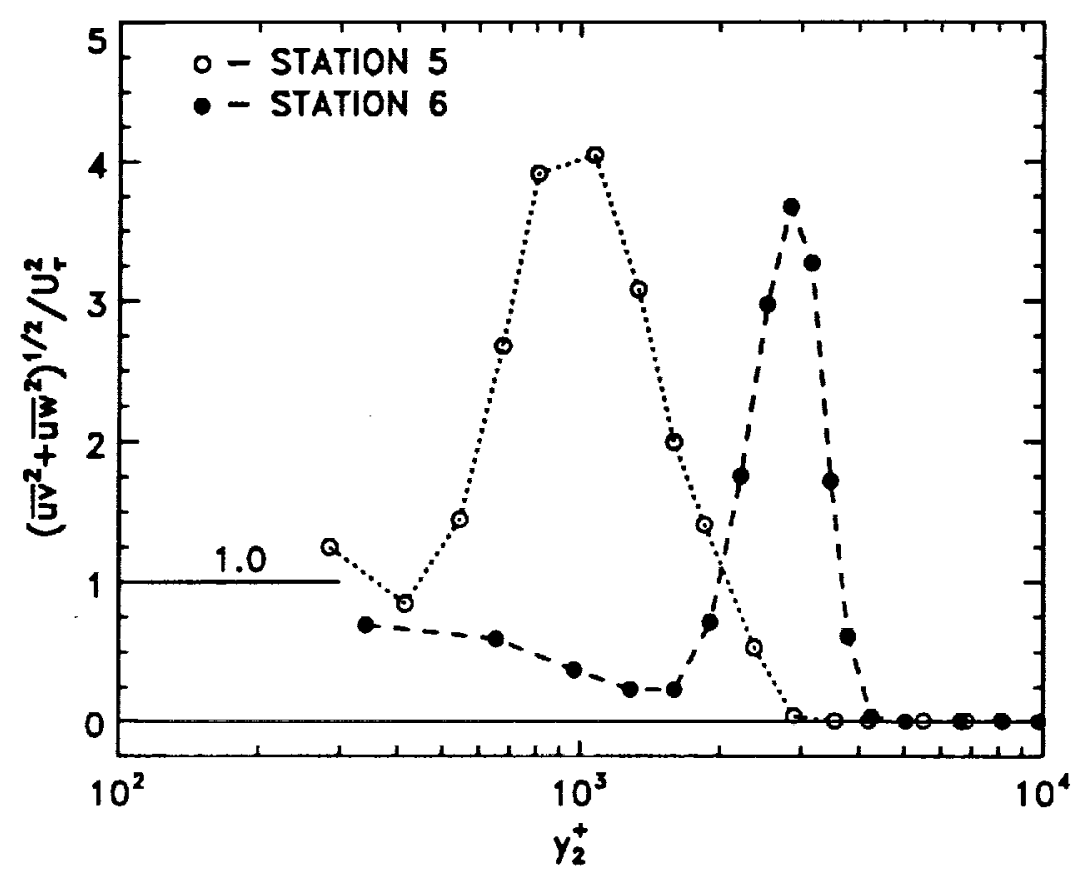

Fig. 5.51. $\left(\overline{u v}^{2}+\overline{u w}^{2}\right)^{1 / 2} / U_{\tau}^{2}$ distributions along semi-major axis.

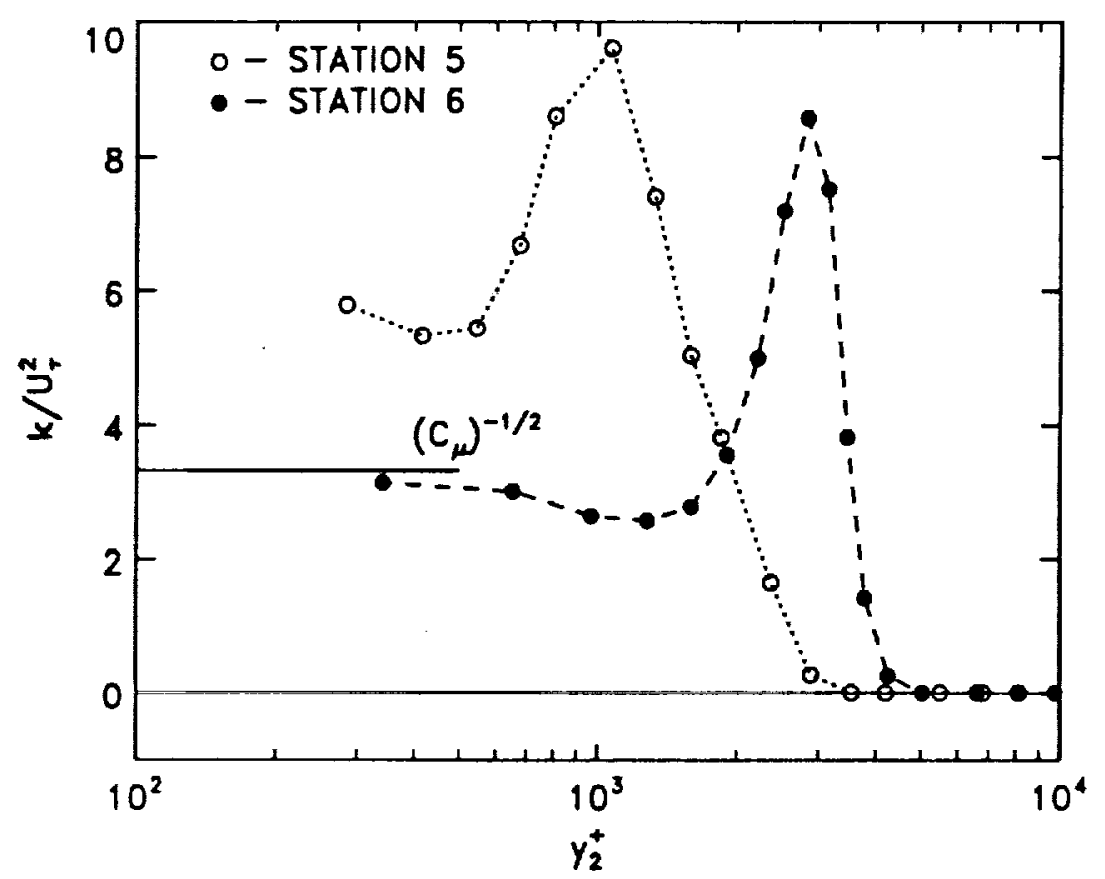

Fig. 5.52. $\mathrm{k} / \mathrm{U}_{\tau}^{2}$ distributions along semi-major axis. 


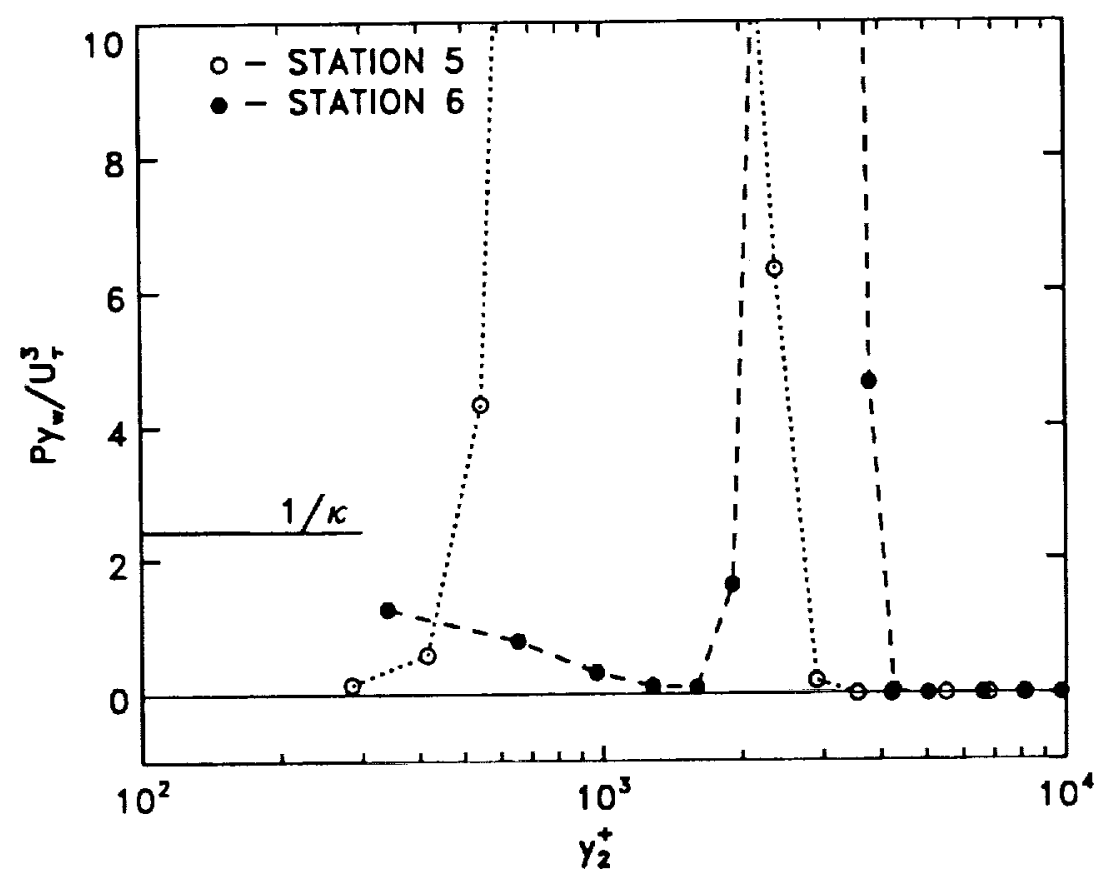

Fig. 5.53. $P y_{w} / U_{\tau}^{3}$ distributions along semi-major axis. 


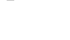




\section{CHAPTER 6}

\section{CONCLUSIONS AND RECOMMENDATIONS}

\subsection{Conclusions}

Incompressible, turbulent, swirl-free flow through a circular-to-rectangular transition duct has been studied experimentally. Mean flow measurements are presented at five axial stations and the complete Reynolds stress tensor is presented at three axial stations for operating bulk Reynolds numbers of 88,000 and 390,000 . No significant Reynolds number dependence was observed and excellent symmetry prevailed through the entire test section. The results of the study may be summarized as follows:

1) The inlet conditions to the duct, which correspond to partially developed turbulent pipe flow, proved to be an interesting case, in itself, considering the large differences between results of various studies on the subject. The present results showed that the boundary layer was significantly thinner than that reported by other investigators over nearly equivalent development lengths $(x / D \approx 9)$. Reynolds stress levels also differed. These discrepancies are generally attributable to inlet conditions at the pipe entrance. Excellent law-of-the-wall behavior was observed in the pipe and the skin friction was very near the fully-developed value.

2) The results of the transition duct measurements show that streamwise vorticity generated in the first half of transition strengthens and concentrates along the duct sidewall in the second half of the transition resulting in a contra-rotating vortex pair (common flow away from surface) centered about the duct semi-major axis. The upwash action between the vortices creates a region of low wall shear stress. The vortex pair significantly distorts the mean flow and turbulence fields.

3) In the transition section, transverse flow velocities of approximately $32 \%$ of the inlet bulk velocity were observed. In the constant area duct extension, the vortex pair causes a secondary flow to persist that peaks at about $12 \%$ of the inlet bulk velocity.

4) In the vicinity of the sidewall vortices, a large velocity deficit exists. The large primary rates-of-strain associated with this velocity deficit dominate much of the vortex region. These large strain rates produce a region of relatively high turbulence intensity.

5) In the vicinity of the vortex cores, nearly isotropic turbulence exists which is dramatically attenuated by lateral convergence effects. 
6) Along the horizontal walls at the end of transition, a peculiar trend in the primary turbulent shear stress was observed which is a result of surface curvature and lateral divergence effects. This anomaly was manifested in a dramatic reduction in the shear stress midway across the boundary layer as the wall is approached. The measurements did not, however, resolve the near-wall region to determine if the shear stress recovered in the inner layer.

7) At the end of transition, law-of-the-wall behavior indicates that the flow is in local equilibrium over only a very small region near the wall. Trends in turbulence-based wall function behavior at this station tend to support the local equilibrium assumption, but the present measurements do not fully resolve the near-wall region. At the end of the transition duct extension, local equilibrium is observed over a fairly large region near the wall.

The present results can serve as an experimental database for CFD code calibration/verification, inasmuch as the results demonstrate the appropriate symmetry and are based on well-defined inlet conditions. The results of this study should also provide a stringent test for turbulence models. To facilitate comparisons, the transition duct geometric variables are available in Appendix A. Tabulated flow data in machine readable form are available from the author.

\subsection{Recommendations}

Between the time that the present study was initiated and completed, a number of studies dealing with circular-to-rectangular transition ducts appeared in the literature. Most notable are those of Reichert et al. [4] and Miau et al. [9]. Despite these additions, there are still some areas which have not been fully addressed. First among these is the case with inlet swirl. Inlet swirl may be beneficial from the standpoint of reduced noise and thermal plume. Although Reichert has provided mean flow data at several axial stations in a CR transition duct, a large region in the vicinity of the sidewall was not accessible. Supplementary mean flow data in this region and turbulence measurements in the entire cross-plane will provide a more complete picture for the swirling case. Another area of interest is the case of laminar flow through a $\mathrm{CR}$ transition duct. This is of interest because laminar flow data are useful for code validation purposes. Having both laminar and turbulent data allows the code developer to distinguish between deficiencies in the numerical scheme and in the turbulence model. Finally, measurements in a different geometry transition duct may be useful. In particular, investigation of an identical duct to the one used in the present study, but without the area variation, may give further insight to the development of streamwise vortices, inasmuch as it has been observed that ducts which have no area expansion are devoid of vortices. Discrete vortices have been observed, however, in ducts which have an area expansion, for different aspect ratios and transition lengths. All of the above research extensions could be made in the 
present facility with minimal hardware change. The swirling case requires only the addition of turning vanes in the inlet pipe or, preferably, a swirl generator similar to the one proposed by Reichert which very nearly approximates a solidbody rotation inlet condition. For the laminar flow case, in addition to removing the boundary layer trip, a means for reducing the flow rate through the facility would be required to ensure that the flow remained laminar through the entire test section. This could be accomplished by providing a controllable bypass flow downstream of the test section. 


\section{REFERENCES}

1) Stevens, H.L., Thayer, E.B., and Fullerton, J.F., "Development of the MultiFunction 2-D/C-D Nozzle," AIAA Paper 81-1491, 1981.

2) Patrick, W.P., and McCormick, D.C., "Circular-to-Rectangular Duct Flows: A Benchmark Experimental Study," SAE Technical Paper 871776, 1987.

3) Patrick, W.P., and McCormick, D.C., "Laser Velocimeter and Total Pressure Measurements in Circular-to-Rectangular Transition Ducts," Report No. 87-41, United Technology Research Center, East Hartford CT, June 1988.

4) Reichert, B.A., Hingst, W.R. and Okiishi, T.H., "An Experimental Comparison of Nonswirling and Swirling Flow in a Circular-to-Rectangular Transition Duct," AIAA Paper 91-0342, 1991.

5) Mayer, E., "Effect of Transition in Cross-Sectional Shape on the Development of the Velocity and Pressure Distribution of the Turbulent Flow in Pipes," NACA TM903, 1939.

6) Taylor, A.M.K.P., Whitelaw, J.H., and Yianneskis, M., "Turbulent Flow in a Square-to-Round Transition," NASA Contractor Report CR-3447, July, 1981.

7) Burley II, J.R., and Carlson, J.R., "Circular-to-Rectangular Transition Ducts for High-Aspect Ratio Nonaxisymmetric Nozzles," AIAA Paper 851346, 1985.

8) Burley II, J.R., Bangert, L.S., and Carlson, J.R., "Investigation of Circularto-Rectangular Transition Ducts for High-Aspect Ratio Nonaxisymmetric Nozzles," NASA Technical Paper TP-2534, March, 1986.

9) Miau, J.J., Leu, T.S., Chou, J.H., Lin, S.A. and Lin, C.K., "Flow Distortion in a Circular-Rectangular Transition Duct," AIAA Journal, Vol.28, August 1990,pp.1447-1456.

10) Schwartz, I.R., "Minimization of Jet and Core Noise of a Turbojet Engine by Swirling the Flow," AIAA Paper 75-503, 1975.

11) Beer, J.M., and Chigier, M.A., Combustion Aerodynamics, Applied Science Publishers, London, 1972, pp. 100-146.

12) Der, J. Jr., Chu, C., Hunt, B.L. and Lorincz, D.J., "Evolution of Swirl in a Two-Dimensional Nozzle Flow," Journal of Aircraft, Vol.19, April 1982, pp.347-349. 
13) Chu, C., Der, J.Jr. and Wun, W., "A Simple 2D Nozzle Plume for IR Analysis," AIAA Paper 80-1808, Aug. 1980.

14) Chu, C. and Der, J.Jr., "Modelling of 2D Nozzle Plume for IR Signature Predictions Under Static Conditions," AIAA Paper 80-1808, Aug. 1980.

15) Sobota, T.H., and Marble, F.E, "Swirling Flows in an Annular-to-Rectangular Transition Section," Journal of Propulsion and Power, Vol.5, No.3, May-June 1989, pp.334-340.

16) Vu, T.C. and Shyy, W., "Navier-Stokes Flow Analysis for Hydraulic Turbine Draft Tubes," Journal of Fluids Engineering, Vol.12, June 1990,pp.199-204.

17) Bradshaw, P., "Effects of Streamline Curvature on Turbulent Flow," AGARDograph 169, 1973.

18) So, R.M.C., and Mellor, G.L., "Experiment on Convex Curvature Effects in Turbulent Boundary Layers," Journal of Fluid Mechanics, Vol.60, Part 1, 1973, pp. 43-62.

19) So, R.M.C., and Mellor, G.L., "Experiment on Turbulent Boundary Layers on a Concave Wall," Aeronautical Quarterly, Vol.26, 1975, pp. 35-50.

20) Meroney, R.N., and Bradshaw, P., "Turbulent Boundary Layer Growth over a Longitudinally Curved Surface," AIAA Journal, Vol.13, No.11, 1975, pp.1448-1453.

21) Hoffmann, P.H., Muck, K.C., and Bradshaw, P., "The Effect of Concave Surface Curvature on Turbulent Boundary Layers," Journal of Fluid Mechanics, Vol.161, 1985, pp. 371-403.

22) Moser R.D., and Moin, P., "The Effects of Curvature in Wall-Bounded Turbulent Flows," Journal of Fluid Mechanics, Vol.175, 1987, pp. 479-510.

23) Gibson, M.M, "Effects of Surface Curvature on the Law-of-the-Wall," NearWall Turbulence, Hemisphere Pub. Corp., New York, 1990, pp.157-171.

24) Hoffmann, P.H., and Bradshaw, P., "Turbulent Boundary Layers on Surfaces of Mild Longitudinal Curvature," Imperial College Aeronautics Report, 7804, 1978.

25) Gillis, J.C., and Johnston, J.P., "Turbulent Boundary Layer Flow and Structure on a Convex Wall and its Redevelopment on a Flat Wall," Journal of Fluid Mechanics, Vol.135, 1983, pp. 123-153.

26) Smits, A.J., Young, S.T.B, and Bradshaw, P., "The Effect of Short Regions of High Surface Curvature on Turbulent Boundary Layers," Journal of Fluid Mechanics, Vol.94, Part 2, 1979, pp. 209-242. 
27) Hunt, I.A., and Joubert, P.N., "Effects of Small Streamline Curvature on Turbulent Duct Flow," Journal of Fluid Mechanics, Vol.91, Part 4, 1979, pp. 633-659.

28) Muck, K.C., Hoffmann, P.H., and Bradshaw, P., "The Effect of Convex Surface Curvature on Turbulent Boundary Layers," Journal of Fluid Mechanics, Vol.161, 1985, pp. 347-369.

29) Shabaka, I.M.M.A, Mehta, R.D. and Bradshaw, P., "Longitudinal Vortices Imbedded in Turbulent Boundary Layers. Part 1. Single Vortex," Journal of Fluid Mechanics, Vol.155, 1985, pp.37-57.

30) Mehta, R.D. and Bradshaw, P., "Longitudinal Vortices Imbedded in Turbulent Boundary Layers. Part 2. Vortex Pair with 'Common Flow' Upwards," Journal of Fluid Mechanics, Vol.188, 1988, pp.529-546.

31) Pauley, W.R. and Eaton, J.K., "The Fluid Dynamics and Heat Transfer Effects of Streamwise Vortices Embedded in a Turbulent Boundary Layer," Standford University Thermosciences Report MD-51, 1988.

32) Pauley, W.R. and Eaton, J.K., "Boundary Layer Turbulence Structure in the Presence of Embedded Streamwise Vortices," Seventh Symposium on Turbulent Shear Flows, Stanford University, 1989.

33) Liandrat, J., Aupoix, B and Cousteix, "Calculation of Longitudinal Vortices Imbedded in a Turbulent Boundary Layer," Turbulent Shear Flows 5, Springer-Verlag, 1987, pp.253-258.

34) Bradshaw, P., "Turbulent Secondary Flows", Annual Review of Fluid Mechanics, Vol.19, 1987, pp.53-74.

35) Bansod, P. and Bradshaw, P., "The Flow in S-Shaped Ducts," Aeronautical Quarterly, Vol.23, Part 2, 1972, pp.131-140.

36) Taylor, A.M.K.P., Whitelae, J.H. and Yianneskis, M., "Developing Flow in S-Shaped Ducts, Part II - Circular Cross Section Duct," NASA CR 3759, Feb. 1984.

37) Smits, A.J., Eaton, J.A., and Bradshaw, P., "The Response of a Turbulent Boundary Layer to Lateral Divergence," Journal of Fluid Mechanics, Vol.94, Part 2, 1979, pp. 243-268.

38) Morel, T., " Comprehensive Design of Axisymmetric Wind Tunnel Contractions," Journal of Fluids Engineering, Vol.97, June 1975, pp.225-233.

39) Towne, C.E. and Hoffman, J.D, "Computation of Viscous Flow in Planar and Axisymmetric Ducts by an Implicit Marching Procedure," NASA TM 83567 (also AIAA Paper 84-0256), 1984. 
40) Eppich, H. M., "An Experimental Investigation of the Turbulence Structure in the Inner Region of a Three-Dimensional Streamwise Corner," M.S. Thesis, Dept. of Mech. Eng., University of Washington, 1982.

41) Chue, S.H., "Pressure Probes for Fluid Measurments," Progress in Aerospace Sciences, Vol. 16, No. 2, 1975, pp. 147-223.

42) Comte-Bellot, G., Strohl, A. and Alcaraz, E., "On Aerodynamic Disturbances Caused by Single Hot-Wire Probes," Journal of Applied Mechanics, Vol.38, 1971, pp. 767-774.

43) Head, M.R. and Vasanta Ram, V., "Simplified Presentation of Preston Tube Calibration," Aeronautical Quarterly, Vol. 22, Part 3, 1971, pp. 295-300.

44) Patel, V.C., "Calibration of the Preston Tube and Limitations on it's Use in Pressure Gradients," Journal of Fluid Mechanics, Vol. 23, 1965, pp. 185208.

45) Al-Beirutty, M. H., "Development of a Hot-Wire Measurement Technique for Moderate Intensity Three-Dimensional Flows," Ph.D. Dissertation, Dept. of Mech. Eng., University of Washington, 1987.

46) Al-Beirutty, M. H., Arterberry, S.H., and Gessner, F.B., "A Hot-Wire Technique for Complex Turbulent Flows," Proc. of the 1st National Fluid Dynamics Congress, Part 3, AIAA Publications, New York, 1988, pp 1479-1486.

47) Arterberry, S. H., "An Improved Method of Flow Field Determination in Three-Dimensional Flows of Low Intensity and Moderate Skewness," M.S. Thesis, Dept. of Mech. Eng., University of Washington, 1982.

48) Klein, A., "REVIEW: Turbulent Developing Pipe Flow," Journal of Fluids Engineering, Vol.103, June 1981, pp.243-249.

49) Barbin, A.R and Jones, J.B., "Turbulent Flow in the Inlet Region of a Smooth Pipe," Journal of Basic Engingeering, Vol.85, No.1, March 1963, pp.29-39.

50) Richman, J.W. and Azad, R.S.,"Developing Turbulent Flow in a Smooth Pipe," Applied Scientific Research, Vol.28, 1973, pp. 419-441.

51) Reichert, J.K. and Azad, R.S., "Nonasymptotic Behavior of Developing Turbulent Pipe Flow," Canadian Journal of Physics, Vol.54, 1976, pp. 268-278.

52) Martinuzzi, R., and Pollard, A., "Comparative Study of Turbulence Models in Predicting Turbulent Pipe Flow, Part I: Algebraic Stress and k- $\epsilon$ Models," AIAA Journal, Vol.27, Jan. 1989, pp. 29-36.

53) Pollard, A. and Martinuzzi, R., "Comparative Study of Turbulence Models in Predicting Turbulent Pipe Flow, Part II: Reynolds Stress and $k-\epsilon$ Models," AIAA Journal, Vol.27, Dec. 1989, pp. 1714-1721. 
54) Schlichting, H., Boundary-Layer Theory, 7th ed., McGraw-Hill, New York, 1979, pp.565-566.

55) Fritsch, F.N. and Carlson, R.E., "Piecewise Cubic Interpolation Methods," Lawerence Livermore Laboratory Report No. UCRL-81230, November, 1978.

56) Bradshaw, P, Ferris, D.H. and Atwell, N.P., "Calculation of Boundary Layer Development Using the Turbulent Energy Equation," Journal of Fluid Mechanics, Vol.28, 1967, pp.593-616.

57) Lumley, J.L. and Newman, G.R, "The Return to Isotropy of Homogeneous Turbulence," Journal of Fluid Mechanics, Vol.82, Part 1, 1977, pp.161-178.

58) Rotta, J.C. "Statistische theorie nichthomogener turbulenz," MitteilungZeitschrift für Physik, Vol.129, 1951, pp.547-572.

59) Gessner, F.B., Eppich, H.M. and Lund, E.G., "The Near-Wall Structure of Turbulent Flow Along a Streamwise Corner," Proc. of the 8th Symposium on Turbulent Shear Flows, Munich, Germany, Sept. 1991.

60) Beyer, W.H.' ed., CRC Standard Mathematical Tables, 25th ed., CRC Press Inc., 1978.

61) De Grande, G. and Kool, P., "An Improved Experimental Method to Determine the Complete Reynolds Stress Tensor with a Single Rotating Slanting Wire," Journal of Physics, Part E, Vol.14, 1981, pp.196-201.

62) Page, R. J., "An Experimental Study of Developing Turbulent Flow in a Square Duct," M.S. Thesis, Dept. of Mech. Eng., University of Washington, 1971.

63) Friehe, C.A, and Schwarz, W.H., "Deviations from the the Cosine Law for Yawed Cylindrical Anemometer Sensors," Journal of Applied Mechanics, Vol.35, No.4, Dec. 1968, pp.655-662.

64) Lawn, C.J., "The Determination of the Rate of Dissipation in Turbulent Pipe Flow," Journal of Fluid Mechanics, Vol.48, Part 3, 1971, pp.477-505. 



\section{APPENDIX A}

\section{TRANSITION DUCT GEOMETRY}

At any given streamwise station, the cross section of the transition duct can be described by the equation of a superellipse, namely:

$$
(y / a)^{\eta}+(z / b)^{\eta}=1
$$

where $a$ and $b$ are the semi-major and semi-minor axes, respectively, of the superellipse. Special cases of the superellipse include a circle $(a=b, \eta=2)$, an ellipse $(a \neq b, \eta=2)$ and a rectangle $(a \neq b, \eta=\infty)$. The cross-sectional area at a given streamwise location is given by [60]:

$$
A_{c s}=\frac{\Gamma(1 / \eta)^{2}}{\Gamma(1 / \eta)}(2 / \eta)(4 a b)
$$

where $\Gamma$ refers to the "gamma function", defined as:

$$
\Gamma(\eta)=\int_{0}^{\infty}\left(e^{-t} t^{\eta-1}\right) d t \quad(\eta>0)
$$

The design procedure for the transition duct is to specify the streamwise variation of the semi-major and semi-minor axes (which define the side and upper (lower) walls of the superellipse) and the streamwise variation, if any, of the cross-sectional area. Once $a(x), b(x)$ and $A_{c s}(x)$ are known, equation $(A .2)$ is then solved numerically to determine the streamwise variation of $\eta$.

For the transition duct used in this study, the variation of the semi-major and semi-minor axes are specified by fifth-order polynomial functions of $x / R$. These functions provide zero derivatives up to the second order at the beginning and end of transition. The aspect ratio $(A R)$ and the length ratio $(L / D)$ for the present duct are 3 and 1.5 , respectively. The cross-sectional area begins to increase at the start of transition to a maximum of $15 \%$ above the inlet cross-sectional area, occurring approximately at the mid-point of transition. From there the area smoothly decreases until the end of transition where the cross-sectional area is equal to the inlet cross-sectional area. After solving equation (A.2), the variation of $\eta$ is then approximated by a fifth-order polynomial function. Table A.1 contains the polynomial coefficients for the variation of $a / R, b / R$ and $\eta$ for the transition duct. Table A.2 contains tabulated values of $a / R, b / R, \eta, A_{c s}$ and the aspect ratio $A R=a / b$ along the transition duct. Also indicated in Table A.2 are the locations of the six data stations used in the present study. 
Table A.1. Transition Duct Polynomial Coefficients

\begin{tabular}{|c|c|c|c|}
\hline & $a / R$ & $b / R$ & $\eta$ \\
\hline & \multicolumn{3}{|c|}{$x / R \leq 1.0$} \\
\hline$C_{0}$ & 1.000000000 & 1.000000000 & 2.000000000 \\
\hline$C_{1}$ & 0.000000000 & 0.000000000 & 0.000000000 \\
\hline$C_{2}$ & 0.000000000 & 0.000000000 & 0.000000000 \\
\hline$C_{3}$ & 0.000000000 & 0.000000000 & 0.000000000 \\
\hline$C_{4}$ & 0.000000000 & 0.000000000 & 0.000000000 \\
\hline \multirow[t]{2}{*}{$C_{5}$} & 0.000000000 & 0.000000000 & 0.000000000 \\
\hline & \multicolumn{3}{|c|}{$1.0 \leq x / R \leq 1.96$} \\
\hline$C_{0}$ & 0.87568217 & 1.1102199 & 8.4833465 \\
\hline$C_{1}$ & 0.38699868 & -0.34310201 & -21.329880 \\
\hline$C_{2}$ & -0.38783144 & 0.34380721 & 26.148548 \\
\hline$C_{3}$ & 0.096543991 & -0.085532496 & -15.173079 \\
\hline$C_{4}$ & 0.041969148 & -0.037245857 & 4.3614143 \\
\hline \multirow[t]{2}{*}{$C_{5}$} & -0.013362557 & 0.011853259 & -0.49034940 \\
\hline & \multicolumn{3}{|c|}{$1.96 \leq x / R \leq 2.92$} \\
\hline$C_{0}$ & -0.37336560 & 2.2162779 & 1.0971109 \\
\hline$C_{1}$ & 3.3510193 & -2.9683112 & 2.3623579 \\
\hline$C_{2}$ & -3.2796590 & 2.9056348 & -2.8785242 \\
\hline$C_{3}$ & 1.5523217 & -1.3754753 & 2.0620912 \\
\hline$C_{4}$ & -0.33664930 & 0.29831302 & -0.64136301 \\
\hline \multirow[t]{2}{*}{$C_{5}$} & 0.027262234 & -0.024157751 & 0.080685615 \\
\hline & \multicolumn{3}{|c|}{$2.92 \leq x / R \leq 4.125$} \\
\hline$C_{0}$ & 14.292266 & -10.787227 & -1858.4412 \\
\hline$C_{1}$ & -19.643522 & 17.419488 & 2828.7353 \\
\hline$C_{2}$ & 11.204615 & -9.9360792 & -1704.8063 \\
\hline$C_{3}$ & -3.0380988 & 2.6941403 & 508.59846 \\
\hline$C_{4}$ & 0.39689283 & -0.35195770 & -74.960370 \\
\hline \multirow[t]{2}{*}{$C_{5}$} & -0.020128978 & 0.017849940 & 4.3651652 \\
\hline & \multicolumn{3}{|c|}{$x / R \geq 4.125$} \\
\hline$C_{0}$ & 1.5463591 & 0.51544988 & 10.000000 \\
\hline$C_{1}$ & 0.0000000 & 0.00000000 & 0.0000000 \\
\hline$C_{2}$ & 0.0000000 & 0.00000000 & 0.0000000 \\
\hline$C_{3}$ & 0.0000000 & 0.00000000 & 0.0000000 \\
\hline$C_{4}$ & 0.0000000 & 0.00000000 & 0.0000000 \\
\hline$C_{5}$ & 0.0000000 & 0.00000000 & 0.0000000 \\
\hline
\end{tabular}


Table A.2. Transition Duct Geometry Variables

\begin{tabular}{|c|c|c|c|c|c|c|}
\hline $\begin{array}{l}\text { Data } \\
\text { Station }\end{array}$ & $x / R$ & $a / R$ & $b / R$ & $\eta$ & $A R$ & $A_{c s} / A_{c s, i}$ \\
\hline 1 & $\begin{array}{c}-1.00 \\
\Downarrow\end{array}$ & $\begin{array}{c}1.0000 \\
\Downarrow\end{array}$ & $\begin{array}{c}1.0000 \\
\Downarrow\end{array}$ & $\begin{array}{c}2.0000 \\
\Downarrow\end{array}$ & $\begin{array}{c}1.0000 \\
\Downarrow\end{array}$ & $\begin{array}{c}1.0000 \\
\Downarrow\end{array}$ \\
\hline \multirow[t]{10}{*}{2} & 1.00 & 1.0000 & 1.0000 & 2.0000 & 1.0000 & 1.0000 \\
\hline & 1.12 & 1.0003 & 0.9997 & 2.1047 & 1.0006 & 1.0192 \\
\hline & 1.24 & 1.0025 & 0.9978 & 2.2154 & 1.0047 & 1.0376 \\
\hline & 1.36 & 1.0078 & 0.9831 & 2.3331 & 1.0251 & 1.0555 \\
\hline & 1.48 & 1.0174 & 0.9746 & 2.4588 & 1.0439 & 1.0729 \\
\hline & 1.60 & 1.0316 & 0.9719 & 2.5934 & 1.0614 & 1.0897 \\
\hline & 1.72 & 1.0509 & 0.9548 & 2.7383 & 1.1006 & 1.1056 \\
\hline & 1.84 & 1.0752 & 0.9333 & 2.8947 & 1.1520 & 1.1198 \\
\hline & 1.96 & 1.1041 & 0.9177 & 3.0645 & 1.2031 & 1.1319 \\
\hline & 2.08 & 1.1371 & 0.8784 & 3.2497 & 1.2945 & 1.1410 \\
\hline \multirow[t]{5}{*}{3} & 2.20 & 1.1734 & 0.8462 & 3.4528 & 1.3867 & 1.1464 \\
\hline & 2.32 & 1.2123 & 0.8117 & 3.6770 & 1.4935 & 1.1475 \\
\hline & 2.44 & 1.2527 & 0.7759 & 3.9261 & 1.6145 & 1.1441 \\
\hline & 2.56 & 1.2936 & 0.7396 & 4.2050 & 1.7491 & 1.1362 \\
\hline & 2.68 & 1.3341 & 0.7037 & 4.5199 & 1.8958 & 1.1241 \\
\hline \multirow[t]{10}{*}{4} & 2.80 & 1.3729 & 0.6693 & 4.8791 & 2.0512 & 1.1087 \\
\hline & 2.92 & 1.4093 & 0.6370 & 5.2932 & 2.2124 & 1.0911 \\
\hline & 3.04 & 1.4423 & 0.6078 & 5.7775 & 2.3730 & 1.0725 \\
\hline & 3.16 & 1.4712 & 0.5821 & 6.3524 & 2.5274 & 1.0544 \\
\hline & 3.28 & 1.4954 & 0.5606 & 7.0484 & 2.6675 & 1.0381 \\
\hline & 3.40 & 1.5147 & 0.5435 & 7.9106 & 2.7869 & 1.0248 \\
\hline & 3.52 & 1.5290 & 0.5308 & 9.0131 & 2.8806 & 1.0152 \\
\hline & 3.64 & 1.5385 & 0.5224 & 10.000 & 2.9451 & 1.0083 \\
\hline & 3.76 & 1.5439 & 0.5176 & 10.000 & 2.9828 & 1.0026 \\
\hline & 3.88 & 1.5460 & 0.5157 & 10.000 & 2.9979 & 1.0003 \\
\hline \multirow[t]{2}{*}{5} & 4.00 & 1.5464 & 0.5154 & 10.000 & 3.0000 & 1.0000 \\
\hline & $\Downarrow$ & $\Downarrow$ & $\Downarrow$ & $\Downarrow$ & $\Downarrow$ & $\Downarrow$ \\
\hline 6 & 8.00 & 1.5464 & 0.5154 & 10.000 & 3.0000 & 1.0000 \\
\hline
\end{tabular}




\section{•}




\section{APPENDIX B}

\section{HOT-WIRE DATA ACQUISITION SYSTEM}

An all new integrated hot-wire data acquisition system was acquired for the present study. This system differs from previous ones used within the M.E. department in that, rather than operating directly on the analog anemometer output, data reduction operations are performed on a digital representation of the analog signal. This appendix contains a description of the system and an overview of some of the more important operating features.

\section{B.1 Hardware}

The primary components of the system are a Thermal Systems Incorporated (TSI) IFA 100 Intelligent Flow Analyzer, a TSI IFA 200 analog-to-digital (A/D) converter and a Digital Equipment Corporation (DEC) VAXLab/VSII Scientific Workstation computer. A block schematic of this equipment is illustrated in Fig. B.1.

The IFA 100 used in this study is a two-channel unit consisting of a Model 158 slave cabinet housing two Model 150 constant temperature anemometers and two Model 157 signal conditioners. The signal conditioners contain a low-pass filter, a high-pass filter and an amplifier capable of applying a DC offset (when the high-pass filter is in the DC mode) and gain to the input (anemometer output) signal. The usable range of the Model 158 digital display and the input voltage range of the IFA200 digitizer is \pm 5 volts. Most wire and film sensors will have a voltage span much less than this. To utilize as much of this range as possible, the transducer signal must be offset and amplified.

The IFA 200 provides high-speed simultaneous A/D conversions of one or more anemometer output signals. Selectable A/D conversions rates of up to $50,000 \mathrm{~Hz}$ per channel provide the capacity of collecting data on rapidly changing signals. The computer controls the IFA 200 by sending ASCII characters through an RS-232 serial communication link. The parameters that are controlled through this link are the starting address (channel to be sampled first), the number of active channels and the sampling rate. For each sample, the $A / D$ converter generates a 16-bit data word. The low 12-bits contain the binary representation of the transducer voltage. The high 4-bits contain the digitizer channel address which must be stripped from the 16-bit data word before converting the binary number to a hexadecimal voltage. The 16-bit data word is loaded into a 64word-deep first-in, first-out (FIFO) memory. The data that enter the FIFO pass

to the FIFO output and are sent via a DMA (Direct Memory Access) interface cable to a DRQ3B DMA controller card in the computer. 
The DRQ3B Parallel DMA I/O module is a high-speed memory interface that allows real-time collection of 16-bit parallel digital data at transfer rates of up to $1.3 \mathrm{megaHz}$. The DRQ3B has a unique feature, referred to as double buffering, that can significantly increase data transfer rates as compared to conventional single buffer DMA transfers. With single buffer DMA, when a buffer fills, it is dequeued, but requires a software call to enqueue the next buffer. During the time between dequeueing the previous buffer and enqueueing the next buffer, no data are transferred. This time between buffers must not exceed the time it takes to fill the 64-word-deep FIFO memory in the IFA200, or data will be lost. To improve this situation, the DRQ3B allows two or more buffers to be enqueued before any data are transferred. Now, when a buffer fills, the DRQ3B automatically begins filling the next buffer without any software intervention. The result is less down time between buffers, enabling higher transfer rates.

\section{B.2 Buffer-Swinging}

Transferring data at high sampling rates can quickly exceed the available memory in the computer. To avoid this, the digitized data are forwarded directly to the 71 megabyte hard disk in the computer by means of the buffer-swinging method of data acquisition. This feature is used to capture a continuous stream of data and operates in the following manner. Two or more memory buffers are enqueued to the DRQ3B prior to any data transfer. When the last buffer is enqueued, data transfer begins to the first buffer. When the first buffer fills, the DRQ3B automatically begins filling the second buffer. While the second buffer is filling, the data in the first buffer are written directly to the hard disk. When the second buffer fills and first buffer's data have been transferred to disk, the process repeats as the second buffer's data are sent to disk and the first buffer again receives data.

\section{B.3 Handshaking}

Synchronization of data transfer between the IFA 200 and the DRQ3B controller card is accomplished by an interlocked two-wire handshake. When properly implemented, the handshake prevents data overrun and underrun conditions from occurring. The FUNCT OUT 0, STROBE and ACK pins on the DRQ3B input port are connected, via the DMA Interface Cable, to the READY, CYCLE REQUEST and BUSY pins, respectively, on the IFA 200 output port. $\dagger$ For data

$\dagger$ At the time the data acquisition equipment was acquired, TSI did not support DMA transfers from the IFA 200 to the DRQ3B controller card. To accomplish this, the DMA cable had to be modified for compatibility with the DRQ3B. The pin-to-pin connections between the J1 and J2 output connectors on the IFA 200 DMA Interface Cable and the J2 input port on the DRQ3B DMA card are listed in Table B.1. 
transfer to begin, the computer asserts the FUNCT OUT 0 pin on the DRQ3B high. When the IFA 200 senses this on the READY line, it turns on the sampling strobe, begins filling its 64-word-deep FIFO memory and asserts the CYCLE REQUEST line low, indicating to the DRQ3B that valid data are available. Until the last buffer is enqueued on the DRQ3B by the computer, the DRQ3B asserts the ACK signal low, indicating that it is not ready to receive data. When the last buffer is enqueued, the DRQ3B releases the ACK line allowing it to go high, indicating that it is now ready to receive data.

Once the digitizer has been activated and the DMA buffers have been enqueued, the following sequence of events occur for data transfer:

1. When the DRQ3B receives STROBE low from the CYCLE REQUEST line of the IFA 200, it reads the data and places the value in its 512-word-deep FIFO memory. The DRQ3B then asserts the ACK signal low, indicating to the IFA 200 that it has received the data value.

2. When the IFA 200 receives BUSY low from the ACK line of the DRQ3B, it sets the CYCLE REQUEST line high, indicating to the DRQ3B that valid data are not available, and prepares to transmit the next data word.

3. When the DRQ3B receives STROBE high from the CYCLE REQUEST line of the IFA 200, it releases the ACK line allowing it to go high (unless the DRQ3B FIFO is full, in which case ACK is held low until a word is read out of FIFO and into memory). ACK high indicates that the DRQ3B is ready for the next word.

4. When the IFA 200 receives BUSY high from the ACK line of the DRQ3B, it places valid data on the data lines and asserts CYCLE REQUEST low, starting the cycle over.

\section{B.4 Software}

Control of the DRQ3B is accomplished through the use of DEC's VAXlab subroutine library. This software package provides useful programs for all aspects of the experimental study, including, real-time data acquisition, signal processing, plotting and data analysis. The subroutines are called from user supplied main programs written in the FORTRAN language. Control of the IFA200 is accomplished by sending ASCII characters, via an RS-232 serial communication link, by means of the FORTRAN WRITE statement.

\section{B.5 Digital Sampling}

The hot-wire techniques employed in the present study (see Appendix C) rely on an accurate evaluation of the mean and variance $\left(\bar{E}\right.$ and $\left.\overline{e^{2}}\right)$ of the analog output signal from the hot-wire anemometer. The usual assumption that the velocity, and therefore the anemometer output voltage, is an ergodic random variable is made here. If the instantaneous voltage is decomposed into a mean and 
fluctuating component, $E=\bar{E}+e$, the true temporal average can be estimated by a finite average of discrete samples, provided that a sufficiently large number of samples $N$ are taken:

$$
\begin{aligned}
& \bar{E}=\lim _{T \rightarrow \infty} \frac{1}{T} \int_{t_{0}}^{t_{0}+T} E(t) d t \approx \frac{1}{N} \sum_{n=1}^{N} E_{n}(n \Delta t) \\
& \overline{e^{2}}=\lim _{T \rightarrow \infty} \frac{1}{T} \int_{t_{0}}^{t_{0}+T}(E(t)-\bar{E})^{2} d t \approx \frac{1}{N} \sum_{n=1}^{N}\left(E_{n}(n \Delta t)-\bar{E}\right)^{2}
\end{aligned}
$$

where $E_{n}$ are the individual digitized samples, $T$ is the total sampling time and $\Delta t$ is the time increment between samples. For a fixed sampling time duration $\mathrm{T}$, the number of samples is $N=T / \Delta t$. Since only single statistical quantities are required for the hot-wire measurements, it was not necessary to "capture" the waveform by digitizing at very high sampling rates. For the turbulence measurements, the sampling rate and duration time were set at $10 \mathrm{kHz}$ and 15 seconds, respectively, which yields a total of 150,000 samples for each data point. The convergence of the mean and mean-square values was monitored for each data point taken and it was observed that the above transfer rate and sampling time were more than adequate for all regions of the transition duct flowfield. 
Table B.1. DMA Interface Cable Pin Connections

\begin{tabular}{|c|c|c|c|c|c|}
\hline & IFA 200 & & & DRQ3B & \\
\hline $\begin{array}{l}\text { Signal } \\
\text { Name } \\
\end{array}$ & $\begin{array}{l}\text { Port } \\
\text { No. }\end{array}$ & $\begin{array}{l}\text { Pin } \\
\text { No. }\end{array}$ & $\begin{array}{l}\text { Pin } \\
\text { No. }\end{array}$ & $\begin{array}{l}\text { Port } \\
\text { No. }\end{array}$ & $\begin{array}{l}\text { Signal } \\
\text { Name } \\
\end{array}$ \\
\hline$\overline{\text { CYCLE REQUEST }}$ & $\mathrm{J} 1$ & 02 & 17 & $\mathrm{~J} 2$ & STROBE \\
\hline READY & $\mathrm{J} 1$ & 06 & 22 & $\Downarrow$ & FUNCT OUT 0 \\
\hline GND & $\mathrm{J} 2$ & 01 & 42 & & GND \\
\hline BUSY & $\Downarrow$ & 02 & 18 & & ACK \\
\hline GND & & 03 & 41 & & GND \\
\hline GND & & 04 & 40 & & GND \\
\hline GND & & 07 & 39 & & GND \\
\hline GND & & 11 & 38 & & GND \\
\hline GND & & 12 & 37 & & GND \\
\hline GND & & 13 & 36 & & GND \\
\hline GND & & 15 & 35 & & GND \\
\hline GND & & 17 & 34 & & GND \\
\hline GND & & 19 & 32 & & GND \\
\hline GND & & 20 & 31 & & GND \\
\hline GND & & 21 & 29 & & GND \\
\hline GND & & 22 & 28 & & GND \\
\hline GND & & 23 & 27 & & GND \\
\hline GND & & 24 & 26 & & GND \\
\hline DATA OUT 00 & & 39 & 01 & & DATA IN 00 \\
\hline DATA OUT 01 & & 37 & 02 & & DATA IN 01 \\
\hline DATA OUT 02 & & 35 & 03 & & DATA IN 02 \\
\hline DATA OUT 03 & & 33 & 04 & & DATA IN 03 \\
\hline DATA OUT 04 & & 31 & 05 & & DATA IN 04 \\
\hline DATA OUT 05 & & 29 & 06 & & DATA IN 05 \\
\hline DATA OUT 06 & & 27 & 07 & & DATA IN 06 \\
\hline DATA OUT 07 & & 25 & 08 & & DATA IN 07 \\
\hline DATA OUT 08 & & 26 & 09 & & DATA IN 08 \\
\hline DATA OUT 09 & & 28 & 10 & & DATA IN 09 \\
\hline DATA OUT 10 & & 30 & 11 & & DATA IN 10 \\
\hline DATA OUT 11 & & 32 & 12 & & DATA IN 11 \\
\hline DATA OUT 12 & & 34 & 13 & & DATA IN 12 \\
\hline DATA OUT 13 & & 36 & 14 & & DATA IN 13 \\
\hline DATA OUT 14 & & 38 & 15 & & DATA IN 14 \\
\hline DATA OUT 15 & $\mathrm{~J} 2$ & 40 & 16 & $\mathrm{~J} 2$ & DATA IN 15 \\
\hline
\end{tabular}

Note: Unlisted pins are not connected. 


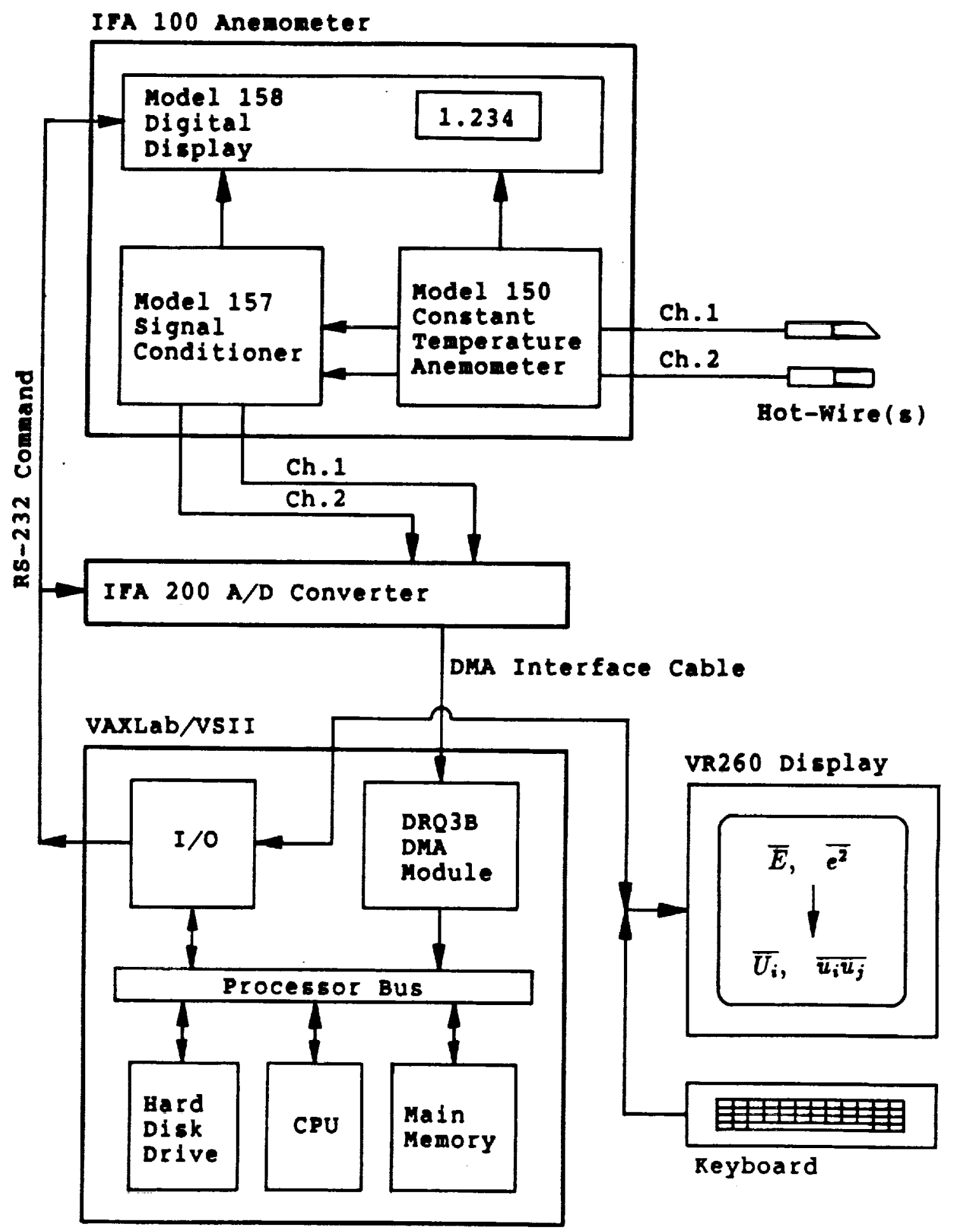

Fig. B.1. Schematic of hot-wire data acquisition system. 


\section{APPENDIX C}

\section{HOT-WIRE TECHNIQUES}

\section{C.1 Introduction}

The derivation of the mean and turbulence hot-wire response equations for the Method $A$ technique are presented in this appendix. The working form of the mean and turbulence response equations developed by Al-Beirutty (Method B) are presented in this chapter without derivation. For details of the development of these equations, the reader is referred to references [45] and [46].

The Method A single rotatable hot-wire technique which is proposed here is applicable for the measurement of the mean flow and Reynolds stress field in situations when the primary flow is nominally normal to the probe axis of rotation. This, in itself, is not unique. De Grande and Kool [61] proposed a hotwire technique in which a single rotatable slant-wire is used to deduce the mean flow and turbulence field under similar conditions. Their technique is not based on an empirical cooling law relation, but rather relies on extensive pitch and yaw calibration. The time required for calibration becomes prohibitive when large amounts of data are being accumulated and frequent recalibration is necessary. Their technique has the advantage that commercially available probes can be used. The use a single slant-wire probe limits the range of sensitivity to the individual mean velocity and stress components. This is usually compensated for by obtaining data at a large number of rotational positions and using leastsquares error minimization criteria to solve for the flow variables. The hot-wire technique proposed here relies on the sequential use of custom-made normal and slant-wire probes in order to maximize sensitivity to the flow variables. The response of the hot-wires to pitch and yaw flow angles is governed by an effective cooling velocity relation so that only a simple calibration procedure is required.

Before continuing, it is important to note a restriction associated with Method $A$ that is not present with Method $B$. Whereas with Method B, for zeroto-moderate skew angles, the probe body can be rotated $360^{\circ}$ with little or no interference effects from the probe supports, there is a significant region of rotation associated with Method $A$ where interference effects from the probe supports cannot be neglected. Within this region, no valid data can be expected; however, the angular sweep of this region can be minimized and the angular position shifted by suitable design of the hot-wire probe itself. Two possible slant-wire probe configurations, along with an estimate of their regions of interference for use with Method A are illustrated in Fig. C.1. The impact of this restriction on Method $A$ will be discussed as the response equations are developed. 


\section{C.2 Generalized Empirical Cooling Law Relation}

The starting point for the derivation of the mean and turbulence response equations for both Method $A$ and Method $B$ is the empirical cooling law relation. The form of this equation is dependent on whether a linearized or non-linearized hot-wire anemometer system is used. For a non-linearized system, the relation is given by:

$$
\left(E_{N L}\right)^{2}-\bar{E}_{0}^{2}=B_{N L} U_{e}^{n}
$$

where $E$ is the instantaneous bridge voltage and $U_{e}$ is the instantaneous cooling velocity; $\bar{E}_{0}^{2}$ and $B$ are the intercept and slope of the wire calibration curve when the calibration stream is normal to the wire. Conversely, when the anemometer output is linearized, the cooling relation is given by:

$$
E_{L}=B_{L} U_{e}
$$

These equations can be presented in a general form applicable to both linearized and non-linearized signals as:

$$
\left(E^{2}-A\right)^{2 / m}=B^{2 / m} U_{e}^{2}
$$

where, for a linearized system:

$$
E^{2}=\left(E_{L}-\bar{E}_{0}\right)^{2}, \quad A=0, \quad B=B_{L}^{2}, \quad m=2
$$

and for a non-linearized system:

$$
E^{2}=E_{N L}^{2}, \quad A=\bar{E}_{0}^{2}, \quad B=B_{N L}, \quad m=n
$$

In the following analysis, the mean and turbulence response equations are developed for the non-linearized case, from which the linearized system equations are extracted as a special case.

\section{C.3 Mean Flow Equation Development}

The mean velocity vector can be evaluated in magnitude and direction when a hot-wire is rotated into four angular positions, such as Positions $1,2,3$ and 4 shown in Fig. C.2, and the corresponding mean bridge voltage is recorded. For Method $A$, this can be accomplished by rotating an inclined wire about the $y$ axis to Positions 1 and 2, and by rotating a normal wire about the same axis to Positions 3 and 4 . Without knowing the mean velocity direction, however, there is the risk that the wire will be rotated into a position where interference from one of the probe supports will occur, rendering the data invalid. We will discuss later how this situation can be avoided, if beforehand, an estimate of the transverse flow angle in the $x-z$ plane, $\gamma$, defined as shown in Fig. C.2, is known. 
The reader should keep in mind that this is strictly a physical limitation and not a restriction inherent in the response equations themselves.

For Method B, the four wire positions shown in Fig. C.2 can be achieved by rotating a single inclined wire about the $x$-axis. Unlike Method $A$, for zeroto-moderate skewness levels, the problem of probe support interference does not present itself with Method $B$, and, therefore, no knowledge of the mean flow direction is required apriori, other than that the flow is nominally in the $x$ direction and that $V$ and $W$ are less than $U$.

The primary difference between Method $A$ and Method $B$ is in the expressions for the effective cooling velocity term in equation (C.1.a). In the remainder of this section, the mean flow response equations for both Method $A$ and Method $B$ will be presented. Except where noted, the following discussion is applicable to both of these methods.

When the time-averaged form of equation (C.1.a) is applied at each wire position shown in Fig. C.2, the following four response equations result:

$$
\begin{aligned}
& \overline{\left(E_{1}{ }^{2}-\bar{E}_{0}^{2}\right)^{2 / m}}=B^{2 / m} \overline{\left(U_{e}^{2}\right)_{1}} \\
& \overline{\left(E_{2}{ }^{2}-\bar{E}_{0}^{2}\right)^{2 / m}}=B^{2 / m} \overline{\left(U_{e}^{2}\right)_{2}} \\
& \overline{\left(E_{3}{ }^{2}-\bar{E}_{0}^{2}\right)^{2 / m}}=B^{2 / m} \overline{\left(U_{e}^{2}\right)_{3}} \\
& \left.\overline{\left(E_{4}{ }^{2}-\bar{E}_{0}^{2}\right)^{2 / m}}=B^{2 / m} \overline{\left(U_{e}{ }^{2}\right.}\right)_{4}
\end{aligned}
$$

Combining equations (C.2.a) \& (C.2.b) and equations (C.2.c) \& (C.2.d) yields:

$$
\begin{aligned}
& \left.\left.\overline{\left(E_{1}^{2}-\bar{E}_{0}^{2}\right)^{2 / m}} / \overline{\left(E_{2}^{2}-\bar{E}_{0}^{2}\right)^{2 / m}}=\overline{\left(U_{e}{ }^{2}\right.}\right)_{1} / \overline{\left(U_{e}{ }^{2}\right.}\right)_{2} \\
& \left.\left.\overline{\left(E_{3}{ }^{2}-\bar{E}_{0}^{2}\right)^{2 / m}} / \overline{\left(E_{4}{ }^{2}-\bar{E}_{0}^{2}\right)^{2 / m}}=\overline{\left(U_{e}{ }^{2}\right.}\right)_{3} / \overline{\left(U_{e}{ }^{2}\right.}\right)_{4}
\end{aligned}
$$

Rearranging equations (C.3.a) and (C.3.b) gives:

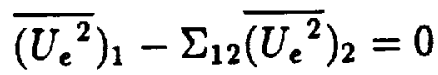

$$
\begin{aligned}
& \overline{\left(U_{e}^{2}\right)_{3}}-\Sigma_{34}\left(\overline{U_{e}^{2}}\right)_{4}=0
\end{aligned}
$$

where, for convenience, the following definitions are employed:

$$
\begin{aligned}
& \Sigma_{12}=\overline{\left(E_{1}^{2}-\bar{E}_{0}^{2}\right)^{2 / m}} \overline{\left(E_{2}{ }^{2}-\bar{E}_{0}^{2}\right)^{2 / m}} \\
& \Sigma_{34}=\overline{\left(E_{3}{ }^{2}-\bar{E}_{0}^{2}\right)^{2 / m}} \overline{\left({\left.E_{4}{ }^{2}-\bar{E}_{0}^{2}\right)^{2 / m}}^{2}\right.}
\end{aligned}
$$

By eliminating the dependence on the wire calibration slope $(B)$ in equations (C.4.a) and (C.4.b), we have placed the restriction that the same hot-wire must 
be used in Positions 1 and 2, and, likewise, in Positions 3 and 4. We do not require, however, that the hot-wire that is used in Positions 1 and 2 be the same as the one used in Positions 3 and 4, so long as the appropriate calibration intercept values are used in equations (C.5.a) and (C.5.b).

In order to solve equations (C.4.a) and (C.4.b) for the mean velocity components, the effective cooling velocity $\left(U_{e}\right)$ must be expressed in terms of the mean velocity components $(U, V, W)$, and the voltage terms $\left(\Sigma_{12}, \Sigma_{34}\right)$ must be expressed in terms of measurable mean $(\bar{E})$ and mean-square $\left(e^{2}\right)$ voltages.

The voltage terms, $\Sigma_{12}$ and $\Sigma_{34}$, in equations (C.4.a) and (C.4.b) can be expressed in terms of measurable voltages by substituting $E=\bar{E}+e$ into $\left(E^{2}-\right.$ $\left.\bar{E}_{0}^{2}\right)^{2 / m}$, expanding the resulting expression in a binomial series, and truncating at second order:

$$
\left(E^{2}-\bar{E}_{0}^{2}\right)^{2 / m}=\left(\bar{E}^{2}-\bar{E}_{0}^{2}\right)^{2 / m}\left[1+(4 / m) \frac{e \bar{E}}{\bar{E}^{2}-\bar{E}_{0}^{2}}+\frac{C e^{2}}{\bar{E}^{2}-\bar{E}_{0}^{2}}\right]
$$

where:

$$
C=2 / m+(4 / m)\left(\frac{2}{m}-1\right)\left(\frac{\bar{E}^{2}}{\overline{\bar{E}}^{2}-\bar{E}_{0}^{2}}\right)
$$

Time-averaging this expression yields:

$$
\overline{\left(E^{2}-\bar{E}_{0}^{2}\right)^{2 / m}}=\left(\bar{E}^{2}-\bar{E}_{0}^{2}\right)^{2 / m}\left[1+\frac{C \overline{e^{2}}}{\bar{E}^{2}-\bar{E}_{0}^{2}}\right]
$$

Substituting equation (C.6.c) into equations (C.5.a) and (C.5.b) gives:

$$
\begin{aligned}
& \Sigma_{12}=\Sigma_{1} / \Sigma_{2} \\
& \Sigma_{34}=\Sigma_{3} / \Sigma_{4}
\end{aligned}
$$

where:

$$
\begin{aligned}
& \Sigma_{1}=\left(\bar{E}_{1}^{2}-\bar{E}_{0}^{2}\right)^{2 / m}\left[1+\frac{C_{1} \overline{e_{1}^{2}}}{\bar{E}_{1}^{2}-\bar{E}_{0}^{2}}\right] \\
& \Sigma_{2}=\left(\bar{E}_{2}^{2}-\bar{E}_{0}^{2}\right)^{2 / m}\left[1+\frac{C_{2} \overline{e_{2}{ }^{2}}}{\bar{E}_{2}^{2}-\overline{\bar{E}_{0}^{2}}}\right] \\
& \Sigma_{3}=\left(\bar{E}_{3}^{2}-\bar{E}_{0}^{2}\right)^{2 / m}\left[1+\frac{C_{3} \overline{e_{3}^{2}}}{\bar{E}_{3}^{2}-\bar{E}_{0}^{2}}\right] \\
& \Sigma_{4}=\left(\bar{E}_{4}^{2}-\bar{E}_{0}^{2}\right)^{2 / m}\left[1+\frac{C_{4} \overline{e_{4}{ }^{2}}}{\bar{E}_{4}^{2}-\bar{E}_{0}^{2}}\right]
\end{aligned}
$$

The exact expression for the effective cooling velocity term, $\overline{U_{e}{ }^{2}}$, in equations (C.4.a) and (C.4.b) can be expressed in terms of the mean velocity components 
$(U, V, W)$ and the Reynolds stresses $\left(\overline{u_{i} u_{j}}, i, j=1,2,3\right)$ at the four wire positions shown in Fig. C.2. as follows (recalling that different expressions are required for Method $A$ and Method B):

Method A (see Appendix D for derivation)

$$
\begin{aligned}
\left(\overline{U_{e}{ }^{2}}\right)_{1}= & K_{0,1} U^{2}\left\{K_{1,1}\left[1+\frac{\overline{u^{2}}}{U^{2}}\right]+\left[\left(\frac{V}{U}\right)^{2}+\frac{\overline{v^{2}}}{U^{2}}\right]\right. \\
& \left.+K_{3,1} h_{B N}^{2}\left[\left(\frac{W}{U}\right)^{2}+\frac{\overline{w^{2}}}{U^{2}}\right]+2 K_{2,1}\left[\left(\frac{V}{U}\right)+\frac{\overline{u v}}{U^{2}}\right]\right\} \\
\left(\overline{U_{e}^{2}}\right)_{2}= & K_{0,2} U^{2}\left\{K_{1,2}\left[1+\frac{\overline{u^{2}}}{U^{2}}\right]+\left[\left(\frac{V}{U}\right)^{2}+\frac{\overline{v^{2}}}{U^{2}}\right]\right. \\
& \left.+K_{3,2} h_{B N}^{2}\left[\left(\frac{W}{U}\right)^{2}+\frac{\overline{w^{2}}}{U^{2}}\right]-2 K_{2,2}\left[\left(\frac{V}{U}\right)+\frac{\overline{u v}}{U^{2}}\right]\right\} \\
\left(\overline{U_{e}^{2}}\right)_{3}= & K_{0,3} U^{2}\left\{\frac{1}{2}\left(K_{1,3}+h_{B N}^{2} K_{3,3}\right)\left[1+\frac{\overline{u^{2}}}{U^{2}}\right]\right. \\
& +\left[\left(\frac{V}{U}\right)^{2}+\frac{\overline{v^{2}}}{U^{2}}\right]+\frac{1}{2}\left(K_{1,3}+h_{B N}^{2} K_{3,3}\right)\left[\left(\frac{W}{U}\right)^{2}+\frac{\overline{w^{2}}}{U^{2}}\right] \\
& \left.-\left(K_{1,3}-h_{B N}^{2} K_{3,3}\right)\left[\left(\frac{W}{U}\right)+\frac{\overline{u w}}{U^{2}}\right]\right\} \\
\left(\overline{U_{e}{ }^{2}}\right)_{4}= & K_{0,4} U^{2}\left\{\frac{1}{2}\left(K_{1,4}+h_{B N}^{2} K_{3,4}\right)\left[1+\frac{\overline{u^{2}}}{U^{2}}\right]\right. \\
& +\left[\left(\frac{V}{U}\right)^{2}+\frac{\overline{v^{2}}}{U^{2}}\right]+\frac{1}{2}\left(K_{1,4}+h_{B N}^{2} K_{3,4}\right)\left[\left(\frac{W}{U}\right)^{2}+\frac{\overline{w^{2}}}{U^{2}}\right] \\
& \left.+\left(K_{1,4}-h_{B N}^{2} K_{3,4}\right)\left[\left(\frac{W}{U}\right)+\frac{\overline{u w}}{U^{2}}\right]\right\}
\end{aligned}
$$

Method B (see Appendix A, reference [47] for derivation)

$$
\begin{aligned}
\left(\overline{U_{e}^{2}}\right)_{1}= & K_{0,1} U^{2}\left\{\left[1+\frac{\overline{u^{2}}}{U^{2}}\right]+K_{1,1}\left[\left(\frac{V}{U}\right)^{2}+\frac{\overline{v^{2}}}{U^{2}}\right]\right. \\
& \left.+K_{3,1} h_{B N}^{2}\left[\left(\frac{W}{U}\right)^{2}+\frac{\overline{w^{2}}}{U^{2}}\right]+2 K_{2,1}\left[\left(\frac{V}{U}\right)+\frac{\overline{u v}}{U^{2}}\right]\right\}
\end{aligned}
$$




$$
\begin{gathered}
\left(\overline{U_{e}^{2}}\right)_{2}=K_{0,2} U^{2}\left\{\left[1+\frac{\overline{u^{2}}}{U^{2}}\right]+K_{1,2}\left[\left(\frac{V}{U}\right)^{2}+\frac{\overline{v^{2}}}{U^{2}}\right]\right. \\
\left.+K_{3,2} h_{B N}^{2}\left[\left(\frac{W}{U}\right)^{2}+\frac{\overline{w^{2}}}{U^{2}}\right]-2 K_{2,2}\left[\left(\frac{V}{U}\right)+\frac{\overline{u v}}{U^{2}}\right]\right\} \\
\left(\overline{U_{e}^{2}}\right)_{3}=K_{0,3} U^{2}\left\{\left[1+\frac{\overline{u^{2}}}{U^{2}}\right]+K_{3,3} h_{B N}^{2}\left[\left(\frac{V}{U}\right)^{2}+\frac{\overline{v^{2}}}{U^{2}}\right]\right. \\
\left.+K_{1,3}\left[\left(\frac{W}{U}\right)^{2}+\frac{\overline{w^{2}}}{U^{2}}\right]+2 K_{2,3}\left[\left(\frac{W}{U}\right)+\frac{\overline{u w}}{U^{2}}\right]\right\} \\
\left(\overline{U_{e}^{2}}\right)_{4}=K_{0,4} U^{2}\left\{\left[1+\frac{\overline{u^{2}}}{U^{2}}\right]+K_{3,4} h_{B N}^{2}\left[\left(\frac{V}{U}\right)^{2}+\frac{\overline{v^{2}}}{U^{2}}\right]\right. \\
\left.+K_{1,4}\left[\left(\frac{W}{U}\right)^{2}+\frac{w^{2}}{U^{2}}\right]-2 K_{2,4}\left[\left(\frac{W}{U}\right)+\frac{\overline{u w}}{U^{2}}\right]\right\}
\end{gathered}
$$

where $K_{0}, K_{1}, K_{2}$ and $K_{3}$ (the second subscript denotes wire position) are functions of the wire inclination angle $(\alpha)$, the normal cooling coefficient $\left(h_{N}\right)$ and the tangential cooling coefficient $(k)$; and $h_{B N}$ is the binormal cooling coefficient. The functional forms for these variables are given in Appendix D. When equations (C.8.a) through (C.8.d) or equations (C.9.a) through (C.9.d) are substituted into equations (C.4.a) and (C.4.b), the following non-linear algebraic equations, applicable to both $M$ ethod $A$ and Method $B$, result:

$$
\begin{gathered}
K_{0 v}+K_{1 v}(V / U)+K_{2 v}(V / U)^{2}+K_{3 v}(W / U)^{2}+C_{v}=0 \\
K_{0 w}+K_{1 w}(W / U)+K_{2 w}(W / U)^{2}+K_{3 w}(V / U)^{2}+C_{w}=0
\end{gathered}
$$

where

$$
\begin{gathered}
C_{v}=K_{0 v}\left(\frac{\overline{u^{2}}}{U^{2}}\right)+K_{1 v}\left(\frac{\overline{u v}}{U^{2}}\right)+K_{2 v}\left(\frac{\overline{v^{2}}}{U^{2}}\right)+K_{3 v}\left(\frac{\overline{w^{2}}}{U^{2}}\right) \\
C_{w}=K_{0 w}\left(\frac{\overline{u^{2}}}{U^{2}}\right)+K_{1 w}\left(\frac{\overline{u w}}{U^{2}}\right)+K_{2 w}\left(\frac{\overline{w^{2}}}{U^{2}}\right)+K_{3 w}\left(\frac{\overline{v^{2}}}{U^{2}}\right)
\end{gathered}
$$

and where, for convenience, the following definitions have been employed:

$$
\text { Method A }
$$

$$
\begin{aligned}
& K_{0 v}=K_{0,1} K_{1,1}-K_{0,2} K_{1,2} \Sigma_{12} \\
& K_{1 v}=2 K_{0,1} K_{2,1}+2 K_{0,2} K_{2,2} \Sigma_{12} \\
& K_{2 v}=K_{0,1}-K_{0,2} \Sigma_{12} \\
& K_{3 v}=h_{B N}^{2}\left(K_{0,1} K_{3,1}-K_{0,2} K_{3,2} \Sigma_{12}\right)
\end{aligned}
$$


and,

$$
\begin{aligned}
K_{0 w}= & \left(\frac{1}{2}\right)\left[K_{0,3}\left(K_{1,3}+h_{B N}^{2} K_{3,3}\right)\right. \\
& -K_{0,4}\left(K_{1,4}+h_{B N}^{2} K_{3,4} \Sigma_{34}\right] \\
K_{1 w}= & -\left[K_{0,3}\left(K_{1,3}-h_{B N}^{2} K_{3,3}\right)\right. \\
& +K_{0,4}\left(K_{1,4}-h_{B N}^{2} K_{3,4} \Sigma_{34}\right] \\
K_{2 w}= & K_{0 w} \\
K_{3 w}= & K_{0,3}-K_{0,4} \Sigma_{34}
\end{aligned}
$$

Method B

$$
\begin{aligned}
& K_{0 v}=K_{0,1}-K_{0,2} \Sigma_{12} \\
& K_{1 v}=2 K_{0,1} K_{2,1}+2 K_{0,2} K_{2,2} \Sigma_{12} \\
& K_{2 v}=K_{0,1} K_{1,1}-K_{0,2} K_{1,2} \Sigma_{12} \\
& K_{3 v}=h_{B N}^{2}\left(K_{0,1} K_{3,1}-K_{0,2} K_{3,2} \Sigma_{12}\right)
\end{aligned}
$$

and,

$$
\begin{aligned}
& K_{0 w}=K_{0,3}-K_{0,4} \Sigma_{34} \\
& K_{1 w}=2 K_{0,3} K_{2,3}+2 K_{0,4} K_{2,4} \Sigma_{34} \\
& K_{2 w}=K_{0,3} K_{1,3}-K_{0,4} K_{1,4} \Sigma_{34} \\
& K_{3 w}=h_{B N}^{2}\left(K_{0,3} K_{3,3}-K_{0,4} K_{3,4} \Sigma_{34}\right)
\end{aligned}
$$

The terms $C_{v}$ and $C_{w}$ in equations (C.10.a) and (C.10.b) account for the errors which would result from neglecting the fluctuating velocity components relative to mean components in the expression for the effective cooling velocity.

In a low turbulence intensity field $(\leq 10 \%)$, the correction terms $C_{v}$ and $C_{w}$ in equations (C.10.a) and (C.10.b) can be neglected without introducing serious errors in the computed mean velocity ratios $(V / U)$ and $(W / U)$. Under these conditions, the mean response equations reduce to:

$$
\begin{gathered}
K_{0 v}+K_{1 v}(V / U)+K_{2 v}(V / U)^{2}+K_{3 v}(W / U)^{2}=0 \\
K_{0 w}+K_{1 w}(W / U)+K_{2 w}(W / U)^{2}+K_{3 w}(V / U)^{2}=0
\end{gathered}
$$

Equations (C.14.a) and (C.14.b) represent two coupled non-linear algebraic equations which can be solved simultaneously for the relative mean velocity components $(V / U)$ and $(W / U)$. When second-order mean velocity terms in equations (C.14.a) and (C.14.b) are neglected, solutions for $(V / U)$ and $(W / U)$ which are first-order accurate may be obtained directly as:

$$
\begin{aligned}
(V / U)_{0} & =-K_{0 v} / K_{1 v} \\
(W / U)_{0} & =-K_{0 w} / K_{1 w}
\end{aligned}
$$


which are appropriate for evaluating $(V / U)$ and $(W / U)$ in slightly skewed flows. For the more general case, however, equations (C.10.a) and (C.10.b) represent two non-linear algebraic equations in seven unknowns $\left(V / U, W / U, u^{2} / U^{2}, \overline{v^{2}} / U^{2}\right.$, $\left.\overline{w^{2}} / U^{2}, \overline{u v} / U^{2}, \overline{u w} / U^{2}\right)$. Therefore, five more independent equations are required for closure, which, in turn, requires rotation of the slant and/or normal-wire to at least five different positions. If this is done arbitrarily, then the coefficient matrix may become ill-conditioned. In order to overcome this difficulty, equations (C.14.a) and (C.14.b) are first solved for the mean component ratios $(V / U)$ and $(W / U)$ after initial estimates are made using equations (C.15.a) and (C.15.b). The calculated results are then used to solve the turbulence response equations (to be developed shortly) for the complete Reynolds stress tensor. The calculated stresses can then be used to correct the mean velocity components by simultaneously solving equations (C.10.a) and (C.10.b). The improved mean velocity values are subsequently used to update the Reynolds stress tensor. This iteration procedure is repeated until convergence is achieved.

Once the mean velocity ratios $(V / U)$ and $(W / U)$ are known, the axial mean velocity $(U)$ can be determined by averaging its calculated value with the wire at each of the four positions shown in Fig. C.2. When the expression for the effective cooling velocity at Position 1 (equation (C.8.a) or (C.9.a)) is substituted into equation (C.2.a), the following equation for the axial velocity with the wire at Position 1 is obtained, after some rearrangement:

Method A

$$
\begin{aligned}
U_{1}= & \left\{\left(\bar{E}_{1}^{2}-\bar{E}_{0}^{2}\right)^{2 / m}\left[1+C_{1} \overline{e_{1}^{2}} /\left(\bar{E}_{1}^{2}-\bar{E}_{0}^{2}\right)\right] /\left(B_{c}\right)^{2 / m}\right\}^{1 / 2} \\
& \left\{K_{1,1}\left[1+\frac{\overline{u^{2}}}{U^{2}}\right]+\left[\left(\frac{V}{U}\right)^{2}+\frac{\overline{v^{2}}}{U^{2}}\right]+K_{3,1} h_{B N}^{2}\left[\left(\frac{W}{U}\right)^{2}+\frac{\overline{w^{2}}}{U^{2}}\right]\right. \\
& \left.+2 K_{2,1}\left[\left(\frac{V}{U}\right)+\frac{\overline{u v}}{U^{2}}\right]\right\}^{-1 / 2}
\end{aligned}
$$

Method B

$$
\begin{aligned}
U_{1}= & \left\{\left(\bar{E}_{1}^{2}-\bar{E}_{0}^{2}\right)^{2 / m}\left[1+C_{1} \overline{e_{1}^{2}} /\left(\bar{E}_{1}^{2}-\bar{E}_{0}^{2}\right)\right] /\left(B_{c}\right)^{2 / m}\right\}^{1 / 2} \\
& \left\{\left[1+\frac{\overline{u^{2}}}{U^{2}}\right]+K_{1,1}\left[\left(\frac{V}{U}\right)^{2}+\frac{\overline{v^{2}}}{U^{2}}\right]+K_{3,1} h_{B N}^{2}\left[\left(\frac{W}{U}\right)^{2}+\frac{\overline{w^{2}}}{U^{2}}\right]\right. \\
& \left.+2 K_{2,1}\left[\left(\frac{V}{U}\right)+\frac{\overline{u v}}{U^{2}}\right]\right\}^{-1 / 2}
\end{aligned}
$$

where $B_{c}$, the slope of the calibration curve, is either:

$$
B_{c}=B
$$


when the calibration stream is normal to the wire, or:

$$
B_{c}=B\left(K_{0}\right)^{m / 2}
$$

when the calibration stream is aligned with the probe body (as in Method B), or:

$$
B_{c}=B\left(K_{0} K_{1}\right)^{m / 2}
$$

when the calibration stream is aligned normal to the probe body (as in Method $A$ ). Similar expressions for the axial mean velocity can be derived for wire Positions 2,3 and 4 .

The total mean velocity $\left(U_{0}\right)$ is related to the axial mean velocity $(U)$ by:

$$
U_{0}=U / \cos \phi
$$

where,

$$
\begin{gathered}
\phi=\tan ^{-1}\left(V_{r} / U\right) \\
V_{r} / U=\left[(V / U)^{2}+(W / U)^{2}\right]^{1 / 2}
\end{gathered}
$$

This section will be concluded with an explanation of how the possible probe support interference associated with Method $A$ can be avoided if data are taken by means of probe rotation about the $y$-axis and an estimate of the transverse flow angle $\gamma$ in the $x-z$ plane is known.

Interference can be avoided if, rather than recording data relative to the primary coordinate system, data are collected relative to a another coordinate system which is rotated about the $y$-axis through an estimated yaw flow angle $\gamma_{0}$, which is close to the actual flow angle $\gamma$, such that the transverse velocity component in the $x-z$ plane is small. This allows the use of a hot-wire probe body designed to tolerate small amounts of unknown yaw skewness. The mean velocity components are computed by the method described above, relative to the secondary coordinate system, and then they are transformed to the primary coordinate system. Estimates of $\gamma_{0}$ can be established, at least to first-order accuracy, by pressure nulling of a two-tube Conrad probe, or by means of the hot-wire nulling technique described by Page [62].

\section{C.4 Turbulence Equation Development}

As was the case for the development of the mean response equations, the starting point for the turbulence response equations is the instantaneous empirical cooling relation:

$$
\left(E^{2}-\bar{E}_{0}^{2}\right)^{2 / m}=B^{2 / m} U_{e}^{2}
$$


Also, as before, the left-hand side is expanded by means of the binomial theorem and truncated at second-order:

$$
\left(E^{2}-\bar{E}_{0}^{2}\right)^{2 / m}=\left(\bar{E}^{2}-\bar{E}_{0}^{2}\right)^{2 / m}\left[1+(4 / m) \frac{e \bar{E}}{\bar{E}^{2}-\bar{E}_{0}^{2}}+\frac{C e^{2}}{\bar{E}^{2}-\bar{E}_{0}^{2}}\right]
$$

where,

$$
C=2 / m+(4 / m)\left(\frac{2}{m}-1\right)\left(\frac{\bar{E}^{2}}{\bar{E}^{2}-\bar{E}_{0}^{2}}\right)
$$

In contrast to the mean velocity development, the effective cooling velocity term in equation (C.1.c), is derived relative to another orthogonal coordinate system. For Method $A$, this coordinate system, $x^{\prime}, y, z^{\prime}$, is generated by a rotation about the $y$-axis such that the $x^{\prime}$-axis coincides with the resultant mean yaw velocity vector, $U_{r}$ (refer to Fig. C.3.a). For Method $B$, a coordinate system, $x, y^{\prime}, z^{\prime}$, is generated by a rotation about the $x$-axis such that the $z^{\prime}=0$ plane coincides with the resultant mean transverse velocity vector, $V_{r}$ (refer to Fig. C.3.b). These new coordinates are employed here because the Reynolds stress components, when calculated relative to these new coordinates, do not involve the solution of a full $6 \times 6$ system of equations under certain conditions. The exact forms of the instantaneous effective cooling velocity relative to these new coordinates are:

Method A (see Appendix E)

$$
\begin{aligned}
U_{e}^{2}=K_{0} U_{r}^{2}\left\{f_{m}\right. & +f_{u}\left(u^{\prime} / U_{r}\right)+f_{v}\left(v / U_{r}\right)-f_{w}\left(w^{\prime} / U_{r}\right) \\
& +K_{12}\left(u^{\prime 2} / U_{r}^{2}\right)+\left(v^{2} / U_{r}^{2}\right)+K_{14}\left(w^{\prime 2} / U_{r}^{2}\right) \\
& +2 K_{10}\left(u^{\prime} v / U_{r}^{2}\right)-2 K_{13}\left(u^{\prime} w^{\prime} / U_{r}^{2}\right) \\
& \left.-2 K_{11}\left(v w^{\prime} / U_{r}^{2}\right)\right\}
\end{aligned}
$$

where,

$$
\begin{aligned}
f_{m} & =K_{12}+2 K_{10}\left(V / U_{r}\right)+\left(V / U_{r}\right)^{2} \\
f_{u} & =2 K_{12}+2 K_{10}\left(V / U_{r}\right) \\
f_{v} & =2 K_{10}+2\left(V / U_{r}\right) \\
f_{w} & =2 K_{13}+2 K_{11}\left(V / U_{r}\right)
\end{aligned}
$$

Method B (see Appendix B, reference [45])

$$
\begin{aligned}
U_{e}^{2}=K_{0} U^{2}\left\{f_{m}\right. & +f_{u}(u / U)+f_{v}\left(v^{\prime} / U\right)+f_{w}\left(w^{\prime} / U\right)+\left(u^{2} / U^{2}\right) \\
& +K_{12}\left(v^{\prime 2} / U^{2}\right)+K_{14}\left(w^{\prime 2} / U^{2}\right)+2 K_{10}\left(u v^{\prime} / U^{2}\right) \\
& \left.+2 K_{11}\left(u w^{\prime} / U^{2}\right)+2 K_{13}\left(v^{\prime} w^{\prime} / U^{2}\right)\right\}
\end{aligned}
$$


where,

$$
\begin{aligned}
f_{m} & =1+2 K_{10}\left(V_{r} / U\right)+K_{12}\left(V_{r} / U\right)^{2} \\
f_{u} & =2+2 K_{10}\left(V_{r} / U\right) \\
f_{v} & =2 K_{10}+2 K_{12}\left(V_{r} / U\right) \\
f_{w} & =2 K_{11}+2 K_{13}\left(V_{r} / U\right)
\end{aligned}
$$

where $K_{10}, K_{11}, K_{12}, K_{13}, K_{14}$ are functions of the probe rotation angle, $\theta$, and of $K_{1}, K_{2}$ and $K_{3}$, which, in turn, are functions of $h_{N}, k$ and $\alpha$ (refer to Appendix $\mathrm{D}$ and $\mathrm{E}$ for explicit forms), and $u^{\prime}, v^{\prime}$ and $w^{\prime}$ are velocity fluctuations along the $x^{\prime}, y^{\prime}$ and $w^{\prime}$ axes, respectively, as shown in Figs. C.3.a and C.3.b. If equation (C.6.a) and either equation (C.20) or (C.22) is substituted into equation (C.1.c) and subsequently time-averaged, then the resulting expression would not take into account first-order effects in the fluctuations and would, therefore, not be appropriate for analyzing low intensity flows. Also, the resulting set of equations would form a $6 \times 6$ system of equations whose solution can lead to inaccuracies in the calculated Reynolds stresses. In order to avoid these shortcomings, consider equation (C.1.c) which is first time-averaged to yield:

$$
\overline{\left(E^{2}-\bar{E}_{0}^{2}\right)^{2 / m}}=B^{2 / m} \overline{U_{e}^{2}}
$$

and then consider the difference between equations (C.1.c) and (C.24) in a normalized form which eliminates the contribution of the slope factor, $B^{2 / m}$, namely:

$$
\frac{\left(E^{2}-\bar{E}_{0}^{2}\right)^{2 / m}-\overline{\left(E^{2}-\bar{E}_{0}^{2}\right)^{2 / m}}}{\overline{\left(E^{2}-\bar{E}_{0}^{2}\right)^{2 / m}}}=\frac{U_{e}{ }^{2}-\overline{U_{e}{ }^{2}}}{\overline{U_{e}{ }^{2}}}
$$

On the basis of equations (C.6.a) and (C.20) or (C.22), the time-averaged terms in equation (C.25) can be written as:

$$
\overline{\left(E^{2}-\bar{E}_{0}^{2}\right)^{2 / m}}=\left(\bar{E}^{2}-\bar{E}_{0}^{2}\right)^{2 / m}\left[1+\frac{C \overline{e^{2}}}{\bar{E}^{2}-\bar{E}_{0}^{2}}\right]
$$

and,

Method A

$$
\begin{aligned}
\overline{U_{e}{ }^{2}}=K_{0} U_{r}^{2}\left\{f_{m}+K_{12}\left(\overline{u^{\prime 2}} / U_{r}^{2}\right)\right. & +\left(\overline{v^{2}} / U_{r}^{2}\right)+K_{14}\left(\overline{w^{\prime 2}} / U_{r}^{2}\right) \\
+2 K_{10}\left(\overline{u^{\prime} v} / U_{r}^{2}\right) & -2 K_{13}\left(\overline{u^{\prime} w^{\prime}} / U_{r}^{2}\right) \\
& \left.-2 K_{11}\left(\overline{v w^{\prime}} / U_{r}^{2}\right)\right\}
\end{aligned}
$$

Method B

$$
\begin{aligned}
\overline{U_{e}{ }^{2}}=K_{0} U^{2}\left\{f_{m}+\left(\overline{u^{2}} / U^{2}\right)+K_{12}\left(\overline{v^{\prime 2}} / U^{2}\right)+K_{14}\left(\overline{w^{\prime 2}} / U^{2}\right)\right. \\
\left.+2 K_{10}\left(\overline{u v^{\prime}} / U^{2}\right)+2 K_{11}\left(\overline{u w^{\prime}} / U^{2}\right)+2 K_{13}\left(\overline{v^{\prime} w^{\prime}} / U^{2}\right)\right\}
\end{aligned}
$$


If the appropriate terms developed thus far are substituted into equation (C.25) an expression which has the following functional form results:

$$
f\left(e, e^{2}, \overline{e^{2}}, u_{i}, u_{i} u_{j}, \overline{u_{i} u_{j}}\right)=0, \quad i, j=1,2,3
$$

since all mean flow field variables are known from the previous mean flow analysis. Equation (C.29) implies that in order to retain first-order fluctuation effects, equation (C.25) should be squared and time-averaged:

$$
\frac{\overline{\left[\left(E^{2}-\bar{E}_{0}^{2}\right)^{2 / m}-\overline{\left(E^{2}-\bar{E}_{0}^{2}\right)^{2 / m}}\right]^{2}}}{\left[\overline{\left(E^{2}-\bar{E}_{0}^{2}\right)^{2 / m}}\right]^{2}}=\frac{\overline{\left[U_{e}{ }^{2}-\overline{U_{e}{ }^{2}}\right]^{2}}}{\left[\overline{U_{e}{ }^{2}}\right]^{2}}
$$

Now, if the appropriate terms are substituted into equation (C.30), and the squaring and time-averaging performed, then, retaining only second-order terms, the following generalized turbulence response equations result:

Method A (see Appendix E)

$$
\begin{aligned}
& F_{u u}\left(\overline{u^{\prime 2}} / U_{r}^{2}\right)+F_{v v}\left(\overline{v^{2}} / U_{r}^{2}\right)+F_{w w}\left(\overline{w^{\prime 2}} / U_{r}^{2}\right) \\
& +F_{u v}\left(\overline{u^{\prime} v} / U_{r}^{2}\right)-F_{u w}\left(\overline{u^{\prime} w^{\prime}} / U_{r}^{2}\right)-F_{v w}\left(\overline{v w^{\prime}} / U_{r}^{2}\right)=F_{m m}
\end{aligned}
$$

where,

$$
\begin{aligned}
F_{u u} & =f_{u}{ }^{2}-2 f_{m} \Pi K_{12} \\
F_{v v} & =f_{v}{ }^{2}-2 f_{m} \Pi \\
F_{w w} & =f_{w}{ }^{2}-2 f_{m} \Pi K_{14} \\
F_{u v} & =2\left(f_{u} f_{v}-2 f_{m} \Pi K_{10}\right) \\
F_{u w} & =2\left(f_{u} f_{w}-2 f_{m} \Pi K_{13}\right) \\
F_{v w} & =2\left(f_{v} f_{w}-2 f_{m} \Pi K_{11}\right) \\
F_{m m} & =f_{m}{ }^{2} \Pi
\end{aligned}
$$

Method B (see Appendix B, reference [45])

$$
\begin{aligned}
& F_{u u}\left(\overline{u^{2}} / U^{2}\right)+F_{v v}\left(\overline{v^{\prime 2}} / U^{2}\right)+F_{w w}\left(\overline{w^{\prime 2}} / U^{2}\right) \\
& +F_{u v}\left(\overline{u v^{\prime}} / U^{2}\right)+F_{u w}\left(\overline{u w^{\prime}} / U^{2}\right)+F_{v w}\left(\overline{v^{\prime} w^{\prime}} / U^{2}\right)=F_{m m}
\end{aligned}
$$

where,

$$
\begin{aligned}
F_{u u} & =f_{u}{ }^{2}-2 f_{m} \Pi \\
F_{v v} & =f_{v}{ }^{2}-2 f_{m} \Pi K_{12} \\
F_{u w} & =f_{w}{ }^{2}-2 f_{m} \Pi K_{14} \\
F_{u v} & =2\left(f_{u} f_{v}-2 f_{m} \Pi K_{10}\right) \\
F_{u w} & =2\left(f_{u} f_{w}-2 f_{m} \Pi K_{11}\right) \\
F_{v w} & =2\left(f_{v} f_{w}-2 f_{m} \Pi K_{13}\right) \\
F_{m m} & =f_{m}{ }^{2} \Pi
\end{aligned}
$$


with (applicable to both Method $A$ and Method B),

$$
\Pi=\left(16 / m^{2}\right) \frac{\left[\overline{e^{2}} \bar{E}^{2} /\left(\bar{E}^{2}-\bar{E}_{0}^{2}\right)^{2}\right]}{\left[1+2 C \overline{e^{2}} /\left(\bar{E}^{2}-\bar{E}_{0}^{2}\right)^{2}\right]}
$$

and $f_{m}, f_{u}, f_{v}$ and $f_{w}$ are defined by equations (C.21) or (C.23). The component $e^{2}$ in the numerator of equation (C.35) is a consequence of the first-order fluctuation (e) in equation (C.6.a), while $\overline{e^{2}}$ in the denominator is a consequence of the second-order fluctuation $\left(e^{2}\right)$ in this equation.

\section{C.4.1 Low Turbulence Intensity Flow}

For flows of low turbulence intensity, the second-order terms in equations (C.6.a) and (C.20) or (C.22) can be neglected in comparison to the first-order terms. Substitution of the first- order forms of equations (C.6.a) and (C.20) or (C.22) into equation (C.30) yields the following turbulence response equations applicable to low intensity flows:

Method A (see Appendix E)

$$
\begin{aligned}
& f_{u}{ }^{2}\left(\overline{u^{\prime 2}} / U_{r}{ }^{2}\right)+f_{v}{ }^{2}\left(\overline{v^{2}} / U_{r}{ }^{2}\right)+f_{w}{ }^{2}\left(\overline{w^{\prime 2}} / U_{r}{ }^{2}\right) \\
& +2 f_{u} f_{v}\left(\overline{u^{\prime} v} / U_{r}{ }^{2}\right)-2 f_{u} f_{w}\left(\overline{u^{\prime} w^{\prime}} /{U_{r}}^{2}\right)-2 f_{v} f_{w}\left(\overline{v w^{\prime}} / U_{r}{ }^{2}\right)=\pi f_{m}{ }^{2}
\end{aligned}
$$

Method B (see Appendix B, reference [45])

$$
\begin{aligned}
& f_{u}{ }^{2}\left(\overline{u^{2}} / U^{2}\right)+f_{v}{ }^{2}\left(\overline{v^{\prime 2}} / U^{2}\right)+f_{w}{ }^{2}\left(\overline{w^{\prime 2}} / U^{2}\right) \\
& +2 f_{u} f_{v}\left(\overline{u v^{\prime}} / U^{2}\right)+2 f_{u} f_{w}\left(\overline{u w^{1}} / U^{2}\right)+2 f_{v} f_{w}\left(\overline{v^{\prime} w^{1}} / U^{2}\right)=\pi f_{m}{ }^{2}
\end{aligned}
$$

with (applicable to both Method A and Method B),

$$
\pi=\left(16 / m^{2}\right)\left[\bar{e}^{2} \bar{E}^{2} /\left(\bar{E}^{2}-\bar{E}_{0}^{2}\right)^{2}\right]
$$

In principle, the six components of the Reynolds stress tensor relative to the $x^{\prime}, y, z^{\prime}$ coordinates shown in Fig. C.3.a or to the $x, y^{\prime}, z^{\prime}$ coordinates shown in Fig. C.3.b could be determined by applying data taken at six fixed probe positions to equation (C.36) or (C.37), respectively, for low intensity flows. This is possible, since, in general, the wire will be sensitive to all three fluctuating velocity components at a given rotation angle. The resulting $6 \times 6$ system of equations may, however, become ill-conditioned if these fixed probe positions are specified in an arbitrary manner.

To increase the accuracy of his calculations, Al-Beirutty employed a sensitivity minimization criteria to Method $B$ which significantly improves the condition number of the coefficient matrix. He accomplished this by observing that 
at certain rotation angles ("optimum planes"), the wire is either insensitive to a particular transverse velocity fluctuation, $v^{\prime}$ or $w^{\prime}$, or is equally sensitive to both. As will be shown shortly, when data are taken at these optimum planes, the resulting $6 \times 6$ system of equations becomes partially decoupled, and, as a result, the problem is reduced to the solution of several lower-order systems of equations. The behavior of the sensitivity coefficients in equation (C.30) will be examined to determine if similar criteria can be applied to Method $A$ as well.

The sensitivity coefficients $f_{u}, f_{v}$ and $f_{w}$ appearing in equations (C.36) and (C.37) can be written in terms of the probe rotation angle $\theta$ when the appropriate expressions for the $K_{10}$ through $K_{14}$ coefficients (given by equation (E.7) in Appendix E) are substituted into equations (C.21) and (C.23):

Method A

$$
\begin{aligned}
& f_{u}=2\left(K_{1} \cos ^{2} \theta+h_{B N}^{2} K_{3} \sin ^{2} \theta\right)+2 K_{2} \cos \theta\left(V / U_{r}\right) \\
& f_{v}=2 K_{2} \cos \theta+2\left(V / U_{r}\right) \\
& f_{w}=2\left(K_{1}-h_{B N}^{2} K_{3}\right) \sin \theta \cos \theta+2 K_{2} \sin \theta\left(V / U_{r}\right)
\end{aligned}
$$

Method B

$$
\begin{aligned}
f_{u} & =2+2 K_{2} \cos \theta\left(V_{r} / U\right) \\
f_{v} & =2 K_{2} \cos \theta+2\left(K_{1} \cos ^{2} \theta+h_{B N}^{2} K_{3} \sin ^{2} \theta\right)\left(V_{r} / U\right) \\
f_{w} & =2 K_{2} \sin \theta+2\left(K_{1}-h_{B N}^{2} K_{3}\right) \sin \theta \cos \theta\left(V_{r} / U\right)
\end{aligned}
$$

In order to examine the wire's sensitivity to the fluctuating velocity components, it is expedient to plot the Reynolds stress coefficients appearing in equations (C.36) and (C.37) as a function of probe rotation angle $(\theta)$ for various levels of skewness. The normal and slant-wire cases for Method $A$ are shown in Figs. C.4 and C.5, respectively, and for Method B in Figs. C.6 and C.7, respectively, utilizing typical values for the cooling coefficients: $k=0.15, h_{N}=1.0$ and $h_{B N}$ $=0.9$. Note that for Method $B$, only positive skewness angles need be considered since negative angles can be transformed to positive angles by a $180^{\circ}$ rotation of the probe.

\section{Method A}

In order for the hot-wire to be insensitive to the $w^{\prime}$ fluctuating velocity component, the coefficient $f_{w}$ must be equal to zero. An examination of Fig. C.5 reveals that $f_{w}$ is zero for a slant-wire at any skewness level when the wire is in the 0 and $180^{\circ}$ positions. These locations will be denoted as Positions $A$ and $B$, respectively. The offset prong slant-wire configuration (refer to Fig. C.1.b) was designed to operate in these positions with minimal probe support interference. 
For a normal-wire (see Fig. C.4), $f_{w}$ is zero at $\theta=0^{\circ}$ and $270^{\circ}$, although the former position is not admissible due to interference effects. The latter position is designated as position $N_{A}$. The above conditions allow three of the Reynolds stresses to be calculated independent of the remaining three. The $\overline{u^{\prime 2}}, \overline{v^{2}}$ and $\overline{u^{\prime} v}$ stress components can be obtained by applying normal-wire data at position $N_{A}$ and slant-wire data at Positions $A$ and $B$ to equation (C.36) and solving the resulting $3 \times 3$ system of equations.

With three stress components known, attention is turned to the condition when the wire is insensitive to the $v$ fluctuating component, which occurs when $f_{v}$ is zero. For Method $A$, this condition proves of little use, inasmuch as for the limiting case when the skewness is zero, the slant-wire positions where $f_{v}=0$ correspond to positions where $f_{w}=0$, namely; $\theta=90$ and $270^{\circ}$. Also, for the normal-wire, in general, there is no location where $f_{v}=0$. The condition where the slant-wire is equally sensitive to both transverse fluctuating velocity components is also of little use. The reason for this is because the angular locations where these conditions occur do not always coincide with, or are even near, the maximum sensitivities of the unknown stress components. Therefore, for Method $A$, it appears that fixed probe positions are necessary.

With reference to Fig. C.5.b, there are four fixed positions, $\theta=45,135,225$ and $315^{\circ}$, designated as Positions $E_{A}, F_{A}, G_{A}$ and $H_{A}$, where the slant-wire is always strongly sensitive to the $\overline{v w^{\prime}}$ stress component, at least over the skewness range considered. The straight prong slant-wire configuration (refer to Fig. C.1.c) was designed to operate in each of these positions with minimal probe support interference. Slant-wire data taken at these four positions can be used to evaluate the $\overline{v w^{\prime}}$ stress component directly. An algebraic expression (derived in Appendix E) for this component is given as:

$$
\frac{\overline{v w^{\prime}}}{{\overline{U_{r}}}^{2}}=\frac{-\left[\left(f_{u} f_{w}\right)_{F_{A}}\left(\pi_{E_{A}}-\pi_{H_{A}}\right)\left(f_{m}{ }^{2}\right)_{E_{A}}-\left(f_{u} f_{w}\right)_{E_{A}}\left(\pi_{F_{A}}-\pi_{G_{A}}\right)\left(f_{m}{ }^{2}\right)_{F_{A}}\right]}{\left(2 f_{u} f_{w}\right)_{F_{A}}\left(2 f_{v} f_{w}\right)_{E_{A}}-\left(2 f_{u} f_{w}\right)_{E_{A}}\left(2 f_{v} f_{w}\right)_{F_{A}}}
$$

At this point, expressions for four of the six Reynolds stresses have been developed and there are no more wire positions available that will further decouple the response equations. Therefore, in order to get the remaining two Reynolds stresses $\left(\overline{w^{\prime 2}}\right.$ and $\left.\overline{u^{\prime} w^{\prime}}\right)$, at least two additional probe positions are required, and, ideally, these positions should be at rotation angles where the sensitivity to the unknown stresses is at or near a maximum. With reference to Fig. C.4.b, for the normal-wire, two fixed positions exist where the wire is nearly equally sensitive to both $u^{\prime}$ and $w^{\prime}$ fluctuations, namely; $\theta=225$ and $315^{\circ}$, which are denoted as Positions $C_{A}$ and $D_{A}$, respectively. When normal-wire data at Positions $C_{A}$ and $D_{A}$ and the four known Reynolds stresses are applied to equation (C.36), then a $2 \times 2$ system of equations results, which, when solved, yields the $\overline{w^{\prime 2}}$ and $\overline{u^{\prime} w^{\prime}}$ Reynolds stresses. 


\section{Method B}

With reference to Fig. C.7, two slant-wire probe positions result when the condition $f_{w}=0$ is applied to Method $B: \theta=0$ and $180^{\circ}$, which are denoted as Positions $A$ and $B$, respectively. The normal-wire also has two positions where $f_{w}=0: \theta=0$ and $90^{\circ}$ (refer to Fig. C.5). For the normal-wire, $\theta=0$ has the additional benefit that $f_{v}=0$ as well. This position is denoted as position $N_{B}$. When normal-wire data at position $N_{B}$ are applied to equation (C.37) a single algebraic equation for the $\overline{u^{2}}$ stress component results. The $\overline{v^{\prime 2}}$ and $\overline{u v^{\prime}}$ stress components can be obtained by applying slant-wire data at Positions $A$ and $B$ to equation (C.37) and solving the resulting $2 \times 2$ matrix.

When the condition $f_{v}=0$ is applied to Method $B$, two discrete slantwire positions result, designated as $C_{B}$ and $D_{B}$ in Fig. C.7.a. At these positions, which are not fixed, but rather vary with skewness level, the slant-wire is sensitive only to $u$ and $w^{\prime}$ fluctuations. If the $\overline{u^{2}}$ stress component, which is known from previous calculations, and slant-wire data at Positions $C_{B}$ and $D_{B}$ are applied to equation (C.37), then a $2 \times 2$ matrix results which may be solved for the $\overline{w^{\prime 2}}$ and $\overline{u w^{\prime}}$ stress components. The remaining unknown stress component, $\overline{v^{\prime} w^{\prime}}$, is determined by solving an algebraic expression which utilizes slant-wire data at Positions $E_{B}, F_{B}, G_{B}$ and $H_{B}$ shown in Fig. C.7.b. These four wire positions correspond to locations where the wire is equally sensitive to both transverse fluctuating velocity components, or, in other words, where $f_{v}{ }^{2}=f_{w}{ }^{2}$. The expression for $\overline{v^{\prime} w^{\prime}}$ (derived in Appendix $\mathrm{C}$ of reference [45]) is given as:

$$
\frac{\overline{v^{\prime} w^{\prime}}}{U^{2}}=\frac{\left(f_{u} f_{w}\right)_{F_{B}}\left(\pi_{E_{B}}-\pi_{H_{B}}\right)\left(f_{m}{ }^{2}\right)_{E_{B}}-\left(f_{u} f_{w}\right)_{E_{B}}\left(\pi_{F_{B}}-\pi_{G_{B}}\right)\left(f_{m}{ }^{2}\right)_{F_{B}}}{\left(2 f_{u} f_{w}\right)_{F_{B}}\left(2 f_{v} f_{w}\right)_{E_{B}}-\left(2 f_{u} f_{w}\right)_{E_{B}}\left(2 f_{v} f_{w}\right)_{F_{B}}}
$$

For all slant-wire measurements referred to Method $B$, the straight prong probe configuration shown in Fig. C.1.c is adequate, since probe interference effects do not occur with the wire in Positions $A$ through $N_{B}$, provided that the skewness level does not exceed $30^{\circ}$ in a low intensity flow and $20^{\circ}$ in a moderate intensity flow [45].

\section{C.4.2 Moderate Turbulence Intensity Flow}

The preceding analysis is applicable only to low turbulence intensity flows. For the more general case, equation (C.31) or (C.32), as applicable, must be solved. Second-order velocity fluctuations which appear in the expressions for the effective cooling velocity (equation (C.20) or (C.21)) prevent the decoupling of the stress components, so that a full $6 \times 6$ system of linear equations must be solved. 


\section{Method A}

For Method $A$, slant-wire data at Positions $A, B, C_{A}$ and $D_{A}$, in addition to normal-wire data at position $N_{A}$, result in a system of five coupled linear equations. The sixth equation is obtained by utilizing slant-wire data obtained at Positions $E_{A}, F_{A}, G_{A}$ and $H_{A}$. The generalized response equation (derived in Appendix E) is given as:

$$
\begin{aligned}
& \left(s_{1} K_{12, E_{A}}-s_{2} K_{12, F_{A}}\right)\left(\overline{u^{\prime 2}} / U_{r}^{2}\right)+\left(s_{1}-s_{2}\right)\left(\overline{v^{2}} / U_{r}^{2}\right)+ \\
& \left(s_{1} K_{14, E_{A}}-s_{2} K_{14, F_{A}}\right)\left(\overline{w^{\prime 2}} / U_{r}^{2}\right)+2\left(s_{1} K_{10, E_{A}}-s_{2} K_{10, F_{A}}\right)\left(\overline{u^{\prime} v} / U_{r}^{2}\right)- \\
& \left(S_{1 v w}-S_{2 v w}\right)\left(\overline{v w^{\prime}} / U_{r}^{2}\right)=-\left(s_{1} f_{m, E_{A}}-s 2 f_{m, F_{A}}\right) / 2
\end{aligned}
$$

where

$$
\begin{aligned}
& s_{1}=-8\left[\left(f_{u} f_{w}\right)_{F_{A}}-\left(f_{m} K_{13}\right)_{F_{A}}\left(\Pi_{F_{A}}+\Pi_{G_{A}}\right)\right]\left(\Pi_{E_{B}}-\Pi_{H_{B}}\right)\left(f_{m}\right)_{E_{A}} \\
& s_{2}=-8\left[\left(f_{u} f_{w}\right)_{E_{A}}-\left(f_{m} K_{13}\right)_{E_{A}}\left(\Pi_{E_{A}}+\Pi_{F_{A}}\right)\right]\left(\Pi_{F_{A}}-\Pi_{G_{A}}\right)\left(f_{m}\right)_{F_{A}}
\end{aligned}
$$

and,

$$
\begin{aligned}
S_{1 v w}= & 16\left[\left(f_{u} f_{w}\right)_{F_{A}}-K_{13, F_{A}}\left(f_{m}\right)_{F_{A}}\left(\Pi_{F_{A}}+\Pi_{G_{A}}\right)\right] \times \\
& {\left[\left(f_{v} f_{w}\right)_{E_{A}}-K_{11, E_{A}}\left(f_{m}\right)_{E_{A}}\left(\Pi_{E_{A}}+\Pi_{H_{A}}\right)\right] } \\
S_{2 v w}= & 16\left[\left(f_{u} f_{w}\right)_{E_{A}}-K_{13, E_{A}}\left(f_{m}\right)_{E_{A}}\left(\Pi_{E_{A}}+\Pi_{H_{A}}\right)\right] \times \\
& {\left[\left(f_{v} f_{w}\right)_{F_{A}}-K_{11, F_{A}}\left(f_{m}\right)_{F_{A}}\left(\Pi_{F_{A}}+\Pi_{G_{A}}\right)\right] }
\end{aligned}
$$

and where $\Pi$ is given by equation (C.35) and $K_{10}$ through $K_{14}$ are given by equation (E.7).

\section{Method B}

For Method $B$, slant-wire data at Positions $A, B, C_{B}$ and $D_{B}$, in addition to normal-wire data at position $N_{B}$, result in a system of five coupled linear equations. The sixth equation is obtained by utilizing slant-wire data obtained at Positions $E_{B}, F_{B}, G_{B}$ and $H_{B}$. The generalized response equation (derived in Appendix $\mathrm{C}$ of reference [45]) is given as:

$$
\begin{aligned}
& \left(s_{1}-s_{2}\right)\left(\overline{u^{2}} / U^{2}\right)+\left(s_{1} K_{12, E_{B}}-s_{2} K_{12, F_{B}}\right)\left(\overline{v^{\prime 2}} / U^{2}\right)+ \\
& \left(s_{1} K_{14, E_{B}}-s_{2} K_{14, F_{B}}\right)\left(\overline{w^{\prime 2}} / U^{2}\right)+2\left(s_{1} K_{10, E_{B}}-s_{2} K_{10, F_{B}}\right)\left(\overline{u w^{\prime}} / U^{2}\right)+ \\
& \left(S_{1 v w}-S_{2 v w}\right)\left(\overline{v^{\prime} w^{\prime}} / U^{2}\right)=-\left(s_{1} f_{m, E_{B}}-s 2 f_{m, F_{B}}\right) / 2
\end{aligned}
$$

where

$$
\begin{aligned}
& s_{1}=-8\left[\left(f_{u} f_{w}\right)_{F_{B}}-K_{11, F_{B}}\left(f_{m}\right)_{F_{B}}\left(\Pi_{F_{B}}+\Pi_{G_{B}}\right)\right]\left(\Pi_{E_{B}}-\Pi_{H_{B}}\right)\left(f_{m}\right)_{E_{B}} \\
& s_{2}=-8\left[\left(f_{u} f_{w}\right)_{E_{B}}-K_{11, E_{B}}\left(f_{m}\right)_{E_{B}}\left(\Pi_{E_{B}}+\Pi_{H_{B}}\right)\right]\left(\Pi_{F_{B}}-\Pi_{G_{B}}\right)\left(f_{m}\right)_{F_{B}}
\end{aligned}
$$


and,

$$
\begin{aligned}
S_{1 v w}= & 16\left[\left(f_{u} f_{w}\right)_{F_{B}}-K_{11, F_{B}}\left(f_{m}\right)_{F_{B}}\left(\Pi_{F_{B}}+\Pi_{G_{B}}\right)\right] \times \\
& {\left[\left(f_{v} f_{w}\right)_{E_{B}}-K_{13, E_{B}}\left(f_{m}\right)_{E_{B}}\left(\Pi_{E_{B}}+\Pi_{H_{B}}\right)\right] } \\
S_{2 v w}= & 16\left[\left(f_{u} f_{w}\right)_{E_{B}}-K_{11, E_{B}}\left(f_{m}\right)_{E_{B}}\left(\Pi_{E_{B}}+\Pi_{H_{B}}\right)\right] \times \\
& {\left[\left(f_{v} f_{w}\right)_{F_{B}}-K_{13, F_{B}}\left(f_{m}\right)_{F_{B}}\left(\Pi_{F_{B}}+\Pi_{G_{B}}\right)\right] }
\end{aligned}
$$

and where $\Pi$ is given by equation (C.35) and $K_{10}$ through $K_{14}$ are given by equation (E.7).

\section{C.5 Solution of Mean and Turbulence Equations}

\section{C.5.1 Low Turbulence Intensity Flow}

For low intensity flows the velocity ratios $V / U$ and $W / U$ and, in turn, $V / U_{r}$ (Method $A$ ) or $V_{r} / U$ (Method $B$ ), are found by applying the Newton-Raphson procedure to the simultaneous equations (C.14.a) and (C.14.b). First-order approximations given by equations (C.15.a) and (C.15.b) are used as initial estimates in the calculation procedure. Once $V / U_{r}$ or $V_{r} / U$ (as applicable) is known, the optimum planes locations are determined from equations (C.39) or (C.40), as applicable. Data can then be taken at these locations or interpolated to these positions from data taken in fixed increments. These data are applied to equations (C.36) and (C.41), or (C.37) and (C.42) from which the Reynolds stress components are computed relative to the secondary turbulence coordinate system. The Reynolds stress tensor is then transformed to the primary coordinate system.

\section{C.5.2 Moderate Turbulence Intensity Flow}

For moderate intensity flows, the velocity ratios $V / U$ and $W / U$ are computed by simultaneously solving equations (C.10.a) and (C.10.b). These equations, however, require knowledge of the Reynolds stress components. To alleviate this problem, $V / U$ and $W / U$ are first estimated by solving equations (C.14.a) and (C.14.b). From these results, optimum planes are established and Reynolds stresses relative to the secondary coordinate system are computed using either equations (C.31) and (C.43), or (C.33) and (C.44), as applicable. The stress tensor is then transformed to the primary coordinates and substituted into equations (C.10.a) and (C.10.b) from which improved estimates of $V / U$ and $W / U$ are calculated. These improved estimates are, in turn, used to update the Reynolds stresses and so on until convergence is achieved.

A disadvantage of the above procedure is that two slant-wire probe configurations are required for Method $A$ in order to obtain data at Positions $A, B$ and $E_{A}$ through $H_{A}$. An alternative procedure would be to obtain slant-wire data at three (or more) fixed positions; $\theta=45,225$ and $315^{\circ}$, all of which are attainable with the offset prong slant-wire probe configuration shown in Fig. C.1.b. By 
applying slant-wire data at these positions and normal-wire data at Positions $C_{A}$ and $D_{A}$ to equation (C.36) the stress components, $\overline{w^{\prime 2}}, \overline{u^{\prime} w^{\prime}}$ and $\overline{v w^{\prime}}$ can be solved so as to minimize the error (in a least-squares sense) associated with the over-specified conditions. It should also be noted that in many practical flows, the secondary shear stress $\overline{v w}$ is at least an order of magnitude less than the other stress components and can be neglected. Under these circumstances there is no need for the straight prong slant-wire probe shown in Fig. C.1.c. 

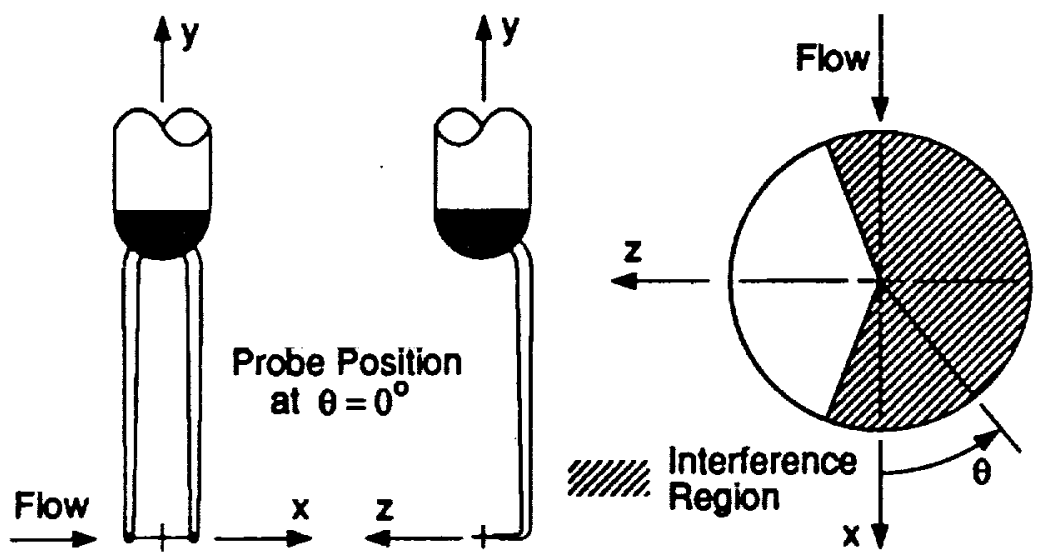

a) Normal-wire.
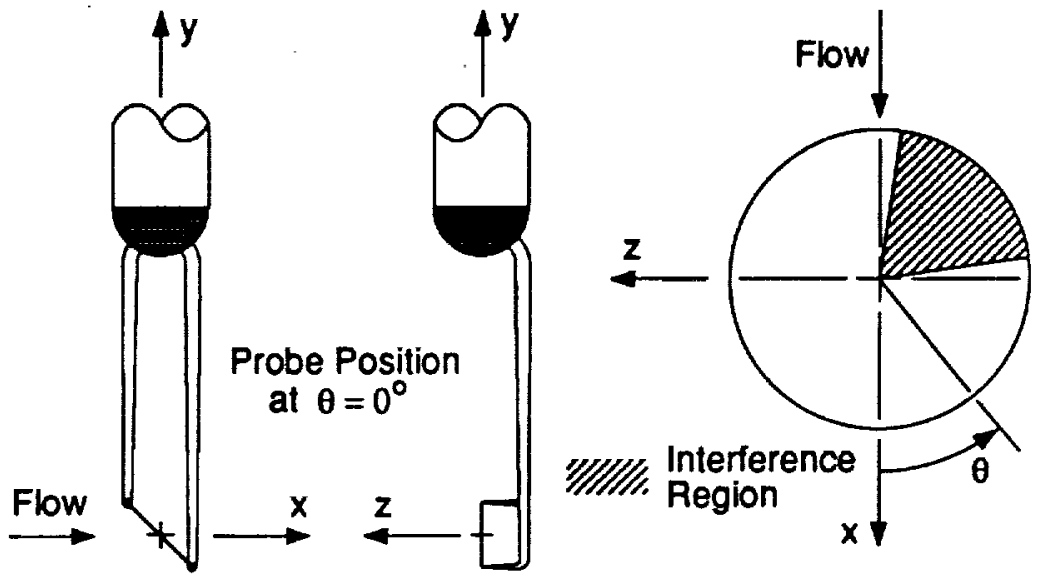

b) Offset prong slant-wire.
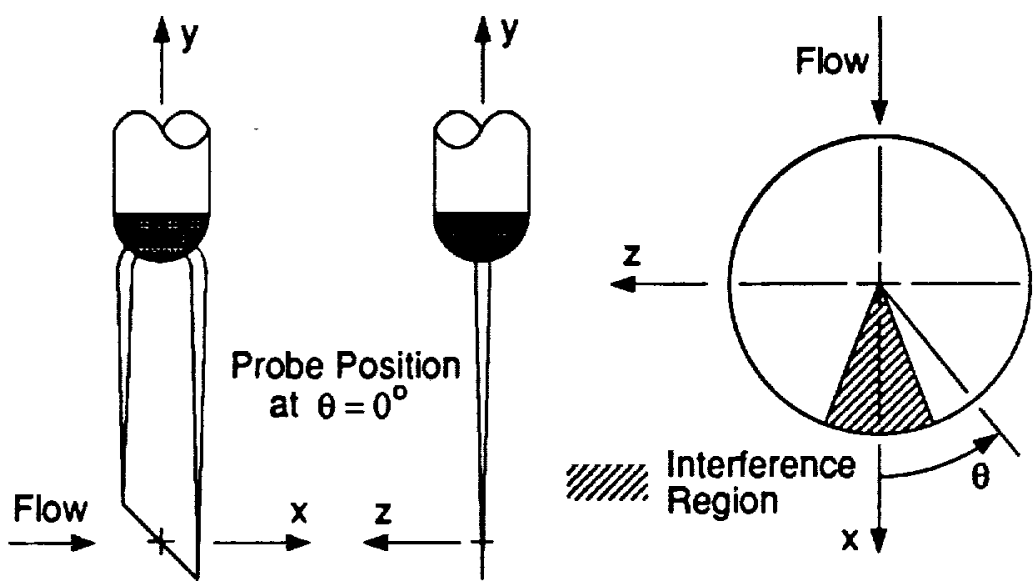

c) Straight prong slant-wire.

Fig. C.1. Hot-wire probe configurations for Method A. 


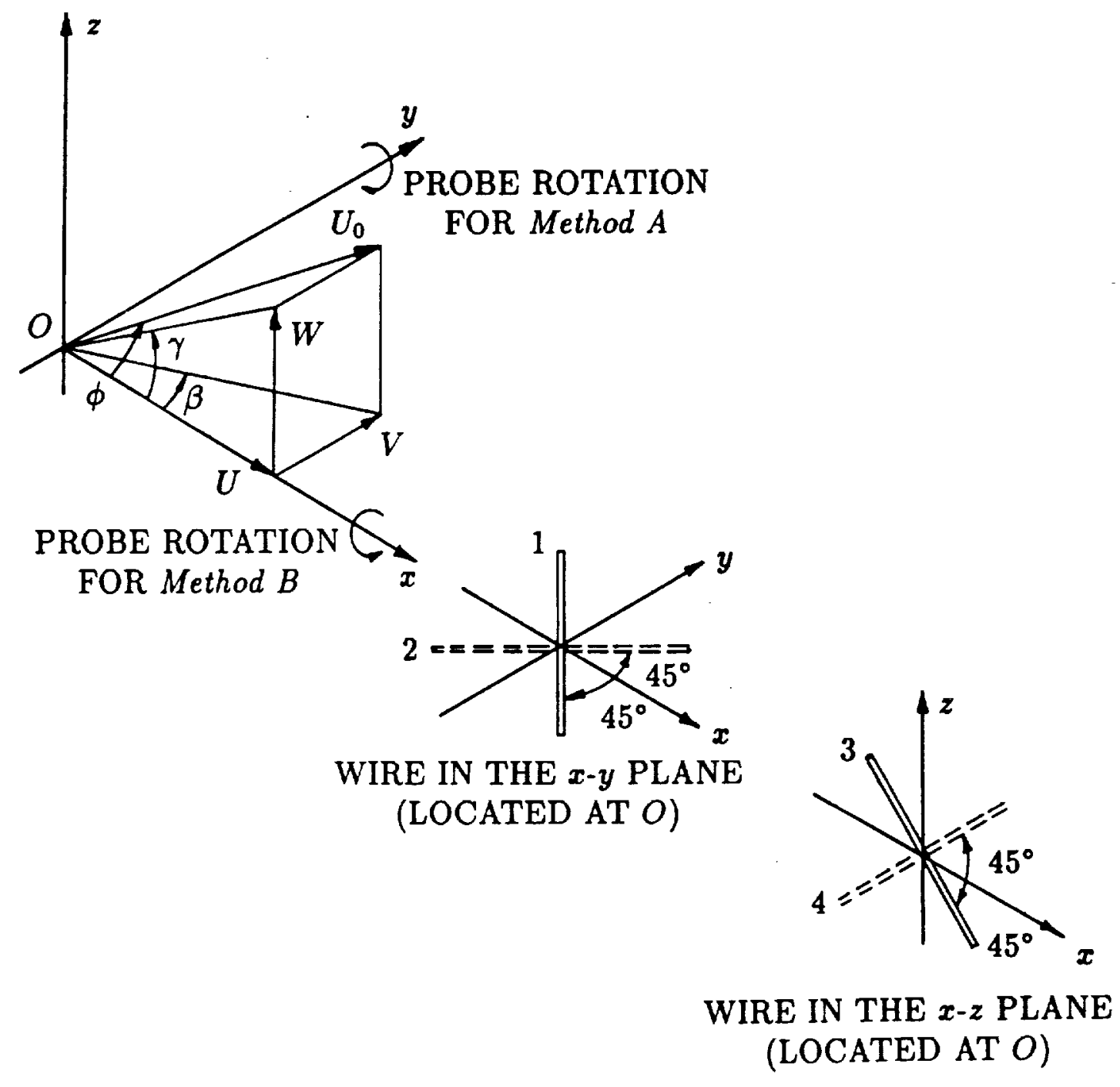

Fig. C.2. Hot-wire positions for mean flow determination. 


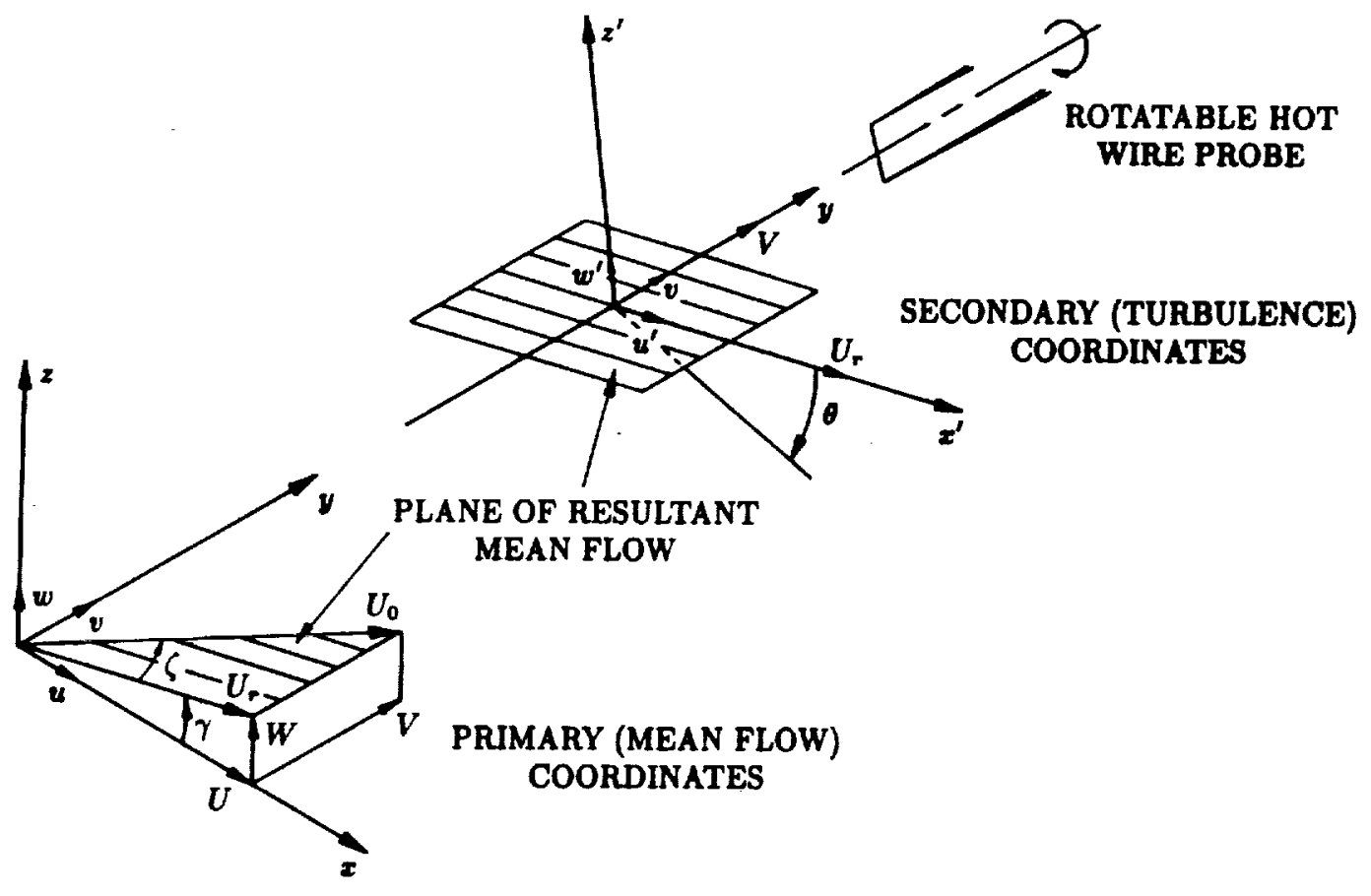

a) Method A.

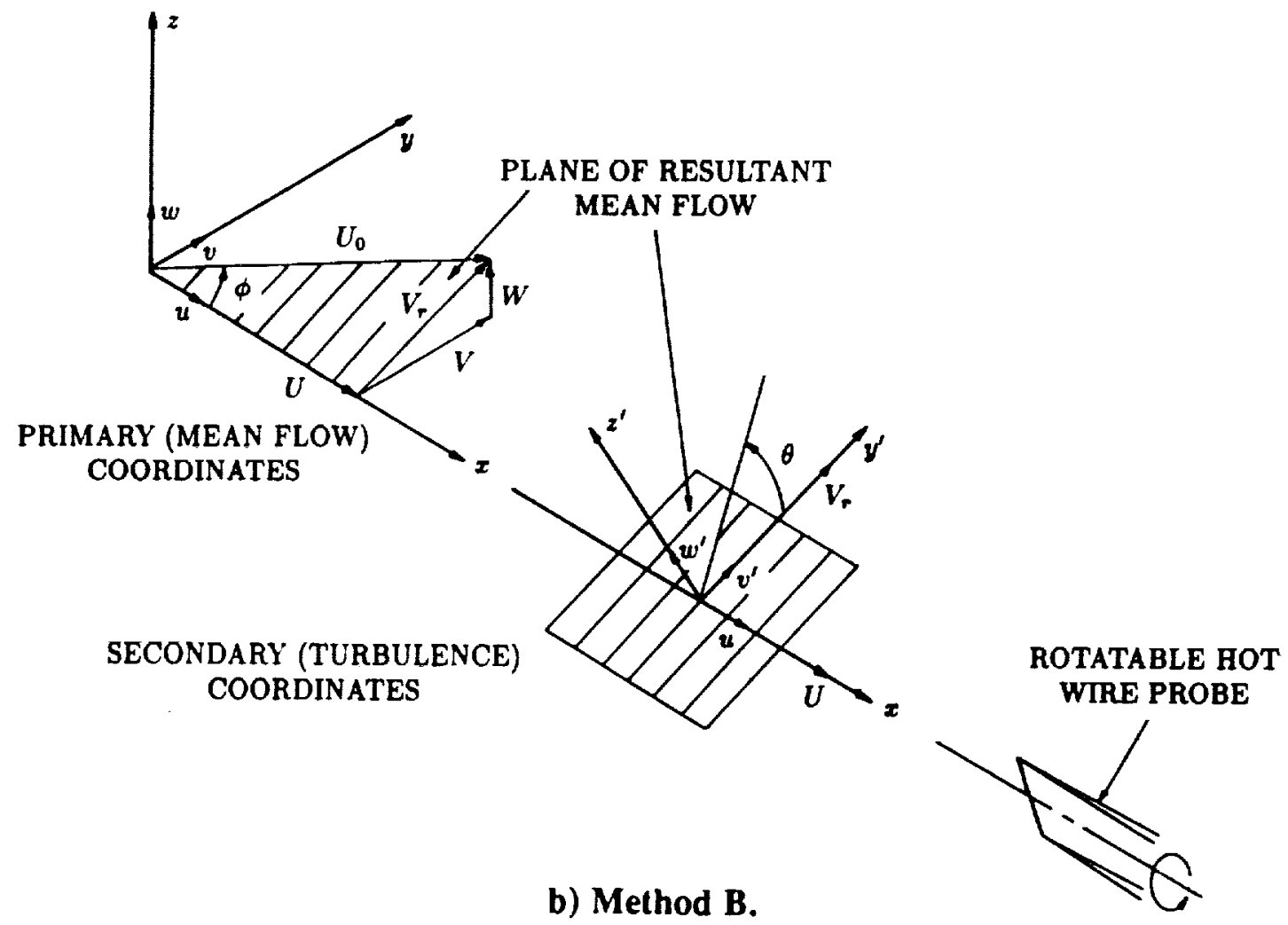

Fig. C.3. Reference coordinates for Reynolds stress determination. 


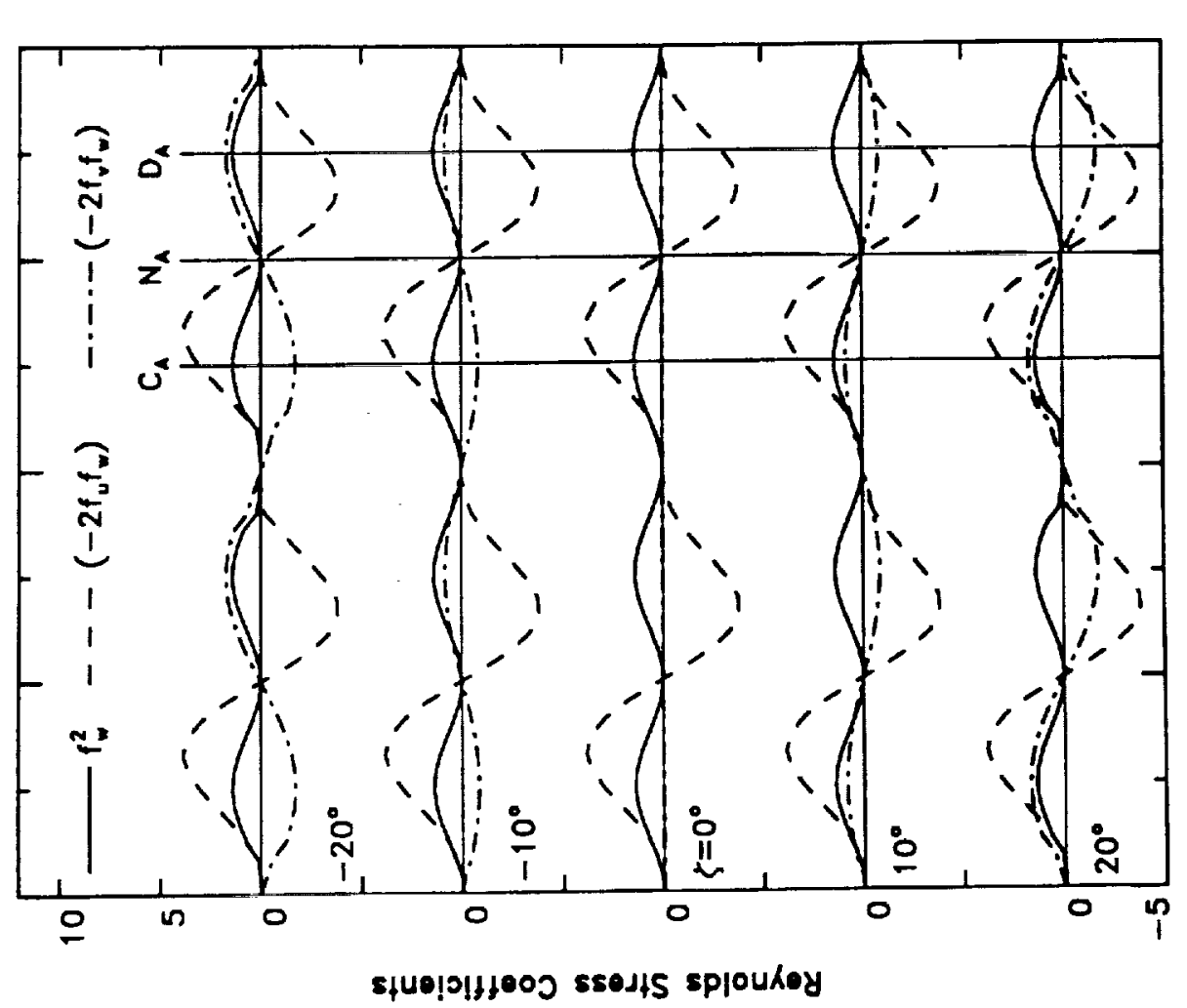

$\stackrel{\circ}{\circ}$
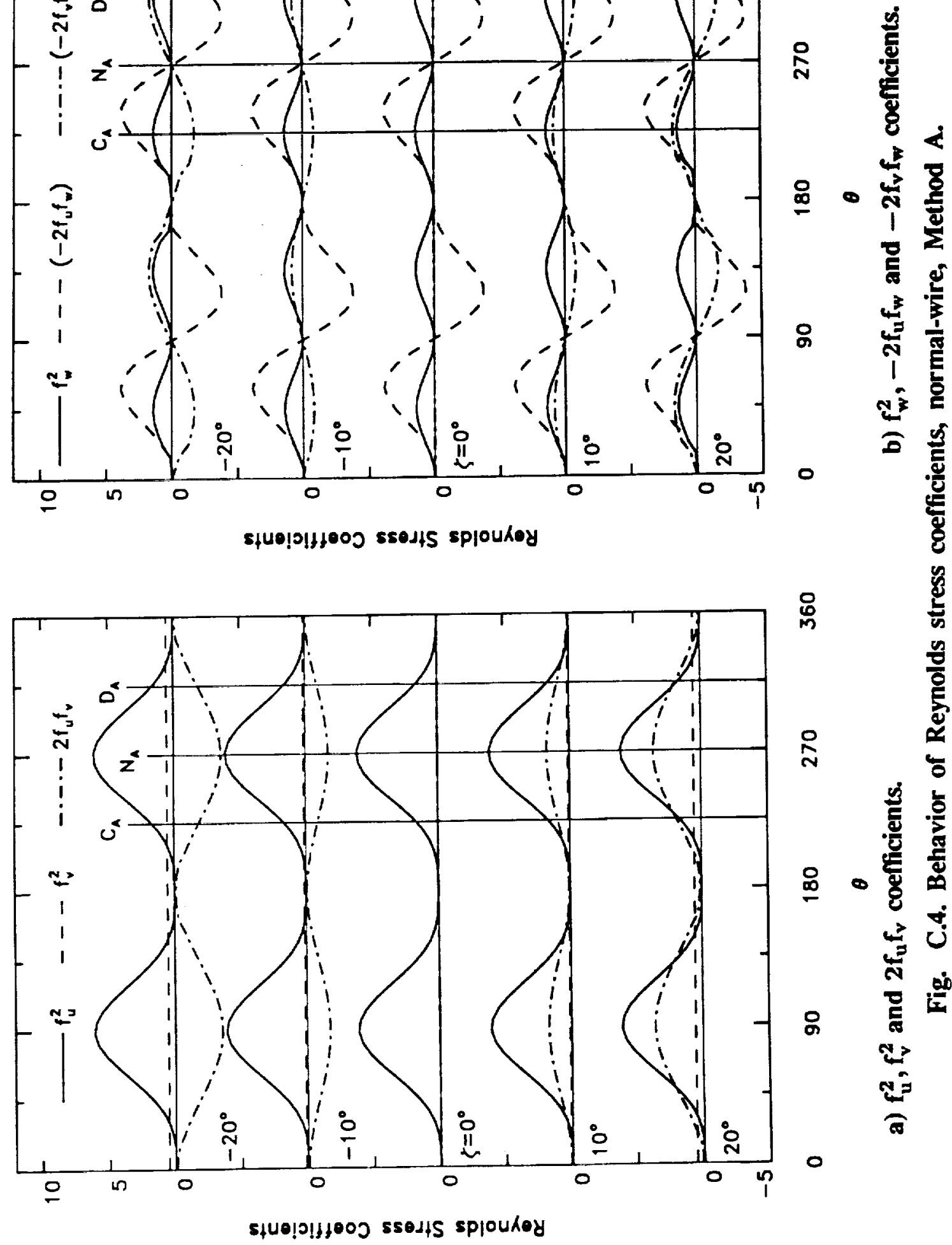

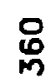

8

흥

$\stackrel{0}{N}$

요욜 


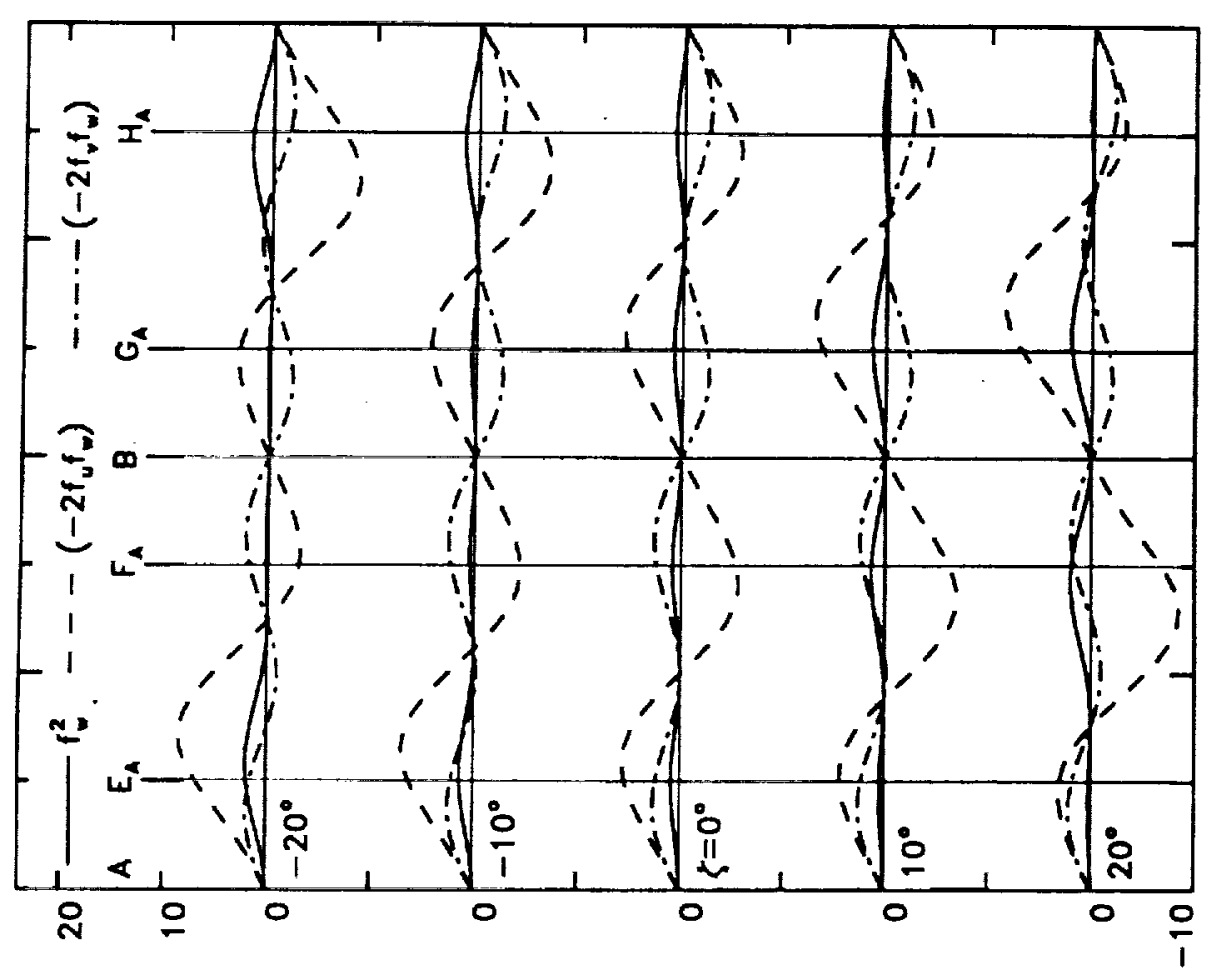

웅

squejगIf)og ssedtS sploukey


słuelopłpoj ssedts sploukey 


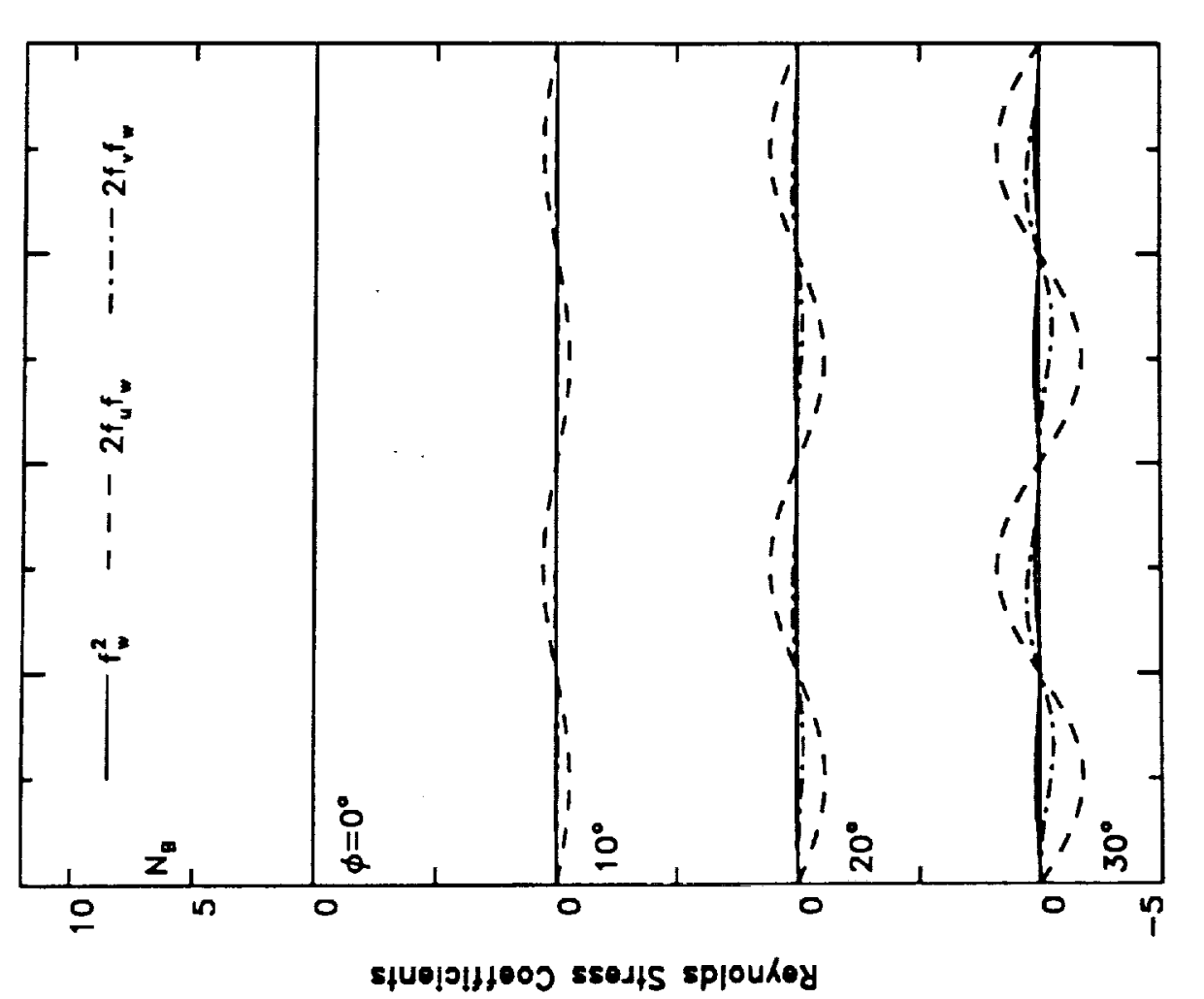

옹
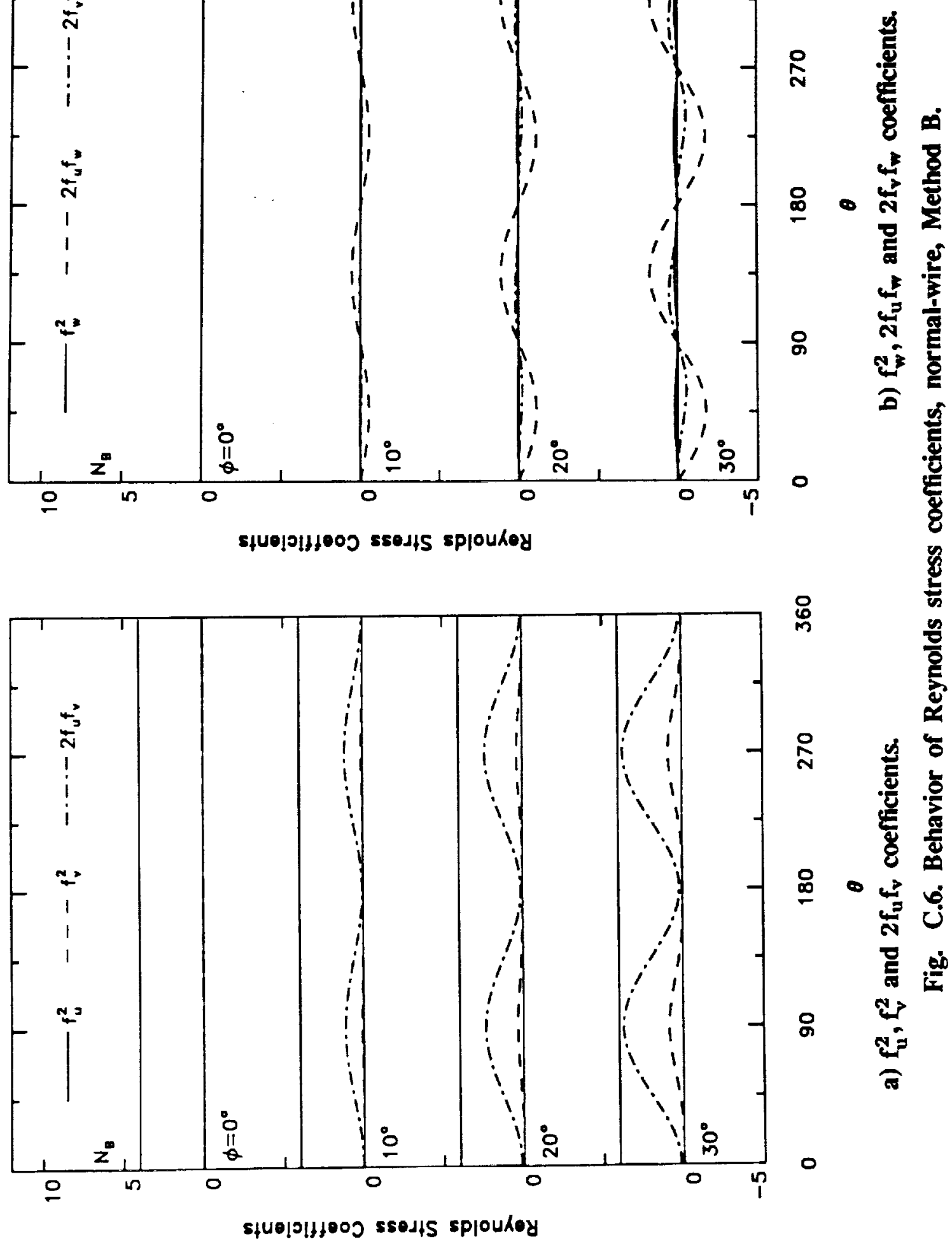


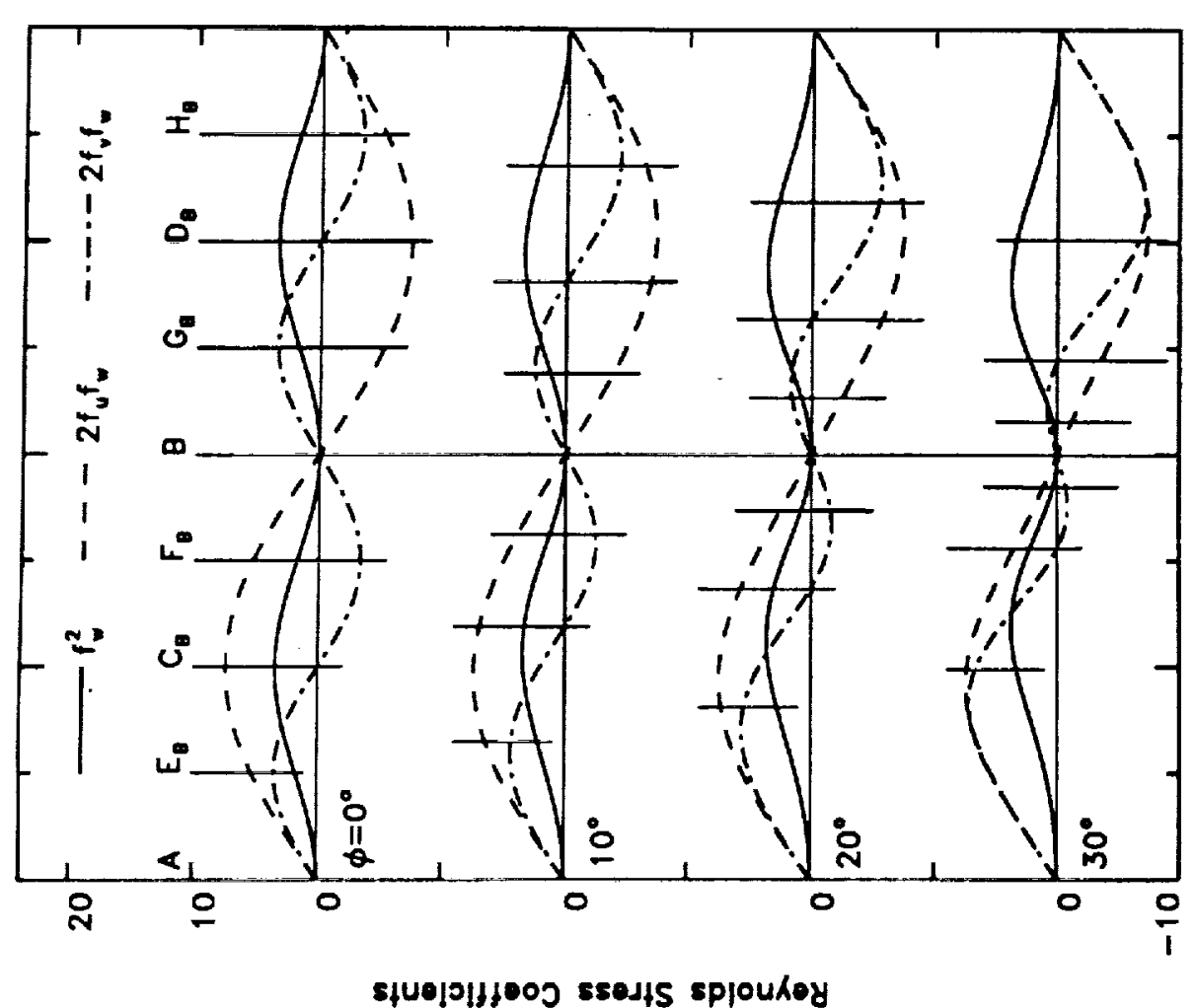

是

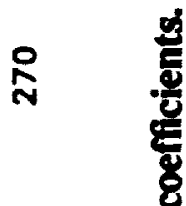

$\stackrel{0}{\circ} 0$

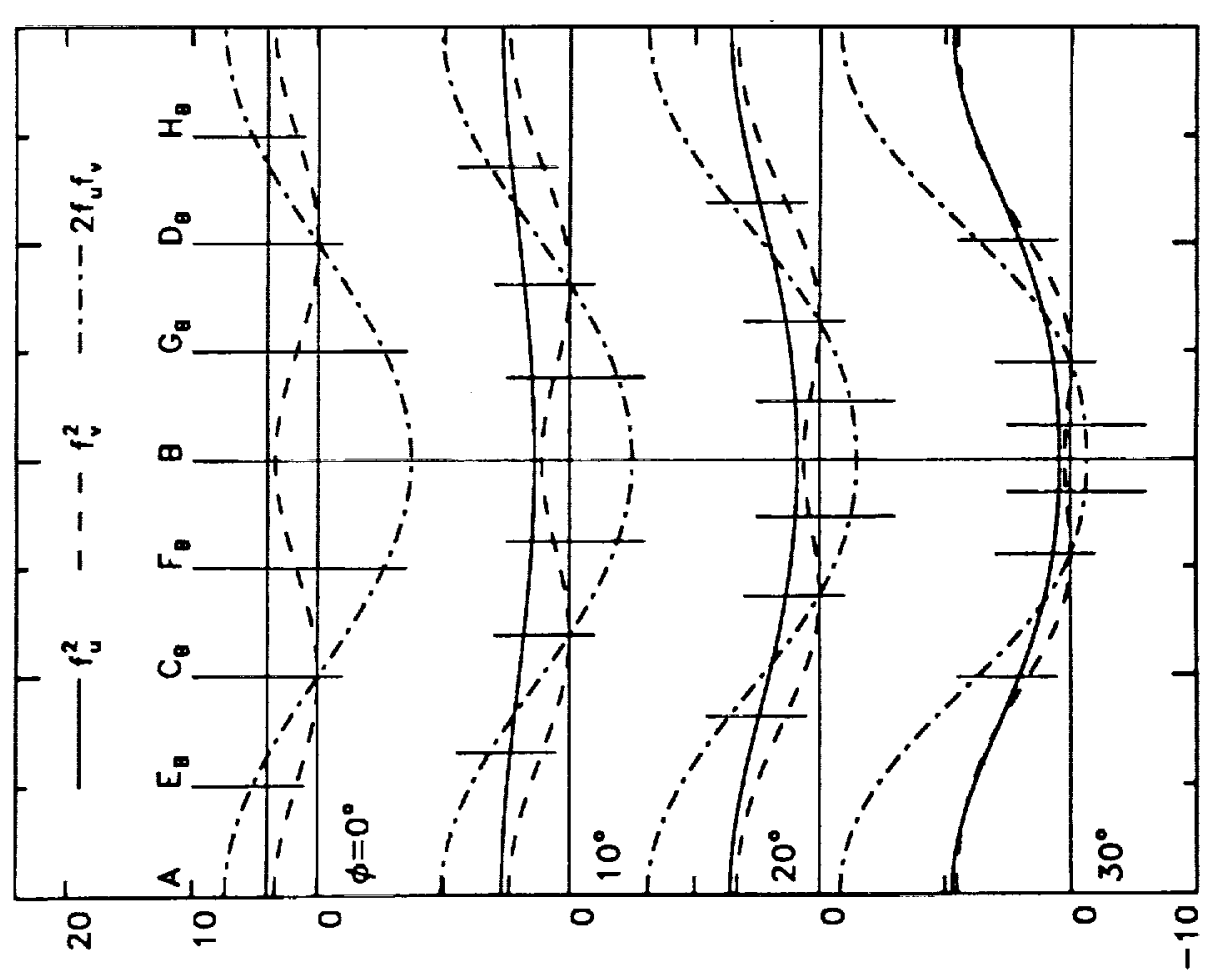

웅

- 8

용

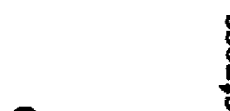

$=$

0 


\section{APPENDIX D \\ SUPPLEMENTARY ON MEAN FLOW EQUATION DEVELOPMENT}

This appendix contains supplementary information on the derivation of the mean flow response equations for Method $A$. The corresponding information for Method $B$ can be found in Appendix A of reference [45].

\section{D.1 Effective Cooling Velocity}

In order to develop an expression for the effective cooling velocity, the instantaneous total velocity vector must be decomposed into components which act normal and tangential to the wire. Fig. D.1 shows these decomposed components acting on a wire positioned in the $x^{\prime \prime}-y$ plane. In Fig. D.1, the $U_{1}, U_{2}$ and $U_{3}$ velocity components are directed along the $x^{\prime \prime}, y, z^{\prime \prime}$ coordinate axes, respectively, which rotate with the wire about the $y$-axis and are related to the laboratory (primary) $x, y, z$ coordinate system through the rotation angle $\lambda$. The normal, parallel and binormal effective cooling velocity components acting on a wire which is rotated through an arbitrary angle $\lambda$ about the $y$-axis relative to the $x, y, z$ primary coordinate system are:

$$
\begin{aligned}
\left(U_{e}\right)_{N} & =h_{N}\{[(U+u) \cos \lambda-(W+w) \sin \lambda] \sin \alpha+(V+v) \cos \alpha\} \\
\left(U_{e}\right)_{P} & =k\{[(U+u) \cos \lambda-(W+w) \sin \lambda] \cos \alpha-(V+v) \sin \alpha\} \\
\left(U_{e}\right)_{B N} & =h_{B N}\{(U+u) \sin \lambda+(W+w) \cos \lambda\}
\end{aligned}
$$

where $h_{N}, k$, and $h_{B N}$ represent weighting factors in the normal, parallel and binormal directions, respectively. The total effective cooling velocity is the Euclidian sum of the individual components:

$$
U_{e}^{2}=\left(U_{e}\right)_{N}^{2}+\left(U_{e}\right)_{P}^{2}+\left(U_{e}\right)_{B N}^{2}
$$

Substitution of equations (D.1), (D.2) and (D.3) into (D.4) yields the exact expression for the effective cooling velocity for a hot-wire rotated an arbitrary angle 
$\lambda$ about the $y$-axis:

$$
\begin{aligned}
U_{e}^{2}=K_{0} U^{2} & \left\{K_{12}\left[1+2\left(\frac{u}{U}\right)+\left(\frac{u^{2}}{U^{2}}\right)\right]\right. \\
+ & {\left[\left(\frac{V^{2}}{U^{2}}\right)+2\left(\frac{v}{U}\right)\left(\frac{V}{U}\right)+\left(\frac{v^{2}}{U^{2}}\right)\right] } \\
+ & K_{14}\left[\left(\frac{W^{2}}{U^{2}}\right)+2\left(\frac{w}{U}\right)\left(\frac{W}{U}\right)+\left(\frac{w^{2}}{U^{2}}\right)\right] \\
+ & 2 K_{10}\left[\left(\frac{V}{U}\right)+\left(\frac{v}{U}\right)+\left(\frac{u}{U}\right)\left(\frac{V}{U}\right)+\left(\frac{u v}{U^{2}}\right)\right] \\
& -2 K_{13}\left[\left(\frac{W}{U}\right)+\left(\frac{w}{U}\right)+\left(\frac{u}{U}\right)\left(\frac{W}{U}\right)+\left(\frac{u w}{U^{2}}\right)\right] \\
& -2 K_{11}\left[\left(\frac{V}{U}\right)\left(\frac{W}{U}\right)+\left(\frac{v}{U}\right)\left(\frac{W}{U}\right)+\left(\frac{w}{U}\right)\left(\frac{V}{U}\right)+\left(\frac{v w}{U^{2}}\right)\right]
\end{aligned}
$$

where,

$$
\begin{aligned}
& K_{10}=K_{2} \cos \lambda \\
& K_{11}=K_{2} \sin \lambda \\
& K_{12}=K_{1} \cos ^{2} \lambda+h_{B N}^{2} K_{3} \sin ^{2} \lambda \\
& K_{13}=\left(K_{1}-h_{B N}^{2} K_{3}\right) \sin \lambda \cos \lambda \\
& K_{14}=K_{1} \sin ^{2} \lambda+h_{B N}^{2} K_{3} \cos ^{2} \lambda
\end{aligned}
$$

and,

$$
\begin{aligned}
& K_{0}=\left(h_{N}^{2} \cos ^{2} \alpha+k^{2} \sin ^{2} \alpha\right) \\
& K_{1}=\left(h_{N}^{2} \sin ^{2} \alpha+k^{2} \cos ^{2} \alpha\right) / K_{0} \\
& K_{2}=\left(h_{N}^{2}-k^{2}\right) \sin \alpha \cos \alpha / K_{0} \\
& K_{3}=1 / K_{0}
\end{aligned}
$$

Time-averaging equation (D.5) yields:

$$
\begin{aligned}
\overline{U_{e}^{2}}=K_{0} U^{2} & \left\{K_{12}\left[1+\frac{\overline{u^{2}}}{U^{2}}\right]+\left[\left(\frac{V}{U}\right)^{2}+\frac{\overline{v^{2}}}{U^{2}}\right]\right. \\
& +K_{14}\left[\left(\frac{W}{U}\right)^{2}+\frac{\overline{w^{2}}}{U^{2}}\right]+2 K_{10}\left[\left(\frac{V}{U}\right)+\frac{\overline{u v}}{U^{2}}\right] \\
& \left.-2 K_{13}\left[\left(\frac{W}{U}\right)+\frac{\overline{u w}}{U^{2}}\right]-2 K_{11}\left[\left(\frac{V}{U}\right)\left(\frac{W}{U}\right)+\frac{\overline{v w}}{U^{2}}\right]\right\}
\end{aligned}
$$

The expressions for the effective cooling velocity at wire positions $1,2,3$ and 4 in Fig. 4.1 are obtained by substituting the corresponding rotation angles $(\lambda=$ $0,180,45$ and $315^{\circ}$ ) into equation (D.8). At wire positions 1 and 2, where a 
slant-wire $\left(\alpha=45^{\circ}\right)$ is utilized, the following expressions result:

$$
\begin{aligned}
\left(\overline{U_{e}^{2}}\right)_{1}= & K_{0,1} U^{2}\left\{K_{1,1}\left[1+\frac{\overline{u^{2}}}{U^{2}}\right]+\left[\left(\frac{V}{U}\right)^{2}+\frac{\overline{v^{2}}}{U^{2}}\right]\right. \\
& \left.+K_{3,1} h_{B N}^{2}\left[\left(\frac{W}{U}\right)^{2}+\frac{\overline{w^{2}}}{U^{2}}\right]+2 K_{2,1}\left[\left(\frac{V}{U}\right)+\frac{\overline{u v}}{U^{2}}\right]\right\} \\
\left(\overline{U_{e}^{2}}\right)_{2}= & K_{0,2} U^{2}\left\{K_{1,2}\left[1+\frac{\overline{u^{2}}}{U^{2}}\right]+\left[\left(\frac{V}{U}\right)^{2}+\frac{\overline{v^{2}}}{U^{2}}\right]\right. \\
& \left.+K_{3,2} h_{B N}^{2}\left[\left(\frac{W}{U}\right)^{2}+\frac{\overline{w^{2}}}{U^{2}}\right]-2 K_{2,2}\left[\left(\frac{V}{U}\right)+\frac{\overline{u v}}{U^{2}}\right]\right\}
\end{aligned}
$$

A normal-wire $\left(\alpha=0^{\circ}\right)$ is utilized at positions 3 and 4 , for which the coefficient $K_{2}=0$. The expressions for the effective cooling velocity at these positions are:

$$
\begin{aligned}
\left(\overline{U_{e}^{2}}\right)_{3}= & K_{0,3} U^{2}\left\{\frac{1}{2}\left(K_{1,3}+h_{B N}^{2} K_{3,3}\right)\left[1+\frac{\overline{u^{2}}}{U^{2}}\right]\right. \\
& +\left[\left(\frac{V}{U}\right)^{2}+\frac{\overline{v^{2}}}{U^{2}}\right]+\frac{1}{2}\left(K_{1,3}+h_{B N}^{2} K_{3,3}\right)\left[\left(\frac{W}{U}\right)^{2}+\frac{\overline{w^{2}}}{U^{2}}\right] \\
& \left.-\left(K_{1,3}-h_{B N}^{2} K_{3,3}\right)\left[\left(\frac{W}{U}\right)+\frac{\overline{u w}}{U^{2}}\right]\right\} \\
\left(\overline{U_{e}^{2}}\right)_{4}= & K_{0,4}^{0} U^{2}\left\{\frac{1}{2}\left(K_{1,4}+h_{B N}^{2} K_{3,4}\right)\left[1+\frac{\overline{u^{2}}}{U^{2}}\right]\right. \\
& +\left[\left(\frac{V}{U}\right)^{2}+\frac{\overline{v^{2}}}{U^{2}}\right]+\frac{1}{2}\left(K_{1,4}+h_{B N}^{2} K_{3,4}\right)\left[\left(\frac{W}{U}\right)^{2}+\frac{\overline{w^{2}}}{U^{2}}\right] \\
& \left.+\left(K_{1,4}-h_{B N}^{2} K_{3,4}\right)\left[\left(\frac{W}{U}\right)+\frac{\overline{u w}}{U^{2}}\right]\right\}
\end{aligned}
$$

\section{D.2 Cooling Coefficient Modeling}

In general, the cooling coefficients $h_{N}, k$ and $h_{B N}$ are functions of the wire rotation angle. The tangential cooling coefficient, $k$, is modeled by a correlation developed by Friehe and Schwarz [63]. This correlation accounts for the dependency of $k$ on the wire orientation angle $(\psi)$ :

$$
k(\psi)=\left\{\left[1-b_{r}\left(1-\cos ^{1 / 2} \psi\right)\right]^{4}-\cos ^{2} \psi\right\}^{1 / 2} / \sin \psi
$$

where,

$$
b_{r}=\frac{1-\left[k_{r}^{2} \sin ^{2} \alpha+\cos ^{2} \alpha\right]^{1 / 4}}{\left(1-\cos ^{1 / 2} \alpha\right)}
$$


and where $\psi$ is the angle of incidence between the total velocity vector and the normal to the axis of the wire. The functional dependency of $\psi$ on probe rotation angle for Method $A$ is given by:

$$
\psi=\pi / 2-\cos ^{-1}\left[\frac{\cos \alpha \cos \lambda-(V / U) \sin \alpha-(W / U) \cos \alpha \sin \lambda}{\left(1+(V / U)^{2}+(W / U)^{2}\right)^{1 / 2}}\right]
$$

The reference value of $k_{r}$ is determined from a calibration procedure in fullydeveloped pipe flow. This procedure is described in detail by Al-Beirutty in Appendix $\mathrm{E}$ of reference [45].

In order to demonstrate the behavior of $k$, it is expedient to examine its behavior relative to the $x^{\prime}, y, z^{\prime}$ coordinate system shown in Fig. C.3.a. In this coordinate system, the mean transverse velocity component directed along the $w^{\prime}$-axis has been nulled out. The probe rotation angle for the $x^{\prime}, y, z^{\prime}$ coordinate system is specified as $\theta$ and is related to the rotation angle in the $x, y, z$ coordinate system by:

$$
\theta=\lambda+\tan ^{-1}(W / U)
$$

Relative to this new coordinate system, the dependence of the wire orientation angle $(\psi)$ on probe rotation angle $(\theta)$ is given by:

$$
\psi=\pi / 2-\cos ^{-1}[\cos \zeta \cos \alpha \cos \theta-\sin \zeta \sin \alpha]
$$

where $\zeta$ is the pitch flow angle defined as:

$$
\zeta=\tan ^{-1}\left(V / U_{r}\right)
$$

A plot of $k$ versus probe rotation angle $\theta$ for various values of $\zeta$ is shown in Fig. D.2.a for a normal-wire, and in Fig. D.2.b for a slant-wire.

For Method $B$, Arterberry [47] determined that the normal and binormal cooling coefficients could be specified as constants; $\left(h_{N}=1.0, h_{B N}=0.9\right)$. This is a reasonable assumption since for low-to-moderate skew angles, the normal velocity component is much larger then the binormal component, regardless of probe rotation angle. For Method $A$, however, equations (D.1) and (D.3) show that the normal and binormal velocity components for the slant-wire are strongly dependent on probe rotation angle, even under unskewed conditions. For example, consider a slant-wire in unskewed flow conditions $(V=W=0)$ in the absence of turbulence fluctuations. In the limit as $\lambda$ approaches 0 or $180^{\circ}$ (refer to Fig. D.1 and equations (D.1) and (D.3)), the effective normal velocity component $\left(U_{e}\right)_{N}$ approaches $h_{N} U \sin \alpha$ and the effective binormal component is zero. For this case, $h_{N}=1.0$ and $h_{B N}=0.9$, since these positions are equivalent to positions associated with Method $B$. In the limit as $\lambda$ approaches $\pm 90^{\circ}$, the normal velocity component approaches zero and the effective binormal component approaches 
the total velocity i.e., $\left(U_{e}\right)_{B N}=h_{B N} U=U_{0}$. For this case, $h_{N}=0.9$ and $h_{B N}=1.0$. These limiting cases for the slant-wire were accounted for in the present study by specifying $h_{N}=1.0$ and $h_{B N}=0.9$ when $\left|U_{N}\right|>\left|U_{B N}\right|$, and $h_{N}=0.9$ and $h_{B N}=1.0$ when $\left|U_{B N}\right|>\left|U_{N}\right|$. For the normal-wire, over the useful range of rotation, and for zero-to-moderate skewness levels, the binormal velocity component is always significantly greater than the normal component, so that the $h_{N}$ and $h_{B N}$ can be specified as 0.9 and 1.0 , respectively. 

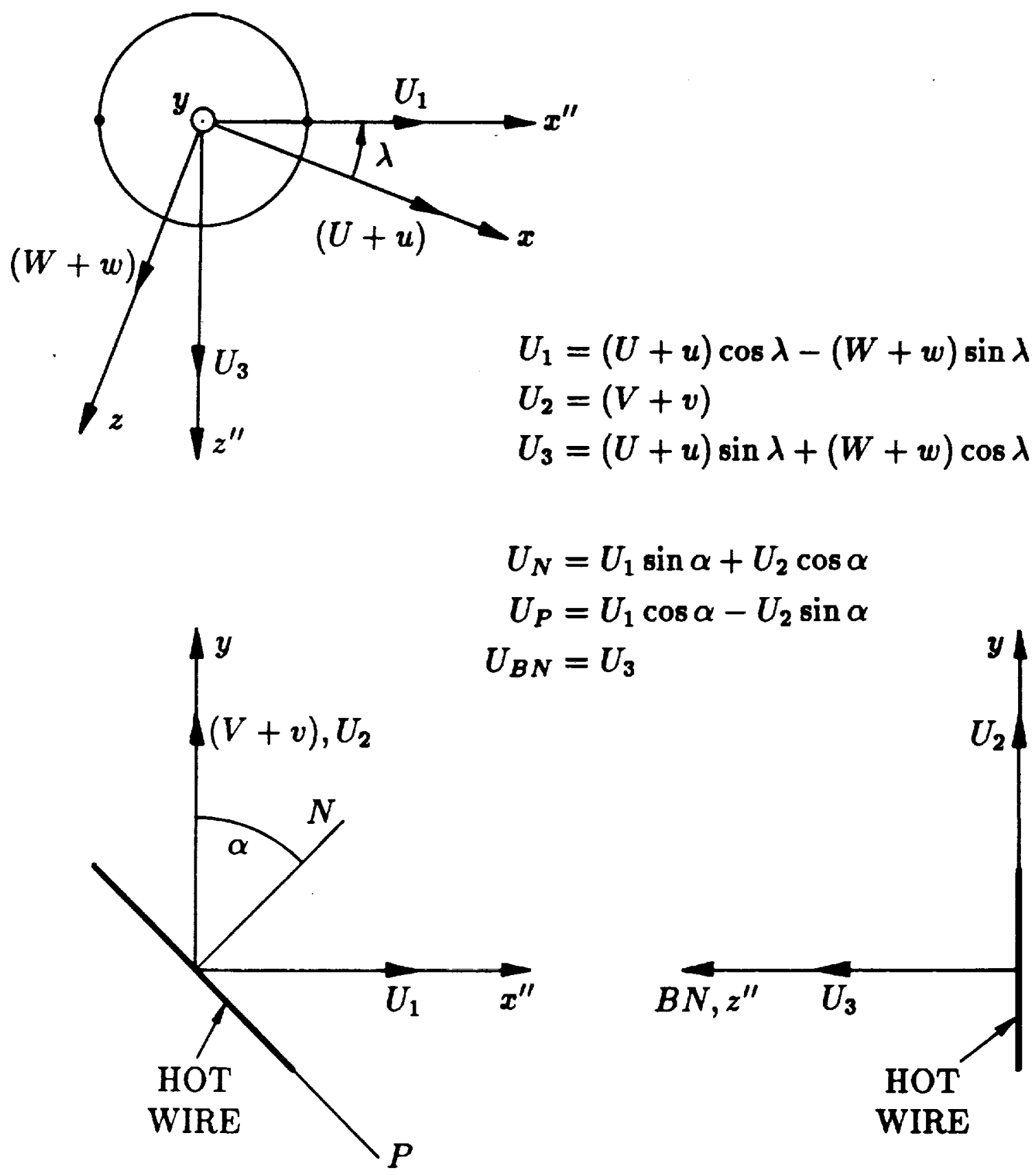

Fig. D.1. Effective cooling velocity for mean flow calculations. 


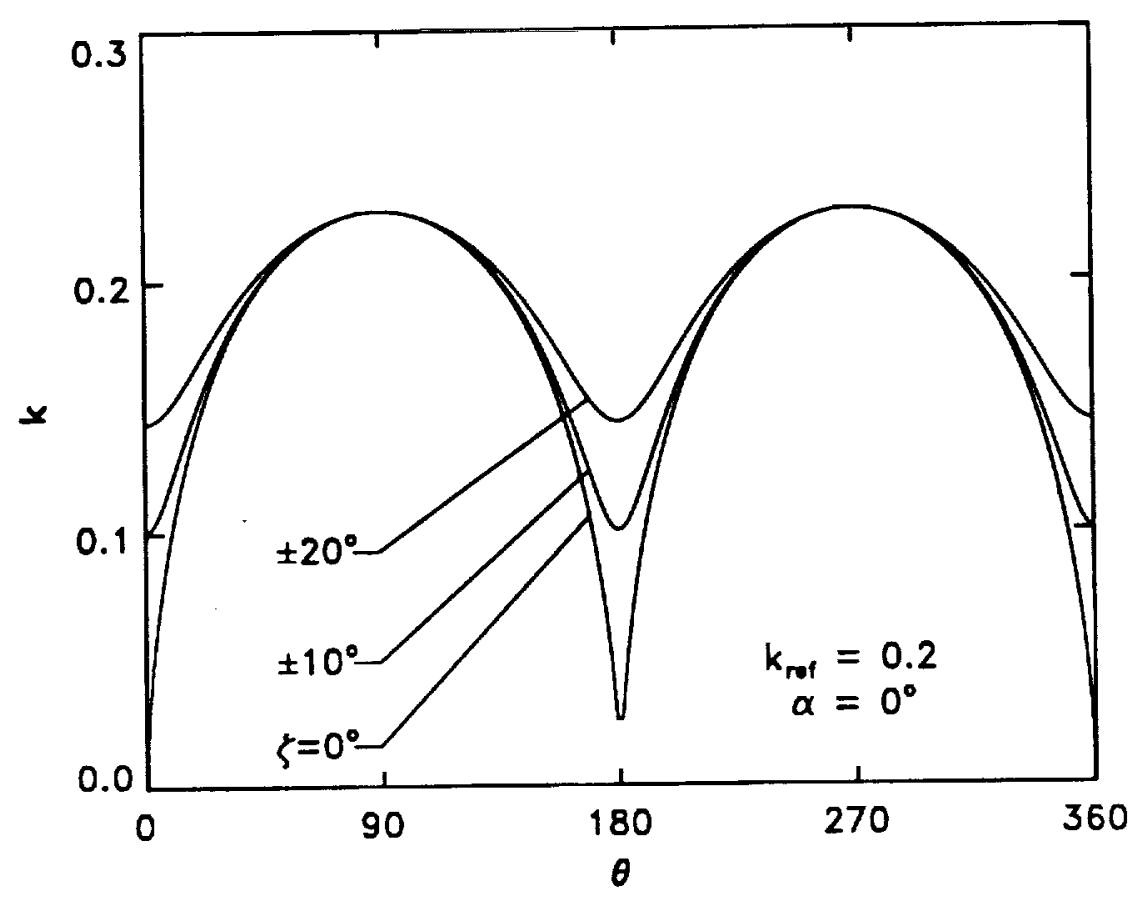

a) Normal-wire.

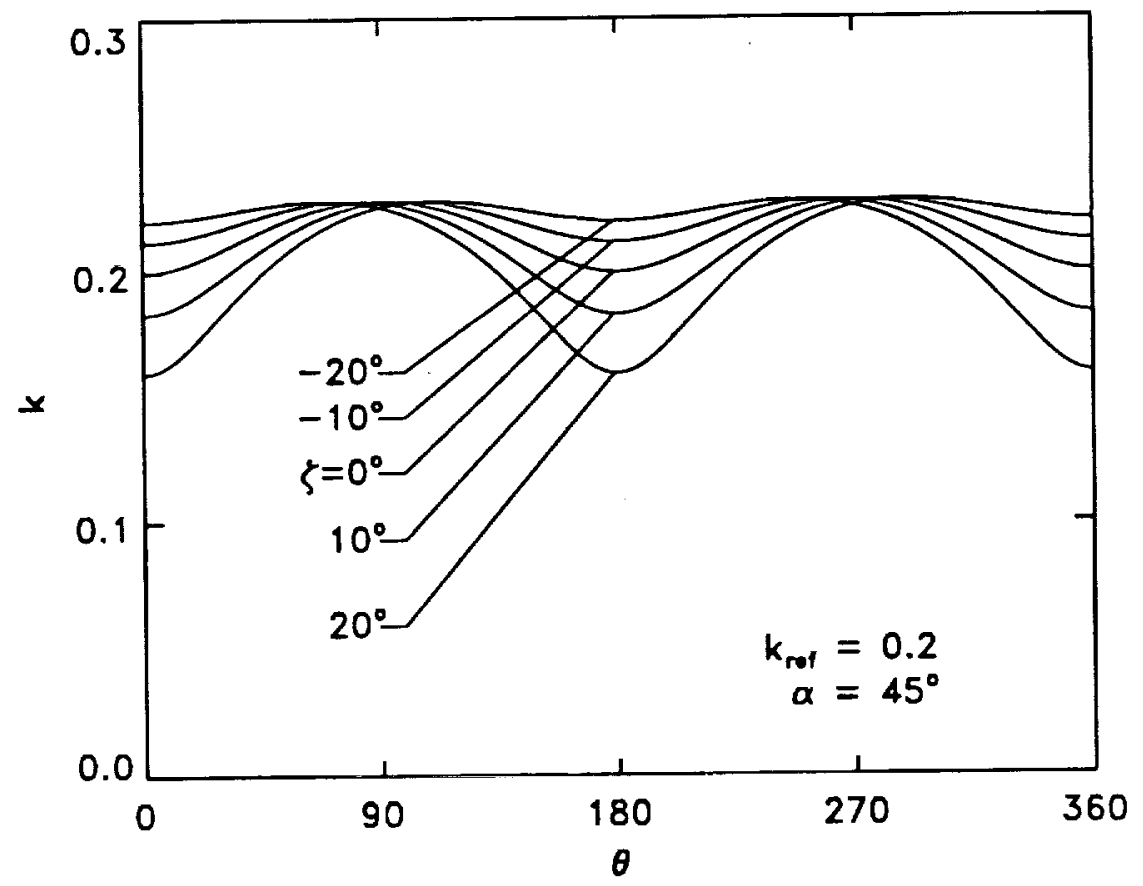

b) Slant-wire.

Fig. D.2. Tangential cooling coefficient behavior. 



\section{APPENDIX E \\ SUPPLEMENTARY ON TURBULENCE EQUATION DEVELOPMENT}

This appendix contains supplementary information on the derivation of the turbulence response equations for Method $A$. The corresponding information for Method B can be found in Appendices B and C of reference [45].

\section{E.1 Effective Cooling Velocity}

In order to develop an expression for the effective cooling velocity relative to the secondary turbulence coordinate system, the instantaneous total velocity vector must be decomposed into components which act normal and tangential to the wire. Fig. E.1 shows these decomposed components acting on a wire positioned in the $x^{\prime \prime}-y$ plane. In Fig. E.1, the $U_{1}, U_{2}$ and $U_{3}$ velocity components are directed along the $x^{\prime \prime}, y, z^{\prime \prime}$ coordinate axes, respectively, which rotate with the wire about the $y$-axis and are related to the turbulence (secondary) $x, y, z$ coordinate system through the rotation angle $\theta$. The normal, parallel and binormal effective cooling velocity components acting on a wire which is rotated through an arbitrary angle $\theta$ about the $y$-axis relative to the $x^{\prime}, y, z^{\prime}$ secondary coordinate system are:

$$
\begin{aligned}
\left(U_{e}\right)_{N} & =h_{N}\left\{\left[\left(U_{r}+u^{\prime}\right) \cos \theta-w^{\prime} \sin \theta\right] \sin \alpha+(V+v) \cos \alpha\right\} \\
\left(U_{e}\right)_{P} & =k\left\{\left[\left(U_{r}+u^{\prime}\right) \cos \theta-w^{\prime} \sin \theta\right] \cos \alpha-(V+v) \sin \alpha\right\} \\
\left(U_{e}\right)_{B N} & =h_{B N}\left\{\left(U_{r}+u^{\prime}\right) \sin \theta+w^{\prime} \cos \theta\right\}
\end{aligned}
$$

where $h_{N}, k$, and $h_{B N}$ represent weighting factors in the normal, parallel and binormal directions, respectively. The total effective cooling velocity is the Euclidian sum of the individual components:

$$
U_{e}^{2}=\left(U_{e}\right)_{N}^{2}+\left(U_{e}\right)_{P}^{2}+\left(U_{e}\right)_{B N}^{2}
$$

Substitution of equations (E.1), (E.2) and (E.3) into (E.4) yields the exact expression for the effective cooling velocity for a hot-wire rotated an arbitrary angle $\theta$ about the $y$-axis:

$$
\begin{aligned}
U_{e}^{2}=K_{0} U_{r}{ }^{2}\left\{f_{m}\right. & +f_{u}\left(u^{\prime} / U_{r}\right)+f_{v}\left(v / U_{r}\right)-f_{w}\left(w^{\prime} / U_{r}\right) \\
& +K_{12}\left(u^{\prime 2} / U_{r}{ }^{2}\right)+\left(v^{2} / U_{r}{ }^{2}\right)+K_{14}\left(w^{\prime 2} / U_{r}{ }^{2}\right) \\
& +2 K_{10}\left(u^{\prime} v / U_{r}{ }^{2}\right)-2 K_{13}\left(u^{\prime} w^{\prime} / U_{r}{ }^{2}\right) \\
& \left.-2 K_{11}\left(v w^{\prime} / U_{r_{0}}{ }^{2}\right)\right\}
\end{aligned}
$$


where,

$$
\begin{aligned}
f_{m} & =K_{12}+2 K_{10}\left(V / U_{r}\right)+\left(V / U_{r}\right)^{2} \\
f_{u} & =2 K_{12}+2 K_{10}\left(V / U_{r}\right) \\
f_{v} & =2 K_{10}+2\left(V / U_{r}\right) \\
f_{w} & =2 K_{13}+2 K_{11}\left(V / U_{r}\right)
\end{aligned}
$$

and,

$$
\begin{aligned}
& K_{10}=K_{2} \cos \theta \\
& K_{11}=K_{2} \sin \theta \\
& K_{12}=K_{1} \cos ^{2} \theta+h_{B N}^{2} K_{3} \sin ^{2} \theta \\
& K_{13}=\left(K_{1}-h_{B N}^{2} K_{3}\right) \sin \theta \cos \theta \\
& K_{14}=K_{1} \sin ^{2} \theta+h_{B N}^{2} K_{3} \cos ^{2} \theta
\end{aligned}
$$

and where the coefficients $K_{0}$ through $K_{3}$ are given by equation (D.7).

\section{E.2 Turbulence Response Equation}

The working expression for the mean-square voltage response of a hot-wire transducer in terms of the effective cooling velocity is given by equation (C.30) as:

$$
\frac{\overline{\left[\left(E^{2}-\bar{E}_{0}^{2}\right)^{2 / m}-\overline{\left(E^{2}-\bar{E}_{0}^{2}\right)^{2 / m}}\right]^{2}}}{\left[\overline{\left(E^{2}-\bar{E}_{0}^{2}\right)^{2 / m}}\right]^{2}}=\frac{\overline{\left[U_{e}^{2}-\overline{U_{e}^{2}}\right]^{2}}}{\left[\overline{U_{e}^{2}}\right]^{2}}
$$

Substitution of equation (E.5) and its time-averaged form into the RHS numerator of equation (E.8) yields:

$$
\begin{aligned}
\overline{\left[U_{e}{ }^{2}-\overline{U_{e}{ }^{2}}\right]^{2}}= & K_{0}{ }^{2} U_{r}{ }^{4}\left\{f_{u}{ }^{2}\left(\overline{u^{\prime 2}} / U_{r}{ }^{2}\right)+f_{v}{ }^{2}\left(\overline{v^{2}} / U_{r}{ }^{2}\right)\right. \\
& +f_{w}{ }^{2}\left(\overline{w^{\prime 2}} / U_{r}{ }^{2}\right)+2 f_{u} f_{v}\left(\overline{u^{\prime} v} / U_{r}{ }^{2}\right) \\
& \left.-2 f_{u} f_{w}\left(\overline{u^{\prime} w^{\prime}} / U_{r}{ }^{2}\right)-2 f_{v} f_{w}\left(\overline{v w^{\prime}} / U_{r}{ }^{2}\right)\right\} \\
& +O\left[2 f_{u} K_{12} K_{0}{ }^{2} U_{r}{ }^{4}\left(\overline{u^{\prime 3}} / U_{r}{ }^{3}\right)\right]
\end{aligned}
$$

and the RHS denominator of equation (E.8) becomes:

$$
\begin{aligned}
{\left[\overline{U_{e}{ }^{2}}\right]^{2}=} & {K_{0}}^{2} U_{r}^{4}\left\{f_{m}{ }^{2}+2 f_{m}\left[K_{12}\left(\overline{u^{\prime 2}} / U_{r}^{2}\right)+\left(\overline{v^{2}} / U_{r}^{2}\right)\right.\right. \\
& +K_{14}\left(\overline{u^{\prime 2}} / U_{r}{ }^{2}\right)+2 K_{10}\left(\overline{u^{\prime} v} / U_{r}{ }^{2}\right)-2 K_{13}\left(\overline{u^{\prime} w^{\prime}} / U_{r}{ }^{2}\right) \\
& \left.\left.-2 K_{11}\left(\overline{v w^{\prime}} / U_{r}{ }^{2}\right)\right]\right\}+O\left[{K_{0}}_{K_{12}} U_{r}{ }^{2}\left(\overline{u^{\prime 2}} / U_{r}{ }^{2}\right)\right]^{2}
\end{aligned}
$$

where the last terms on the RHS of equations (E.9) and (E.10) represent the order of the truncated terms. The first term in the LHS numerator of equation (E.8) is given by equation (C.6) as:

$$
\left(E^{2}-\bar{E}_{0}^{2}\right)^{2 / m}=\left(\bar{E}^{2}-\bar{E}_{0}^{2}\right)^{2 / m}\left[1+(4 / m) \frac{e \bar{E}}{\bar{E}^{2}-\bar{E}_{0}^{2}}+\frac{C \epsilon^{2}}{\bar{E}^{2}-\bar{E}_{0}^{2}}\right]
$$


where,

$$
C=2 / m+(4 / m)\left(\frac{2}{m}-1\right)\left(\frac{\bar{E}^{2}}{\bar{E}^{2}-\bar{E}_{0}^{2}}\right)
$$

Substitution of equation (E.11) and its time-averaged form into the LHS numerator of equation (E.8) yields:

$$
\overline{\left[\left(E^{2}-\bar{E}_{0}^{2}\right)^{2 / m}-\overline{\left.\left(E^{2}-\bar{E}_{0}^{2}\right)^{2 / m}\right]^{2}}\right.}=\left(\bar{E}^{2}-\bar{E}_{0}^{2}\right)^{4 / m}\left[(4 / m)^{2} \frac{\overline{e^{2}} \bar{E}^{2}}{\left(\bar{E}^{2}-\bar{E}_{0}^{2}\right)^{2}}\right]
$$

and the LHS denominator of equation (E.8) becomes:

$$
\left.\overline{\left[\left(E^{2}-\bar{E}_{0}^{2}\right)^{2 / m}\right.}\right]^{2}=\left(\bar{E}^{2}-\bar{E}_{0}^{2}\right)^{4 / m}\left[1+\frac{2 C \overline{e^{2}}}{\left(\bar{E}^{2}-\bar{E}_{0}^{2}\right)}\right]
$$

where third and higher-order voltage terms have been neglected in the development.

Substituting equations (E.9), (E.10), (E.13) and (E.14) into equation (E.8), and retaining only second-order terms, the following generalized turbulence response equation results:

$$
\begin{aligned}
& F_{u u}\left(\overline{u^{\prime 2}} / U_{r}{ }^{2}\right)+F_{v v}\left(\overline{v^{2}} / U_{r}^{2}\right)+F_{w w}\left(\overline{w^{\prime 2}} / U_{r}^{2}\right) \\
& +F_{u v}\left(\overline{u^{\prime} v} / U_{r}{ }^{2}\right)-F_{u w}\left(\overline{u^{\prime} w^{\prime}} / U_{r}{ }^{2}\right)-F_{v w}\left(\overline{v w^{\prime}} / U_{r}{ }^{2}\right)=F_{m m}
\end{aligned}
$$

where,

$$
\begin{aligned}
F_{u u} & =f_{u}{ }^{2}-2 f_{m} \Pi K_{12} \\
F_{v v} & =f_{v}{ }^{2}-2 f_{m} \Pi \\
F_{w w} & =f_{w}{ }^{2}-2 f_{m} \Pi K_{14} \\
F_{u v} & =2\left(f_{u} f_{v}-2 f_{m} \Pi K_{10}\right) \\
F_{u w} & =2\left(f_{u} f_{w}-2 f_{m} \Pi K_{13}\right) \\
F_{v w} & =2\left(f_{v} f_{w}-2 f_{m} \Pi K_{11}\right) \\
F_{m m} & =f_{m}{ }^{2} \Pi
\end{aligned}
$$

and,

$$
\Pi=\left(16 / m^{2}\right) \frac{\left[\overline{e^{2}} \bar{E}^{2} /\left(\bar{E}^{2}-\bar{E}_{0}^{2}\right)^{2}\right]}{\left[1+2 C \bar{e}^{2} /\left(\bar{E}^{2}-\bar{E}_{0}^{2}\right)^{2}\right]}
$$

and $f_{m}, f_{u}, f_{v}$ and $f_{w}$ are defined by equation (E.6), and where $K_{10}$ through $K_{14}$ are given by equation (E.7). 


\section{E.3 Algebraic Expression for $\overline{v w^{\prime}} / U_{r}^{2}$}

When slant-wire data taken at positions $E_{A}, F_{A}, G_{A}$ and $H_{A}$ are applied to equation (E.15), the following four equations result:

$$
\begin{aligned}
& \left(F_{u v}\right)_{E_{A}}\left(\overline{u^{\prime 2}} / U_{r}^{2}\right)+\left(F_{v v}\right)_{E_{A}}\left(\overline{v^{2}} / U_{r}^{2}\right) \\
& +\left(F_{w w}\right)_{E_{A}}\left(\overline{w^{\prime 2}} / U_{r}^{2}\right)+\left(F_{u v}\right)_{E_{A}}\left(\overline{u^{\prime} v} / U_{r}^{2}\right) \\
& -\left(F_{u w}\right)_{E_{A}}\left(\overline{u^{\prime} w^{\prime}} / U_{r}^{2}\right)-\left(F_{v w}\right)_{E_{A}}\left(\overline{v w^{\prime}} / U_{r}^{2}\right)=\left(F_{m m}\right)_{E_{A}} \\
& \left(F_{u u}\right)_{F_{A}}\left(\overline{u^{\prime 2}} / U_{r}^{2}\right)+\left(F_{v v}\right)_{F_{A}}\left(\overline{v^{2}} / U_{r}^{2}\right) \\
& +\left(F_{w w}\right)_{F_{A}}\left(\overline{w^{\prime 2}} / U_{r}^{2}\right)+\left(F_{u v}\right)_{F_{A}}\left(\overline{u^{\prime} v} / U_{r}^{2}\right) \\
& -\left(F_{u w}\right)_{F_{A}}\left(\overline{u^{\prime} w^{\prime}} / U_{r}^{2}\right)-\left(F_{v w}\right)_{F_{A}}\left(\overline{v w^{\prime}} / U_{r}^{2}\right)=\left(F_{m m}\right)_{F_{A}} \\
& \left(F_{u u}\right)_{G_{A}}\left(\overline{u^{\prime 2}} / U_{r}^{2}\right)+\left(F_{v v}\right)_{G_{A}}\left(\overline{v^{2}} / U_{r}^{2}\right) \\
& +\left(F_{w w}\right)_{G_{A}}\left(\overline{w^{\prime 2}} / U_{r}^{2}\right)+\left(F_{u v}\right)_{G_{A}}\left(\overline{u^{\prime} v} / U_{r}^{2}\right) \\
& -\left(F_{u w}\right)_{G_{A}}\left(\overline{u^{\prime} w^{\prime}} / U_{r}^{2}\right)-\left(F_{v w}\right)_{G_{A}}\left(\overline{v w^{\prime}} / U_{r}^{2}\right)=\left(F_{m m}\right)_{G_{A}} \\
& \left(F_{u u}\right)_{H_{A}}\left(\overline{u^{\prime 2}} / U_{r}^{2}\right)+\left(F_{v v}\right)_{H_{A}}\left(\overline{v^{2}} / U_{r}^{2}\right) \\
& +\left(F_{w w}\right)_{H_{A}}\left(\overline{w^{\prime 2}} / U_{r}^{2}\right)+\left(F_{u v}\right)_{H_{A}}\left(\overline{u^{\prime} v} / U_{r}^{2}\right) \\
& -\left(F_{u w}\right)_{H_{A}}\left(\overline{u^{\prime} w^{\prime}} / U_{r}^{2}\right)-\left(F_{v w}\right)_{H_{A}}\left(\overline{v w^{\prime}} / U_{r}^{2}\right)=\left(F_{m m}\right)_{H_{A}}
\end{aligned}
$$

where the expressions for $F_{u u}, \ldots F_{v w}$ are given by equation (E.16). Subtraction of equation (E.21) from (E.18) and (E.20) from (E.19) yields the following combined equations:

$$
\begin{aligned}
& \left.\left[\left(F_{u v}\right)_{E_{A}}-\left(F_{u u}\right)_{H_{A}}\right] \overline{\left(u^{\prime 2}\right.} / U_{r}^{2}\right)+\left[\left(F_{v v}\right)_{E_{A}}-\left(F_{v v}\right)_{H_{A}}\right]\left(\overline{v^{2}} / U_{r}^{2}\right) \\
& \left.+\left[\left(F_{w w}\right)_{E_{A}}-\left(F_{w w}\right)_{H_{A}}\right]\left(\overline{w^{\prime 2}} / U_{r}^{2}\right)+\left[\left(F_{u v}\right)_{E_{A}}-\left(F_{u v}\right)_{H_{A}}\right] \overline{u^{\prime} v} / U_{r}^{2}\right) \\
& \left.-\left[\left(F_{u w}\right)_{E_{A}}-\left(F_{u w}\right)_{H_{A}}\right] \overline{u^{\prime} w^{\prime}} / U_{r}^{2}\right)-\left[\left(F_{v w}\right)_{E_{A}}-\left(F_{v w}\right)_{H_{A}}\right]\left(\overline{v w^{\prime}} / U_{r}^{2}\right) \\
& =\left[\left(F_{m m}\right)_{E_{A}}-\left(F_{m m}\right)_{H_{A}}\right] \\
& \left.\left[\left(F_{u u}\right)_{F_{A}}-\left(F_{u u}\right)_{G_{A}}\right] \overline{\left(u^{\prime 2}\right.} / U_{r}^{2}\right)+\left[\left(F_{v v}\right)_{F_{A}}-\left(F_{v v}\right)_{G_{A}}\right]\left(\overline{v^{2}} / U_{r}^{2}\right) \\
& \left.+\left[\left(F_{w w}\right)_{F_{A}}-\left(F_{w w}\right)_{G_{A}}\right] \overline{w^{\prime 2}} / U_{r}^{2}\right)+\left[\left(F_{u v}\right)_{F_{A}}-\left(F_{u v}\right)_{G_{A}}\right]\left(\overline{u^{\prime} v} / U_{r}^{2}\right) \\
& -\left[\left(F_{u w}\right)_{F_{A}}-\left(F_{u w}\right)_{G_{A}}\right]\left(\overline{u^{\prime} w^{\prime}} / U_{r}^{2}\right)-\left[\left(F_{v w}\right)_{F_{A}}-\left(F_{v w}\right)_{G_{A}}\right]\left(\overline{v w^{\prime}} / U_{r}^{2}\right) \\
& =\left[\left(F_{m m}\right)_{F_{A}}-\left(F_{m m}\right)_{G_{A}}\right]
\end{aligned}
$$

Multiplication of equation (E.22) by $\left[\left(F_{u w}\right)_{F_{A}}-\left(F_{u w}\right)_{G_{A}}\right]$ and equation (E.23) by $\left[\left(F_{u w}\right)_{E_{A}}-\left(F_{u w}\right)_{H_{A}}\right]$ and subtraction of the resulting equations yields:

$$
\begin{aligned}
& \left(S_{1 u u}-S_{2 u u}\right)\left(\overline{u^{\prime 2}} / U_{r}^{2}\right)+\left(S_{1 v v}-S_{2 v v}\right)\left(\overline{v^{2}} / U_{r}^{2}\right) \\
& +\left(S_{1 w w}-S_{2 w w}\right)\left(\overline{w^{\prime 2}} / U_{r}^{2}\right)+\left(S_{1 u v}-S_{2 u v}\right)\left(\overline{u^{\prime} v} / U_{r}^{2}\right) \\
& -\left(S_{1 v w}-S_{2 v w}\right)\left(\overline{v w^{\prime}} / U_{r}^{2}\right)=\left(S_{1 m m}-S_{2 m m}\right)
\end{aligned}
$$


where,

$$
\begin{aligned}
& S_{1 i j}=\left[\left(F_{u w}\right)_{F_{A}}-\left(F_{u w}\right)_{G_{A}}\right]\left[\left(F_{i j}\right)_{E_{A}}-\left(F_{i j}\right)_{H_{A}}\right] \\
& S_{2 i j}=\left[\left(F_{u w}\right)_{E_{A}}-\left(F_{u w}\right)_{H_{A}}\right]\left[\left(F_{i j}\right)_{F_{A}}-\left(F_{i j}\right)_{G_{A}}\right]
\end{aligned} \quad(i, j=u, v, w, m)
$$

By observing the behavior of the coefficients $f_{u}, f_{v}, f_{w}, f_{m}, K_{10}, K_{11}, K_{12}, K_{13}$ and $K_{14}$ at positions $E_{A}, F_{A}, G_{A}$ and $H_{A}$, it can be shown that:

$$
\begin{aligned}
\left(f_{u}^{2}\right)_{E_{A}}=\left(f_{u}^{2}\right)_{H_{A}} ; \quad\left(f_{u}^{2}\right)_{F_{A}}=\left(f_{u}^{2}\right)_{G_{A}} \\
\left(f_{v}^{2}\right)_{E_{A}}=\left(f_{v}^{2}\right)_{H_{A}} ; \quad\left(f_{v}^{2}\right)_{F_{A}}=\left(f_{v}^{2}\right)_{G_{A}} \\
\left(f_{w}^{2}\right)_{E_{A}}=\left(f_{w}^{2}\right)_{H_{A}} ; \quad\left(f_{w}^{2}\right)_{F_{A}}=\left(f_{w}^{2}\right)_{G_{A}} \\
\left(f_{m}^{2}\right)_{E_{A}}=\left(f_{m}^{2}\right)_{H_{A}} ; \quad\left(f_{m}^{2}\right)_{F_{A}}=\left(f_{m}^{2}\right)_{G_{A}} \\
\left(f_{u} f_{v}\right)_{E_{A}}=\left(f_{u} f_{v}\right)_{H_{A}} ; \quad\left(f_{u} f_{v}\right)_{F_{A}}=\left(f_{u} f_{v}\right)_{G_{A}} \\
\left(f_{u} f_{w}\right)_{E_{A}}=-\left(f_{u} f_{w}\right)_{H_{A}} ; \quad\left(f_{u} f_{w}\right)_{F_{A}}=-\left(f_{u} f_{w}\right)_{G_{A}} \\
\left(f_{v} f_{w}\right)_{E_{A}}=-\left(f_{v} f_{w}\right)_{H_{A}} ; \quad\left(f_{v} f_{w}\right)_{F_{A}}=-\left(f_{v} f_{w}\right)_{G_{A}}
\end{aligned}
$$

and,

$$
\begin{array}{rll}
\left(K_{10}\right)_{E_{A}}=\left(K_{10}\right)_{H_{A}} ; & \left(K_{10}\right)_{F_{A}}=\left(K_{10}\right)_{G_{A}} \\
\left(K_{11}\right)_{E_{A}}=-\left(K_{11}\right)_{H_{A}} ; & \left(K_{11}\right)_{F_{A}}=-\left(K_{11}\right)_{G_{A}} \\
\left(K_{12}\right)_{E_{A}}=\left(K_{12}\right)_{H_{A}} ; & \left(K_{12}\right)_{F_{A}}=\left(K_{12}\right)_{G_{A}} \\
\left(K_{13}\right)_{E_{A}}=-\left(K_{13}\right)_{H_{A}} ; & \left(K_{13}\right)_{F_{A}}=-\left(K_{13}\right)_{G_{A}} \\
\left(K_{14}\right)_{E_{A}}=\left(K_{14}\right)_{H_{A}} ; & \left(K_{14}\right)_{F_{A}}=\left(K_{14}\right)_{G_{A}}
\end{array}
$$

By imposing conditions (E.25.a) and (E.25.b) on equation (E.24), the following final form is obtained:

$$
\begin{aligned}
& \left.\left(s_{1} K_{12, E_{A}}-s_{2} K_{12, F_{A}}\right) \overline{\left(u^{\prime 2}\right.} / U_{r}^{2}\right)+\left(s_{1}-s_{2}\right)\left(\overline{v^{2}} / U_{r}^{2}\right)+ \\
& \left(s_{1} K_{14, E_{A}}-s_{2} K_{14, F_{A}}\right)\left(\overline{w^{\prime 2}} / U_{r}^{2}\right)+2\left(s_{1} K_{10, E_{A}}-s_{2} K_{10, F_{A}}\right)\left(\overline{u^{\prime} v} / U_{r}{ }^{2}\right)- \\
& \left(S_{1 v w}-S_{2 v w}\right)\left(\overline{v w^{\prime}} / U_{r}^{2}\right)=-\left(s_{1} f_{m, E_{A}}-s_{2} f_{m, F_{A}}\right) / 2
\end{aligned}
$$

where

$$
\begin{aligned}
& s_{1}=-8\left[\left(f_{u} f_{w}\right)_{F_{A}}-\left(f_{m} K_{13}\right)_{F_{A}}\left(\Pi_{F_{A}}+\Pi_{G_{A}}\right)\right]\left(\Pi_{E_{B}}-\Pi_{H_{B}}\right)\left(f_{m}\right)_{E_{A}} \\
& s_{2}=-8\left[\left(f_{u} f_{w}\right)_{E_{A}}-\left(f_{m} K_{13}\right)_{E_{A}}\left(\Pi_{E_{A}}+\Pi_{F_{A}}\right)\right]\left(\Pi_{F_{A}}-\Pi_{G_{A}}\right)\left(f_{m}\right)_{F_{A}}
\end{aligned}
$$

and,

$$
\begin{aligned}
S_{1 v w}= & 16\left[\left(f_{u} f_{w}\right)_{F_{A}}-K_{13, F_{A}}\left(f_{m}\right)_{F_{A}}\left(\Pi_{F_{A}}+\Pi_{G_{A}}\right)\right] \times \\
& {\left[\left(f_{v} f_{w}\right)_{E_{A}}-K_{11, E_{A}}\left(f_{m}\right)_{E_{A}}\left(\Pi_{E_{A}}+\Pi_{H_{A}}\right)\right] } \\
S_{2 v u^{\prime}}= & 16\left[\left(f_{u} f_{w}\right)_{E_{A}}-K_{13, E_{A}}\left(f_{m}\right)_{E_{A}}\left(\Pi_{E_{A}}+\Pi_{H_{A}}\right)\right] \times \\
& {\left[\left(f_{v} f_{w}\right)_{F_{A}}-K_{11, F_{A}}\left(f_{m}\right)_{F_{A}}\left(\Pi_{F_{A}}+\Pi_{G_{A}}\right)\right] }
\end{aligned}
$$


and where the expression for $\Pi$ is given by equation (E.17). An algebraic expression for $\overline{v w^{\prime}}$, applicable to low turbulence intensity flows, can be derived from equation (E.24) in a similar manner; however, it can also be extracted as a special case from equation (E.24) by substituting $F_{i i}=f_{i} f_{i} ; \quad(i=u, v, w)$, $F_{i j}=2 f_{i} f_{j} ; \quad(i, j=u, v, w ; \quad i \neq j)$ and $F_{m m}=f_{m}{ }^{2} \pi$ into equation (E.24), which yields:

$$
\begin{aligned}
& S_{1 u u}=S_{1 v v}=S_{1 w w}=S_{1 u v}=0 \\
& S_{2 u u}=S_{2 v v}=S_{2 w w}=S_{2 u v}=0
\end{aligned}
$$

and,

$$
\begin{aligned}
S_{1 v w} & =\left[2\left(f_{u} f_{w}\right)_{F_{A}}-2\left(f_{u} f_{w}\right)_{G_{A}}\right]\left[2\left(f_{v} f_{w}\right)_{E_{A}}-2\left(f_{v} f_{w}\right)_{H_{A}}\right] \\
& =\left(4 f_{u} f_{w}\right)_{F_{A}}\left(4 f_{v} f_{w}\right)_{E_{A}} \\
S_{2 v w} & =\left(4 f_{u} f_{w}\right)_{E_{A}}\left(4 f_{v} f_{w}\right)_{F_{A}} \\
S_{1 m m} & =\left(4 f_{u} f_{w}\right)_{F_{A}}\left(f_{m}^{2}\right)_{E_{A}}\left(\pi_{E_{A}}-\pi_{H_{A}}\right) \\
S_{2 m m} & =\left(4 f_{u} f_{w}\right)_{E_{A}}\left(f_{m}{ }^{2}\right)_{F_{A}}\left(\pi_{F_{A}}-\pi_{G_{A}}\right)
\end{aligned}
$$

Substitution of these coefficients back into (E.24) yields, after some rearrangement:

$$
\frac{\overline{v w^{\prime}}}{U_{r}{ }^{2}}=\frac{-\left[\left(f_{u} f_{w}\right)_{F_{A}}\left(\pi_{E_{A}}-\pi_{H_{A}}\right)\left(f_{m}{ }^{2}\right)_{E_{A}}-\left(f_{u} f_{w}\right)_{E_{A}}\left(\pi_{F_{A}}-\pi_{G_{A}}\right)\left(f_{m}{ }^{2}\right)_{F_{A}}\right]}{\left(2 f_{u} f_{w}\right)_{F_{A}}\left(2 f_{v} f_{w}\right)_{E_{A}}-\left(2 f_{u} f_{w}\right)_{E_{A}}\left(2 f_{v} f_{w}\right)_{F_{A}}}
$$



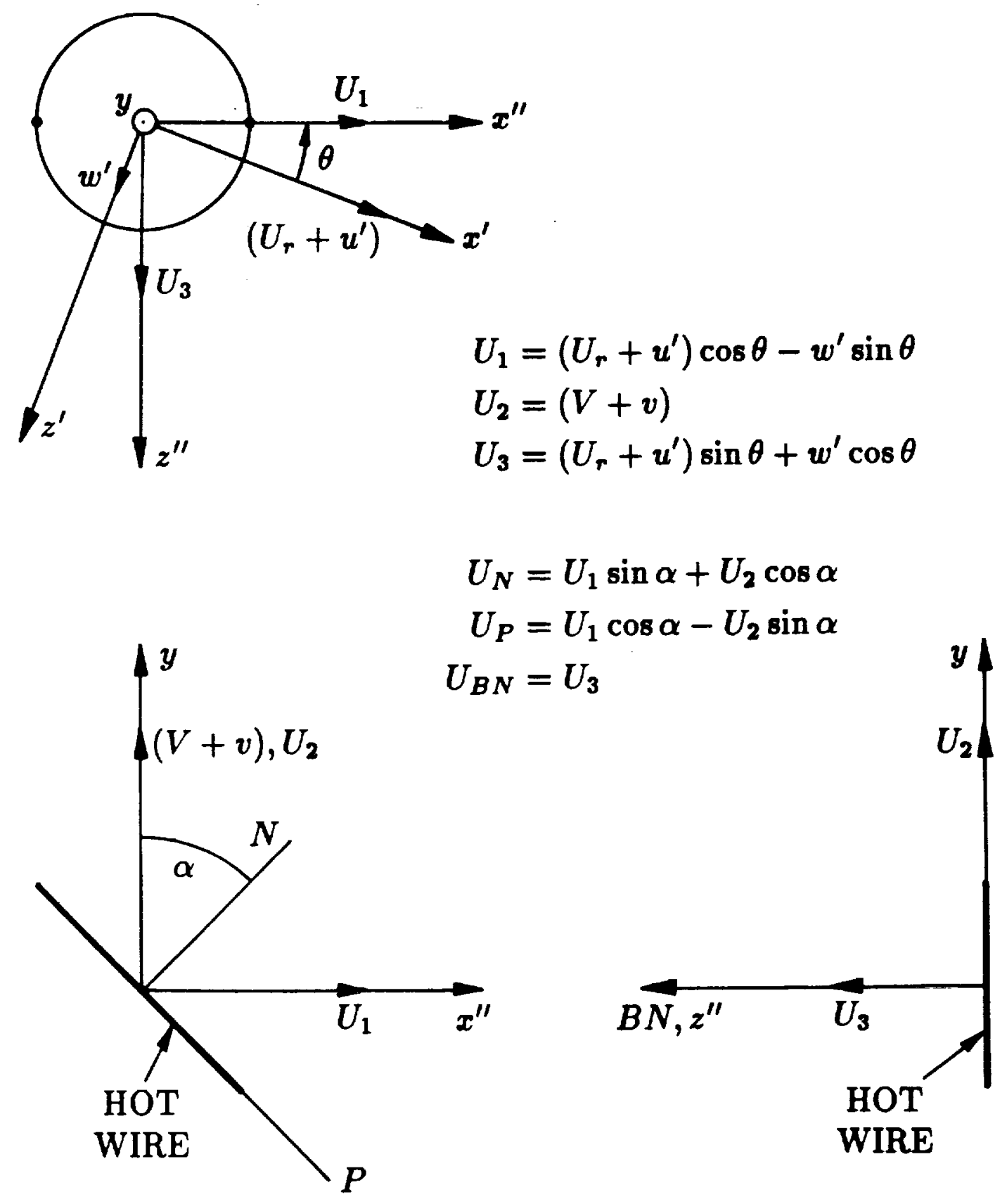

Fig. E.1. Effective cooling velocity for turbulence flow calculations. 


\section{APPENDIX F}

\section{VERIFICATION OF THE HOT-WIRE TECHNIQUE}

The low-intensity form of the Method $A$ hot-wire technique proposed in the Appendix $C$ has been partially verified by means of measurements in fullydeveloped turbulent pipe flow under simulated skewed flow conditions. In addition, the implementation of the Method $B$ technique into the present study was verified in pipe flow for the unskewed case. The pipe flow facility, as well as the full verification procedure for Method $B$, is described in detail in references [45] and [47].

\section{F.1 Fully-developed pipe flow}

A schematic of the pipe flow verification setup for Method $A$ is shown in Fig. F.1. The probes are inserted into the flow through a slot $(6.5 \times 13 \mathrm{~mm})$ located one diameter upstream from the pipe exit. The outside edges of the slot were chamfered to allow the probe to be skewed \pm 20 degrees in the pitch direction. The verification measurements were made on the far side of the pipe to minimize disturbances due to the slot. The diameter of the pipe is $10.16 \mathrm{~cm}$ and all the verification measurements were made at an operating Reynolds number of 125,000 based on the centerline velocity and the pipe diameter. The development length for the pipe flow is $L / D=136$.

Along a radial traverse of turbulent pipe flow, azimuthal symmetry imposes the condition that the $\overline{u w}$ and the $\overline{v w}$ Reynolds stress components (relative to pipe flow coordinates) are identically zero. When the flow is fully-developed, the $\overline{u v}$ shear stress component, when normalized by the friction velocity, is a linear function of the radial location if the very near-wall region is excluded i.e.,:

$$
\frac{\overline{u v}}{U_{\tau}^{2}}=\frac{r}{R}
$$

where the friction velocity is given as:

$$
U_{\tau} / U_{b}=\sqrt{f / 8}
$$

and $U_{b}$ (bulk velocity) and $f$ (friction factor) are determined from:

$$
1 / \sqrt{f}=2.0 \log _{10}\left(R e_{b} \sqrt{f}\right)-0.8
$$

with,

$$
\begin{aligned}
U_{b} / U_{c l} & =R e_{b} / R e_{c l} \\
R e_{c l} & =U_{c l} D / \nu
\end{aligned}
$$


and where the Reynolds number ratio is given by a correlation proposed by Eppich [40]:

$$
R e_{b} / R e_{c l}=0.045 \log _{10}\left(R e_{c l}\right)+0.600
$$

For the present study, the friction velocity was calculated from equation (F.2) and also measured independently with a Preston tube using the calibration given by equation (3.3). The results from the two methods were virtually the same:

$$
U_{\tau} / U_{b}=0.04726 @ R e_{c l}=125,000
$$

\section{F.2 Verification approach}

Since, for low intensity flows, the yaw angle $\gamma$ (refer to Fig. C.2.a) can be determined with sufficient accuracy by nulling of a two-tube Conrad probe or by the normal hot-wire nulling technique described by Page [62], the low intensity verification procedure for Method $A$ was initially performed for unknown pitch angles $\zeta$ only. For this case, the transverse velocity component $W$ is zero and the primary (mean flow) coordinates and secondary (turbulence) coordinates coincide (see Fig. C.3.a). Wire Positions 1,2,3 and 4 in the primary coordinate system (see Fig. C.2), then, are equivalent to wire Positions $A, B, C_{A}$ and $D_{A}$ in the secondary coordinate system, respectively. For the following discussion, the probe rotation angle will be denoted by $\theta$ and the prime (') superscript associated with the secondary (turbulence) coordinate system will be dropped. The probe rotation angle $\theta$ is defined as shown in Fig. F.2.

The proposed procedure for determining the mean velocity components and Reynolds stress components is presented in detail in Appendix $\mathrm{C}$ and is summarized here. The $V / U$ and $W / U$ mean velocity ratios are determined by applying data obtained with the offset prong slant-wire probe (Fig. C.1.b) at positions $\theta=0$ and $180^{\circ}$ and normal-wire data obtained at $\theta=225$ and $315^{\circ}$ to equation (C.14). Once the mean velocity ratios are known, the axial velocity component $U$ is evaluated at wire Position 1 by means of equation (C.16), and it's equivalent for wire Positions 2,3 and 4, and then the four values are averaged. The procedure for determining the components of the Reynolds stress tensor is as follows:

1) $\overline{u^{2}}, \overline{v^{2}}, \overline{u v}$ - Data obtained with a normal-wire at $\theta=270^{\circ}$ and with the offset prong slant-wire configuration (Fig. C.1.b) at $\theta=0$ and $180^{\circ}$, when applied to equation (C.36), results in a $3 \times 3$ system of equations which, when solved, yields the $\overline{u^{2}}, \overline{v^{2}}$ and $\overline{u v}$ Reynolds stress components.

2) $\overline{v w}$ - Data obtained with the straight prong slant-wire probe (Fig. C.1.c) at $\theta=45,135,225$ and $315^{\circ}$, when applied to equation (C.41), yields an algebraic expression for the $\overline{v w}$ stress component. 
3) $\overline{w^{2}}, \overline{u w}$ - Normal-wire data obtained at $\theta=225$ and $315^{\circ}$ and the stress components from 1) and 2) above, when applied to equation (C.36), results in a $2 \times 2$ system of equations which, when solved, yields the $\overline{w^{2}}$ and $\overline{u w}$ Reynolds stress components.

By considering pitch skewness only in the fully-developed pipe flow verification, the $\overline{u w}$ and $\overline{v w}$ stress components are identically zero regardless of the pitch angle. Further, the $\overline{w^{2}}$ stress component is invariant as the pitch angle is changed.

\section{F.3 Hot-wire probe design and construction}

The proposed Method A hot-wire technique requires the sequential use of two and possibly three probe configurations which are illustrated in Fig. C.1. All the probes for the Method $A$ technique were constructed from scratch with the basic dimensions and materials as shown in Fig. F.3. Detail dimensions of the probe prongs for the three probe configurations are shown in Fig. F.4. One of the constraints imposed on the probe design was that it had to fit through a $4.57 \mathrm{~mm}(0.180 \mathrm{in})$ access hole which corresponds to the diameter of a standard Thermal Systems Inc. (TSI) probe holder. This constraint places a limitation on the amount of offset that can be applied to a probe such as the slant-wire probe shown in Fig. F.4.b. Given a diameter that the probe must pass through without interference, a compromise must be made between probe offset and the distance between the probe prong tips (hot-wire length).

\section{F.4 Calibration}

The hot-wire probes were calibrated in the core flow at Data Station 1 of the transition duct flow facility (see Fig. 3.2). A Pitot tube placed adjacent to the hot-wire probe and the local wall static pressure provided a reference calibration velocity. The normal-wire was calibrated in the $\theta=270^{\circ}$ position

and the straight prong slant-wire was calibrated in the $\theta=0^{\circ}$ position. The offset prong slant-wire was calibrated in both the $\theta=0$ and $180^{\circ}$ positions. A typical plot of an offset prong slant-wire probe calibration is shown in Fig. F.5. Clearly, the calibrations are not equivalent. There are two possible reasons for the differences. First, unlike Method $B$ where, for unskewed flow, the stagnation line for the normal velocity component on the hot-wire is unchanged regardless of rotational position, for Method $A$ the stagnation line rotates around the hot-wire as the probe is rotated. Waviness or other imperfections in the wire may cause it to respond differently when the flow is exposed to the front or back "side" of the wire. The second cause is the influence of the upstream probe prong when the wire is in the $\theta=180^{\circ}$ position. There are indications that the latter cause is responsible for the discrepancy. If wire imperfections were responsible, then the calibration curves should vary randomly with each different wire. Calibration with separate but similar probes and several different wires has shown that the 
calibration curve for Position $\mathrm{B}\left(\theta=180^{\circ}\right)$ consistently rides slightly higher then the curve for Position A. If it is assumed that the wire is exposed to the true calibration velocity when in Position $A$ and that the wire itself is perfect, that is, it will respond the same in any orientation so long as the effective cooling velocity remains constant, then Fig. F.5 indicates that the wire is exposed to a higher velocity when in Position B than when in Position A. It is plausible that the higher velocity in Position B is a result of the local acceleration of the flow as it is displaced about the upstream probe prong. This behavior is illustrated in Fig. F.6.

The question arises as to whether the prong influence is significant enough to preclude accurate flow measurements. The effect of different calibration slopes in the $\theta=0$ and $180^{\circ}$ positions can be taken into account in the mean flow response equations by replacing equation (C.7.a) by:

$$
\Sigma_{12}=\left(B_{2} / B_{1}\right)^{2 / m}\left(\Sigma_{1} / \Sigma_{2}\right)
$$

and by using the appropriate value of $B$ in equation (C.16) or its equivalent for wire Position 2. The turbulence response equations are unchanged since they are cast into a non-dimensional form in which the calibration slope is eliminated (see equation (C.25)). By accounting for the different calibration characteristics of the offset slant-wire, it should be possible to obtain accurate mean flow and turbulence data for unskewed flow conditions $(V=W=0)$. The effect of the probe prong interference under skewed conditions is uncertain and requires further study.

\section{F.5 Unskewed flow verification}

The mean velocity and Reynolds stress components in the fully-developed pipe flow were measured by means of the Method $A$ and Method $B$ hot-wire techniques. The measurements by the Method $B$ technique were performed to demonstrate proper operation of the data acquisition equipment and correct programming of the response equations. For the Method A measurements, the offset slant-wire data were reduced using the calibration in Position A only (uncorrected) and also using the calibration in Positions A and B (corrected) as described in the previous section. The measured axial mean velocity profile and transverse flow angles are shown in Fig. F.7.a and Fig. F.7.b, respectively. In Fig. F.7.a, a reference velocity distribution measured with a flattened Pitot probe is shown for comparison. The axial velocity results for Method $A$ were calculated from the output of the slant-wire only, rather then averaging the slant and normal-wire results, in order to illustrate more clearly the effect of the calibration anomaly. The corrected Method $A$ results and the Method $B$ results show very good agreement with the reference distributions. The uncorrected Method $A$ results show the need to include the calibration of the offset prong slant-wire

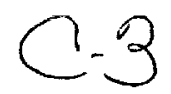


in both Positions A and B. All subsequent Method $A$ results will be based on calibrations in both positions.

The six components of the Reynolds stress tensor for unskewed conditions were measured by means of both the Method $A$ and Method $B$ techniques. The normal stress and shear stress profiles are shown in Fig. F.8.a and F.8.b, respectively. The normal stress distributions are compared to the fully-developed pipe flow results due to Lawn [64], which were taken at a comparable Reynolds number. The shear stresses are compared with the theoretical values. Overall, the results for both techniques show good agreement with the reference distributions. It should be noted here that agreement of the measured $\overline{u w}$ and $\overline{v w}$ shear stresses with the zero theoretical value is really only a partial verification. This is due to the fact that when the yaw angle is zero, the response equations will yield zero values for these components, regardless of whether or not there are probe disturbances present, so long as the probe output exhibits rotational symmetry about the $\theta=0^{\circ}$ position. Since the normal-wire probe is used to measure the $\bar{w}^{2}$ stress component as well as the $\overline{u w}$ component, there is justification to assume that interference effects associated with the normal-wire probe are minimal, inasmuch as the $\overline{w^{2}}$ stress component is predicted well. The straight prong slant-wire, however, is used solely to measure the $\overline{v w}$ stress component. Full verification of measurements with this probe must be made by simulating yaw and pitch flow conditions.

The unskewed flow case is used to determine the reference tangential cooling coefficient $k_{\text {ref }}$ for the hot-wires. For both Method $A$ and Method $B, k_{\text {ref }}$ for the slant-wires is varied until the best agreement with the theoretical $\overline{u v}$ shear stress distribution is obtained. This is accomplished by fitting a straight line (least-squares fit) through the $\overline{u v} / U_{\tau}^{2}$ results and adjusting $k_{\text {ref }}$ until a slope of one is obtained. To avoid probe prong disturbances, the straight prong slant-wire of Method $A$ had to be used in the Method $B$ mode to determine its value of $k_{\text {ref }}$. For the normal-wire used in Method $A, k_{\text {ref }}$ is adjusted until the best agreement with the Method $B$ or Lawn's $\overline{w^{2}}$ distribution is obtained. Specification of $k_{\text {ref }}$ for the normal-wire used in Method $B$ is not critical, inasmuch as the tangential velocity component is always much smaller than the normal component for zeroto-moderate skewness levels. Typical values for $k_{\text {ref }}$ were within the range of 0.05-0.2.

\section{F.6 Skewed flow verification}

With good results achieved for the unskewed case, the next step was to repeat the measurements under simulated pitch skewness conditions. Verification measurements were obtained at pitch angles of $\zeta=-20,-10,0,+10,+20^{\circ}$. The $\overline{v w}$ stress component for the skewed conditions was not measured because of the aformentioned problem of verification under simulated pitch skewness only. For 
calculation of the $\overline{w^{2}}$ and $\overline{u w}$ components (step 3 in Section F.2), the $\overline{v w}$ stress component was set to its theoretical value of zero.

Measurements of the mean flow field under simulated pitch conditions are shown in Fig. F.9. The axial velocity profiles shown in Fig. F.9.a have been transformed back to the unskewed (pipe flow) coordinate system to facilitate comparison with the Pitot tube results. Overall the agreement is fairly good. The spread in the data increases slightly as the pipe wall is approached $(r / R=$ 1.0) and is probably related to the higher turbulence intensity in that region. The measured transverse flow angles are shown in Figs. F.9.b and F.9.c. Generally the technique predicts the set flow angles (solid-line distributions) well, but slight deviations as the wall is approached are again evident.

Five components of the Reynolds stress tensor were measured (the $\overline{v w}$ component was not measured) using the Method $A$ technique under simulated pitch skewness conditions. The results are shown transformed to the unskewed coordinate system. The normal stresses are compared with Lawn's data and the shear stresses are compared to the theoretical distribution. The results of the turbulence measurements are shown in Fig. F.10. These results show that good agreement is obtained near the centerline of the pipe, but that systematic deviations occur as the wall is approached. Some conclusions can be drawn from the manner in which the stresses are calculated. Since the $\overline{u w}$ and $\overline{v w}$ stress components are zero for all of these measurements and the coordinate rotation is about the $z$-axis, the only stresses which are affected by the transformation are the $\overline{u^{2}}, \overline{v^{2}}$ and $\overline{u v}$ components, which are computed independently of the remaining stresses (see step 1 in Section F.2). The output from the normal-wire probe in the $\theta=270^{\circ}$ position (from which the streamwise stress component is primarily derived) was observed to be the same for all pitch angles. This is expected since probe prong disturbances are unlikely for this configuration and the effective cooling velocity is invariant with pitch angle. This implies that the deviations are attributable to the offset prong slant-wire probe. This, of course, shouldn't be surprising since a prong interference effect was clearly present in the calibration results. The $\overline{w^{2}}$ stress component deviates from Lawn's data because it's calculation is coupled with the $\overline{u^{2}}, \overline{v^{2}}$ and $\overline{u v}$ components. It was considered that perhaps the deviations from the reference distributions for the skewed flow case were due to an error either in the derivation of the response equations or in the implementation of the equations into a computer program. However, since no errors have been detected and the fact that the results agree well near the center of the pipe, it is believed that the progressive deviation as the wall is approached is a result of the increasing turbulence intensity, coupled with the probe prong interference effects. 


\section{F.7 Conclusions and recommendations}

A hot-wire technique for measuring the mean flow and Reynolds stresses in a low turbulence intensity flow with moderate mean flow skewness has been proposed. Preliminary verification measurements have been obtained under simulated pitch angle conditions. The results are encouraging but further work is needed. In particular, the technique is adequate for determination of the mean velocity components for pitch angles up to \pm 20 degrees. Indeed, the results indicate that this range can probably be extended. The technique is also adequate for determination of the Reynolds stress tensor for small skew angles (less than \pm 10 degrees), but systematic deviations were observed for moderate skew levels.

It has been concluded that the source of errors is attributable to the design of the offset slant-wire probe. Specifically, the hot-wire is apparently sensing the local acceleration of the flow around the longer probe prong when it is positioned upstream of the center of the hot-wire. The effect of the prong disturbance has been at least partially compensated for by calibrating the wire in two positions. In order to reduce the effect of prong disturbances, a new offset slant-wire probe design is proposed here. This new probe configuration, which is illustrated in Fig. F.11, has the following advantages:

1) The offset distance is greater while still remaining within the clearance envelope.

2) In the rotational positions $\theta=0$ and $180^{\circ}$, the probe prongs are not upstream of the sensing element. Even if some prong influence does occur, it should be the same in both positions, thereby eliminating the need for two calibrations.

3) By closely spacing the prongs and filling between them with epoxy, a more aerodynamic shape is achieved which will reduce the chance of prong vibration due to vortex shedding.

4) The relocation of the longer prong changes the rotational sweep where prong interference will occur. An estimate of the interference region is shown in Fig. F.12. The need for the straight prong slant-wire probe may be eliminated.

The primary disadvantage of this probe is that it is considerably more difficult to construct.

It is felt that further refinement of this technique is justified inasmuch as there are many flow configurations where it is not practical to align the rotational axis of a hot-wire probe with the direction of the primary flow. The simple calibration and the increased sensitivity make this technique an attractive alternate to the technique proposed by De Grande and Kool [61]. Should the new probe design yield improved results, the verification procedure should be expanded to investigate the best way to obtain the secondary shear stress $\overline{v w}$. Once accomplished, a study of the performance of the technique for unknown yaw skewness and moderate turbulence intensity flows should be undertaken. 


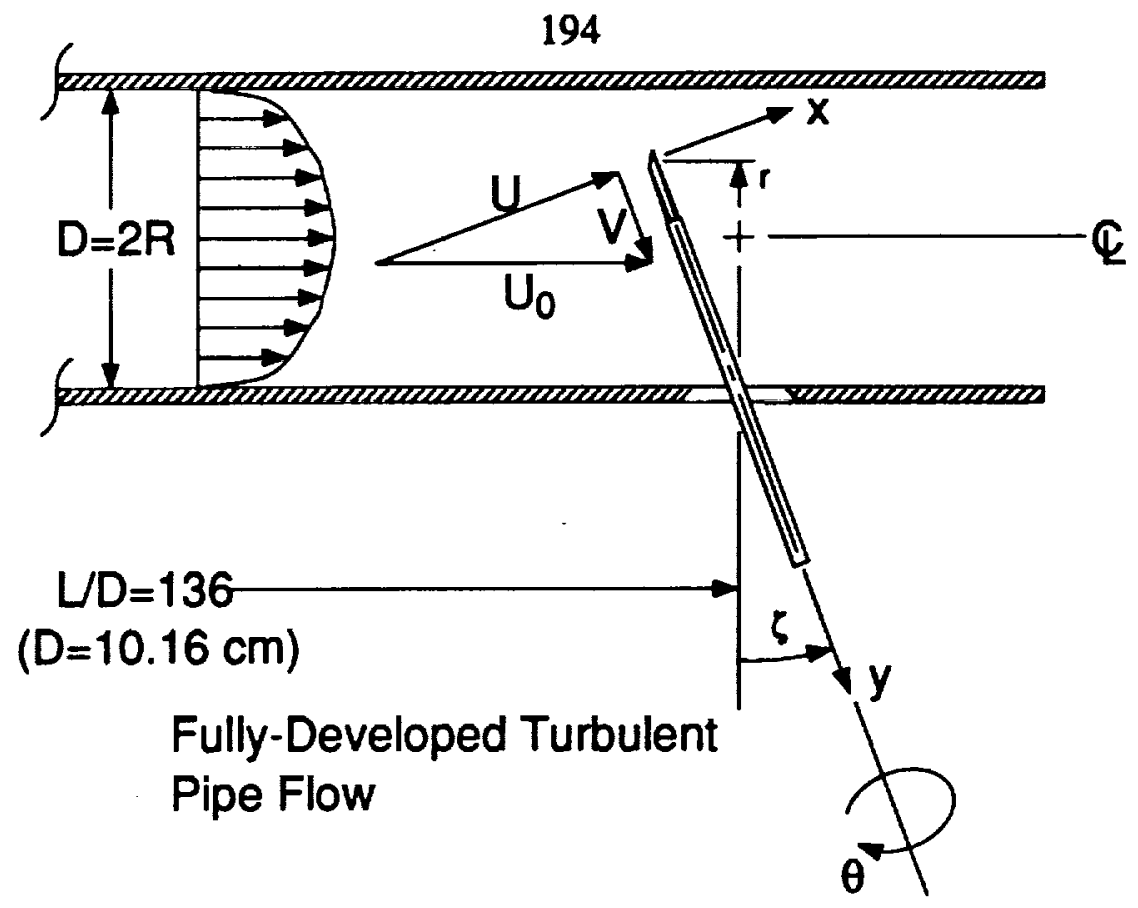

Fig. F.1. Schematic of Method A hot-wire verification setup.

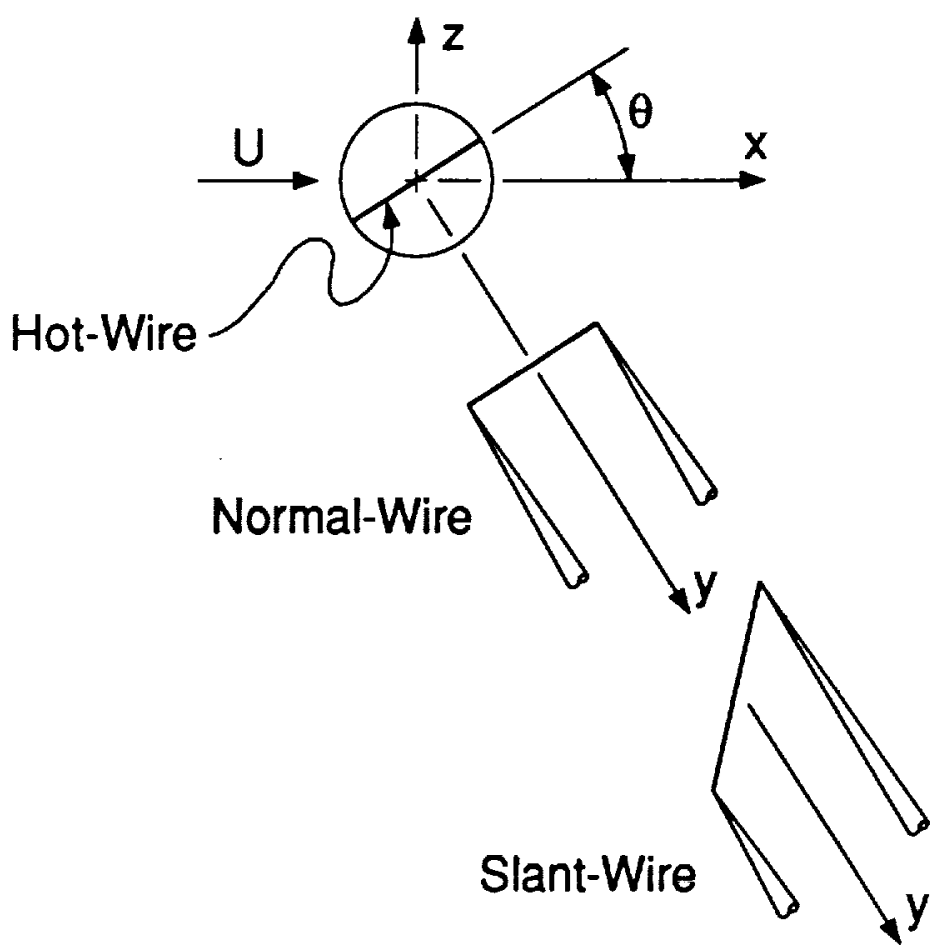

Fig. F.2. Hot-wire orientation for Method A. 


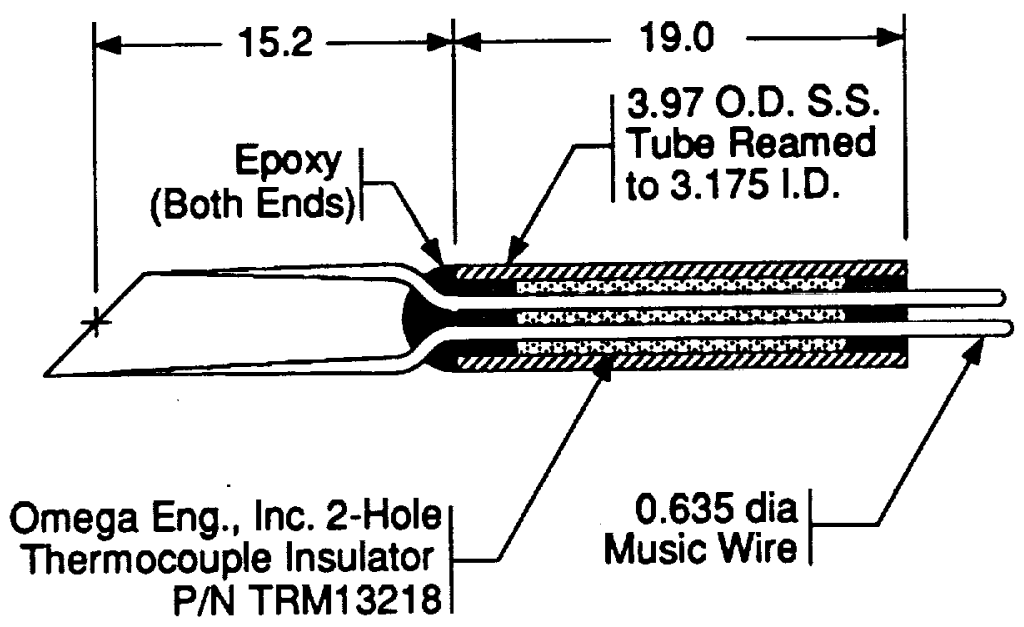

All dimensions in millimeters

Fig. F.3. Typical probe construction for Method A.
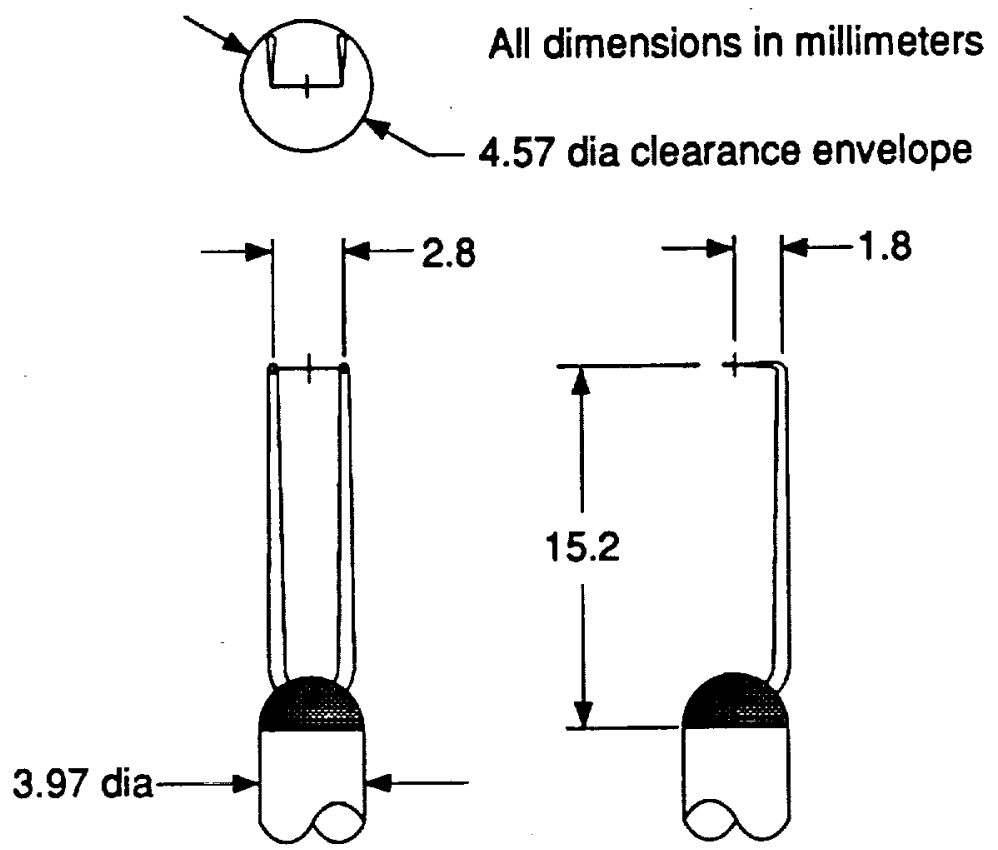

a) Normal-wire.

Fig. F.4. Detail of probe prong geometry for Method A. (Continued ...) 

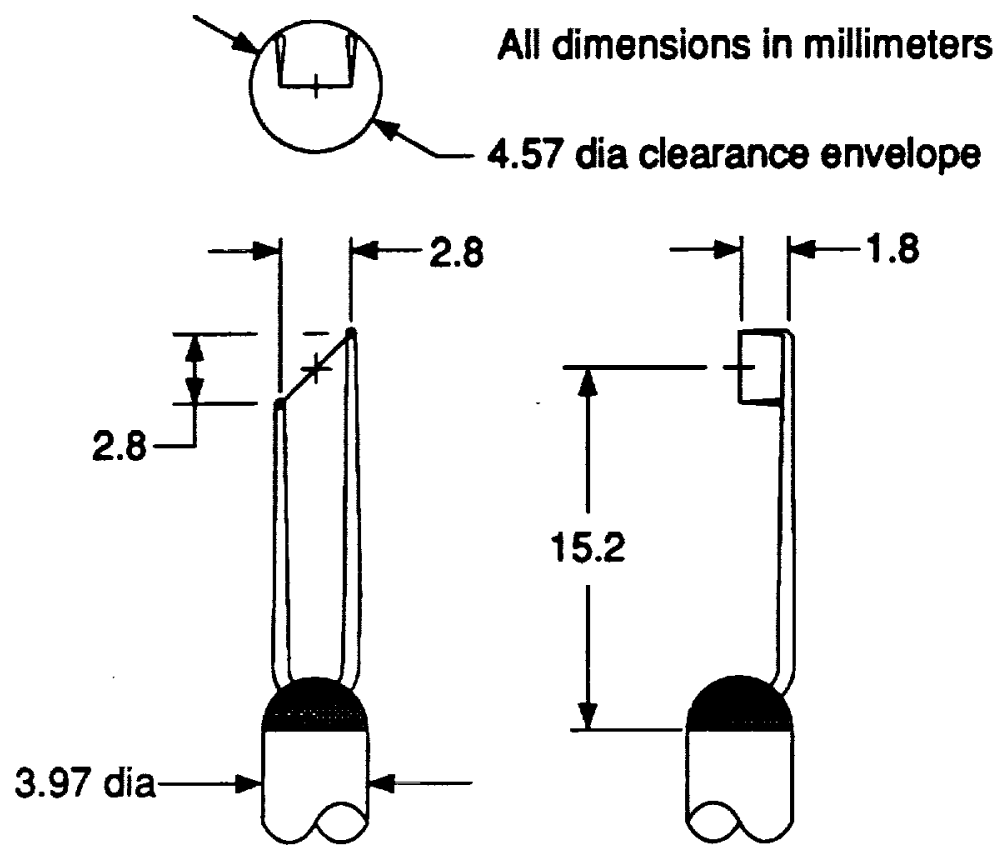

b) Offset prong slant-wire.
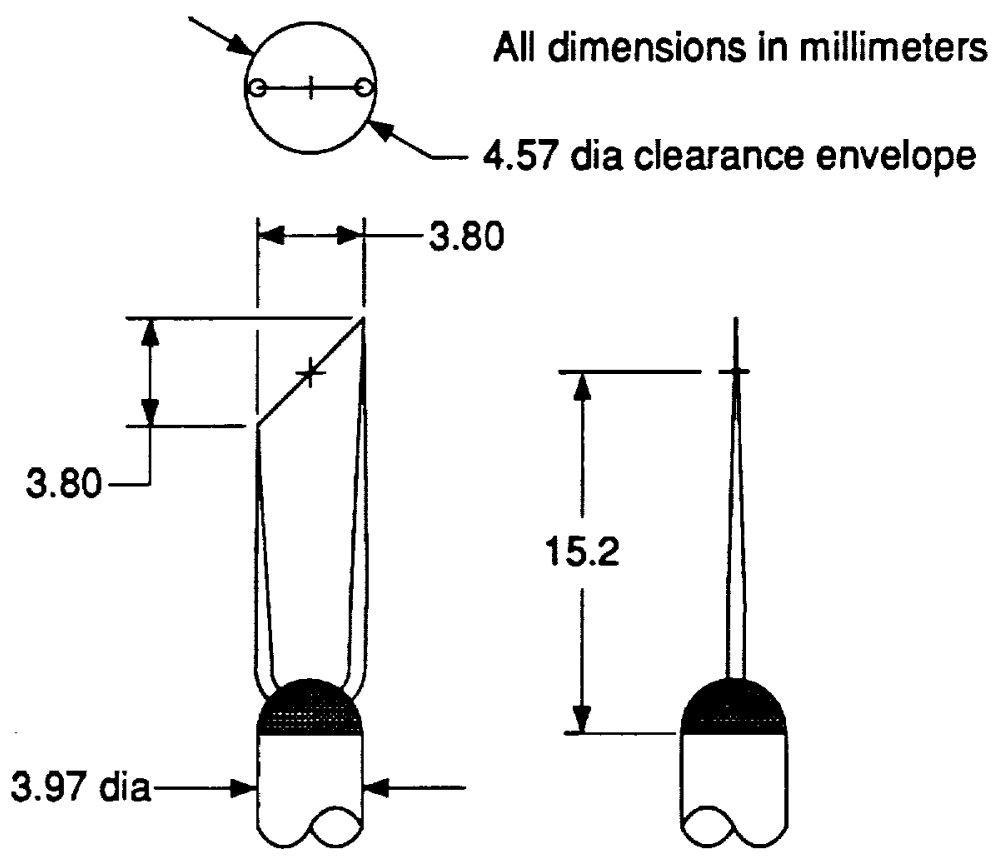

c) Straight prong slant-wire.

Fig. F.4. Detail of probe prong geometry for Method A. 


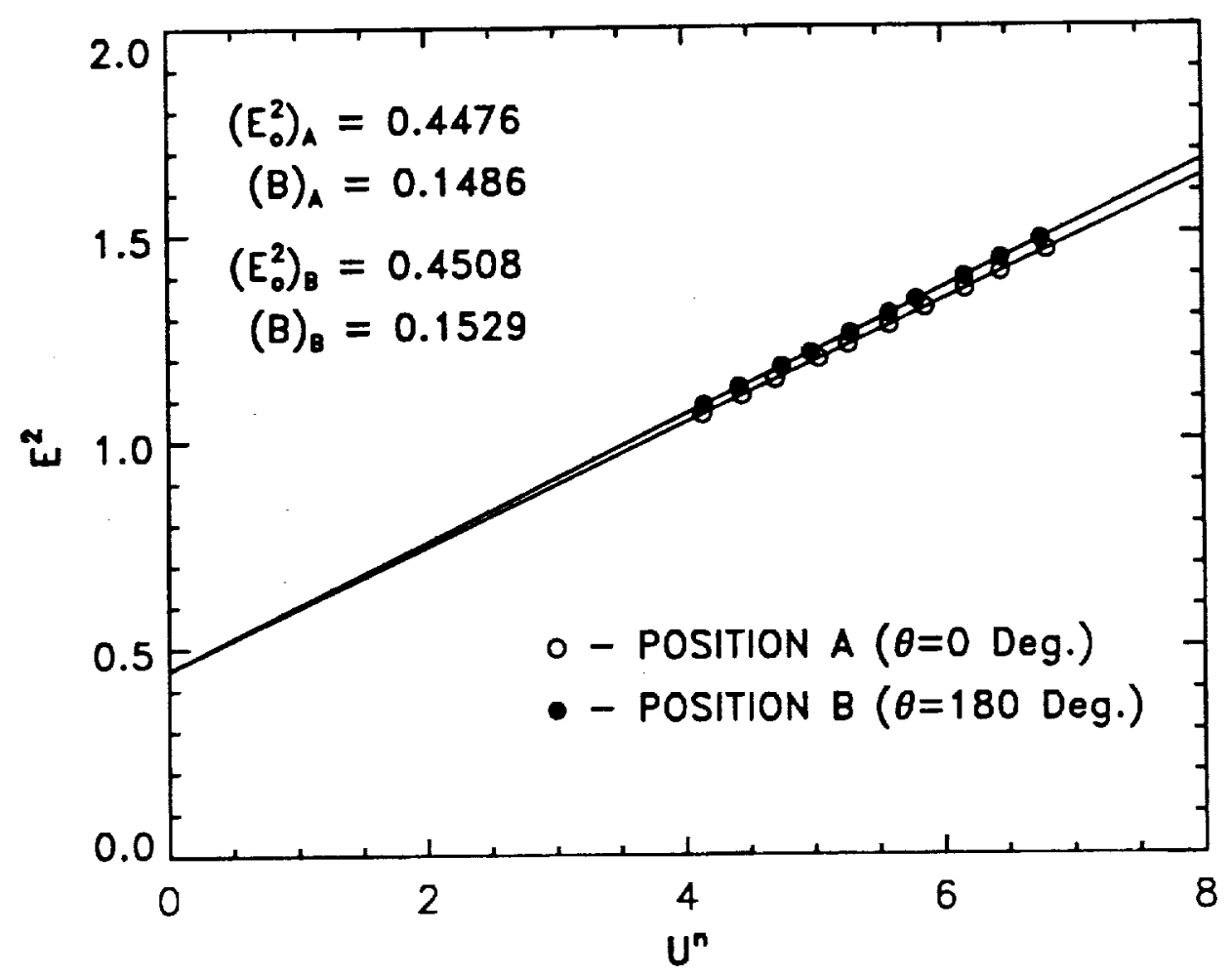

Fig. F.5. Offset slant-wire calibration in Position A and Position B.
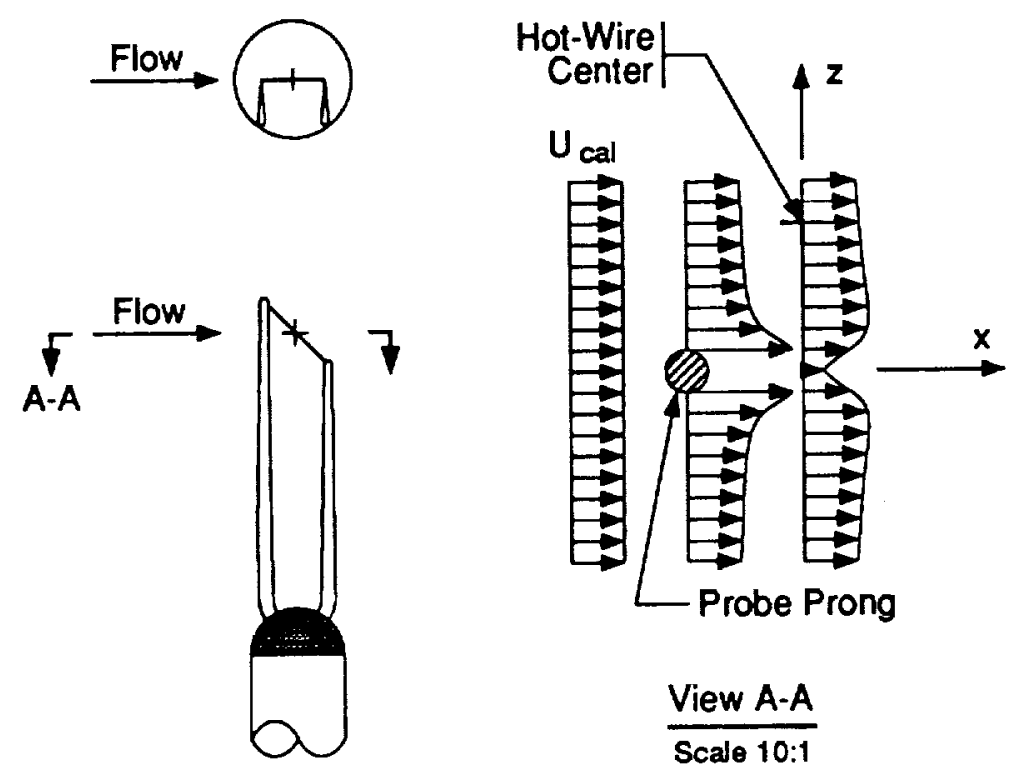

Fig. F.6. Conjectured probe prong influence for offset slant-wire. 


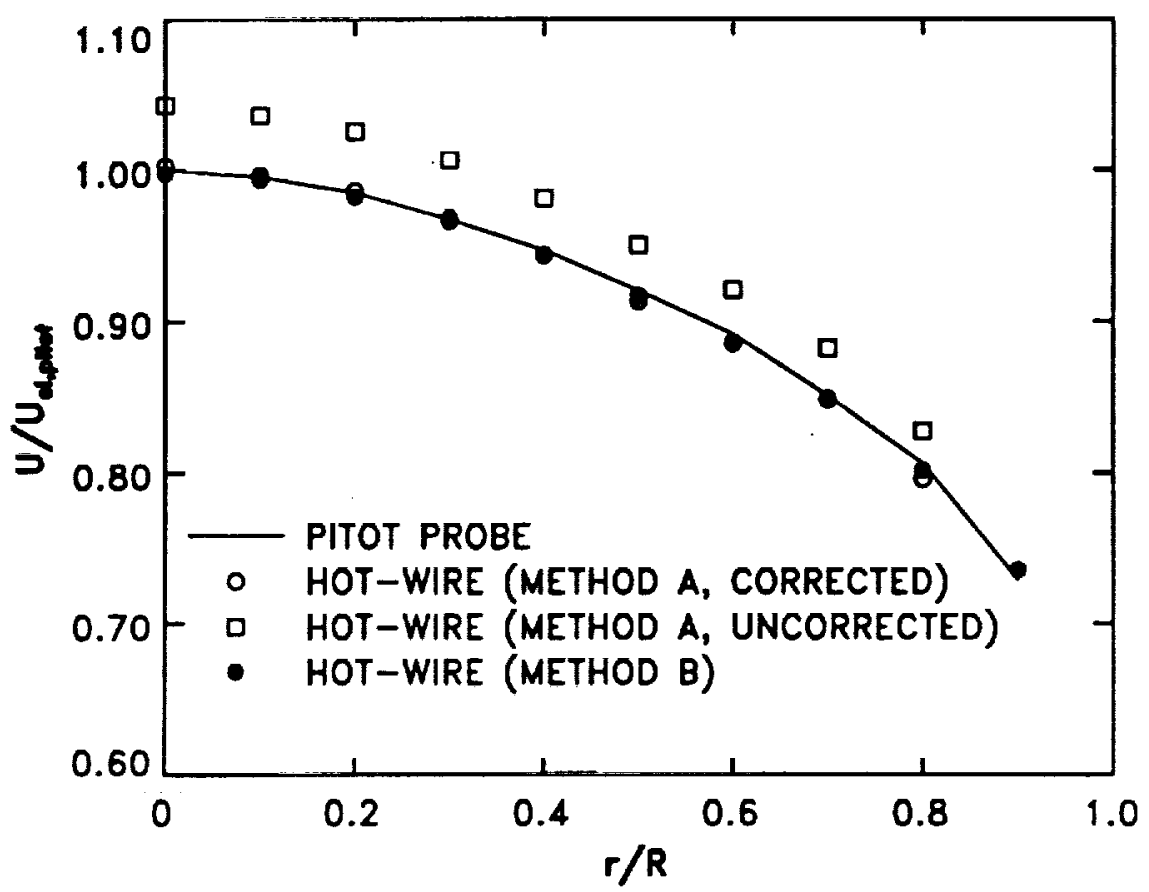

a) Axial velocity profile (from slant-wire only).

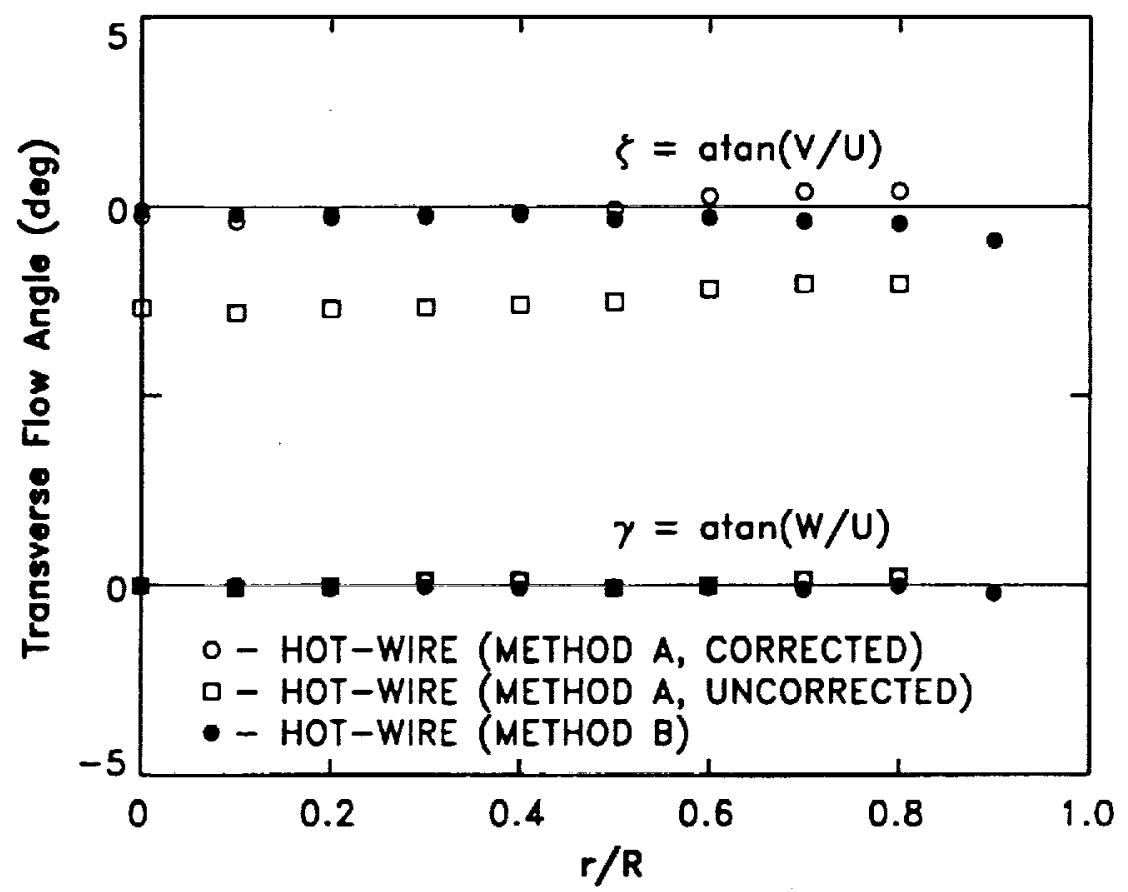

b) Transverse flow angles.

Fig. F.7. Fully-developed mean flow for unskewed conditions. 


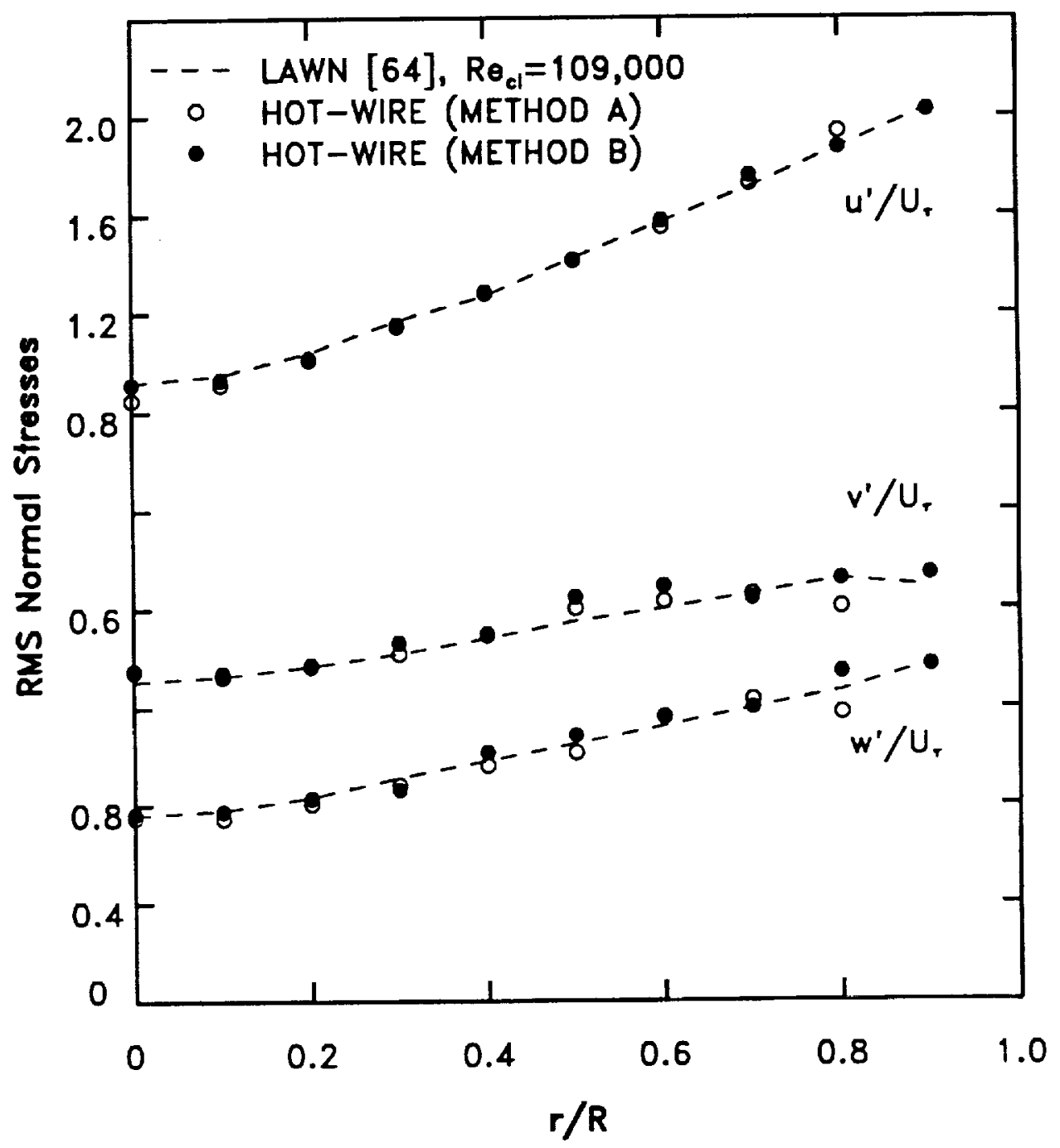

a) Normal stresses.

Fig. F.8. Fully-developed Reynolds stress profiles for unskewed conditions. (Continued ...) 


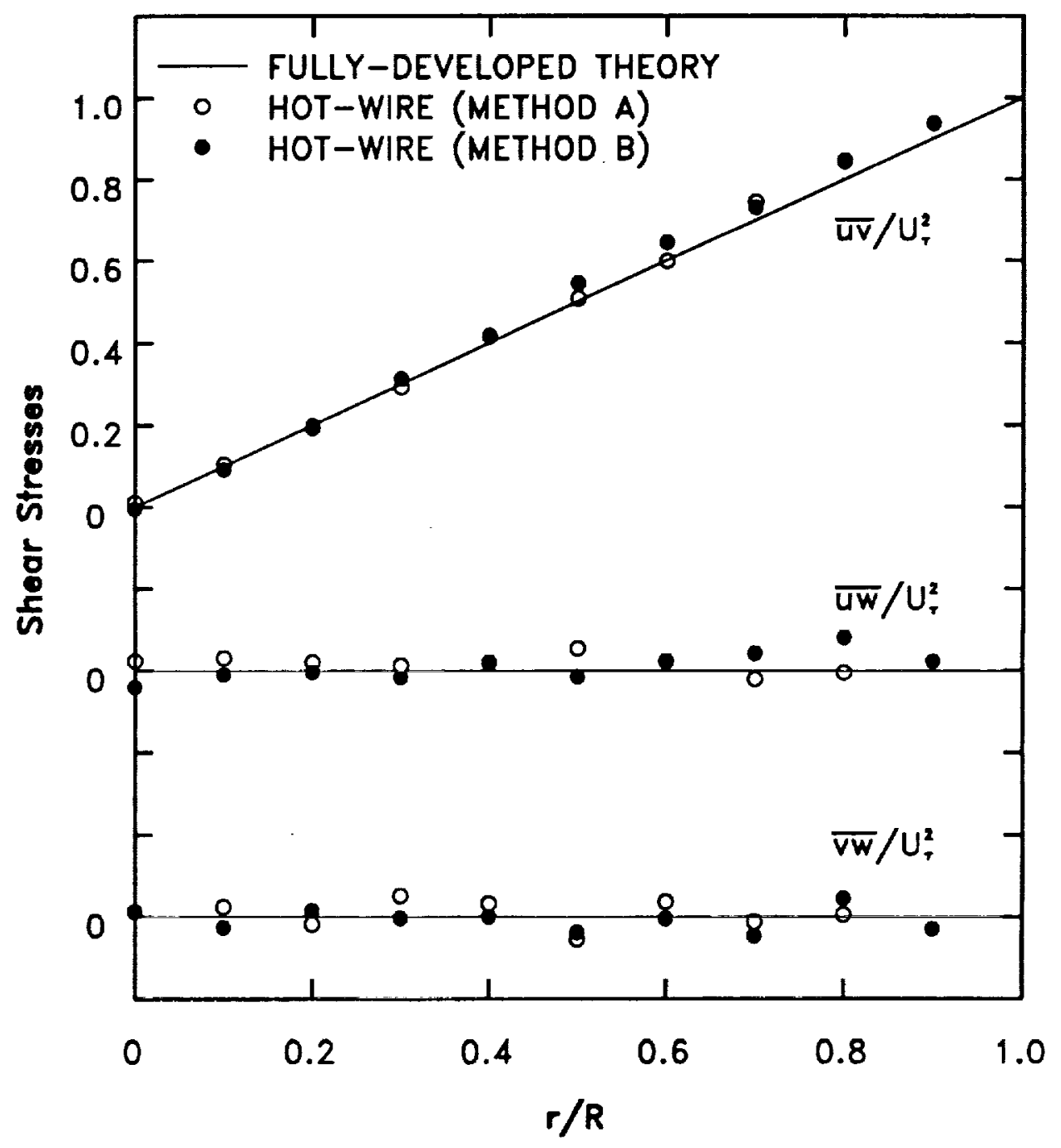

b) Shear stresses.

Fig. F.8. Fully-developed Reynolds stress profiles for unskewed conditions. 


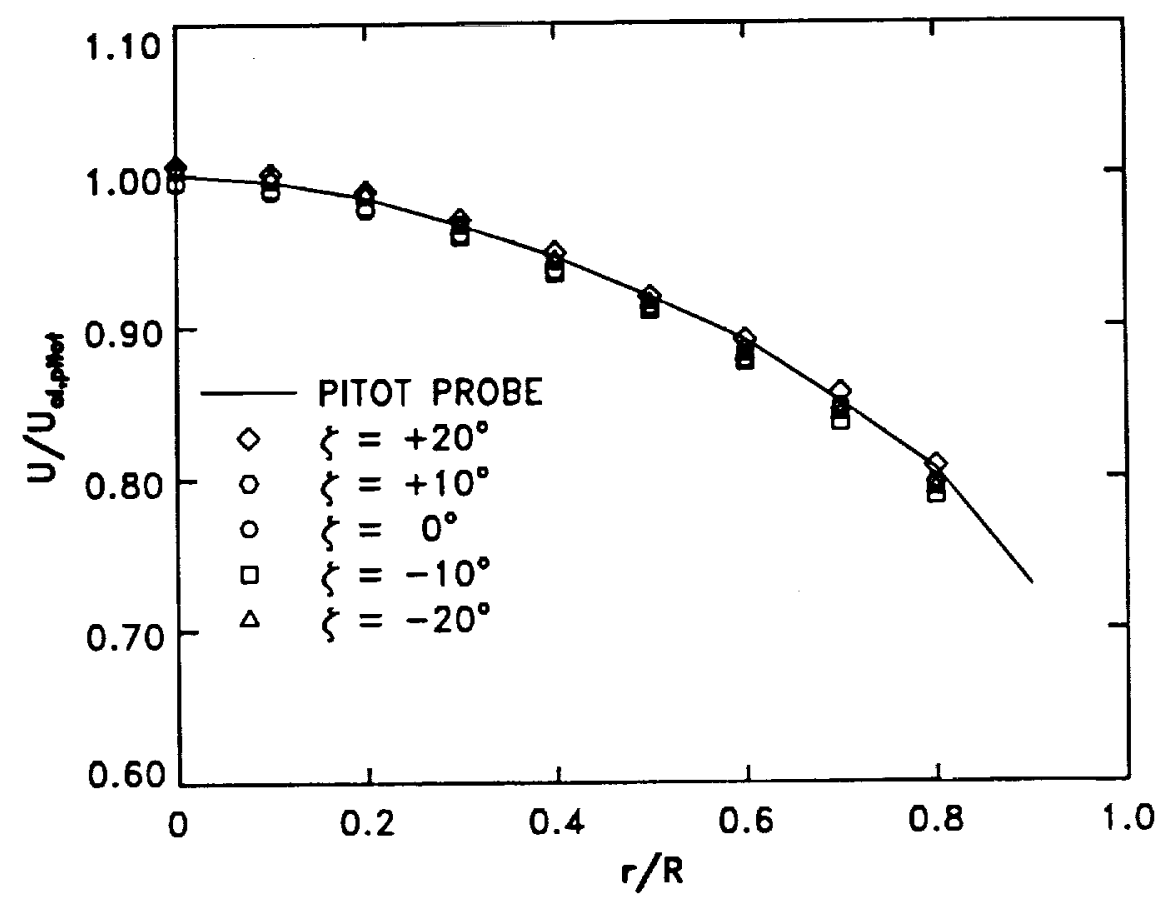

a) Transformed axial velocity profile (from slant-wire only).

Fig. F.9. Fully-developed mean flow for skewed conditions. (Continued ...) 


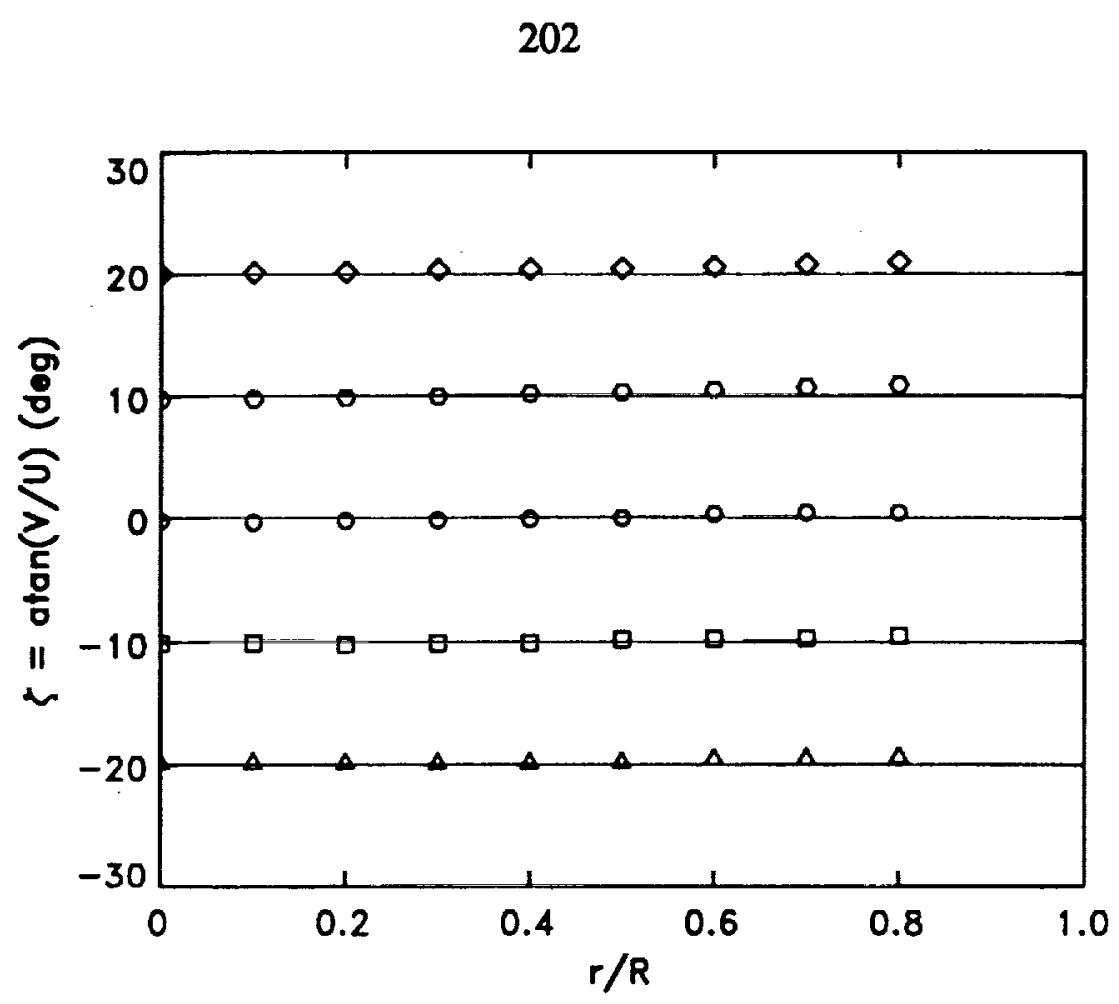

b) Pitch angle.

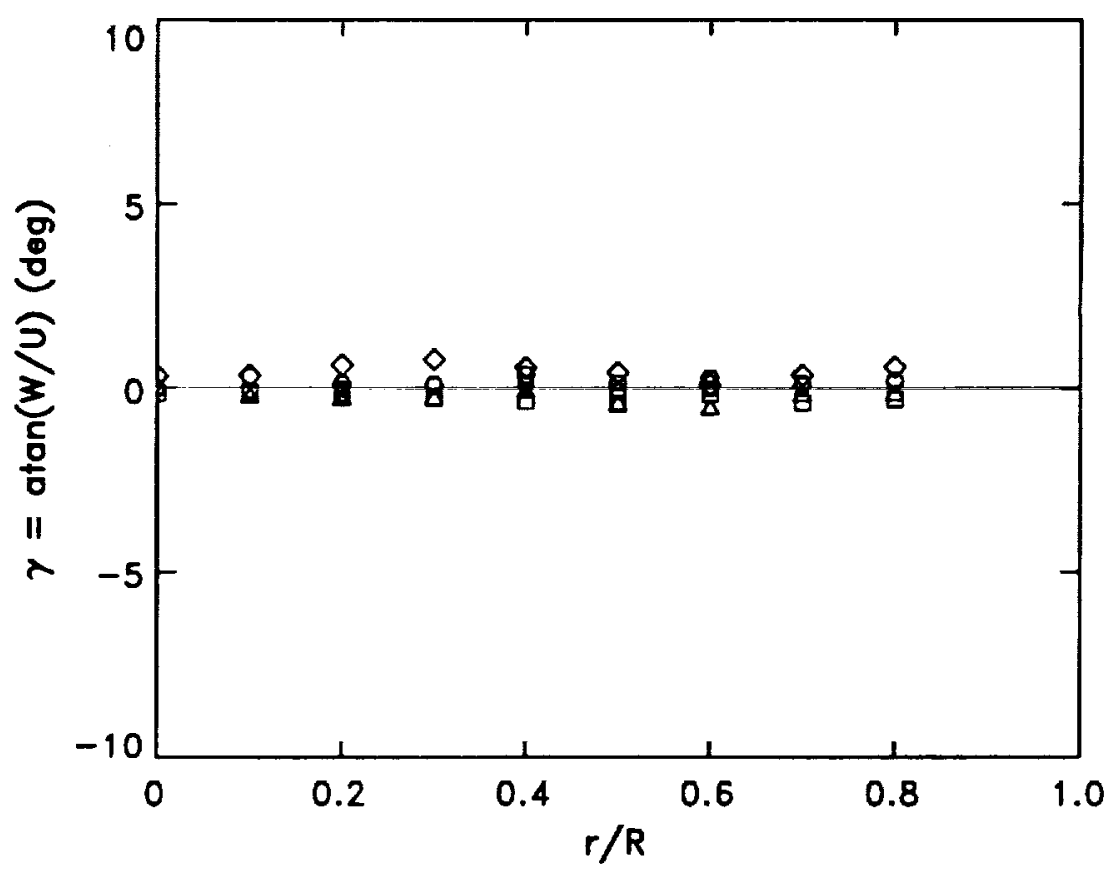

c) Yaw angle.

Fig. F.9. Fully-developed mean flow for skewed conditions. 


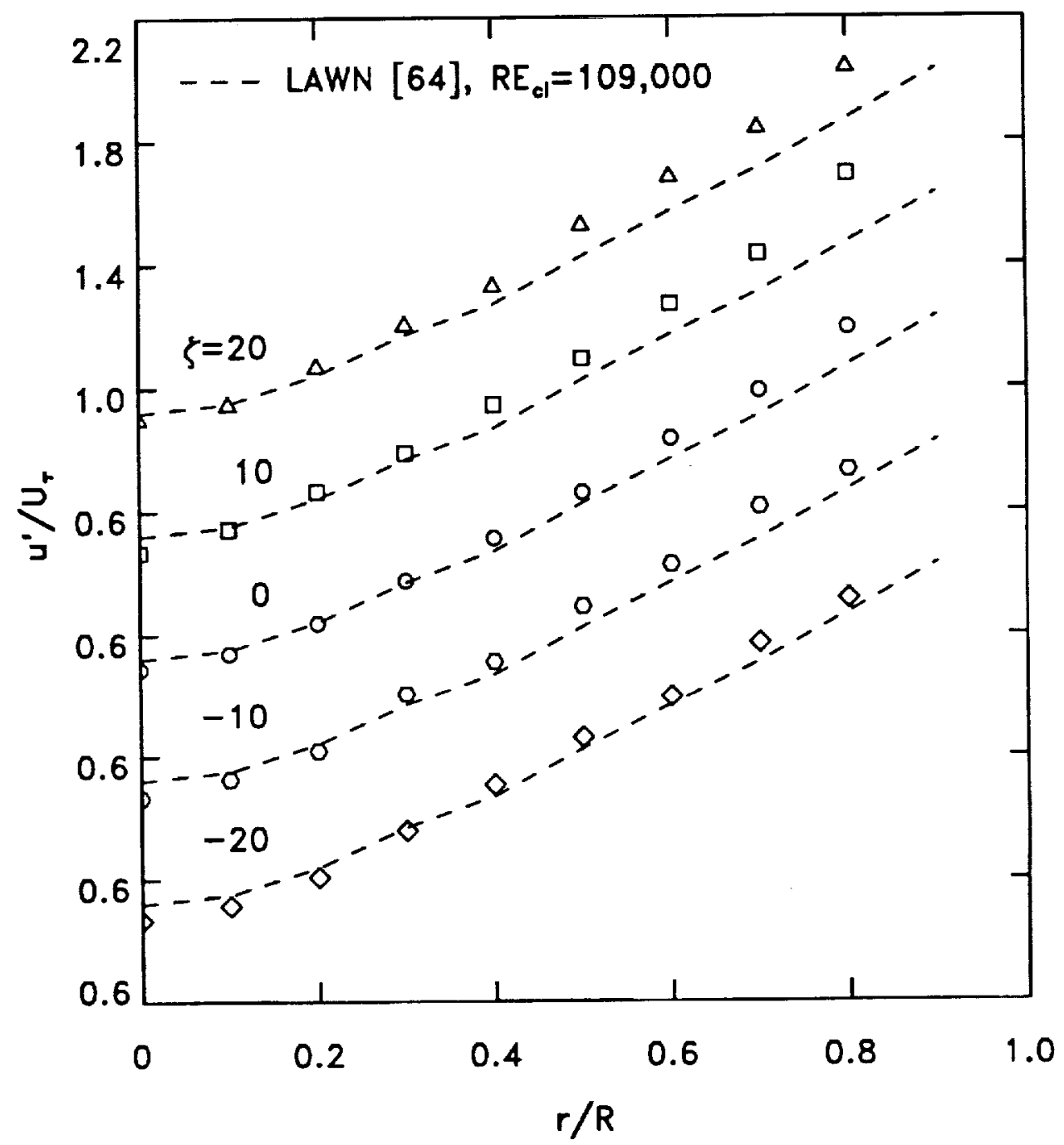

a) $\mathbf{u}^{\prime}$ stress component.

Fig. F.10. Transformed Reynolds stress profiles. (Continued ...) 


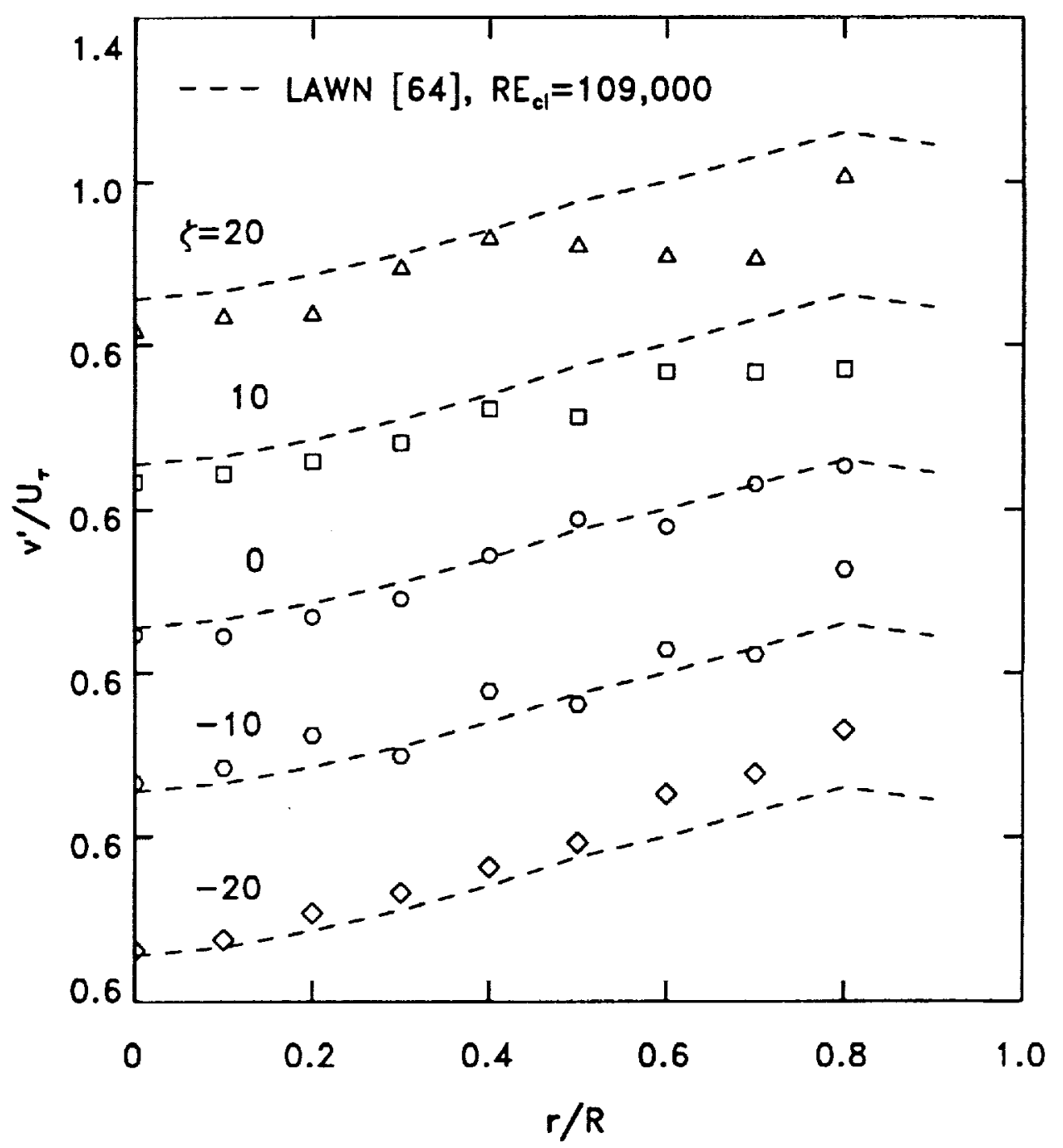

b) $v^{\prime}$ stress component.

Fig. F.10. Transformed Reynolds stress profiles. (Continued ... ) 


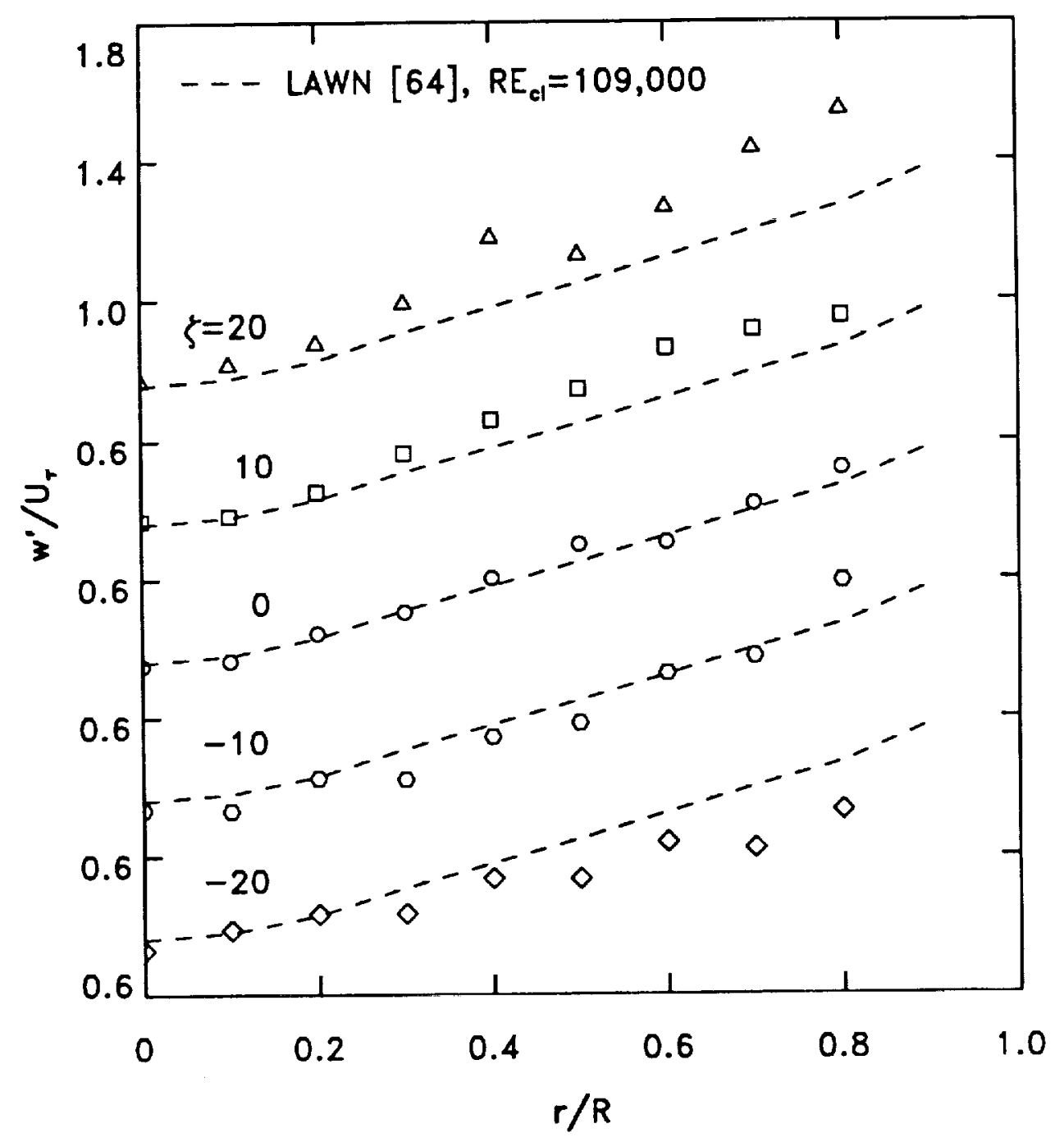

c) $\mathbf{w}^{\prime}$ stress component.

Fig. F.10. Transformed Reynolds stress profiles. (Continued ...) 


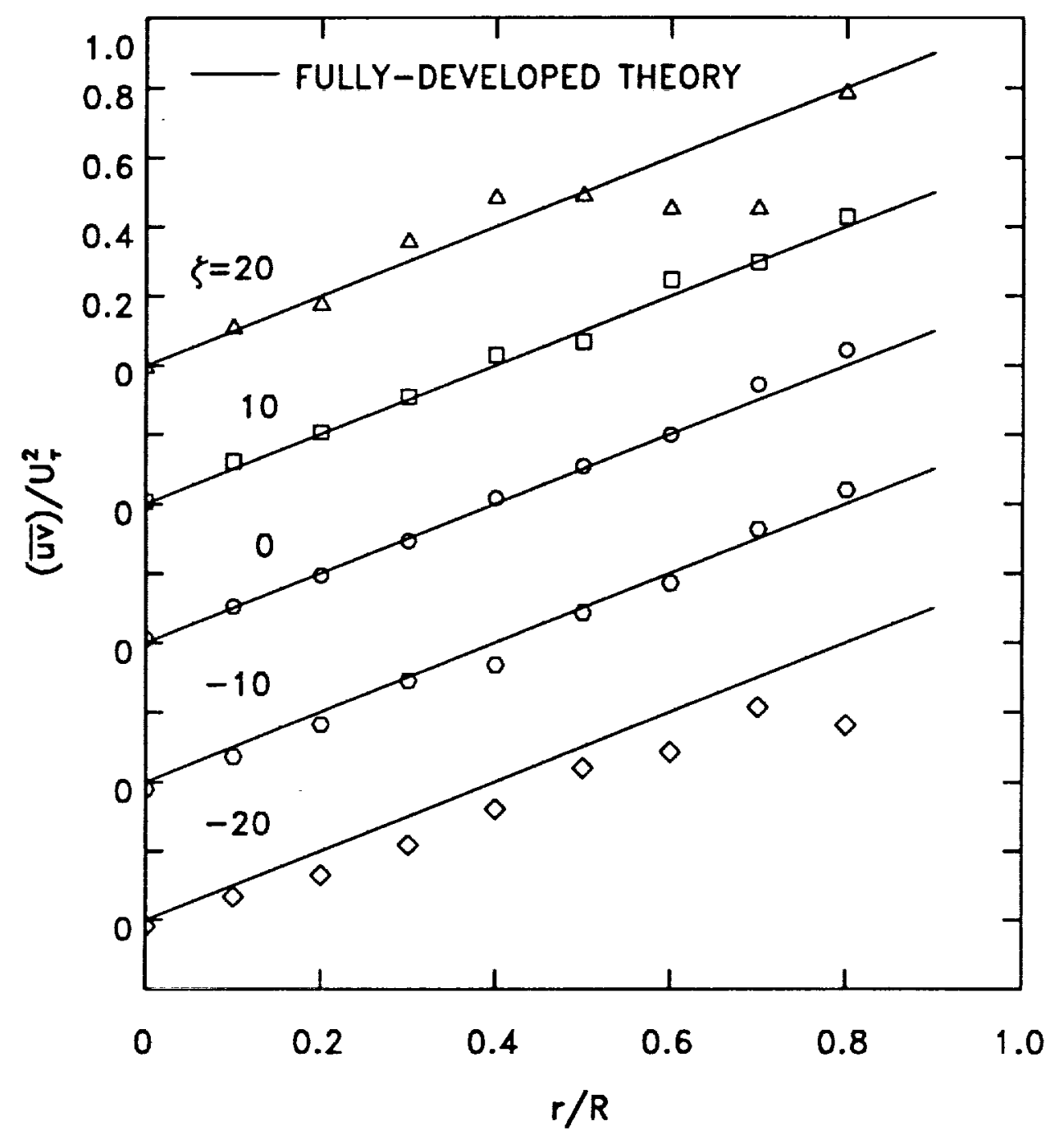

d) $\overline{\text { uv }}$ stress component.

Fig. F.10. Transformed Reynolds stress profiles. (Continued ...) 


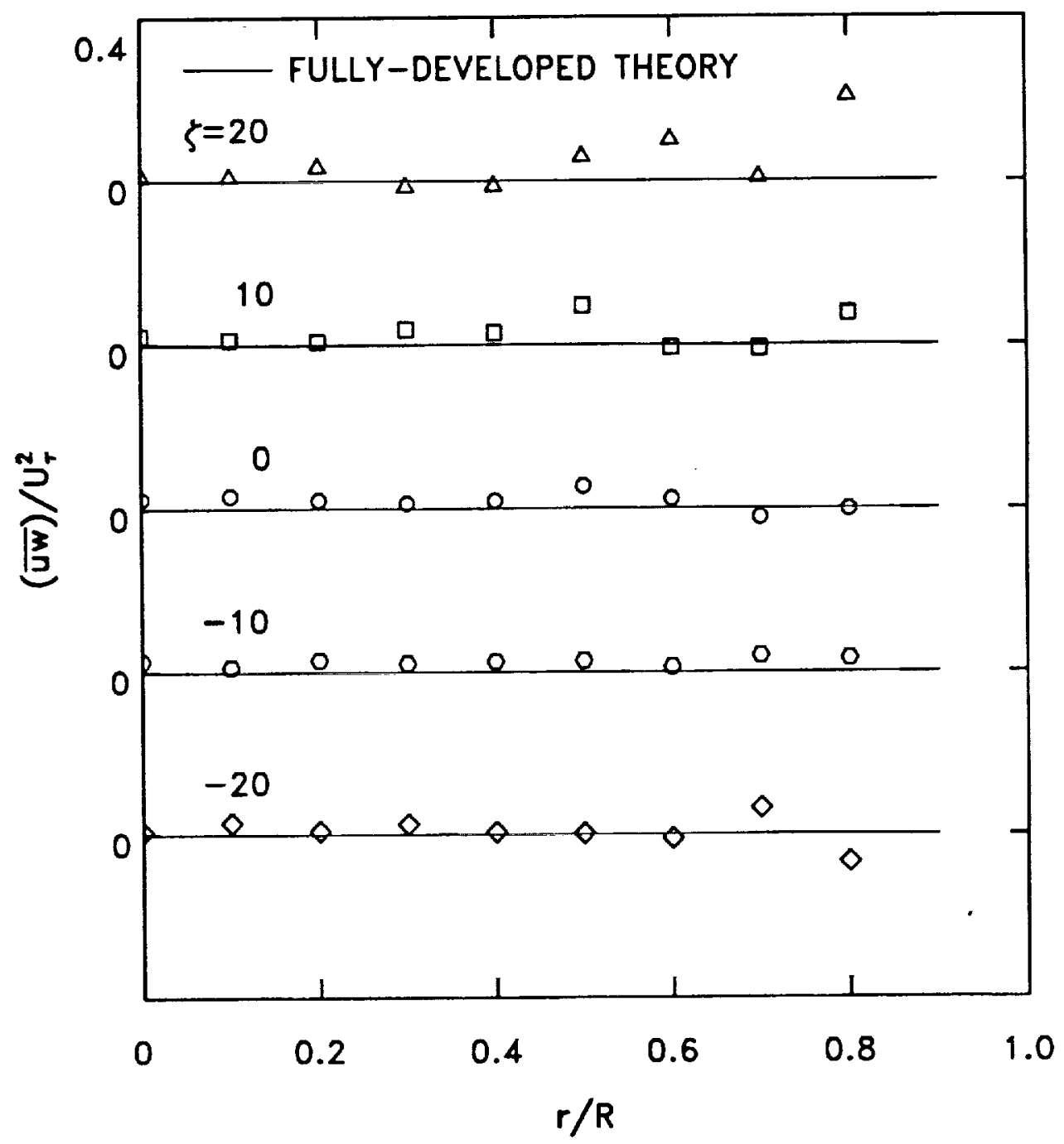

e) $\overline{u w}$ stress component.

Fig. F.10. Transformed Reynolds stress profiles. 

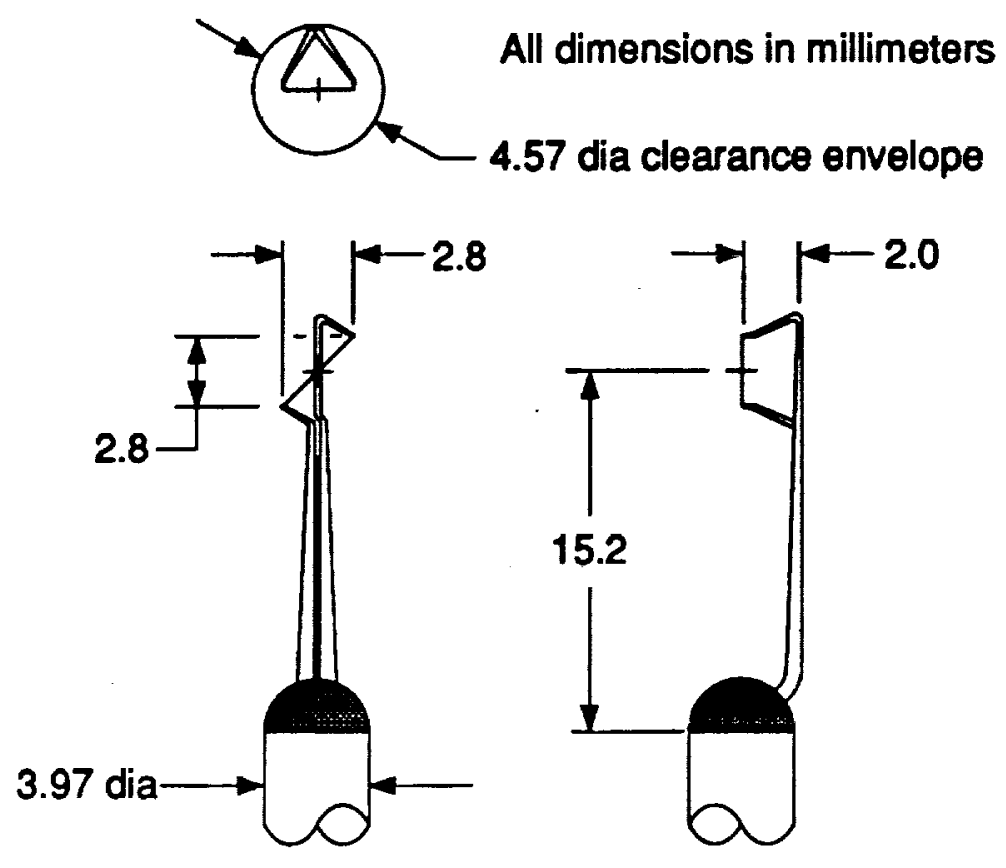

Fig. F.11. Proposed offset prong slant-wire probe.
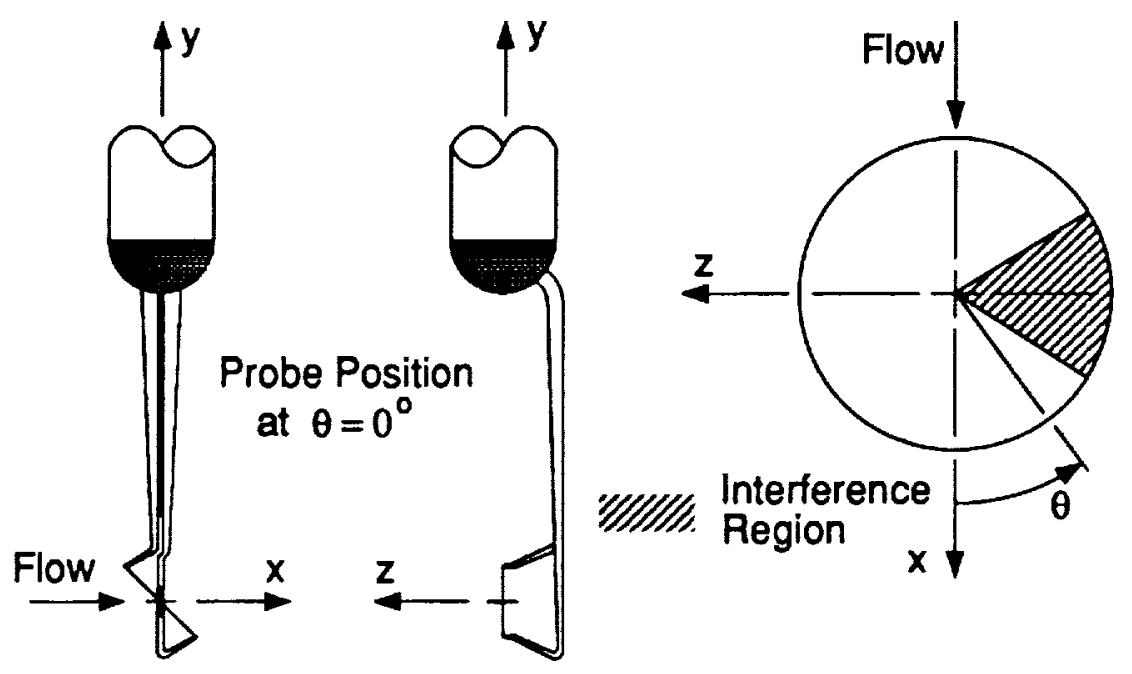

Fig. F.12. Interference region for proposed offset prong slant-wire probe. 


\section{APPENDIX G}

\section{UNCERTAINTY ESTIMATES}

Data presented in this dissertation are deduced from measurements with surface static pressure taps, static pressure probes, total pressure probes and hot-wire anemometry. The uncertainity estimates quoted for a particular variable represent error bounds associated with the peak value observed for that variable.

\section{G.1 Pressure Measurements}

Uncertainties associated with pressure probe design are based on data presented by Chue [41] Static pressure coefficient $\left(C_{p}\right)$ values based on wall tap readings and the static pressure probe are estimated to be accurate to within \pm 0.002 . Skin friction coefficient $\left(C_{f}\right)$ values deduced from Preston tube results at stations 1,5 and 6 are estimated to be accurate to within $\pm 0.0001, \pm 0.0005$ and \pm 0.0002 , respectively. Normalized velocity components $U^{+}=U / U_{\tau}$ based on total pressure probe results at stations 1,5 and 6 are estimated to be accurate to within $\pm 0.2, \pm 0.7$ and \pm 0.3 , respectively.

\section{G.2 Hot-Wire Measurements}

The uncertainties in hot-wire based results were estimated from an error analysis presented by Al-Beirutty [45]. In that analysis, the effects of uncertainties in the slant-wire angle $\alpha$, binormal and tangential cooling coefficients $h_{B N}$ and $k$, normal and slant-wire calibration intercept values $E_{0, n}$ and $E_{0, s}$, slantwire calibration slope $B_{c}$, and the measured normal and slant-wire voltages $\bar{E}_{n}$, $\bar{E}_{s}, \overline{e_{n}^{2}}$ and $\overline{e_{s}^{2}}$ are taken into account.

If the above considerations are applied to the present results, then the uncertainties in hot-wire based values of $U / U_{b}$ and $V_{r} / U_{b}$ are estimated as \pm 0.01 and \pm 0.002 , respectively. Uncertainties in the Reynolds stress components $\overline{u^{2}} / U_{b}^{2}$, $\overline{v^{2}} / U_{b}^{2}, \overline{w^{2}} / U_{b}^{2}, \overline{u v} / U_{b}^{2}, \overline{u w} / U_{b}^{2}$ and $\overline{v w} / U_{b}^{2}$ are estimated as $\pm 0.0001, \pm 0.0002$, $\pm 0.0002, \pm 0.00015, \pm 0.00015$ and \pm 0.0001 , respectively. 


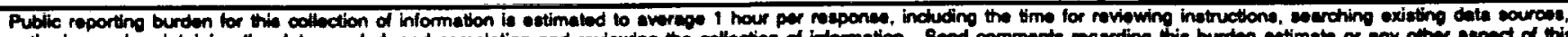

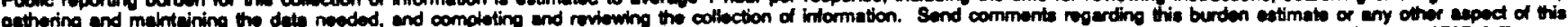
cotherion

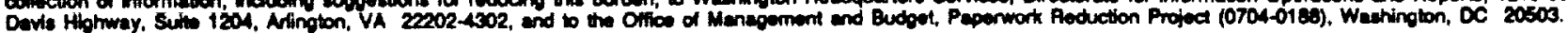

\begin{tabular}{|c|c|c|}
\hline 1. AOENCY USE ONLY (Loavo Blank) & $\begin{array}{l}\text { 2. REPOAT DATE } \\
\text { September } 1991\end{array}$ & 3. REPOAT TYPE \\
\hline
\end{tabular}

4. AUTHOR(8)

David O. Davis
WU-505-62-52

G-NAG3-376

5. FUNDINO MUMBERS

b. PERFormina oroanization REPOAT NUMBER

E-6522

National Aeronautics and Space Administration

Lewis Research Center

Cleveland, Ohio 44135 - 3191

0. SPONSORING/MONTORINO AOENCY NAMES(S) AND ADDRESS(ES)

National Aeronautics and Space Administration

Washington, D.C. 20546-0001

10. SPONSOAINQ/MONTORINO

AOENCY REPORT NUMBER

NASA TM- 105210

11. SUPPLEMENTARY NOTES

Report was submitted as a dissertation in partial fulfillment of the requirements for the degree Doctor of Philosophy to the University of Washington, Seattle, Washington 98195. Responsible person, David O. Davis, (216) 433-8116.

12. DISTAIBUTOON/AVALABILTY BTATEMENT

12b. DISTAIBUTION COOE

Unclassified - Unlimited

Subject Category 02

13. ABSTAACT (Maximum 200 words)

Steady, incompressible, turbulent, swirl-free flow through a circular-to-rectangular transition duct has been studied experimentally. The transition duct has an inlet diameter of $20.43 \mathrm{~cm}$, a length-to-diameter ratio of 1.5, and an exit plane aspect ratio of three. The cross-sectional area remains the same at the exit as at the inlet, but varies through the transition section to a maximum value approximately $15 \%$ above the inlet value. The cross-sectional geometry everywhere along the duct is defined by the equation of a superellipse. Mean and turbulence data were accumulated utilizing pressure and hot-wire instrumentation at five stations along the test section. Data are presented for operating bulk Reynolds numbers of 88,000 and 390,000. Measured quantities include total and static pressure, the three components of the mean velocity vector and the six components of the Reynolds stress tensor. The results show that the curvature of the transition duct walls induces a relatively strong pressure-driven crossflow that produce a contra-rotating vortex pair along the diverging side-walls of the duct. The vortex pair significantly distorts both the mean flow and turbulence field. Local equilibrium conditions at the duct exit, if they exist at all, are confined to a very small region near the wall indicating that care must be taken when using wall functions to predict the flowfield. Analysis of the Reynolds stress tensor at the exit plane shows that streamline curvature and lateral divergence effects distort the non-dimensional turbulence structure parameters. In addition to the transition duct measurements, a bot-wire technique which relies on the sequential use of single rotatable normal and slant-wire probes has been proposed. The technique is applicable for measurement of the total mean velocity vector and the complete Reynolds stress tensor when the primary flow is arbitrarily skewed relative to a plane which lies normal to the probe axis of rotation. Measurement of the mean flow has been verified in fully-developed pipe flow under simulated pitch angles up to $\pm 20^{\circ}$. Measurements of the Reynolds stress tensor has been verified for the unskewed condition. Under skewed conditions, systematic deviations of the stress tensor were observed which are attributed to the geometry of the slant-wire probe. A new slant-wire probe is proposed which is designed to reduce, if not eliminate, the observed deficiencies.

\begin{tabular}{|c|c|c|}
\hline 14. SUBJECT TERMS & & \\
\hline $\begin{array}{l}\text { 17. SECUATV CLASSIFICATION } \\
\text { OF REPORT } \\
\text { Unclassified }\end{array}$ & $\begin{array}{l}\text { 18. SECURTYY CLASSIFICATION } \\
\text { OF THIS PAOE } \\
\text { Unclassified }\end{array}$ & $\begin{array}{l}\text { 19. SECUATY CLASSIFICATIOH } \\
\text { OF ABSTRACT } \\
\text { Unclassified }\end{array}$ \\
\hline
\end{tabular}

